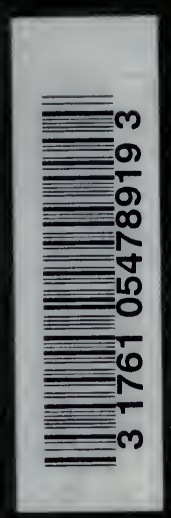


SOLUBILITIES OF BASES AND SALTS IN WATER AT $18^{\circ}$.

\begin{tabular}{|c|c|c|c|c|c|c|c|c|c|c|c|}
\hline & $\mathbf{K}$ & $\mathrm{Na}$ & $\mathrm{Li}$ & $\mathrm{Ag}$ & $\mathrm{TI}$ & $\mathrm{Ba}$ & . $\mathrm{Sr}$ & $\mathrm{Ca}$ & $\mathrm{Mg}$ & $\mathrm{Zn}$ & $\mathrm{Pb}$ \\
\hline $\mathrm{Cl}$ & $\begin{array}{c}32.95 \\
3.9\end{array}$ & $\begin{array}{r}35.86 \\
5.42\end{array}$ & $\begin{array}{l}77.79 \\
13.3\end{array}$ & $\begin{array}{l}0.0_{3} 13 \\
0.0_{5} 9\end{array}$ & $\begin{array}{l}0.3 \\
0.013\end{array}$ & $\begin{array}{c}37.24 \\
1.7\end{array}$ & $\begin{array}{c}51.09 \\
3.0\end{array}$ & $\begin{array}{c}73.19 \\
5.4\end{array}$ & $\begin{array}{c}55.81 \\
5.1\end{array}$ & $\begin{array}{r}203.9 \\
9.2\end{array}$ & $\begin{array}{l}1.49 \\
.0 .05\end{array}$ \\
\hline $\mathrm{Br}$ & $\begin{array}{c}65.86 \\
4.6\end{array}$ & $\begin{array}{c}88.76 \\
6.9\end{array}$ & $\begin{array}{r}168.7 \\
12.6\end{array}$ & $\begin{array}{l}0.041 \\
0.0_{6} 6\end{array}$ & $\begin{array}{l}0.042 \\
0.0215\end{array}$ & $\begin{array}{r}103.6 \\
2.9\end{array}$ & $\begin{array}{c}96.52 \\
3.4\end{array}$ & $\begin{array}{r}143.3 \\
5.2\end{array}$ & $\begin{array}{r}103.1 \\
4.6\end{array}$ & $\begin{array}{r}478.2 \\
9.8\end{array}$ & $\begin{array}{l}0.598 \\
0.02\end{array}$ \\
\hline I & $\begin{array}{r}137.5 \\
-6.0\end{array}$ & $\begin{array}{r}177.9 \\
8.1\end{array}$ & $\begin{array}{r}161.5 \\
8.5\end{array}$ & $\begin{array}{l}0.0635 \\
0.071\end{array}$ & $\begin{array}{l}0.006 \\
0.0317\end{array}$ & $\begin{array}{r}201.4 \\
3.8\end{array}$ & $\begin{array}{r}169.2 \\
3.9\end{array}$ & 200 & $\begin{array}{r}148.2 \\
4.1\end{array}$ & $\begin{array}{l}419 \\
6.9\end{array}$ & $\begin{array}{l}0.08 \\
0.022\end{array}$ \\
\hline $\mathbf{F}$ & $\begin{array}{l}92.56 \\
12.4\end{array}$ & $\begin{array}{l}4.44 \\
1.06\end{array}$ & $\begin{array}{l}0.27 \\
0.11\end{array}$ & $\begin{array}{r}195.4 \\
13.5\end{array}$ & $\begin{array}{l}72.05 \\
3\end{array}$ & $\begin{array}{l}0.16 \\
0.0_{2} 92\end{array}$ & $\begin{array}{l}0.012 \\
0.001\end{array}$ & $\begin{array}{l}0.0016 \\
0.032\end{array}$ & $\begin{array}{l}0.0087 \\
0.0214\end{array}$ & $\begin{array}{l}0.005 \\
0.035\end{array}$ & $\begin{array}{l}0.06 \\
0.002\end{array}$ \\
\hline $\mathrm{NO}_{3}$ & $\begin{array}{c}30.34 \\
2.6\end{array}$ & $\begin{array}{c}83.97 \\
7.4\end{array}$ & $\begin{array}{c}71.43 \\
7.3\end{array}$ & $\begin{array}{r}213.4 \\
8.4\end{array}$ & $\begin{array}{l}8.91 \\
0.35\end{array}$ & $\begin{array}{l}8.74 \\
0.33\end{array}$ & $\begin{array}{c}66.27 \\
2.7\end{array}$ & $\begin{array}{r}121.8 \\
5.2\end{array}$ & $\begin{array}{c}74.31 \\
4.0\end{array}$ & $\begin{array}{r}117.8 \\
4.7\end{array}$ & $\begin{array}{c}51.66 \\
1.4\end{array}$ \\
\hline $\mathrm{ClO}_{3}$ & $\begin{array}{l}6.6 \\
0.52\end{array}$ & $\begin{array}{c}97.16 \\
6.4\end{array}$ & $\begin{array}{r}313.4 \\
15.3\end{array}$ & $\begin{array}{c}12.25 \\
0.6\end{array}$ & $\begin{array}{l}3.69 \\
0.13\end{array}$ & $\begin{array}{c}35.42 \\
1.1\end{array}$ & $\begin{array}{r}174.9 \\
4.6\end{array}$ & $\begin{array}{r}179.3 \\
5.3\end{array}$ & $\begin{array}{r}126.4 \\
4.7\end{array}$ & $\begin{array}{r}183.9 \\
5.3\end{array}$ & $\begin{array}{c}150.6 \\
3.16\end{array}$ \\
\hline $\mathrm{BrO}_{3}$ & $\begin{array}{l}6.38 \\
0.38\end{array}$ & $\begin{array}{c}36.67 \\
2.2\end{array}$ & $\begin{array}{c}152.5 \\
8.20\end{array}$ & $\begin{array}{l}0.59 \\
0.025\end{array}$ & $\begin{array}{l}0.30 \\
0.009\end{array}$ & $\begin{array}{l}0.8 \\
0.02\end{array}$ & $\begin{array}{r}30.0 \\
0.9\end{array}$ & $\begin{array}{c}85.17 \\
2.3\end{array}$ & $\begin{array}{c}42.86 \\
1.5\end{array}$ & $\begin{array}{c}58.43 \\
1.8\end{array}$ & $\begin{array}{l}1.3 \\
0.03\end{array}$ \\
\hline $\mathrm{IO}_{3}$ & $\begin{array}{l}7.62 \\
0.35\end{array}$ & $\begin{array}{l}8.33 \\
0.4\end{array}$ & $\begin{array}{r}80.43 \\
3.84\end{array}$ & $\begin{array}{l}0.004 \\
0.0314\end{array}$ & $\begin{array}{l}0.059 \\
0.0216\end{array}$ & $\begin{array}{l}0.05 \\
0.001\end{array}$ & \begin{tabular}{l|}
0.25 \\
0.0257
\end{tabular} & $\begin{array}{l}0.25 \\
0.007\end{array}$ & $\begin{array}{l}6.87 \\
0.26\end{array}$ & $\begin{array}{l}0.83 \\
0.02\end{array}$ & $\begin{array}{l}0.002 \\
0.043\end{array}$ \\
\hline $\mathrm{OH}$ & $\begin{array}{c}142.9 \\
18\end{array}$ & $\begin{array}{c}116.4 \\
21 .\end{array}$ & $\begin{array}{c}12.04 \\
5.0\end{array}$ & $\begin{array}{l}0.01 \\
0.001\end{array}$ & $\begin{array}{r}40.04 \\
1.76\end{array}$ & $\begin{array}{l}3.7 \\
0.22\end{array}$ & $\begin{array}{l}0.77 \\
0.063\end{array}$ & $\begin{array}{l}0.17 \\
0.02\end{array}$ & $\begin{array}{l}0.001 \\
0.032\end{array}$ & $\begin{array}{l}0.035 \\
0.045\end{array}$ & $\begin{array}{l}0.01 \\
0.034\end{array}$ \\
\hline $\mathrm{SO}_{4}$ & $\begin{array}{r}11.11 \\
.0 .62\end{array}$ & $\begin{array}{r}16.83 \\
1.15\end{array}$ & $\begin{array}{l}35.64 \\
2.8\end{array}$ & $\begin{array}{c}0.55 \\
0.020\end{array}$ & 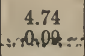 & $\begin{array}{r}0.0323 \\
\because \because 0.0410\end{array}$ & $\begin{array}{c}0.011 \\
\therefore 0.036: .\end{array}$ & $\begin{array}{l}0.20 \\
0.015\end{array}$ & $\begin{array}{c}35.43 \\
2.8\end{array}$ & $\begin{array}{c}53.12 \\
3.1\end{array}$ & $\begin{array}{l}0.0041 \\
0.0313\end{array}$ \\
\hline $\mathrm{CrO}_{4}$ & $\begin{array}{r}63.1 \\
2.7\end{array}$ & $\begin{array}{r}61.21^{\circ} \\
3.30 \\
5 \\
\end{array}$ & $\begin{array}{r}111.6 \\
.6 \% 6\end{array}$ & $\begin{array}{r}0.0025 \\
0.015 \\
4 \\
4\end{array}$ & $\begin{array}{l}0.006 \\
0.051 \\
?\end{array}$ & 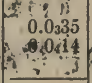 & $\begin{array}{l}0.12 \\
0.006\end{array}$ & $\begin{array}{l}0.4 \\
0.03\end{array}$ & $\begin{array}{r}73.0 \\
4.3\end{array}$ & $\ldots$. & $\begin{array}{l}0.042 \\
0.065\end{array}$ \\
\hline $\mathrm{C}_{2} \mathrm{O}_{4}$ & $\begin{array}{r}30.27 \\
1.6\end{array}$ & \begin{tabular}{l}
$3: 34$ \\
\hdashline-24 \\
$0: 24$
\end{tabular} & 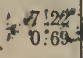 & 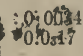 & $1.48 \mathrm{8}$ & $\left\{\begin{array}{l}0.0985 \\
-0.0338\end{array}\right.$ & $\begin{array}{l}0.0046 \\
0.0326\end{array}$ & $\begin{array}{l}0.055 \\
0.0443\end{array}$ & \begin{tabular}{l|}
0.03 \\
0.0027
\end{tabular} & $\begin{array}{l}0.0364 \\
0.044\end{array}$ & $\begin{array}{l}0.0316 \\
0.0554\end{array}$ \\
\hline $\mathrm{CO}_{3}$ & $\begin{array}{r}108.0 \\
5.9\end{array}$ & $\begin{array}{c}19.39 \\
1.8\end{array}$ & $\begin{array}{l}1.3 \\
0.17\end{array}$ & $\begin{array}{l}0.003 \\
0.031\end{array}$ & $\begin{array}{l}4.95 \\
0.10\end{array}$ & $\begin{array}{l}0.0023 \\
0.0311\end{array}$ & $\begin{array}{l}0.0011 \\
0.047\end{array}$ & $\begin{array}{l}0.0013 \\
0.0 \mathrm{~s} 13\end{array}$ & $\begin{array}{l}0.1 \\
0.01\end{array}$ & $\begin{array}{l}0.004 ? \\
0.033 ?\end{array}$ & $\begin{array}{l}0.0_{3} 1 \\
0.043\end{array}$ \\
\hline
\end{tabular}

The upper number in eacth square-gives the number of grams of the anhydrous salt held in solution by 100 c.c. of water. The lower number is the molar solubility, i.e., the number of moles contained in one liter of the saturated solution. The numbers for small solubilities have been abbreviated. Thus $0.0_{6} 4=C .0000004$. For some other solubilities, see page 113 . 
ke) 9

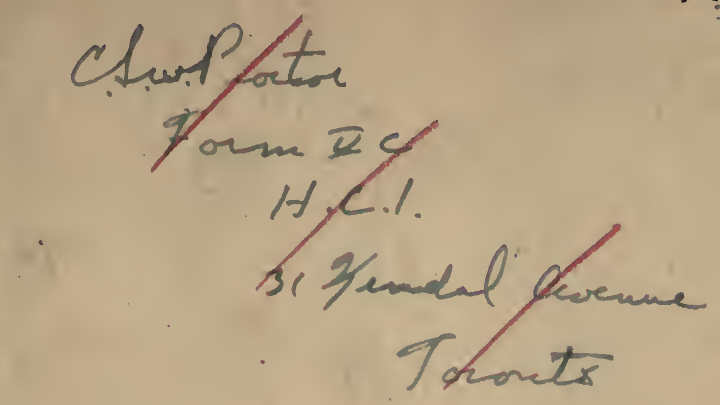







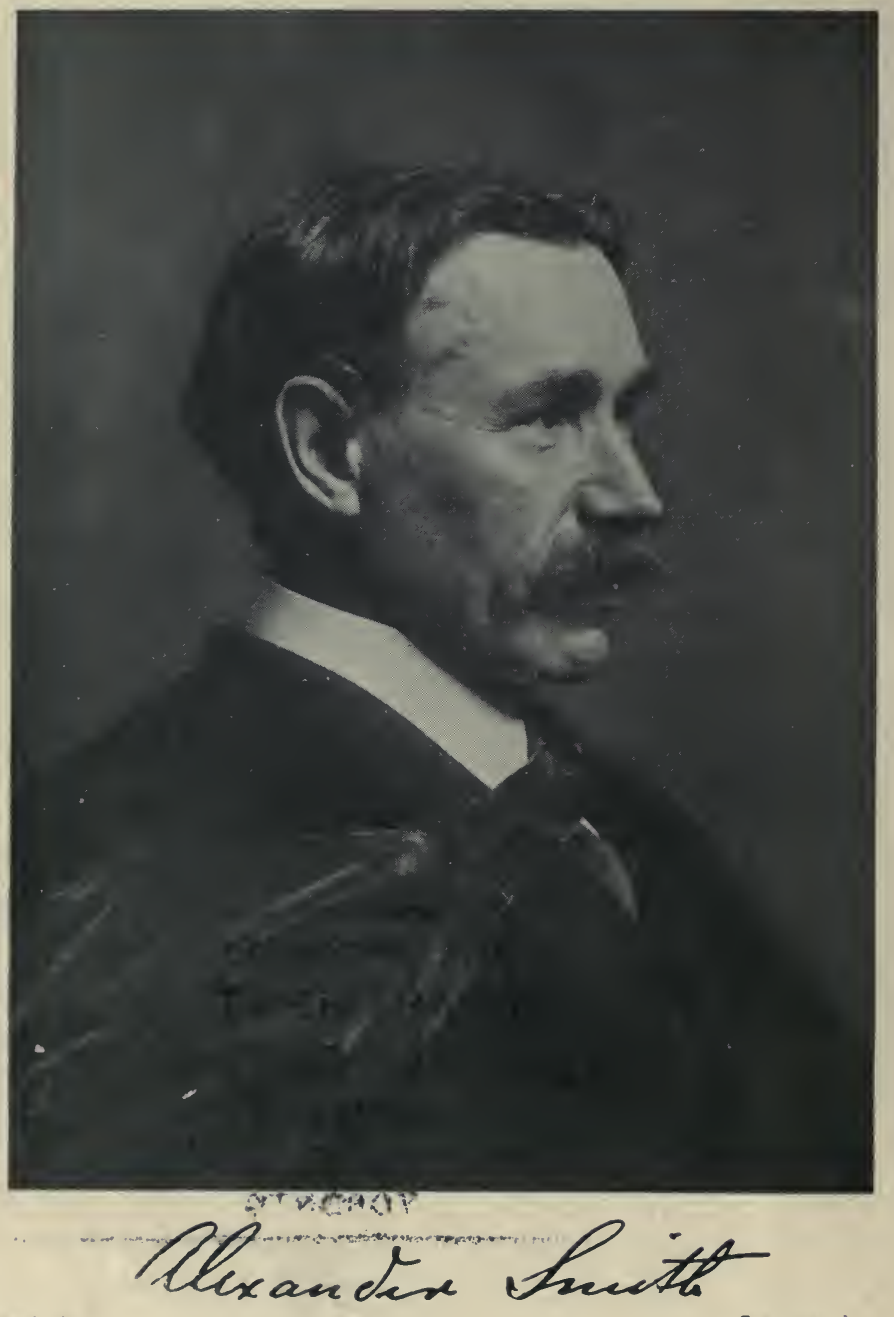




\title{
SMITH'S
}

\section{INTERMEDIATE CHEMISTRY}

\section{REVISED AND REWRITTEN}

\author{
BY \\ JAMES KENDALL \\ Professor of Chemistry and Administrative Head of \\ the Departaent of Chemistry in Washington \\ Square College, New York University \\ AND \\ EDWIN E. SLOSSON \\ Editor of "Science Service," Author of "Creative \\ Chemistry," etc.
}

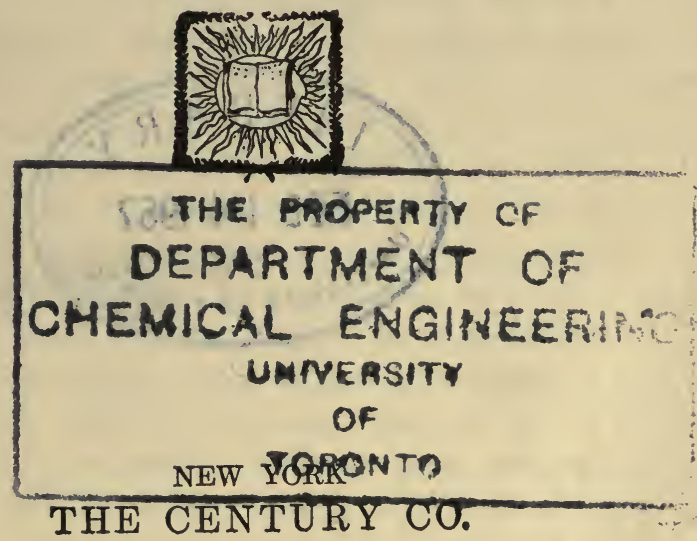


Copyright, 1919, 1922, B Y

THE CENTURY CO 2128

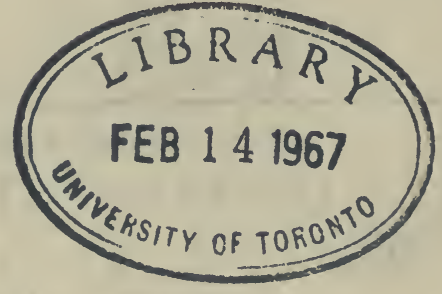

Printed in the U.S.A. 


\section{WHY STUDY CHEMISTRY?}

Chemistry began as a secret science. The early chemists concealed their knowledge - and more often their ignorance - under a cloak of symbols and ciphers of the most mysterious and aweinspiring sort. But now the Black Art has been opened to daylight. The modern chemist is more anxious to tell people what he knows than people are to listen to him. He still uses symbols and has a fondness for long words, but these are designed to reveal, not to conceal.

Still there lingers about chemistry something of the witchery of its antiquity. It has the air of being much harder to understand than it really is. The curious structural formulæ of organic compounds are no more difficult to work out than a Chinese puzzle and quite as much fun.

Chemistry is especially fitted to give training in the scientific method, for it is experimental from the start. Properly taught - or rather properly learned - it inculcates self-reliance and independence of thought. If the pupil will take the teacher's word for the names of things and follow the advice of the book as to what experiments to try, he can find out and think out the most important part of the science for himself. He can work out a system of analysis by testing known, substances in a systematic way and then when he enters upon unknown mixtures he can attack them with the courage of self-confidence. The student of astronomy never gets a chance to handle a star, or even an asteroid. But the substances that the young chemist studies are always weighable, usually tangible, generally visible and frequently smellable. The student of geology never has the opportunity to make a mastodon and all he knows of a volcano or a geyser is the picture of it. But the Freshman chemist makes iii 
oxygen the first week and if he gets through the term without making a volcano or geyser he is lucky.

This is not said to the disparagement of other studies. They all have their peculiar merits which their professors are at liberty to demonstrate. But chemistry has the advantage of most of them in that it points toward the future and instigates to action. The study of the history of man as written in books and of the history of the earth as recorded in rocks gives one a sad sense of irreparableness. What they deal with is dead and gone and all that is left is their lesson which may or may not be applicable to present problems. Meteorology is a discouraging science. "People are always complaining about the weather but nobody ever does anything about it." Astronomy reduces one to a state of impotent awe. It is interesting of course to find out the structure of the solar system, but if you do not like it you cannot change it. You cannot put Saturn in the place of Venus so as to get a brighter evening star with two rings and eight satellites running around it. You cannot even alter the inclination of the axis of the earth so as to give the polar regions a chance to grow bananas. But when you find out the structure of a chemical molecule you can alter it to suit yourself. You can substitute a bromine for a chlorine atom and hook up a carbon chain into a ring.

In this field man is master of his material and the only limitations to his power are his own ignorance and the innate intractability of the elements. Berthelot calls chemistry the most creative of the sciences, because it penetrates most profoundly into the nature of things and deals with the infinitesimal parts of which all substances are composed. The chemist begins by taking things apart (analysis); then he proceeds to put them together again (synthesis). Sometimes he puts them together in a different way and then he gets something quite different, perhaps something that never existed on the earth before. Some 300,000 distinct substances are described in chemical dictionaries and only a small fraction of these are to be found in nature. 
Chemistry is the science of power. All the energy of man and beast and all the energy of machinery, except that furnished by windmills and water wheels, is chemical energy and nearly all of this is derived from two simple and similar chemical reactions, the oxidation of hydrogen to form water and of carbon to form carbon dioxide. Food and fuel plus the oxygen of the air provide the motive force of the modern world. The automobile and the elephant, the airplane and the eagle, the whale and the steamboat, man and his machines would alike be lifeless unless empowered by the oxidation of hydrogen-carbon compounds. Potential chemical energy is peculiarly effective in that it is so compact, portable and permanent. A few bags can contain enough of it to blow up a building or propel a projectile seventyfive miles.

Chemistry has control of life and death. An animal or plant deprived for a time of the proper chemical compounds will starve or if it imbibes a minute amount of the wrong chemical compounds it dies at once. The vital processes of the body are controlled by chemicals, some of which are already known, and a little more or less of them in the blood determines whether we shall be tall or short, bright or dull, handsome or ugly, cheerful or melancholy.

Chemistry is the science of terror and pity. Warfare has been an intermittent branch of applied chemistry ever since the invention of gunpowder, but it was not until 1914-18 that its terrible power of destruction and devastation was realized. But chemistry may heal the wounds it causes, even with medicaments drawn from the same unpromising protean stuff, coal tar, that yields the high explosives and poison gas. Chemistry is the chief weapon of man in his lifelong fight against the bacteria and protozoa that invade his body and ultimately destroy it. It is with chemicals that he combats the insects that eat his crops and infect him with disease. It is chemistry that brings relief to the sleepless and suffering and deprives surgery of its pain. Chemistry 
may, it is true, demolish a building, but it is chemistry that erects it, even the steel-ribbed skyscrapers and mammoth monoliths of concrete.

Chemistry is the democratic science. It bestows upon the poorest what once were the gifts of kings; jewels and brightdyed garments, sugar and scents, vessels of porcelain and metal, foreign fruits and out-of-season foods, books and pictures. Chemistry carries music into farmhouse and tenement on disks of resin and reveals to multitudes from the celluloid film scenes from a distance and past events; thus short-circuiting space and time. Chemistry has brought light into dark dwellings by means of windows and lamps. It supplies food to the hungry, for with nitrogen extracted from the air and phosphorus from the rocks it makes two blades of corn grow where one grew before.

Chemistry is the joyous science. It contributes most to the pleasures of life. Every scent and every savor is due to chemistry. The tints that delight the eye in flower or fruit, in gem or painting, the greens and browns of the forest leaves, the reds and yellows of the brick-built town, the black of print, the dyes of cloth, the blush on the maiden's cheek - whether it be natural or artificial - all colors are chemical. We may not gratify our ears with music more than once a week, but we satisfy our chemical senses three times a day and chemical colors delight our eyes all the hours they are open. The students should realize that chemistry is not exclusively concerned with the stuff in the bottles behind the prescription counter, but has just as much to say about the soda-water side to which they are quite naturally more attracted. They should understand that chemistry includes not merely the process of cooking but the process of digestion. This explains why we have introduced some unconventional topics and experiments in the following pages.

Chemistry is a practical science. None touches everyday life at more points except its sister science, physics, between which and chemistry no clear boundary can be drawn. None has more 
avenues for profitable employment. Some studies can be made a profession only by teaching them. But the qualified chemist has two strings to his bow. If he does not like teaching, he may leave the campus for the industrial field. He may find employment in the big establishments devoted to the reduction of ores, to steel or other manufactures or to the production of foods, fertilizers, dyes, drugs, soaps, gas, rubber, oil, cements or explosives. Here he may be engaged in routine analysis of the raw material and finished products. Or he may be put in control of the factory processes. Or he may be engaged in research to prevent waste, to utilize by-products, to invent new compounds or to contrive new uses. In any case he will be near to the heart of the industry and if he has inventive genius or managerial ability he may rise high. In some fields of chemistry women have almost as much of a chance as men.

On account of its varied opportunities and its expanding field chemistry is drawing an increasing number of college graduates. Many more take the degree of Doctor of Philosophy in chemistry than in any other branch. The American Chemical Society with 15,500 members is the largest special scientific body in the world. When the Government took a census of chemists to see how many were available for war service, 25,000 names were registered, and this did not include them all. No other science is growing so fast through original research. In the files of the National Research Council more than 2100 living Americans are recorded as having made contributions to chemistry. Compared with this, the research men in botany number 1400, in zoölogy 1255 , in geology 750 , and in mathematics 600 , in psychology 450 and in astronomy 250.

But it is not sufficient that the ranks of research chemists and technologists be kept filled. Chemistry has an interest and a usefulness for everyone. What would be the value of an art gallery if nobody entered it but artists? And what would become of the book business if nobody read but authors? Chemistry 
viii

is of value to those who never intend to become professional chemists because it gives one an understanding of household arts and modern manufactures, of the principles of agriculture and bodily processes, but more because it opens the eyes of the mind to the molecular meaning of the marvelous metamorphoses of the visible world.

\author{
E. E. S.
}




\section{CONTENTS}

CHAPTER

PAGE

I. Substances and Properties................... 1

II. Chemical Change and the Methods of Studying It ... 12

III. Air and Oxygen ........................ 26

IV. The Measurement of Gases. The Molecular HyPOTHESIS............................. 44

V. Hydrogen............................ 50

VI. Water............................. 58

VII. Chemical Units of Weight. Formdle........... 72

ViII. Application of the Molecular Hypothesis in ChemISTRY............................... 82

IX. Making of Formula and Equations............. 98

X. Solution............................... 108

XI. Hydrochloric Acid Calculations............ 125

XII. Chlorine. Calculations..................... 139

XIII. Energy and Chemical Change................. 153

XIV. Sodium and Sodium Hydroxide................ 164

XV. Acids, Bases, and Salts.................. 170

XVI. Ionization ......................... 181

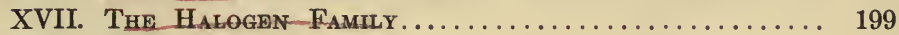

XVIII. Valence........................... 211

XIX. Oxidizing Substances..................... 219

XX. Chemical Equilibrium.................... 230

XXI. SUlpHUR-AND Hydrogen-SULPHIDE............. 248

XXII. Oxides AND Oxygen AcIDS Of Sulphur.......... 257

XXIII. The Periodic System..................... 273

XXIV. Nitrogen. The Atmosphere .................. 286

XXV. АммоліA................................ 299

XXVI. Nitric Acid.............................. 308

XXVII. Phosphorus, Arsenic, Antimony, Bismuth.......... 319

XXVIII. Carbon and the Oxides of Carbon............. 328

XXIX. The Hydrocarbons and their Derivatives. Flame.. 343 ix 
CHAPTER

XXX. Silicon, Boron.......................... 359

XXXI. Compounds of Sodium and Potassidm ........... 365

XXXiI. The Recognition of Substances, I. - A Review of the Non-Metallic Elements.................. 374

XXXIII. Calcium and Its Compounds................. 383

XXXIV. Plant Life. Celldlose, Starch and Sugar......... 395

XXXV. Plant Life. Osmosis. Fertilizers............. 405

XXXVI. Plant Products. Fermentation and Fuels......... 417

XXXVII. Animal Life and Animal Products. Foods........ 428

XXXVIII. Magnesium and Zinc. Ionic EqUilibria......... 446

XXXIX. Aldminidm ............................. 466

XL. Synthetic Organic Products................ 474

XLI. Iron, Nickel, Cobalt................... 486

XLII. Lead and Tin. ......................... 502

XLIII. Copper and Mercury ................... 510

Xliv. Silver, Gold, Platinum.................... 519

XlV. Manganese and Chromidm................. 527

Xlvi. The Recognition of Substances, II. - A Review of the Metallic Elements..................... 536

XLVII. Radidm. Atomic Energy and Atomic Structure..... 542

Appendix.............................. 557

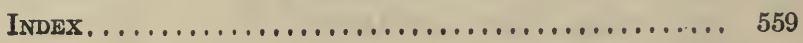




\section{LIST OF ILLUSTRATIONS}

PAGE

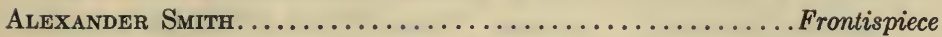

Alexander Smith; B. Sc., Edinburgh, 1886; LL. D., 1919; Ph. D., Munich, 1889. Fellow of the Royal Society of Edinburgh; Member of the National Academy of Sciences; recently Head of the Department of Chemistry, Columbia University, New York City.

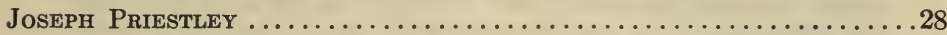

Joseph Priestley (1733-1804) was a nonconformist minister of Birmingham, England, but devoted his leisure and his means to chemical research, the results of which were published in his six volumes on "Experiments and Observations on Different Kinds of Air." He was the first to prepare pure oxygen, which he called "dephlogisticated air." He also made nitrous oxide, nitric oxide, ammonia, hydrochloric acid, sulphur dioxide, and silicon fluoride. $\mathrm{He}$ found that water could be made to absorb carbon dioxide under pressure, thus laying the foundation of the "soda-water" industry. On account of his sympathy with the French Revolution and his heterodox theological views a mob burned his home and laboratory, July 14, 1791. Priestley narrowly escaped with his life and went to America, where he settled at Northumberland, Pennsylvania. Here a group of chemists gathered on August 1, 1874, to celebrate the centenary of the discovery of oxygen, and founded the American Chemical Society, now the largest scientific association in the world.

Antoine LaURent Lavoisier .............................2

Antoine Laurent Lavoisier (1743-1794) is called "the father of modern chemistry" because he placed the science on the solid basis of quantitative experiments and devised the system of naming compounds by their composition. He proved that the air contained two gases; one which was inert and another which supported combustion and united with metals. He called the former gas "azote" because it would not support life and the latter "oxygen" because it formed acids. He was guillotined during the Reign of Terror. The Commission on Weights and Measures petitioned that he might be allowed to complete his work on the metric system, but Coffinhal, vice-president of the revolutionary tribunal, refused, saying: "The republic has no need for savants." 
Svante Arrhenius .................................. 182

Arrhenius put forward his theory of ionization while still a student at Upsala. His own description of its reception is worth repeating: "I came to my professor, Cleve, whom I admire very much, and I said: 'I have a new theory of electrical conductivity as a cause of chemical reactions.' He said: 'That is very interesting,' and then said 'Good-bye.' He explained to me later, when he had to pronounce the reason for my receiving the Nobel Prize for that work, that he knew very well that there are so many different theories formed, and that they are almost all certain to be wrong, for after a short time they disappear; and therefore, by using the statistical manner of forming his ideas, he concluded that my theory also would not exist very long."

It scarcely needs to be added here that the theory is still very much alive!

The Electric Current is Passed through Salt Water in These Nelson Cells to Obtain Caustic Soda and Chlorine Gas.....................................

In the U. S. Government Chlorine Plant at Edgewood Arsenal during the Great War there were eight cell rooms similar to that shown in the picture, with a total capacity of 100 tons chlorine per day. Each cell room consists of six circuits -74 cells per circuit, or a total of 444 cells per room.

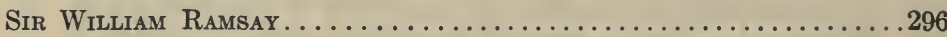

Sir William Ramsay (1852-1916) will always be famous for his work on the inert gases of the atmosphere and for his discoveries in the field of radioactivity. He first became interested in chemistry in a rather unusual way. While a boy at school, he broke his leg in a football game, and was kept on a couch for several weeks. To kill time, he read Graham's Chemistry, hoping to find out how to make fireworks. For the next few years his bedroom was full of bottles and test-tubes, and often full of strange smells and startling noises.

The First Helium-Filled Dirigible After Its Landing at WashINGTON

When Ramsay was presented with the Longstaff Medal for his work on the inert gases, the President of the London Chemical Society remarked: "If I may say a word of disparagement, it is that these elements are hardly worthy of the position in which they are placed. If other elements were of the same unsociable character, Chemistry would not exist." 
This very unsociability of helium, however, renders it the ideal gas for balloons and avoids all the risk of fire or explosion attendant upon the use of hydrogen. Helium exists in small quantity in many natural gases (see p. 345), and is obtained pure by liquefying out the other components.

\section{Chemical Smoke-Screens Against Submarines. A Vessel Using}

Silicon Tetrachloride. . . . . . . . . . . . . . . . . 360

The silicon tetrachloride is contained in a cylinder fixed on the deck at the stern of the ship, and is forced out into a long funnel, in the form of a fine spray, by the pressure of liquid carbon dioxide. Ammonia gas issues from a second cylinder, and rapid mixture is effected in the presence of excess of moist air by means of an electric fan placed at one end of the funnel. The current of air produced drives the smoke out at the other end of the funnel.

\section{Chemical Smoke-Screens Against Submarines. A Vessel Using}

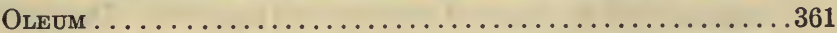

Oleum (fuming sulphuric acid) was also employed to conceal ships against submarines during the Great War. A finely-divided spray of oleum was injected into the smoke-stacks of the vessel and carried by the hot gases issuing therefrom into the atmosphere in the form of vapor. In contact with the cool moist air over the ocean, this vapor condensed to give tiny droplets of sulphuric acid, which hung over the surface as a very persistent mist (compare pp. 263-4), extremely difficult to distinguish from a natural fog.

Koppers Benzol Recovery Plant, River Furnace Co., CleveLAND, ОнIо. ............................424

The recovery of valuable hydrocarbons of the aromatic series (benzene, toluene, naphthalene, etc.) from coal gas is a feature of every modern coke and gas plant. The bulk of the peace-time demand for "benzols" - as the light oils extracted by washing the gas are called in industry - is for employment as motor fuels. From one ton of coal approximately 3 gallons of "benzol" is recovered, giving from 20 to 30 per cent more power and mileage than high-grade gasoline, and causing less carbon trouble.

\section{Oil Shale Cliff, Utah.}

Enormous deposits of oil shale (see p. 345) have recently been opened up in the United States, notably in Colorado, Utah, and Nevada. Though at first sight it would appear that the cost of producing oil from shale would prohibit competition with well petroleum, yet the value of the by-products offsets this disadvantage 
to a large extent. In any case, in view of the apprehended exhaustion of the world's petroleum fields in the near future, these shale deposits constitute a most important national asset.

Photomicrographs Showing the Structure of Steel Made By Professor E. G. Mahin of Purdue University ......... 490

1. Cold-worked steel showing ferrite and sorbite (enlarged 500 times).

2. Steel showing pearlite crystals (enlarged 500 times).

3. Structure characteristic of air-cooled steel (enlarged 50 times).

4. The triangular structure characteristic of cast steel showing ferrite and pearlite (enlarged 50 times).

Repairing the Broken Stern-Post of the U. S. S. "Northern PAcific": the Biggest Marine Weld in the World........491

On the right the fractured stern-post is shown. On the left it is being mended by means of thermite (see page 468). Two crucibles each containing 700 pounds of the thermite mixture are seen on the sides of the vessel. From the bottom of these the melted steel flowed down to fill the fracture.

Madame Curie in Her Laboratory.............................. 542

Marie Sklodovska, a Polish refugee stranded in Paris, was first engaged in the physical science department of the Sorbonne to wash bottles and prepare the furnace. Professor Lippmann, the pioneer in color photography, promoted her to setting up apparatus, and put her to do work with Pierre Curie, one of his assistants. In 1895 she became Madame Curie.

In 1903 the results of Madame Curie's work on radium were presented to the faculty as a thesis for the doctor's degree. The thesis, unlike that of Arrhenius, was favorably received, and shortly afterwards the Nobel Prize in Physics was divided between the Curies and Becquerel, whose previous work on uranium had suggested the research.

In 1911 Madame Curie was awarded the Nobel Prize in Chemistry.

Fog-Tracks from RadiUm. . . . . . . . . . . . . . . . . . . .

The positively-charged helium atoms (alpha-particles), thrown off from radium, in passing through the air ionize the molecules with which they collide, and these ionized molecules have the same power that dust possesses (see p. 293) of affording nuclei on which moisture may condense. Hence, when a particle of a radium compound is supported in a flask containing air saturated vith 
moisture, and the air is suddenly cooled by expansion, the paths of the particles become lines of fog. With powerful illumination, the fog-tracks can be photographed and the lengths of the paths can be measured.

The negatively-charged electrons (beta-particles) give fog-tracks which are much fainter and extremely tangled. 



\section{SMITH'S \\ INTERMEDIATE CHEMISTRY}

\section{CHAPTER I \\ SUBSTANCES AND PROPERTIES}

WHEN exact information in regard to any sort of material is required, we hand the material to a chemist. To learn something about the nature of chemistry, let us watch the chemist at work on a typical problem.

Properties.- Suppose that the material is a piece of cloth, and we desire to know whether it is all wool, or partly cotton. The chemist places a piece of the cloth in a test-tube (Fig. 1), and pours in an amount of lye sufficient to cover it. Lye is a solution in water of a white solid, named by chemists, commonly, "caustic soda" and, more formally, sodium hydroxide. The contents of the test-tube are then heated over a flame and are kept at the boiling point for ten minutes. If the cloth dissolves entirely, leaving a liquid, clear like water, then it was composed of nothing but wool. The chemist draws this conclusion because wool, although not affected by the boiling water, has the property of turning into a soluble

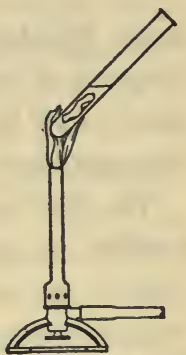

FIG. 1. substance when caustic soda is heated with it. If, on the other hand, the piece of cloth becomes thinner, obviously losing a part of its material, but leaving a part undissolved, then it contained cotton. This conclusion depends on the fact that cotton has the property of not being dissolved by caustic soda solution. 
The same conclusion could have been reached in other ways. For example, some threads could have been taken from the edges of the sample and placed under a microscope. In this case, they would have been seen to be made up of long, hollow fibers or tubes (Fig. 2). But the cotton fibers (A) are smooth on the surface, while the woolen fibers (B) are covered with scales. By this difference in properties, the presence of both kinds, or of only one of the kinds, could quickly be found out. Still again, the chemist knows that wool will "take" almost any dye, while

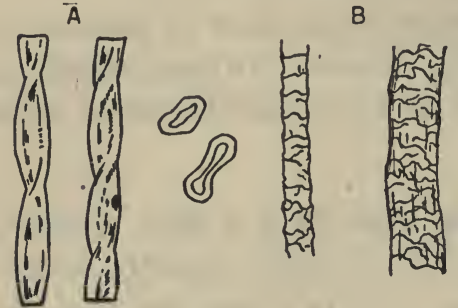

FIG. 2 cotton remains uncolored by the greater number of dyes. He could, therefore, boil a piece of the cloth with a solution of a soluble dye - such as red ink (eosin) - for a few minutes, and then wash the sample thoroughly in clean water. On examining the cloth with a microscope he could then observe whether any fibers were still white. Here wool has the property of uniting with the dye, while cotton has not. The last plan could be used only with white goods, or goods not already strongly dyed.

The first method is the one which the chemist would probably employ in practice, because by its means he can ascertain and report, not only the presence of cotton, but the proportion of cotton present. To do this, he weighs the dry piece of cloth before placing it in the test-tube. Then, after the boiling with caustic soda solution, he washes what is left of the sample very thoroughly in running water, dries it, and weighs it again. The weight of this "residue" is that of the cottcL. The difference between this and the original weight is the weight of the wool. The chemist is then able to state the percentages by weight of cotton and of wool in the original material. 
Substances.- Upon considering these operations, we can discover a general plan which the chemist has devised and employs in his work. Different samples of cloth are of different colors and appearance, even when they contain the same proportions of cotton and wool. A casual inspection is, therefore, of little value. But in certain respects all samples of wool are alike, such as in dissolving in caustic soda, in "taking" certain dyes and in possessing a scaly surface. Those respects in which all samples of wool are alike are called the specific properties of wool. Similarly all samples of cotton are alike in certain respects, which are called the specific properties of cotton. And these properties of cotton and of wool are different, many of them very different indeed. For the purpose of stating what he means, the chemist calls a kind of material, all specimens of which possess a certain set of specific properties, a substance. Wool is one substance,* and cotton another substance. Every part of a specimen of a substance has the same specific properties as any other part. If any portions can be found which have different specific properties, these are portions of another substance, accidentally or intentionally mixed with the first. The chemist calls the foreign matter an impurity, and the specimen an impure sample of the substance of which it is mainly composed.

A substance, then, is a species or kind of matter, and all specimens of it show the same set of specific properties. Any particular substance is recognized by the specific properties which it exhibits when exposed to various tests. Specific properties, conversely, are those qualities which are characteristic of any particular substance.

The plan which the chemist uses in his experimental tests, therefore, is that of ascertaining whether a given material exhibits

* In point of fact, wool contains several substances, but they are all alike in respect to the three properties mentioned above. They differ slightly in respect to other properties, and so can be distinguished from one another. 
the properties of one, or of more than one substance. He then describes it by naming the substances he finds in it.

Another Illustration.- This view-point is peculiar to the chemist. Each art or science has its own view-point - its own way of thinking about a given object. By the geologist, a piece of granite is at once thought of as belonging to the older rocks of the earth, and the geologist considers when it was formed and how it was formed (namely, by solidification of a molten mass). To the builder, it is a very hard stone, expensive to cut and polish, but very ornamental, and very durable. How and when it was formed does not make any difference to the builder.

To the chemist, as a chemist, on the other hand, the expense of cutting granite, and its ornamental character, are of no interest. Instead, the chemist notices at once that it is spotted, and, upon examining it closely, he observes that it appears to be a mixture. He breaks up a portion, and stidies the properties of the fragments. Some are transparent like glass, are very hard, and in fact are in all respects like quartz or rock crystal (Fig. 3). All specimens of rock crystal, from whatever source, are alike in properties,

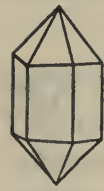

Fig. 3.

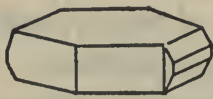

FIG. 4. and quartz is, therefore, a distinct substance or species of matter. Again, certain of the particles in granite are dark, and with a penknife can be easily split into transparent leaves, thinner than paper. These fragments are in all respects like mica (sheets of which, under the name of "isinglass," are used to close the windows of stoves), which is another substance (Fig. 4) well-known to the chemist. Still others of the fragments are less transparent than the quartz and less hard. They can be split, but with much greater difficulty than the mica. They are crystals, ${ }^{*}$

* Crystals are natural forms of a geometrical nature, assumed by solid substances (p. 94). 
oblong in shape. These are pieces of a third substance, felspar (Fig. 5). All the particles in the granite belong to one or other of these three kinds. The chemist, then, studies the specific properties, such as the hardnesses and the crystalline forms of various parts of the specimen and seeks to state or describe the nature of the specimen in terms of the substances he finds in it.

A Third Illustration. - When flour is examined by the chemist, it appears to the eye to be all alike. Under the microscope, even, all he can learn is that it consists largely of grains, which have the characteristic appearance of grains of starch (see Fig. 99, p. 399). He places some flour on a square piece of cheesecloth and encloses it by tying with a thread (Fig. 6). On kneading the little bag in a vessel of water, the water becomes milky. When the milky water stands, the white material settles to the bottom, the water can be poured off, and the deposit can be dried. This white substance, when boiled with water, gives an almost clear liquid which jellies on cooling. This is another property of starch. A little tincture of iodine (solu-

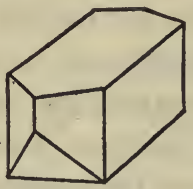

FIG. 5.

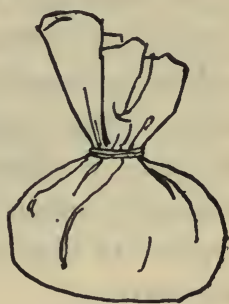

FIG. 6. tion of iodine in alcohol), dropped on a part of the starch, causes the latter to turn blue. This is a very characteristic property of (and therefore test for) starch. When the bag of flour is kneaded persistently in water which is frequently changed, the material finally ceases to render the water milky. The starch has all been washed out. When the bag is now opened, a sticky material is found in it. This is called gluten.

The chemist therefore finds that flour contains starch and gluten. He learns this by separating these two different substances. 
The Law of Component Substances.-Every material consists of certain substances, each of which has a definite set of specific properties. In terms of these properties the material can be described. This is the first and simplest law of chemistry, and at the same time the most fundamental.

Mixtures and Impurities.-A material containing more than one substance is called a mixture. The characteristic of a mixture is that each of the substances present, although mixed with the others, possesses exactly the same properties as if it were present alone. No one of the ingredients affects any other ingredient, or alters any of its properties. Granite and flour are typical mixtures.

When a specimen is composed mainly of one substance, and contains only minute amounts of one or more other substances, it is frequently spoken of as a specimen of the main substance containing certain specified substances as impurities. To be called an impurity, the foreign matter need not be dirty or offensive. Thus, common salt usually contains a little magnesium chloride, a white crystalline solid, as an impurity, and it is this impurity which becomes damp in wet weather. Again, compounds of lime and magnesium are common impurities in drinking water.

Component.-The ingredients of a mixture are called the components (Latin, put with), because they are simply placed together, without change, and can be separated without change.

Other Specific Properties.-Beside the specific properties which happen to have occurred in these illustrations, there are others which are constantly found useful by the chemist. Thus, in the case of solids, besides the hardness and crystalline form, he gives special attention to the temperature at which the substance melts (the melting-point), the specific gravity or density (weight 
of 1 c.c., see Appendix I), the color and the solubility or non-solubility in water. In the case of liquids, the temperature at which the liquid boils under atmospheric pressure (the boiling-point), the specific gravity, the mobility, the odor and the color (if any) are never exactly the same for any two different liquids. We shall learn more about these properties as occasions for using them arise.

Attributes and Conditions. - It should be noted that mass is not a specific property. Different specimens of a given substance may have different masses, but they all have the same specific properties. The mass of each, although fixed, is a quality of that particular specimen only. So is it with the volume and the dimensions of a specimen. These are attributes of a specimen.

Again the temperature is not a specific property. There is no particular temperature peculiar to quartz. Even the very same specimen may be at different temperatures at different times. Temperature and pressure are variable and are called conditions. The temperature or the pressure of a specimen may be changed at will. But the specific properties of a substance under any given conditions cannot be changed, so long as we have to do with the same substance.

Law of Chemical Change. - When the wool was boiled with caustic soda solution, it was in some way acted upon by the caustic soda. It became soluble, and disappeared into the liquid. Wool will not dissolve in boiling water. But in the former operation its material acquired at least one new property, namely that of being soluble. Since we have defined a substance as a species of matter, with a definite set of specific properties, we are compelled to decide that when a piece of material changes its properties, it has, in doing so, become a new substance. This experiment, then, calls our attention to the second of the fundamental laws of chemistry, namely that material forming one or 
more substances, without ceasing to exist, may be changed into one or more new and entirely different substances. When such an alteration occurs, it is called a chemical change or reaction. To learn more about this most remarkable fact, we shall take up in the next chapter some examples of a simple and long familiar nature.

Definition of Law. - In science, a law, or generalization or rule, is a statement describing some general fact or constant mode of behavior. Its uses are to condense a great many similar facts into one statement, and thus to make the whole set of facts more easy to remember.

Constituents. - As we have seen, we speak of the substances in a mixture as the components. When we wish to refer to the forms of matter which are chemically united in a compound, we call them the constituents (Lat., standing together) of the compound substance. Thus, iron and oxygen are the constituents of rust.

The chemist separates (p. 13) the components of a mixture, for that is all that is necessary. He liberates the constituents of a compound, however, because they are bound together in chemical combination.

The names given to compounds are usually devised so as to indicate the nature of the constituents. Thus, iron-rust is oxide of iron (or ferric oxide, from Lat., ferrum, iron). The yellowish powder obtained when lead is heated in air is lead oxide or oxide of lead, and the white powder similarly derived from tin is oxide of tin.

A Condensed Form of Statement.-We may represent a chemical combination, or indeed any kind of chemical change, in a condensed form, thus:

Iron + Oxygen $\rightarrow$ Oxide of iron (ferric oxide) 
Each name stands for a substance. Two substances in contact with one añother (mixed), but not united chemically, are connected by the + sign. The arrow shows where the chemical change comes in, and the direction of the change. We read the statement thus: Iron and oxygen brought together under suitable conditions undergo chemical change into oxide of iron, called also ferric oxide. Similarly we may write:

$$
\begin{gathered}
\text { Lead }+ \text { Oxygen } \rightarrow \text { Oxide of lead. } \\
\text { Tin }+ \text { Oxygen } \rightarrow \text { Oxide of tin. }
\end{gathered}
$$

Explanation of Rusting.-Experiment shows that the process of rusting is accompanied by a slow increase in the weight of the solid, due to the gradual addition of oxygen to the metal. Now, this increase in weight ceases of its own accord, when a certain maximum has been reached. This occurs when the last particles exhibiting the properties of the metal have disappeared. Thus, lead gains in weight until every 100 parts of the metal have gained 7.72 parts of oxygen, and tin until every 100 parts have gained 26.9 parts of oxygen. When these increases have occurred, the metal is found to have been all used up, and prolonged heating and stirring cause no further union with oxygen and no further change in weight. This fact, that each substance limits itself of its own accord to combining with a fixed proportion of the other substance, in forming a given compound, is one of the most striking facts about chemical combination. In mixtures, any proportions chosen by the experimenter may be used. In chemical union, the experimenter has no choice; the proportions are determined by the substances themselves. Thus, 100 parts of iron when turning into ordinary red rust take up 43 parts of oxygen, no more and no less.

This fact enables us to make our condensed statements more specific and complete by including in them the proportions by wreight used in the chemical change: 
Iron (100) + Oxygen (43) $\rightarrow$ Ferric oxide (143).

Lead (100) + Oxygen (7.72) $\rightarrow$ Oxide of 'ead (107.72).

The following numbers, which represent the same proportions by weight, are the ones commonly used by chemists:

$$
\begin{aligned}
& \text { Iron (223.36) + Oxygen (96) } \rightarrow \text { Ferric oxide (319.36). } \\
& \text { Lead (414.4) + Oxygen (32) } \rightarrow \text { Oxide of lead (446.4). }
\end{aligned}
$$

Summary. - Thus far, we have learned that chemistry deals with substances and their specific properties, and with the changes which substances undergo. We have discussed and defined a number of important words expressing fundamental chemical ideas. Finally, we have touched upon the weights of the materials used in chemical change, a subject of great importance which will be more fully developed in a later chapter.

Exercises.-1. Describe the following, by mentioning some of their specific properties: (a) water, (b) wool, (c) cotton (pp. 1-3).

2. If any of the following are mixtures, mention the facts which show them to contain more than one substance (p. 3): (a) muddy water, (b) an egg, (c) milk.

3. State and illustrate the first two laws of chemistry (pp. 6, 7).

4. Make definitions of "pure" and "impure" as applied to a sample of a substance (p. 6).

5. Give a list of specific properties mentioned in this chapter.

6. In recognizing a specimen to be quartz, does the chemist consider (a) the weight, (b) the temperature, (c) the length of the specimen (p. 7 )? If not, why not?

7. Take one by one the words or phrases printed in black type and the titles of the sections in this chapter, and endeavor to recollect what you have read about each. In each case try, (a) to recall the meaning and to state it in your own words; $(b)$ to recall the facts associated with, and the reasoning which lead up to the point in question; $(c)$ to recall examples illustrating the conception and to apply the conception in detail to each example. 
Whenever memory fails to give a perfectly clear report of the matter in hand, the text must be read and re-read until the essential point can be repeated from memory.

Use the same method in all future chapters. A useful practice is to employ a pencil as you read and to underline systematically all the important facts and statements, and then to go back and apply to each marked place the process described above.

8. Define the following terms: Specific gravity, tenacity, melting-point, specific physical property, pure body, vacuum.

9. Is it logical to say "pure substance?"

10. Why do we decide that granite is a mixture and iron a single substance?

11. What weight of oxygen would be required to convert 25 grams of lead into oxide of lead?

12. Make a list of the technical words we have defined, and place the definition opposite to each. 


\section{CHAPTER II}

\section{CHEMICAL CHANGE AND THE METHODS OF STUDYING IT}

WE must now take up two new examples of chemical change. They will aid us in introducing one or two additional conceptions and laws. These are continually used by the chemist, and without them we cannot begin the systematic study of the science.

\section{Another Case of Combination: Iron and Sulphur.-} Since oxygen is an invisible gas, there is a slight difficulty in realizing that rusting consists in the union of two substances - this gas and a metal. The present example is less interesting historically, but it is simpler because both substances are visible and are easily handled. The case of iron and sulphur will enable us to

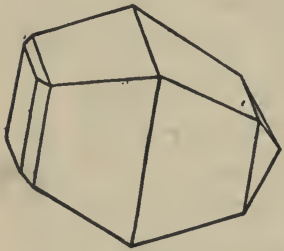

FIg. 7 illustrate the same point of view and to practice the application of the same technical words. It will also introduce us to two manipulations - filtration and evaporation - which are frequently used by the chemist.

We begin by observing and contrasting the specific properties of the two substances. Sulphur is a påle-yellow substance of low specific gravity (sp. gr. 2). It is easily melted (m.-p. $114.5^{\circ}$ C.). It does not dissolve in water - that is, it does not mix completely with and disappear in water, as sugar does on stirring. It does dissolve readily in certain other liquids, such as carbon disulphide, however. It crystallizes in rhombic forms (Fig. 7). It is not attracted by a magnet. Iron is a lustrous greyish substance of much higher specific gravity (sp. gr. 7.8). 
It is very difficult to melt it (m.-p. over $1500^{\circ}$ C.). It does not dissolve in any common liquids at ordinary temperatures. It crystal izes in cubes, and is attracted strongly by a magnet.

Study of the Mixture, before Combination.-Now, if some iron filings and pulverized sulphur are stirred together in a mortar, the result is a mixture. True, the color is not that of either substance, but with a lens particles of both substances can be seen. Passing a magnet over the mixture will easily remove a part of the iron, and with the help of a lens and a needle the mixture could be picked apart particle by particle, completely. We can separate the components of the mixture more expeditiously, however, by using manipulations based upon other more suitable properties. Thus, sulphur dissolves in carbon disulphide while iron does not. If, therefore, a part of the mix-

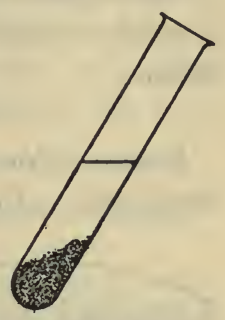

FIG. 8 ture is placed in a dry test-tube along with some carbon disulphide (Fig. 8), and is shaken, the liquid dissolves the sulphur and leaves the iron. To complete the separation, the

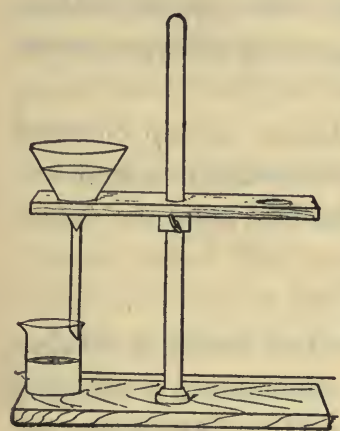

FIG. 9 iron must be removed from the liquid by filtration, and the sulphur recovered by evaporation of the carbon disulphide.

Filtration.- Iron, or any solid, when it is mixed with a liquid or with a solution (like the solution of sulphur in carbon disulphide) is said to be suspended in the liquid. If the solid is one that settles rapidly, the liquid may be separated from the solid, in a rough way, by pouring off as much of the clear, supernatant liquid as possible. This is called decantation. A complete separation is effected by pouring the mixture on to a 
cone of filter paper supported in a glass funnel (Fig. 9). The liquid, together with anything that may be dissolved in it, runs through the pores of the paper and down the hollow stem of the funnel. The liquid is then called the filtrate. The particles of the suspended solid are too large to pass through the pores, and so collect on the surface of the filter paper. This operation, like everything the chemist does, takes advantage of differences in the specific properties of the various materials.

The material remaining on the paper (the residue), when dry, is wholly attracted by a magnet and shows all the other properties of iron.

Evaporation.- To recover the sulphur, the solution in carbon disulphide - the filtrate - is poured into a porcelain evaporating dish. (Carbon disulphide is very in-

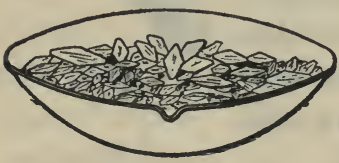

FIG. 10 flammable! Keep flames away). When the vessel is set aside, the liquid gradually passes off in vapor (e-vapor-ates). Sulphur, however, does not evaporate at room temperature and remains as a residue, in the form of crystals of rhombic outline in the bottom of the dish (Fig. 10). Here, again, differences in specific properties have been utilized.

Since the physical properties of two substances are not changed by mixing, we have thus used the properties of the iron and sulphur so as to separate them once more. The iron is on the paper; the sulphur is in the dish.

Combination of Iron and Sulphur. $\longrightarrow$ But iron and sulphur are capable of combining to form a new substance, if we alter the conditions by raising the temperature. When we place some of the original mixture of iron and sulphur into a clean test-tube and warm it, we soon notice a rather violent development of heat taking place, the contents begin to glow, and what appears to 
be a form of combustion spreads through the mass. The heating employed at the start falls far short of accounting for the much greater heat produced. When these phenomena have ceased, and the test-tube has been allowed to cool, we find that it now contains a somewhat porous-looking, black solid. This material is brittle; it is not magnetic; it does not dissolve in carbon disulphide; and close examination, even under a microscope, does not reveal the presence of different kinds of matter. This substance is known to chemists as ferrous sulphide and, as we see, its properties are entirely different from those of its constituents.

In this connection we must not omit to notice that, as in rusting, a certain fixed proportion will be used in forming the compound.

\section{Iron (55.84) + Sulphur (32.06) $\rightarrow$ Ferrous sulphide (87.90)}

If more iron is put into the original mixture, then some unused iron will be found in the mass after the action. If too much sulphur is employed, some may be driven off as vapor by the heat and any that remains, beyond the correct proportion, can be dissolved out of the ferrous sulphide with carbon disulphide. The sulphur which has combined with the iron, however, is no longer present as sulphur - it has no longer the properties of sulphur, and therefore cannot be dissolved out.

Another Illustration: Mercuric Oxide.- It has long been known that air contains an active and an inactive gas. The Chinese called them yin and yang, respectively. Mayow (16431679) showed that the active gas caused rusting, that it was absorbed by paint (really by the linseed oil) in " drying," that it supported combustion of wood and sulphur, and that it is necessary to life, being absorbed by the blood from the air entering the lungs. It was not until 1774 , however, that a pure specimen of this gas was obtained, by Bayen, and was recognized to be a special kind of gas different from ordinary air. 
The gas (later to be named oxygen) was made by Bayen from mercuric oxide, a bright red, rather heavy powder. When

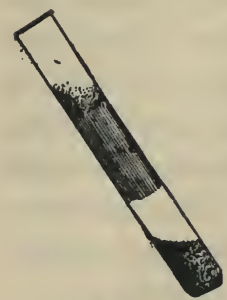

Fig. 11 the oxide is heated, (Fig. 11), we find that a gas is given off. This gas is easily shown to be different from air, since a glowing splinter of wood is instantly relighted on being immersed in it. The gas is pure oxygen. During the heating, we notice also that a metallic coating appears on the sides of the tube, in the form of a sort of mirror. Apparently the vapor of some metal is coming off with the oxygen and condensing on the cool parts of the tube. As this shining substance accumulates it takes the form of globules, which may be scraped together. It is, in fact, the metal mercury, or quicksilver. If the heating continues long enough, the whole of the red powder eventually disappears, and is converted into these two products.

\section{Second Variety of Chemical Change: Decomposition.-} Bayen's experiment introduces to us a second, and very common kind of chemical action. The first variety was combination or union (p. 14). The second is called decomposition. It consists in starting with a single substance (here mercuric oxide) and splitting it into two (or more) substances, which differ in properties from the substance taken and from one another. Here, the red powder gave mercury, a liquid metal, and oxygen, a colorless gas.

Simple and Compound Substances. - We have seen that two (or more) substances, like lead and oxygen, can combine to form a compound substance. Are all substances, then, compounds? We find that some are not. We have never succeeded in obtaining lead, or oxygen, or iron, or tin, or sulphur by combining any two substances. We can decompose mercuric oxide by heat, and we have other ways of decomposing compounds like oxide of tin and ferrous sulphide, but we have never succeeded in 
decomposing the mercury or the oxygen, the iron or the sulphur themselves. Substances which we are not able, by chemical means, to decompose into, or to make by chemical union from, other substances are called simple or elementary substances. The distinction between simple and compound substances was first drawn by Boyle in 1678. Later, and independently, it was stated very clearly by Lavoisier (1789).

Several substances, regarded in Lavoisier's time as elementary, have since been shown to be compounds. Thus, quicklime was a simple substance until Davy, in 1808, prepared the metal calcium and showed that quicklime was the oxide of this metal. Hence, we do not say that the substances regarded as simple cannot be decomposed, but only that they are substances which we " are not able" (at present) to decompose.

The phrase "by chemical means" is also important. Although by chemical methods we are not able to effect any decomposition of the elements, yet we cannot regard them as absolutely unalterable and permanent. The element radium cannot be decomposed by chemical means, but it undergoes continuous and spontaneous "disintegration" into the elements helium and lead (see p. 546). It has recently been discovered by Rutherford that other elements, such as nitrogen, and aluminum, may also be disrupted to give hydrogen, under the action of the tremendous forces of the swiftly-moving particles ejected from radium in the course of this disintegration. Such phenomena, however, do not affect our conception of elements as applied to ordinary chemical reactions.

Elements. - The word element is used in two senses. It is applied to the simple substance. Thus we speak of "the element iron," meaning the metal iron. It is applied also to the ironmatter contained in ferrous sulphide or in ferric oxide. The reader should note that it is correct usage to speak of the element iron and the element sulphur in ferrous sulphide, but a chemist 
would never say that this compound contained the simple substances iron and sulphur. If he did, we should understand him to mean that it was a mixture, and we should expect parts of the material to be magnetic like iron, and other parts to be yellow and soluble in carbon disulphide, which is not the case. In the same way the name of an element (such as iron) is applied both to the material in combination and to the free substance. Thus "iron " may mean free, uncombined, metallic iron, or iron-matter in some compound. The sense in which the word is employed must be inferred from the context or circumstances. When a chemist speaks, as he sometimes does, colloquially, of "iron " in a drinking water, for example, we know at once that he refers to iron in the form of some compound, for metallic iron does not dissolve in water.

The word element, then, means one of the simple forms of matter, either free or in combination.

In formally describing a body or specimen, the chemist always avoids the ambiguity just referred to by naming the components, i.e., the substance or substances it contains. He assumes that the nature and constituents of these substances will be known to anyone hearing or reading the description. If he says the body contains zinc and sulphur, it is understood that the body is a mixture of these simple substances. If it contained these elements in combination, the chemist would report that it was sulphide of zinc.

The Common Elements.-Thousands of different compound substances are known but, when they are decomposed, it is found that the number of different elements contained in them is not great. Dozens of substances contain iron, hundreds contain sulphur, thousands contain oxygen. In fact, by combining a limited number, two, three, or four, of simple substances together, in varying proportions by weight, an almost unlimited number of different compound substances could be produced. 
A list of the elements appears on the inside of the cover, at the end of this book, and contains about eighty names. Of these, a large number are rare, and seldom encountered. More than 99 per cent of terrestrial material is made up of eighteen or twenty elements and their compounds. Only about twenty elements occur in nature in their simple, uncombined condition. Three-fourths of the whole number are found in combination exclusively, and must be liberated by some chemical action.

Taking the atmosphere, all terrestrial waters, and the earth's crust, so far as it has been examined, F. W. Clarke has estimated the plentifulness of the various elements. The first twelve, with the quantity of each contained in one hundred parts of terrestrial matter, and constituting together 99 per cent, are as follows:

\begin{tabular}{|c|c|c|}
\hline .49 .85 & Calcium. & Hydrogen. \\
\hline .26 .0 & Sodium. & Titanium. \\
\hline Aluminium & Potassium. & Chlorine. \\
\hline Iron...... & Magnesium......2.11 & Carbon. . \\
\hline
\end{tabular}

The significance of these figures is more clearly shown in Fig. 12. It will be seen that oxygen accounts for nearly one-half of the whole mass. Silicon, the oxide of which when pure is quartz and in less pure form constitutes ordinary sand, makes up half of the remainder. Valuable and useful elements, like gold, silver, sulphur, and mercury, are among the less plentiful which, all taken together, furnish the remaining one per cent.

Law of Definite Proportions. - In the decomposition of mercuric oxide (p. 16)* we find that, for every 100 parts of

* References to previous pages are used in order to save needless repetition in writing. The beginner requires endless repetition in his reading, however, and must form the habit of examining, in conjunction with the current text, the parts referred to. The passages cited are, by the reference, made part of the current text, which will usually not be clear without them. The same remark applies to topics referred to by name. Such topics must be sought in the index.

All terms, and especially those borrowed from physics, if not perfectly familiar, must be looked up in a work on physics or in a dictionary. 
mercury liberated, almost exactly 8 parts of oxygen by weight are set free. Using the numbers commonly employed in chemistry, which represent the same proportion by weight:

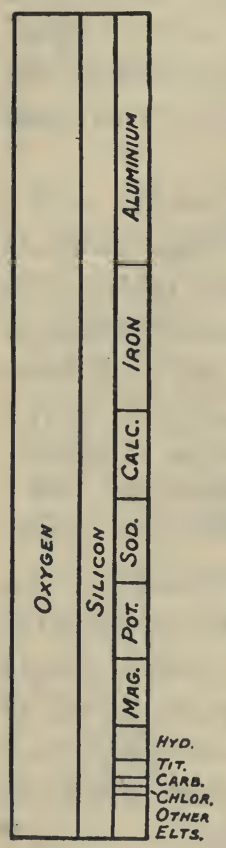

Fig. 12

Mercuric oxide (216.6) $\rightarrow$ Mercury (200.6) +

Oxygen (16).

We find also that mercury and oxygen can be made to combine to form mercuric oxide, and the proportions by weight required are the same. Moreover, every sample of mercuric oxide, whether made by combination, or in any of the other possible ways, always contains this proportion of the two elements. We have already seen that the oxides of lead and tin contain fixed proportions (p. 9) of the metal and oxygen and that ferrous sulphide has a constant composition by weight. The same principle is found to apply to all chemical compounds, and is stated in the law of definite or constant proportions: In every sample of any compound substance, formed or decomposed, the proportion by weight of the constituent elements is always the same.

Certain elements have recently been shown to exist in two or more forms (isotopes), which would, if separable, give compounds possessing the same specific properties yet differing in composition. Detailed discussion of this point must be deferred to a later chapter (pp. 550-552).

Conservation of Mass. - The most painstaking chemical work seems to show that, if all the substances concerned in a chemical change are weighed before and after the change, there is no evidence of any alteration in the quantity of matter. The two weights, representing the sums of the constituents and of the products, respectively, are, indeed, never absolutely identical, but 
the more careful the work and the more delicate the instrument used in weighing, the more nearly do the values approach identity. We are able to state, therefore, that the mass of a system is not affected by any chemical change within the system.

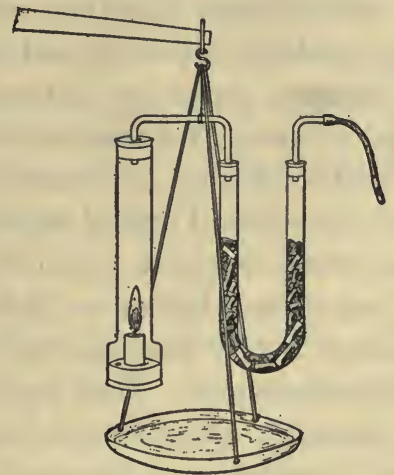

FIG. 13

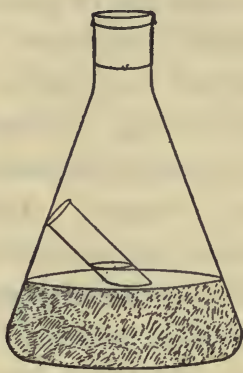

Frg. 14

This statement simply means that the great law of the conservation of mass holds true in chemistry as it does in physics. Chemical changes, thoroughgoing as they are in respect to all other qualities, do not affect the mass; an element carries with it its weight, entirely unchanged, through the most complicated chemical transformations.

Superficial observation, as of a growing tree, might seem to give evidence of the very opposite of conservation of matter. But here the carbon dioxide gas in the air, the most important source of nourishment for plants, is overlooked. Similarly, the gradual disappearance of a candle by combustion seems to illustrate the destruction of matter. But if we catch the gases which rise through the flame (Fig. 13), we find that the gases weigh even more than the part of the candle which has been sacrificed in making them. When we take account of the weight of the oxygen obtained from the air which sustains the combustion, we find that there is really neither loss nor gain in weight. If we carry 
out chemical changes in closed vessels (Fig. 14), which permit neither escape nor access of material, we find that the weight does not alter.

Physics in Chemistry. - It will be seen that one cannot accomplish anything in chemistry without acquiring and using some knowledge of physics. We measure quantities by means of the physical attributes, weight and volume. We produce chemical change by arranging the physical conditions, for example, by mixing, heating, or using an electric current. Physical means are the only means we possess for producing, stopping, or modifying chemical changes. Again, we ascertain whether a chemical change has taken place or not by observing the physical properties of the materials before and after the experiment. Thus, we noted that the red, powdery oxide of mercury, when heated, gave a liquid metal and a gas. All the phenomena of chemistry are physical. A phenomenon is literally something that is seen or, more generally, something that affects any of the senses. Observing physical phenomena is, therefore, our sole means of studying chemical changes. Chemical work is, in fact, entirely dependent upon the skilful use of physical agencies, and upon the close observation of physical phenomena for its success.

It is only the inference, following the experiment and the observation, that is strictly chemical. If one substance gives two different substances, or if two substances give one different substance, for example, we infer that a chemical change has occurred. We then try to recognize the substances by their properties and name them.

Changes like that of ice into water, or of water into steam, and vice versa, are not regarded as chemical changes. These are called changes of state (see p. 64).

Law: Explanation: Scientific Method.-There is a widely spread impression that a science, like chemistry is a part of 
the natural order of the universe. It is thought that we are trying to find the boundaries of chemistry, as they have been predetermined by nature, and to discover the facts, relations of facts, and laws which nature has provided as a means of classifying the content of the science. Now, the situation is precisely the reverse of this. Nature provides only the materials and the phenomena, and man is attempting to classify them. He divides the whole into groups, such as physics, chemistry, botany, etc. Then he classifies the facts within each group, in order that he may more easily remember them and perceive their relations. He often finds that, when new facts are discovered, parts of the classification have to be changed.

In the preceding pages, we have discussed some of the ways that have been invented for classifying the materials and facts assigned to chemistry. Thus, we pick out a number of facts of a like nature and try to make a single statement which will cover all these facts. For example, we find about one hundred thousand different substances and, in the case of each substance, every specimen that we have examined contains the same proportions of the constituent elements. So we formulate the law of constant proportions.

A law or generalization in chemistry is a brief statement describing some general fact or constant mode of behavior. We must remember, however, that laws are only true so long as no facts in conflict with them are known. There are no laws in nature. Nature presents materials and phenomena as she pleases. The laws are parts of science, which is made by man, and is a description of natural facts as man knows them.

One section (p. 9) was entitled: "Explanation of rusting." If that paragraph be now re-read, it will be found that, in the ordinary (as distinct from the scientific) sense of the word, no explanation was given! When we ask a man to "explain" some feature in his conduct, we recognize that he might have chosen to act otherwise, and we wish to know why he acted precisely as he 
did. Nature, however, has no free will, and cannot tell why she presents certain phenomena, and not others.

On examining the explanation, we find that it simply shows that when iron rusts it combines with oxygen from the air. This is an additional fact. It shows how iron rusts, namely, by taking up oxygen, but not why it is able to unite with oxygen. We simply do not know why iron can combine with oxygen gas and platinum cannot.

Explanations in chemistry are of three kinds. (1) We usually try to show that the phenomenon is not an isolated one. Thus, we show that other metals rust. This reconciles us to some extent to the fact that iron rusts, and we feel some mental satisfaction. This is the method of showing that the fact to be explained is a member of a large class of similar facts. (2) Next, we try to get more information about the fact to be explained. Thus, when, to the acquaintance with the outward manifestations of rusting, we add the further information that there is an increase in weight, and that this is due to union of oxygen from the air with the iron, we feel increased satisfaction, and say that the fact has been "explained." (3) If we are still dissatisfied, and can discover no further useful facts, we imagine a state of affairs which, if true, would classify the fact or add to what we know about it. This step we call explaining by means of an hypothesis. We then devote our attention to trying to verify the hypothesis.

The formulation of laws and the making of attempts to explain facts are part of what is called the scientific method. The purpose of this method is to convert the subject matter into a science, that is, into an organized body of knowledge.

Summary. - In this chapter we have learned: (1) that, while there are many substances, there is a limited number of entirely different kinds of matter (elements); (2) that, in addition to definite specific properties, each substance has a constant composition by weight. We have also learned that physical 
properties are utilized in manipulations, like filtration and evaporation, as well as for identifying substances, and that physical attributes are used for measuring quantities in chemistry and physical conditions for guiding chemical change. Finally, we have seen that a science is not a natural, but a manufactured product, and that the science of chemistry is still in the making.

Exercises.*-1. What physical properties are used (a) in filtration, (b) in evaporation, (c) in the separation and identification of the products from heating mercuric oxide (p. 15)?

2. Describe: (a) a red-hot rod of iron, $10 \mathrm{~cm}$. long by $1 \mathrm{~cm}$. diameter, weighing 58.5 g.; (b) a solution of $5 \mathrm{~g}$. of sulphur in 20 c.c. ( 26 g.) of carbon disulphide at $18^{\circ} \mathrm{C}$. In doing so, divide the description into attributes, conditions, and properties.

3. Consider the following materials and state whether, so far as you can now judge, each is a single substance or a mixture: (a) a candle, (b) a cake of soap, (c) an egg.

4. What are the two most direct ways of showing a substance to be a compound? Illustrate each.

5. If we say that quicklime contains calcium, do we mean the element or the simple substance calcium?

6. What explanation was given, $(a)$ of the disappearance of mercuric oxide when heated, $(b)$ of the absence of iron and sulphur, as substances, from ferrous sulphide? Which of the three kinds of explanation was used in each case?

7. What weight of oxygen will be required to combine with 15 grams of lead (p. 10)?

8. If 5 grams of lead and 4 grams of oxygen were heated together, which of the two would remain in part unused? How much of this one would remain (p. 10)?

* The exercises should in all cases be studied with minute care. They not only serve as tests to show that the chapter has been understood, but very frequently (as in No. 4) also call attention to ideas which might not be acquired from the text alone, or (as in Nos. 1, 2, 5) assist in elucidating ideas given in the text which, without the exercises, might not be fully grasped. 


\section{CHAPTER III}

\section{AIR AND OXYGEN}

$\mathrm{WE}_{\mathrm{E}}$ have seen that metals absorb a gas, called oxygen, from the air, and turn into a rust or oxide. Let us now consider what happens to the air during this process.

The Nature of Air. - Can a metal, like iron, when rusting, absorb the whole of a sample of air, or does it select a part of the air only? If we sprinkle some powdered iron in a test-tube,

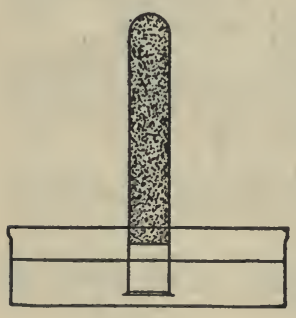

FIg. 15 having first moistened the interior to cause the powder to adhere to the inside surface, and then set the tube, mouth downwards, in a dish of water (Fig. 15), we obtain before long an answer to this question. As the iron slowly removes the oxygen, the pressure of the atmosphere outside pushes the water up the tube. But, after ascending only about one-fifth of the total height of the tube, the water comes to rest. Inspection shows reddened particles where rusting has taken place, but much of the iron is still dark grey, and is as little able to rust in the remaining gas as in a vacuum. Four-fifths of the air, then, is composed of gases which do not combine with iron, and only one-fifth is oxygen. The four-fifths is in fact almost all (99 per cent) nitrogen, a substance which combines with very few materials, while the balance ( 1 per cent) is made up of argon and other gases which do not enter into combination with any known substances. Oxygen, on the contrary, combines with almost all simple substances, although in many cases, as in those of lead and tin, heating is required to hasten the process. 
Activity and Stability.-A substance which enters into combination easily, is called active, so that oxygen is spoken of as an active element, nitrogen as a relatively inactive or indifferent element. An active element, since it combines greedily, holds tenaciously to that with which it has combined. An active element means, therefore, also, one which is in general difficult to liberate from combination. Its compounds are in general relatively stable, or inactive. The compounds of more indifferent elements, on the other hand, are in general relatively easy to decompose. In other words, they are unstable or active.

Law of the Influence of Heating.- Even oxygen, active as it is, does not combine visibly with tin, when both are cold. Lead rusts very slowly at the ordinary temperature. Iron rusts very much faster when heated than when cold. In every chemical change we find that raising the temperature hastens the process very considerably. Other things being equal, it causes a greater quantity of reacting material to undergo the change in a given interval of time.

As exceptions to this law, radioactive disintegrations (see p. 545) must be noted. The rate at which reactions of this type proceed is in every case absolutely independent of the temperature. The speed of a photochemical reaction (a chemical change induced by the action of light; see p. 498) is also, in general, very little affected by raising the temperature. For normal chemical changes, however, the influence of temperature is very marked, a rise of only ten degrees (approximately) causing most reactions to proceed at twice their original rate.

Oxygen.- We cannot do better than begin the more systematic study of chemistry with oxygen, for it is a most interesting as well as useful substance. It is the active component of the air. We depend upon it for life, since in its absence we suffocate, for heat, 
since wood, coal, and gas will not burn without it, and even for light where oil, gas, or a candle is used.

We wish to know with which substances it can combine, as well as the substances on which it has no action. This information will show us how to work, in future, without interference from the oxygen in the air and whether oxygen has probably played a part in some experiment or not.

Let us take up, then, (1) the history of the element, (2) what materials contain oxygen (occurrence), (3) how we can obtain it in a pure state (preparation), (4) what its specific physical properties as a substance are, and (5) what it does, and what it cannot do in nature and in the laboratory (chemical properties). The classification of the facts about this, and other substances under five heads, is somewhat mechanical, but has the advantage of enabling the reader quickly to find any required information.

History of Oxygen.- Leonardo da Vinci (1452-1519) seems to be the first European to mention the presence of two gases in the air. Mayow (1669) measured the proportion of oxygen in the air and discussed fully its uses in combustion, rusting, vinegarmaking, and respiration, but did not make a pure sample. Hales (1731) made it from saltpeter, and measured the amount obtainable, but did not see any connection between it and the air! Bayen (Apr., 1774) was the first to make it by heating mercuric

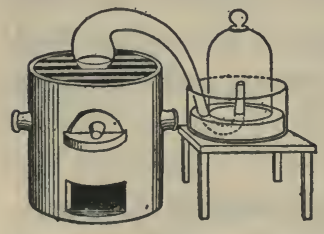

FIG. 16 oxide. Priestley (Aug. 1, 1774) made it by heating the same substance and quite purposelessly, as he admits, thrust a lighted candle into it and was delighted with the extreme brilliance of the flame. Scheele, a Swedish apothecary, had made it in 1771-2 from no less than seven different substances and understood clearly that atmospheric oxygen combined with metals, phosphorus, hydrogen, linseed oil and many other substances. Finally, Lavoisier 


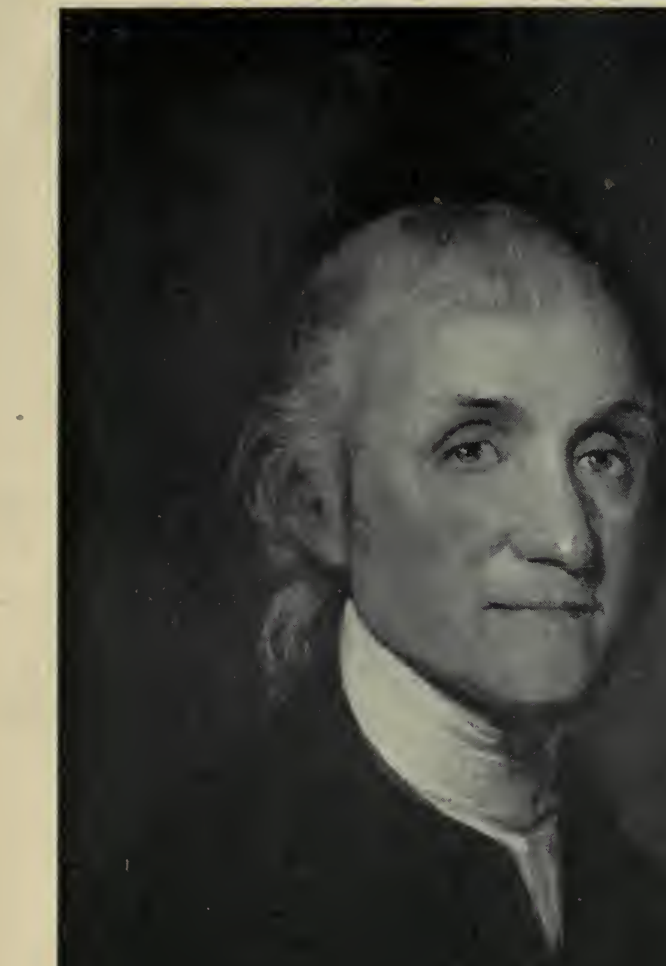

JOSEPH PRIESTLEY

See page xi 

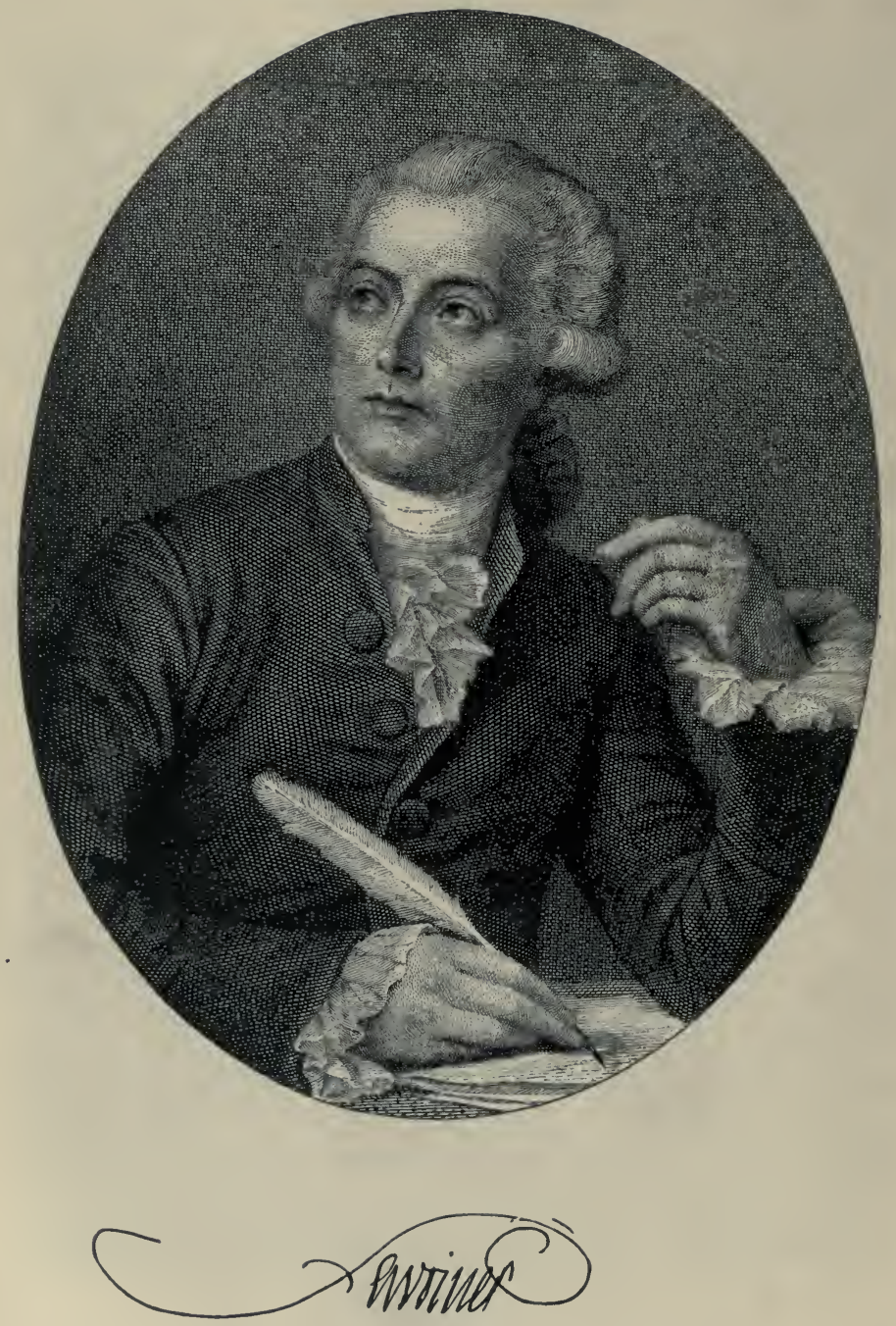

See page xi 
(1777) heated mercury in a retort (Fig. 16), the neck of which projected into a jar standing in a larger dish of mercury. The air, thus enclosed within the jar and the retort, during twelve days lost one-fifth of its volume. Simultaneously, red particles of mercuric oxide accumulated on the surface of the mercury in the retort. The residual gas, nitrogen, no longer supported life or combustion. The oxide, on being heated more strongly, by itself, gave off a gas whose volume exactly corresponded with the shrinkage undergone by the enclosed air, and this gas possessed in an exaggerated degree the properties which the air had lost. The proof that oxygen was a component of the atmosphere was therefore complete. Later, Lavoisier, in the mistaken belief that the new element was an essential constituent of all sour substances, named it oxygen (Greek, acidproducer).

Occurrence.-As we have seen, nearly 50 per cent of terrestrial matter is oxygen. Water contains about 89 per cent, the human body over 60 per cent, and common materials like sandstone, limestone, brick, and mortar more than 50 per cent of this element. One-fifth by volume (nearly one-fourth by weight) of the air is free oxygen.

Preparation of Oxygen.- 1. The oxygen of commerce is now made chiefly from liquefied air. The liquid oxygen boils at $-182.5^{\circ}$, but the nitrogen boils at an even lower temperature $\left(-194^{\circ}\right)$. Since the liquid air has a temperature of about $-190^{\circ}$, somewhat above that of boiling nitrogen, the latter evaporates much more freely than does the oxygen. After a time, when the remaining liquid is almost pure

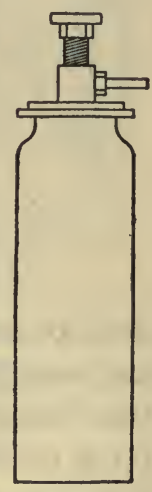

FIG. 17 oxygen (96 per cent), the gas coming off is compressed by pumps into the steel cylinders (Fig. 17) in which it is sold. In medicine, 
patients suffering from pneumonia or suffocation obtain some relief by inhaling it in this form. It is also used in feeding flames, instead of air, when intense heat is required (see acetylene torch, p. 352, and calcium light, p. 385).

2. Unfortunately, it is difficult to liberate oxygen from natural substances. Saltpeter (potassium nitrate), for example, which is found in many soils and can be dissolved out with water, gives off oxygen only when raised to a bright red heat by the Bunsen flame or blast lamp. But, even at this temperature, it gives up only one-third of the oxygen it contains.

3 . In practice, we are compelled to use manufactured substances. Amongst the artificial substances are mercuric oxide, expensive but historically interesting (p. 15), potassium chlorate, perhaps the most convenient for laboratory use, and sodium peroxide. Potassium chlorate is a white crystalline substance

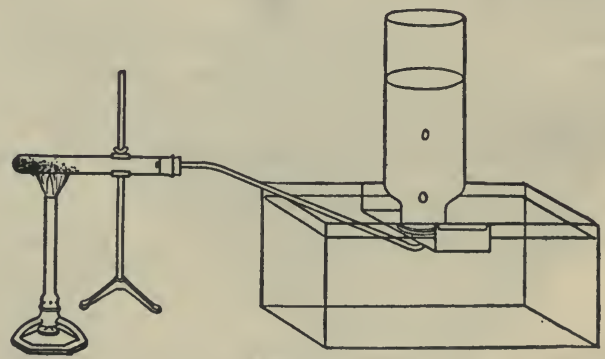

FIG. 18

used, on account of the oxygen it contains, in large quantities in the manufacture of matches and fireworks. When heated in a tube similar to that in Fig. 11, it first melts $\left(357^{\circ}\right)$ and then, on being more strongly heated, it effervesces and gives off a very large volume of oxygen. Examination shows that the whole of the oxygen it contains (39 per cent) can be driven out. The white material which remains after the heating is identical with the mineral sylvite. To the chemist it is known as potassium chloride. 
The change, together with the weights of the materials, is as follows:

Potassium chlorate (122.56) $\rightarrow$ Potassium chloride (74.56) + Oxygen (48)

Potassium (39.1)

Chlorine (35.46)

Potassium (39.1)

Oxygen (48)

Chlorine (35.46)

A peculiarity of this action is that admixture of manganese dioxide (the mineral pyrolusite) increases very markedly the speed with which the decomposition of the potassium chlorate takes place. Hence powdered manganese dioxide is generally mixed with the chlorate in laboratory experiments (Fig. 18), and in its presence a sufficient stream of oxygen is obtained at a relatively low temperature (below $200^{\circ}$ ).

4. Oxygen can be obtained conveniently from sodium peroxide and water by means of generators (Fig. 19) similar to the acetylene generators used on automobiles. When the metal sodium is burned in air, sodium peroxide is obtained as a powder. This powder, after being melted, solidifies in compact, solid form, and is sold as oxone. The oxone is bought in a small, sealed tin can, the ends of which are perforated in several places just before use. When the valve $(B)$ is opened, so that the oxygen escapes, the water, which fills the generator

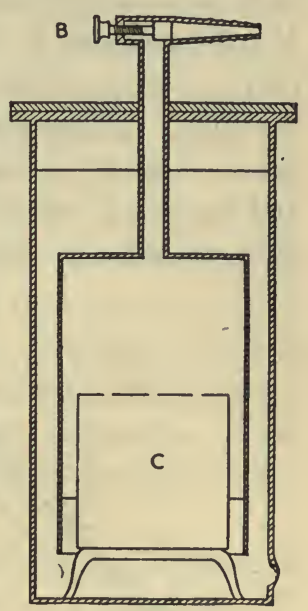

FIG. 19 almost to the top, enters the can $(C)$ by the holes in the bottom and interacts with the oxone. When the valve is shut, the gas continues to be generated until it has driven the water down again below the level of the bottom of the can.

Sodium peroxide (78) + Water (18) $\rightarrow$ Sodium hydroxide (80) + Oxygen (16)

Sodium (46)

Oxygen (32)

Hydrogen (2.016)

Oxygen (16)
Sodium (46)

Oxygen (32)

Hydrogen (2.016)

This method is convenient because it works at room temperature 
and can be started and stopped at will. The sodium hydroxide produced is very soluble in water and remains dissolved.

Catalytic or Contact Action.- The influence of manganese dioxide in causing the potassium chlorate to decompose more easily (p. 31) well deserves nòtice. The effect is very striking if some pure potassium chlorate is melted carefully, to avoid super, heating, in a wide-mouth flask (Fig. 20). The flask is provided with a wide exit tube, from which a rubber tube may lead to a bottle inverted in a trough filled with water as in Fig. 18. A little manganese dioxide is contained in the upper, closed tube. No effervescence of the chlorate can be seen at its melting-point $\left(357^{\circ}\right)$ - only a little air, expanded by the heating, issues from the tube. When, however, the closed tube containing the manganese dioxide is rotated into a vertical position (see dotted lines), and the black powder falls into the chlorate, the oxygen comes off in torrents, in consequence of the enormous acceleration of the

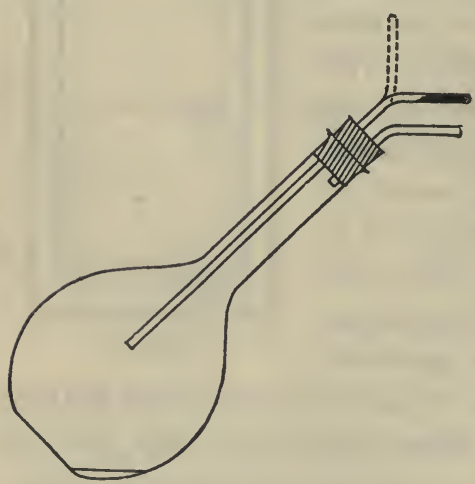

Frg. 20 decomposition. As a precaution against injury from an explosion of the flask, it is advisable to wrap the latter in a towel before turning the tube.

It must also be noted that the manganese dioxide is not itself permanently altered. If the material left after the action is shaken with water, the potassium chloride dissolves, while the dioxide does not. Filtration (p. 13) thus enables us to recover the latter, and to ascertain that it has been changed neither in quantity nor in properties.

The only effect of the dioxide is to hasten the decomposition of the chlorate, which would otherwise be too slow at $200^{\circ}$ (p. 31), 
or even at $357^{\circ}$ (its m.-p.) to be of any practical value. Substances which hasten a chemical action without themselves undergoing any permanent change are called contact agents, catalytic agents, or catalysts. The process is called contact action or catalysis (Greek, decomposition, not a very fortunate choice of words). Such substances are frequently used in chemistry. The addition of a suitable catalyst is one of the conditions (p. 7) for carrying out actions in which a contact agent is necessary. Many substances of this class are secreted by animals and plants and play an important part in digestion, fermentation, and other physiological changes. Their presence often enables very complex chemical actions to proceed rapidly at rather low temperatures.

The oxone, mentioned above, always contains a catalyst, a trace of cuprous oxide, which hastens the action on water.

Specific Properties of Two Kinds, Physical and Chemical. - We have learned that every substance has its own set of specific properties. In describing a substance, it is convenient to divide the properties into two classes. The list of substances with which the given substance can enter into chemical combination, for example, we place under specific chemical properties. Relations of the substance to any of the varieties of chemical change belong to this class.

On the other hand, we do not consider melting or boiling to be chemical changes, so we place the temperatures at which the substance melts (m.-p.) and boils (b.-p.), its color, etc. (for list, see p. $6)$, under specific physical properties.

Properties of either class may be used for recognizing a substance.

Specific Physical Properties of Oxygen.- Oxygen resembles air in having neither color, taste, nor odor. The density of a substance is, strictly speaking, the weight of 1 cubic centimeter (1 c.c.). In the case of a gas, we frequently prefer to give the weight of 1000 c.c. (1 liter), at $0^{\circ}$ and $760 \mathrm{~mm}$. (1 atmosphere) barometric 
pressure. For oxygen this weight is 1.42900 grams (Morley). The corresponding weight for air is 1.293 , so that oxygen is slightly heavier, bulk for bulk, than air (in the ratio $1.105: 1$ ). Oxygen can be liquefied by compression, provided its temperature is first reduced below $-118^{\circ}$, which is its critical temperature.* The gas is only slightly soluble in water, the solubility at $20^{\circ}$ being 3 volumes of gas in 100 volumes of water (at $0^{\circ}, 4.9: 100$ ).

The solubility of oxygen in water, although slight, is in some respects its most important physical property. Fish obtain oxygen for their blood from that dissolved in the water. With airbreathing animals (like man), the oxygen could not be so readily taken into the system, if it did not first dissolve in the moisture contained in the walls of the air sacs of the lungs, and then pass inwards in a dissolved state to the blood.

Liquid oxygen, first prepared by Wroblevski, has a pale-blue color. At one atmosphere pressure, that is, in an open vessel, it boils at $-182.5^{\circ}$. Its density (weight of 1 c.c.) is 1.13 , so that it is slightly denser than water. By cooling with a jet of liquid hydrogen, Dewar froze the liquid to a snow-like, pale-blue solid. A tube of liquid oxygen is noticeably attracted by a magnet.

Six Specific Physical Properties of Each Gas.-Although every substance has many physical properties, we shall mention only those which are used in chemical work, with occasionally the addition of any peculiar or unexpected quality. It will aid the memory to recall the physical properties of a gas, if we note that, as a rule, only six such properties are mentioned: (1) color, (2) taste, (3) odor, (4) density, (5) liquefiability, defined by the critical temperature, (6) solubility, usually in water only.

* Each gas has an individual critical temperature (p. 91) above which no pressure, however great, will produce liquefaction. The farther the temperature of a specimen of the gas is below the critical point, the less will be the pressure required to liquefy it. 
Specific Chemical Properties of Oxygen.-The chemical properties of pure oxygen are like those of atmospheric air, only more pronounced.

Reactions with Non-metallic Elements. Sulphur, when raised in advance to the temperature necessary to start the action, unites vigorously with oxygen, giving out much heat and producing a familiar gas having a pungent odor (sulphur dioxide). This odor is frequently spoken of as the "smell of sulphur," but in reality sulphur itself has no odor, and neither has oxygen. The odor is a property of the compound of the two. The mode of experimentation can be changed and the oxygen led into sulphur vapor through a tube. The oxygen then appears to burn with a bright flame, giving the same product as before.

Phosphorus, when set on fire, blazes in oxygen very vigorously, forming a white, powdery, solid oxide - phosphorus pentoxide. Burning carbon, in the form of charcoal or hard coal, glows brilliantly and is soon burnt up. It leaves an invisible, odorless gas - carbon dioxide. At high temperatures, oxygen combines readily with one or two other non-metals (e.g., silicon, boron, and arsenic), and to a small extent ( 1 per cent at $1900^{\circ}$ ) with nitrogen. It will not combine directly with chlorine, bromine, or iodine, although oxides of the first and last can be prepared by using other varieties of chemical change. With the six members of the helium family of which no compounds are known, and with fluorine, oxygen forms no compounds.

Sulphur (32.06) + Oxygen (32) $\rightarrow$ Sulphur dioxide (64.06) Phosphorus (62.08) + Oxygen (80) $\rightarrow$ Phosphorus pentoxide(142.08). Carbon (12) + Oxygen (32) $\rightarrow$ Carbon dioxide (44).

Reactions with Metallic Elements. Iron, as we have seen, rusts exceedingly slowly in air and, even when red-hot, gives hammerscale, the black solid which is broken off on the anvil, rather deliberately. In pure oxygen, a bundle of picture-wire, if once ignited, will burn with surprising brilliancy, throwing off sparkling 
globules of the oxide, melted by the heat. This oxide is a black, brittle substance, identical with hammer-scale, and different from rust (ferric oxide). It contains, in fact, a smaller proportion of oxygen than does the latter, and is called magnetic oxide of iron. Iron (167.52) + Oxygen (64) $\rightarrow$ Magnetic oxide of iron (231.52).

All the familiar metals, excepting gold, silver, and platinum, when heated, combine with oxygen, some more vigorously, others less vigorously than does iron. Oxides of the three metals just named can also be made, but only by varieties of chemical change other than direct combination.

Compound substances, if they are composed largely or entirely of elements which combine with oxygen, are able themselves to interact with oxygen. Usually, they produce a mixture of the same oxides which each element, separately, would give. Hence, wood, which is composed of carbon and hydrogen with some oxygen, when burnt in oxygen, produces carbon dioxide and water (oxide of hydrogen) in the form of vapor. Again, carbon disulphide burns readily, giving carbon dioxide and sulphur dioxide, just as do carbon and sulphur, separately. Ferrous sulphide gives, similarly, sulphur dioxide and magnetic oxide of iron.

Tests. A Test for Oxygen.-A test is a property which, because it is easily recognized (a strong color, for example), or because it is especially distinctive, is commonly employed in recognizing a substance.

Oxygen, when pure, is recognized by the fact that a splinter of wood, glowing at one end, bursts into flame when introduced into the gas. Only one other gas (see nitrous oxide) behaves similarly.

\section{The Measurement of Combining Proportions.-In a} number of condensed statements we have given the proportions by weight of the materials combining. It is now desirable that 
we should know how the necessary measurements are made. The most exact measurement of the proportions in which the elements combine to form compounds involves manipulations too elaborate to be gone into here. One or two brief statements, diagrammatic rather than accurate, will show the principles, however.

If we take a weighed quantity of iron in a testtube and heat it with more than enough sulphur (an excess of sulphur), we get free sulphur along with the ferrous sulphide ( $p$.

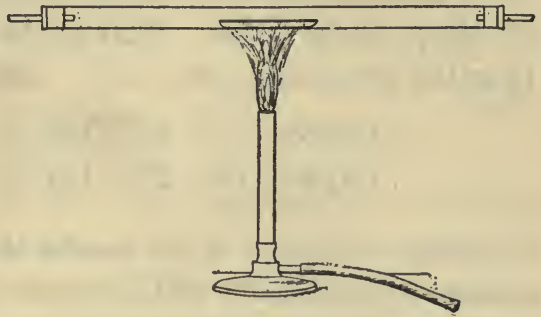

FIG. 21

15), and no free iron survives. We may remove the free sulphur by washing the solid with carbon disulphide. The difference between the weights of the ferrous sulphide and the iron gives the amount of sulphur combined with the known quantity of the latter.

As an example of the study of the combination of a metal with oxygen, we may weigh a small amount of copper in the form of powder in a porcelain boat and pass oxygen over the heated metal until it is completely converted into black cupric oxide (Fig. 21). The original weight of the copper, and the increase in weight, representing oxygen, give us then the data for determining the composition of this oxide. The data furnished by one rough lecture-experiment, for example, were as follows:

Weight of boat + copper. $\ldots \ldots \ldots \ldots \ldots \ldots \ldots \ldots \ldots$. $4.278 \mathrm{~g}$.

Weight of boat empty................... $3.428 \mathrm{~g}$.

Difference $=$ weight of copper $\ldots \ldots \ldots \ldots \ldots \ldots \ldots, \overline{0.850 \mathrm{~g}}$.

Weight after addition of oxygen............... $4.488 \mathrm{~g}$.

Weight without oxygen................. $4.278 \mathrm{~g}$.

Difference $=$ weight of oxygen $\ldots \ldots \ldots \ldots \ldots \ldots \ldots \overline{0.210 \mathrm{~g}}$. 
The proportion of copper to oxygen, so far as this one measurement goes, is therefore $85: 21$.

The results of quantitative experiments are often recorded in the form of parts in one hundred. To find the percentage of each constituent, we observe that the proportion of copper is $85: 85+$ 21 , or $\frac{85}{106}$ of the whole. That of the oxygen is $\frac{21}{106}$ of the whole. Thus the percentages are:

$$
\begin{array}{ll}
\text { Copper, } 106: 85:: 100: x . & x^{\prime}=80.2 . \\
\text { Oxygen, } 106: 21:: 100: x^{\prime} . & x^{\prime}=19.8 .
\end{array}
$$

Naturally, the mean of the results of a number of more carefully managed experiments will be nearer the true proportion. The percentages at present accepted as most accurate are 79.9 and 20.1.

In the case of mercuric oxide, we may decompose a known weight of the oxide (p. 16), collect the mercury and weigh it, and ascertain the oxygen by difference.

The names of the constituent elements in a compound, together with the proportion by weight in which they are present, are called the composition of the substance. Thus, the composition of cupric oxide is copper : oxygen :: $79.9: 20.1$. This is the percentage composition, but other numbers expressing the same proportion (such as $63.57: 16$ ) will serve the purpose.

All experiments involving measurement, such as those used in determining composition, are called quantitative experiments.

\section{Another Quantitative Experiment.-The following will} show how the combining proportions may be measured when the product is a gas, the weight of which must be ascertained. Sulphur burns in oxygen to form sulphur dioxide. A known weight of sulphur is placed in a porcelain boat in a hard-glass combustion tube (Fig. 22). The U-shaped tube to the right contains a solution of potassium hydroxide, which is capable of absorbing the resulting gas. The oxygen enters from the left. When the sulphur is heated, it burns in the oxygen, and the gain in weight of 
the U-tube shows the weight of the compound produced. By subtracting from this the weight of the sulphur taken, we get the quantity of oxygen with which it has combined.

In one experiment, the weight of sulphur was $1.21 \mathrm{~g}$. The weight gained by the U-tube was $2.42 \mathrm{~g}$. The difference (= oxygen) is 1.21 . The proportion of sulphur to oxygen in sulphur dioxide is therefore $1.21: 1.21$

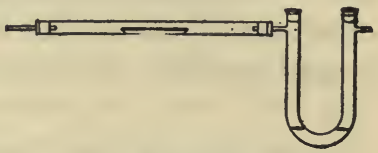

Fig. 22 or $1: 1$ or, in percentages, $50: 50$. This proportion is very close to the accepted value, $32.06: 32$.

The same method could be used for carbon, for the carbon dioxide produced would be absorbed in the solution of potassium hydroxide.

Combustion.- Violent union with oxygen is called, in popular language, combustion or burning. Yet, since similar vigorous interactions with other gases are common, the term has no scientific significance. Even the union of iron and sulphur gives out light and heat, and is quite similar in the chemical point of view to combustion.

A misleading term often used in this connection is kindling temperature. It gives the impression that there is a definite temperature at which combustion will start. But the temperature is only one of the conditions which produce combustion. Finely powdered iron will start burning at a lower temperature than will an iron wire, because it presents relatively more surface to the gas. Again, if the oxygen is at less than one atmosphere pressure, the wire will require to reach a higher temperature before combustion will begin. Finally, the vapor of methyl alcohol and air requires to be raised above a red heat before combustion starts, but a pocket cigar-lighter sets fire to this very mixture by means of a contact agent (a thin platinum wire) without any other means of heating being required. Therefore, the conditions under which 
combustion begins involve the physical condition of the solid, the pressure of the gas or vapor, the presence or absence of a contact agent and the nature of the contact agent, as well as the temperature. No definite kindling temperature can be given, unless the other conditions are specified also. Kindling conditions involve several variables, of which the temperature is only one.

Oxidation.- The slower union with oxygen which occurs in rusting is called oxidation. We shall see later, however, that it has been found convenient to stretch this term so as to cover combinations of other elements than oxygen, and even to include actions not involving combination. At this point we can discuss only oxidation by oxygen.

This process of slow oxidation by oxygen, although less conspicuous than combustion, is really of greater interest. Thus the decay of wood is simply a process of oxidation whereby the same products are formed as by the more rapid ordinary combustion. Sewage is mixed with large volumes of river water, the object being, not simply to dilute the sewage, but to mix it with water containing oxygen in solution. This has an oxidizing power like that of oxygen gas and, through the agency of bacteria, quickly renders dissolved organic matters innocuous by converting them for the most part into carbon dioxide and water. Thus, a few miles further down the stream, the water may become as suitable for drinking as it was before the sewage entered. In our own bodies we have likewise a familiar illustration of slow oxidation. Avoiding details, it is sufficient to say that the oxygen, from the air taken into the lungs, combines with the hæmoglobin in the red blood-corpuscles. In this form of loose combination, it is carried by the blood throughout our tissues and there oxidizes the foodstuffs which have been absorbed during digestion. The material products are carbon dioxide and water, of which the former is carried back to the lungs by the blood, and finally reaches the air during exhalation. The important product, however, is 
not material, but the heat, given out by the oxidation, which keeps the body warm.

The opposite of oxidation, the removal of oxygen, is spoken of in chemistry as reduction. But this term, also, has been stretched to cover other similar kinds of chemical change.

9.7. Spontaneous Combustion.- Sometimes a mere slow oxidation develops into a combustion, which is then known as spontaneous combustion. To understand this, we must note the fact that a given weight of material, say, iron, in combining with oxygen to form a given oxide, will liberate the same total amount of heat whether the union proceeds rapidly or slowly. If the action proceeds slowly, and the material being oxidized is freely exposed to the air, the latter will become heated and will carry off the heat as fast as it is produced. Thus, no particular rise in temperature will occur. If, however, the material is a poor conductor of heat, like hay or rags, and there is sufficient air for oxidation, but not enough to carry off the heated air, the heat may accumulate and a temperature sufficient to start combustion may be reached.

Such a situation sometimes arises in hay-stacks. It occurs also when rags, saturated with oils used in making paints (linseed oil and turpentine) are left in a heap. These oils, in "drying," combine with oxygen from the air and turn into a tough, resinous material. The rags, being poor conductors of heat, may finally become hot enough to burst into flame, and serious conflagrations often owe their origin to causes such as this. Oily rags should always be disposed of by burning, or should at least be placed in a closed can of metal. Fires in coal bunkers of ships arise from the same cause - slow oxidation, with accumulation of the resulting heat. That coal does undergo slow oxidation, especially when freshly mined, is shown by the fact that such coal, if left exposed to the air for months, may lose from 2 to 5 per cent or more of its heating value. 
Uses of Oxygen.- In the foregoing sections we have referred to its use in breathing, its role in decay, which is a beneficient process because it removes much useless matter which might otherwise cause disease, and its value in the disposal of sewage. Power and heat for commercial purposes are almost all obtained by the burning of coal, in which oxygen from the air plays a large part. If we had to purchase the oxygen as well as the coal, we should require at least three tons of oxygen for every ton of coal.

Oxygen in cylinders and oxygen generators are used to restore the supply in the atmosphere of submarine boats, and to assist the respiration of aviators at very great altitudes.

Substances Indifferent to Oxygen.-Finally, since the atmosphere contains so large a proportion of oxygen, substances which do not oxidize and, when heated, do not burn, have many uses. Gold, silver, and platinum are of this kind (p. 36), and are used for ornaments. The last is used for crucibles in which bodies are heated in the laboratory. Although iron burns in pure oxygen, it does not oxidize rapidly in the air even when heated, and so is used for making vessels for cooking and in constructing fireproof buildings.

Substances, already fully oxidized, are naturally not combustible. Of this nature are sandstone, granite, brick, porcelain, glass, and water. All these are, therefore, fireproof. Moreover these substances do not give off oxygen when heated. It is this inactivity which renders glass and porcelain suitable materials for laboratory apparatus, since they experience no change in weight when heated.

Exercises.- 1. What percentage by weight of free oxygen is obtained by heating: (a) mercuric oxide, (b) potassium nitrate, (c) potassium chlorate? At $\$ 1.50, \$ 0.15$, and $\$ 0.15$ per kilogram, respectively, which is the cheapest source of oxygen?

2. Using the data on p. 34, calculate the weight of oxygen dissolved by 100 c.c. of water at $0^{\circ}$ under one atm. pressure. 
3. Why does a forced draft make a fire burn more rapidly?

4. Why does a naked flame sometimes cause an explosion in a mine, when the air of the mine is filled with coal dust?

5. The substances, like phosphorus and sulphur, which burn rapidly in ordinary oxygen, combine very, very slowly with oxygen which has been freed from moisture by careful drying. How is this effect of water to be classified?

6. Air is 20 per cent oxygen. Why does iron burn brilliantly in pure oxygen, but not in air?

7. What weight of copper would be required to combine with the amount of oxygen contained in 100 grams of mercuric oxide? 


\section{CHAPTER IV}

\section{THE MEASUREMENT OF GASES. THE MOLECULAR HYPOTHESIS}

AfTER the discussion in regard to the proportions of oxygen in the air and the measurement of the volume of oxygen removed (Fig. 15, p. 26), it will readily be imagined that measuring the volume of a sample of gas is a common operation in chemistry. Indeed, it is much easier to measure quantities of gas by noting their volumes than by weighing them. Some facts have to be taken account of, however, in order that the measurements of the volume may be of value.

A sample of gas diminishes in volume when the pressure increases, and it increases in volume when the temperature rises. Hence, when the volume of the gas is measured, the pressure and the temperature must also be stated.

Measurement of Pressure.- In order that the pressure may be easily measured, we arrange the sample of gas so that its pressure is the same as that of the atmosphere at the

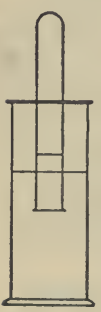

Fig. 23 moment. To do this, if, for example, the gas is contained in the narrow tube (Fig. 23), we bring the water inside the tube to the same level as that outside by lowering the tube. Then we read the volume by means of the graduation (not shown) on the tube and at the same time we ascertain the atmospheric pressure from the height of the barometer.

A simple form of barometer is shown in Fig. 33, p. 61 (tube on left of diagram only). A glass tube, about 1 meter long and closed at one end, is completely filled with mercury and carefully inverted, with its open end dipping into a mercury reservoir. The mercury falls inside the tube, leaving a vacuum 
at the top, until the pressure of the mercury column inside balances the pressure of the air outside. The atmospheric pressure is therefore expressed by the barometer as equivalent to the pressure exerted by so many millimeters of mercury. For accurate work, all readings obtained must be reduced to a standard temperature $\left(0^{\circ} \mathrm{C}\right.$.), by correcting for the expansion of mercury above that temperature.

Correction of the Volume to $760 \mathrm{~mm}$. Pressure.- Since the barometer varies in height, and the volume of a sample of gas therefore varies also, it is convenient to "correct" the volume further by "reducing" it to that which the gas would occupy at "standard" pressure, namely $760 \mathrm{~mm}$. Now, the volume of a sample of gas varies inverseiy with the pressure (Boyle's law). If, for example, the volume is 23 c.c. and the observed pressure of the barometer is $745 \mathrm{~mm}$., Boyle's law enables us to calculate the volume the same sample of gas would occupy at $760 \mathrm{~mm}$. The volume changes in the ratio of these two pressures. If the pressure of the gas were actually changed to $760 \mathrm{~mm}$.,- a greater pressure - the gas would assume a smaller volume. Hence, the new volume $=23 \times \frac{745}{760}=22.5$ c.c. That is to say, if the new volume is to be less, we place the smaller pressure in the numerator.

If a sample of gas occupies 15 c.c. at $850 \mathrm{~mm}$., what volume will it occupy at $500 \mathrm{~mm}$.? Here the new pressure is smaller, and the new volume therefore greater. New volume $=15 \times \frac{850}{500}=25.5$ c.c.

Correction of the Volume to $0^{\circ}$ C. - All gases at $0^{\circ}$ are found to gain $1 / 273$ of their volume when heated 1 degree, $2 / 273$ for 2 degrees and $273 / 273$ for 273 degrees. Thus at $273^{\circ}$ the volume is doubled. When cooled below $0^{\circ}$, every gas similarly loses $1 / 273$ of its volume for each degree. At $-273^{\circ}$, if the 
regular contraction continued so far, the sample would, by calculation, at least, lose all its volume. This temperature (the lowest temperature that could possibly be attained) is called the absolute zero. In point of fact, however, all gases liquefy before the temperature has fallen to $-273^{\circ}$.

The rule contained in these statements is known as Charles' law. By applying an arithmetical device, we can state the law in a form which makes its use in calculations quite easy. The device consists in adding 273 algebraically to all temperatures. The temperature, when 273 has been added, is called the absolute temperature. The rule then reads: The volume of a sample of any gas is directly proportional to the absolute temperature.

Thus, a sample of gas occupies 45 c.c. at $15^{\circ}$, what would be its volume at $10^{\circ}$ ? After we have applied the device, this reads: a sample of gas occupies 45 c.c. at $15+273=288^{\circ}$ Abs., what would be its volume at $10+273=283^{\circ}$ Abs.? The volume changes in the ratio of these absolute temperatures. Since the new temperature $\left(10^{\circ} \mathrm{C}\right.$. or $283^{\circ} \mathrm{Abs}$.) is lower, the volume becomes smaller. Therefore, putting the smaller number in the numerator, the volume at $283^{\circ}=45 \times \frac{283}{288}=44.2$ c.c.

Again, a sample of gas occupies 125 c.c. at $25^{\circ}$, what will be its volume at $-15^{\circ}$ ? The absolute temperatures are $25+273=$ $298^{\circ}$ Abs., and $-15+273=258^{\circ}$ Abs. As the new temperature is lower, the volume will be less. Hence the new volume $125 \times \frac{258}{298}=108.2$ c.c.

In practice, when a sample of gas is measured, we read the existing temperature, and correct the volume to that which the sample specimen would occupy at $0^{\circ} \mathrm{C}$. For example, the volume is 102 c.c. at $18^{\circ}$, what is it at $0^{\circ}$ ? The absolute temperatures are $18+273=291^{\circ} \mathrm{Abs}$, and $0+273=273^{\circ} \mathrm{Abs}$. The new volume will be smaller. Hence, the new volume is $102 \times$ 
$\frac{273}{291}=95$ c.c. This correction enables us to compare all volumes of gases as if they had been measured at the same standard temperature, namely at $0^{\circ}$.

Corrections for Pressure and Temperature Combined. Since the volume changes, due to alterations in pressure and in temperature, are independent of one another, the corrections may be made either separately or together. The latter is more convenient. Thus, a sample of gas occupies 190 c.c. at $17^{\circ}$ and $750 \mathrm{~mm}$., what will be the volume under standard conditions, namely $0^{\circ}$ and $760 \mathrm{~mm}$. pressure?

$$
\text { New volume }=190 \times \frac{273}{290} \times \frac{750}{760}=176.6 \text { c.c. }
$$

Correction for the Tension of Aqueous Vapor.- When a gas is measured over mercury, the latter gives off practically no vapor at room temperature, and the foregoing are the only corrections required. If, however, the sample of gas is standing over water, then the volume is not that of the gas, but that of the gas plus a certain amount of water vapor. The latter must be subtracted. To do this we have to remember that, in a gaseous mixture, each one of the several gases (or vapors) exercises the same pressure as if it were present alone (Dalton's law of partial pressures). Now the pressure of the water vapor, in a gas standing over water, at each temperature is known (see p. 62 and Appendix IV). It varies from $13.5 \mathrm{~mm}$. at $16^{\circ}$ to $23.5 \mathrm{~mm}$. at $25^{\circ}$. When, therefore, the gas is measured over water, the pressure of the water vapor is subtracted from the barometric reading, before the above-mentioned corrections are applied.

For example, a specimen of a gas, standing over water, occupies 175 c.c. at $19^{\circ}$ and $752 \mathrm{~mm}$., what is the volume of the same gas at $0^{\circ}$ and $760 \mathrm{~mm}$. when the gas is free from water? At $19^{\circ}$ 
the aqueous tension is $16 \mathrm{~mm}$. The pressire of the gas alone is therefore $752-16=736 \mathrm{~mm}$. The fully corrected volume $=$ $175 \times \frac{736}{760} \times \frac{273}{292}=158.4$ c.c.

Molecular Hypothesis. - The relations between pressure and volume (Boyle's law) and between either of these and temperature (Charles' law) in gases may be explained by the molecular hypothesis. According to this idea, all matter is composed of minute particles called molecules, those of any given substance being all alike in nature and in mass.

In solids and liquids these molecules are closely packed together. In gases, however, they are widely scattered, with much vacant space between them. A gas is in fact a vacuum, with numerous relatively minute particles scattered through it. When a gas is compressed, only the spaces between the molecules are reduced. By assuming further that, in gases, the molecules are in rapid motion, and produce pressure by striking the walls of the vessel, and that this motion is increased by raising the temperature, all the laws of gases can be completely explained (see p. 88-93).

Exercises.- 1. Show that, if the levels of the water inside and outside the tube (Fig. 15, p. 26) are equal, the pressure of the gas inside must be equal to the atmospheric pressure.

2. Find the volume that 48 c.c. of gas at $732 \mathrm{~mm}$. would occupy at $760 \mathrm{~mm}$.

3. Reduce 48 c.c. of gas at $780 \mathrm{~mm}$. to standard pressure (760 mm.).

4. Find the volume which 28 c.c. of gas at $775, \mathrm{~mm}$. would occupy if the pressure changed to $730 \mathrm{~mm}$.

5. Find the volume which 320 c.c. of gas at $20^{\circ}$ would occupy at $0^{\circ}$ (pressure unaltered).

6. Reduce 600 c.c. at $25^{\circ}$ and $760 \mathrm{~mm}$. to standard conditions ( $0^{\circ}$ and $760 \mathrm{~mm}$.). 
THE MEASUREMENT OF GASES. MOLECULAR HYPOTHESIS 49

7. What change in volume would occur if 1 liter of gas at $200^{\circ}$ were cooled to $0^{\circ}$.

8. Reduce 260 c.c. of gas at $10^{\circ}$ and $742 \mathrm{~mm}$. to standard conditions.

9. Reduce 35 c.c. of gas standing over water at $21^{\circ}$ and $732 \mathrm{~mm}$. to dry gas under standard conditions.

10. Give the feature of the molecular hypothesis which corresponds to each of the following facts about gases:

(a) A specimen of gas can be compressed to $1 / 2000$ th of the volume it occupies at $760 \mathrm{~mm}$.

(b) A gas, even if heavier than air, gradually leaves an open cylinder.

(c) A specimen of a gas exercises pressure equally in all directions.

(d) Crowding the specimen of gas into one-third of its original volume triples its pressure.

(e) When the temperature is raised, the pressure of a specimen of gas increases (if the volume remains unchanged).

11. When we drink lemonade through a straw, what causes the liquid to flow up into our mouths? 


\section{CHAPTER V}

\section{HYDROGEN}

AFTER considering the atmosphere, and particularly its most active component, we naturally turn to water, which, like air, is so closely associated with our daily life. We find that water is a compound of oxygen with hydrogen, and the latter element, therefore, next claims our attention. Hydrogen is of interest upon its own account. It is often used for filling balloons. Illuminating gas, of the kind (water gas) used in most large cities, consists of hydrogen to the extent of about 40 per cent.

Preparation by the Action of Metals on Water.- Hydrogen is not easily liberated from water, since this oxide of hydrogen, like many other oxides, is very stable. It is necessary to use some element with which oxygen will combine even more

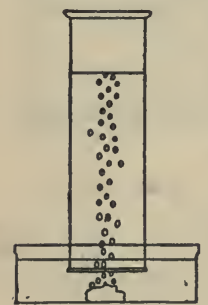

FIG. 24 eagerly than with hydrogen, and to offer this element in exchange for the hydrogen.

If a piece of one of the very active metals, such as potassium, sodium, or calcium is thrown into water, hydrogen is liberated and comes off in bubbles. The former two metals are lighter than water, and run about on the surface. The action with potassium is so violent that the hydrogen usually catches fire, and both with sodium and potassium much heat is produced. The action often ends with a slight explosion, so that a glass plate should be held up to protect the eyes. This experiment is too dangerous to be tried by a novice. With calcium the action is rapid, but not violent, and there is no danger. The metal sinks to the bottom of the vessel (Fig. 24), so that a testtube or bottle filled with water can be inverted over the metal to catch the gas as it ascends. 
The metals, of course, act upon a small part only of the whole vesselful of water. In each case the metal displaces one-half of the hydrogen from that part of the water upon which it acts: Sodium (23) + Water (18) $\rightarrow$ Hydrogen (1) + Sodium hydroxide (40).

$\begin{array}{ll}\text { Hydrogen } & \text { Sodium } \\ \text { Hydrogen } & \text { Hydrogen } \\ \text { Oxygen } & \text { Oxygen }\end{array}$

The products are hydrogen, along with potassium hydroxide, sodium hydroxide, or calcium hydroxide. The first two hydroxides are very soluble in water, but most of the calcium hydroxide is not dissolved, and may be seen suspended in the liquid.

Magnesium will liberate hydrogen from water, provided the latter is hot. If steam be passed through a heated tube containing iron filings, a mixture of hydrogen, with unused steam, issues at the other end. The magnetic oxide of iron, not hydroxide, remains in the tube:

Iron $+\underset{\substack{\text { Hyder } \\ \text { Oxygen }}}{\text { Waten }} \rightarrow$ Hydrogen $+\underset{\substack{\text { Magnetic oxide of iron. } \\ \text { Oxygen }}}{\text { Mron }}$

This is a method much used in making hydrogen for commercial purposes.

Silver, gold and platinum, which do not combine directly with pure oxygen, and even copper and mercury, which do, are all unable to form oxides and to liberate hydrogen when heated in steam.

Displacement.- The foregoing actions, in which hydrogen is liberated, present us with a new - the third - variety of chemical change. Here an element displaces one of the elements from a compound, setting it free, and unites with the rest of the constituents of the compound. Thus, calcium displaces part of the hydrogen, and unites with the oxygen and the rest of the hydrogen.

Preparation by the Action of Metals upon Dilute Acids.All the metals which displace hydrogen from water or steam will also act upon cold dilute acids and displace the hydrogen they contain. This action is the one most commonly employed in the laboratory 
(Fig. 25). The gas, being much lighter than air, is collected by downward displacement of the air (Fig. 26b). Heavy gases are collected by upward displacement of air (Fig. 26a).

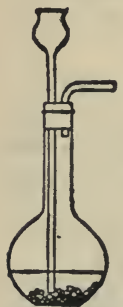

Extremely pure zinc is almost inactive, but commercial zinc, on account of the contact action of the slight impurities it contains, gives a steady, not too violent, evolution of hydrogen. Sulphuric acid and hydrochloric acid, in each case diluted with water, are convenient acids. Iron shows about the same activity, but, on account of the impurities Fig. 25 usually present in iron filings or wire, the hydrogen contains other gases which exhibit a distinct odor.

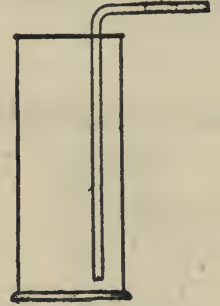

Frg. 26a

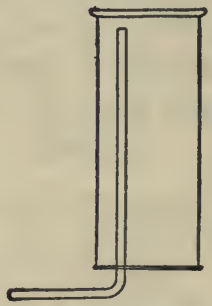

Fig. $26 \mathrm{~b}$

Zinc (65.4) + Sulphuric acid (98) $\rightarrow$ Hydrogen (2) + Zinc sulphate (161.4). Hydrogen $\left\{\begin{array}{l}\text { Sulphur } \\ \text { Oxygen }\end{array}\right.$

Zine $\left\{\begin{array}{l}\text { Sulphur } \\ \text { Oxygen }\end{array}\right.$ Iron (56) + Hydrochloric acid (72.9) $\rightarrow$ Hydrogen $(2)+\underset{(126.9)}{\text { Ferrous chloride }}$ Hydrogen Chlorine

Iron Chlorine

It will be seen that the action is of the form we have ealled displacement (p. 51).

The Proportions by Weight. - It may be well to remind ourselves that the weights of the various materials (given in brackets) are obtained by laboratory measurements. It is always found that the total weight of the product is exactly equal to that of the materials used (p. 20). Also, that a given weight of the metal, say zinc, will always displace and liberate the same weight 
of hydrogen. Also, that the proportions by weight of the constituent elements in the compound produced are always the same (p. 20). If we place a weighed piece of zinc in hydrochloric acid, and wait until the zinc has all disappeared, we can then boil away the water and unused acid, and weigh the white, solid zinc chloride. We find that 65.4 grams of zinc always leave 136.3 grams of zinc chloride. The difference, 70.9 , is the chlorine, and whatever weights we take, the proportion of zinc to chlorine in the zinc chloride is always in the ratio $65.4: 70.9$.

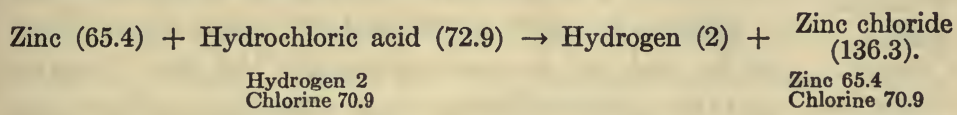

Chemically Equivalent Quantities. - It will be observed that 65.4 parts of zinc displace 2 parts of hydrogen, whether: the acid used is sulphuric acid or hydrochloric acid. The propor. tion is, in fact, the same with every acid. Hence 65.4 parts of zinc and 2 parts of hydrogen are spoken of as chemically equivalent quantities. The quantities of the displacing and of the displaced element are in all cases referred to as chemically equivalent.

The term equivalents is applied also to the quantities liberated by decomposition of a compound like the 100 parts of mercury and the 8 parts of oxygen (p. 19). It is likewise used of the proportions combining when chemical union takes place, as in the case of phosphorus 31 parts and oxygen 40 parts (p. 35). The proportions of the elements in zinc chloride (see above) are also chemically equivalent.

Chemically equivalent quantities (or, simply, equivalents) of two substances are exact quantities which enter into or result from a chemical reaction.

The Order of Activity of the Metals. - It will greatly aid us in remembering a number of the facts already given, as well as many others, if we compare carefully with those facts the 
order in which the metals stand in the adjoining list. The most active metals are at the top. All above hydrogen displace this element from dilute acids (and, with more difficulty, from water); those below it do not.

The first displaces the hydrogen from water so violently that the gas catches fire, the second displaces it vigorously, the third less vigorously. Magnesium requires hot water and

ORDER OF ACTIVITY. Metals

Potassium

Sodium

Calcium

Magnesium

Aluminium

Manganese

Zine

Chromium

Iron

Nickel

Lead

Tin

N.B-Hydrogen

Copper

Bismuth

Antimony

Mercury

Silver

Platinum

Gold

iron superheated steam. Copper and the metals following it do not liberate hydrogen from water.

Again, the upper metals act too violently on dilute acids, and zinc is used to prepare the gas. Copper and the metals following it do not displace hydrogen from dilute acids.

Still again, we recall the fact (p. 36) that, when we heat metals in pure oxygen, the last three do not become oxidized at all. Those preceding silver do combine with pure oxygen - mercury with difficulty, and the others more and more vigorously as we ascend the list. On the other hand, if we start with the oxides of all the metals, we find that those at the foot of the list, up to and including mercuric oxide, lose all their oxygen when heated, leaving the metal.

Other facts of a similar nature will be mentioned as we encounter them. Meantime, it may be noted that the metals found uncombined in nature are those following hydrogen. Again, the metals known to have been first used by the human race were gold and silver. In the "bronze age" means of liberating copper from its ores had been discovered. Lead, tin, and iron came later. The list, read from the bottom

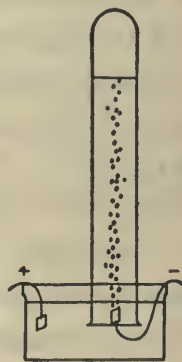

FIG. 27 up, gives, therefore, roughly, the historical order in which the metals came into use. 
Preparation by Electrolysis of a Dilute Acid.- A convenient way of obtaining pure hydrogen is by passing a current of electricity through a dilute acid (Fig. 27). The gas is liberated at the negative wire (cathode) and collects in the tube (also filled with the dilute acid). The direct, 110-volt current, passing through a 16-c.-p. lamp placed in series with the electrolytic apparatus, may liberate 50 c.c. of hydrogen in 7-8 minutes. Every acid contains hydrogen, combined with other elements. The other elements are carried to the positive plate (anode) and therefore do not interfere with the collection of pure hydrogen. What may be liberated at the positive plate depends upon the acid used. With hydrochloric acid, it is chlorine; with sulphuric acid, oxygen comes off and sulphuric acid is regenerated.

Hydrochloric acid (in Aq. Soln.) $\rightarrow$ Hydrogen (neg. plate) + Chlorine (pos. plate).

The process is called electrolysis, from the Greek, meaning decomposed by electricity.

Physical Properties of Hydrogen. - The gas is colorless, odorless, and tasteless. It is, bulk for bulk, the lightest known gas, the density of air being about 14.5 times as great. It can be liquefied by compression below $-234^{\circ}$ (its critical temperature). It dissolves in water to the extent of 1.8 volumes in 100 volumes of water at $15^{\circ}$.

The lightness of the gas may be shown by pouring it upwards from one jar to another, or by balancing an inverted beaker with shot, and allowing hydrogen to flow in and displace the air.

Several metals can adsorb ("occlude") hydrogen gas. Iron takes up about 19 times its own volume, platinum 50 volumes, and palladium from 500 to 800 volumes.

Liquid hydrogen, when allowed to evaporate rapidly, freezes to a colorless solid, which melts again at $-260^{\circ}$. 
Chemical Properties of Hydrogen.- That hydrogen, when it burns in the air, forms water, was first shown by Cavendish (1781). A test-tube or beaker, filled with cold water and held over a flame of burning hydrogen, will condense the steam to droplets of water (Fig. 28).

\section{Hydrogen + Oxygen $\rightarrow$ Water.}

The union is very violent, so that when a mixture of hydrogen with pure oxygen is set on fire (they do not unite when cold) much heat is liberated in the explosion. The gases can be made

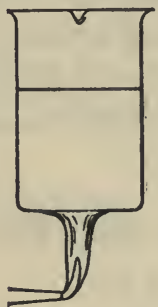

Fig. 28

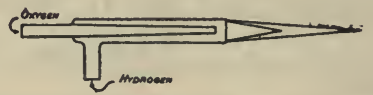

Fra. 29

to burn quietly, but with an exceedingly hot flame, by the use of an oxy-hydrogen burner (Fig. 29), which is constructed like a blast lamp. Iron melts and burns in the flame. A piece of
quicklime, held in the flame, glows with a brillant
white light - the calcium light (Drummond light or
lime-light). For such uses the gases are obtained in
compressed form in iron cylinders (Fig. 30).
Hydrogen combines vigorously with chlorine, giving
hydrogen chloride, a gas of which hydrochloric acid is
a solution. It unites with the three most active
metals in the list (p. 54). Calcium hydride is sold
under the name of hydrolyte, and is used, on ac-
count of its action on water, as a source of hydro-
gen.

Hydrogen acts upon many compounds containing oxygen, removing the latter to form water. Thus when the 
oxide of iron or of copper is heated in a tube in a stream of hydrogen, water is produced and the metal remains:

$\underset{\substack{\text { Mron } \\ \text { Oxygen }}}{\text { Magnetic oxide of iron }}+$ Hydrogen $\rightarrow$ Iron $+\underset{\substack{\text { Wyder. } \\ \text { Oxygen }}}{\text { Waten }}$

Oxides of metals above iron in the "order of activity" (p. 54), however, are very stable. Hydrogen is unable to remove the oxygen from such oxides and leave the metal.

Reduction.- The removal of oxygen from a compound by its union with some other substance is called reduction and the substance (in the foregoing instance, hydrogen) is called a reducing agent. Carbon, in the form of coal or coke, is the agent of this kind most commonly used in chemical industries. The term reduction is applied to some other chemical actions, in which oxygen is not concerned. In all cases, however, reduction is the opposite of oxidation (p. 41).

Exercises. - 1. Name three varieties of chemical change (pp. $8,16,51)$ and explain the difference between them.

2. What do you infer as to the composition of a substance when it is named: (a) an oxide, (b) an hydroxide (p. 51)?

3. What are the equivalent quantities of: (a) carbon and oxygen (p. 35), (b) zinc and sulphur?

4. What law shows that the ratio of chemically equivalent quantities of any two substances must be constant?

5. Name the metals which: (a) do not liberate hydrogen from water or dilute acids, (b) are found free in nature.

6. Why does hydrogen gas, when poured out, flow upwards? Why is it an excellent gas for filling balloons? 


\section{CHAPTER VI}

\section{WATER}

As oxygen is necessary to the life of plants and animals, so also is water. The human body is saturated with it, and water to make up for evaporation, as well as to aid in digestion, is a most necessary part of our food. The ocean covers about threefourths of the surface of the earth, and the "dry " land would be uninhabitable if it were really dry. The air always contains more or less water vapor.

Measurement of the Composition by Weight.-An arrangement by which the proportion by weight of hydrogen and

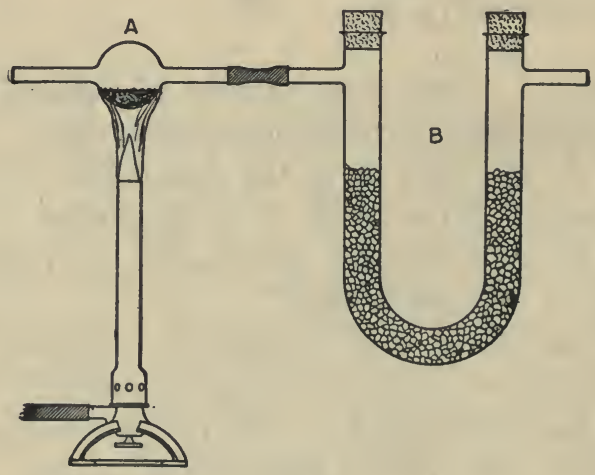

FIG. 31

oxygen in water can be determined is shown in Fig. 31 . The bulb $A$ contains cupric oxide, which is heated. Hydrogen from a generator or cylinder enters on the left and reduces the oxide: forming copper and water:

Hydrogen + Cupric oxide $\rightarrow$ Water + Copper. 
The water is carried as vapor by the excess of hydrogen and passes into the $U$-tube $B$. This tube contains calcium chloride, a substance which absorbs water greedily and is used therefore for drying gases. Here the water is all caught, while the hydrogen passes on. The tubes $A$ and $B$ with their contents are weighed just before, and again just after, the experiment. The loss of weight in $A$ is the weight of the oxygen. The gain in weight in $B$ is the water. The difference between these numbers is the hydrogen. It is found that the weights of hydrogen and oxygen thus ascertained always stand in a ratio close to 1 (Hyd.) : 7.94 (Ox.) or $1.008: 8$, the proportions accurately determined by Morley.

\section{Measurement of the Composition by Vol-} ume.-The proportions by volume in which hydrogen and oxygen combine may be shown by introducing the two gases into a tube, filled with mercury and inverted in a cylinder of mercury (Fig. 32). The volumes, at atmospheric pressure, are read by lowering the tube, after the introduction of each gas, until the levels of the mercury inside and outside are alike. A spark from an induction coil passed between the platinum wires inserted at the top of the tube causes the union of the gases. The water condenses to a slight dew and the volume of the single gas which remains is measured. Thus, if 19.5 c.c. of oxygen and 20 c.c. of hydrogen

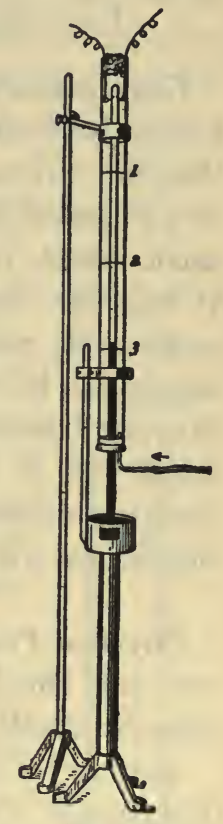

Fig. 32 are taken, the volume of gas remaining is 9.5 c.c. and this gas is afterwards found to be oxygen (test, p. 36). The volumes consumed were therefore $19.5-9.5=10$ c.c. of oxygen and 20 c.c. of hydrogen. The ratio by volume is therefore $2 \mathrm{Hyd}$ : $1 \mathrm{Ox}$. If these exact proportions are used, the mercury fills the tube 
after the explosion, but is apt to break it by striking the top violently.

By taking the gases in the exact ratio $1: 2$, and surrounding the tube by a wider one through which steam passes, the condensation of the resulting steam is prevented. It is found that, when all the gases are measured at the same temperature (here about $100^{\circ}$ ), a shrinkage of one-third occurs. That is to say:

1 vol. Oxygen +2 vols. Hydrogen $\rightarrow 2$ vols. Steam.

Gay-Lussac's Law of Volumes.-When other chemical actions between gases are studied in the same way, it is found that, in every case, the volumes of the gases used and produced in a chemical change can always be represented by the ratio of small whole numbers. This fact is exceedingly interesting. It was first discovered by Gay-Lussac in 1808. There is no such simple relation amongst the proportions by weight, which usually can be expressed only by large numbers (see p. 36) or by irregular fractions, so that this evidence of the existence of a simple rule in regard to combining proportions is the first we have encountered and is very welcome. The use made of it by the chemist will be developed in the next chapter.

Physical Properties. - Water is without odor or taste. It is very pale blue in color, as is shown when we look through a considerable depth of water.

Melting ice and freezing water have the same temperature. The point at which the mercury column of a thermometer stands, when the instrument is immersed in such a mixture of ice and water, is marked $0^{\circ}$ on the centigrade scale. This is the freezingpoint of water. The density of ice is little over nine-tenths that of water.

When water is heated, it gives off vapor more and more freely until-finally it boils. This point is recognized by the fact that 
bubbles of vapor form within the liquid, rise through it, and burst on the surface. The temperature of the water (if it is pure), and of the steam, are now found to be identical, and this thermometer reading is marked $100^{\circ} \mathrm{C}$., the boiling-point. Water is thus the standard substance used in the graduating of thermometers. When so used for fixing the temperature of $100^{\circ}$, the atmospheric pressure must be normal, that is $760 \mathrm{~mm}$., for the temperature of boiling is lower when the pressure is lower.

When water has reached the boiling-point, the temperature ceases to rise, and the heat supplied is used in changing the water into steam. The evaporation of 1 gram of water consumes 537 heat units or calories (heat of vaporization). The melting of 1 gram of ice consumes 79 calories (heat of fusion). The calorie is the average amount of heat required to raise 1 gram of water one degree in temperature between $0^{\circ}$ and $100^{\circ}$.

The amount of heat required to raise the temperature of a given mass of water one degree is greater than that required for an equal mass of any other common material. Hence, the temperature of the sea changes more slowly, and within a smaller range, than that of the rocks which compose the land. For this reason the climate of islands surrounded by much water is less variable from season to season within the year than is that of the continents.

Steam.- The tendency of water to evaporate at various temperatures is best measured by the gaseous pressure it exercises. A little water introduced into a barometric vacuum ( $B$, Fig. 33$)$ will depress the mercury, and the difference in height is a measure of the vapor pressure of the water. The

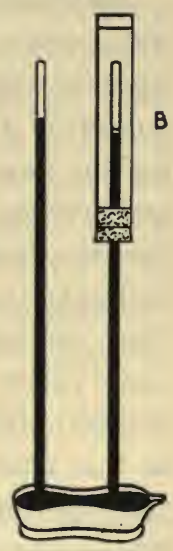

Fig. 33 temperature may be changed by putting hot water into the tube surrounding the barometer, and thus the increase in vapor pressure with rising temperature may be shown. At $0^{\circ}$ the column 
of mercury is depressed $4.6 \mathrm{~mm}$, at $10^{\circ}$ the vapor pressure becomes $9.2 \mathrm{~mm}$., at $20^{\circ} 17.4 \mathrm{~mm}$. (see Appendix IV). At $100^{\circ}$ the level of the mercury in the tube would be depressed $760 \mathrm{~mm}$., and would sink to the level of that in the trough. If a little air is first placed above dry mercury, causing it to fall, the additional depression produced by adding water is the same as if the air had been absent (p. 47).

Steam at $100^{\circ}$, subjected to a pressure over one atmosphere, is condensed to water. Steam cannot be condensed by pressure, however, if the temperature is over $374^{\circ}$, its critical temperature. At $100^{\circ}$ the steam occupies nearly 1600 times the volume of an equal weight of water.

Steam is a perfectly invisible gas. The visible cloud of fog, issuing from a valve when steam escapes, is composed of minute drops of water formed by condensation.

Molecular Relations of Liquid and Vapor.-When the water was introduced above the barometric column, the vapor, or gaseous water, could have resulted only from the spontaneous motion of the molecules in the liquid. Some of the molecules, moving near the surface, went off into the space above the water and became gaseous. To be consistent, we must also conclude that the vapor above the water is not composed of the same set of molecules one minute as it was during the preceding minute. Their motions must cause many of them to plunge into the liquid, while others emerge and take their places. When the water is first introduced, there are no molecules of vapor in the space at all, so that emission from the water predominates. The pressure of the vapor increases as the concentration of the molecules of vapor becomes greater, hence the mercury column falls steadily. At the same time the number of gaseous molecules plunging into the water per second must increase in proportion to the degree to which they are crowded in the vapor. The rate at which molecules return to the water thus begins at zero, and increases 
steadily; the rate at which molecules leave the water maintains a constant value. Hence the rate at which vapor molecules enter the water must eventually equal that at which other water molecules leave the liquid. At this point, occasion for visible changes ceases and the mercury comes to rest. We are bound to think, however, of the exchange as still going on, since nothing has occurred to stop it. The condition is not one of rest but of rapid and equal exchange. Such, described in terms of molecules, is the state of affairs which is characteristic of a condition of equilibrium. The condition is dynamic, and not static.

Equilibrium.- This term is used so often in chemistry, and is used in so unfamiliar a sense, that the reader should consider attentively what it implies. Three things are characteristic of a state of equilibrium:

1. There are always two opposing tendencies which, when equilibrium is reached, balance each other. In the foregoing instance, one of these is the hail of molecules leaving the liquid, which is constant throughout the experiment. It represents the vapor tension of the liquid. The other is the hail of returning molecules, which, at first, increases steadily as the concentration of the vapor becomes greater. This is the vapor pressure of the vapor. These have the effect of opposing pressures and, when the latter becomes equal to the former, equilibrium is established. In all cases of equilibrium we shall symbolize the two opposing tendencies by two arrows, thus:

$$
\text { Water (liq.) } \rightleftarrows \text { Water (vapor). }
$$

2. Although their effects thus neutralize each other at equilibrium, both tendencies are still in full operation. In the case in point, the opposing hails of molecules are still at work, but neither can effect any visible change in the system. Equilibrium is a state, not of rest, but of balanced activities.

3. (and this is the chief mark of equilibrium.) A slight change

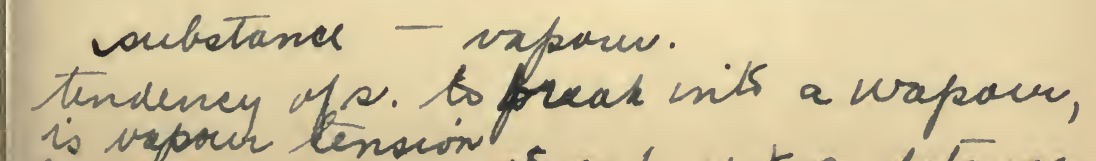


in the conditions produces, never a great or sharp change, but always, and instantly, a corresponding small change in the state of the system. The change in the conditions accomplishes this by favoring or disfavoring one of the two opposing tendencies. Thus, for example, when the temperature of a liquid is raised, the motion of its molecules is increased, the rate at which they leave its sur- face beeomes greater, the vapor tension increases and, hence, a greater concentration of vapor can be maintained. The system, therefore, quickly reaches a new state of equilibrium in which a higher vapor pressure exists.

In the preceding illustration, the evaporating tendency was favored by a rise in temperature. As an example of a change in conditions disfavoring one tendency, take the case where the liquid is placed in an open, shallow vessel. Here the condensing tendency is markedly discouraged, for there is less chance of return of the emitted molecules. Hence complete evaporation finally takes place. Elevation of the temperature hastens the process. A draft insures the diffusion of the vapor away from the surface of the liquid, and has therefore the same effect. The two methods of assisting the displacement of an equilibrium, and particularly the second, in which the opposed process is weakened and the forward process triumphs solely on this account, should be noted carefully. They are applied with surprising effectiveness in the explanation of chemical phenomena.

The States of Matter.- Most substances are known in at last three different states, namely, a crystalline (or solid), a liquid, and a gaseous form. There is no magic about the number three, however. Very many substances are known in more states than three. Thus sulphur has a vapor state, two liquid states, and several different crystalline forms. There are no fewer than five forms of ice, different in physical but identical in chemical properties.

When we wish to transform a substance from one state to 
another, we change its conditions (see p. 7). Thus under atmospheric pressure water is converted to ice by reducing the temperature below $0^{\circ}$, and to steam by raising it above $100^{\circ}$. Under a pressure of 100 atmospheres, however, water freezes at $-1^{\circ}$, and boils only at $330^{\circ}$. With still higher pressures, the change in the freezing-point is much more marked. Thus under 2000 atmospheres pressure water does not freeze above $-20^{\circ}$. If the pressure is increased further, different crystalline forms of ice make their appearance, and the freezing-point rises again until under 20,000 atmospheres pressure water is found to freeze at $+78^{\circ}$ !

Water as a Solvent: Natural Waters.-Water has a remarkable power of dissolving many other substances, and is said, therefore, to have great power as a solvent. Rain is the purest natural water. As it is formed by condensation of water vapor, and has been in contact with the atmosphere only, it contains only oxygen and other gases dissolved from the air, together with a little dust. Sea water contains the greatest amount of dissolved material, namely about 3.5 per cent. River and, especially, well waters contain materials in solution which have been dissolved from the soil and the rocks. These materials act chemically upon soap so that well waters are more or less hard, while rain water is soft. We have already learned (p. 40) that natural waters may also contain bacteria, which give rise to putrefaction and disease. Many river waters contain large amounts of clay and other insoluble substances suspended in them. The suspended matter can be seen, as it renders the water turbid, but the bacteria are invisible, so organic matter and bacteria may be present in waters which look perfectly clear.

Purification of Water.- The suspended impurities, including the bacteria, may be removed by filtration. City waters are often filtered through extensive beds of gravel, but this treatment 
will not remove all bacteria. In many cases, small amounts of alum, or alum and lime, are added to the water, and the suspended matter is then allowed to settle, which it does very quickly, in large basins or reservoirs. By this coagulation

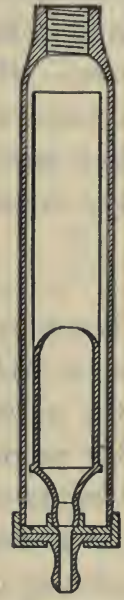

FIG. 34 method (p. 470), all but a few of the bacteria are removed. Sometimes the remaining organisms are destroyed by adding a little bleaching powder before the water is distributed (p. 226).

In the household, the simplest appliance is the Pasteur filter. It consists of a tube of unglazed porcelain, closed at one end (Fig. 34), through the pores of which the water is forced by its own pressure. The cylinder (" bougie") should be cleaned daily with a brush to remove the mud and the organisms which collect in its surface.

All forms of filters must be cleaned at short intervals. If this is not done, the organisms multiply and soon the filter pollutes the water instead of purifying it.

Filtration does not remove dissolved matter, and therefore does not soften hard water (p. 13). For this latter purpose washing powders are used in the laundry (see p. 390-1).

All organisms can be killed by boiling unfiltered water, but the boiling should continue for at least 10 to 15 minutes to be effective.

Pure water for chemical purposes is prepared by distillation (Fig. 35). Dissolved solids remain in the flask (or boiler). The steam is condensed by cold water circulating in the jacket of the condenser. Freshly distilled water contains only gases dissolved from the air. If kept in a vessel, however, such water quickly dissolves traces of glass or porcelain. The purest water is made by using a platinum tube in the condenser and a platinum bottle as the receiver.

Chemical Properties: Stability.-As we should infer from the vigor with which its constituents combine, water is a very 
stable substance. When steam is superheated, hardly a trace of decomposition occurs. Even when the temperature reaches $2000^{\circ}$ (far above a white heat), only 1.8 per cent of the vapor is

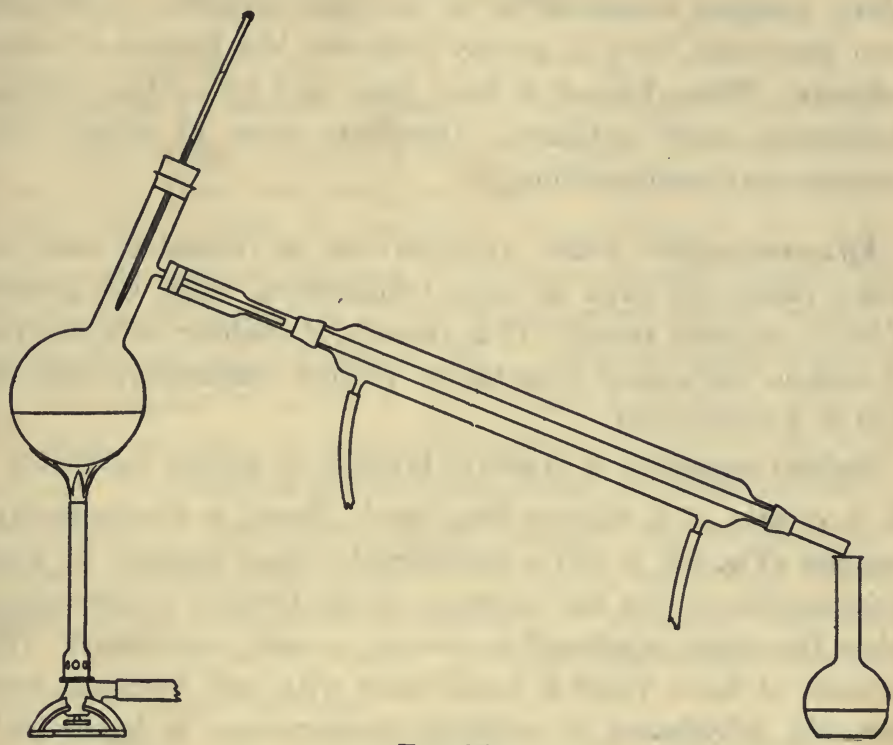

FIG. 35

broken up into oxygen and hydrogen. When the steam cools, these elements recombine:

$$
\text { Water } \rightleftarrows \text { Hydrogen }+ \text { Oxygen. }
$$

Hydrates.-Many substances unite with water to give compounds called hydrates. Thus if we take zinc sulphate (p. 52) and dissolve it in water and allow the excess of the latter to evaporate, the solid appears in long transparent crystals. When these are dried with blotting paper and heated in a test-tube, they give off a large amount of steam. The hydrate of zinc sulphate decomposes and leaves anhydrous (Greek, deprived of water) zinc sul- 
phate. The latter, when once more moistened, changes back into the hydrate.

Zinc sulphate + Water $\rightleftarrows$ Hydrate of Zinc sulphate.

Many common chemicals are in fact such hydrates. Thus common blue-stone, used in gravity batteries, is a hydrate of cupric sulphate. When heated, it loses water and leaves the colorless, anhydrous cupric sulphate. These are cases of simple combination and decomposition.

Efflorescence.-Some hydrates are so unstable that the water passes off, even at room temperature, when the hydrate is left in an open vessel. Thus crystals of washing soda (hydrate of sodium carbonate) crumble to powder (effloresce) when not kept in a closed vessel.

Sodium carbonate + Water $\rightleftarrows$ Hydrate of sodium carbonate.

If a crystal of a hydrate like this is placed in the barometric vacuum (Fig. 33, p. 61) a considerable vapor pressure of water is indicated, so that the tendency of the hydrate to decompose, when this vapor is allowed to escape, is easily understood. The pressure of water vapor in equilibrium with such hydrates, when partially dehydrated at ordinary temperatures, is found to be greater than the average pressure of water vapor in the atmosphere.

On the other hand, when anhydrous cupric sulphate and zinc sulphate, obtainable from the hydrates by heating only, are spread out in the air, they return slowly to the hydrated condition. They combine with the moisture in the air. The vapor pressure of water in the air is greater than the pressure of water vapor in equilibrium with these hydrates and their anhydrous products at ordinary temperatures. Calcium chloride absorbs water vapor (p. 59) because of its tendency to form a hydrate.

Other Chemical Properties.- Water combines directly with some oxides. Its union with quicklime yields slaked lime or 
calcium hydroxide. Other oxides, for example sulphur dioxide or phosphorus pentoxide, give acids (sulphurous acid or phosphoric acid). Cases in which water acts chemically upon substances dissolved in it will be noted later (p. 145).

Reversible Chemical Actions. - The contrary effect upon an unstable hydrate of leaving the bottle open or closed, referred to on p. 68, deserves a moment's notice. When understood, it explains many things in chemistry. The hydration and dehydration are opposite directions of the same chemical change, and the condensed statements of the actions were written with the double arrow to indicate this (p. 68). When the bottle is closed, the water vapor is unable to escape and recombines with the anhydrous particles as fast as other particles of the hydrate decompose. A reversible action therefore can not complete itself, if the products of the action are kept together and not allowed to separate. On the other hand, a reversible action will go to completion, if one of the products escapes, as the water vapor does when the bottle is left open. This idea enables us to answer several interesting questions.

For example, why does steam decompose to the extent of 1.8 per cent at 2000 degrees, but not any further? All its parts are alike and are therefore equally capable of decomposing. The answer is, because neither the oxygen nor the hydrogen is removed, or can easily be removed, from the steam, and so the completion of the decomposition is prevented by continual recombination of these gases.

When a reversible action has come to a standstill, we say that equilibrium has been reached. This means that two opposing tendencies are neutralizing one another's effects.

When will reversible actions go to completion? The products must be of such a nature that they separate easily. In practice this happens when one is a gas or vapor, like the water vapor 
coming from a hydrate, while the other is not. The settling of one product as a precipitate, while the other stays in solution, is, as we shall see (pp. 127, 129), another common way in which the separation occurs.

When iron and water are heated in a closed vessel, the hydrogen and oxide of iron which are produced (p. 51) react with one another to give back water and iron.

Iron $+\underset{\substack{\text { Steam } \\ \text { Oxygen }}}{\text { Strongen }} \underset{\text { Hydrogen }}{\rightleftarrows}+$ Magnetic oxide of iron.

In a closed vessel we could never use this method for preparing any quantity of hydrogen. To prepare hydrogen by this action we must leave the tube open, and let the steam sweep the hydrogen out. This separates it effectually from the oxide of iron, and prevents the reversal of the action.

Devices depending on mechanical principles like this are continually used in chemistry for securing easy methods of preparing substances.

The reader can now answer for himself the question why we are able to prepare oxygen by heating mercuric oxide (p. 15), in spite of the fact that the action is reversible (p. 29).

Exercises.- 1. If 50 c.c. of hydrogen and 37 c.c. of oxygen are exploded in a closed tube, which gas remains and what volume of it is left?

2. Why do not bubbles of steam ordinarily form in water and rise through it at temperatures below $100^{\circ}$ ?

3. How many calories of heat would be required to change 5 grams of ice at $0^{\circ}$ into steam at $100^{\circ}$ ?

4. How could one find out how much solid matter was dissolved in a sample of water?

5. Define: filtration, distillation, efflorescence, chemical equilibrium. 
6. Explain how water, even at room temperature, gradually dries up (p. 64).

7. Why does a strong wind hasten the evaporation of water (p. 64)?

8. Why does the lower surface of a sheet of ice on the pavement melt in the sun before the upper one? 


\section{CHAPTER VII}

\section{CHEMICAL UNITS OF WEIGHT. FORMULAE}

As we have seen (p. 60), when the volumes occupied by substances in the gaseous condition, rather than the weights, are taken as the basis of measurement, the combining proportions are simple and are expressible by small whole numbers (Gay-Lussac's law). This shows that there must be some relationship, connected with chemical combination, between the amounts of different substances contained as gases in equal volumes. It suggests that we might do well to take such amounts (weights of equal volumes) as the standard or unit quantities for chemical purposes. Now this is precisely what the chemist has found it in practice most convenient to do, and the present chapter deals with the units of material based upon comparing equal volumes.

Illustrations of Gay-Lussac's Law. - Let us first familiarize ourselves with the volume-measuring point of view in chemical actions. The following are a few observed facts, beginning with the union of hydrogen and oxygen already discussed (p. 59):

(1) Hydrogen (2 vols.) + Oxygen (1 vol.) $\rightarrow$ Steam (2 vols.).

(2) Hydrogen (1 vol.) + Chlorine (1 vol.) $\rightarrow$ Hydrogen chloride ( 2 vols.).

(3) Chlorine monoxide (2 vols.) $\rightarrow$ Chlorine (2 vols.) + Oxygen (1 vol.).

(4) Mercuric oxide (not volatile) $\rightarrow$ Mercury (2 vols.) + Oxygen (1 vol.).

(5) Phosphorus (1 vol.) + Oxygen (5 vols.) $\rightarrow$ Phosphorus pentoxide ( 1 vol.).

(6) Zinc (at $1000^{\circ}, 2$ vols.) + Sulphur (at $1000^{\circ}, 1$ vol.) $\rightarrow$ Zinc sulphide (not volatile) 
It will be noted that in some cases, like (2), there is no change in the total volume. In others there is a shrinkage, as in (1) and (5). In still others, like (3), where chlorine monoxide decomposes, there is an increase in volume. In (5), in order that all the materials may be gaseous, the whole experiment must be done at a very high temperature (and at some suitable pressure). In (4) the mercuric oxide itself does not become gaseous, but decomposes, so that its own relative volume cannot be given. In (6) the zinc and sulphur can be combined as vapors at $1000^{\circ}$. The product (zinc sulphide) will not remain gaseous at any temperature at which its volume could be measured, however, and so its volume is not recorded.

It must be kept constantly in mind that the law applies to volumes in the state of gas or vapor only. There is no rule about the proportions by volume required for the chemical combination of liquids and solids.

One can read these illustrations in different ways. For example: (1) A given volume of steam is formed by union of the same volume of hydrogen with half as great a volume of oxygen. (4) Mercuric oxide, when decomposed by heating, gives two volumes of mercury vapor and one volume of oxygen in every three volumes of the escaping gases. (5) One volume of phosphorus vapor, together with an equivalent quantity of oxygen, will give one volume of the vapor of phosphorus pentoxide, all being measured at the same temperature. In fact, whenever two vaporizable substances are amongst the factors and products of a chemical change, their volumes thus ${ }^{\text {are }}$ either equal, or are to one another in the ratio of whole numbers.

The Standard or Unit Volume.- The volumes in the foregoing paragraph are simply relative, and the statements are true of gaseous volumes of any actual dimensions (large or small), provided only they bear the proper relationship, such as $2: 1$, $1: 1$, or $1: 5$, in each case. An actual value has been chosen, 
however, for the volume which is the standard or unit in chemistry. This is the volume occupied by 32 grams of oxygen, which is $\mathbf{2 2 . 4}$ liters at $0^{\circ}$ and $760 \mathrm{~mm}$. This volume is equal to that of a cube about 11 inches in height (Fig. 36). At other temperatures and pressures this volume, in order to contain the same amount of material, alters its value, in accordance with the laws of Boyle and Charles (p. 45). The reason for selecting this particular volume will be readily seen, so soon as we shall have

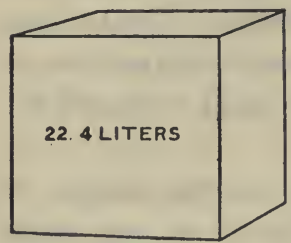

FIG. 36 presented the actual weights of various materials which fill it.

\section{The Weights Filling the Unit Vol-} ume, 22.4 Liters. - The following table contains a few sample substances, and gives the weight (in grams) of each which, in the gaseous condition, at $0^{\circ}$ and $760 \mathrm{~mm}$., occupies the cube -22.4 liters. In the cases of compound substances, like water, we have given also the weights of the constituent elements which together make up the total weight of the compound filling the unit volume:

Weights of Gases in 22.4 Liters (at $0^{\circ}$ and 760 mm.).

\begin{tabular}{|c|c|c|c|c|}
\hline Substance & Total wt. & Wt. ox. & Wt. hyd. & Wt. chlor. \\
\hline $\begin{array}{l}\text { Oxygen............. } \\
\text { Water.............. } \\
\text { Hydrogen........ } \\
\text { Hydrogen chloride } \\
\text { Chlorine......... } \\
\text { Chlorine monoxide }\end{array}$ & $\begin{array}{l}32 \\
18.016 \\
2.016 \\
36.468 \\
70.92 \\
86.92\end{array}$ & $\begin{array}{c}32 \\
16 \\
\cdots \cdots \\
\cdots \cdots \\
\cdots \cdots \\
16\end{array}$ & $\begin{array}{c}2.016 \\
2.016 \\
1.008 \\
\ldots \ldots \ldots \\
\ldots \ldots \ldots\end{array}$ & $\begin{array}{l}35.46 \\
70.92 \\
70.92\end{array}$ \\
\hline
\end{tabular}

The first column ("Total wt.") gives the number of grams of each substance occupying, as a gas, 22.4 liters at $0^{\circ}$ and $760 \mathrm{~mm}$. This is the standard or unit weight of that substance. In the case of water, which is a liquid at room temperatures and pressures, a known volume of steam is weighed. The volume is reduced by 
rule to $0^{\circ}$ and $760 \mathrm{~mm}$., and the weight of 22.4 liters is calculated from the reduced volume and the measured weight.

These standard or unit weights of substances are commonly called molecular weights (see p. 83).

Unit or Standard Weights of the Elements.- Let us now examine the weights of the constituent elements making up a cubeful of each substance, as shown in the last three columns of the above table. We must first be sure we understand what these numbers are. They are combining proportions, such as we have given on previous occasions (e.g., pp. 15, 20,31). They are equivalents (p. 53). We can use them in our condensed form for representing chemical changes:

Oxygen (16) + Hydrogen (2.016) $\rightarrow$ Water (18.016).

Hydrogen (1.008) + Chlorine (35.46) $\rightarrow$ Hydrogen chloride (36.468).

We observe at once that the weights in the oxygen column, or the chlorine column, for example, are not identical. There was no reason to expect that they would be alike, since different substances differ in composition. But we do observe that the weights of any one element are all exact multiples of the smallest number in its column, either by unity or some other whole number. Thus, for hydrogen, the weights are: 2.016, 2.016 and 1.008. The unit weight of water contains exactly the same weight of hydrogen as does the unit weight of hydrogen itself, and exactly twice as much as does the unit weight of hydrogen chloride. Similar relations hold in the oxygen and chlorine columns. This is a very surprising, natural fact, and, better still, one for which we instantly perceive a use. This fact greatly simplifies our task of finding some way of expressing the compositions of substances in a simple manner. The fact does not apply to a few compounds only. If our table had included all the hundreds of compounds of chlorine (for example) which are capable of being converted into vapor, we should have found, indeed, many multiples of 35.46 larger than the two units (70.92) in chlorine monoxide, but no number smaller 
than 35.46 and none which was not a multiple of 35.46 by a whole number. Clearly we shall find it convenient to accept 35.46 as the unit or standard weight of chlorine for expressing the compositions of its chemical compounds. Its use will enable us to state the exact composition of any given compound by simply giving the whole number $(1,2,3$, etc.) by which the basal weight 35.46 is to be multiplied in the given case.

Similarly, we take 1.008 as the unit weight of hydrogen and 16 as the unit weight of oxygen. By including volatile compounds of other elements in our investigation, we can similarly pick out the most convenient unit for each element. Those unit weights for elements are often called also combining or reacting weights, and still more frequently atomic weights (see p. 86). A complete list of their values for all the known elements is given in a table inside the rear cover of this book. These numbers will hereafter be in constant use.

The following table presents the results obtained from a larger number of substances and gives their formulæ (see p. 78):

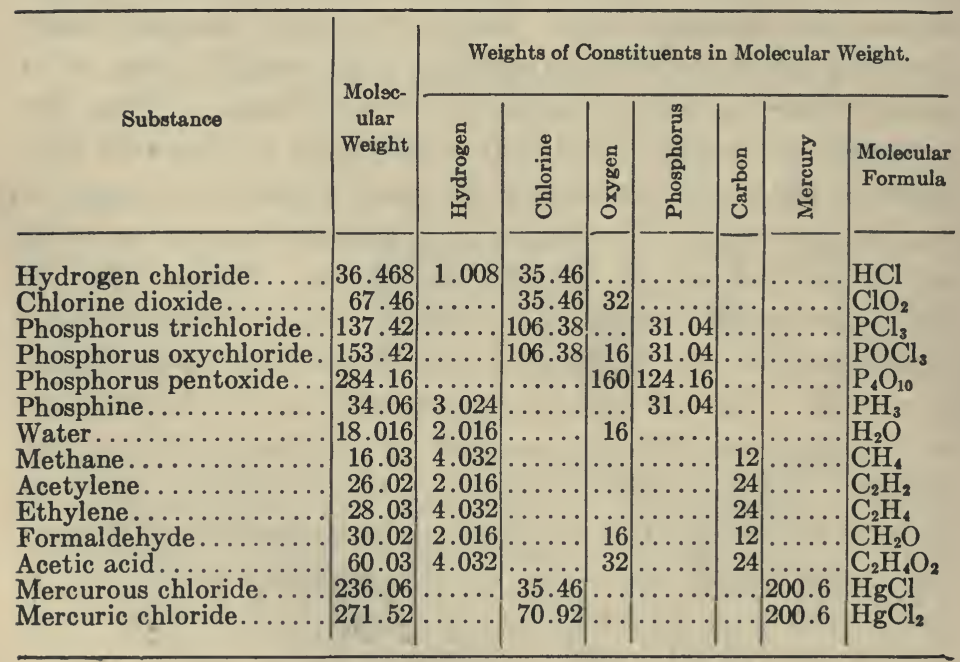


The Case of Non-volatile Compounds.- The same elements enter also into many compounds which are not volatile. But the unit weights of such elements, determined by the use of volatile compounds, are found (by using multiples, when necessary) to express the composition of the involatile compounds also.

For example, the unit weight for oxygen (16) and that for mercury (200.6), both formed by studying volatile compounds, are found correctly to express the composition of mercuric oxide (p. 20), which is not volatile.

In the cases of some elements no easily vaporizable compound is known, and the unit weight cannot be determined by the present method. In such instances, an entirely different way of obtaining the value of the unit weight is employed (see p. 87).

The Law of Combining Weights. - The general fact which we have developed in the preceding sections is known as the law of combining weights: All the proportions in which the elements combine with one another may be represented by a set of numbers (one for each element) or by multiples of these numbers by whole numbers.

Definition of Reacting or Atomic Weight. - Many different values for each element would satisfy the law of combining weights as stated above (see, for example, the values given for oxygen on p. 10). The particular values chosen as units in this chapter, however, fulfil an additional condition which fixes the value in each case, absolutely. The chosen reacting or atomic weight of an element is the smallest weight of the element found in 22.4 liters (at $0^{\circ}$ and $760 \mathrm{~mm}$.) of the vapor of any volatile compound of that element (p. 74). The amounts of the element in 22.4 liters of other compounds are all either the same amount or multiples thereof by a whole number.

Simplification of Condensed Expressions for Chemical Actions - Symbols. - Our condensed expressions for chemical 
changes showed the name of each substance and also weights to indicate the combining proportions. Having now found a unit weight for each element, we can condense the statement still further, by using a letter or pair of letters, called a symbol, to stand for one chemical unit weight of each element. Thus $\mathrm{O}$ stands for one unit or 16 parts of oxygen, $\mathrm{H}$ for one unit or 1.008 parts of hydrogen, $\mathrm{Cl}$ for one unit or 35.46 parts of chlorine. A pair of letters is required when the names of several elements begin with the same letter. Thus, C stands for 12 parts of carbon, Ca for 40 parts of calcium, $\mathrm{Cr}$ for 52 parts of chromium, each of these amounts being one chemical unit of the element. These symbols are international, and are alike in all languages. In some instances they are based upon the Latin name for the element. Thus, Fe stands for 55.84 parts of iron (ferrum), Sn for 119 parts of tin (stannum), Ag for 107.88 parts of silver (argentum) and $\mathrm{Hg}$ for 200.6 parts of mercury (hydrargyrum). Again K stands for 39.1 parts of potassium (German, kalium) and $\mathrm{Na}$ for 23 parts of sodium (German, natrium).

Formulae. - The composition* of a substance can be shown briefly by putting together the symbols of the constituent elements, and using numbers for the multiples of the unit weights, where such are required. The resulting expression is called a formula. In the last column of the table on p. 76 the formulæ of the various substances therein considered are indicated. Thus water contains oxygen 16 parts $(0)$ and hydrogen 2.016 parts $(=2 \times 1.008=$ $\mathrm{H}_{2}$ ), and receives the formula $\mathrm{H}_{2} \mathrm{O}$. Hydrogen chloride contains hydrogen 1.008 parts $(\mathrm{H})$ and chlorine 35.46 parts $(\mathrm{Cl})$, and its formula is $\mathrm{HCl}$.

It must be noted particularly, that the formula, to be consistent, must represent also the total weight of the unit quantity of the substance (wt. of 22.4 1.) in the gaseous state. Thus, $\mathrm{HCl}(1.008$

* The composition means the names of the elements contained in the substance, and also the proportion by weight of each element (see p. 38). 
$+35.46=36.468$ ) is correct, since $36.468 \mathrm{~g}$. is the amount filling the cube. Again, the formula for oxygen gas itself must represent $32 \mathrm{~g}$. $(=2 \times 16)$, the weight of $22.4 \mathrm{l}$., and is therefore $\mathrm{O}_{2}$. Half as much as this may enter into a compound, $\mathrm{H}_{2} \mathrm{O}, \mathrm{ZnO}$, etc., but, logically, the formula for free oxygen must record the double weight required to fill the cube when the gas is present alone, in the free condition (see pp. 86, 105). Similarly the formula for hydrogen gas is $\mathrm{H}_{2}(2 \times 1.008=2.016$, the weight of 22.41 .). The formula of every volatile substance must thus be written so as to show the weight of the chemical unit quantity (the molecular weight, p. 84). When the substance is not easily volatilized, this unit cannot be measured, and the simplest formula is employed.

Information Contained in Each Formula.-A formula thus contains, in condensed form, several items of information. It shows:

1. The elements making up the substance,

2. The proportion by weight of those elements,

3. The total unit weight (molecular weight) of the substance.

Given the formula, we can read these facts in it.

Thus, if we are given the formula of carbon dioxide, $\mathrm{CO}_{2}$, we consult the table of reacting or atomic weights (inside rear cover), and learn that $\mathrm{C}=12$ parts by weight of carbon and $\mathrm{O}_{2}=2 \times 16$ parts by weight of oxygen. The proportion by weight of the elements in this compound is, therefore, 12 of carbon to 32 of oxygen. The total weight (molecular weight) is $12+32=44$, and this must be the weight filling 22.41 . (at $0^{\circ}$ and $760 \mathrm{~mm}$.).

Why was 22.4 Liters Chosen as the Unit Volume? - It is clearly advantageous to employ a unit volume of such dimensions that no element shall receive a unit weight smaller than unity. At first sight, indeed, it would seem self-evident that we ought to choose, for our own convenience, a unit volume based on 
a smallest unit weight of exactly 1 . Now hydrogen is the element which enters in the smallest proportions into chemical combination, and we have seen that in 22.41. of some compounds there is as little as $1.008 \mathrm{~g}$. of this element (see table, p. 76).

If a slightly smaller unit volume (22.2 1.) had been chosen, hydrogen would possess a unit weight of exactly 1 . Why was not, it will be asked, this volume selected instead of 22.41 . as our standard? It was, in fact, at one time the standard, the unit weight of oxygen being then about 15.9 and the unit weights for all of the other elements being correspondingly reduced. The transfer to the present scale $\mathrm{O}=16$ was made for a purely practical reason; because of the greater activity of oxygen (see p. 27), the exact determinations of the combining proportions of most other elements are generally given by experiment on an oxygen, not on a hydrogen, basis. It so happens, also, that the majority of atomic weights on the scale $\mathrm{O}=16$ are very close to whole numbers (see table), an aid to calculations being thereby afforded which does not exist under the standard $H=1$. Recent work on the " complexity" of elements, it may be added, has given further justification of our choice of the oxygen standard here adopted (see p. 552).

The accepted scale is, therefore, that of 32 for the unit weight of oxygen gas; $22.4 \mathrm{l}$. (the volume of $32 \mathrm{~g}$. of free oxygen) for the chemical unit of volume; and 16 for the unit weight of oxygen in compounds containing that element. The unit weights of all the other compounds and elements are based upon this scale.

Further Discussion. - At this point we have completed the explanation of the derivation of the units of weight, and of the symbols and formulæ used in chemistry. We are now in a position to proceed with the application of these conceptions, as they are developed in Chapter IX. This would be possible, at least so far as strict logic is concerned. But, in shaping our course so as to reach the results by the shortest route, we have omitted 
a number of interesting and useful ideas. These were not indispensable links in our reasoning, but, now that we have covered the essential steps, a consideration of these ideas will be of great value as a review of the subject and a means of mastering it thoroughly. Formulæ and their uses come up so constantly in every part of the science that we must not omit any means of securing a perfect understanding of them. The following chapter is therefore devoted to restating in other ways certain parts of the same subject. 


\section{CHAPTER VIII}

\section{APPLICATION OF THE MOLECULAR HYPOTHESIS IN CHEMISTRY}

Avogadro's Hypothesis. - We have seen (p. 48) that the physical behavior of matter, and particularly of gases, may be explained by the conception that matter is composed of molecules. The easy compressibility of gases is, under this view, a consequence of the smallness of the molecules and the relative vastness of the empty space between them. The pressure exerted by a gas is regarded as due to the innumerable blows which the molecules deliver when they strike the boundary walls of the space in which the gas is confined. Now, when gases interact chemically, the volumes required for complete interaction are either equal or stand in the ratio of small whole numbers (Gay-Lussac's law, p. 72). Although that is a chemical, and not merely a physical fact, the molecular hypothesis can explain it also.

Since matter is composed of molecules, a chemical action between two kinds of matter must consist really in an interaction of the molecules of each kind. Molecules of the two kinds must meet and they may then either combine to form a compound molecule, or they may exchange material in some fashion. Since equal volumes are often the exact quantities required for the action, it appears most likely that in equal volumes of different gases (at the same temperature and pressure) the numbers of molecules present are equal. This addition to the molecular hypothesis was first suggested by an Italian physicist, Avogadro (1811). When two gases interact in equal volumes (like hydrogen and chlorine), one molecule of each is all that is required for a small sample of the change in question. Since two volumes of hydrogen are required to unite with one volume of oxygen (p. 60), clearly the interaction involves two molecules of hydrogen for 
every one of oxygen. Since two volumes of steam are produced, evidently the two molecules of hydrogen and one of oxygen yield two molecules of water.

This hypothesis of Avogadro helps us to a clearer notion of how these chemical changes take place. The idea that all gases contain equal numbers of molecules in equal volumes also explains why all gases behave alike when subjected to equal pressures, or to equal changes in temperature (Boyle's and Charles' laws, p. 45). No facts which conflict with this hypothesis are known, and all the known facts confirm it. Hence, Avogadro's hypothesis has been accepted by chemists, and since 1858 has been the keystone of chemical theory.

\section{Consequences of Avogadro's Hypothesis-Molecular} Weights. - Equal volumes of the same gas (at the same tem. perature and pressure) have equal weights. But equal volumes of different gases have different weights. The differences are often very great. Thus, bulk for bulk, oxygen is sixteen times as heavy as hydrogen, and mercury vapor one hundred times as heavy. Now, if equal volumes of different gases contain equal numbers of molecules, these differences must be due to the differing weights of the several kinds of molecules. Thus, measuring the weights of equal volumes of different gases will give us the relative weights of their molecules. For example, since 22.4 l. of oxygen weigh $32 \mathrm{~g}$. (p. 74), while the same volume of hydrogen weighs $2.016 \mathrm{~g}$. and of water vapor $18.016 \mathrm{~g}$., and these are the weights of equal numbers of molecules, the individual molecules must differ in weight in the ratio $32: 2.016: 18.016$. These are the relative weights of the three kinds of molecules. In chemistry the weights of 22.41 . (at $0^{\circ}$ and $760 \mathrm{~mm}$.) of various gases are called the molecular weights of those gases. The unit quantities of various substances are therefore spoken of, technically, as the molecular weights of those substances. The unit volume, 22.4 l., is called the gram-molecular volume (G.M.V.). 
The numoer of molecules actually contained in the G.M.V. has been determined by several independent methods, the agreement of the results obtained furnishing very strong support for the validity of the molecular hypothesis. The value at present accepted as most accurate is that of Millikan, $6.06 \times 10^{23 *}$. It is of importance that the student should obtain some idea of the significance of this stupendous number, in order to appreciate the more detailed discussion of the properties of gases in the following sections (p. 88-93). Imagine a G.M.V. of a gas at $0^{\circ}$ and 760 $\mathrm{mm}$. to be divided equally among the inhabitants of the United States (say 110,000,000). Each person would receive about onefifth of a cubic millimeter as his share. Imagine, further, that the market price of a million molecules of this gas was one cent. Few of the recipients would think it worth while to cash in their microscopic sample; those doing so, however, would benefit to the extent of over 50 million dollars!

Molecular Weight, Measurement of.-The molecular weight is measured by weighing any convenient volume of the gas (say 200 c.c.), and calculating by proportion the weight of 22.41 . If the gas or vapor was not measured at $0^{\circ}$ and $760 \mathrm{~mm}$., the measured volume must be reduced by rule to standard conditions before the weight of 22.41 . is calculated. $\dagger$

* This is the method used in physics and chemistry to express numbers so large as to be cumbrous and incomprehensible if written in the ordinary way. It means that the unit is to be followed by the number of zeros indicated by the exponent. In this case, the quantity written out at length would be $606,000,000,000,000,000,000,000$.

† In practice, owing to the fact that Boyle's and Charles' laws do not describe the behavior of any known gas exactly (they apply only to a "perfect" gas), certain additional, small corrections have to be applied when very precise values are required. It may be noted that, in order to make the fundamental significance of the numerical data presented in the preceding pages immediately intelligible to the beginner, all of the gases so far considered have been assumed to be "perfect." The actual experimental values differ only very slightly from those given. 
For example, 190 c.c. of a gas at $0^{\circ}$ and $760 \mathrm{~mm}$. weigh $1.23 \mathrm{~g}$ If $x$ be the weight of 22.4 liters ( $=22,400$ c.c.),

$$
190: 1.23:: 22,400: x \text { (= } 145 \text { g.). }
$$

Again, 210 c.c. of a vapor at $100^{\circ}$ and $743 \mathrm{~mm}$. weigh $1.12 \mathrm{~g}$. This volume at $0^{\circ}$ and $760 \mathrm{~mm}$. would become:

$$
210 \times \frac{273}{373} \times \frac{743}{760}=150.3 \text { c.c. }
$$

and

$$
150.3: 1.12:: 22,400: x \text { (=167 g.). }
$$

Molecular Weight and Density. - Density is the term used in physics for the weight of 1 cubic centimeter of a substance. Thus, the density of water at $4^{\circ} \mathrm{C}$. is 1 , because at $4^{\circ} \mathrm{C}$., 1 c.c. of water weighs $1 \mathrm{~g}$. The density of ammonia gas (wt. in g. of 1 c.c. at $0^{\circ}$ and $760 \mathrm{~mm}$.) is 0.000759 .

Since the molecular weight is the weight of 22.41 ., or 22,400 c.c., the molecular weight of a gas is obtained by multiplying the density (if known) by 22,400 . Thus, the molecular weight of ammonia is $0.000759 \times 22,400=17.0$.

Densities are often given on the scale, density-of-air $=1$. Now 22.4 liters of air weigh $28.95 \mathrm{~g}$. If a gas has a density twice that of air, 22.4 liters of this gas would weigh $28.95 \times 2 \mathrm{~g}$. For example, the density of carbon dioxide (air $=1$ ) is 1.52 . The molecular weight is therefore $28.95 \times 1.52$, or 44.0 (see p. 79 ).

Atomic Weights.-Since a compound substance can be formed by union of the elementary substances, and decomposed to give these substances, its molecule may be assumed to contain those constituent elements as distinct parts of the mass. Those elementary parts of a molecule are called atoms. When two elementary substances combine, the process involves the union of the two kinds of atoms to form compound molecules. Now, in the table (p. 76), we recorded the weights of the constituent elements which together made up the molecular weights of the com- 
pounds. Then, upon examining the weights of any one element cortained in molecular weights of different compounds, we saw (p. 77) that one (the smallest) could be taken as the unit weight, of which the others were multiples by some whole number. Obviously then, if the molecular weights are the relative weights of different kinds of molecules, the unit weights of the elements (35.46 for chlorine, etc.) are the relative weights of the atoms of the different elements. The relative weights of the different kinds of atoms, such as 1.008 for hydrogen, 16 for oxygen, 35.46 for chlorine, are called the atomic weights of the respective elements. These weights, as we have seen (p. 80), are relative to the weights of the atom of oxygen, when the weight of the latter is taken as 16, and the weight of the molecule of oxygen is taken as 32 .

A compound molecule may contain one, or more than one, atom of each of the elements forming the compound. In the molecular weight of compounds, weights of elements which are smaller than the atomic weight (p. 77), are never found to occur. This indicates that, in chemical change, fractions of atoms play no part. The name atom (Greek, not cut, or not divided) records this fact.

The Atomic Hypothesis and Definite Proportions.-The idea of atoms furnishes at once an explanation of the law of definite proportions. Evidently, every molecule of a given substance must always contain the same numbers and the same kinds of atoms, so that the proportions by weight of the constituents of the compound as a whole must be alike in all samples.

Dulong and Petit's Law.- When an element gives no volatile compounds, as is the case with calcium, we can still find the atomic weight. We first find for the element an equivalent weight ( $p$. 53). Thus, the weight of calcium which combines with 35.46 parts of chlorine is 20 parts. This weight, or some multiple of it by a whole number, does in fact always correctly represent the 
amount of calcium combined with atomic weights of elements whose atomic weights are known. Hence 20, or some multiple of 20 is the required atomic weight. Dulong and Petit (1819) discovered a rule by which the correctness of such an atomic weight could be checked. When the atomic weight of an element is multiplied by the specific heat of the element in the solid form, the product is roughly 6.4. Thus, the known atomic weight of magnesium is 24.3 , its specific heat 0.245 , and the product is 5.95. Again, the known atomic weight of mercury is 200.6, and its specific heat 0.0335 , and the product 6.7 . Now the specific heat of calcium is 0.170 , and the product $20 \times 0.170$ is equal to 3.4. But $2 \times 20 \times 0.170=6.8$. The value $2 \times 20$, or 40 for the atomic weight is therefore the correct one. The following table contains additional illustrations of this law:

\begin{tabular}{|c|c|c|c|c|c|c|c|}
\hline Element & $\begin{array}{l}\text { Atomic } \\
\text { Wt. }\end{array}$ & Sp. Ht. & $\begin{array}{l}\text { Prod- } \\
\text { uct }\end{array}$ & Element & $\begin{array}{c}\text { Atomic } \\
\text { Wt. }\end{array}$ & Sp. Ht. & $\begin{array}{c}\text { Prod- } \\
\text { uct }\end{array}$ \\
\hline Lithium. & 7 & 0.94 & 6.6 & Iron. . & 56 & 0.112 & 6.3 \\
\hline Sodium. & 23 & 0.29 & 6.7 & Zinc & 65.4 & 0.093 & 6.1 \\
\hline Magnesium & 24.3 & 0.245 & 6.0 & Bromine (Solid) & 80 & 0.084 & 6.7 \\
\hline Silicon........ & 28.3 & 0.16 & 4.5 & Gold.... & 197 & 0.032 & 6.3 \\
\hline Phosphorus & & & & Mercury (Solid) & 200 & 0.0335 & 6.7 \\
\hline (Yellow).. & 31 & 0.19 & 5.9 & Uranium....... & 238.5 & 0.0276 & 6.6 \\
\hline Calcium....... & 40 & 0.170 & 6.8 & & & & \\
\hline
\end{tabular}

Another way of expressing this law will give it greater chemical significance. The specific heats are the amounts of heat required to raise one gram, that is one physical unit, of each element through one degree. When we multiply this by the atomic weight, we obtain the amount of heat required to raise one gram-atomic weight of the element, that is, one chemical unit, through one degree. The values of this product are approximately equal. Since there are equal numbers of atoms in one gram-atomic weight of each element, it follows that: Equal amounts of heat raise equal number of atoms of all elements in the solid form through equal intervals of temperature. 
Relations between the Structure and Behavior of MATTER

We have seen that matter is composed of exceedingly minute particles called molecules. Just as we can thoroughly understand the behavior of a watch or an automobile engine only if we know the details of its structure, and how the parts work, so we can understand the physical and chemical behavior of matter in masses only if we are familiar with its ultimate mechanism. Hence, we must now take up the structure of matter in its three states, the gaseous, the liquid, and the crystalline (or solid). In doing this, we shall keep constantly in view the connection between the molecular relations and the general behavior of the matter.

The Properties of Gases. - The most remarkable thing about a gas, considering the looseness with which its material is packed, is the total absence in it of any tendency to settling or subsidence. Since the molecules cannot be at rest upon one another, as the great compressibility shows, we are driven to conclude that they are widely separated from one another, and that they occupy the space, otherwise a complete vacuum, by constantly moving about in all directions. But a moving aggregate of particles which does not even finally settle must be in perpetual motion. We must, therefore, believe the molecules to be wholly unlike larger particles of matter in having perfect elasticity, in consequence of which they undergo no loss of energy after a collision. They must continually strike the walls of the vessel and one another and rebound, yet without loss of motion. The fact that each gas is homogeneous, efforts to sift out lighter or heavier samples having failed, requires the supposition that all the molecules of a pure gas are closely alike.

The diffusibility of gases is due to the motion of the molecules, and their permeability to the space available to receive molecules of another gas. These two modes of behavior involve no additional molecular properties. The word "diffusion" is often 
thought to mean the property of a given mass of gas in virtue of which another gas can mix with the given mass. This property is not diffusibility but permeability. It is the other gas, which makes its way into the given gas, which is diffusing. Diffusion is spontaneous motion of the parts of a gas away from their original location. Unless this motion is into an empty space, the diffusing molecules must, of course, move into another body of gas. In the case of the jars of hydrogen and air (p. 55), each gas moved in part out of its original jar (diffused), and each received parts of the other gas into its jar (was permeated).

\section{Boyle's Law and Charles' Law.- Passing now to Boyle's} law (p. 45), the thing to be accounted for is that when a sample of a gas diminishes in volume, its pressure increases in the same proportion. Let the diagram (Fig. 37) represent a cylinder with a movable piston, upon which weights may be placed to resist the pressure. Now the pressure exercised by the gas under the piston cannot be like the pressure of the hand upon a table, since we have just assumed that the particles are not even approximately at rest, and the spaces between them are enormous compared with the size of the molecules themselves. The gaseous pressure must therefore be attributed to the colossal hailstorm which their innumerable impacts upon the piston produce. If this is the case, the compressing of a gas must consist simply in moving the partition downwards, so that the particles as they fly about are gradually restricted to a smaller and smaller space. Their paths become on an average shorter and shorter. Their impacts upon the walls become more and more frequent. So the pressure which this causes becomes greater and greater, and is proportional to

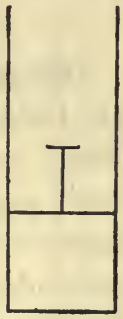

FIa. 37 the degree of crowding (the concentration) of the molecules.

There are two other points to be added. When we diminish the volume to one-half, we find from experience that the pressure 
becomes exactly, or almost exactly, twice as great. This must mean that, although the particles are becoming crowded, they do not interfere with one another's motion, excepting of course where actual collision causes a rebound. Only in the absence of interference would doubling the number of molecules per unit of volume give exactly double the number of impacts on the walls. Hence the molecules must have practically no tendency to cohesion. Finally, the molecules must be supposed to move in straight lines between collisions.

Boyle's law therefore adds four more details concerning molecular behavior, namely, that the impacts of the particles produce the pressure, that the crowding of the molecules represents the concentration of the material and that the particles move in straight lines and show almost no cohesion, since pressure and concentration are very closely proportional to one another.

How, now, can we account for Charles' law (p. 46), according to which an increase in volume (or in pressure, if the volume is kept constant) results from heating a mass of rapidly moving molecules? The action of a particle colliding with a surface is measured in physics in terms of its mass and its velocity. It is evident that heating a cloud of molecules would not increase the mass of each, and it must therefore increase the velocity.

Diffusion.- It is found by experiment that the lighter a gas is, the faster its particles move by diffusion (p. 89) in any direction. Exact measurement shows that the rate is inversely proportional to the square root of the density of the gas. From the results obtained for any particular gas, the average speed at which the molecules of which it is composed are moving can be calculated. For the hydrogen molecule at room temperature, this speed is 1840 meters per second, or faster than a rifle bullet. The speed of the oxygen molecule, which is almost exactly 16 times as heavy, is consequently the square root of one-sixteenth (that is, one quarter). of this value, or $460 \mathrm{~m}$. per sec. 
Each one of the enormous number of molecules in any given sample of gas is moving, therefore, with an enormous velocity, and the pressure exerted by gases (explained above as due to the impact of the molecules on the walls of the containing vessel) becomes more intelligible. In air, the number of molecules striking a single square centimeter of surface per second would fill no less than 12 liters.

\section{Liquefaction of Gases: Critical Temperature.-All gases} can be liquefied by sufficient cooling and compression. This fact compels us to suppose that, after all, even gaseous molecules have some tendency to cohesion. This cohesion is, in general, scarcely perceptible so long as the gas is warm and is diffuse but it is possible, by careful measurement, to establish the fact that it does cause slight deviations from Boyle's Law even under such conditions. Thus, 2 liters of oxygen at 1 atmosphere pressure, when subjected to 2 atmospheres pressure, give 0.9991 liters instead of 1 liter. The additional contraction of 0.0009 liters ( 0.9 c.c.) is to be regarded as due to the effect of cohesion when the molecules are thus crowded closer together. The gases which are more easily liquefied than is oxygen show greater effects. Thus, 2 liters of sulphur dioxide at $760 \mathrm{~mm}$., when subjected to 2 atmospheres pressure, give only 0.974 liters, showing a contraction due to cohesion of 26 c.c. These data refer to $0^{\circ}$. At lower temperatures the contractions due to cohesion become rapidly greater.

We can readily understand, therefore, that when the velocity of the molecules is sufficiently reduced by cooling, and the molecules are brought sufficiently close together, the tendency of the molecules to cohere causes the gas to condense and assume the liquid form. In 1869

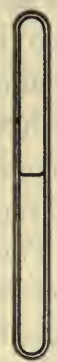

FIG. 38 Andrews found that carbon dioxide could be liquefied at $0^{\circ}$ by 38 atmospheres pressure, and at $30^{\circ}$ by 71 atmospheres, but that above $31.35^{\circ}$ it could not be liquefied by any pressure. 
He discovered that each gas has a critical temperature, as he called it. For carbon dioxide, this temperature can be observed by placing a heavy-walled, glass tube (Fig. 38), half-filled with liquid carbon dioxide, in a beaker of water, and gradually raising the temperature of the latter. At $31.35^{\circ}$, the surface between the liquid and gas becomes hazy and vanishes. At this temperature the liquid state disappears, merging into the gaseous. When the temperature falls once more, the surface marking the boundary between liquid and gas reappears.

The critical temperature of oxygen is $-118^{\circ}$, of hydrogen $-234^{\circ}$, of sulphur dioxide $156^{\circ}$, of water $358^{\circ}$.

Another Deviation from the Laws of Gases. A Perfect Gas.- It is also found by experiment that when a gas is already under very high pressure, and therefore very closely packed, an increase in the pressure does not produce quite as great a diminution in volume as Boyle's law leads us to expect. This reminds us that we are diminishing only the space between the molecules, and not the volumes of the molecules themselves, and therefore not the total volume of the gas. When, on severe compression, the volume occupied by the molecules themselves has become an appreciable fraction of the whole volume, additional compression does not affect the whole volume, and the contraction is smaller than Boyle's law would indicate. Thus, 200 liters of hydrogen, at $16^{\circ}$ and under one atmosphere pressure, when subjected to 200 atmospheres pressure, give 1.134 liters, instead of 1 liter.

The last two effects (namely, those due to the tendency to cohesion of, and the space occupied by the molecules) are called deviations from the laws of gases. In consequence of these individual deviations, there are not exactly equal numbers of molecules in equal volumes of any two different gases, at the same temperature and pressure. An imaginary gas, which exhibits neither deviation, called a perfect gas, is often referred to in discussing the behavior of gases (see footnote, p. 84). 
Summary. - We may now summarize the principal facts about gases in mass, with the corresponding features of the molecular relations.

\section{Facts About Gases in Mass}

Compressibility

Diff usibility

Permeability

Non-settling

Homogene

Pressure.................

Boyle's law...............

Charles' law...........

Gay-Lussac's law

Law of diffusion.........

Gases can be liquefied, and show lower compressibility at high pressures.
Corresponding Relations of Molecules

Vacuum + molecules widely separated.

Molecules in rapid motio-:

Empty space relatively large.

Molecules perfectly elastic.

Molecules of any one substance closely alike.

Due to impacts of molecules.

Pressure proportional to concentration of the molecules. Molecules move in straight lines and, when widely scattered, show no tendency to cohesion.

Rise in temperature increases the velocity of the molecules.

Avogadro's law.

Speed of molecules inversely proportional to square root of mass.

Molecules do possess some tendency to cohesion, and do occupy an appreciable fraction of the space when pressure is very great.

The Properties of Liquids. - The fact that even great pressures produce little diminution in the volume of a liquid shows that the free space, so predominant in gases, is relatively unimportant in liquids. This is only natural in view of the smaller volume occupied by substances in the liquid state. Thus we have seen (p. 62) that steam at $100^{\circ}$ occupies nearly 1600 times the volume of an equal weight of water. To reduce the volume of water to four-fifths requires, not raising the pressure to fivefourths as in the case of steam, but increasing it from 1 to 10,000 atmospheres. Evidently the molecules are already very closely packed.

This close packing of the molecules causes cohesion to be much more pronounced in liquids than in gases. A small amount of liquid, when poured into a vessel, does not fill the whole of 
the available space, but forms coherent drops, the curvature of the surface of which indicates that tremendous forces are existent, pulling the outside molecules towards the interior of the liquid. Nevertheless, molecules do escape outwards from a liquid surface to form vapor (see p. 62-4). We must therefore conclude that the molecules of a liquid are still in rapid motion. Similarly, when liquids which are capable of mixing (e.g., alcohol and water) are placed in separate layers in the same vessel, they do mix, slowly, by diffusion. The rate of dispersion of the molecules, although much impeded by their close packing, has not been annihilated.

A liquid still possesses, therefore, in a modified degree, many of the properties exhibited by a gas. For any particular substance, the differences between the behavior of the liquid and the gaseous states grow less and less as the temperature is raised, until at the critical temperature (see p. 91) the two states become identical.

The Properties of Solids. - True solids are sharply distinguished from liquids by their crystalline forms (Figs. 39-43, see

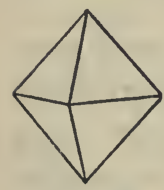

FIG. 39

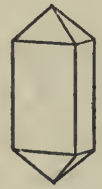

Fig. 40

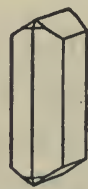

FIG. 41

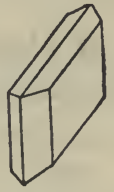

Frg. 42

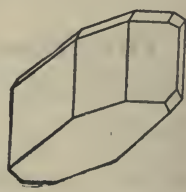

Frg. 43

Octahedron Square Pris- Rhombic Monosymmetric (Alum) matic (Niter)

Asymmetric (Hydrated cupric sulphate)

also pp. 4, 5 and 12), which possess definite planes of cleavage and by their behavior towards light and $\mathrm{X}$-rays give further evidence of regular structure. Substances such as glass, which do not exhibit any specific crystalline form, although commonly called solids, are strictly speaking still in an extremely viscous liquid state. In such substances, as in liquids and gases, the cohesive forces 
between adjacent molecules are exercised equally in all directions, with the result that the molecules are all in haphazard, unordered positions relative to one another and no particular arrangement of particles in space can persist. Substances of this character are therefore called amorphous (Greek, without form). In crystals, on the other hand, since each substance shows an individual structure, the forces between adjacent particles must be exercised in definite directions.

By using crystals of different substances as X-ray gratings (see p. 548), W. H. and W. L. Bragg (1914) have been able to show that crystals are composed of particles arranged in rows, the spacing of these rows with respect to one another determining the geometrical form of the crystal. They have also succeeded in proving that the particles, which so arrange themselves in definite patterns in crystals, are not molecules, much less aggregates of molecules, but atoms of the constituent elements of the substance. Thus when common salt (sodium chloride), which crystallizes in cubes, is examined by reflecting X-rays from an appropriate plane, it is found to consist of alternate rows of sodium atoms and

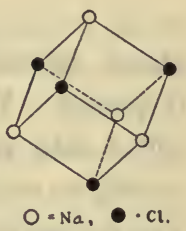

FIG. 44 chlorine atoms, the rows being arranged in space in such a way as to build up a cubical structure. The framework of a unit cube of sodium chloride is shown in Fig. 44. The actual length of a side of this cube is approximately only one hundred millionth of an inch! However, by imagining other cubes to be packed all round this, with atoms of sodium and of chlorine placed at their alternate corners, the reader will obtain for himself an idea of the ultimate structure of a crystal of common salt. Most substances crystallize in less simple geometrical forms and consequently possess a much more complex structure. The framework of the carbon atoms in a diamond, for example, is indicated in Fig. 45.

In a substance in the crystalline state, therefore, definite molec- 
ular units no longer exist. Thus, in sodium chloride, no sodium atom can be said to be combined specifically with any one chlorine atom. It is, instead, imprisoned by a number of chlorine atoms, stationed at definite intervals around it, and among these chlorine

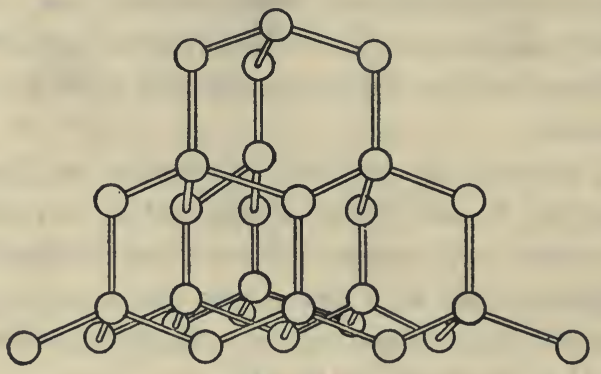

Fia. 45

atoms its combining forces must be regarded as impartially dispersed. Very powerful forces, it will be evident, must be called into play in order to constrain the separate atoms in a crystal to retain their regular positions with respect to one another. The nature of such forces will be discussed in a later chapter (see p. 554-5).

The resistance offered by the atoms in a crystal to forcible changes of position is shown by the very low compressibility of matter in the crystalline state. Nevertheless, some degree of motion of the particles must still persist, since many crystalline substances show a measurable vapor pressure. Some vapors, indeed (e.g., phosphorus, iodine), can be condensed directly to crystals without passing through the intermediate liquid state.

The results of X-ray work indicate that this motion of the atoms of a crystal consists of rapid vibrations about a mean position. Atoms vibrating violently at the surface are evidently exposed to the risk of breaking away altogether, after which they combine to form molecules of vapor. Similarly, molecules of vapor striking the crystal surface may stick thereto, their constituent atoms 
arranging themselves in a continuation of the crystal pattern. Equilibrium relations between crystals and vapor resemble closely, therefore, those between liquid and vapor discussed in an earlier chapter (p. 62-4), to which reference should here be made.

Exercises. - 1. State the change which takes place in the total volume of the gases or vapors in each of the six actions mentioned, p. 72.

2. If 1 liter of oxygen at $0^{\circ}$ and $760 \mathrm{~mm}$. weighs $1.429 \mathrm{~g}$., what is the molecular weight (pp. 74, 84)?

3. Calculate the molecular weight of a gas, 200 c.c. of which at $0^{\circ}$ and $760 \mathrm{~mm}$. weigh $2.1 \mathrm{~g}$. (p. 85).

4. Find the molecular weight of a gas, of which 250 c.c. at $18^{\circ}$ and $752 \mathrm{~mm}$. weigh $2.5 \mathrm{~g}$. (p. 85).

5. The following are the weights of sulphur contained in the molecular weights of several of its compounds: $32.06,64.12$, 96.18. What is the atomic weight of sulphur (pp. 74, 76)?

6. What information is contained in each of the formulæ: $\mathrm{CS}_{2}$, $\mathrm{PCl}_{3}, \mathrm{Al}_{2} \mathrm{O}_{3}$ (p. 79)?

7. If two gases combine in the ratio $5: 2$ by volume, in what relative numbers do their molecules interact (p. 82)?

8. The density of sulphur dioxide is 0.00286 . What is its molecular weight (p. 85)?

9. The density of a gas, air $=1$, is 2.3 . What is its molecular weight (p. 85)?

10. An element combines with hydrogen in the proportion $10.03: 1$, and its specific heat is 0.2 . What is its atomic weight (p. 87)?

11. The molecular formula of a gas is $\mathrm{CH}_{4}$. What is the grammolecular weight? What volume does this weight occupy? What is the weight of 1 liter of the gas? 


\section{CHAPTER IX}

\section{MAKING OF FORMULAE AND EQUATIONS}

THE formula (p. 78) is a condensed statement of the composition of a substance. Before we can make (i.e., calculate) the formula for a substance, we must (1) measure the proportions by weight of the constituent elements. Then, we must (2) express these proportions in multiples of the known atomic weights of the elements.

Analysis and Synthesis.- In the case of water $\mathrm{H}_{2} \mathrm{O}$ we saw (p. 58) how the weights of hydrogen and of oxygen required to give a measured amount of water were determined. The composition of the water was found out by putting the substance together out of the elements. This method is called synthesis (Greek, putting together).

In the case of mercuric oxide we can take a weighed amount of the oxide, decompose it, and weigh the mercury formed. The difference is the weight of the oxygen. This process, of decomposing a substance to learn its composition, is called analysis, the Greek word for decomposition.

One or other - sometimes both - of these plans can be used with every compound.

Some of the results of such experiments have been given in the earlier chapters. For example:

Tin (100) + Oxygen (26.9) $\rightarrow$ Tin oxide (p. 9).

Lead (100) + Oxygen (7.72) $\rightarrow$ Lead oxide (p. 10).

Iron (100) + Oxygen (43) $\rightarrow$ Ferric oxide (p. 10).

Zinc $(2.04)+$ Sulphur $(1) \rightarrow$ Zinc sulphide.

Mercuric oxide (108) $\rightarrow$ Mercury (100) + Oxygen (8) (pp. 15, 19). 
Making Formulae.- In the formulæ, these proportions are to be replaced by multiples of the atomic weights by whole numbers. We therefore divide the quantity of each element by the corresponding atomic weight. This operation gives us the factors by which the atomic weights are to be multiplied. The atomic weights we find in the table, where the values determined by the most expert chemists are collected.

For example. - In the case of tin oxide the proportion of tin to oxygen is $\frac{100}{26.89}$. The atomic weights are 119 and 16, respectively. $100 \div 119=0.84$, and $26.89 \div 16=1.68$. The proportion $\frac{100}{26.89}$ now becomes $\frac{119 \times 0.84}{16 \times 1.68}$.

Now this proportion - like all chemical proportions - must be expressed in multiples of the atomic weight by whole numbers. Hence, we next find the greatest common measure of the two factors. It is 0.84 . Indeed, in this simple instance, we can see that the ratio of the factors is $1: 2$. Dividing above and below by 0.84 , we get $\frac{119 \times 1}{16 \times 2}$.

Now, the symbols stand for the atomic weights. Substituting these symbols, the proportion becomes $\frac{\mathrm{Sn} \times 1}{\mathrm{O} \times 2}$. The formula is therefore $\mathrm{SnO}_{2}$.

Applying the same process to the lead oxide, we get

$$
\frac{100}{7.72}=\frac{207.1 \times 0.483}{16 \times 0.483}=\frac{207.1 \times 1}{16 \times 1}=\frac{\mathrm{Pb} \times 1}{\mathrm{O} \times 1}, \text { or } \mathrm{PbO} .
$$

Treating the other data in the same manner, we find: ferric oxide $\mathrm{Fe}_{2} \mathrm{O}_{3}$, zinie sulphide $\mathrm{ZnS}$ and mercuric oxide $\mathrm{HgO}$.

If the composition of the substance is given in percentages, the same process is used. Thus, the case of sodium sulphate works out as follows: 


\begin{tabular}{|c|c|c|c|c|c|c|c|}
\hline Elements & Percentages & At. wt. & Quotient & $\div$ & Formu & & \\
\hline $\begin{array}{l}\text { Sodium } \ldots \ldots \ldots \ldots \ldots \ldots \\
\text { Sulphur } \ldots \ldots \ldots \ldots \ldots \\
\text { Oxygen } \ldots \ldots \ldots \ldots \ldots \ldots\end{array}$ & $\begin{array}{l}32.43 \\
22.55 \\
45.02\end{array}$ & $\begin{array}{l}23 \times \\
32 \times \\
16 \times\end{array}$ & $\begin{array}{l}1.41 \\
0.705 \\
2.814\end{array}$ & $\begin{array}{l}0.705 \\
0.705 \\
0.705\end{array}$ & $\begin{array}{l}\mathrm{Na} \\
\mathrm{S} \\
\mathrm{O}\end{array}$ & $\begin{array}{l}X \\
\times\end{array}$ & 4 \\
\hline
\end{tabular}

The formula is therefore $\mathrm{Na}_{2} \mathrm{SO}_{4}$.

Making Equations.- The condensed statements of chemical changes which we have been using can now be still further simplified by using the formula in place of the names of the substances (p. 78). Thus

$$
\text { . } \mathrm{Sn}+\mathrm{O}_{2} \rightarrow \mathrm{SnO}_{2}
$$

This is to be read: 119 parts (or 1 atomic weight) of tin, acting chemically with $2 \times 16$ parts (or two atomic weights) of oxygen, give 151 parts of stannic oxide. We may also read it thus: 1 atom of tin with 1 molecule of oxygen gives 1 molecule of stannic oxide.

In making an equation there are four stages or steps:

1. Find out by experiment what the substances used and produced are.

2. Learn the molecular formula of each substance.

3. Set down the molecular formulæ in the form of a skeleton equation. Place the formulæ of the initial substances on the left, and those of the products on the right.

4. Adjust, or balance the equation.

For example:

(1) When hydrogen and oxygen combine, water is formed.

(2) The molecular formulæ are $\mathrm{H}_{2}, \mathrm{O}_{2}$, and $\mathrm{H}_{2} \mathrm{O}$. .

(3) Skeleton equation: $\mathrm{H}_{2}+\mathrm{O}_{2} \rightarrow \mathrm{H}_{2} \mathrm{O}$.

(4) In accordance with the law of conservation of mass, the numbers of atomic weights (or atoms) of each element must be 
the same after the action as before it. Now the skeleton equation shows two atomic weights of oxygen before, and, thus far only one after the action, whereas there ought to be two there also. With $\mathrm{O}_{2}(2 \times 16$ parts $)$ we have enough oxygen to give $2 \mathrm{H}_{2} \mathrm{O}$, which contains $2 \times 16$ parts of oxygen. But this will require us to take $2 \mathrm{H}_{2}$ to " balance" the equation. The final equation is, therefore:

Balanced Equation: $\quad 2 \mathrm{H}_{2}+\mathrm{O}_{2} \rightarrow 2 \mathrm{H}_{2} \mathrm{O}$.

Observe, we could not adjust the difficulty by writing $\mathrm{H}_{2}+\mathrm{O}$ $\rightarrow \mathrm{H}_{2} \mathrm{O}$, because each substance must be represented by its molecular formula, which stands for the weight of the substance in the standard volume of 22.4 liters, or one chemical unit weight, and in the case of oxygen this is $\mathrm{O}_{2}(=32 \mathrm{~g}$.). Putting this in terms of the hypothesis, each formula must represent 1 molecule, and the molecules of oxygen contain 2 atoms. Hence we could not divide the oxygen molecule. But we could take more than one molecule of hydrogen, so we took 2 molecules of this substance.

The coefficients in front of formulæ multiply the whole formula. $2 \mathrm{H}_{2} \mathrm{O}$ is equivalent to $2\left(\mathrm{H}_{2} \mathrm{O}\right)$, or two whole molecules of water.

Balancing Equations. - Learning to balance equations correctly comes only by practice. Take, again, the case of iron rusting. The substances are iron $(\mathrm{Fe})$, oxygen $\left(\mathrm{O}_{2}\right)$ and ferric oxide $\left(\mathrm{Fe}_{2} \mathrm{O}_{3}\right)$. The skeleton equation is

$$
\mathrm{Fe}+\mathrm{O}_{2} \rightarrow \mathrm{Fe}_{2} \mathrm{O}_{3} \text {. }
$$

We are not permitted to alter these formulæ themselves, but we may put coefficients in front of any of them to make the number vi atomic weights alike on_both sides. A good rule is to pick out the largest formula and reason back from that. Here, this is $\mathrm{Fe}_{2} \mathrm{O}_{3}$. To get oxygen atoms in threes, we must clearly take $3 \mathrm{O}_{2}(=60)$. That will give us $2 \mathrm{Fe}_{2} \mathrm{O}_{3}$. This, in turn, will require $4 \mathrm{Fe}$ :

Balanced:

$$
4 \mathrm{Fe}+3 \mathrm{O}_{2} \rightarrow 2 \mathrm{Fe}_{2} \mathrm{O}_{3} \text {. }
$$


Equations for Actions Already Studied.-In the preparation of oxygen (p. 15) we used mercuric oxide and got mercury and oxygen:

Skeleton:

Balanced:

$$
\begin{aligned}
\mathrm{HgO} & \rightarrow \mathrm{Hg}+\mathrm{O}_{2} . \\
2 \mathrm{HgO} & \rightarrow 2 \mathrm{Hg}+\mathrm{O}_{2} .
\end{aligned}
$$

Potassium chlorate has a composition shown by the formula $\mathrm{KClO}_{3}$. It gives (p. 29) potassium chloride ( $\mathrm{KCl}$ ) and oxygen $\left(\mathrm{O}_{2}\right)$.

Skeleton:

$$
\mathrm{KClO}_{3} \rightarrow \mathrm{KCl}+\mathrm{O}_{2} .
$$

Balanced:

$$
2 \mathrm{KClO}_{3} \rightarrow 2 \mathrm{KCl}+3 \mathrm{O}_{2} \text {. }
$$

The variety of chemical change, where one substance gives two (or more) substances, decomposition (p. 16), is readily recognized in these equations.

Substances, like the manganese dioxide (catalytic agent) used here, and the water so often employed as a solvent, when they undergo no chemical change, are omitted from the equation.

When the water takes part in the action, however, it must, of course, be included. Thus sodium peroxide $\left(\mathrm{Na}_{2} \mathrm{O}_{2}\right)$ and water $\left(\mathrm{H}_{2} \mathrm{O}\right)$ interact (p. 31) to give sodium hydroxide and oxygen:

$$
2 \mathrm{Na}_{2} \mathrm{O}_{2}+2 \mathrm{H}_{2} \mathrm{O} \rightarrow 4 \mathrm{NaOH}+\mathrm{O}_{2} \text {. }
$$

The Preparation of Hydrogen (p. 50) from sodium (Na) and water gives sodium hydroxide $(\mathrm{NaOH})$ and hydrogen $\left(\mathrm{H}_{2}\right)$ :

$$
2 \mathrm{Na}+2 \mathrm{H}_{2} \mathrm{O} \rightarrow 2 \mathrm{NaOH}+\mathrm{H}_{2} \text {. }
$$

When steam is passed over iron (p. 51), we get hydrogen and magnetic oxide of iron $\left(\mathrm{Fe}_{3} \mathrm{O}_{4}\right)$ :

$$
3 \mathrm{Fe}+4 \mathrm{H}_{2} \mathrm{O} \rightleftarrows \mathrm{Fe}_{3} \mathrm{O}_{4}+4 \mathrm{H}_{2} \text {. }
$$

The liberation of hydrogen by the action of zinc $(\mathrm{Zn})$ upon sulphuric acid $\left(\mathrm{H}_{2} \mathrm{SO}_{4}\right)$, where the products (p. 52) are hydrogen and zinc sulphate, is shown thus:

$$
\mathrm{Zn}+\mathrm{H}_{2} \mathrm{SO}_{4} \rightarrow \mathrm{H}_{2}+\mathrm{ZnSO}_{4} \text {. }
$$


Again, iron and hydrochloric acid $(\mathrm{HCl})$ give hydrogen and ferrous chloride $\left(\mathrm{FeCl}_{2}\right)$ :

$$
\mathrm{Fe}+2 \mathrm{HCl} \rightarrow \mathrm{H}_{2}+\mathrm{FeCl}_{2} \text {. }
$$

In the last two equations the variety of chemical changes called displacement (p. 51), where one elementary substance displaces another from a compound, is well illustrated.

The equation for the formation of water by union of hydrogen and oxygen,

$$
2 \mathrm{H}_{2}+\mathrm{O}_{2} \rightarrow 2 \mathrm{H}_{2} \mathrm{O} \text {, }
$$

has already ( $p .59$ ) been discussed. The reduction of an oxide, such as magnetic oxide of iron or cupric oxide, by hydrogen (p. 57), gives the metal and water:

$$
\begin{aligned}
& \mathrm{Fe}_{3} \mathrm{O}_{4}+4 \mathrm{H}_{2} \rightleftarrows 3 \mathrm{Fe}+4 \mathrm{H}_{2} \mathrm{O} . \\
& \mathrm{CuO}+\mathrm{H}_{2} \rightarrow \mathrm{Cu}+\mathrm{H}_{2} \mathrm{O} .
\end{aligned}
$$

Upon examining these equations for reductions, we perceive that they are illustrations of displacement also.

Reversible Actions (p. 69), like the decomposition of water by heating, and the recombination of the elements on cooling (p. 67), are shown by using two arrows:

$$
2 \mathrm{H}_{2} \mathrm{O} \rightleftarrows 2 \mathrm{H}_{2}+\mathrm{O}_{2} \text {. }
$$

The equation may be read from either end. The decomposition and formation of hydrates (p. 68) are also reversible actions. In the case of zinc sulphate, the equation is

$$
\mathrm{ZnSO}_{4}+7 \mathrm{H}_{2} \mathrm{O} \rightleftarrows \mathrm{ZnSO}_{4}, 7 \mathrm{H}_{2} \mathrm{O} \text {. }
$$

Reaction Formulae.- In the foregoing formula for the hydrate of zinc sulphate, it will be seen that we do not add together all the atoms of oxygen, and write $\mathrm{ZnH}_{14} \mathrm{SO}_{11}$. The latter would show the composition of the substance correctly, but it would show nothing more. Now chemists find it convenient, frequently, to alter the formula so that it shall indicate also some important chemical property or reaction of the substance. Hence 
the formula $\mathrm{ZnSO}_{4}, 7 \mathrm{H}_{2} \mathrm{O}$, which indicates at a glance the relationship of the substance to zinc sulphate $\left(\mathrm{ZnSO}_{4}\right)$. The hydrate is made from zinc sulphate by adding water, and is easily decomposed into these two substances again. The reaction formula hints at this familiar reaction. Note, however, that the comma (,) does not indicate a mixture of the materials, such as $\mathrm{ZnSO}_{4}$ and $\mathrm{H}_{2} \mathrm{O}$, but a single substance composed of both. The plus $(+)$ sign is used between the formulæ of different, uncombined substances in a mixture.

In accordance with this plan, washing soda, hydrate of sodium carbonate (p. 68), is written $\mathrm{Na}_{2} \mathrm{CO}_{3}, 10 \mathrm{H}_{2} \mathrm{O}$, and bluestone ( $\mathrm{p}$. 68), hydrate of cupric sulphate, $\mathrm{CuSO}_{4}, 5 \mathrm{H}_{2} \mathrm{O}$.

Dissociation.-A decomposition which, like that in p. 67, is reversible is called a dissociation. When heat is the agent producing the change, it is sometimes called a thermal dissociation. Not all decompositions are reversible. Thus potassium chlorate decomposes to give potassium chloride and oxygen, but these products will not combine under any known conditions, directly, to give potassium chlorate.

Molecular Formulce. - In this chapter, for the sake of simplicity, we have so far left in the background the fact that the formula must represent a molecular weight of the substance, as well as its composition. The total weight, for which the symbols in a formula stand, must be equal to the weight of the substance occupying the gram-molecular volume. In other words, the formula-weight must represent one cube-full (Fig. 36, p. 74) of the substance. This is true, as we have seen, of the formulæ $\mathrm{H}_{2} \mathrm{O}$ and $\mathrm{HCl}$ (see p. 74).

In the cases of tin oxide $\left(\mathrm{SnO}_{2}\right)$ and ferric oxide $\left(\mathrm{Fe}_{2} \mathrm{O}_{3}\right)$, we have substances which cannot be converted into vapor or dissolved, so that their molecular weights are unknown. In such cases, we use the simplest formula that will show the correct proportions. 
Molecular Formulae of Simple Substances.-With oxygen $\left(\mathrm{O}_{2}\right)$ and hydrogen $\left(\mathrm{H}_{2}\right)$, however, the double formulæ are used, instead of the simpler $\mathrm{O}$ and $\mathrm{H}$, because the weights $2 \times 16$ and $2 \times 1.008$ are the ones which fill the cube. The molecules of all elements are not diatomic, however. Thus the cube-full of mercury vapor weighs only 200.6 , the same as the atomic weight, and the correct molecular formula of the element is therefore Hg. Similarly, the correct formulæ are $\mathrm{Na}$ (sodium), K (potassium), and $\mathrm{Zn}$ (zinc). But the weight of 22.4 liters requires us to write $\mathrm{Cl}_{2}$ for chlorine, $\mathrm{N}_{2}$ for nitrogen, $\mathrm{P}_{4}$ for phosphorus vapor and $S_{2}$ for sulphur vapor (above $1000^{\circ}$ ).

After all, there is nothing surprising in the fact that the molecules even of elementary substances should, in some cases, contain several atoms. All that it means, in the case of an element with diatomic molecules, such as oxygen $\left(\mathrm{O}_{2}=32\right)$, is that, when oxygen combines with another element, each molecule of oxygen will be divided between two molecules of the product if the latter contain only 16 parts of oxygen each.

As an illustration, the union of hydrogen and oxygen to form steam (p. 61) may be considered.

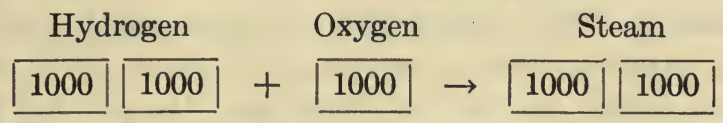

If each of the above rectangles represents a small volume containing 1000 molecules of gas, then 2000 molecules of hydrogen and 1000 molecules of oxygen give 2000 molecules of water vapor. Since each molecule of water vapor must contain at least one atom (see p. 86) of oxygen, at least 2000 atoms of oxygen were required, and must have been furnished by the 1000 molecules of oxygen. Each of these molecules must therefore have split into at least two atoms. We have no reason, however, for supposing that there are more than two atoms in the oxygen molecule. Hence we accept the formula $\mathrm{O}_{2}$ as correct. 
Similar confirmation of the formulæ $\mathrm{H}_{2}$ and $\mathrm{Cl}_{2}$ will be found on p. 148.

Molecular Formulae of Compounds.-The need of attention to making our formulæ molecular comes out also in the cases of many compounds. Thus, formaldehyde (a disinfectant) has the composition $\mathrm{CH}_{2} \mathrm{O}$, and its molecular weight is 30 , so that $\mathrm{CH}_{2} \mathrm{O}(=12+2+16)$ is the correct formula. But acetic acid (the sour substance in vinegar) has the same composition, $\mathrm{CH}_{2} \mathrm{O}$, only its molecular weight is 60 , and the formula is therefore written $\mathrm{C}_{2} \mathrm{H}_{4} \mathrm{O}_{2}(=24+4+32)$.

For gaseous and volatile substances the correct molecular formulæ are always used. Thus, for phosphorus pentoxide (p. 72), $\mathrm{P}_{4} \mathrm{O}_{10}$ is preferred to $\mathrm{P}_{2} \mathrm{O}_{5}$ because the molecular weight of the substance in the state of vapor is 284 and not 142.

The correct equation for reaction $5, \mathrm{p} .72$, is therefore:

$$
\mathrm{P}_{4}+5 \mathrm{O}_{2} \rightarrow \mathrm{P}_{4} \mathrm{O}_{10} \text {. }
$$

Only by bearing in mind the true molecular formulæ can we include the volume and molecular proportions of the reacting substances in the vapor state, as well as their weight and atomic proportions (p. 100), in our condensed statement of a reaction.

Often, however, when a reaction takes place wholly between solids and liquids, we use for convenience the simplest possible formulæ throughout. Thus for the combination of iron and sulphur (p. 15) we write: $\mathrm{Fe}+\mathrm{S} \rightarrow \mathrm{FeS}$.

Warnings.-Always place the formulæ of the products on the right-hand side of the equation, and the formulæ of the reacting substances on the left.

Point the arrow in the direction of the reaction; that is, towards the products.

Use the molecular formulæ for elementary substances $\left(\mathrm{O}_{2}, \mathrm{H}_{2}\right.$, $\mathrm{N}_{2}$, etc.). The molecular formulæ, when they are known, are the only ones given in the text. The symbols of the elements, as 
given in the table on the rear cover, must be made into molecular formulæ before use in equations.

Exercises.-1. Using the data given on p. 35, calculate the formulæ of sulphur dioxide, phosphorus pentoxide, and carbon dioxide.

2. Using the results of 1 , make and balance the equations for the union of each of the three elements, sulphur, phosphorus, and carbon, with oxygen.

3 . Using the information on p. 36 , make and balance the equations for the interaction with oxygen of: $(a)$ carbon disulphide $\left(\mathrm{CS}_{2}\right)$; (b) zinc sulphide (zinc oxide is $\mathrm{ZnO}$ ), and (c) wood (assuming the formula of the latter to be that of cellulose, $\mathrm{C}_{6} \mathrm{H}_{10} \mathrm{O}_{5}$ ).

4. Make and balance the equations for the actions on water of: (a) potassium (giving $\mathrm{KOH}$ ), and (b) calcium (giving $\mathrm{CaO}_{2} \mathrm{H}_{2}$, usually written $\mathrm{Ca}(\mathrm{OH})_{2}$ ).

5. Using the data in regard to the action of zinc on hydrochloric acid, given on p. 53, calculate the formula of zinc chloride and make the equation.

6. Make equations for the action of magnesium and aluminium upon hydrochloric acid (giving $\mathrm{MgCl}_{2}$ and $\mathrm{AlCl}_{3}$ ) and upon sulphuric acid (giving $\mathrm{MgSO}_{4}$ and $\left.\mathrm{Al}_{2}\left(\mathrm{SO}_{4}\right)_{3}\right)$.

7. Make a molecular equation for the decomposition of hydrochloric acid by electrolysis (p. 55).

8. Make equations for the formation of the hydrates of sodium carbonate $\left(\mathrm{Na}_{2} \mathrm{CO}_{3}, 10 \mathrm{H}_{2} \mathrm{O}\right)$ and of cupric sulphate $\left(\mathrm{CuSO}_{4}, 5 \mathrm{H}_{2} \mathrm{O}\right)$, by union of the anhydrous substances with water. 


\section{CHAPTER $\mathrm{X}$}

\section{SOLUTION}

THE property that many substances have of dissolving in others is a most interesting and valuable one. The value lies chiefly in the fact that some substances are easily soluble in a given liquid and others are, practically, not soluble in it at all. These differences in solubility enable us to accomplish, both in the laboratory and in chemical industry, many things otherwise impossible. Thus, we separated sulphur from iron (p. 13), by using carbon disulphide $\left(\mathrm{CS}_{2}\right)$ to dissolve the former. In the same way the refining of silver (its separation from the lead in which it is contained) is carried out on a large scale in actual practice by the use of molten zinc as a solvent. We must first learn precisely what is meant by a solution, and then we shall be ready to understand the uses and properties of solvents and solutions.

Solution.-We distinguish carefully between a solution and a mere mixture, also between a solution and a compound.

A mechanical mixture, such as that of iron and sulphur, can never be perfect, as will be evident from what has been said in an earlier chapter (p. 84) regarding the size of molecules. However finely we may powder up such a mixture, we cannot possibly bring about a sufficiently intimate dispersion of the particles of its components among one another to justify us in believing that the whole mass has become homogeneous in its ultimate structure. In any true solution, however, intermingling of the particles of the separate components down to molecular magnitudes has actually been accomplished. In a solution of salt in water, for example, the dissolved substance is completely and permanently dissipated throughout the liquid. However long the solution is allowed to 
stand, salt never settles out. Only by evaporating off all the water can a complete separation be effected.

Practically speaking, there is no limit to the amount of dissipation which may thus be produced. Thus a single small crystal of potassium permanganate, a common disinfectant which gives a very deep purple solution in water, may be dissolved in a liter or even in a hundred liters of water, and the purple tinge which it imparts to the liquid will still be perfectly perceptible in every portion of the solution. We may note here the distinguishing characteristic of a solution as opposed to a compound. Compounds contain definite proportions by weight (p. 19) and simple atomic ratios (p. 86) of their constituent elements. The composition of a solution, on the other hand, can within certain limits (see p. 110) be varied continuously.

Sometimes, when we shake up a finely-divided solid with a liquid, the latter becomes dull, or cloudy, or muddy. The solid particles are here simply suspended in the liquid, not dissolved, and will eventually settle out. Sand, shaken with water, settles at once. Flour, mixed with water, settles more slowly. The particles of flour can be readily separated from the water, however, by filtration (p. 13), the flour remaining on the paper while the water runs through. Such mixtures are called suspensions.

In exceptional cases, the subdivision of a suspended substance in a liquid, while not approaching molecular magnitudes, is so minute as to make its retention by filter-paper impossible, or even to prohibit it from settling out in any reasonable time. Solutions of soap, starch and gelatine in water are of this nature. Such suspensions are known as colloidal suspensions. To the unaided eye, they appear to be true solutions. Their main properties, however, are essentially different from those of true solutions, as will be seen later (pp. 440-1).

Milk owes its cloudy, white appearance largely to droplets of oily matter, which reflect much light from their surfaces. They pass easily through filter paper. But when milk is allowed to 
stand they slowly rise to the top, being lighter than the water in which they are not dissolved, but suspended. A mixture of two liquids of this nature is called an emulsion.

Chemists commonly call the dissolved substance the solute and the substance in which it is dissolved the solvent. In many cases. however, (when we take two liquids such as alcohol and water, for example) the terms solvent and solute are interchangeable. Gases, liquids, and solids may all be solutes, and dissolve in suitable gaseous, liquid, or solid solvents.

Solvents. - Water is by far the commonest and most useful solvent. Very many inorganic substances dissolve in it easily. The fact that many (like sulphur and sand) do not, enables us to separate the components of a mixture containing a soluble and an insoluble substance.

Many organic substances, such as fats, paraffin, petroleum, tar, rubber, cotton, paper, shellac, and so forth, do not dissolve to any measurable extent in water. But fats dissolve readily in ether $\left(\mathrm{C}_{4} \mathrm{H}_{10} \mathrm{O}\right)$, in carbon disulphide $\left(\mathrm{CS}_{2}\right)$, in carbon tetrachloride $\left(\mathrm{CCl}_{4}\right)$, and in chloroform $\left(\mathrm{CHCl}_{3}\right)$. For this reason these substances remove grease which has accidentally got into cloth. Paraffin, petroleum, and tar dissolve in gasoline (petrol), and in benzene $\left(\mathrm{C}_{6} \mathrm{H}_{6}\right)$. Cotton and pure paper (like filter paper) will dissolve in strong sulphuric acid. Alcohol $\left(\mathrm{C}_{2} \mathrm{H}_{6} \mathrm{O}\right)$ dissolves shellac (to make varnish).

Again, water dissolves little carbon disulphide, chloroform, carbon tetrachloride, gasoline or benzene. But it dissolves alcohol in any amount, and ether in limited quantity. Some organic substances, like sugar, dissolve easily in water, but hardly at all in the other solvents just mentioned. Hence candy or molasses can be taken out of cloth by water, but not by solvents for fats.

Saturation.- As a rule, not more than a certain amount of a solute is dissolved by a given quantity of the solvent. By 
shaking the solute with the solvent for a sufficient length of time, this maximum amount will finally be dissolved. The solvent is then said to be saturated by the solute in question. Thus, 100 c.c. of water at $18^{\circ}$ will dissolve as much as $6.6 \mathrm{~g}$. of potassium chlorate, but not more. The same amount of water will dissolve $213.4 \mathrm{~g}$. of silver nitrate, however, before the solvent becomes saturated. On the other hand, a saturated solution of chalk (calcium carbonate) in water will contain only $0.0013 \mathrm{~g}$. in 100 c.c.

To describe these cases we should say that potassium chlorate is only moderately soluble in water," silver nitrate very soluble, and chalk insoluble. But no substance is absolutely insoluble.

The number of grams of the solute required to saturate 100 c.c. of the solvent we call the solubility of the substance (at the existing temperature). The solubilities at $18^{\circ}$ of one hundred and forty-two substances in water are given in a table printed inside the cover, at the front of this book. A few additional examples are given below (p. 113).

In some cases there is no limit to the solubility, and therefore no possibility of the solution reaching saturation. Thus alcohol or glycerine and water will dissolve in one another in any proportion. Such pairs of substances are said to be miscible in all proportions.

Dilute and Concentrated Solutions.-A dilute solution is one containing little dissolved matter, whether the matter is naturally very soluble or not. A concentrated solution is one containing much of the dissolved substance, and such a solution can be made with very soluble solutes only.

Conditions affecting the Solubility of a Gas.- When the dissolving substance is a gas, led through, or confined above the liquid at a definite pressure, the gas dissolves until a state of equilibrium between dissolving and emission is reached, for example, Oxygen (gas) $\rightleftarrows$ Oxygen (dissolved), and the liquid is then saturated with the gas. 
It is found, as the molecular theory would lead us to expect, that the concentration of the saturated solution of a gas is proportional to the pressure at which the gas is supplied (Henry's law).

This equilibrium, Gas (gaseous) $\rightleftarrows$ Gas (dissolved), can be reached, naturally, from the other direction, namely by starting with a solution of the gas and a space above the solution containing, at first, none of the gas. The gas leaves the solution until the rates of emission and return become equal. Hence, a gas may be entirely removed from solution by bubbling a foreign gas through the liquid. The bubbles furnish the space to receive the emitted gas, and have a large surface, so that the process goes on rapidly. The bubbles also escape, and carry with them the emitted gas, so that, in this case, there is no re-solution. This is a case of nullifying one of the two opposed tendencies (p. 64).

When a mixture of two gases is shaken with a liquid, the gases behave independently of each other (Dalton's law, p. 47). Each has the same pressure, and therefore the same solubility, as it would possess if it alone occupied the whole space above the liquid.

\section{Two Immiscible Solvents: Law of Partition.-An inter-} esting application of the same ideas may be made to a case which occurs very commonly in chemical work. If we shake up a small particle of iodine with water, we find that it dissolves slowly, giving eventually a saturated but very dilute solution. If now ether in sufficient quantity be shaken with the aqueous solution, the greater part of the iodine will find its way into the ether, and be contained in the brown layer which rises to the top. The process of removing a substance practically from solution in one solvent and securing it in another is called extraction. We find in such cases that neither solvent can entirely deprive the other of the whole of the dissolved substance, if the latter is soluble in both independently. A state of equilibrium is finally reached: 
$\mathrm{I}_{2}$ (in water) $\rightleftarrows \mathrm{I}_{2}$ (in ether). The partition of the substance takes place in proportion to its solubility in each solvent. It is found that any amount of the solute, up to the maximum the system can contain, provided this does not involve too high a concentration in either solvent, is divided so that the ratio of the concentration in the two solvents is always the same. In the case of iodine divided between water and ether, this ratio is about $1: 200$.

This principle is used in Parke's process for extracting silver from molten lead, by means of melted zinc as the second solvent. It is employed in separating interesting compounds from animal secretions and vegetable extracts, and in purifying such compounds. Nicotine from tobacco and cocaine from coca leaves are secured in this way.

Temperature and Solubility. - The solubility of every substance in any solvent varies more or less with the temperature. The solubility of niter (potassium nitrate $\mathrm{KNO}_{3}$ ) in water shows great variation, namely $13 \mathrm{~g}$. in 100 c.c. at $0^{\circ}, 26$ g. at $20^{\circ}, 140 \mathrm{~g}$. at $70^{\circ}$. On the other hand, the solubility of common salt (sodium chloride $\mathrm{NaCl}$ ) is nearly constant, $35.5 \mathrm{~g}$. at $0^{\circ}, 36.5 \mathrm{~g}$. at $20^{\circ}, 38 \mathrm{~g}$. at $70^{\circ}, 40 \mathrm{~g}$. at $100^{\circ}$.

Usually, as in these two cases, the solubility of solids in liquids (and of liquids in liquids) increases with rise in temperature, but in a few cases it diminishes. Thus, the solubility in water of slaked lime (calcium hydroxide $\mathrm{Ca}(\mathrm{OH})_{2}$, used to make lime water) is $0.175 \mathrm{~g}$. at $20^{\circ}$ and $0.079 \mathrm{~g}$. at $100^{\circ}$, and that of anhydrous sodium sulphate $\left(\mathrm{Na}_{2} \mathrm{SO}_{4}\right)$ is $55 \mathrm{~g}$. at $32.5^{\circ}$ and $42 \mathrm{~g}$. at $100^{\circ}$.

The solubility of gases in liquids diminishes with rising temperature. This may be illustrated by heating cold tap-water in a beaker. The dissolved gases, originally obtained from the air, appear in bubbles on the bottom and sides as the temperature rises. 
Crystallization.- If the solvent has been saturated while warm, and the substance is one that is less soluble at lower temperatures, then, when the temperature falls, the solute begins to come out of solution. The amount appearing, of course, is only the excess beyond what is needed to saturate the solvent at the lower temperature.

If the solute is liquid at the new temperature, it appears at first as a cloud of drops, rendering the liquid milky. This may be shown by cooling a solution of phenol (carbolic acid) in hot water.

If the solute is solid, then the particles, as they appear, take the form of crystals (p. 94). These grow by taking on more of the separating solute. If the cooling goes on slowly, very large crystals can finally be obtained. On the other hand, with rapid cooling, new crystals are continually formed, and a fine crystalmeal falls to the bottom of the solution. The crystals in this meal, however, when viewed through a lens, are seen to be just as perfect as the larger ones.

When a more dilute solution is used, instead of a saturated one, crystals may still be obtained. A part of the solvent must first be removed, however. This may be done, either by boiling the solution for some time, or by leaving it to evaporate in a wide dish in which a large surface is exposed.

When the dissolved substance can form a compound with the solvent (e.g., a hydrate, see p. 67) which is stable at the temperature of crystallization, the crystals are composed of this compound.

The whole of the solvent may be boiled off. But in this case, good crystals of the solute are never obtained - the residue is usually a crust composed of imperfect crystals.

When the substance is more soluble in cold than in hot water, then crystallization is produced by raising the temperature.

Crystallization from a Melted Mass.-In this connection, it should be noted that there is another way of obtaining crystals. 
This is to melt the substance (without any solvent), and allow the mass to cool slowly. When a part has solidified, the rest of the liquid is rapidly poured off. Metals and many other fusible substances give good crystals in this way. Water itself, when it freezes, deposits radiating, hexagonal crystals of ice.

Supersaturation.- When a hot, saturated solution is cooled, there is quite commonly some delay before the crystals begin to appear. The solution, pending the appearance of the crystals, is then said to be supersaturated. In most cases the crystals soon appear in due course, especially if the liquid is shaken or stirred. But certain substances have a tendency to remain indefinitely in the state of supersaturated solution. The hydrates of sodium sulphate $\left(\mathrm{Na}_{2} \mathrm{SO}_{4}, 10 \mathrm{H}_{2} \mathrm{O}\right)$ and of sodium thiosulphate (photographers" "hypo " $\mathrm{Na}_{2} \mathrm{~S}_{2} \mathrm{O}_{3}, 5 \mathrm{H}_{2} \mathrm{O}$ ) give solutions in water of this nature. The addition of a minute crystal of the substance concerned ("inoculation"), however, always starts the crystallization (Fig. 46).

Many pure liquids, similarly, when cooled below their freezing-point, do not always crystallize out at once. Thus water can be taken down to $-10^{\circ}$ without the appearance of ice. In this condition it is said to be supercooled. Shaking, or stirring, or (better still) inoculating with a fragment of ice, induces crystallization in this case also. It may be noted that the opposite phenomenon has never been observed; ice invariably melts sharply at $0^{\circ}$ under atmospheric pressure.

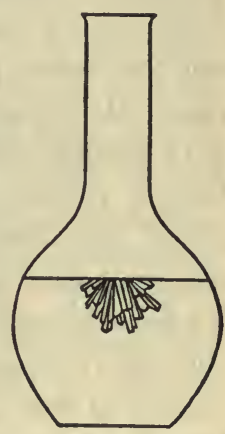

FIg. 46

Heat of Solution.- Most substances absorb heat when they dissolve, making the solution cooler, and give out heat when they crystallize. Thus, in the cases of the two sodium salts last mentioned, when the crystallization is brought about in the cool, supersaturated solutions, the rise in temperature is considerable. 
This fact has been utilized in devising a sort of hot-water bottle. The bottle is made of rubber and contains a supersaturated solution of sodium acetate. Whenever the heat is wanted, the stopper is taken out, rubbed with the finger, and screwed back. The rubbing spreads on the inner surface of the stopper, next the liquid, some of the crystals adhering to the screw, and so starts the crystallization. The bottle then becomes warm and remains so for a considerable time. After it has cooled, it is placed, without being opened, in boiling water to redissolve the crystals, and, when cold, is ready for use again.

Influence of the Solute upon the Solvent.-The dissolving of a substance alters the properties of the solvent. The observed changes may be divided into two classes.

In the first class, the amount of the change varies with the substance dissolved. Very striking and difficult to explain, for example, are the erratic changes in volume which occur when solution takes place. Specific effects of this class show that chemical changes often accompany solution. For example, 58.5 g. of sodium chloride (volume 27.5 c.c.) and 10,000 c.c. of water have a volume, totalling $10,027.5$ c.c., but, when they are dissolved, the solution measures only $10,016.5$ c.c. This is a very dilute solution (about $\frac{1}{2}$ per cent), so that the contraction of 11 c.c. is relatively considerable. On the other hand, $214 \mathrm{~g}$. of ammonium chloride (volume 142.5 c.c.) and 843.5 c.c. of water have a total volume of 986 c.c., but when dissolved give 1000 c.c. of solution. Here there is an expansion of 14 c.c. In the case of table-sugar and water, however, there is almost no change in volume.

Another important property of solutions in which the influence of the solute is specific is conduction of electricity. Pure water is an exceedingly poor conductor. A solution of table-sugar in water is also practically non-conducting. But when acetic acid is dissolved in water a solution is obtained which conducts the current fairly well, while a solution of sodium chloride is an ex- 
ceedingly good conductor. The significance of these differences in behavior will be taken up later ( $p .118$ ).

In the case of many properties of solutions, however, it has been found that equal numbers of dissolved molecules of different substances produce the same amount of change. The effect appears here to be due essentially to physical causes, and is discussed in the following sections in the light of the molecular hypothesis. Before attacking these sections, the student is recommended to refer back to p. 62-4 and read these pages through again carefully, noting that the equilibrium relationships between liquid water and water vapor, therein discussed, can obviously be extended to any volatile substance in contact with its own vapor.

Vapor Pressure of Solutions. - When we take equal quantities of a volatile liquid (e.g., benzene, $\mathrm{C}_{6} \mathrm{H}_{6}$ ) and add to each equal weights of different non-volatile solutes (e.g., naphthalene, anthracene, camphor; three organic solids which are practically non-volatile at ordinary temperatures) we find that the vapor pressures of all the resulting solutions are less than that of the pure solvent, but the depression is different in each case. But if, instead of adding equal weights of the different solutes, we add equal numbers of molecules (as we can do by dissolving, for example, $1 \mathrm{~g}$. molecular weight of each substance in $1000 \mathrm{~g}$. of benzene), we find that the depression is the same in every case. The depression is proportional, moreover, to the fraction of solute molecules in the solution. This very striking fact is explained by the molecular hypothesis as follows.

Every molecule at the surface of a pure volatile liquid has an equal chance to escape into the vapor above the liquid. But as soon as we add to such a liquid a solute which is practically non-volatile, we have a liquid in which some of the molecules have no tendency to pass into the state of vapor, but are fixed in the liquid state. Suppose, for instance, we consider a solution in which one molecule in every ten is non-volatile; the intensity 
of the hail of molecules leaving the liquid will evidently be reduced by one-tenth. Equilibrium between liquid and vapor over such a solution will be re-established only when the intensity of the hail of vapor molecules returning to the liquid is also reduced by one-tenth, since otherwise more molecules will be returning than leaving. This means that the vapor pressure of the solution must be one-tenth less than that of the pure solvent.

It is important to note that the nature of the solute is here immaterial, the essential factor is the number of molecules it furnishes to the solution. We have here a method of determining the molecular weights of non-volatile substances. By dissolving a known weight of such a substance in a known weight of a suitable solvent and determining the relative depression of vapor pressure thereby produced, we learn what fraction of the molecules in the solution belong to the solute, and hence can calculate its molecular weight.

All aqueous solutions show a lower tension of water vapor than does pure water. With conducting solutes (e.g., sodium chloride), indeed, the vapor pressure depressions obtained are abnormally large, and do not agree with the accepted molecular weights. This is a point to which we shall return later (p. 177).

If a substance is very soluble in water, the solution may give a vapor pressure of water less even than that commonly present in the atmosphere. Such a solution, placed in an open vessel, will not evaporate. On the contrary, it will take up moisture from the air and increase in bulk. For this reason very soluble substances are commonly moist and, when exposed to the air, extract water from the latter and dissolve in this water. This behavior is called deliquescence, and is shown, for example, by the hydrate of calcium chloride $\mathrm{CaCl}_{2}, 6 \mathrm{H}_{2} \mathrm{O}$, used to dry gases (p. 59).

Boiling-Points of Solutions. - The boiling-point of a liquid is that temperature at which the vapor pressure reaches $760 \mathrm{~mm}$. 
(see p. 62). Since the addition of a non-volatile solute lowers the vapor pressure of a pure liquid, it naturally raises the boilingpoint to a higher temperature.

In dilute non-conducting solutions, equal numbers of molecules of different solutes raise the boiling-point of a given solvent to the same extent. Thus, one molecular weight of sugar $\left(\mathrm{C}_{12} \mathrm{H}_{22} \mathrm{O}_{11}=342 \mathrm{~g}\right.$. $)$ or of glycerine $\left(\mathrm{C}_{3} \mathrm{H}_{8} \mathrm{O}_{3}=92 \mathrm{~g}\right.$.), dissolved in $1000 \mathrm{~g}$. of water, will each raise the boiling-point from $100^{\circ}$ to $100.52^{\circ}$.

Molecular weights of non-volatile, non-conducting substances can therefore be determined by finding out what weight of the substance, when dissolved in $1000 \mathrm{~g}$., is required to raise the boilingpoint of water from $100^{\circ}$ to $100.52^{\circ}$.

Freezing-Points of Solutions. - The addition of a solute similarly tends to prevent the freezing of the solution, for freezing means the separation of a part of the pure solvent in the form of ice. Hence solutions can be frozen only at temperatures below those of the pure solvents. Thus, one molecular weight of a substance, such as sugar (342 g.) or glycerine (92 g.), dissolved in $1000 \mathrm{~g}$. of water, will cause the water to freeze at $-1.86^{\circ}$ instead of $0^{\circ}$. Molecular weights can be measured by this method also.

This behavior explains why sea water is frozen in cold weather much less often than fresh water.

It explains also why salt thrown on ice will cause the latter to melt. Saturated salt solution freezes only at $-21^{\circ}$, to give a mixture of pure ice and pure salt, both in solid form. Hence, ice and salt can not permanently exist together above that temperature. When the outside temperature is below $-21^{\circ}$, salt will no longer melt the ice. But calcium chloride, which is more soluble, will do so. A mixture of ice and salt, giving the temperature $-21^{\circ}$, is called a freezing mixture. Such a mixture is used in freezing ice cream and ices. 


\section{Definition of a Saturated Solution: A Warning.-To} avoid a common misconception, it must be noted that solution is not a process of filling the pores of the liquid. If that were true, approximately equal weights of all substances would find accommodation in equal volumes of water. The fact is that, for example, 100 c.c. of water can dissolve $195 \mathrm{~g}$. of silver fluoride, but only $0.00000035 \mathrm{~g}$. of silver iodide, although the space available (if there is any such space) is the same in both cases.

The same conclusion is reached when we consider that two forms of the same salt may have different solubilities. Thus, at $20^{\circ}$, $\mathrm{Na}_{2} \mathrm{SO}_{4}, 10 \mathrm{H}_{2} \mathrm{O}$ can give about $18 \mathrm{~g}$. of $\mathrm{Na}_{2} \mathrm{SO}_{4}$ to 100 c.c. of water. But anhydrous sodium sulphate $\mathrm{Na}_{2} \mathrm{SO}_{4}$ at $20^{\circ}$ gives $59 \mathrm{~g}$. to the same amount of water.

The reader is also warned against the frequent definition of a saturated solution as one containing all of the solute that it can hold. A supersaturated solution evidently holds more. The saturated solution under any given conditions is that solution which, when placed in contact with excess of the solute, is found to be in equilibrium.

The molecular hypothesis may again be called to our assistance in this connection. When we have a solute (either crystalline, or liquid or gaseous) in contact with its saturated solution, and therefore in equilibrium with it, two opposing tendencies must balance each other at the surface of contact. One of these is the tendency of the solute particles to escape into solution, the other is the tendency of the solute particles already in solution to return back to the solute. The first of these tendencies (the intensity of the hail of particles thrown off from the surface of the solute into a given solvent, under given conditions of temperature and pressure) we may regard as constant. The second tendency (the intensity of the hail of particles returning from the solution to the surface of the solute) will increase steadily as the concentration of the solute particles in the solution increases. At one definite concentration only, therefore, can these two opposing tendencies 
counterbalance, namely that of the saturated solution. The rate at which solute particles are returning from the solution just equals, in this case, the rate at which they are entering. With unsaturated solutions, containing less solute, the number of returning particles will be deficient, and the solute will continue to dissolve until it all disappears or saturation is reached. With supersaturated solutions, on the other hand, containing more solute, the number returning will be in excess, and deposition of home-coming particles on the solute surface will continue until this excess is wiped out.

If, however, we have a supersaturated solution in which no free solute is present, the solute particles in the solution have no home to return to, no surface.upon which they can deposit themselves. They are therefore compelled to continue wandering around and around in the solution, having lost their equilibrium completely. By violent shaking or stirring we may succeed in inducing crystallization in such a solution, but the only certain means of establishing equilibrium conditions is inoculation with a small fragment of the solute.

Units Used in Expressing Concentrations.- The concentrations of solutions, saturated and otherwise, are sometimes expressed in physical, and sometimes in chemical, units of weight. When physical units are employed, we give the number of grams of the solute held in solution by one hundred grams of the solvent.

When chemical units of weight are employed, two different plans are possible, and both are in use. Either the equivalent (p. 53) or the atomic weights may be taken as a basis of measurement. In the former case, the solutions are called normal solutions, and in the latter, molar solutions.

\section{A normal solution contains one gram-equivalent of the solute} in one liter of solution (not in $1 \mathrm{l}$. of solvent). The word " equivalent " has been used hitherto only of elements, and this application of the expression involves an extension of its meaning. An 
equivalent weight of a compound is that amount of it which will interact with one equivalent of an element. Thus, a formulaweight of hydrochloric acid $\mathrm{HCl}(36.5 \mathrm{~g}$.) is also an equivalent weight, for it contains $1 \mathrm{~g}$. of hydrogen, and this amount of hydrogen is displaceable by. one equivalent weight of a metal. A formula-weight of sulphuric acid $\mathrm{H}_{2} \mathrm{SO}_{4}(98 \mathrm{~g}$.), however, contains two equivalents of the compound, and a formula-weight of aluminium chloride $\mathrm{AlCl}_{3}$ (133.5 g.) three equivalents. Hence normal solutions of these three substances contain, respectively, $36.5 \mathrm{~g} . \mathrm{HCl}, 49 \mathrm{~g} . \mathrm{H}_{2} \mathrm{SO}_{4}$, and $44.5 \mathrm{~g}$. $\mathrm{AlCl}_{3}$ per liter of solution. The special property of normal solutions is, obviously, that equal volumes of two of them contain the exact proportions of the solutes which are required for complete interaction. Solutions of this kind are much used in quantitative analysis. We frequently use also decinormal or one-tenth normal solutions $(0.1 N$ or $N / 10)$, and seminormal $(0.5 N$ or $N / 2)$, and six times normal solutions $(6 N)$, and so forth.

\section{A molar solution contains one mole (gram-molecular weight)} of the solute in one liter of solution (not in 11 . of solvent). When molecular formulæ (p. 78) are used, this means one gram-formula weight per liter. In the cases cited above, the molar solution contains 36.5 g. $\mathrm{HCl}, 98$ g. $\mathrm{H}_{2} \mathrm{SO}_{4}$, and 133.5 g. $\mathrm{AlCl}_{3}$ per liter.

Is Dissolving a Physical or a Chemical Change? - This is a question still much discussed amongst chemists. Probably in simple, typical cases, like dissolving paraffin in gasoline or benzene, the process may be considered purely physical, and the solution contains both components in unchanged chemical condition.

On the other hand, when water is used, as it is more frequently than any other solvent, chemical changes undoubtedly take place. The water itself, at least, is always changed. Water in the liquid state is not simply $\mathrm{H}_{2} \mathrm{O}$. Its physical properties indicate that it is an associated liquid, extensive combination having taken 
place between simple $\mathrm{H}_{2} \mathrm{O}$ molecules to form more complex molecules with the general formula $\left(\mathrm{H}_{2} \mathrm{O}\right)_{n}$. Dissolving any substance in water must upset the equilibrium amongst these different kinds of molecules:

$$
\left(\mathrm{H}_{2} \mathrm{O}\right)_{\mathrm{n}} \rightleftarrows \mathrm{nH}_{2} \mathrm{O}
$$

and produce more of one and less of the other kind. This is the extent of the chemical change in the water.

The dissolved substance probably combines also, in the majority of cases, with part of the water. The nature of the compounds is hard to determine, and no simple statement can as yet be made about them. But the compounds, whatever they are, are physically dissolved in the rest of the water.

Dissolving, therefore, is partly a chemical, and only partly a pure physical process. The striking differences in solubility already mentioned (p. 110) may consequently be accounted for partly on a chemical, and partly on a physical basis. The main chemical factor is compound formation between the components of the solution. The more extensive this is, the greater, in general, is the solubility. Thus substances which form definite hydrates with water are mostly extremely soluble, while substances which are only difficultly soluble in water invariably crystallize out from an aqueous solution in an anhydrous state. The main physical factor is the relative magnitude of the cohesive forces between the various types of molecules present in the solution. Thus in the case of water and benzene, the water molecules attract one another much more strongly than they do the benzene molecules. Molecules of benzene endeavoring to intermingle with water molecules encounter, therefore, very considerable resistance, and are almost certain to be squeezed out. The two liquids, indeed, are found to be practically immiscible.

Exercises. - 1. Give two ways of separating a mixture, consisting of a suspended solid and a liquid (p. 109).

2. If you had a spot on your clothing consisting of: (a) grease, 
(b) sugar, or (c) sugar and grease together, or (d) varnish, how should you proceed in each case to remove the spot?

3. If chalk ( $5 \mathrm{~g}$.) and potassium chlorate ( $5 \mathrm{~g}$.) were mixed, how should you separate them (p. 13)? Explain how you could secure each substance.

4. Could you make (a) a concentrated, (b) a saturated solution of chalk in water (p. 111)? Of alcohol in water?

5. If you saturated 200 c.c. of water at $70^{\circ}$ with (a) salt, or (b) potassium nitrate, and then cooled the clear liquid to $20^{\circ}$, what weight of the solid substance would separate out in each case (p. 113)?

6. To make as concentrated a solution of lime water as possible, should you use hot water or cold (p. 113)?

7. Explain why boiled water has a slightly different taste from tap-water that has not been boiled (p. 66).

8. If $100 \mathrm{~g}$. of a non-volatile substance, dissolved in $1000 \mathrm{~g}$. of benzene $\left(\mathrm{C}_{6} \mathrm{H}_{6}\right)$, lower the vapor pressure from 74.8 to 68.0 $\mathrm{mm}$., what is the molecular weight of the substance (pp. 117-8)?

9. Explain why potassium carbonate becomes wet, and finally dissolves, when exposed to moist air. How must calcium chloride be preserved from becoming moist?

10. If $52 \mathrm{~g}$. of a substance dissolved in $1000 \mathrm{~g}$. of water gives a solution boiling at $100.26^{\circ}$, what is the molecular weight of the substance (p. 119)?

11. If $68.5 \mathrm{~g}$. of a substance, dissolved in $500 \mathrm{~g}$. of water gives a solution freezing at $-1.86^{\circ}$, what is the molecular weight of the substance (p. 119)?

12. How much glycerine $\left(\mathrm{C}_{3} \mathrm{H}_{8} \mathrm{O}_{3}\right)$ could you dissolve in $1000 \mathrm{~g}$ of water, and still be able to freeze the water with ice and salt (p. 119)?

13. Explain why a sodium acetate hot-water bottle can be used over and over again. What is the source of the heat it gives out each time it is used? 


\section{CHAPTER XI \\ HYDROCHLORIC ACID. CALCULATIONS}

THus far, the substances we have studied have been mainly air and its components and water and its constituents. Another of the simpler, familiar substances, common salt or sodium chloride $(\mathrm{NaCl})$ may now be taken up. Large amounts of it are used in the household, in cooking and in making freezing mixtures. Still larger quantities are consumed in manufacturing washing soda and soap, for both of which it supplies the necessary sodium. It is employed also to furnish the chlorine for bleaching materials. We shall consider it first as a means of making compounds of chlorine.

Preparation of Hydrogen Chloride.- When a few drops of commercial, concentrated sulphuric acid $\left(\mathrm{H}_{2} \mathrm{SO}_{4}\right)$ are poured upon common salt in an open dish, vigorous effervescence begins. This indicates that a gas is forming bubbles upon the salt and that the bubbles are rising through the layer of acid and bursting. The gas is itself invisible, but when we breathe upon the contents of the vessel, a heavy fog is produced. This is due to condensation of water vapor (in the breath) to droplets of water, in which the gas has dissolved. The fog is composed, in fact, of drops of a solution of hydrogen chloride $(\mathrm{HCl})$ in water, which receives the name of hydrochloric acid (in commerce, muriatic acid).

In order to handle the gas more readily, the sulphuric acid may be allowed to fall from a funnel, drop by drop, upon salt contained in a flask (Fig. 47). Soon the air in the flask is all displaced by the gas, and the latter issues from the open delivery tube. If a U-tube containing some water is attached to the 125 
delivery tube, the gas dissolves in the water as fast as it is formed.

If the correct proportions of the materials are used, then, when the action is over, all that remains in the flask is a white solid, different from salt, and called sodium-hydrogen sulphate $\mathrm{NaHSO}_{4}$.

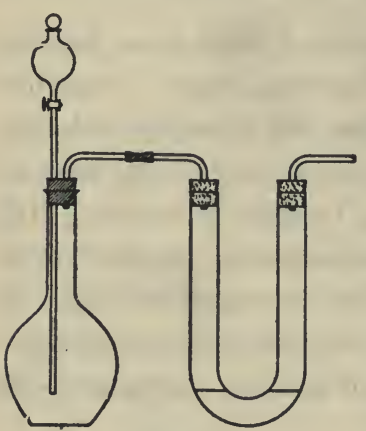

FIG. 47 A part of this may be in solution in a little water, contained originally in the commercial sulphuric acid, of which water commonly forms from 6 to 7 per cent. The equation is easy to make from the formulæ given,

$\mathrm{NaCl}+\mathrm{H}_{2} \mathrm{SO}_{4} \rightarrow \mathrm{HCl} \uparrow+\mathrm{NaHSO}_{4}$,

and requires no further balancing. (An arrow pointing upward is used in equations to indicate that the substance to which it refers removes itself from the reaction by escaping in the form of a gas.)

Other Sources of Hydrogen Chloride.-Chlorides of other metals could be substituted for the sodium chloride in this action, and all but the less soluble ones would give hydrogen chloride freely. Common salt is employed because it is the cheapest of the chlorides.

While theoretically any acid would, like sulphuric acid, furnish the required hydrogen, and liberate hydrogen chloride, yet in practice no other acid works so well. Some, like phosphoric acid $\mathrm{H}_{3} \mathrm{PO}_{4}$, act too slowly, because they do not dissolve sodium chloride so readily. Others, like hydrofluoric acid HF, are too volatile, and the heat of the action would send them over with the hydrogen chloride in the form of vapor. Others, like nitric acid $\mathrm{HNO}_{3}$, would react chemically with hydrogen chloride. Still others, like hydriodic acid HI, being gases, could be used only in aqueous solution, and the water would dissolve the hydrogen chloride produced, and prevent its escape from the vessel. Aside 
from these objections, all the other acids are more expensive than sulphuric acid.

\section{The Molecular View of the Interaction of Sulphuric Acid} and Salt.- One who has used the above-described methods for making hydrogen chloride without reflection would not realize the complexity of the machinery by which the result is achieved. The means are apparently very simple. Yet the mechanical features of this experiment, when laid bare, are extremely curious and interesting. A single fact will show the possibilities which are concealed in it.

If we take a saturated solution of sodium-hydrogen sulphate in water and add to it a concentrated solution of hydrogen chloride in water (concentrated hydrochloric acid), we shall perceive at once the formation of a copious precipitate. This is composed entirely of minute cubes of sodium chloride:

$$
\mathrm{NaHSO}_{4}+\mathrm{HCl} \rightarrow \mathrm{H}_{2} \mathrm{SO}_{4}+\mathrm{NaCl} \downarrow \text {. }
$$

(An arrow pointing downwards is used in equations to indicate that the substance to which it refers removes itself from the reaction in the form of a precipitate.) Now this action is nothing less than the precise reverse of (1), yet it proceeds with equal success. In fact, this chemical interaction is not only reversible (p. 103), but can be carried virtually to completion in either direction. It is only in presence of a large amount of water, sufficient to keep both the hydrogen chloride and the salt all in solution, that it stops midway in its career and is valueless for securing a complete transformation in either direction:

$$
\mathrm{NaHSO}_{4}+\mathrm{HCl} \rightleftarrows \mathrm{H}_{2} \mathrm{SO}_{4}+\mathrm{NaCl} \text {. }
$$

In an action which is reversible, if the products remain as perfectly mixed and accessible to each other as were the initial substances, their interaction will continually undo a part of the work of the forward direction of the change. Hence, in such a case the 
reaction must, and does, come to a standstill while as yet only partly accomplished; but this was not the case with action. (1) and (2). Let us examine the means by which the premature cessation of each was avoided.

In equation (1) the salt dissolved to some extent in the sulphuric acid, $\mathrm{NaCl}$ (solid) $\rightleftarrows \mathrm{NaCl}$ (dslvd.), and so, by intimate contact of the two kinds of molecules in the resulting solution, the products $\mathrm{HCl}$ and $\mathrm{NaHSO}_{4}$ were formed. On the other hand, the hydrogen chloride, being practically insoluble in sulphuric acid, escaped as fast as it was formed: $\mathrm{HCl}$ (dslvd.) $\rightleftarrows \mathrm{HCl}$ (gas). Hence, in that case, almost no reverse action was possible, and the double decomposition went on virtually to completion. With all the sodium-hydrogen sulphate in the bottom of the flask, and most of the hydrogen chloride in the space above, the two products might as well have been in separate vessels so far as any efficient re-interaction was concerned. This plan, in which water is purposely excluded, forms therefore the method of making hydrogen chloride.

In equation (2), on the other hand, the hydrogen chloride was taken in aqueous solution, and was mixed with a concentrated solution of sodium-hydrogen sulphate. The acid was, therefore, kept permanently in full contact with the sodium-hydrogen sulphate. It had in this case, every opportunity to interact with the latter and no chance of escape. Every molecule of each ingredient could reach every molecule of the other with equal ease. Furthermore, the sodium chloride, produced as a result of their activity, is not very soluble in concentrated hydrochloric acid (far less so than in water), and so it came out as a precipitate: $\mathrm{NaCl}$ (dslvd.) $\rightleftarrows \mathrm{NaCl}$ (solid). But this was almost the same as if it had gone off as a gas. It meant that the greater part of the salt was in the solid form. In this form, it was no longer able to interact effectively molecule to molecule with the other product, the sulphuric acid. Hence, there was little reverse action to impede the progress of the primary one. Thus (2) is 
nearly as perfect a way of liberating sulphuric acid as (1) is of liberating hydrogen chloride.

Precipitation.- When two soluble substances are dissolved separately in water, and the solutions are mixed, chemical interaction frequently is evident between the dissolved materials. If one of the products is not very soluble, then a supersaturated solution (p. 115) of this product may be thus produced. As a rule, this substance immediately becomes visible as a fine powder, called a precipitate, suspended in the liquid. More or less rapidly, according to its fineness of dispersion, this precipitate settles out, leaving the solution clear. Equation (2) in the preceding paragraph is an example of such a reaction.

Often the precipitated product can be recognized by the physical appearance of the precipitate, and so this sort of action is used as a test for one of the original substances. Thus, precipitates are classified according to their color. Again, precipitates of the same color differ in degree of dispersion, and may be described as gelatinous, curdy, pulverulent, or crystalline. In the two former cases, at least, the precipitation is so sudden that there is not time for crystals to be formed, and the product is amorphous (see p. 95).

Physical Properties of Hydrogen Chloride.- Hydrogen chloride is a colorless gas. It is sour in taste, and has a sharp odor. It is irritating, but not poisonous in small amounts.

The gas is exceedingly soluble in water, one volume of which, at $15^{\circ}$, will dissolve no less than 455 volumes of the gas. The saturated solution at $15^{\circ}$ contains nearly 43 per cent of the gas by weight. The concentrated hydrochloric acid of commerce contains about 35 per cent.

The density of the gas (weight of 1 c.c.) is 0.001628 . Of more interest to the chemist is the weight of 22,400 c.c. or 22.4 liters (the gram-molecular volume), namely 36.468 grams. This is the 
molecular weight of the substance. As we have seen (p. 74): it is made up of $1.008 \mathrm{~g}$. of hydrogen combined with $35.46 \mathrm{~g}$. of chlorine.

Is the gas heavier or lighter than air? This question is answered at once if we recall the fact that the 22.4-liter cube-full of air weighs $28.95 \mathrm{~g}$. (p. 85). The gas is one-fourth heavier. It may therefore be collected by upward displacement (Fig. 26a, p. 52).

The gas can be liquefied by pressure alone at any temperature below $52^{\circ}$ (its critical temperature). One atmosphere pressure will liquefy it at $-84^{\circ}$, which is therefore the boiling-point of liquefied hydrogen chloride.

When the concentrated aqueous solution is heated, it is the hydrogen chloride and not the water which is vaporized, for the most part. When the concentration has been reduced to 20.2 per cent, the rest of the mixture distils unchanged at $110^{\circ}$. This occurs because, at this concentration, the hydrogen chloride is carried off in the bubbles of steam in the same proportion in which it is present in the liquid. If a dilute solution is used, water is the chief product of distillation (about $100^{\circ}$ ), but gradually the boiling-point rises and, when the concentration has reached 20.2 per cent once more, the same hydrochloric acid of constant boiling-point $\left(110^{\circ}\right.$ at $760 \mathrm{~mm}$.), as it is called, forms the residue.)

Chemical Properties of Hydrogen Chloride.- In the case of a compound, the chemical property in regard to which we first enquire is its stability (p. 27). Is it easy or difficult to decompose by heating? Hydrogen chloride must be heated above $1500^{\circ}$ before even a trace of it is dissociated into hydrogen and chlorine. Pure hydrogen chloride is therefore a very stable and, from a chemical point of view, rather an inactive substance. It has no action on non-metals, such as phosphorus, carbon, sulphur, etc. However, many of the more active metals (see p. 54), such as potassium, sodium, and magnesium, decompose it. Hydrogen is set free, and the chloride of the metal is formed.

$$
2 \mathrm{~K}+2 \mathrm{HCl} \rightarrow 2 \mathrm{KCl}+\mathrm{H}_{2} \uparrow \text {. }
$$


When hydrogen chloride is mixed with ammonia $\mathrm{NH}_{3}$ the gases unite to form a cloud of fine, solid particles of ammonium chloride.

$$
\mathrm{HCl}+\mathrm{NH}_{3} \rightarrow \mathrm{NH}_{4} \mathrm{Cl} \downarrow \text {. }
$$

Chemical Properties of Hydrochloric Acid.- The solution of hydrogen chloride in water is an entirely different substance in its behavior from hydrogen chloride. (1) The solution is sour in taste. (2) It changes the color of litmus, a vegetable coloring matter, from blue to red. (3) It is a conductor of electricity, and is decomposed by the current, hydrogen being liberated at the negative wire (p. 55). (4) When the metals preceding hydrogen in the order of activity (p. 54) are introduced into hydrochloric acid, hydrogen is displaced and liberated.

In a later chapter (ch. xv) we shall see that these four properties of hydrogen chloride in aqueous solution are properties common to all substances called acids. We may sum up the main properties of a solution of hydrogen chloride in water in one word, therefore, by saying that it is an acid.

Hydrochloric acid interacts with many other compounds in solution. In some instances, one of the new substances produced can be seen, because it appears as a precipitate. One such example has already been discussed in detail (see equation 2, p. 127). When hydrochloric acid is added to a solution of silver nitrate $\left(\mathrm{AgNO}_{3}\right.$ lunar caustic), a precipitate of silver chloride $(\mathrm{AgCl})$ is obtained, which is white and curdy in appearance. The other product, nitric acid $\left(\mathrm{HNO}_{3}\right)$, remains dissolved and invisible:

$$
\mathrm{HCl}+\mathrm{AgNO}_{3} \rightarrow \mathrm{AgCl} \downarrow+\mathrm{HNO}_{3} .
$$

Uses of Hydrochloric Acid.- This substance is used as a source of chlorine. It is employed for cleaning metals. Although present in very small proportions (about 1 part in 500) in the gastric juice of the stomach, it is a most important component of this fluid. It is sometimes given as a medicine, when the natural supply is too small. 
Double Decomposition. - In this chapter we have met for the first time with another variety of chemical change. If we examine the equation for the action of silver nitrate on hydrochloric acid (p. 131), we shall see that the silver nitrate decomposed as if it had been made up of two parts, namely $(\mathrm{Ag})$ and $\left(\mathrm{NO}_{3}\right)$. The hydrochloric acid similarly separated into its two parts $(\mathrm{H})$ and $(\mathrm{Cl})$. The $(\mathrm{Ag})$ then united with the $(\mathrm{Cl})$ and the $(\mathrm{H})$ with the $\left(\mathrm{NO}_{3}\right)$.

$$
(\mathrm{Ag})\left(\mathrm{NO}_{3}\right)+(\mathrm{H})(\mathrm{Cl}) \rightarrow(\mathrm{Ag})(\mathrm{Cl})+(\mathrm{H})\left(\mathrm{NO}_{3}\right) .
$$

Since both original substances decomposed, this whole change is called a double decomposition. A sort of exchange between the halves of the decomposing substances took place.

The hydrogen chloride was prepared by an action (p. 126), which, if we write it as follows, is seen to be of the same class:

$$
(\mathrm{Na})(\mathrm{Cl})+(\mathrm{H})\left(\mathrm{HSO}_{4}\right) \rightarrow(\mathrm{H})(\mathrm{Cl})+(\mathrm{Na})\left(\mathrm{HSO}_{4}\right) .
$$

The Varieties of Chemical Change.-Almost all chemical changes belong to one or other of the varieties we have already met with and defined (pp. 14, 16, 51). These, along with one example of each, are now placed together:

1. Combination: $\mathrm{Zn}+\mathrm{S} \rightarrow \mathrm{ZnS}$.

2. Decomposition: $2 \mathrm{KClO}_{3} \rightarrow 2 \mathrm{KCl}+3 \mathrm{O}_{2}$.

3. Displacement: $\mathrm{Zn}+\mathrm{H}_{2} \mathrm{SO}_{4} \rightarrow \mathrm{H}_{2}+\mathrm{ZnSO}_{4}$.

4. Double Decomposition: $\mathrm{AgNO}_{3}+\mathrm{HCl} \rightarrow \mathrm{AgCl}+\mathrm{HNO}_{3}$.

In the first, 2 substances give 1 substance.

In the second, 1 substance gives 2 (or more) substances.

In the third, 1 element and 1 compound give 1 element and 1 compound.

In the fourth, 2 compounds give 2 compounds.

This classification suffices for most purposes. But, for special kinds of cases, some other names are used. Thus, a dissociation 
(p. 104) is an action which belongs to both of the first two classes, because it is reversible. For example,

$$
2 \mathrm{H}_{2} \mathrm{O} \rightleftarrows 2 \mathrm{H}_{2}+\mathrm{O}_{2} \text {. }
$$

Again oxidation (p. 40) and reduction (p. 57) are connected with the particular substances, such as oxygen, which are concerned in the action. The first classification (Nos. 1 to 4 ) paid no attention to the kinds of elements which were present. Thus, every decomposition is a decomposition. If it is reversible, then it is also a dissociation. If oxygen is set free, then it is a reduction as well.

\section{Calculations}

Calculations Connected with Formulae. - In a previous chapter (p. 99) we have seen that formulæ represent the composition of substances; and we have seen how the formula of each substance is worked out from the data obtained by experiment. Some ways in which the information contained in formulæ can be used may now be taken up.

The Composition from the Formula.- Take, for example, the formula for silver nitrate, $\mathrm{AgNO}_{3}$. To learn the composition of this compound, we look up the atomic weights (see rear cover of this book). We find $\mathrm{Ag}=107.88$ parts of silver, $\mathrm{N}=14.008$ parts of nitrogen, $\mathrm{O}_{3}=3 \times 16$ or exactly 48 parts of oxygen. The proportions of the constituents, in the same order, therefore, are $107.88: 14.008: 48$.

What is the proportion of oxygen to nitrogen alone? It is $48: 14.008$, or $3.427: 1$.

Significant Figures.- The division of 14.008 into 48 really gives the quotient 3.426613. But no atomic weights have been measured so accurately that we know the values of the numbers beyond the third place of decimals. For many elements, we do 
not know even the first place accurately. Hence 3.427 is just as likely to be the exact value as the longer number. In the most exact calculation we round the number off, usually, at the second decimal. For rougher purposes the first decimal place is sufficient.

The Formula-Weight. - The sum of the weights of the constituents indicated in the formula is called the formula-weight. For silver nitrate this has the value, $107.88+14.008+48$, or 169.89 (Query: Why not 169.888 ?).

If the substance is a gas, or is easily volatile, the formula-weight will be also the molecular weight. Thus, acetylene gas (used in lighting) has the formula $\mathrm{C}_{2} \mathrm{H}_{2}$. The composition is $\mathrm{C}_{2}=2 \times$ 12.005 or 24.010 parts of carbon and $\mathrm{H}_{2}=2 \times 1.008$ or 2.016 parts of hydrogen. The molecular weight is $24.010+2.016$ $=26.026$. Again, hydrogen peroxide (used in medicine), a liquid, is decomposed when boiled, but it dissolves in water and depresses the freezing-point. Its molecular weight has therefore been determined (p. 119) and is $\mathrm{H}_{2} \mathrm{O}_{2}$. The molecular weight on which this formula is based is $2.016+32$, or 34.016 .

When the substance is neither volatile nor soluble, the simplest formula is always used, and therefore only the formula-weight can be ascertained.

To Find the Percentage Composition.- In silver nitrate the proportions are 107.88 of silver, 14.008 of nitrogen, and 48 of oxygen in a total of 169.89 . In one hundred parts, the silver will be $\frac{107.88}{169.89} \times 100$, or 63.50 ; the nitrogen $\frac{14.008}{169.89} \times 100$, or 8.25 , and the oxygen $\frac{48}{169.89} \times 100=28.25$.

The same results may be obtained by the rule of proportion. Thus, for the silver, $169.89: 107.88:: 100: x$ where $x$ is the percentage of silver. 
Calculations by Use of Equations.-We frequently desire to know what weight of a product can be obtained from a given weight of the necessary materials. For example, what weight of zinc sulphide can be made with $100 \mathrm{~g}$. of sulphur? It is understood, of course, that the necessary zinc is available.

In such calculations mistakes are easily made. The following rules must be strictly followed:

First, write down the equation:

$$
\mathrm{Zn}+\mathrm{S} \rightarrow \mathrm{ZnS} \text {. }
$$

Second, place beneath each formula the weight for which it stands:

$$
\underset{65.37}{\mathrm{Zn}}+\underset{32.06}{\mathrm{~S}} \rightarrow \underset{97.43}{\mathrm{ZnS}}
$$

Third, read the whole statement. In this case it reads: 65.37 parts by weight of zinc combine with 32.06 parts of sulphur to give 97.43 parts of zinc sulphide.

Fourth, re-read the original problem. Then place the amount given in the problem (100 g. of sulphur) under the formula of the substance concerned. Then observe that the problem asks "What weight of zinc sulphide?" and place an interrogation point or an $x$ under the formula of that substance:

$$
\begin{gathered}
\mathrm{Zn} \\
65.37 \mathrm{~S}
\end{gathered} \begin{array}{cc}
32.06 & \mathrm{ZnS} \\
100 & ? \text { or } x
\end{array}
$$

Fifth, read the problem as it now appears in this expanded equation: $32.06 \mathrm{~g}$. of sulphur will give $97.43 \mathrm{~g}$. of zinc sulphide, therefore $100 \mathrm{~g}$. of sulphur will give $x \mathrm{~g}$. of zinc sulphate.

Sixth. The answer may be now obtained by stating the proportion in the same order:

$$
32.06: 97.43:: 100: x(=303.9) .
$$


If the expanded equation has been prepared correctly, this final statement is purely mechanical. It will be seen that only two of the three quantities in the equation were really used.

Alternative to the Sixth Step.-We may also say: If $32.06 \mathrm{~g}$. of sulphur will give $97.43 \mathrm{~g}$. of zine sulphide, $1 \mathrm{~g}$. of sulphur will give $\frac{97.43}{32.06} \mathrm{~g}$. (= $=3.039 \mathrm{~g}$.) of zinc sulphide. Then, if $1 \mathrm{~g}$. of sulphur gives $3.039 \mathrm{~g}$. of zinc sulphide, the $100 \mathrm{~g}$. of sulphur will give $100 \times 3.039 \mathrm{~g}$. ( $=303.9 \mathrm{~g}$.) of zinc sulphide.

Warnings.- In solving the exercises at the end of the chapter, beware of three kinds of mistakes commonly made by beginners.

1. Conquer a tendency to say that the symbols $\mathrm{Zn}$ and $\mathrm{S}$ stand for " 1 part " of zinc or of sulphur. They stand for 1 chemical unit, or atomic weight, or atom, in each case, - that is to say, for 65.37 " parts" and 32.06 " parts," respectively.

2. Follow the rules laid down above. When one has once become familiar with the art of solving such problems, running through the rules takes only a few seconds. The chemist does it almost unconsciously. The beginner always thinks he can ignore these rules, and he fails in consequence. Writing the equation in the expanded form, and then reading the problem into it are absolutely essential steps.

3. Do not read the original problem carelessly and make the equation backwards, that is, with the sides reversed. If there seems to be confusion somewhere, when the last steps are reached, this hint will probably show the cause of the difficulty.

Another Example. - What weight of hydrogen is required to reduce $45 \mathrm{~g}$. of magnetic oxide of iron to metallic iron?

Following the rules, as before, we reach the expanded equation:

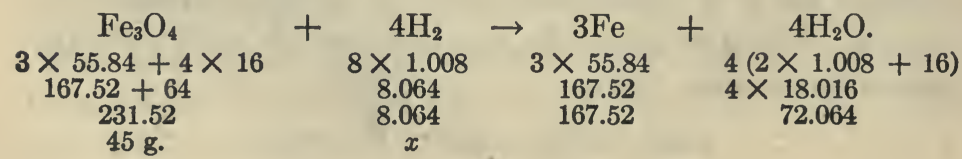


Observe that the atomic weights are multiplied by the sub-numbers, so that, for example, $\mathrm{Fe}_{3}=3 \times 55.84$. Observe also that the formula weights are multiplied by the coefficients, when such occur, in front of the formula, so that, for example, $4 \mathrm{H}_{2} \mathrm{O}=4$ $\times 18.016$.

The proportion $231.52: 8.064:: 45: x(=1.57)$ supplies the answer, 1.57 grams of hydrogen.

Using the alternative plan (p. 130): If $231.52 \mathrm{~g}$. of magnetic oxide are reduced by $8.064 \mathrm{~g}$. of hydrogen, $1 \mathrm{~g}$. will be reduced by $\frac{8.064}{231.52}$ g. ( $=0.035$ g.) of hydrogen. Hence, if $1 \mathrm{~g}$. of magnetic oxide is reduced by $0.035 \mathrm{~g}$. of hydrogen, $45 \mathrm{~g}$. will be reduced by $45 \times 0.035 \mathrm{~g}$. ( $=1.57 \mathrm{~g}$.) of hydrogen.

Exercises.- 1. Complete the equation $\mathrm{ZnCl}_{2}+\mathrm{H}_{2} \mathrm{SO}_{4} \rightarrow$ $\mathrm{ZnSO}_{4}+$, and attach the name of the substance to each formula in it.

2. Point out the differences in physical properties between oxygen and hydrogen chloride.

3. Make equations for the displacement of hydrogen from hydrochloric acid by zinc and by sodium (pp. 51 and 52 ).

4. Give additional examples of the four varieties of chemical change (p. 132).

5. Classify (p. 132) the following actions: (a) the action of steam on iron (p. 51); (b) the rusting of iron; (c) the electrolysis of dilute hydrochloric acid (p. 55); (d) the effect of heating the hydrate of cupric sulphate (p. 68).

6 . Is the decomposition of mercuric oxide a case of: (a) dissociation, or (b) oxidation or reduction?

7. What is the proportion of: (a) sodium to one part of chlorine in salt; (b) one part of hydrogen to nitrogen in nitric acid $\left(\mathrm{HNO}_{3}\right)$ ?

8. Calculate the percentage composition of: (a) sulphuric acid; (b) acetylene $\mathrm{C}_{2} \mathrm{H}_{2}$.

9. What weight of hydrogen is displaced by the action of $100 \mathrm{~g}$. of zinc upon an excess of hydrochloric a.cid $\left(\mathrm{ZnCl}_{2}\right.$ is formed)? 
10. What weight of silver chloride is formed by the interaction of $5 \mathrm{~g}$. of sodium chloride with silver nitrate?

11. What should be the difference in cost of $1000 \mathrm{~g}$. of oxygen according as it is obtained by decomposing mercuric oxide at $\$ 3.00$ per kilog. or potassium chlorate at 30 cents per kilog.? 


\section{CHAPTER XII}

\section{CHLORINE. CALCULATIONS}

Chlorine was discovered by Scheele (1774). It was supposed to be a compound containing oxygen, until the contrary was proved by Davy (1809-1818). It is used in immense quantities for making bleaching and disinfecting agents, explosives and dyestuffs. For its use in gas warfare, see Chapter XL.

Occurrence.- Many compounds of chlorine occur in nature, but the most plentiful is common salt $(\mathrm{NaCl})$. Most of the matter dissolved in sea water is sodium chloride. Salt also occurs underground, either in strata in almost pure form, or mixed with rocky material. Near such deposits, wells and springs of salt water are common.

Preparation by Electrolysis of a Chloride.-Chlorine is liberated by passing a current of electricity through a concentrated aqueous solution of a chloride, such as hydrogen chloride or sodium chloride (see Fig. 27, p. 54). Much of it is, in fact, manufactured by electrolyzing natural brines. The chlorine is liberated, first in solution and later as a gas, at the positive wire (anode).

Other Products of Electrolysis of Chlorides. - In electrolyzing hydrochloric acid, the hydrogen is set free at the negative wire (cathode). With a solution of sodium chloride we might expect to get free sodium at this wire. It will be recalled, however, that sodium is very much more active than is hydrogen, and indeed displaces hydrogen from water. Hence the electrical energy sets free the more easily liberated element - the hydrogen - 
and the sodium remains in the solution as sodium hydroxide $(\mathrm{NaOH})$. The process is best shown by a diagram:

$$
\begin{aligned}
& \dddot{2} \dddot{\mathrm{Na}}: \mathrm{Cl} \rightarrow \mathrm{Cl}_{2} \uparrow \text { (pos. wire) } \\
& \text { (neg. wire) } \uparrow \mathrm{H}_{2} \leftarrow 2 \mathrm{H} \vdots \mathrm{OH} \vdots
\end{aligned}
$$

The chlorine $\mathrm{Cl}_{2}$ and hydrogen $\mathrm{H}_{2}$ being liberated, leave behind in the solution the constituents of $2 \mathrm{NaOH}$ :

$$
2 \mathrm{NaCl}+2 \mathrm{H}_{2} \mathrm{O}+\text { Elect. } \rightarrow \mathrm{H}_{2}+\mathrm{Cl}_{2}+2 \mathrm{NaOH} \text {. }
$$

\section{Preparation from Oxygen and Hydrogen Chloride.-} When hydrogen chloride and oxygen gases are heated, they interact very slowly to give water and chlorine. The action is greatly hastened by contact with copper chloride. Lumps of pumice,

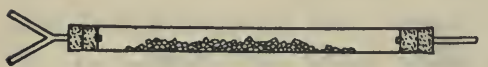

FIG. 48 saturated with a solution of this catalyst (see p. 32), are placed in a tube. When the mixture of gases is passed over the heated pumice (Fig. 48), steam, chlorine, and about 20 per cent of unchanged oxygen and hydrogen chloride issue at the other end:

Skeleton equation: $\mathrm{HCl}+\mathrm{O}_{2} \rightleftarrows \mathrm{H}_{2} \mathrm{O}+\mathrm{Cl}_{2}$.

Balanced equation: $4 \mathrm{HCl}+\mathrm{O}_{2} \rightleftarrows 2 \mathrm{H}_{2} \mathrm{O}+2 \mathrm{Cl}_{2}$.

Longer heating does not alter the proportion of the materials successfully transformed. This is Deacon's process.

That 80 per cent is changed, and 20 per cent unchanged, is due to the fact that the action is reversible. If we lead pure chlorine and steam through the tube (read the equation backwards), 20 per cent of hydrogen chloride and oxygen are formed. No more than 20 per cent is formed, because these products are continually being used up again and reproduce steam and chlorine. If one product could be separated (p. 128) from the other, to prevent the backward action, the yield could be raised to 100 per 
cent. But all the four substances are gases (in the hot tube), and mix perfectly.

The results here noted are interesting, because they show that, under the conditions of the experiment, oxygen is somewhat more active than chlorine in combining with hydrogen. The precise proportions of the four gases present at equilibrium depend on the temperature at which the experiment is carried out. In the commercial application of Deacon's process, this is near $345^{\circ}$.

\section{Preparation from Hydrochloric Acid and an Oxidizing} Agent.- The best way to make a supply of chlorine in the laboratory is to place potassium permanganate crystals $\left(\mathrm{KMnO}_{4}\right)$ in a flask (Fig. 49) and allow concentrated hydrochloric acid, previously mixed with an equal volume of water, to fall upon them drop by drop. The gas is rather soluble in water, and is best collected by displacing the air from bottles. When one bottle is full, it is stoppered and a fresh one substituted. To

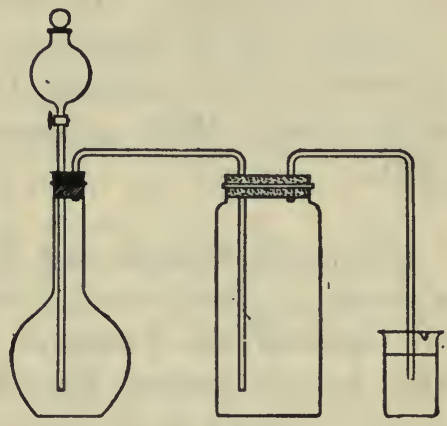

FIG. 49 avoid the escape of the very irritating gas into the room the tube from the collecting bottle dips beneath sodium hydroxide solution.

The essential feature of this reaction is that the oxygen of the potassium permanganate unites with the hydrogen of the hydrochloric acid to give water. The potassium and manganese take as much chlorine as they require to form their chlorides, $\mathrm{KCl}$ and $\mathrm{MnCl}_{2}$. The rest of the chlorine is liberated.

Skeleton: $\mathrm{KMnO}_{4}+\mathrm{HCl} \rightarrow \mathrm{H}_{2} \mathrm{O}+\mathrm{KCl}+\mathrm{MnCl}_{2}+\mathrm{Cl}_{2}$.

To convert all the oxygen of the $\mathrm{KMnO}_{4}$ to water, we require $8 \mathrm{HCl}$. The formation of $\mathrm{KCl}$ and $\mathrm{MnCl}_{2}$ uses up 3 of the 
8 atoms of chlorine thereby made available, leaving $5 \mathrm{Cl}$ to be liberated. Since this chlorine is obtained as $\mathrm{Cl}_{2}$, it is necessary to double our quantities throughout.

Balanced: $2 \mathrm{KMnO}_{4}+16 \mathrm{HCl} \rightarrow 8 \mathrm{H}_{2} \mathrm{O}+2 \mathrm{KCl}+2 \mathrm{MnCl}_{2}+5 \mathrm{Cl}_{2}$.

This action is an oxidation of the hydrogen chloride by the permanganate. The potassium permanganate, which supplied the oxygen, is called the oxidizing agent. Since the permanganate lost oxygen, it was itself reduced. In all oxidations one substance is oxidized and another reduced.

Deacon's process (p. 140) is also an oxidation of hydrogen chloride (by free oxygen). The oxygen is reduced to water.

Chlorine may be prepared by using other substances to oxidize hydrochloric acid. Amongst those which are suitable are manganese dioxide $\mathrm{MnO}_{2}$, potassium chlorate $\mathrm{KClO}_{3}$ and red lead $\mathrm{Pb}_{3} \mathrm{O}_{4}$.

Manganese Dioxide and Hydrogen Chloride.- The action of manganese dioxide upon hydrochloric acid is an instructive one. It is a general rule, of which we shall meet many applications, that when an acid interacts with an oxide of a metal, there are two constant features in the result, namely: (1) The oxygen of the oxide combines with the hydrogen of the acid to form water, and (2) the metal of the oxide combines with the acid radical of the acid. Here the skeleton equation should be $\mathrm{MnO}_{2}+\mathrm{HCl} \rightarrow$ $\mathrm{H}_{2} \mathrm{O}+\mathrm{MnCl}_{4}$. With $\mathrm{O}_{2}$, to form water, $4 \mathrm{HCl}$ is required, and the product is $2 \mathrm{H}_{2} \mathrm{O}$. Hence the equation is

Balanced: $\quad \mathrm{MnO}_{2}+4 \mathrm{HCl} \rightarrow 2 \mathrm{H}_{2} \mathrm{O}+\mathrm{MnCl}_{4}$.

This is, undoubtedly, what happens in the first place. The products actually obtained on heating the mixture, however, are water, manganous chloride $\mathrm{MnCl}_{2}$ and chlorine. We owe the chlorine to the fact that the tetrachloride is unstable. At low temperatures it decomposes into manganese trichloride $\left(\mathrm{MnCl}_{3}\right)$ 
and chlorine. When the mixture is warmed, the $\mathrm{MnCl}_{3}$ breaks down further into $\mathrm{MnCl}_{2}$ and chlorine. The complete series of reactions may be represented in one equation as follows:

$$
\mathrm{MnO}_{2}+4 \mathrm{HCl} \rightarrow 2 \mathrm{H}_{2} \mathrm{O}+\mathrm{MnCl}_{2}+\mathrm{Cl}_{2} \text {. }
$$

If we had used manganous oxide $\mathrm{MnO}$, we should have had a double decomposition:

$$
\mathrm{MnO}+2 \mathrm{HCl} \rightarrow \mathrm{H}_{2} \mathrm{O}+\mathrm{MnCl}_{2},
$$

but we should have got no chlorine. The difference between these two actions will be discussed in a later chapter (p. 215).

Physical Properties. - Chlorine is a greenish-yellow gas, and takes its name from the Greek for this color. It has an exceedingly disagreeable odor and irritates the lining of the nose and throat. Alcohol vapor or ammonia, when breathed, relieves the irritation.

The density of the gas is recorded in the formula $\mathrm{Cl}_{2}$. The 22.4-liter-cube-full weighs $70.92 \mathrm{~g}$., against $28.95 \mathrm{~g}$. for air, so that chlorine is about $2 \frac{1}{2}$ times heavier. Two volumes of the gas dissolve in one volume of water at $20^{\circ}$. The solution is called chlorine-water.

The gas is liquefied by pressure below $146^{\circ}$ (its critical temperature), the pressure required at $20^{\circ}$ being 6.6 atmospheres. The liquid boils at $-33^{\circ}$, and solidifies at $-102^{\circ}$ (its meltingpoint).

Chemical Properties. - Chlorine is an element with about the same degree of activity as oxygen (compare p. 141), and it unites with very much the same list of other elements. The compounds are called chlorides.

Unites with Metals. - When powdered antimony (cold) or iron powder (warmed) are thrown into chlorine, they combine with it, and red hot particles of the chlorides, $\mathrm{SbCl}_{3}$ or $\mathrm{FeCl}_{3}$, fall to the bottom. Copper leaf (Dutch metal, used in "gilding "), 
or heated copper foil, burns in the gas, giving a fog of solid cupric chloride $\mathrm{CuCl}_{2}$.

Skeleton: $\quad \mathrm{Sb}+\mathrm{Cl}_{2} \rightarrow \mathrm{SbCl}_{3}$.

Balanced: $\quad 2 \mathrm{Sb}+3 \mathrm{Cl}_{2} \rightarrow 2 \mathrm{SbCl}_{3}$.

Sodium burns brilliantly in chlorine, giving sodium chloride. That a shining metal and a poisonous irritant like chlorine, in uniting, should yield a mild, household article like common salt illustrates very well the extraordinary nature of chemical change.

When thoroughly freed from moisture, chlorine no longer combines with metals like copper and iron. Water seems to be needed as a contact agent, in these, as well as in hundreds of other chemical actions. Hence, carefully dried chlorine in compressed liquid form can be, and is, stored and sold in iron cylinders (see detinning, p. 508).

Unites with Hydrogen.-A jet of burning hydrogen, lowered into a bottle of chlorine, continues to burn, giving hydrogen chloride $\mathrm{HCl}$, the presence of which is shown by the fog produced by allowing the gas to come in contact with moist air:

$$
\mathrm{H}_{2}+\mathrm{Cl}_{2} \rightarrow 2 \mathrm{HCl} \text {. }
$$

Hydrogen and chlorine, mixed, do not combine when cold, provided strong light is excluded. But sunlight, or light from burning magnesium ("flashlight powder"), starts the combination, which occurs with explosive violence. Plunging a lighted taper into the mixture has, of course, the same effect.

Acts upon Compounds Containing Hydrogen.-Because of its activity toward hydrogen, chlorine removes hydrogen from many compounds. Thus, if a lighted wax taper be plunged into chlorine, it continues to burn, though with a feebler flame. Dense smoke, composed of particles of free carbon, rise from the flame. 
Blowing the breath into the jar, afterwards, gives the fog due to hydrogen chloride. Thus the presence of hydrogen and carbon in the wax of the taper is proved. From this we learn, also, that chlorine has a relatively small tendency to combine with carbon. A few drops of warm turpentine $\left(\mathrm{C}_{10} \mathrm{H}_{16}\right)$ upon a slip of filter paper will blaze up in chlorine, giving hydrogen chloride and an immense cloud of soot:

Skeleton: $\quad \mathrm{C}_{10} \mathrm{H}_{16}+\mathrm{Cl}_{2} \rightarrow \mathrm{C}+\mathrm{HCl}$.

Balanced: $\quad \mathrm{C}_{10} \mathrm{H}_{16}+8 \mathrm{Cl}_{2} \rightarrow 10 \mathrm{C}+16 \mathrm{HCl}$.

Acts upon Water. - We have seen that chlorine acts on steam, reversing Deacon's reaction to the extent of 20 per cent. It acts also upon cold water, when dissolved in the latter, although in a similarly incomplete way. With half-saturated chlorinewater at $10^{\circ}$, about one-third of the chlorine is transformed. One of the products, hypochlorous acid $\mathrm{HOCl}$, is of especial interest, because it is an exceedingly active substance, much used as an oxidizing agent (see p. 223) and for bleaching:

$$
\mathrm{Cl}_{2}+\mathrm{H}_{2} \mathrm{O} \rightleftarrows \mathrm{HCl}+\mathrm{HOCl} \text {. }
$$

Unites with Non-metals.- Phosphorus burns in the gas, giving the vapor of phosphorus trichloride $\mathrm{PCl}_{3}$. This substance, with excess of chlorine, forms the pentachloride $\mathrm{PCl}_{5}$. When phosphorus pentachloride is heated, it vaporizes and dissociates again partially into the trichloride and chlorine, according to the reversible reaction:

$$
\mathrm{PCl}_{5} \rightleftharpoons \mathrm{PCl}_{3}+\mathrm{Cl}_{2} \text {. }
$$

Sulphur, when heated with chlorine, combines slowly, giving sulphur monochloride $\mathrm{S}_{2} \mathrm{Cl}_{2}$, used in vulcanizing rubber.

Although chlorine does not combine very readily with carbon, several compounds of carbon and chlorine are very important, such as chloroform $\mathrm{CHCl}_{3}$, carbon tetrachloride $\mathrm{CCl}_{4}$ (p. 332) and phosgene $\mathrm{COCl}_{2}$ (p. 484). 
Composition of Hydrogen Chloride.- Now that we are familiar with the properties of chlorine, as well as with those

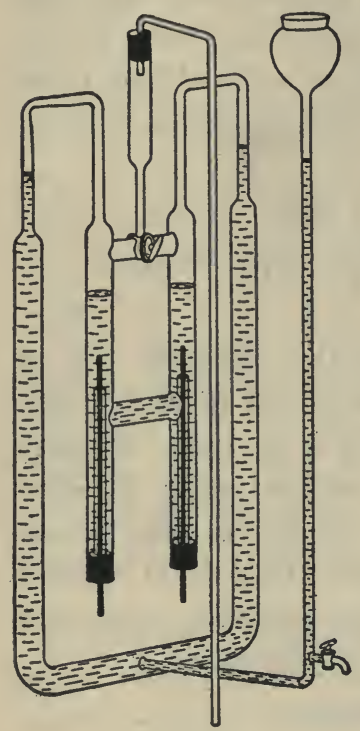

FIG. 50 of hydrogen, we may return to the question of the proportion by volume in which they are produced by decomposition of hydrogen chloride. When we electrolyze hydrochloric acid in the apparatus shown in Fig. 27 (p. 54), we find that the chlorine dissolves to a large extent in the liquid, and its true volume as gas is not easily ascertained. The apparatus (Brownlee's) in Fig. 50 avoids the difficulty by enabling us to saturate the liquid with chlorine before observing the collected gases. The volumes of the two gases are found to be equal.

A simpler apparatus (Fig. 51) may be used to show the same fact. The gases are generated, by electrolysis, in the testtube, pass through the straight tube, driving the air before them, and finally bubble through sodium hy-

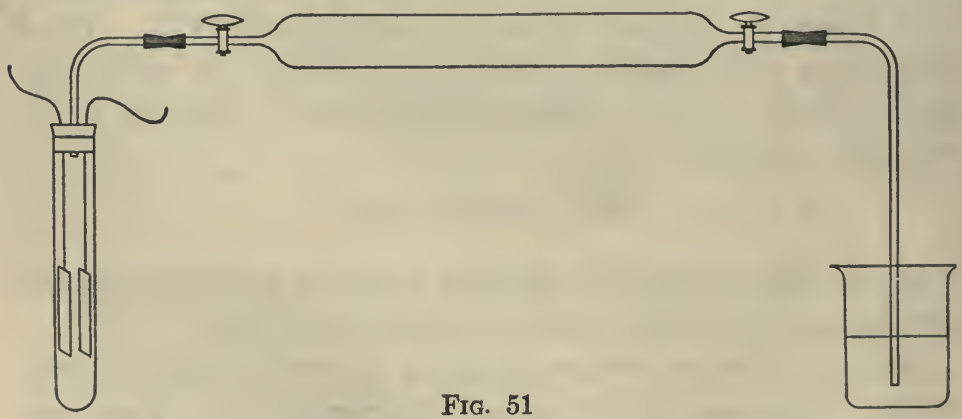

droxide solution. The whole apparatus must be covered with a dark cloth to exclude light, and handled in diffused light. In fifteen 
minutes, chlorine ceases to be dissolved in the test-tube, and the gases come off in their natural proportions. In half-an-hour more they have filled the tube. The stop cocks are now closed, the tube is set in a tall cylinder containing potassium iodide (KI) solution (Fig. 52) and the lower stop cock is opened. The potassium iodide acts upon the chlorine, giving potassium chloride and free iodine which is a solid,

$$
2 \mathrm{KI}+\mathrm{Cl}_{2} \rightarrow 2 \mathrm{KCl}+\mathrm{I}_{2}
$$

and the liquid rises until it fills half the tube. The remaining gas burns and is hydrogen.

The volume of the hydrogen chloride, in relation to the volumes of the constituents, may be learned by using a different apparatus (Fig. 53). A testtube of heavy glass is filled with dry hydrogen chloride and closed with a rubber stopper greased with vaseline. A little sodium amalgam (solution

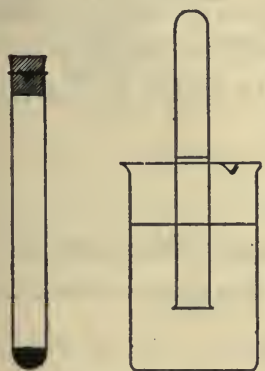

Fig. 53 of sodium in mercury) is introduced, and the stopper instantly replaced. When the contents are shaken for one

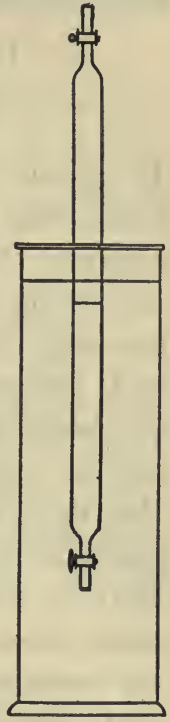

Fig. 52 or two minutes, the sodium combines with the chlorine and the hydrogen is liberated and remains. The mouth of the test-tube is then immersed in a jar of mercury, and the stopper withdrawn. The mercury rises and fills about half the tube.

Conclusion. The hydrogen has half the volume of the hydrogen chloride, and the volume of the chlorine is equal to that of the hydrogen. Therefore:

1 vol. hydrogen +1 vol. chlorine $\rightarrow 2$ vols. hydrogen chloride. This result further illustrates Gay-Lussac's law (p. 60). 
Confirmation of the Formula $\mathrm{Cl}_{2}$ and $\mathrm{H}_{2}$ - - According to Avogadro's hypothesis, there are equal numbers of molecules in equal volumes of these gases. Let the rectangles represent small volumes, containing 1000 molecules each:

\begin{tabular}{|c|c|c|c|c|c|}
\hline Hydroge & Chloride & & ydroge & & Chlorine \\
\hline 1000 & 1000 & $\leftarrow$ & 1000 & + & 1000 \\
\hline
\end{tabular}

It appears from this that 2000 molecules of hydrogen chloride come from 1000 molecules of hydrogen and 1000 molecules of chlorine. Now, each molecule of hydrogen chloride contains at least one atom of hydrogen, so that the 1000 molecules of hydrogen must have given at least 2000 atoms of hydrogen, one for eich molecule of the compound. Hence each molecule of hydrogen contains at least two atoms. The same is true of each molecule of chlorine. We have no reason, however, for supposing that there are more than two atoms in either molecule; no substance is known which contains less than $1.008 \mathrm{~g}$. hydrogen or 35.46 g. chlorine in its unit weight (p. 75). Hence the conclusion is confirmed which we reached before (p. 79), namely, that the formulæ of the free gases are $\mathrm{H}_{2}$ and $\mathrm{Cl}_{2}$, and that single atoms of the elements occur only in combination (as in $\mathrm{NaCl}, \mathrm{HCl}$, etc.).

\section{Calculations}

Calculations Involving Volumes of Gases.- For such calculations the simplest method is always to use the gram-molecular volume - the 22.4-liter cube.

Relative Densities of Gases.- What are the relative weights of equal volumes of chlorine and hydrogen chloride? The formulæ record the weights of 22.4 liters: $\mathrm{Cl}_{2}=2 \times 35.46=70.92$, and $\mathrm{HCl}=1.008+35.46=36.468$. The required relation is $70.92: 36.468$, so that chlorine is almost twice as heavy, bulk for bulk, as hydrogen chloride. 
Density Compared with Air.-We have seen (p. 85) that 22.4 liters of air weigh $28.95 \mathrm{~g}$. Is water vapor heavier or lighter, at the same temperature and pressure? The formula, $\mathrm{H}_{2} \mathrm{O}$, shows that 22.4 liters of steam weigh $2 \times 1.008+16=18.016$. Air is heavier, in the raiio of $29: 18$, approximately.

The Volume of Gas from Given Weights of Material.What volume of oxygen at $0^{\circ}$ and $760 \mathrm{~mm}$. is obtained by heating 15 g. of potassium chlorate? Follow the rules given (p. 135) for calculating weights:

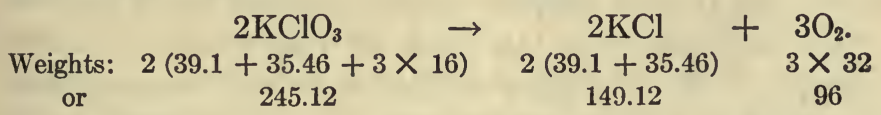

Now, insert the volumes in the case of substances which are gases. Here oxygen is the only one. Remembering that $\mathrm{O}_{2}(=32 \mathrm{~g}$.) occupies 22.4 liters, we see that the volume of the oxygen is $3 \times$ 22.4 liters, or 67.2 liters. As the next step, add the data given and the $x$ in their proper places. The $x$ here goes under the volume of oxygen:

$\begin{gathered}2 \mathrm{KClO}_{3} \\ 245.12 \mathrm{~g} . \\ 15 \mathrm{~g} .\end{gathered} \rightarrow \underset{149.12 \mathrm{~g} .}{2 \mathrm{KCl}}+\underset{96 \mathrm{~g} . \text { (or } 67.2 \text { liters) }}{3 \mathrm{O}_{2} .}$

The problem now reads thus: If $245.12 \mathrm{~g}$. of potassium chlorate give 67.2 liters of oxygen, $15 \mathrm{~g}$. of potassium chlorate will give $x$ liters of oxygen:

$$
245.12: 67.2:: 15: x(=4.07),
$$

where $x$, the answer, is 4.07 liters of oxygen.

Observe that, when we use the gram-molecular volume, one proportion gives the answer. It is not necessary to make two steps in the calculation, by finding first the weight of the oxygen, and then its volume. The beginner always tends to do this, until he learns by experience that it takes twice as long to solve the problem in this way and that the chance of obtaining the wrong answer_by making arithmetical errors is greatly increased. 
Another Example. - What weight of copper will combine with 15.2 liters of chlorine (at $0^{\circ}$ and $760 \mathrm{~mm}$.)?
$\mathrm{Cu}$
$63.57 \mathrm{~g}$.
$x$
$+$
$\mathrm{Cl}_{2}$
70.92 g. or $22.4 \mathrm{l}$.
15.2 liters
$\mathrm{CuCl}_{2}$.
$134.49 \mathrm{~g}$.

The proportion is:

$$
22.4: 63.57:: 15.2: x(=43.14 \text { g.). }
$$

At Other than Standard Conditions. - If the problem concerns a gas at some temperature and pressure not $0^{\circ}$ and 760 $\mathrm{mm}$., then correction must be made as a separate calculation. For example, if the $\mathbf{1 5 . 2}$ liters of chlorine, in the foregoing illustration, had been at $15^{\circ}$ and $742 \mathrm{~mm}$., then the volume would first have to be reduced to $0^{\circ}$ and $760 \mathrm{~mm}$. (See p. 47.)

Questions of Volume Alone.-When weights are not mentioned in the question, but volumes only, the calculation is very simple. For example: What are the relative volumes of oxygen and hydrogen chloride used in Deacon's process?

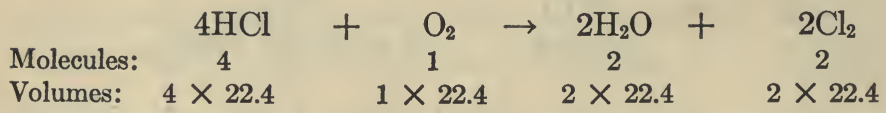

Remembering that equal numbers of molecules occupy equal volumes, and that $4 \mathrm{HCl}=4$ molecules, $\mathrm{O}_{2}=1$ molecule, etc., we perceive that 4 volumes of $\mathrm{HCl}$ will be required for 1 volume of oxygen.

Does the volume change during the process? Yes, $4+1$ volumes become $2+2$, or 5 volumes give 4 .

Warnings. - The commonest mistake made in these calculations is to neglect to use, in the equations, the molecular formulæ. We must use $\mathrm{H}_{2}, \mathrm{O}_{2}, \mathrm{C}_{2} \mathrm{H}_{2}$ (acetylene), etc., (and not $\mathrm{H}$, $\mathrm{O}$, or $\mathrm{CH}$ ), because the 22.4 liters hold the weights represented by the molecular formulæ. 
Before calculating the volume (gaseous) of a substance represented in an equation, consider whether it is a volatile substance. Only volumes of gases can be calculated by the rules given above.

Exercises.-1. What would be the results of electrolyzing aqueous solutions of: (a) potassium chloride; (b) cupric chloride (cf. p. 54).

2. How should you separate the chlorine and the steam produced by Deacon's process?

3. Make equations showing the interactions with hydrochloric acid of: (a) manganese dioxide; (b) potassium chlorate; (c) red lead. The metals form $\mathrm{MnCl}_{2}, \mathrm{KCl}$, and $\mathrm{PbCl}_{2}$, respectively.

4. What would be the pressure in a cylinder of liquid chlorine at $20^{\circ}$ ?

5. Make equations for the union of chlorine with: (a) copper; (b) sodium; (c) iron; (d) phosphorus; (e) sulphur.

6. When plunged into chlorine, a jet of illuminating gas continues to burn. A stream of soot rises from the flame, however, and blowing the breath into the jar, afterwards, produces a fog. What do you infer as to the constituents of illuminating gas?

7. What information is conveyed by the fact that the formula of the chloride of sulphur is written $\mathrm{S}_{2} \mathrm{Cl}_{2}$, and not $\mathrm{SCl}$ ?

8. Make the molecular equation for the action of sodium upon hydrogen chloride (p. 131). Why does not the mercury interact with the latter (cf. p. 54)?

9. What facts led us, in Chap. VIII, to the conclusion that the molecular weight of chlorine was 70.92 while its atomic weight was 35.46 ?

10. What are the relative densities (p. 85) of: (a) oxygen and chlorine; (b) hydrogen and hydrogen chloride; (c) hydrogen and air; (d) acetylene $\mathrm{C}_{2} \mathrm{H}_{2}$ and air?

11. What volume of hydrogen chloride at $0^{\circ}$ and $760 \mathrm{~mm}$. is obtained by the interaction of $65 \mathrm{~g}$. of sodium chloride, and an excess of sulphuric acid (p. 126)? 
12. What weight of zine is required to make 100 liters of hydrogen, at $10^{\circ}$ and $750 \mathrm{~mm}$., by displacement from hydrochloric acid (p. 53)?

13. What are the relative volumes of the factors and of the products in the interaction between: (a) turpentine vapor and chlorine: (b) oxygen and carbon disulphide vapor (giving $\mathrm{SO}_{2}$ and $\mathrm{CO}_{2}$ )?

14. What are the relative volumes of the products in the decomposition of: (a) mercuric oxide; (b) chlorine monoxide $\mathrm{Cl}_{2} \mathrm{O}$ (chlorine and oxygen are formed)?

15. What are the relative volumes of the volatile substances concerned in the action of water vapor on iron (p. 102)?

16. Using the relative volumes in which oxygen and hydrogen combine to form steam, prove that each molecule of free oxygen is composed of at least two atoms (cf. p. 105).

17. (a) Calculate the weight of chlorine dissolved by 100 c.c. of water at room temperature (p. 143). (b) In half-saturated chlorine-water, what is the concentration of hypochlorous acid (p. 145)? 


\section{CHAPTER XIII \\ ENERGY AND CHEMICAL CHANGE}

IN the description of chemical changes, the fact that heat was evolved has frequently been mentioned. In several instances a current of electricity has been used to produce chemical change. It is now necessary to collect these scattered facts and classify them for future use.

\section{Physical Accompaniments of Chemical Change.-When} iron and sulphur combined (p. 14), and when iron burned in oxygen or copper in chlorine, much heat was developed. On the other hand, the decomposition of mercuric oxide, as was pointed out (p. 15), owed its continuance to the persistent application of heat and ceased as soon as the source of heat was withdrawn. Here, apparently, heat was consumed during the progress of the change, and the chemical action was limited by the amount of heat supplied. The production or consumption of heat may, therefore, be a feature of chemical change.

In the burning of iron or magnesium in oxygen, and in the actions of chlorine on copper and turpentine, light was also produced. Conversely, silver chloride can be kept any length of time in the dark, but in sunlight it becomes first bluish and then brown, simultaneously giving off chlorine gas and finally leaving only silver as a fine powder. Silver bromide or iodide, in photographic plates, films, and paper, is changed by light in a similar way, liberating the bromine or iodine. It would appear, therefore, that light may be given out or consumed in connection with chemical change.

We have seen (p. 55) that a current of electricity may be employed to decompose hydrochloric acid and other chlorides, and 
the battery, or other source of the current must be kept going or the chemical change stops. The inverse of this is likewise familiar.

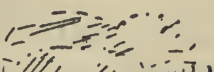

जill/

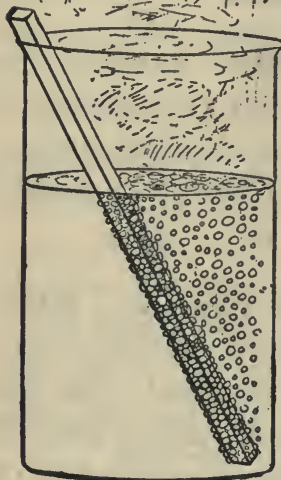

FIG. 54

If we place in dilute sulphuric acid a stick of the metal zinc, we find that hydrogen is given off (Fig. 54), that the zinc goes into solution as zinc sulphate (p. 102), and that a large amount of heat is developed. The presence of small quantities of other metals in the zinc catalyzes this reaction. If impure zinc in fine particles, with much surface, is used, the temperature of the liquid may even rise spontaneously to the boilingpoint. This form of the action produces heat. If, however, we attach the same stick of zinc to a copper wire and, having provided a plate of platinum also connected with a wire, immerse the two simultaneously in the acid (Fig. 55), then a galvanometer, with which the wires are connected, shows at once

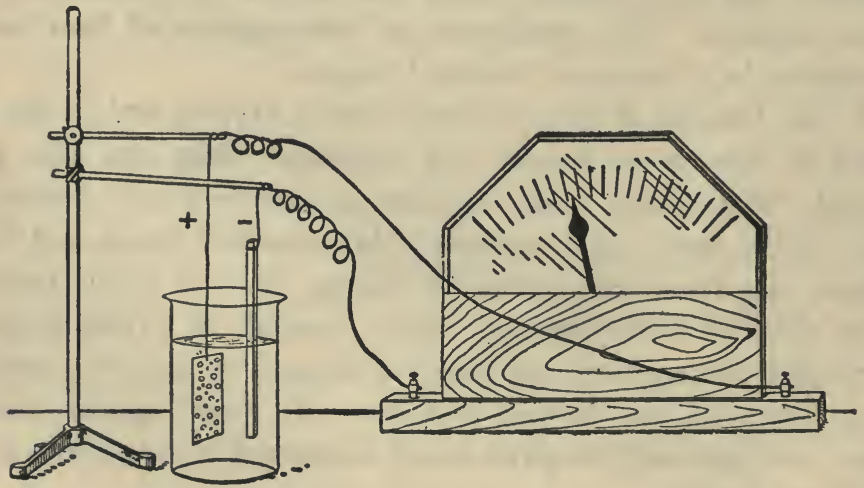

FIg. 55

the passage of a current of electricity round the circuit. Exactly the same chemical change goes on as before. The sole difference 
is that the gas appears to arise from the surface of the platinum. It is easy to show, however, that the platinum by itself is not acted upon by dilute acids and, in this case, undergoes no change whatever; it serves simply as a suitable conductor for the electricity. Here, then, in place of the heat which the first plan produced, we get an electric current. The arrangement is, in fact, a battery-cell, for a battery is a system in which a chemical action which would otherwise give heat furnishes electricity instead. Thus, electrical energy may be consumed or produced in connection with a change in composition.

Even violent rubbing in a mortar, in the case of some substances, can effect an appreciable amount of decomposition in a few minutes. In this way silver chloride can be separated into silver and chlorine, just as by light. It is the mechanical energy which is the agent, and part of it is consumed in producing the change, and only the balance appears as heat. Conversely, the production of mechanical energy, as the result of chemical change, is seen in the behavior of explosives and in the working of our muscles. Thus, mechanical energy may be used up or produced in chemica. changes.

Summing up our experience, we may state that no change in composition occurs without some accompaniments, such as the production or consumption of heat, light, electrical energy, or, in some cases, mechanical energy.

\section{Classification of the Accompaniments of Change in} Composition: Energy. - The problem of classifying (i.e., placing in a suitable category) things like heat, light, and electricity has occupied much attention. In all changes in composition, one of these natural accompaniments is given out or absorbed, sometimes in great amount, yet in none is any alteration in weight observed.* There are many things which are real,

* Electrons (see p. 195) do possess mass, but it is very small compared with that of the materials concerned. 
however, even if they are not affected by gravitation. In the present instance we reason as follcws:

A brick in motion is different from a brick at rest. The former can do some things that the latter cannot. Furthermore, we can easily make a distinction in our minds. The brick can be deprived of the motion and be endowed with it again. Thus, we can get the idea of motion as a separate conception. Similarly, we observe that a piece of iron behaves differently when hot, and when cold, when bearing a current of electricity, and when bearing none. We conceive then of the brick or the iron as having a certain amount and kind of matter which is unalterable, and as having motion, heat, or electricity added to this or removed. Thus, we describe our observations, by using two categories, one of which includes the various kinds of matter, and the other, various things whose association with matter seems to be invariable and is often so conspicuous. The latter we call the forms of energy.

\section{The Practical Importance of Energy in Chemistry.-} The absorption or liberation of energy accompanying a chemical transformation of matter is often, of the two, the more important feature. We do not burn coal in order to manufacture carbon dioxide gas. We are glad to get rid of the material product through the chimney. It is the heat we want. We do not buy gasoline (petrol) for an automobile in order to obtain various gases to expel through the muffler. We really pay for the mechanical energy. It is the same with burning illuminating-gas or magnesium powder when we want light, and with eating food, which we do, chiefly, to get energy to sustain our activity. We do not run electricity for hours into a storage battery, in order to make a particular compound (lead dioxide, for example), but in order to save and store the energy for future use. In industry and life fully half the total arnount of chemical change involved is set in motion by us, solely on account of the energy changes it involves. 
But the production of energy in chemical change is not only thus of practical importance; it is also of scientific interest, as will be seen in the section on energy and chemical activity (see below).

\section{Interconvertibility of Forms of Energy: Conservation.-} At first sight, these different forms of energy seem to be quite unrelated. But a relation between them can be found. If the heat of a Bunsen flame or of the sun is brought under a hot-air motor (Fig. 56) violent motion results. Again, if the motor is connected with a dynamo, electricity may be generated. Still again, if the current from the dynamo flows through an incandescent lamp, heat and light are evolved. Conversely, when motion of the hot-air motor is impeded by a brake, heat appears. When a current of electricity is run through the dynamo, the armature of the latter turns and motion results. But the most significant facts are still to be mentioned. The heat absorbed by the motor is found to be greater when the machine is permitted to move and do work, than when it is not. Thus, it is found that when work is done some heat disappears, and this heat is, in fact, transformed into work. Similarly, when the poles of the dynamo are properly connected and electricity is being produced, and only

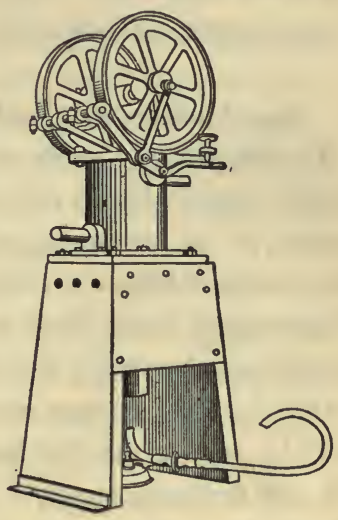

Fig. 56 then, motion is used up. This is shown by the effort required to turn the armature under these circumstances, and the ease with which it is turned when the circuit is open. So, with a conductor like the filament in the lamp, unless it offers resistance to the current and destroys a sufficient amount of electrical energy, it gives out neither light nor heat. Finally, motion gives no heat unless the brake is set, and effort is then demanded to 
maintain the motion. These experiences lead us to believe that we have here a set of things which are fundamentally of the same kind, for each form can be made from any of the others. We have, therefore, invented the conception of a single thing, of which heat, light, electricity, and motion are forms, and to it we give the name energy: energy is work and every other thing which can arise from work and be converted into work.

Closer study shows that equal amounts of electrical or mechanical energy always produce equal amounts of heat. No loss is ever observed in the transformations of energy, any more than in the transformations of matter. Hence we have been led to the conclusion that in a limited system no gain or loss of energy is ever observed. This brief statement of the results of many experiments is called the law of the conservation of energy.

\section{Application of the Conception of Energy in Chemistry.-} At first sight it looks as if the statement that energy is conserved is not applicable in chemistry. Heat and electricity, for example, seem to be produced and consumed, in connection with changes in composition, in a mysterious manner. We trace light in an incandescent lamp back to the electricity, and this in turn to the mechanical energy, and this again to the heat in the engine. But what form of energy gave the heat developed by the combustion of the coal under the boiler, or by the union of iron and sulphur in our first experiment? Since we do not perceive any electricity, light, heat, or motion, in the original materials, and yet wish to create an harmonious system, we are bound to conceive of the iron and the sulphur, and the coal and the air, as containing another form of energy, which we call internal energy. Similarly, when heat is used up in decomposing mercuric oxide, or light in decomposing silver chloride, we regard the energy as passing into, and being stored in the products of decomposition in the form of internal energy.

These conclusions compel us, for the sake of consistency, to 
think of all our materials as repositories of energy as well as of matter, each of these two constituents being equally real and equally important. A piece of the substance known as "iron" must thus be held to contain so much iron matter and so much internal energy. So ferrous sulphide contains sulphur matter, iron matter, and internal energy. Thus, by a substance we mean a distinct species of matter, simple or compound, with its appropriate proportion of internal energy. During the progress of a chemical change, like the union of iron and sulphur, the internal energy of the system also changes. The total energy which can thus be made available as the result of a chemical action, and converted (through, say, heat or electrical energy) into work, is called the free energy of the reaction.

In the course of this discussion it has become clear that it is characteristic of chemical phenomena that, besides a change in the nature of the matter, there is always an alteration in the amount of internal energy in the system. This alteration involves the production of internal energy from, or the transformation of internal energy into some other form of energy.

Energy and Chemical Activity.- Other things being equal, when the free energy of a reaction is large, the reaction proceeds rapidly; that is to say, a large proportion of the reacting materials are changed in the unit of time. Those reactions in which the change of internal energy is small proceed more slowly. The speed of a chemical change, and the quantity of energy available because of it, are therefore closely related. Now, we are accustomed to speak of materials which, like iron and sulphur, interact rapidly and with liberation of much energy as "chemically active," or as possessing great "chemical affinity" for one another. Thus, relative chemical activity or affinity may be estimated, (1) by observing the speed of a change or, in many cases (2) by measuring the heat developed or (3) by ascertaining the electromotive force of the current, when the materials are 
arranged in the form of a battery-cell. The order of activity of the metals, given on p. 54, is obtained by this third method.

It is evident that the chemical activity or affinity of a given substance will not be the same towards all others. Thus, iron unites much more vigorously with chlorine than with sulphur and, with identical amounts of iron, more heat is liberated in the former case than in the latter. With silver, sodium, and many other substances, iron does not unite at all. One of the tasks of the chemist is to make such comparisons as this. He calls the results, the specific chemical properties of the substances in question.

Care must be observed, however, in making comparisons by the above methods. Although it is true that most chemical changes that take place readily develop heat, yet at high temperatures compounds can be formed by the direct union of their elements with absorption of heat (see, for example, p. 312). Reversible reactions must also be accounted for. In such reactions, one of the chemical changes taking place necessarily absorbs just as much heat as the other develops, yet both have a definite speed under any given conditions, as is shown by the fact that a fixed point of equilibrium is reached. Deacon's process (p. 140) is a good instance. As we shall see later (chapter $\mathrm{XX}$ ), the relative concentrations of the reacting substances, as well as their affinities, must be taken into consideration.

\section{The Cause of Chemical Activity or Affinity.-The} reader will undoubtedly be inclined to inquire whether we can assign any cause for the tendency which substances have to undergo chemical change. Why do iron and sulphur unite to form ferrous sulphide, while other pairs of elements taken at random will frequently be found to have no effect upon one another under any circumstances? A final answer to this question cannot, of course, be given. As the facts regarding chemical activity or affinity, however, become better known, we may arrive at a stage,where a logical explanation (see p. 23) can be advanced which, 
basing itself on these facts, affords a means of classifying them and supplies us with a new and useful hypothesis regarding matter in its various forms (iron, sulphur, etc.) and the energy contained therein. A brief discussion of such a hypothesis will be found in the concluding chapter (pp. 552-5).

\section{The Speed of Chemical Actions: a Means of Measuring} Activity.- One means of measuring the relative chemical activities of several substances is to observe the speed with which they undergo the same chemical change. Thus we may compare the activities of the various metals by allowing them separately to interact with hydrochloric acid and collecting and measuring the hydrogen liberated per minute by each. It will be seen, even in the roughest experiment, that magnesium is thus much more active than zinc. The comparison must be made with such precautions, however, as will make it certain that the conditions under which the several metals act are all alike. Thus, in spite of the heat evolved by the action, means must be used, by suitable cooling, to keep the temperature at some fixed point during the experiment, for all actions become more rapid when the temperature rises (p. 27). Again, the pieces of the various metals must be arranged so that equal surfaces are exposed to the acid in each case. It is found that the order in which this comparison places the metals is much the same as that in which they are placed by a study of other similar actions. A single table, showing the order of activity (p. 54), suffices, therefore, for all purposes.

Thermochemistry. - Chemical changes in which heat is liberated are called exothermal. Those in which heat is continuously absorbed (pp. 15, 30) are called endothermal changes. Since the activities, or affinities of two substances (say, two metals) may often be compared by observing the amounts of heat liberated when each combines with a third substance (say, oxygen), it will be instructive now to consider some of the elementary facts of thermochemistry. 
The chemical interactions to be studied thermally are arranged so that they may be carried out in a small vessel which can be placed inside another containing water. The whole apparatus is called a calorimeter (Greek, heat-measurer). The heat developed raises the temperature of this water. Where gases like oxygen are concerned, a closed bulb of platinum forms the inner vessel. The quantity of heat capable of raising one gram of water one degree in temperature at $15^{\circ}$ Centigrade is called a calorie.

Thermochemical Equations. - While in physics the unit of quantity is the gram, in chemistry the unit which we select is naturally a gram-atomic weight or a gram-molecular weight of the substance. Thus, the heat of combustion of carbon means the heat produced by combining twelve grams of carbon with thirtytwo grams of oxygen, and is sufficient to raise nearly 100,000 grams of water one degree. This is expressed as follows:

$$
\mathrm{C}+\mathrm{O}_{2} \rightarrow \mathrm{CO}_{2}+96,900 \text { cal. }
$$

In other words, the combustion of less than half an ounce of carbon will raise over two pounds of water from $0^{\circ}$ to the boilingpoint.

When the action is one which absorbs heat, this fact is indicated by the negative sign preceding the number of calories. Thus for the dissociation of water vapor into hydrogen and oxygen we have the thermochemical equation:

$$
2 \mathrm{H}_{2} \mathrm{O} \rightarrow 2 \mathrm{H}_{2}+\mathrm{O}_{2}-116,200 \text { cal. }
$$

If the action is reversible, as this one is, the heat absorbed when it proceeds in one direction is equal to that liberated when it goes in the other direction:

$$
2 \mathrm{H}_{2}+\mathrm{O}_{2} \rightarrow 2 \mathrm{H}_{2} \mathrm{O}+116,200 \text { cal. }
$$

Answers to Possible Questions. - It is always found that the same quantities of any given chemical substances, undergoing the same chemical change under the same conditions, produce 
or absorb, according as the action is excthermal or endothermal, amounts of heat which are equal.

The rate at which a given chemical action is allowed to take place has no influence on the total amount of heat ccnsumed or produced. It may not at first sight appear obvious that rusting evolves heat, but a delicate thermometer will show that a heap of rusting nails is somewhat higher in temperature than surrounding bodies. Poor conductors, like oily rags and ill-dried hay, show a tendency to spontaneous combustion owing to accumulation of the slowly developing heat of oxidation (p. 41). The warmth of our own bodies is due to the same cause.

It should be noted that production or absorption of heat is not, in itself, an evidence of chemical action. Physical changes are all likewise accompanied by the same phenomena. Thus, the evaporation of water absorbs heat, and condensation of a vapor and the crystallization of a supercooled liquid liberate heat.

Exercises.-1. Which form of energy is delivered as such, and paid for as such, in most cities?

2. How many calories are required to raise $500 \mathrm{~g}$. of a substance of specific heat 0.5 from $15^{\circ}$ to $37^{\circ}$ ?

3. The combustion of $1 \mathrm{~g}$. of sulphur to sulphur dioxide develops 2220 calories. What is the heat of combustion of sulphur? Write the thermochemical equation. 


\section{CHAPTER XIV}

\section{SODIUM AND SODIUM HYDROXIDE}

IN our study of common salt we have taken up one of its constituents, namely, chlorine, and its commonest derivative hydrochloric acid. The latter is a good example of an acid. We now turn to the other constituent, sodium, and one of its familiar compounds, namely sodium hydroxide $(\mathrm{NaOH})$. The latter is an example of a different kind of substances, called alkalies or bases. Salt, hydrochloric acid, and sodium hydroxide are examples of the three largest and most important classes of substances known to inorganic chemistry.

Preparation of the Metal Sodium.-Sodium cannot be made by displacement (like hydrogen), because it is close to the

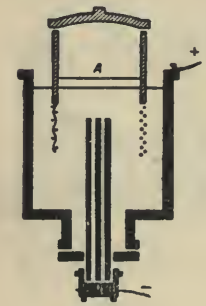

Fig. 57 top of the activity list (p. 54) of the metals, and no more active, and at the same time easily obtained, metal is available to displace it. It was first prepared by Davy (1807) by electrolyzing melted sodium hydroxide, and is still manufactured in this way. The aqueous solution of a sodium compound, such as sodium chloride, cannot be used, because, as we have seen (p. 140), hydrogen from the water is liberated in place of the metal.

The dry sodium hydroxide is melted (about $318^{\circ}$ ) in an iron vessel (Fig. 57), which is connected with the positive wire from the dynamo, and the oxygen is liberated on the iron (anode). The negative wire is connected with rods of carbon which stand up through the bottom of the vessel, and here (cathode) the sodium and the hydrogen are set free. Being lighter than the fused com164 
pound, the hydrogen rises in bubbles, and the sodium in melted globules, to the surface. Here they collect under an iron cylinder. Tha latter is made of wire-gauze at the lower part, to permit circulation of the liquid, but prevent the escape of the globules of sodium. It is closed at the top, to prevent the heated sodium from burning, as it would do if air could reach it. The melted sodium is ladeled into cylindrical moulds, and the sticks of the metal are preserved in air-tight tin boxes.

Physical Properties of Sodium.- Sodium is a silver-like metal, of specific gravity 0.97 . It is soft and can be cut with a knife. It melts at $95.6^{\circ}$ and boils at $742^{\circ}$. The gram-molecular volume of sodium vapcr weighs $23 \mathrm{~g}$., the same as the atomic weight, so that the molecular formula is $\mathrm{Na}$.

Chemical Properties.-Sodium burns in chlorine, giving sodium chloride $\mathrm{NaCl}$. It burns also in oxygen (or air) to form sodium peroxide $\mathrm{Na}_{2} \mathrm{O}_{2}$. It acts violently on water, as we have seen (p. 50), displacing hydrogen and forming sodium hydroxide. Skeleton: $\mathrm{Na}+\mathrm{H}_{2} \mathrm{O} \rightarrow \mathrm{NaOH}+\mathrm{H}_{2}$.

Balanced:

$$
2 \mathrm{Na}+2 \mathrm{H}_{2} \mathrm{O} \rightarrow 2 \mathrm{NaOH}+\mathrm{H}_{2} \text {. }
$$

For this reason it tarnishes quickly in moist air. In the laboratory small amounts are kept under kerosene, which contains no compounds of oxygen.

Uses.-Sodium is used in the manufacture of many complex organic compounds. By contact action, it converts isoprene $\left(\mathrm{C}_{5} \mathrm{H}_{8}\right)$ into caoutchouc $\left(\mathrm{C}_{10} \mathrm{H}_{16}\right)_{n}$ or raw rubber (see p. 480). This is a method of making rubber artificially. It cannot yet be carried out so cheaply as to compete with the natural product under ordinary circumstances, but in Germany during the war, when the supply of natural rubber was cut off entirely, considerable quantities were manufactured by this synthetic method. 
Sodium peroxide (" oxone"), made by burning sodium, is used as a source of oxygen (p. 31).

Preparation of Sodium Hydroxide.-As we have seen, this compound is formed by the action of water on sodium, and may -be obtained by evaporating the solution to dryness. But manufacturing it from an expensive substance like sodium is out of the question.

Much sodium hydroxide is made by boiling an aqueous solution of sodium carbonate $\mathrm{Na}_{2} \mathrm{CO}_{3}$ with slaked lime (calcium hydroxide, $\left.\mathrm{Ca}(\mathrm{OH})_{2}\right)$ :

\section{$\mathrm{Ca}(\mathrm{OH})_{2}+\mathrm{Na}_{2} \mathrm{CO}_{3} \rightleftarrows 2 \mathrm{NaOH}+\mathrm{CaCO}_{3} \downarrow$.}

The calcium carbonate, which is chemically the same substance as lime-stone and chalk, is much less soluble than any of the other substances taking part in the reaction, and is consequently precipitated out of the solution. This reaction is another example of a double decomposition (p. 132). All double decompositions are reversible, but here the precipitation of the calcium carbonate prevents it from acting upon the sodium hydroxide and reversing the action to any appreciable extent. After the precipitate has settled, the solution of sodium hydroxide is drawn off and evaporated to dryness.

Electrolytic Process. - Sodium hydroxide is also manufactured by electrolysis of sodium chloride solution (pp. 139-40), the other product, chlorine, being of great commercial value also. The Nelson cell (Fig. 58) is now most extensively used. A porous diaphragm of asbestos separates the perforated steel cathode from the carbon anode, which is immersed in a current of brine flowing through the cell between the electrodes. Chlorine is liberated at the anode and rises in bubbles to the surface of the solution. It is drawn off, dried, and compressed to liquid form in iron cylinders, or is made directly into bleaching compounds (p. 224). Sodium, as we have seen in the discussion of electroly- 
sis on pp. 139-40, remains in the solution as sodium hydroxide, which collects around the cathode and flows out into a catch basin. It is purified from residual sodium chloride by fractional

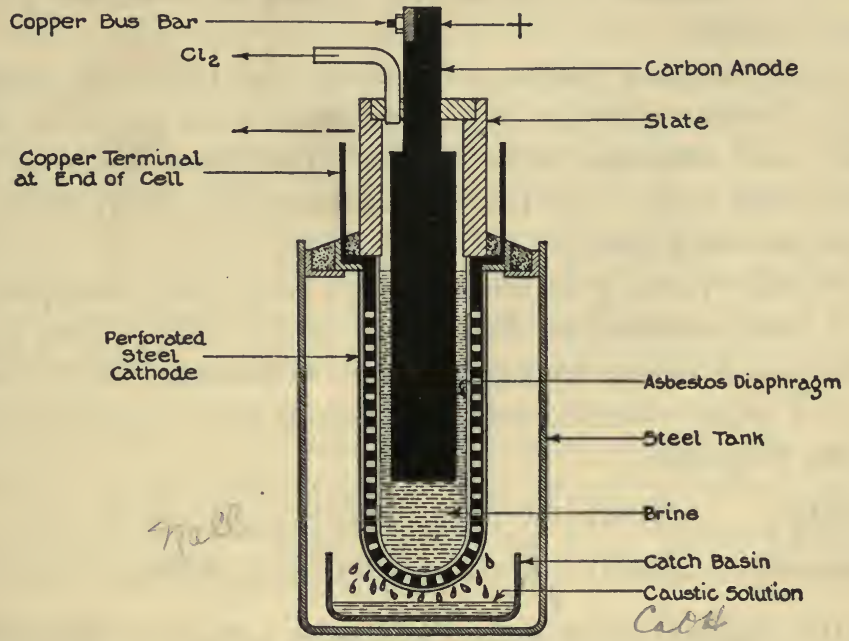

FIG. 58

crystallization. Hydrogen is also liberated at the cathode, and is a valuable by-product of the process.

Physical Properties of Sodium Hydroxide.-The substance is a white crystalline solid. Generally it shows the form of the iron drums, into which it is run when melted, or of the sticks into which it is cast. It is exceedingly soluble in water. Its solution gives to objects the smooth, soapy feeling which is characteristic of alkalies. The solution is sometimes called soda-lye, and the solid, caustic soda.

Chemical Properties.-Sodium hydroxide is exceedingly stable, being melted, but not decomposed, by heating.

- The aqueous solution possesses the following important prop- 
erties. (1) The solution has an acrid taste, like soap or borax. (2) It changes the color of litmus, reddened by a trace of an acid, back again from red to blue. (3) It is a conductor of electricity, and is decomposed by the current, oxygen being liberated at the positive wire.

In the following chapter we shall see that these three properties of sodium hydroxide in aqueous solution are properties common to all substances called alkalies. The reader should at this point refer back to p. 131, and contrast these properties with those exhibited by acids.

Sodium hydroxide in solution enters into double decomposition with many substances. Frequently one of the products is insoluble, and appears as a precipitate. For example, with a solution of cupric chloride, sodium hydroxide gives a precipitate of cupric hydroxide.

\section{Skeleton: $\quad \mathrm{NaOH}+\mathrm{CuCl}_{2} \rightarrow \mathrm{Cu}(\mathrm{OH})_{2} \downarrow+\mathrm{NaCl}$. \\ Balanced: $\quad 2 \mathrm{NaOH}+\mathrm{CuCl}_{2} \rightarrow \mathrm{Cu}(\mathrm{OH})_{2} \downarrow+2 \mathrm{NaCl}$.}

As this equation shows, sodium hydroxide behaves in such actions as if composed of two parts, namely $(\mathrm{Na})$ and $(\mathrm{OH})$ (compare p. 132). The reaction consists, essentially, of a transfer of $(\mathrm{OH})$ groups from $(\mathrm{Na})$ to $(\mathrm{Cu})$.

Alkalies and Bases. - It will be seen that the chemical properties of sodium hydroxide solution may be summed up by saying that it is an alkali.

Solutions of the alkalies also act upon animal matter, e.g. wool (p. 1), especially when hot, converting it largely into soluble substances. For this reason they are called caustic alkalies. They likewise act slowly upon the components of glass. For this reason a precipitate is often visible in the caustic soda reagent bottle, and the inner surface of the glass is always etched.

A very delicate test for an alkali is given by phenolphthatein, a colorless organic substance. One drop of phenolphthalein 
solution added to an alkali in water solution produces an intense red (when dilute, pink) coloration. Addition of excess acid renders the solution colorless again.

The alkalies, however, are simply the more active members of a much larger class of substances called bases. Solutions of the less soluble bases, of which cupric hydroxide is an extreme example, do not show, distinctly, all the properties exhibited by alkalies. Thus, those which are least soluble have, naturally, no taste, do not visibly affect litmus, do not conduct the electric current very well in solution, and are not soapy to the touch or corrosive towards glass. But they all show the tendency to double decomposition, in which the group $(\mathrm{OH})$ is transferred, as it was from $\mathrm{NaOH}$ to $\mathrm{Cu}(\mathrm{OH})_{2}$ in the foregoing example.

Uses.- Sodium hydroxide is used in immense quantities along with fats, in the manufacture of soap. Some bleaching liquids are made by saturating it with chlorine. It is employed also in making many other sodium compounds which are used in the arts.

Exercises.- 1. In the electrolysis of sodium hydroxide, why is the metal not liberated as a solid or as a vapor, but as a liquid?

2. Make molecular equations for the burning of sodium in chlorine and in oxygen. 


\section{CHAPTER XV}

\section{ACIDS, BASES, AND SALTS}

As we saw at the opening of the preceding chapter, acids, bases, and substances with certain chemical properties similar to those of common salt form the vast majority of the compounds met with in inorganic chemistry. On account of their resemblance to common salt, the substances of the third class are called salts. We shall first recall the names and formulæ of some of the examples of these. classes that we have already had occasion to mention.

Some Familiar Acids. - The following are some of the acids we have mentioned:

Hydrochloric acid .........HCl

Sulphuric acid ............ $\mathrm{H}_{2} \mathrm{SO}_{\mathrm{O}}$

Phosphoric acid........... $\mathrm{H}_{3} \mathrm{PO}_{4}$
Nitric acid...............HNO Hypochlorous acid.........HOCl Acetic acid. ............ $\mathrm{HCO}_{2} \mathrm{CH}_{8}$

Familiar Bases. - The bases mentioned, with one or two additions (in italics), \& re:

Sodium hydroxide....... NaOH

Potassium hydroxide....... KOH

Calcium hydroxide ... $\mathrm{Ca}(\mathrm{OH})_{2}$

Ammonium hydroxide..... $\mathrm{NH}_{4} \mathrm{OH}$

Cupric hydroxide..... Cu $(\mathrm{OH})_{2}$

Zinc hydroxide ....... $\mathrm{Zn}(\mathrm{OH})_{2}$

Familiar Salts. - Some of those already mentioned are:

Sodium chloride......... NaCl

Sodium sulphate.......... $\mathrm{Na}_{2} \mathrm{SO}_{4}$

Potassium nitrate.......... $\mathrm{KNO}_{3}$

Ammonium chloride......... $\mathrm{NH}_{4} \mathrm{Cl}$

Cupric sulphate........... $\mathrm{CuSO}_{4}$
Potassium chlorate......... $\mathrm{KClO}_{3}$

Zine sulphide............ ZnS

Sodium peroxide.......... $\mathrm{Na}_{2} \mathrm{O}_{2}$

Sodium carbonate........ $\mathrm{Na}_{2} \mathrm{CO}_{3}$

Lead sulphide...........PbS

Radicals.- Upon comparing the formulæ in the lists, we notice that: 
1. Every acid contains hydrogen, combined with one or more other atoms, "constituting a group called a radical.

2. The very same radicals, such as $\mathrm{SO}_{4}$, appear also in a number of salts.

3. Every base contains $\mathrm{OH}$ (the radical hydroxyl), and usually one atom, but occasionally more than one (as in $\mathrm{NH}_{4}$ ) in the other radical.

4. The same radicals that are combined in bases with $\mathrm{OH}$ appear also in numerous salts.

5. Radicals may be simple, like $\mathrm{H}, \mathrm{Na}$, and $\mathrm{Cl}$, or compound, like $\mathrm{SO}_{4}$ and $\mathrm{NH}_{4}$.

Positive and Negative Radicals. - When the electric current is passed through an aqueous solution of an acid, such as hydrochloric acid (p. 55), the acid is decomposed and hydrogen is liberated at the negative electrode. Since this electrode attracts positively charged particles, hydrogen is called a positive radical. (In all circumstances, unlike charges of electricity attract, and like charges repel one another.) The rest of the acid molecule, such as $\mathrm{Cl}$, is attracted to the positive electrode, and is therefore called a negative radical. Similarly, when we electrolyze a solution of a base, such as sodium hydroxide, oxygen is always liberated at the positive electrode. This is due to the decomposition of the hydroxyl radical $\mathrm{OH}$, which is consequently also a negative radical.

$$
4 \mathrm{OH} \rightarrow \mathrm{O}_{2}+2 \mathrm{H}_{2} \mathrm{O} \text {. }
$$

The rest of the alkali molecule, such as $\mathrm{Na}$, is attracted to the negative electrode and is therefore a positive radical.

In the same way every salt contains a positive radical, other than $\mathrm{H}$, combined with a negative radical, other than $\mathrm{OH}$. The name of each salt indicates the radicals of which it is composed.

As regards the salts in the second column, they show positive radicals, like $\mathrm{K}, \mathrm{Zn}$, and $\mathrm{Na}$, found in the list of bases. The negative radicals $\mathrm{ClO}_{3}, \mathrm{~S}, \mathrm{O}_{2}$, and $\mathrm{CO}_{3}$ are not in the list of acids 
we have met with. But there are well-known acids corresponding to them, namely, chloric acid $\mathrm{HClO}_{3}$, hydrogen sulphide $\mathrm{H}_{2} \mathrm{~S}$, hydrogen peroxide $\mathrm{H}_{2} \mathrm{O}_{2}$, and carbonic acid $\mathrm{H}_{2} \mathrm{CO}_{3}$.

In general, then, all positive radicals combine with $\mathrm{OH}$ to give bases, all negative radicals combine with $\mathrm{H}$ to give acids. In general, also, each positive radical will combine with any negative radical to give a salt. In a few exceptional cases only, the compound cannot be formed, presumably because it is unstable under ordinary conditions.

Reaction Formulue. - The formulæ of acids, bases, and salts are written in a uniform manner to show the behavior of the substances represented, when in solution. Thus, the radical written first is usually the positive one which, when a solution is electrolyzed, is attracted by the negative wire, and the negative radical follows. Again, in a compound like calcium hydroxide, the formula $\mathrm{CaO}_{2} \mathrm{H}_{2}$ would conceal the existence of the hydroxyl group. So the radicals are written in brackets, with the coefficient outside $-\mathrm{Ca}(\mathrm{OH})_{2}$. Thus we write also $\mathrm{Cu}\left(\mathrm{NO}_{3}\right)_{2}$, and not $\mathrm{CuN}_{2} \mathrm{O}_{6}$, and $\left(\mathrm{NH}_{4}\right)_{2} \mathrm{SO}_{4}$, not $\mathrm{N}_{2} \mathrm{H}_{8} \mathrm{SO}_{4}$. These formulæ are all reaction formulæ. That is, they indicate, not simply the composition, but also the parts into which the compounds decompose, and from which they are formed in double decompositions (p. 132, and p. 173, below).

\section{Properties Common to Acids, Bases and Salts in Solution.} - There are four of these properties, all of which have come up previously.

1. Displacement. - A simple radical belonging to an acid, base, or salt in solution can be displaced by another element, and is thereby obtained in the free state. Thus we have already seen (p. 51) that hydrogen is liberated from acids by the addition of the more active metals:

$$
\mathrm{Zn}+2 \mathrm{HCl} \rightarrow \mathrm{H}_{2}+\mathrm{ZnCl}_{2} \text {. }
$$


Exactly the same type of reaction takes place when we add to the solution of a salt any metal higher up in the activity series than the positive radical of the salt. For example, zinc will displace copper from a solution of cupric sulphate or any other soluble cupric salt:

$$
\mathrm{Zn}+\mathrm{CuSO}_{4} \rightarrow \mathrm{ZnSO}_{4}+\mathrm{Cu} .
$$

The copper is obtained as a red precipitate. This principle is extensively used in the purification of the more valuable metals at the foot of the activity series (p. 54). Thus copper will displace silver, and silver will displace gold, from solutions of their respective salts.

Similarly, a simple negative radical can be displaced by a more active element. Thus the iodide radical in potassium iodide is displaced by gaseous chlorine, iodine being liberated:

$$
2 \mathrm{KI}+\mathrm{Cl}_{2} \rightarrow 2 \mathrm{KCl}+\mathrm{I}_{2} \text {. }
$$

2. Double Decomposition.- Several examples of this type of reaction between acids, bases and salts in solution have already been discussed (pp. 126, 132, 166 and 168). In fact, whenever two solutions of such substances, which contain no radical in common, are mixed, a double decomposition occurs. Any acid or base will therefore react with any salt of a different acid or base. Any acid will give a reaction with any base. Salts containing no common radical will also react in pairs.

Very frequently we obtain instant evidence of such reactions by the appearance of a precipitate, one of the products formed being only slightly soluble in water. For example:

Salt and acid: $\quad \mathrm{AgNO}_{3}+\mathrm{HCl} \rightarrow \mathrm{AgCl} \downarrow+\mathrm{HNO}_{3}$

Salt and base: $\mathrm{CuCl}_{2}+2 \mathrm{NaOH} \rightarrow \mathrm{Cu}(\mathrm{OH})_{2} \downarrow+2 \mathrm{NaCl}$

Acid and base: $\mathrm{H}_{2} \mathrm{SO}_{4}+\mathrm{Ca}(\mathrm{OH})_{2} \rightarrow \mathrm{CaSO}_{4} \downarrow+2 \mathrm{H}_{2} \mathrm{O}$

Salt and salt: $\quad \mathrm{AgNO}_{3}+\mathrm{NaCl} \rightarrow \mathrm{AgCl} \downarrow+\mathrm{NaNO}_{3}$

Such precipitations furnish us with very useful tests for establishing the presence or absence of certain radicals in an unknown 
substance. For example, a curdy white precipitate of silver chloride is obtained not only by adding to a solution of silver nitrate a solution of hydrochloric acid (equation 1) or sodium chloride (equation 4), but by addition of any solution containing the radical $\mathrm{Ag}$ to any solution containing the radical $\mathrm{Cl}$. If we suspect that a solution contains $\mathrm{Ag}$, we add, therefore, a solution of any chloride. If we suspect that a solution contains $\mathrm{Cl}$, we add a solution of any soluble silver salt. In either case a negative result is conclusive evidence of the absence of the radical tested for. If a positive result is obtained, the precipitate must be examined further, to prove whether or not it is silver chloride.

Even when no precipitate appears, however, some interaction takes place. Thus a solution obtained by mixing sodium nitrate and potassium chloride solutions is identical in all its properties with a solution obtained by mixing sodium chloride and potassium nitrate solutions. The appearance of a precipitate is not, therefore, an essential feature of a double decomposition. The most important point for us to notice is that, in all such reactions, each substance present behaves exactly as if it consisted of two distinct radicals. We may therefore regard double decompositions as a simple result of the liberty of radicals to interchange pariners. Normally, this exchange will not be complete. Thus in the instance cited immediately above we have the reversible reaction:

$$
(\mathrm{Na})\left(\mathrm{NO}_{3}\right)+(\mathrm{K})(\mathrm{Cl}) \rightleftharpoons(\mathrm{Na})(\mathrm{Cl})+(\mathrm{K})\left(\mathrm{NO}_{3}\right)
$$

Whichever pair of salts we start with, we reach the same result on mixing their solutions. Every double decomposition is similarly reversible in theory, and gives an equilibrium mixture. But because, in many mixtures, one of the four possible compounds withdraws from the exchange of partners by disappearing from the solution either as a gas (p. 126) or as a precipitate (p. 127), the reaction in such cases becomes practically complete in one direction.

Another significant point may now be noted. Not only does every acid, base and salt in solution behave, in double decom- 
positions, as if it consisted of two distinct radicals, but it can be shown to possess two independent sets of properties, one referring to each radical. Thus a solution of cupric chloride in water exhibits one set of properties which can be referred directly to the cupric radical, and which is accordingly not peculiar to cupric chloride, but is common to all cupric salts in aqueous solution. To mention only two of these; (1) the color of the solution when diluted with water, is blue, and (2) the addition of a base gives a pale blue, gelatinous precipitate of cupric hydroxide (see equation 3 above). The same solution exhibits a second set of properties which can be referred directly to the chloride radical and which is consequently common to all chlorides. To mention only two of these again; (1) when the solution is heated with concentrated sulphuric acid, $\mathrm{HCl}$ is evolved (see p. 126), and (2) the addition of a silver salt gives a precipitate of silver chloride.

The properties of acids (p. 131) are properties of the hydrogen radical. The properties of bases (p. 167) are properties of the hydroxyl radical $\mathrm{OH}$.

A radical, then, is an atom, or group of atoms, which behaves as a distinct unit in double decompositions, and which confers a definite independent set of properties upon solutions of all acids, bases or salts of which it forms one constituent. Simple radicals also behave as separate units in displacements.

3. Conductivity. - Solutions of acids, bases, and salts in water are all conductors of electricity. Acids, bases and salts are therefore called "electrolytes." In all cases the solution is decomposed by the passage of the current. The positive and negative radicals of which the solute is composed are attracted to the opposite electrodes. There, unless special circumstances prohibit (see for example, the electrolysis of sodium chloride, p. 139), they are liberated. Thus, all acids give hydrogen at the negative pole, the other radical passing to the positive pole.

Of all the properties they have in common, this one of being 
electrolytes is perhaps the most remarkable. It appears more surprising when we consider that no substances, other than acids, bases, and salts, undergo electrolysis in aqueous solution. This is an exclusive property of these classes of bodies.

The conducting power of solutions can be examined, roughly, by the apparatus in Fig. 59. The platinum electrodes are connected with a direct-current circuit. The lamp, which is on one of the wires, serves, by its resistance, to cut down the current. Its glowing also shows when the liquid is a conductor, and by varying brightness indicates roughly the conducting power of the solution.

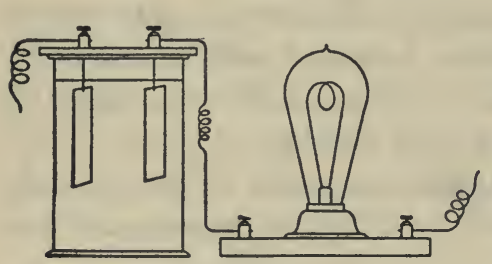

Fig. 59

A solution of sugar in water shows no conductance, and the lamp remains dark. Solutions of acids, bases, and salts in water enable the lamp to glow.

We quickly find that different solutions, when they conduct, do so in different degrees. Solutions of hydrochloric and nitric acids conduct well. So do solutions of sodium and potassium hydroxides. Salt solutions are practically all good conductors. But many acids, like acetic acid, conduct poorly in aqueous solution. The same is true of some bases, like ammonium hydroxide.

Of course, acids, bases, and salts which are only very slightly soluble in water give poorly conducting solutions. But in estimating the conductivity for chemical purposes, we have to take into consideration the amount dissolved. Thus silver chloride, being a salt, is an excellent conductor, when we allow for the extreme diluteness of the solution. A saturated solution of silver chloride, in point of fact, shows a slightly higher conductance than a solution of sodium chloride of the same concentration.

Subștances which give solutions with high conducting power 
are called strong electrolytes. Substances which give poorly conducting solutions are called weak electrolytes.

\section{Vapor Pressures; Boiling-Points; Freezing-Points.-} We have seen (pp.117-9) that equal numbers of molecules of different solutes dissolved in equal weights of water normally depress the vapor pressure, raise the boiling-point, and lower the freezing-point by constant amounts. Thus, one molecular weight of sugar ( $342 \mathrm{~g}$.) or of glycerine (92 g.) dissolved in 1000 g. of water will raise the boiling-point from $100^{\circ}$ to $100.52^{\circ}$, and will lower the freezing-point from $0^{\circ}$ to $-1.86^{\circ}$. But this is uniformly true only of non-conducting solutions, in other words, solutions of substances which are not acids, bases, or salts. Grammolecular weights of substances of these three classes, dissolved in $1000 \mathrm{~g}$. of water, raise the boiling-point more than 0.52 degrees and lower the freezing-point by more than 1.86 degrees. We say they give abnormal elevations of the boiling-point and $a b$ normal lowerings of the freezing-point. Vapor pressure depressions in such solutions are also abnormal.

Thus, a solution of $58.46 \mathrm{~g}$. of sodium chloride in $1000 \mathrm{~g}$. of water boils at $100.97^{\circ}$, and freezes at $-3.42^{\circ}$. The elevation in the boiling-point of the water is $0.97^{\circ}$ instead of $0.52^{\circ}$. The depression of the freezing-point is $3.42^{\circ}$ instead of $1.86^{\circ}$. The effect in each case is nearly twice as great as the normal one. In the same way, a gram-molecular weight of potassium chloride dissolved in $1000 \mathrm{~g}$. of water at $20^{\circ}$ depresses the vapor pressure by 0.554 millimeters, while a gram-molecular weight of mannite (a normal sugar which gives a non-conducting solution) in the same amount of water lowers the vapor pressure by only $0.313 \mathrm{~mm}$. Again the effect is nearly twice the normal.

The only conclusion we can draw from these results is that nearly twice the normal numbers of solute molecules are present in such solutions. In other words, not only do we have two independent sets of properties exhibited by the constituent radicals 
in solutions of electrolytes, but these same solutions actually behave as if the radicals were, to a large extent, uncombined with each other. It appears as if sodium chloride, for example, is largely decomposed in aqueous solution into independent sodium and chloride radicals. Additional evidence in this direction is supplied by the behavior of electrolytes of more complex types, such as sodium sulphate $\mathrm{Na}_{2} \mathrm{SO}_{4}$ or zinc chloride $\mathrm{ZnCl}_{2}$. Both of these substances, in dilute solution, give boiling-point and freezing-point changes which are nearly three times the normal, indicating that they are largely decomposed into their three constituent radicals $\left(2 \mathrm{Na}\right.$ and $\mathrm{SO}_{4} ; \mathrm{Zn}$ and $2 \mathrm{Cl}$ ). In the same way, substances containing four radicals, like ferric chloride $\mathrm{FeCl}_{3}$, give effects approaching four times the normal in dilute solution.

One last point remains to be mentioned. It has been noted above that some acids, like acetic acid, and some bases, like ammonium hydroxide, are only poor conductors in solution. Just these same two classes of substances, it has been found, give only very slightly abnormal changes in the three physical properties of solutions here under examination. The full significance of this difference in behavior will appear in the following chapter.

A Warning. - The reader is urged to keep in mind the fact that it is only in solution (and particularly in aqueous solution) that the special properties of acids, bases, and salts become apparent. Their behavior is often quite different in the absence of a solvent. If, for example, we mix together dry ammonium carbonate $\left(\mathrm{NH}_{4}\right)_{2} \mathrm{CO}_{3}$ and partially dehydrated, solid cupric nitrate $\mathrm{Cu}\left(\mathrm{NO}_{3}\right)_{2}$, and apply heat, a violent interaction begins. This interaction is nothing so simple as a double decomposition, however. An immense cloud of smoke and gas is thrown out of the tube, and the solid remaining is either black or reddish, in parts, according to the proportions of the substances employed. This residue contains black cupric oxide $\mathrm{CuO}$, and sometimes red cuprous oxide $\mathrm{Cu}_{2} \mathrm{O}$. The gas evolved is tinged red by the pres- 
ence of nitrogen peroxide $\mathrm{NO}_{2}$, while a careful analysis would show that it contained also carbon dioxide $\mathrm{CO}_{2}$, nitrogen $\mathrm{N}_{2}$, nitrous oxide $\mathrm{N}_{2} \mathrm{O}$, water vapor $\mathrm{H}_{2} \mathrm{O}$, and perhaps still other products.

The contrast, when the substances are dissolved in water before being brought in contact, is very great. A pale green precipitate is immediately produced, which rapidly settles out and proves, on examination, to be a carbonate of copper. Evaporation of the solution gives us ammonium nitrate.

$$
\left(\mathrm{NH}_{4}\right)_{2} \mathrm{CO}_{3}+\mathrm{Cu}\left(\mathrm{NO}_{3}\right)_{2} \rightarrow \mathrm{CuCO}_{3} \downarrow+2 \mathrm{NH}_{4} \mathrm{NO}_{3} .
$$

The reaction is essentially a double decomposition (although, strictly speaking, the precipitate is not the normal carbonate of copper, but a basic salt, see p. 192), similar in character to those already discussed.

The differences in properties between dry hydrogen chloride and hydrochloric acid (p. 131) furnish another good example of the fundamental changes involved in the addition of water as a solvent. Carefully dried hydrogen chloride has practically none of the properties of an acid. It does not affect litmus, it does not conduct the electric current. It is true that it does react with the more active metals (such as $\mathrm{Na}$ or $\mathrm{K}$ ), hydrogen being displaced and the chloride of the metal formed. But it is probable that even this property would disappear if we could succeed in eliminating the last traces of moisture from our reacting products. A solution of sulphuric acid acts on sodium so vigorously that the mixture explodes, but pure hydrogen sulphate $\mathrm{H}_{2} \mathrm{SO}_{4}$, when very carefully dehydrated, is so inert that metallic sodium floats in it without the slightest evidence of any interaction.

The rules that have been derived in the preceding sections regarding the behavior of acids, bases, and salts in solution will be found to clear the ground wonderfully in the development of subsequent chapters, for the substances which we shall meet with, that are not included in the above groups, are very few indeed. 
Furthermore, since we naturally prefer, when we carry out a reaction either on a small scale in the laboratory or on a large scale in chemical industry, to have it under our control as much as possible, we contrive to perform most chemical changes with our reacting materials in solution. By this means we are enabled, in general, to forecast the course of the reaction accurately, to arrange the conditions so that the reaction will be sure to proceed smoothly, and to isolate without much difficulty those products which we desire to obtain in a pure state. In the absence of a solvent a reaction is much more liable to get out of hand, perhaps explosively, with the result that the proper conditions of the process are not maintained, and we obtain either a poor yield or an impure product.

Exercises. - 1. By what experiments should you determine which were the radicals in substances of the following composition: $\mathrm{Cu}\left(\mathrm{NO}_{3}\right)_{2}, \mathrm{CaCO}_{3}, \mathrm{NH}_{4} \mathrm{Br}, \mathrm{NH}_{4} \mathrm{I}, \mathrm{KClO}_{4}$ and $\mathrm{KMnO}_{4}$.

There are always two ways, and usually three, of determining the radicals - what are they (pp. 171-2)?

2. How should you determine whether a given substance were an acid, base, or salt (electrolyte), or not?

3. Make equations showing the interactions of solutions of aluminium chloride $\mathrm{AlCl}_{3}$ and of cupric sulphate $\mathrm{CuSO}_{4}$ with sodium hydroxide (p. 168). Name the products.

4. Make equations showing the interactions of solutions of zinc chloride $\mathrm{ZnCl}_{2}$ and of ferric chloride $\mathrm{FeCl}_{3}$ with silver sulphate $\mathrm{Ag}_{2} \mathrm{SO}_{4}$. Name the products.

5. What is the action of metallic zinc on solutions of sodium chloride, lead nitrate $\mathrm{Pb}\left(\mathrm{NO}_{3}\right)_{2}$, silver sulphate $\mathrm{Ag}_{2} \mathrm{SO}_{4}$, magnesium sulphate $\mathrm{MgSO}_{4}$, mercuric chloride $\mathrm{HgCl}_{2}$ ? 


\section{CHAPTER XVI}

\section{IONIZATION}

WE have learned in the preceding chapter that acids, bases, and salts in solution exhibit two independent sets of properties, one of which can be referred to the positive, the other to the negative radical. We have seen that the passage of an electric current through such a solution decomposes it, the positive radical of the electrolyte proceeding to the negative electrode, the negative radical to the positive electrode. Finally, we have found that the abnormalities in certain physical properties of these solutions seem to indicate that the radicals actually exist, to a large extent, in an uncombined state in the solution.

Upon these facts a hypothesis has been based which has proved of considerable assistance in explaining the special properties of conducting solutions. The fundamental idea of this hypothesis, which was first definitely advanced by the Swedish chemist Arrhenius in 1887, is now accepted as an established fact. Our knowledge of the field of conducting solutions is still, however, very imperfect, and many important deductions from the hypothesis of Arrhenius remain matters of conjecture and dispute.

The Ionic Hypothesis. - In this hypothesis of Arrhenius it is assumed that the molecules of an electrolyte, such as hydrogen chloride, are largely broken up in solution into their constituent radicals, each radical being electrically charged. These charged radicals have been called ions, and the hypothesis is hence known as the ionic hypothesis. A solution of hydrogen chloride in water is supposed therefore to consist of two parts; (1) an undissociated part, made up of hydrogen chloride molecules $\mathrm{HCl}$; (2) a dissociated or ionized part, made up of equal numbers of 181 
hydrogen atoms carrying a positive charge $\left(\mathrm{H}^{+}\right)$and chlorine atoms carrying a negative charge $\left(\mathrm{Cl}^{-}\right)$. That part of the hydrogen chloride which remains undissociated in solution is understood to be inactive. It plays no part in the conduction of the current, it exerts a normal effect on the physical properties of the solution (vapor pressure, boiling-point, freezing-point changes), it does not convey to the solution any of the properties of an acid, it takes no direct share in displacements or double decompositions. That part of the hydrogen chloride, on the other hand, which breaks up into hydrogen ions $\mathrm{H}^{+}$and chlorine ions $\mathrm{Cl}^{-}$is active. All of the special properties of the solution may therefore be referred to these ions, as we shall see below.

Ionic Equations. - Of course, there is an equilibrium between the undissociated and dissociated parts of an electrolyte in solution, and this equilibrium may be represented by the ionic equation:

$$
\mathrm{HCl} \rightleftharpoons \mathrm{H}^{+}+\mathrm{Cl}^{-} .
$$

Ionization is, therefore, a kind of decomposition or rather dissociation, and is in every sense a chemical change. In ionic equations the charges upon the ions must be shown, as they are essential parts of the ionic substances.

$$
\begin{array}{ll}
\mathrm{NaCl} \rightleftarrows \mathrm{Na}^{+}+\mathrm{Cl}^{-} & \mathrm{FeCl}_{3} \rightleftarrows \mathrm{Fe}^{+++}+3 \mathrm{Cl}^{-} \\
\mathrm{Ca}\left(\mathrm{NO}_{3}\right)_{2} \rightleftarrows \mathrm{Ca}^{++}+2 \mathrm{NO}_{3}^{-} & \mathrm{Na}_{2} \mathrm{SO}_{4} \rightleftarrows 2 \mathrm{Na}^{+}+\mathrm{SO}_{4}=.
\end{array}
$$

The number of positive charges must equal the number of negative charges. This is proved most simply by the fact that the solution of a substance like ferric chloride $\left(\mathrm{FeCl}_{3}\right)$ is electrically neutral, as a whole. Thus some ions carry one charge, like $\mathrm{Na}^{+}$and $\mathrm{Cl}^{-}$ and $\mathrm{H}^{+}$, others two charges, like $\mathrm{Ca}^{++}$and $\mathrm{SO}_{4}=$, and so forth. An ion is an atom, or group of atoms, carrying an electric charge, or a number of such charges.

The positive ions are called the cations, since they move toward 


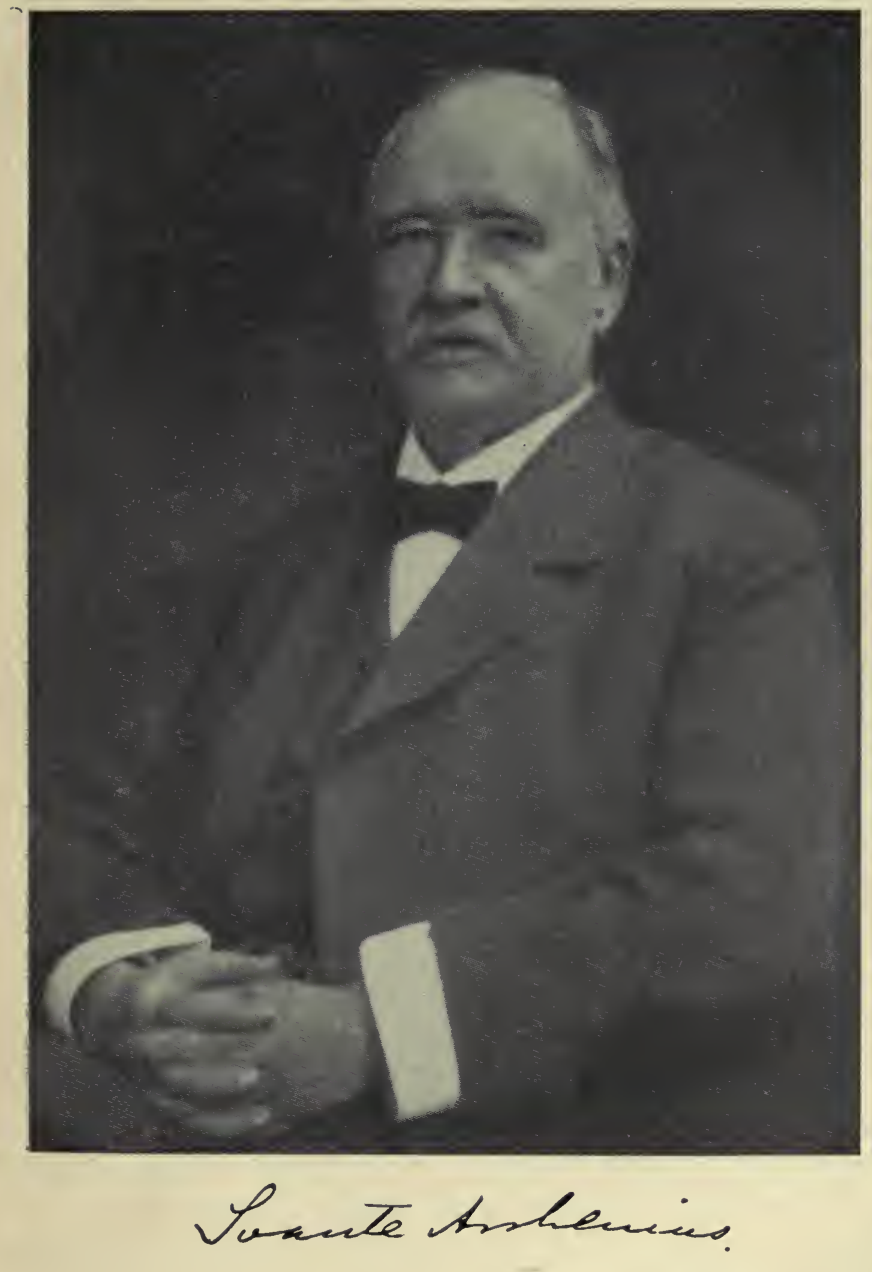

See page xii 


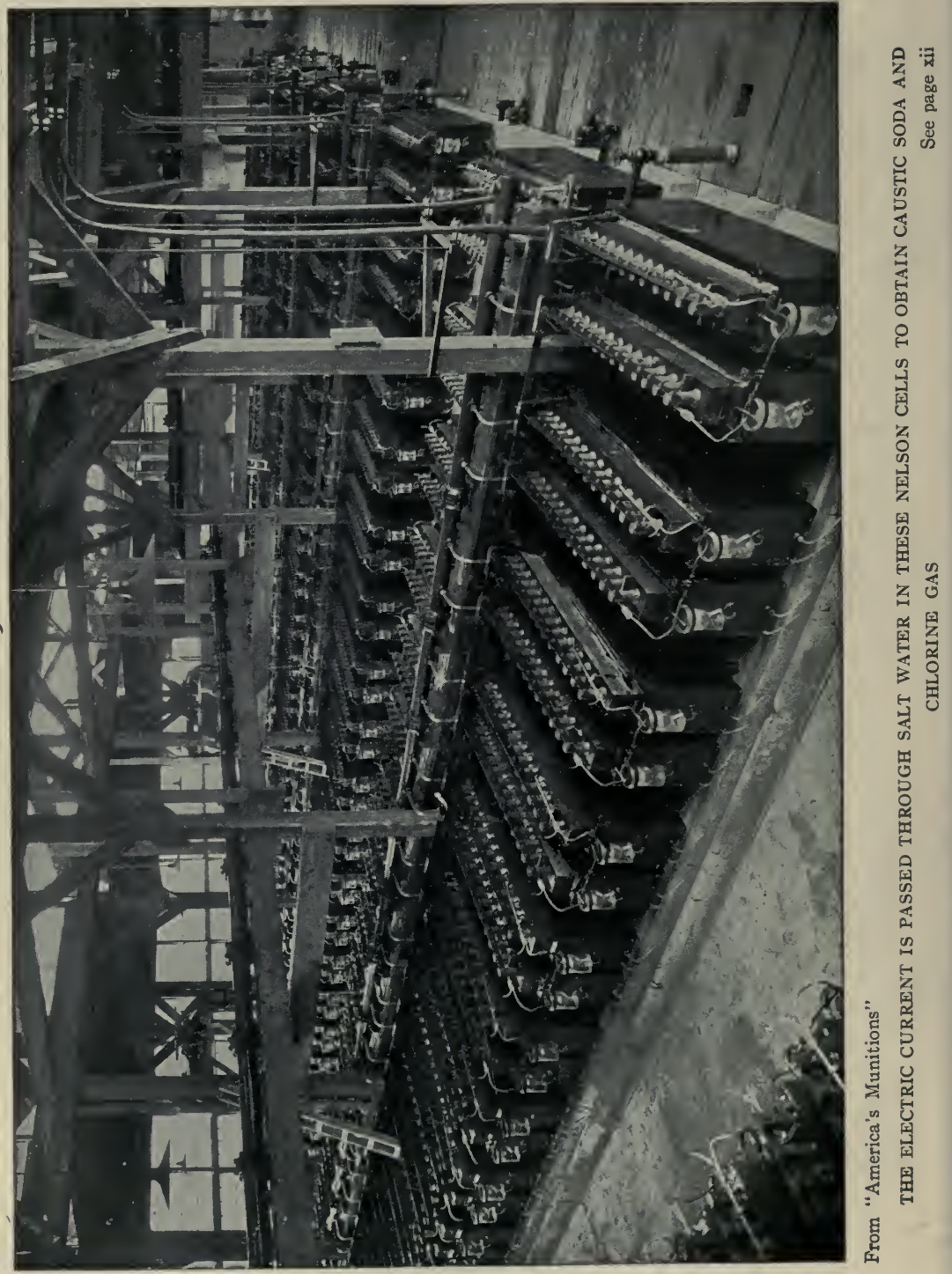


the negative electrode, or cathode. The negative ions are the anions, and move toward the positive electrode, or anode.

We can usually tell which is the positive radical in a formula because it generally consists of one atom of a metallic element $\left(\mathrm{K}^{+}, \mathrm{Cu}^{++}\right.$, etc.) or of hydrogen. The negative radical may contain a metal, like the $\mathrm{Mn}$ in $\mathrm{K}\left(\mathrm{MnO}_{4}\right)$, but always along with a non-metal like oxygen.

Ions and Electrolysis. - Let us first attempt to understand the phenomena of electrolysis in the light of the ionic hypothesis. A solution of $\mathrm{HCl}$ in water contains three kinds of solute; undissociated $\mathrm{HCl}$ molecules, positively charged hydrogen ions $\mathrm{H}^{+}$, and negatively charged chlorine ions $\mathrm{Cl}^{-}$. These are scattered indiscriminately throughout the solution, as indicated in Fig. 60 (only the two ions are there shown, the undissociated part is omitted since it plays no direct

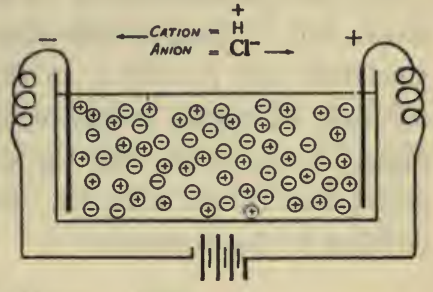

FIg. 60

part in the electrolysis). Equilibrium is kept up by continual dissociation and recombination, according to the equation $\mathrm{HCl} \rightleftarrows \mathrm{H}^{+}+\mathrm{Cl}^{-}$.

As soon as the circuit is completed, all the ions in the solution begin to migrate towards their proper electrodes. The positivelycharged hydrogen ions are attracted towards the negative electrode, the negatively-charged chlorine ions are attracted towards the positive electrode. Two orderly processions of ions, moving in opposite directions, proceed therefore through the solution. Ions (Greek, going) derive their name from this fact.

The rest is easily understood. When a positive ion reaches and touches the negative electrode, its positive charge of electricity is neutralized, and the result is an ordinary atom of hydrogen. The atoms of free hydrogen unite to give molecules $\left(\mathrm{H}_{2}\right)$ 
and these form bubbles of the gas. Simultaneously the negative ions are discharged at the positive electrode, and the atoms of free chlorine unite to give molecules $\left(\mathrm{Cl}_{2}\right)$.

Meanwhile, in the body of the solution, the departure of the ions has disturbed the equilibrium $\mathrm{HCl} \rightleftharpoons \mathrm{H}^{+}+\mathrm{Cl}^{-}$. The undissociated part $\mathrm{HCl}$ therefore continues to break up, attempting to re-establish equilibrium, until the electrolysis is complete.

By electrolysis of a solution of hydrochloric acid, therefore, we obtain hydrogen and chlorine. With some electrolytes the course of events is not so simple, secondary reactions taking place at the electrodes. Thus when we pass a current through a solution of sodium chloride, we obtain chlorine at the positive electrode, but hydrogen instead of sodium is liberated at the negative electrode (see p. 139). In the same way, when we electrolyze a. solution of cupric sulphate, metallic copper is deposited on the negative electrode, but the radical $\mathrm{SO}_{4}$ cannot exist in the free state, and reacts with the water present to liberate oxygen, according to the equation:

$$
2 \mathrm{SO}_{4}+2 \mathrm{H}_{2} \mathrm{O} \rightarrow 2 \mathrm{H}_{2} \mathrm{SO}_{4}+\mathrm{O}_{2} \text {. }
$$

Even when we do not actually isolate the free radicals of an electrolyte by electrolysis, however, we can show that they have migrated with the current in the usual way by the fact that they collect around the electrodes. Thus, in the electrolysis of sodium chloride, the solution around the negative electrode becomes alkaline, owing to accumulation of sodium hydroxide. Similarly, in the electrolysis of copper sulphate, the solution around the positive electrode becomes acid, owing to accumulation of sulphuric acid.

Ions and Displacement. - When a metal acts upon a dilute acid, and hydrogen is liberated, it is the ions alone that are directly concerned in the mechanism of the action. Thus the equation for the action of zinc on hydrochloric acid: $\mathrm{Zn}+2 \mathrm{HCl} \rightarrow$ 
$\mathrm{ZnCl}_{2}+\mathrm{H}_{2} \uparrow$ may be written:

$$
\mathrm{Zn}+2 \mathrm{H}^{+}+2 \mathrm{Cl}^{-} \rightarrow \mathrm{Zn}^{++}+2 \mathrm{Cl}^{-}+\mathrm{H}_{2} \uparrow
$$

or:

$$
\mathrm{Zn}+2 \mathrm{H}^{+} \rightarrow \mathrm{Zn}^{++}+\mathrm{H}_{2} \uparrow .
$$

From this it appears (see however p. 195) that the action simply consists of a transfer of positive charges from hydrogen ions to atomic zinc, free hydrogen being liberated and zinc ion going into solution. Similarly the action of zinc on a solution of a copper salt may now be written:

$$
\mathrm{Zn}+\mathrm{Cu}^{++} \rightarrow \mathrm{Zn}^{++}+\mathrm{Cu} \downarrow
$$

The activity series of the metals (p. 54) expresses, therefore, the order of their preference for assuming the ionic state.

Ions and Double Decomposition.- The mechanism of reactions of this type also becomes much clearer when we write the equations in the ionic form. Thus, for the precipitation of silver chloride by the action of hydrochloric acid on silver nitrate solution, we have:-

$$
\begin{aligned}
\mathrm{AgNO}_{3} & \rightleftharpoons \mathrm{Ag}^{+}+\mathrm{NO}_{3}^{-} \\
\mathrm{HCl} & \rightleftharpoons \mathrm{Cl}^{-}+\mathrm{H}^{+} \\
1 \downarrow & 1 \downarrow \\
\mathrm{AgCl} \downarrow & \mathrm{HNO}_{3} .
\end{aligned}
$$

In the mixed solutions, we have four ions in quantity, $\mathrm{Ag}^{+}$ and $\mathrm{NO}_{3}{ }^{-}$from the $\mathrm{AgNO}_{3}, \mathrm{H}^{+}$and $\mathrm{Cl}^{-}$from the $\mathrm{HCl}$. Before mixing, these were in equilibrium with undissociated $\mathrm{AgNO}_{3}$ and undissociated $\mathrm{HCl}$ respectively. But as soon as we bring all four ions into the same solution, we furnish them with the opportunity of combining with other partners, $\mathrm{Ag}^{+}$with $\mathrm{Cl}^{-}$and $\mathrm{H}^{+}$ with $\mathrm{NO}_{3}-$. Undissociated $\mathrm{AgCl}$ and undissociated $\mathrm{HNO}_{3}$ are also present, therefore, in the mixed solutions, each substance in equilibrium with its respective pair of ions. (This is conveniently indicated by the method of writing the equations which is employed above. The student should copy this method in analyzing 
all double decompositions.) The mixture contains, then, no fewer than eight different solutes, four ionic and four molecular, and four reversible reactions control the equilibrium relationships between them.

Now it so happens that one of the new solutes, $\mathrm{AgCl}$, is practically insoluble in water. Unless the solutions are excessively dilute, therefore, silver chloride separates out from the solution as a precipitate. This precipitation disturbs the existing equilibria by withdrawing practically all silver ions $\mathrm{Ag}^{+}$and chloride ions $\mathrm{Cl}^{-}$from the solution. Undissociated $\mathrm{AgNO}_{3}$ and $\mathrm{HCl}$ continue to break up, in an attempt to re-establish the equilibria, until they also are practically eliminated, and only hydrogen ions $\mathrm{H}^{+}$ and nitrate ions $\mathrm{NO}_{3}{ }^{-}$, in equilibrium with undissociated $\mathrm{HNO}_{3}$, are left in quantity in the solution.

The reader may feel, at this point, that the previous method of writing the reaction (p. 131):

$$
\mathrm{AgNO}_{3}+\mathrm{HCl} \rightarrow \mathrm{AgCl} \downarrow+\mathrm{HNO}_{3}
$$

expresses all this more simply, without the need of worrying about ions. But he must note that any silver salt added to any chloride in solution also gives a precipitate of silver chloride. This can be predicted immediately by ionic equations; any two solutions containing $\mathrm{Ag}^{+}$and $\mathrm{Cl}^{-}$respectively must give a precipitate of $\mathrm{AgCl}$ when mixed. Without the use of ionic equations, however, we should have no guide to the course of any such reaction; we should have to try it out for every mixture, memorize what happened in each case, and then attempt to draw up some empirical rules to assist us in retaining our tremendous mass of isolated facts. The use of the ionic hypothesis obviates all this. We do not require to remember all the properties of hundreds of different acids, bases and salts in solution singly and mixed. We know that each one of these substances exhibits two sets of properties in solution, and that one set can be referred to the positive ion, one set to the negative ion. If, therefore, we learn 
the properties of a few important positive and negative ions, we learn, at the same time, the properties in solution of all the electrolytes of which these ions are constituents. This introduces a great simplification into the study of chemistry.

To obtain the necessary practice in dealing with double decompositions, the student should now return to the section on these reactions in the preceding chapter (p. 173) and rewrite all of the equations there given or referred to in full ionic form. It is of great importance that the principles involved should be thoroughly understood in each case.

Ions and Conductivity. - Pure water is an exceedingly poor conductor of electricity. The high conducting power of a solution of an electrolyte is due, therefore, to the ions present. The actual conductance of any given solution will depend on the number of ions between the electrodes and the rate at which they move. The more numerous the ions are, and the more rapidly they migrate towards the oppositely-charged electrodes, the greater will be the number of discharges taking place per second upon each electrode.

The rate at which the ions move, under given conditions, has been carefully determined by methods which the student will find discussed in text-books on physical chemistry. It will suffice at this stage to give a few results. With electrodes one centimeter apart, and with a difference in electrical potential between the positive and negative electrodes of one volt, the velocities in centimeters per hour in dilute aqueous solution at $18^{\circ}$ are as follows: $\mathrm{H}^{+} 10.8, \mathrm{Na}^{+} 1.26, \mathrm{Ag}^{+} 1.66, \mathrm{OH}^{-} 5.6, \mathrm{Cl}^{-} 2.12, \mathrm{NO}_{3}^{-}$ 1.91. The hydrogen ion is the fastest, the hydroxyl ion holds the second place.

With the help of these figures we can calculate what is the extent of ionization of the electrolyte in any solution containing these ions, hydrogen chloride for example. We have already learnt (p. 85) that a gram molecular weight of hydrogen chloride 
contains $6.06 \times 10^{23}$ molecules. If ionization in solution were complete, therefore, a liter of a normal solution (p. 121) of hydrochloric acid would contain $6.06 \times 10^{23}$ hydrogen ions, and the same number of chlorine ions. These, moving at the rates given above, would give a definite, calculable conductivity. The conductivity actually obtained by experiment with a normal solution of $\mathrm{HCl}$ at $18^{\circ}$, however, is only 78 per cent of this calculated value. The conclusion has been drawn that, in normal solution at $18^{\circ}$, hydrochloric acid contains only 78 per cent of free ions, the remaining 22 per cent of solute contributing nothing towards the conductivity. We may express this conclusion by writing the reversible ionic dissociation in the form:

$$
(22 \%) \mathrm{HCl} \rightleftharpoons \mathrm{H}^{+}+\mathrm{Cl}^{-}(78 \%) .
$$

Equilibrium is reached in a normal solution of hydrochloric acid at $18^{\circ}$, therefore, when 78 per cent of the molecules are broken up into free ions.

At other concentrations different degrees of dissociation would be indicated. For example, a $10 \mathrm{~N}$ solution gives only 17 per cent of the calculated conductivity, a $0.1 \mathrm{~N}$ solution 92 per cent, a $0.01 \mathrm{~N}$ solution 97 per cent. At very high dilutions, therefore, the ionization becomes practically complete.

All electrolytes are not ionized to equal extents at the same concentrations. Thus, a normal solution of acetic acid at $18^{\circ}$ shows only 0.4 per cent of the calculated conductivity for complete ionization. Even a $0.001 \mathrm{~N}$ solution is only 12.5 per cent ionized. A fundamental point in the ionic hypothesis of Arrhenius, however, is that ionization always approaches completion as the solution becomes more and more dilute.

The following table shows the approximate degrees of ionization of a number of typical electrolytes in tenth-normal solution in water at $18^{\circ}$. In the case of acids and bases containing more than one displaceable unit of hydrogen or hydroxyl, the kind of ionization on which the figure is based should be particularly noted. 


\section{FRACTION IONIZED IN $0.1 N$ SOLUTIONS AT $18^{\circ}$}

The 0.92 ACIDS

Nitric acid $\left(\mathrm{H}+, \mathrm{NO}_{3}-\right) \ldots .0 .92$

Sulphuric acid $\left(2 \mathrm{H}+, \mathrm{SO}_{4}=\right) .0 .61$

Oxalic acid $\left(\mathrm{H}^{+}, \mathrm{HC}_{2} \mathrm{O}_{4}^{-}\right) \ldots 0.50$

Phosphoric acid $\left(\mathrm{H}+, \mathrm{H}_{2} \mathrm{PO},-\right) 0.27$

Hydrofluoric acid $\left(\mathrm{H}^{+}, \mathrm{F}^{-}\right) \ldots . .0 .085$

Carbonic acid $\left(\mathrm{H}+, \mathrm{HCO}_{3}-\right) \ldots \ldots 0.0017$

Hydrosulphuric acid $\left(\mathrm{H}^{+}, \mathrm{HS}^{-}\right) .0 .0007$

Boric acid $\left(\mathrm{H}^{+}, \mathrm{H}_{2} \mathrm{BO}_{3}-\right) \ldots \ldots .0 .0001$

\section{BASES}

Sodium hydroxide

$\left(\mathrm{Na}^{+}, \mathrm{OH}^{-}\right)$

Potassium hydroxide

$\left(\mathrm{K}+, \mathrm{OH}^{-}\right)$.

Barium hydroxide $\left(\mathrm{Ba}^{++}, 2 \mathrm{OH}^{-}\right) 0.77$

Ammonium hydroxide

$\left(\mathrm{NH}_{6}+, \mathrm{OH}^{-}\right) \ldots \ldots \ldots \ldots \ldots .013$

\section{SALTS}

Potassium chloride $(\mathrm{K}+, \mathrm{Cl}-) 0.86$ Sodium chloride $\left(\mathrm{Na}+\mathrm{Cl}^{-}\right) 0.85$ Potassium fluoride $\left(\mathrm{K}^{+}, \mathrm{F}^{-}\right) \quad 0.85$ Sodium nitrate $\left(\mathrm{Na}^{+}, \mathrm{NO}_{3}-\right) 0.83$ Silver nitrate $\left(\mathrm{Ag}^{+}, \mathrm{NO}_{3}{ }^{-}\right) \ldots .81$
Barium chloride $(\mathrm{Ba}++, 2 \mathrm{Cl}-1) .0 .76$

Sodium sulphate $\left(2 \mathrm{Na}+, \mathrm{SO}_{4}=\right) \quad .0 .70$

Zinc sulphate $\left(\mathrm{Zn}++, \mathrm{SO}_{4}=\right) \ldots 0.40$

Copper sulphate $\left(\mathrm{Cu}^{++}, \mathrm{SO}_{4}=\right) \ldots 0.40$

Mercuric chloride $\left(\mathrm{Hg}^{++}, 2 \mathrm{Cl}-\right)<0.01$

Ionization and Chemical Activity.- From a consideration of the above figures and the results given by other acids, bases and salts, the following important conclusions may be drawn:

1. Salts, with the exception of a few mercuric salts, are all extensively ionized in $0.1 \mathrm{~N}$ aqueous solution. The salts which ionize most simply show the greatest degree of ionization. Compare, for example, the series sodium chloride, sodium sulphate, copper sulphate.

2. Acids show the most extreme differences in their degrees of ionization. That is to say, equivalent solutions contain very different concentrations of hydrogen ion. Since their activity as acids depends upon this substance (p. 191), it follows that acids will exhibit very marked differences in chemical activity (for example, in their action on metals). In fact, they may be divided roughly into three classes:

(a) Strong acids, such as hydrochloric acid, nitric acid, sulphuric acid. These substances are highly ionized in $0.1 \mathrm{~N}$ solution. Their solutions conduct the electric current excellently, and are chemically most active. 
(b) Transition acids, such as phosphoric acid, hydrofluoric acid. These substances are slightly ionized in $0.1 \mathrm{~N}$ solution. Their solutions conduct fairly well, and show moderate activity.

(c) Weak acids, such as acetic acid, carbonic acid, boric acid. These substances are scarcely ionized at all in $0.1 \mathrm{~N}$ solution. Their solutions conduct the current very poorly, and exhibit little activity as acids.

3. Bases also show very extreme differences in their degrees of ionization. We have two main classes - strong bases, such as sodium hydroxide, barium hydroxide; and weak bases, such as ammonium hydroxide. Between these fall certain transition bases, such as silver hydroxide (which is only very slightly soluble, but which is noticeably stronger than ammonium hydroxide) and some organic derivatives of ammonium hydroxide.

4. Water itself is both an exceedingly weak acid and an exceedingly weak base. It breaks up into the two ions $\mathrm{H}^{+}$and $\mathrm{OH}^{-}$ to a very minute extent indeed. At ordinary temperature the fraction ionized is less than $0.000,000,002$. Pure water consequently conducts the electric current practically not at all. The ionization of water, however, is a factor of vital importance in the explanation of certain reactions, as we shall see later.

\section{Ions and Vapor-Pressure, Boiling-Point and Freezing-} Point Abnormalities. - The cause of the abnormal changes in these three physical properties, exhibited by solutions of electrolytes, will now be evident. A solution of sodium chloride containing one-gram molecular weight of salt in $1000 \mathrm{~g}$. of water gives almost twice the calculated effect in each case, because almost all of the solute is present as $\mathrm{Na}^{+}$and $\mathrm{Cl}^{-}$, instead of as undissociated $\mathrm{NaCl}$. The number of solute molecules present is almost doubled by ionization. Similarly a dilute solution of ferric chloride shows nearly four times the normal change in freezing-point lowering, for example, owing to the fact that the majority of $\mathrm{FeCl}_{3}$ molecules are broken up into four ions, $\mathrm{Fe}^{+\mathrm{H}}$ and $3 \mathrm{Cl}^{-}$. 
Solutions of weak electrolytes, such as acetic acid and ammonium hydroxide, exhibit practically normal results, because only an insignificant fraction of the solute is broken up into ions.

Careful measurements show a close agreement in respect to extents of ionization, as determined by the two independent methods, conductivity ratio and freezing-point depression, throughout the whole list of hundreds of electrolytes. Minor divergences exist in some cases which have not yet been entirely accounted for, but the agreement in general is so remarkably close that it cannot be a chance coincidence. We have here, in fact, very strong confirmation of the truth of the ionic hypothesis.

The Properties of Acids. - The properties of acids (p. 131) are now seen to be properties of hydrogen ion $\mathrm{H}^{+}$. An acid is a substance which contains hydrogen as a positive radical and, in solution, gives hydrogen ion. Strictly speaking, only the conducting solutions of such substances are acids, but for convenience we extend the term sometimes to include the pure substances. For example, $\mathrm{HNO}_{3}$ is usually called nitric acid, not hydrogen nitrate.

Many substances, such as sugar $\mathrm{C}_{12} \mathrm{H}_{22} \mathrm{O}_{11}$, contain hydrogen, but their solutions lack all of the properties of hydrogen ion. They therefore do not contain hydrogen as a radical, and are not acids.

A strong acid is one which is highly ionized in solution, and therefore shows the properties of hydrogen ion very markedly. A weak acid is one which is very little ionized in solution, and consequently exhibits the properties of hydrogen ion only feebly. Solutions of very weak acids (such as boric acid) scarcely affect blue litmus. Water, a still weaker acid, contains just as much hydroxyl ion $\mathrm{OH}^{-}$as hydrogen ion $\mathrm{H}^{+}$, and does not change the color of either blue or reddened litmus. Its acid properties are still evident, however, in its action on the most active metals, such as sodium:

$$
\mathrm{Na}+2 \mathrm{H}^{+} \rightarrow 2 \mathrm{Na}^{+}+\mathrm{H}_{2} \uparrow .
$$


The Properties of Bases. - The properties of bases (p. 167) are properties of hydroxyl ion $\mathrm{OH}^{-}$. A base is a substance which contains hydroxyl as a negative radical and, in solution, gives hydroxyl ion.

Many substances, such as ethyl alcohol $\mathrm{C}_{2} \mathrm{H}_{5} \mathrm{OH}$, contain the hydroxyl group, but their solutions exhibit none of the characteristic properties of a base. They are not bases, since they do not contain hydroxyl as an ionizing radical.

A strong base is one which is highly ionized in solution, and therefore shows the properties of hydroxyl ion very markedly. It will be observed that the most active bases (alkalies) are the hydroxides of those metals $(\mathrm{K}, \mathrm{Na}, \mathrm{Ba})$ which come first on the activity list (p. 54). Weak bases, like ammonium hydroxide and copper hydroxide, are little ionized in solution, and exhibit the properties of hydroxyl ion only feebly. Water is an exceedingly weak base.

The Properties of Salts. - Salts are substances which contain a positive ionizing radical other than hydrogen, combined with a negative ionizing radical, other than hydroxyl. The properties of a salt in solution are the properties of its two ions (see p. 175).

Some salts do indeed contain hydrogen or hydroxyl as a radical, but always in addition to two other radicals. Thus sodium-hydrogen sulphate (p. 126) gives $\mathrm{H}^{+}$in solution, as well as $\mathrm{Na}^{+}$and $\mathrm{SO}_{4}=$. This is an acid salt. The precipitate actually obtained by the action of a soluble carbonate on a cupric salt (p. 179) has the composition $\mathrm{Cu}_{2}(\mathrm{OH})_{2} \mathrm{CO}_{3}$. It is a basic salt.

Neutralization.- This is a special case of double decomposition between electrolytes in solution, the reacting substances being an acid and a base. The products of the reaction are a salt and water. Thus the mixture of equivalent amounts of hydrochloric acid and sodium hydroxide solutions gives a reaction which is complete, although no substance concerned in the reaction 
has escaped either as a precipitate or as a gas. All of the peculiar properties of the original components (such as their action on litmus) disappear, and we are left with a solution of common salt.

The explanation, according to the ionic hypothesis, is very simple. Before mixing the two solutions, we have our acid and base almost entirely broken up into the four ions $\mathrm{H}^{+}, \mathrm{Cl}^{-}, \mathrm{Na}^{+}$ and $\mathrm{OH}^{-}$. But as soon as mixture is effected, practically all $\mathrm{H}^{+}$and $\mathrm{OH}^{-}$ions withdraw from the solution, combining to form water. The minute amounts of undissociated $\mathrm{HCl}$ and $\mathrm{NaOH}$ continue to break up, attempting to regain equilibrium with their respective ions, until they are both eliminated, and only chloride ions $\mathrm{Cl}^{-}$and sodium ions $\mathrm{Na}^{+}$, in equilibrium with undissociated $\mathrm{NaCl}$, remain in the solution.

The reaction may be written in expanded ionic form as follows:

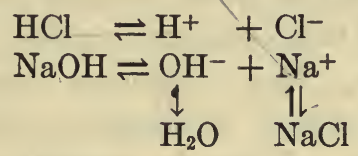

The ionization of water is so minute that it may be neglected. When either the acid or the base employed is weak, however, the tendency of water to break up into $\mathrm{H}^{+}$and $\mathrm{OH}^{-}$must be taken into consideration (see hydrolysis, p. 369).

We may note here a third method of driving reversible reactions between electrolytes to completion. Besides removing one product as a precipitate or as a gas (see pp. 126-7), we may arrange the conditions so that one of the substances formed is practically non-ionized. This is a procedure which is very frequently employed by chemists in carrying out reactions.

The Part Played by the Solvent in Ionization.- So far, we have regarded water as playing merely a physical role in the ionization of electrolytes. The ions in a solution of hydrogen chloride, for example, have been regarded as $\mathrm{H}^{+}$and $\mathrm{Cl}^{-}$, the 
solvent breaking up the molecule $\mathrm{HCl}$ in some way, but not being itself directly concerned in the reaction.

This view, although sufficient for many purposes, will not stand strict investigation. Thus we know by experiment that the ions, when they migrate with the electric current in electrolysis, carry water with them. This indicates that ions are hydrated. The solvent is therefore chemically active in ionization.

Recent work, indeed, suggests that the distinction drawn between solvent and solute in explaining ionization phenomena is entirely misleading. Pure liquefied hydrogen chloride is practically a non-conductor, just like pure water. It is quite an arbitrary procedure, therefore, to ascribe all of the conducting power of a mixture of hydrogen chloride and water to the former substance, and to regard the latter as quite inert. It would be more logical to consider both components of the solution as equally concerned in ionization. Now experiment shows that extensive ionization in solution always accompanies extensive compound formation on admixture. When no interaction at all between the components occurs, the solution is non-conducting. All strong acids, for example, give hydrates with water which are sufficiently stable to be isolated in the solid state. No very weak acids give isolable hydrates. Those mercuric salts which are highly ionized, as mercuric nitrate $\mathrm{Hg}\left(\mathrm{NO}_{3}\right)_{2}$, all yield hydrates, such as $\mathrm{Hg}\left(\mathrm{NO}_{3}\right)_{2}, 8 \mathrm{H}_{2} \mathrm{O}$. Those which are only slightly ionized are all non-hydrated. It is possible, therefore, to regard ionization as due to the formation of solvent-solute complexes. The attractive forces between the constituent groups in such complexes would be considerably weaker than in the simpler molecules of the two components, and dísintegration into oppositely-charged radicals could occur much more readily.

It should be added that ionization is not restricted to solutions of electrolytes in water. Many other solvents, such as liquid ammonia $\mathrm{NH}_{3}$, formic acid $\mathrm{H} . \mathrm{COOH}$, ethyl alcohol $\mathrm{C}_{2} \mathrm{H}_{5} \mathrm{OH}$, 
dissolve many electrolytes to give solutions of excellent conducting power. Water, however, is the solvent most commonly used in chemical operations, and other ionizing solvents need not be considered at this stage.

Ions and Electrons. - The question may be asked: Whence do the ions obtain their electric charges? A brief answer to this question may be attempted here, although for a clear realization of its significance a knowledge of the subject-matter of the final chapter (pp. 552-4) is necessary.

Matter is electrical in its ultimate nature, and the atoms of all elements are more or less complex aggregates of positive and negative electrical units. The positive units (protons) constitute the main mass of the core or nucleus of the atom, and are fixed therein, except in radioactive disintegrations. The outermost shell of the atom consists of a number of negative units (electrons), which are less rigidly held. The atom as a whole, of course, is electrically neutral. The hydrogen atom, to choose the simplest example, is made up of a single proton and a single electron. The structure of the atoms of other elements is, of course, more complex, but all possess, in their outermost shell, a definite small number of electrons, which are relatively loosely held. When two atoms of different elements combine, it may happen that an electron (or a number of electrons) will pass from one atom to the other. Atoms which lose electrons in this way become positive radicals, the departure of an electron leaving the atom as a whole electrically positive. Atoms which gain electrons become negative radicals, arrival of an electron making the atom as a whole electrically negative. Under normal circumstances, the attractive forces between such oppositely-charged radicals will be sufficient, in most cases, to bind them firmly together as electrically neutral molecules. If we weaken these forces, however, as we undoubtedly do when we dissolve an electrolyte in a solvent such as water, separation of the bound radicals into free positive and negative ions 
can be effected much more readily, and extensive ionization may result.

A positive ion, therefore, is a free atom, or group of atoms, which has lost an olectron, or a number of electrons, such as $\mathrm{Na}^{+}$, $\left(\mathrm{NH}_{4}\right)^{+}, \mathrm{Zn}++$. A negative ion is a free atom, or group of atoms, which has of a number of electrons, such as $\mathrm{Cl}-,\left(\mathrm{NO}_{3}\right)^{-}, \mathrm{S}^{=}$.

Some Possible Misunderstandings.-Before we close the chapter, it will be profitable to anticipate some difficulties into which the reader may fall. If the ionic hypothesis is not properly understood, it appears to conflict so strongly with what the student has learnt in previous chapters that he is apt to become hopelessly confused. The following points of possible misunderstanding and the explanations appended should therefore be read through very carefully. For convenience of illustration, sodium chloride is taken as a typical electrolyte in the questions and answers listed below. The student should test his knowledge of the subject by substituting other electrolytes.

1. If sodium chloride is broken up in aqueous solution into sodium and chlorine, why do we not find any of the properties of sodium or of chlorine exhibited by the solution? - This has always been a very common misapprehension of the ionic theory. Many prominent chemists never could understand how sodium (a metal which acts vigorously on water) and chlorine (an obnoxious gas) could exist side by side in a solution of sodium chloride without immediately notifying us of their presence by characteristic reactions. The answer is that free sodium and free chlorine do not exist in sodium chloride solution. The ionic hypothesis has never stated that they do. What it does state is that sodium ion $\mathrm{Na}^{+}$and chloride ion $\mathrm{Cl}^{-}$are present in the solution. These are entirely different substances from atomic sodium $\mathrm{Na}$ and molecular chlorine $\mathrm{Cl}_{2}$. The electric charges on the ions change their properties completely. There is no more reason why they should behave like free sodium and free chlorine than there is 
for crystals of common salt to behave like a mixture of sodium and of chlorine. Metallic sodium Na reacts with water to form a solution of sodium hydroxide. In sodium chloride solution, however, the ionic sodium $\mathrm{Na}^{+}$is already in the same state as it is in sodium hydroxide solution, and is in no need of trying to enter that state.

2. Salt is a very stable substance. The union of sodium and chlorine evolves a great deal of heat. A great deal of work will be required, therefore, to decompose sodium chloride. How can mere addition of water break it up? - We have here the same misunderstanding in another form. It is true that it would be very difficult to decompose sodium chloride into free sodium and free chlorine, but its dissociation into sodium ion and chloride ion is an entirely different question. As a matter of fact, the heat of ionization is extremely small. Sodium chloride is stable only in the solid state. In solution, it reacts with very great facility with many other electrolytes.

3. Why do not the ions $\mathrm{Na}^{+}$and $\mathrm{Cl}^{-}$recombine at once, in response to the attractions of their charges? - The answer is that they do combine. The tendency towards combination is, however, opposed by the tendency of undissociated $\mathrm{NaCl}$ (the attractive forces between the bound radicals of which are weakened in solution) to decompose into free ions. An equilibrium between the two tendencies is, therefore, set up, which we may express by the reversible reaction $\mathrm{NaCl} \rightleftharpoons \mathrm{Na}^{+}+\mathrm{Cl}^{-}$.

4. Why can we not separate sodium ions from chloride ions in a solution of sodium chloride before we pass a current through the solution? Does not this show that it is the electric current which breaks up the sodium chloride? - The charges on the ions are not derived from the electric current. Free sodium ions and free chloride ions are present in the solution the instant the salt is dissolved, whether a current is passing or not. Before we pass a current through the solution, however, any portion of it of sensible magnitude contains just as many sodium ions as chlo- 
ride ions. By making use of the fact that the chloride ion diffuses more rapidly than the sodium ion (into a layer of pure water, for example, carefully poured over the solution) we can bring about a slight separation of the two ions, the water layer becoming negatively charged and the solution positively. The passage of the current does not cause ionization, it merely makes its existence more obvious, effectually separating the ions by forcing them to migrate in different directions towards the oppositely-charged electrodes.

Exercises. - 1. Which are the anions and which the cations in the substances whose formulæ are given on p. 189?

2. Using the models given in p. 182 , make the ionic equations representing the ionization of all the acids, bases, and salts, the formulæ of which are given on p. 170.

3. Make an ionic equation (p. 185) for the displacement: (a) of hydrogen from dilute hydrochloric acid by magnesium; and (b) of copper from cupric sulphate solution by zinc.

4. Rewrite the double decompositions on pp. 166-168 in full ionic form.

5. Why does a solution of $0.1 \mathrm{~N}$ hydrochloric acid conduct the current nearly twice as well as a solution of $0.1 \mathrm{~N}$ sodium hydroxide, and nearly four times as well as a solution of $0.1 \mathrm{~N}$ sodium chloride?

6. From the results given on p. 177 , calculate the degree of ionization of sodium chloride in a solution containing $1 \mathrm{gr}$. mol. wt. $\mathrm{NaCl}$ to $1000 \mathrm{~g}$. water (a) at the boiling-point, and (b) at the freezing-point.

7. From the results given on p. 177, calculate the degree of ionization of potassium chloride in a solution containing $1 \mathrm{gr}$. mol. wt. $\mathrm{KCl}$ to $1000 \mathrm{~g}$. water at $20^{\circ}$. 


\section{CHAPTER XVII \\ THE HALOGEN FAMILY}

THE elements, if we may judge from those studied or mentioned thus far, may be divided into two classes - the metallic or positive elements, like sodium, zinc and magnesium, and the non-metallic or negative elements, like oxygen, chlorine and sulphur. The former give positive ions, such as $\mathrm{Na}^{+}, \mathrm{Mg}^{++}$. The latter give negative ions, such as $\mathrm{Cl}^{-}, \mathrm{S}^{-}$. Hydrogen constitutes the single exception, giving the positive ion $\mathrm{H}^{+}$.

Natural Families of Elements. - We have a simple means of subdividing within each of these two classes. We can place together the elements of like chemical behavior. Thus sodium and potassium or zinc and magnesium resemble one another very closely in their reactions. Also, oxygen and sulphur form one group and chlorine, bromine, iodine and fluorine form another. Groups of this kind are often spoken of as natural families of elements. The last group is called the halogen family, from the Greek for salt-producing, because these elements combine with sodium to give substances all resembling common salt. (Usually, the elements of one family and their corresponding compounds resemble one another in a number of ways, and show at the same time a gradation in properties which it is interesting to study.)

\section{Bromine $\mathrm{Br}_{2}$}

The element was discovered by Balard in 1826 and derives its name from its offensive odor (Greek, $a$ stench).

Preparation.- The salt deposits and natural salt wells of Cheshire, of Germany, and of Michigan, West Virginia, Ohio, and 199

7 amily contuins

have closely selated characteristic. 
Connecticut, contain some bromides, along with large quantities of common salt. When the latter has been largely separated by evaporation and crystallization, the bromides of sodium and magnesium, which are more soluble, collect in the mother liquor.

The bromine can be liberated at the positive electrode by electrolysis. But usually a chemical process is employed.

In one process, chlorine gas is dissolved in the liquor. This displaces the bromine, and the latter can be distilled out by heating:

$$
2 \mathrm{Br}^{-}+\mathrm{Cl}_{2} \rightarrow 2 \mathrm{Cl}^{-}+\mathrm{Br}_{2} \text {. }
$$

In another process, oxidation of a bromide by pulverized manganese dioxide and sulphuric acid is employed, and this method can be used in the laboratory (Fig. 81, p. 308).

The manganese dioxide is reduced to manganous sulphate (compare p. 142), its oxygen combining with hydrogen from $\mathrm{H}_{2} \mathrm{SO}_{4}$ to form water. The sodium bromide used is converted to sodium hydrogen sulphate (compare p. 126) and bromine is liberaied.

Skeleton: $\mathrm{NaBr}+\mathrm{MnO}_{2}+\mathrm{H}_{2} \mathrm{SO}_{4} \rightarrow \mathrm{Br}_{2}+\mathrm{NaHSO}_{4}+\mathrm{MnSO}_{4}+$ $\mathrm{H}_{2} \mathrm{O}$.

Balanced: $2 \mathrm{NaBr}+\mathrm{MnO}_{2}+3 \mathrm{H}_{2} \mathrm{SO}_{4} \rightarrow \mathrm{Br}_{2}+2 \mathrm{NaHSO}_{4}+\mathrm{MnSO}_{4}$ $+2 \mathrm{H}_{2} \mathrm{O}$.

Physical Properties. - Bromine is a liquid of a deep redbrown color and the vapor, of the same color, has a suffocating odor. It boils at $59^{\circ}$. It is moderately soluble in water, giving a 3.2 per cent solution (bromine-water), and is very soluble-in carbon disulphide. The density of the vapor gives it the formula $\mathrm{Br}_{2}$. Great care must be used in handling bromine, as, when spilt upon the skin, it kills the tissues and the sore is very liable to become infected.

Treatment of Burns.- - Burns made by bromine or strong acids should be washed instantly with water and then with bi- 
carbonate of soda solution, and covered thickly with vaseline, or a salve of boric acid in lanoline, to protect them from infection.

Chemical Properties.-A jet of burning hydrogen will continue to burn in bromine vapor, giving hydrogen bromide, a gas which fumes in moist air like hydrogen chloride:

$$
\mathrm{H}_{2}+\mathrm{Br}_{2} \rightarrow 2 \mathrm{HBr} \text {. }
$$

Many of the metals, when thrown in the form of powder, leaf, or foil, into bromine vapor, combine directly, giving bromides. The action is similar to that with chlorine, but less vigorous.

Hydrogen Bromide $\mathrm{HBr}$, Preparation.-Hydrogen and bromine vapor unite much less readily than hydrogen and chlorine. A stream of pure hydrogen bromide is most easily made by moistening red phosphorus with water, and allowing bromine to fall drop by drop into the paste (Fig. 61). To absorb the bromine vapor, carried by the gas, the latter is passed through a U-tube containing dry red phosphorus mixed with broken glass or beads:

$$
2 \mathrm{P}+3 \mathrm{Br}_{2} \rightarrow 2 \mathrm{PBr}_{3} \text {. }
$$

$$
\mathrm{PBr}_{3}+3 \mathrm{H}_{2} \mathrm{O} \rightarrow 3 \mathrm{HBr} \uparrow+\mathrm{H}_{3} \mathrm{PO}_{3} \text {. }
$$

The bromine forms phosphorus tribromide, which is immediately decomposed by the water. The phosphorous acid $\mathrm{H}_{3} \mathrm{PO}_{3}$ remains, dissolved in the water, in the flask. The gas can be collected by upward displacement of air.

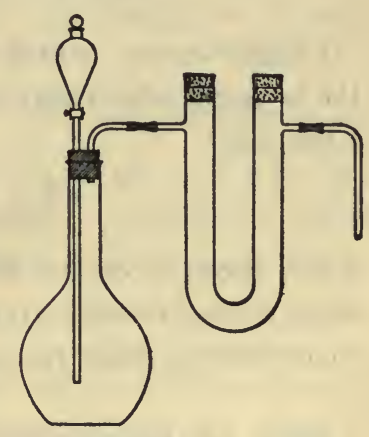

Fig. 61

It might seem that a simpler action would be that of sulphuric acid upon a bromide (compare p. 126):

$$
\mathrm{H}_{2} \mathrm{SO}_{4}+\mathrm{KBr} \rightarrow \mathrm{KHSO}_{4}+\mathrm{HBr} \uparrow \text {. }
$$


This action does take place, but the hydrogen bromide formed, being less stable than $\mathrm{HCl}$, is oxidized rapidly by the concentrated sulphuric acid, so that, although some of the gas escapes oxidation, it is mixed with much free bromine and sulphur dioxide: $\mathrm{H}_{2} \mathrm{SO}_{4}+2 \mathrm{HBr} \rightarrow \mathrm{SO}_{2}+2 \mathrm{H}_{2} \mathrm{O}+\mathrm{Br}_{2}$. This action, indeed, enables us to recognize a bromide, by the color of the bromine vapor and the fuming of the hydrogen bromide produced.

Properties of Hydrogen Bromide.- The gas, like hydrogen chloride, is colorless, and has an irritating effect when breathed. It is extremely soluble in water, and fumes in moist air, giving a fog of $\mathrm{HBr}$ dissolved in water.

Chemically, hydrogen bromide is stable, though not so much so as hydrogen chloride. Its aqueous solution is an active acid. As such, it gives double decomposition with bases and salts. Thus, with a salt of silver, we get a cream-colored precipitate of insoluble silver bromide:

$$
\mathrm{AgNO}_{3}+\mathrm{HBr} \rightarrow \mathrm{AgBr} \downarrow+\mathrm{HNO}_{3} .
$$

Chlorine-water, added to a solution of any bromide, displaces the bromine, which may be recognized by its brown color (test for a bromide):

$$
\mathrm{Cl}_{2}+2 \mathrm{Br}^{-} \rightarrow 2 \mathrm{Cl}^{-}+\mathrm{Br}_{2} .
$$

A few drops of carbon disulphide, shaken with the mixture, will settle to the bottom, carrying the brown bromine with it in a more concentrated, easily recognizable form.

Uses of Compounds of Bromine.-Bromine is manufactured in large amounts in Germany and in the United States. It is employed to make potassium bromide, and other bromides. These are utilized in medicine, and to precipitate silver bromide in the manufacture of photographic films and plates. 


\section{IODINE $I_{2}$}

Sources.-Iodine was formerly all obtained from seaweed (kelp), certain species of which use the traces of organic compounds of iodine in sea water as part of their food. The dried seaweed is carbonized in retorts, and sodium iodide remains in the residue, along with much sodium carbonate and carbon. In an improved process the iodine compounds are dissolved out of the kelp, and from the latter a sort of gelatin, named algin, is extracted.

The greater part of our supply of iodine is at present obtained from sodium iodate $\mathrm{NaIO}_{3}$, which forms about 0.2 per cent of crude Chile saltpeter.

Preparation.- The processes for obtaining iodine from an iodide are precisely the same as those for bromine.

In France chlorine is used to displace the iodine:

$$
\mathrm{Cl}_{2}+2 \mathrm{I}^{-} \rightarrow 2 \mathrm{Cl}^{-}+\mathrm{I}_{2} \downarrow \text {. }
$$

The precipitate of iodine is pressed free from the solution.

In Great Britain the iodide is mixed with manganese dioxide and sulphuric acid and heated:

$3 \mathrm{H}_{2} \mathrm{SO}_{4}+\mathrm{MnO}_{2}+2 \mathrm{NaI} \rightarrow \mathrm{MnSO}_{4}+2 \mathrm{NaHSO}_{4}+2 \mathrm{H}_{2} \mathrm{O}+\mathrm{I}_{2} \uparrow$.

Iodine vapor condenses upon a cold surface, not to the liquid, but directly to the solid, crystalline form. Distillation which gives a solid product is called sublimation. The crude iodine is purified by repetition of this process.

Physical Properties.-Iodine forms black, shining crystals. The vapor, visible even at the ordinary temperature, is violet in color (hence the name of the substance, from Greek, meaning like a violet) and has a density corresponding to the formula $\mathrm{I}_{2}$. The substance is very slightly soluble in water. It dissolves, however, easily in carbon disulphide (violet solution), in alcohol or potassium iodide solution (brown solution), and even in starch, upon 
which a trace of it confers a strong blue color (test for free iodine, p. 400). These colors are shown only by free iodine - the iodides are colorless.

Chemical Properties. - Iodine unites very slowly and incompletely with hydrogen, giving hydrogen iodide. It combines readily with phosphorus $\left(\mathrm{PI}_{3}\right)$ and with many of the metals, giving iodides.

Hydrogen Iodide, Preparation.-The gas is prepared by the process used for hydrogen bromide. Red phosphorus and iodine are mixed, and water is allowed to fall drop by drop upon the mass (Fig. 25, p. 52):

$$
\begin{aligned}
2 \mathrm{P}+3 \mathrm{I}_{2} & \rightarrow 2 \mathrm{PI}_{3} . \\
\mathrm{PI}_{3}+3 \mathrm{H}_{2} \mathrm{O} & \rightarrow 3 \mathrm{HI}+\mathrm{H}_{3} \mathrm{PO}_{3} .
\end{aligned}
$$

The gas is very dense $(\mathrm{HI}=1+127=128 \mathrm{~g}$. per 22.4 liters, against $28.95 \mathrm{~g}$. for air) and can be collected by upward displacement of air.

The action of sulphuric acid upon an iodide does not give pure hydrogen iodide, although the action $\mathrm{NaI}+\mathrm{H}_{2} \mathrm{SO}_{4} \rightarrow \mathrm{NaHSO}_{4}$ $+\mathrm{HI}$.does take place (compare p. 202). Hydrogen iodide, being much less stable than even hydrogen bromide, is a more active reducing agent, and reduces the sulphuric acid to hydrogen sulphide. The odor of this gas is therefore very conspicuous when an iodide is moistened with sulphuric acid:

$$
\mathrm{H}_{2} \mathrm{SO}_{4}+8 \mathrm{HI} \rightarrow \mathrm{H}_{2} \mathrm{~S}+4 \mathrm{H}_{2} \mathrm{O}+4 \mathrm{I}_{2} \text {. }
$$

The violet vapor of iodine becomes visible if the test-tube is warmed. A rough test for an iodide is afforded by this action.

A nother method of making hydrogen iodide is frequently employed when a solution of the gas in water is required, and not the gas itself. Powdered iodine is suspended in water, and hydrogen sulphide gas is introduced through a tube in a continuous stream. The iodine dissolves slowly in the water and acts by 
displacement upon the sulphide ion $\mathrm{S}^{=}$, derived from the solution of $\mathrm{H}_{2} \mathrm{~S}$ in water. Sulphur separates in a fine powder, and a solution of hydrogen iodide (hydriodic acid) is formed in accordance with the equation:

$$
2 \mathrm{H}^{+}+\mathrm{S}^{=}+\mathrm{I}_{2} \rightarrow 2 \mathrm{H}^{+}+2 \mathrm{I}^{-}+\mathrm{S} \downarrow \text {. }
$$

The solution is freed from the deposit of sulphur by filtration, and may be concentrated to 57 per cent of hydriodic acid by distilling off the water.

Properties.- Hydrogen iodide is exceedingly soluble in water, and fumes strongly in moist air, giving a fog of $\mathrm{HI}$ solution. It is colorless.

The aqueous solution is an active acid. The iodide-ion I-, which it contains, gives, with any soluble salt of silver, a precipitate of insoluble yellow silver iodide AgI:

$$
\mathrm{AgNO}_{3}+\mathrm{HI} \rightarrow \mathrm{AgI} \downarrow+\mathrm{HNO}_{3} .
$$

Chlorine-water or bromine-water, added to a solution of this or any other iodide, displaces the iodine:

$$
\mathrm{Cl}_{2}+2 \mathrm{I}^{-} \rightarrow 2 \mathrm{Cl}^{-}+\mathrm{I}_{2} \text {. }
$$

The free iodine, even if present in minute amounts, may be recognized by shaking the liquid with a few drops of carbon disulphide. The iodine gives a violet solution in the latter. A still more delicate test is the addition of a drop of very thin starch paste, which gives a deep-blue tint with free iodine. Filter paper dipped in starch paste and dried can also be used, by touching it with a drop of the solution containing the free iodine.

Uses of Iodine and Its Compounds. - The alcoholic solution (tincture of iodine), painted over the skin, reduces swellings and inflammation. Iodoform $\mathrm{CHI}_{3}$ is a solid used for similar purposes. Iodothyrin is an organic compound found in the human thyroid gland, as well as that of other animals. An extract of sheeps' 
thyroids (thyroxin) is administered with remarkable success in cases of degeneration caused by abnormally small natural development of this gland (cretinism). Potassium iodide is also used in medicine, to cause absorption of blood-clots and effusions of blood, for example in the eye. Silver iodide is contained in the coating on photographic plates and films.

\section{FLUORINE $\mathrm{F}_{2}$}

Compounds of fluorine are found in large quantities as minerals, but the compounds are so stable that the element is very difficult to liberate. The natural compounds, however, have many important uses.

Occurrence.-Calcium fluoride $\mathrm{CaF}_{2}$ (fluorite) occurs in nature in beautiful cubical crystals. Cryolite $\mathrm{AlF}_{3}, 3 \mathrm{NaF}$ is used

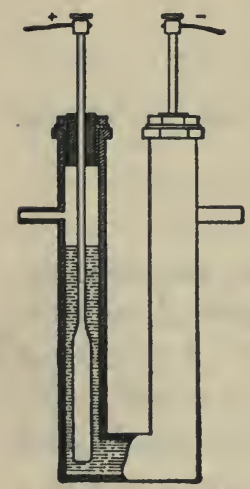

Fig. 62 in the modern manufacture of aluminium (p. 466). Apatite $\mathrm{Ca}_{5}\left(\mathrm{PO}_{4}\right)_{3} \mathrm{~F}$ is a common constituent of rocks and soils. When slowly decomposed, by weathering, it furnishes soluble phosphates. These are absorbed by plants, for which they are a necessary food.

Preparation of Fluorine.-The element is obtained by electrolysis of potassium-hydrogen fluoride $\mathrm{KHF}_{2}$ dissolved in liquefied hydrogen fluoride. The electrodes are made of an alloy of platinum and iridium, with which fluorine has little tendency to combine. The vessel is a U-tube of copper (Fig. 62) and, to prevent vaporization of the hydrogen fluoride (b.-p. $19.4^{\circ}$ ), is kept at $-23^{\circ}$ to $-40^{\circ}$ during the operation. Hydrogen is liberated at one pole and fluorine at the other.

Properties.-Fluorine is a yellow gas, with a density greater than that of air (G.M.V. weighs 38 g.). It is the most difficult of the halogens to liquefy (b.-p. $-187^{\circ}$ ). 
$\therefore$ thees 40 gms.
$\therefore$ THE HALOGEN FAMILY

Fluorine is the most active of the non-metals. It combines with all the metals, but most slowly with platinum and with gold. In the preparation of the gas the copper is protected from serious attack by the layer of fluoride first formed. Fluorine combines also with hydrogen in the cold and, unlike chlorine, without the assistance of light. It combines with most of the non-metals, but not with oxygen, chlorine, nitrogen, or the indifferent gases of the atmosphere.

With water (vapor or liquid) fluorine interacts, giving ozone (see p. 219) and hydrogen fluoride $\mathrm{H}_{2} \mathrm{~F}_{2}$ :

Skeleton:

$\mathrm{F}_{2}+\mathrm{H}_{2} \mathrm{O} \rightarrow \mathrm{H}_{2} \mathrm{~F}_{2}+\mathrm{O}_{3}$.

Balanced: $\quad 3 \mathrm{~F}_{2}+3 \mathrm{H}_{2} \mathrm{O} \rightarrow 3 \mathrm{H}_{2} \mathrm{~F}_{2}+\mathrm{O}_{3}$.

Hydrogen Fluoride $\mathrm{H}_{2} \mathrm{~F}_{2}$, Preparation.- When pulverized calcium fluoride and concentrated sulphuric acid are placed in a retort of platinum or lead and the mixture is warmed, hydrogen fluoride passes over. The vapor is usually led into water, in which it is very soluble (hydrofluoric acid):

$$
\mathrm{CaF}_{2}+\mathrm{H}_{2} \mathrm{SO}_{4} \rightarrow \mathrm{CaSO}_{4}+\mathrm{H}_{2} \mathrm{~F}_{2} \text {. }
$$

The acid is kept in bottles of paraffin or rubber, as glass interacts with it rapidly.

Physical Properties. - The vapor of hydrogen fluoride can be condensed to a colorless liquid boiling at $19.4^{\circ}$. Being very soluble, it fumes strongly in moist air. The vapor density below $40^{\circ}$ corresponds with the formula $\mathrm{H}_{2} \mathrm{~F}_{2}$, but at higher temperatures gradual dissociation to $\mathrm{HF}$ occurs.

Chemical Properties. - The aqueous solution has all the properties of a transition acid (see p. 190). The substance has, in addition, the remarkable property of acting upon silica $\mathrm{SiO}_{2}$ (sand), and silicates, to give silicon tetrafluoride $\mathrm{SiF}_{4}$ (a gas). Hence it attacks glass, which is a mixture of sodium silicate $\mathrm{Na}_{2} \mathrm{SiO}_{3}$ and calcium silicate $\mathrm{CaSiO}_{3}$ :

$$
\begin{aligned}
\mathrm{SiO}_{2}+2 \mathrm{H}_{2} \mathrm{~F}_{2} & \rightarrow \mathrm{SiF}_{4}+2 \mathrm{H}_{2} \mathrm{O} . \\
\mathrm{CaSiO}_{3}+3 \mathrm{H}_{2} \mathrm{~F}_{2} & \rightarrow \mathrm{SiF}_{4}+\mathrm{CaF}_{2}+3 \mathrm{H}_{2} \mathrm{O} .
\end{aligned}
$$


Thus, when glass is covered with melted paraffin to protect the surface, and marks or letters are made by removing the paraffin with a sharp instrument, hydrogen fluoride will decompose the glass at the parts thus exposed (test for fluorine). In this way the graduation on thermometer stems and lettering on glass are frequently made. The vapor gives rough, easily visible depressions, the solution smooth, glossy ones.

On account of this property, hydrofluoric acid is used for removing adhering sand from castings and for cleaning the outsides of granite and sandstone buildings.

\section{The Halogens as a Family}

The reader is recommended to compare carefully the properties of the several halogens and their compounds with hydrogen. It will be found that, while very striking similarities exist throughout the whole halogen family in the case of nearly every physical and chemical property, there is always a regular gradation in properties in the order of the atomic weights, namely $\mathrm{F}, \mathrm{Cl}, \mathrm{Br}, \mathrm{I} . \mathrm{A}$ few examples are noted below; the student should tabulate the rest himself for his own convenience.

Tw Color in liquid state: $\mathrm{F}_{2}$ yellow; $\mathrm{Cl}_{2}$ orange-yellow; $\mathrm{Br}_{2}$ brown; $\mathrm{I}_{2}$ deep violet.

Boiling-point: $\mathrm{F}_{2}-187^{\circ} ; \mathrm{Cl}_{2}-34^{\circ} ; \mathrm{Br}_{2} 59^{\circ} ; \mathrm{I}_{2} 184^{\circ}$.

Action on hydrogen: $\mathrm{F}_{2}$ very rapid action in cold, without light; $\mathrm{Cl}_{2}$ action rapid in cold, only with strong light; $\mathrm{Br}_{2}$ action rapid only when heated; $I_{2}$ action slow and incomplete even when heated. Action on H. gets cueaker as the atomic weight gets yueter.

As an aid to the memory, such a table is exceedingly valuable. But it is really not necessary to attempt to memorize all the properties of each halogen and of each halogen compound separately. The properties of bromine, for example, are all intermediate between those of chlorine and iodine. Indeed, when bromine activity gets hes as atomie veighr gets higher.

The forn imilar componinds wet soduin there rimilur propertie - they ace bleach too 
was first examined by Liebig, he thought it was an unstable compound of chlorine and iodine, and so missed gaining the credit of its discovery as an element.

The activity of the halogens, as is evident from their action on hydrogen, decreases in the order of increasing atomic weight. This is seen also in their displacement reactions in solutions. Thus we have found 'that chlorine displaces bromine from bromides and that bromine displaces iodine from iodides. Fluorine is able to displace even chlorine from chlorides: $2 \mathrm{Cl}^{+}+\mathrm{F}_{2} \rightarrow 2 \mathrm{~F}^{-}+$ $\mathrm{Cl}_{2}$. When we note also that the halogens displace sulphur from sulphides (compare p. 205) and that oxygen displaces iodine from hydriodic acid, we are able to draw up an order of activity for the non-metals, similar to the activity order for the metals. This order of activity expresses the order of preference of the nonmetals for assuming the ionic state. Starting from the top, it is $\mathrm{F}, \mathrm{Cl}, \mathrm{Br}, \mathrm{O}, \mathrm{I}, \mathrm{S}$.

A last point worthy of mention is that some of the properties of fluorides are peculiar. Thus hydrogen fluoride at low temperatures has the formula $\mathrm{H}_{2} \mathrm{~F}_{2}$, and its solution in water is not highly ionized. We shall see later that it is usual for the first member of a family of elements or compounds to exhibit a few peculiarities. In fact we have already noted, at the beginning of this chapter, one peculiarity of the first of all the elements, hydrogen. Although a non-metal, it gives a positive ion $\mathrm{H}^{+}$.

Exercises.-1. Tabulate the properties of fluorine, chlorine, bromine and iodine, and of their compounds with hydrogen.

2. How should you distinguish by chemical reactions the chloride, bromide, iodide, and fluoride, $(a)$ of hydrogen, $(b)$ of sodium from one another?

3. Write equations for the action, (a) of chlorine upon a solution of hydrogen sulphide, $(b)$ of bromine upon a solution of hydrogen iodide, $(c)$ of oxygen upon a solution of hydrogen iodide, (d) of fluorine upon a solution of hydrogen iodide. 
4. Why does hydriodic acid, when left in the air, become brown in color?

5. Make a list of all the acids we have encountered, and note which are weak and which strong.

6. How should you make potassium bromide, starting with, (a) potassium and bromine, (b) hydrogen bromide, (c) potassium iodide?

7. Rewrite the equation for the action of hydrobromic acid on silver nitrate solution in full ionic form. 


\section{CHAPTER XVIII}

\section{VALENCE}

THE differing number of charges on different ions has called our attention vaguely to a subject which must now be explored and set forth more clearly.

Valence.-The formulæ of a number of common compounds, including some that we have met with, are as follows:

$\begin{array}{llll}\mathrm{NaCl} & \mathrm{ZnCl}_{2} & \mathrm{AlCl}_{3} & \mathrm{SnCl}_{4} \\ \mathrm{NaBr} & \mathrm{ZnBr}_{2} & \mathrm{AlBr}_{3} & \mathrm{SnBr}_{4} \\ \mathrm{NaI} & \mathrm{ZnI}_{2} & \mathrm{AlI}_{3} & \end{array}$

We observe that one atomic weight of sodium appears to unite with only one unit of another element, one unit weight of zinc with only two units of another element, a unit of aluminium with not more than three, and a unit of tin with only four units.

It seems that an atomic weighi of each element has a fixed capacity for combining with not more than a certain number of atomic weights of other elements.

Other compounds of only two elements have the formulæ:

\section{$\begin{array}{llllll}\mathrm{HCl} & \mathrm{H}_{2} \mathrm{O} & \mathrm{NH}_{3} & \mathrm{CH}_{4} & \mathrm{Li}_{3} \mathrm{~N} & \mathrm{CCl}_{4} .\end{array}$}

So far as we may judge from this limited list, $\mathrm{Cl}$ combines with only one atom of another element, $\mathrm{O}$ with two atoms, $\mathrm{N}$ with three atoms, C with four atoms. Also an atom of hydrogen combines with not more than one atom of another element, although it may take more than one atom of hydrogen to satisfy the atom of that other element $\left(\mathrm{H}_{2} \mathrm{O}, \mathrm{CH}_{4}\right.$, etc. $)$.

This limited combining capacity of each kind of atomic weight (or atom) is called its valence. 
Marking the Valence.-Until we are familiar with their values in each case, it may be well to mark the valences thus:

$\begin{array}{llllllllll}\mathrm{Na}^{2} & \mathrm{Zn}^{\mathrm{II}} & \mathrm{Al}^{\mathrm{II}} & \mathrm{Sn}^{\mathrm{Iv}} & \mathrm{Cl}^{\mathrm{r}} & \mathrm{Br}^{\mathrm{I}} & \mathrm{I}^{\mathrm{I}} & \mathrm{O}^{\text {II }} & \mathrm{N}^{\mathrm{III}} & \mathrm{C}^{\mathrm{rv}}\end{array}$

As we should expect, an atom with the double capacity can combine with two of the single capacity, or with one of the double capacity, and so forth. Thus we have compounds of oxygen:

\section{$\mathrm{Zn}^{\mathrm{I}} \mathrm{O}^{\mathrm{II}} \quad \mathrm{Sn}^{\mathrm{Iv}} \mathrm{O}_{2}^{\mathrm{II}} \quad \mathrm{Al}_{2} \mathrm{mi}^{\mathrm{m}} \mathrm{O}_{3}^{\mathrm{II}}$}

Briefly stated, the quantities of the two elements which combine must have equal total combining capacities. Thus $\mathrm{Sn}^{\mathrm{rv}}$ has the capacity four, and $\mathrm{O}_{2} \mathrm{II}$ has the total capacity of $2 \times 2$ or 4 : $\mathrm{Al}_{2}{ }^{\mathrm{III}}$ has a total capacity of $2 \times 3$ (or 6$)$ and so has $\mathrm{O}_{3}^{\mathrm{II}}(3 \times 2$ $=6$ ).

( The unit of combining capacity of an atomic weight (or atom) is called a valence. The atomic weights of $\mathrm{H}$ and $\mathrm{Cl}$ are said to be univalent; those of $\mathrm{Zn}$ and $\mathrm{O}$, bivalent; those of $\mathrm{Al}$ and $\mathrm{N}$, trivalent; those of $\mathrm{Sn}$ and C, quadrivalent. The highest valence known is eight.

Valence and Ionic Charges.- Comparison with the formulæ of the ions already given will now show that the valence is equal to the number of charges on the corresponding ions: $\mathrm{H}^{\mathrm{I}} \mathrm{Cl}^{\mathrm{I}}$ gives $\mathrm{H}^{+}+\mathrm{Cl}^{-}$and $\mathrm{Zn}^{\mathrm{II}} \mathrm{Cl}_{2}{ }^{\mathrm{I}}$ gives $\mathrm{Zn}^{++}+2 \mathrm{Cl}^{-}$. Also, of course, the total number of each kind of charges (positive and negative) was equal, just as the total valences of each of two constituents of each compound are equal.

Valence of Radicals. - What has been said applies to compounds of not more than two elements - so called, binary compounds. We cannot, by inspection, tell the valences in a compound of three or more elements, like $\mathrm{H}_{2} \mathrm{SO}_{4}$. But, as we have seen, all electrolytes behave like binary compounds, because they divide into radicals, which move as wholes from one state of combination to another. Hence we can assign a valence to the radical 
$\mathrm{SO}_{4}$ as a whole. It is evidently bivalent, $\mathrm{H}_{2} \mathrm{I}\left(\mathrm{SO}_{4}\right)^{\mathrm{II}}, \mathrm{Zn}^{\mathrm{II}}\left(\mathrm{SO}_{4}\right) \mathrm{II}$. Similarly, in $\mathrm{K}^{\mathrm{I}}\left(\mathrm{NO}_{3}\right)^{\mathrm{I}}$, and in $\mathrm{H}^{\mathrm{I}}\left(\mathrm{NO}_{3}\right)^{\mathrm{I}}$, the $\mathrm{NO}_{3}$ is clearly univalent. $\mathrm{H}_{3} \mathrm{I}\left(\mathrm{PO}_{4}\right)^{\mathrm{III}}$ shows $\mathrm{PO}_{4}$ to be trivalent.

Valence also by Displacement.- In the foregoing instances, we have learned the valence of an element or radical by studying its combinations. But, clearly, if an element is displaced from combination, atoms of equal total valence must take its place. Thus the action:

$$
\mathrm{Zn}+2 \mathrm{HCl} \rightarrow \mathrm{ZnCl}_{2}+\mathrm{H}_{2}
$$

shows $\mathrm{Zn}$ displacing $2 \mathrm{H}^{\mathrm{I}}$, and the valence of $\mathrm{Zn}$ must therefore be two. We see that this is the case for, on displacing the $2 \mathrm{H}$, it combines with $2 \mathrm{Cl}$.

Summary. - We may now sum up all these facts by saying: The valence of an element is a number representing the capacity of one atomic weight of the element to combine with, or displace, atomic weights of other elements, the unit of such capacity being that of one atomic weight of hydrogen or chlorine. To make a corresponding statement for the valence of a radical, we substitute, in the foregoing sentence, the word radical for element, and the word formula-weight for atomic weight.

Application in Making Formula and Equations.-We can see at once that the rule of valence will be of great assistance to us in making formulæ and equations. Suppose, for example, that we burn a piece of aluminium foil in chlorine, and get the white aluminium chloride. What is its formula? Up to this point, we should simply have looked for it in a book. And if, subsequently, we had required the formulæ of the oxide and sulphate of aluminium, we should have looked these up separately also.

But now, all we have to do is to find out the valence of aluminium. Knowing already the valences of $\mathrm{Cl}^{\mathrm{I}}$ and $\mathrm{O}^{\mathrm{II}}$ and $\left(\mathrm{SO}_{4}\right)^{\mathrm{II}}$, we have 
then all the information we require for making the needed formulæ. Suppose we know that the atomic weight of aluminium is trivalent $\mathrm{Al}^{\mathrm{III}}$ (see next section). Making the total valences of each half of the compound alike, we get the formulæ:

$$
\mathrm{Al}^{\mathrm{III}} \mathrm{Cl}_{3} \mathrm{I}, \quad \mathrm{Al}_{2}{ }^{\mathrm{III}} \mathrm{O}_{3} \mathrm{II}, \quad \mathrm{Al}_{2} \mathrm{II}\left(\mathrm{SO}_{4}\right)_{3}{ }^{\mathrm{II}} \text {. }
$$

When we know the valences of the elements and radicals, we can make the formula of any required compound.

The reader must therefore make a special effort always to learn the valences of each element and radical, and always to use them in making formula.

The reader must also always check every formula he writes from memory, to make sure that it is correct. Thus, if he thinks the formula of zinc nitrate is $\mathrm{ZnNO}_{3}$, he must count the valences, $\mathrm{Zn}{ }^{\mathrm{II}}\left(\mathrm{NO}_{3}\right)^{\mathrm{I}}$. Evidently, the correct formula is $\mathrm{Zn}\left(\mathrm{NO}_{3}\right)_{2}$.

How to Learn the Valence of an Element.- To find out the valence of an element, we must obtain the formula of one simple compound of the element, containing another element of known valence. Thus, what is the valence of carbon? Its oxide is $\mathrm{CO}_{2}$. The total valence of oxygen here is $2 \times 2=4$. Carbon $\mathrm{C}^{\mathrm{Iv}}$ is therefore quadrivalent. Hence its chloride must be $\mathrm{C}_{\mathrm{rv}} \mathrm{Cl}_{4}{ }^{\mathrm{I}}$ (carbon tetrachloride), and its compound with hydrogen $\mathrm{CrvH}_{4}{ }^{\mathrm{I}}$ (methane, composing a large part of natural gas). When carbon combines with a trivalent element, equi-valent amounts of each element must be used, as in $\mathrm{Al}_{4}{ }^{\mathrm{III}} \mathrm{C}_{3}{ }^{\mathrm{Iv}}$ (aluminium carbide), where $\mathrm{Al}_{4} \mathrm{III}$ and $\mathrm{C}_{3}{ }^{\mathrm{rV}}$ contain $3 \times 4$, or 12 units of valence each.

Again, when we know the formula of sodium iodide to be $\mathrm{Na}^{\mathrm{I} I}$, or that of hydrogen iodide to be $\mathrm{H}^{\mathrm{II}}$, we infer that iodine is univalent. The formula of silica (sand) $\mathrm{SiO}_{2}{ }^{\mathrm{II}}$ shows silicon to be quadrivalent, and indicates that the chloride must be $\mathrm{SiCl}_{4}$. Similarly the formula of calcium carbonate $\mathrm{Ca}^{\mathrm{II}} \mathrm{CO}_{3}$ shows that the radical $\mathrm{CO}_{3}$, which is common to all carbonates, must be bivalent. The chemist does not memorize the valences themselves: he, 
recovers the valence of an element or radical, when needed, by recalling the formula of a substance containing this element or radical in combination with a more familiar element or radical, such as $\mathrm{Cl}^{\mathrm{I}}$ or $\mathrm{H}^{\mathrm{I}}$.

Elements with More than One Valence.-The rule of valence is not so simple as it has thus far appeared to be. A number of the elements have more than one valence. In other words, the capacity of an atomic weight of such an element may have two (or even more) values, according to the circumstances under which it is combining with other elements.

Thus, antimony is usually trivalent, and gives compounds like $\mathrm{SbCl}_{3}, \mathrm{Sb}_{2} \mathrm{O}_{3}, \mathrm{SbBr}_{3}$. But it can also form compounds in which it is quinquivalent, like $\mathrm{SbCl}_{5}$. Similarly, iron forms two complete series of compounds:

$\begin{array}{lllll}\text { Bivalent: } & \mathrm{FeCl}_{2}, & \mathrm{FeO}, & \mathrm{Fe}(\mathrm{OH})_{2}, & \mathrm{FeSO}_{4} . \\ \text { Trivalent: } & \mathrm{FeCl}_{3}, & \mathrm{Fe}_{2} \mathrm{O}_{3}, & \mathrm{Fe}(\mathrm{OH})_{3}, & \mathrm{Fe}_{2}\left(\mathrm{SO}_{4}\right)_{3} .\end{array}$

Even the halogens, although uniformly univalent in their compounds with hydrogen and other positive radicals, show oxygen compounds of higher valence, such as chlorine dioxide $\mathrm{ClO}_{2}$, iodine pentoxide $\mathrm{I}_{2} \mathrm{O}_{5}$. When an element does give more than one series of compounds, however, we always make a strong point of this fact, so that it may not be overlooked.

No simple rule, for telling, in advance, which valence will be used in a given action, can be stated. But the ions $\mathrm{Fe}^{++}$and $\mathrm{Fe}^{+++}$, for example, have different properties, and are easily recognized in practice.

As a rule, an element passes from one form of combination to another without change of valence. But compounds of elements like tin or manganese can undergo changes in the course of which the valence alters. A case of this kind has already been encountered in the preparation of chlorine (p. 143).

$$
\mathrm{MnO}_{2}+4 \mathrm{HCl} \rightarrow 2 \mathrm{H}_{2} \mathrm{O}+\mathrm{MnCl}_{2}+\mathrm{Cl}_{2} \text {. }
$$


balaneed valeney, the comporindobreaks up 210

\section{SMITH'S INTERMEDIATE CHEMISTRY}

The valence of the atomic weight of manganese changes in the course of this action from 4 to 2. When $\mathrm{MnO}$ acts on $\mathrm{HCl}$, however, manganese is bivalent throughout.

Exceptional Compounds. - A few compounds will be met with in which an element shows an exceptional valence. Thus, nitrogen gives two series of compounds of $\mathrm{NIII}$ and Nv. But there are three oxides, $\mathrm{N}_{2} \mathrm{O}, \mathrm{NO}$, and $\mathrm{NO}_{2}$, in which the valence of nitrogen seems to be one, two, and four, respectively. However, these are single compounds, not belonging to any series, and are the only compounds of nitrogen showing any of those three valences.

Again, $\mathrm{FeO}$ and $\mathrm{Fe}_{2} \mathrm{O}_{3}$ belong to the two regular series of compounds of iron. But there is the magnetic oxide, $\mathrm{Fe}_{3} \mathrm{O}_{4}$, where the valence of iron appears not to be a whole number, but $8 / 3$ or $2 \frac{2}{3}$. In this case the chemist makes the valence regular by supposing the magnetic oxide to be a compound of the other two oxides, and writing its formula, $\mathrm{FeO}, \mathrm{Fe}_{2} \mathrm{O}_{3}$.

A List of Valences and Charges. - The following table contains the valences of some familiar ions and the commonest

\begin{tabular}{|c|c|c|c|c|}
\hline Univalent & & Bivalent & Trivalent & Quadrivalent \\
\hline \multirow{5}{*}{$\begin{array}{l}\mathrm{Na}^{+} \\
\mathrm{K}^{+} \\
\mathrm{H}+ \\
\left(\mathrm{NH}_{4}\right)^{+} \\
\mathrm{Ag}^{+} \\
\mathrm{Cl}- \\
\mathrm{Br}^{-} \\
\mathrm{I}^{-} \\
\mathrm{F}- \\
\left(\mathrm{OH}_{-}^{-}\right. \\
\left(\mathrm{NO}_{3}\right)^{-} \\
\left(\mathrm{ClO}_{3}\right)^{-}\end{array}$} & \multirow[t]{5}{*}{$\varphi$} & \multirow{5}{*}{$\begin{array}{l}\mathrm{Ca}_{+}+ \\
\mathrm{Ba}^{++} \\
\mathrm{Mg}++ \\
\mathrm{Zn}++ \\
\mathrm{Pb}++ \\
\mathrm{Ni}++ \\
\mathrm{Co}++ \\
\mathrm{Mn}++ \\
\mathrm{Cu}++ \text { (cupric) } \\
\mathrm{Fe}^{++} \text {(ferrous) } \\
\mathrm{Hg}++ \text { (mercuric) } \\
\mathrm{Sn}^{++} \text {(stannous) } \\
\mathrm{O}^{+} \\
\left(\mathrm{SO}_{4}\right)= \\
\mathrm{S}= \\
\left(\mathrm{CO}_{3}\right)= \\
\mathrm{O}_{2}=\text { (peroxide) }\end{array}$} & \multirow{5}{*}{$\begin{array}{l}\mathrm{Al}+++ \\
\mathrm{Fe}+++ \text { (ferric) } \\
\mathrm{Cr}+++ \\
\mathrm{Sb}+++ \\
\mathrm{Bi}+++ \\
\left(\mathrm{PO}_{4}\right) \equiv \\
\mathrm{As}\left(\mathrm{AsH}_{3}\right) \\
\mathrm{B}\left(\mathrm{B}_{2} \mathrm{O}_{3}\right) \\
\mathrm{N}\left(\mathrm{NH}_{3}, \mathrm{~N}_{2} \mathrm{O}_{3}\right) \\
\mathrm{P}\left(\mathrm{PH}_{8}\right)\end{array}$} & $\begin{array}{l}\text { Snt++ (stannic }) \\
\left(\mathrm{SiO}_{4}\right)== \\
\mathrm{C} .\left(\mathrm{CH}_{4}, \mathrm{CO}_{2}\right)\end{array}$ \\
\hline & & & & Quinquivalent \\
\hline & & & & $\begin{array}{l}\mathrm{N}\left(\mathrm{N}_{2} \mathrm{O}_{5}\right) \\
\mathrm{P}\left(\mathrm{P}_{2} \mathrm{O}_{5}\right) \\
\text { As }\left(\mathrm{As}_{2} \mathrm{O}_{5}\right)\end{array}$ \\
\hline & & & & Sexivalent \\
\hline & & & & $\mathrm{S}\left(\mathrm{SO}_{3}\right)$ \\
\hline
\end{tabular}


valences of some elements. Many of these elements, however, possess other regular valences, in addition to those shown, so that the list does not pretend to be complete.

Where no charges are indicated, the element, by itself, does not ordinarily form an ion.

(Valence and Electrons.) We stated in our discussion on the source of the charges on ions (p. 195) that the outermost shell $\lambda^{\prime}$ of an atom of any element contained a definite small number of negative electrical units (electrons), relatively loosely held. We also assumed that, when atoms of unlike elements combined or reacted, a transfer of electrons from one atom, or group of atoms, to another might take place.

On the basis of electrons, our idea of valence becomes somewhat more definite. The valence of an element is the fnumber of electrons that an atom of that element loses, or takes up, in entering into combination with atoms of other elements.) An atom of hydrogen has only one electron to lose, the hydrogen ion $\mathrm{H}^{+}$consists of nothing but the residual proton. Consequently hydrogen is univalent. $\mathrm{Zn}$, however, can lose two electrons to form $\mathrm{Zn}^{++}$, and $\mathrm{Al}$ can lose three to give $\mathrm{Al}^{+++}$. $\mathrm{Cl}$ can gain one electron to form $\mathrm{Cl}^{-}, \mathrm{S}$ can gain two to give $\mathrm{S}=$.

The relation of valence to atomic structure in the case of these and other elements will be taken up in detail in the final chapter.

A Suggestion.-Having just discussed the conception of valence, we have now considered all the laws of chemical composition. At this point the reader should pause and review thoroughly the subjects of the first eighteen chapters. The understanding of the fundamental principles which this retrospect will give will greatly lighten the task of understanding the new and more complex substances we shall have to consider, and the new kinds of reactions and new conceptions we shall encounter, in the chapters immediately following. 
Exercises. - 1. Mark the valences in the formulæ: $\mathrm{InCl}_{3}$, $\mathrm{V}_{2} \mathrm{O}_{5}, \mathrm{OsO}_{4}, \mathrm{PtCl}_{4}$.

2. Mark the valences of the radicals in the formulæ: $\mathrm{Zn}\left(\mathrm{SeO}_{4}\right)$, $\mathrm{Al}_{2}\left(\mathrm{TeO}_{4}\right)_{3}, \mathrm{H}_{3}\left(\mathrm{AsO}_{4}\right), \mathrm{H}\left(\mathrm{SbO}_{3}\right)$.

3. If $26 \mathrm{~g}$. of chromium displace $1 \mathrm{~g}$. of hydrogen from hydrochloric acid, what is the valence of chromium in this displacement (see Table of Atomic Weights)?

4. Correct the following formulæ: $\mathrm{CaNO}_{3}, \mathrm{CaPO}_{4}, \mathrm{Al}\left(\mathrm{PO}_{4}\right)_{2}$, $\mathrm{LiO}, \mathrm{PbF}, \mathrm{Bi}\left(\mathrm{NO}_{3}\right)_{2}$.

5. One gram of a quadrivalent element unites with $0.27 \mathrm{~g}$. of oxygen. What is the atomic weight of the element? 


\section{CHAPTER XIX \\ OXIDIZING SUBSTANCES}

IN the preceding chapters we have encountered several rather confusing oxidizing reactions. For example, in the preparation of chlorine by the action of $\mathrm{KMnO}_{4}$ on $\mathrm{HCl}$ (p. 142), it was stated that the hydrogen chloride was oxidized to chlorine and the potassium permanganate reduced to manganous chloride. The student may have found it difficult to understand how we can regard a substance as oxidized when no oxygen is added to it. In order to explain oxidations more clearly, particularly in connection with the conception of valence, we must now learn more about oxidation in general. We can do this best through the study of three oxidizing substances which are of a simpler nature and are all in common use. These are ozone, hydrogen peroxide, and hypochlorous acid.

\section{OzONe $\mathrm{O}_{3}$}

When oxygen is blown from a small nozzle through the tip of a Bunsen flame, a part of it is turned into ozone. The same thing happens when a platinum wire, heated by an electric current, is held under liquefied oxygen. This shows us that, to get ozone, we must add energy (for example, by strong heating) to oxygen. We learn, also, that the ozone must be cooled at once and kept cold. If it lingers in the cooler (but not cold) region round the flame, it decomposes again.

Ozone is also obtained by the action of fluorine on water ( $p$. 207).

Preparation of Ozone.- In practice electrical energy, developed by passing a "silent discharge" through the oxygen, is 219 
employed. This discharge gives ozone very easily, because its use involves no rise in temperature whatever.

$$
3 \mathrm{O}_{2} \rightarrow 2 \mathrm{O}_{3} \text {. }
$$

The poles of the induction coil are attached to the tinfoil upon the outside of the outer tube and the inside of the inner tube (Fig. 63). The " discharge" therefore passes through two layers

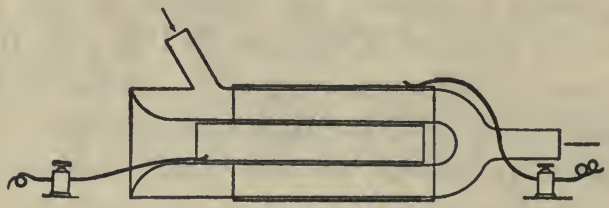

Fig. 63

of glass, as well as through the oxygen. The oxygen, from a cylinder of the gas, flows slowly through the space between the tubes. At best, only 6 to 7 per cent of the oxygen is usually changed into ozone.

Physical Properties.-Ozone (Greek, to smell) is a gas of deep blue color, with a fresh, highly individual odor. It is more easily liquefied (b.-p. $-119^{\circ}$ ) than is oxygen, and is also much more soluble in water. Its density is one-half greater than that of oxygen, and the formula $\mathrm{O}_{3}$ records this fact. When a simple substance shows more than one form in the same state, like oxygen and ozone, we call them allotropic modifications. Ice (p. 64) exists in at least five allotropic modifications.

Chemical Properties.-Ozone is at rather low temperatures (e.g., from $10^{\circ}$ to $500^{\circ}$ ) the less stable form of the element. Upon standing, and more quickly when warmed, it changes into oxygen, with liberation of the additional energy it contains.

Being possessed of more internal energy than oxygen, ozone oxidizes the same substances as does oxygen, only more rapidly and vigorously. For the same reason it oxidizes many substances 
not affected by ordinary oxygen. Thus, it rusts silver to black silver peroxide $\mathrm{Ag}_{2} \mathrm{O}_{2}$.

$$
2 \mathrm{Ag}+2 \mathrm{O}_{3} \rightarrow \mathrm{Ag}_{2} \mathrm{O}_{2}+2 \mathrm{O}_{2} \text {. }
$$

Ozone also oxidizes a number of organic compounds which are unchanged in atmospheric oxygen. For example, when ozonized oxygen is bubbled through a dilute indigo solution, a yellow substance, of much paler tint, isatin, is formed, and the indigo is said to have been bleached. Indigo is taken, for illustration, because it is a most widely used dye, employed in dying navy-blue and blue-black goods, and is totally unaffected by light, and by oxygen, soap, and other ordinary substances:

$$
\underset{\text { indigo }}{\mathrm{C}_{16} \mathrm{H}_{10} \mathrm{~N}_{2} \mathrm{O}_{2}}+2 \mathrm{O}_{3} \rightarrow \underset{\text { isatin }}{2 \mathrm{O}_{2}}+\underset{5}{2 \mathrm{C}_{8} \mathrm{H}_{5}} \mathrm{NO}_{2} \text {. }
$$

Litmus, and the traces of coloring matter in wax, starch, flour, and ivory are all oxidized by ozone to colorless, or nearly colorless, substances. For this reason it is used commercially in bleaching the last-named materials.

Ozone is sometimes recommended for use, in connection with ventilation, as a means of destroying minute organisms in the air. Recent investigations have shown, however, conclusively, that when thus diluted with air, it has little value as a germicide. It is employed by some cities for sterilizing the water supply.

\section{Hydrogen Peroxide $\mathrm{H}_{2} \mathrm{O}_{2}$}

Preparation.- Sodium peroxide $\mathrm{Na}_{2} \mathrm{O}_{2}$, produced by burning sodium in dry air, can be dissolved, a little at a time, in ice-cold water. When this solution is acidified with hydrochloric or sulphuric acid, a double decomposition takes place:

$$
\mathrm{Na}_{2} \mathrm{O}_{2}+2 \mathrm{HCl} \rightarrow 2 \mathrm{NaCl}+\mathrm{H}_{2} \mathrm{O}_{2}
$$

and a dilute solution of hydrogen peroxide (mixed with common salt) is obtained. The nature of the action shows the product to be an acid, with the negative radical $\mathrm{O}_{2}{ }^{\mathrm{II}}$. 
For manufacturing purposes it is more convenient to use barium peroxide $\mathrm{BaO}_{2}$, suspended in water, and sulphuric acid:

$$
\mathrm{BaO}_{2}+\mathrm{H}_{2} \mathrm{SO}_{4} \rightarrow \mathrm{BaSO}_{4} \downarrow+\mathrm{H}_{2} \mathrm{O}_{2}
$$

because the precipitation of insoluble barium sulphate carries the reaction to completion. The precipitate is filtered off and a clear solution of hydrogen peroxide obtained.

In pharmacy a 3 per cent solution is the one commonly sold. As hydrogen peroxide decomposes rapidly at $100^{\circ}$, the pure substance can be obtained only by distilling off the water under reduced pressure.

Properties. - Hydrogen peroxide is a colorless, syrupy

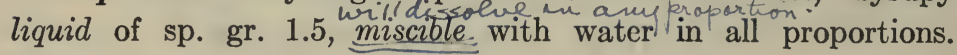
Dilute solutions have a metallic taste.

Chemical Properties.-1. In water solution, hydrogen peroxide is a weak acid. It enters into double decomposition, particularly with bases, giving salts containing the bivalent radical $\mathrm{O}_{2}$ :

$$
\mathrm{Ca}(\mathrm{OH})_{2}+\mathrm{H}_{2} \mathrm{O}_{2} \rightarrow \mathrm{CaO}_{2} \downarrow+2 \mathrm{H}_{2} \mathrm{O} \text {. }
$$

2. When the solution is heated, the compound decomposes, with evolution of heat, giving water and oxygen:

$$
2 \mathrm{H}_{2} \mathrm{O}_{2} \rightarrow 2 \mathrm{H}_{2} \mathrm{O}+\mathrm{O}_{2} \uparrow \text {, }
$$

Contact agents hasten the decomposition. Thus, it takes place with frothing when the cold solution is applied, as an antiseptic, to cuts or sores, or when powders, such as manganese dioxide, are thrown into the solution.

3. Since hydrogen peroxide, like ozone, gives off oxygen with liberation of energy, it is an oxidizing agent also. In this respect its behavior is very similar to that of ozone. It oxidizes colored organic compounds to colorless ones, and is, therefore, used in bleaching hair, feathers, silk, and ivory. It is also fatal to microorganisms, and is, therefore, employed in medicine to disinfect wounds and as a throat wash.

In oil paintings the high lights are produced in part with white lead (carbonate of lead). These disappear and the picture darkens 
with age, because hydrogen sulphide in the air turns the white lead into the black lead sulphide, $\mathrm{PbS}$. In picture restoring the latter is oxidized to lead sulphate, which is white, by treatment with hydrogen peroxide solution.

$$
\mathrm{PbS}+4 \mathrm{H}_{2} \mathrm{O}_{2} \rightarrow \mathrm{PbSO}_{4}+4 \mathrm{H}_{2} \mathrm{O} .
$$

4. The following reaction is used as a test for hydrogen peroxide. When a solution of potassium dichromate $\mathrm{K}_{2} \mathrm{Cr}_{2} \mathrm{O}_{7}$ is acidified with sulphuric acid and a drop of the mixture is added to aqueous hydrogen peroxide, an unstable substance possessing a deep, brilliant blue color is formed. By this test the presence of hydrogen peroxide in rain water can often be demonstrated.

\section{Hypochlorous ACID $\mathrm{HOCl}$}

Pure Hypochlorous Acid.-A pure solution of the acid may be made by dissolving chlorine monoxide $\mathrm{Cl}_{2} \mathrm{O}$ in water. Chlorine monoxide is a brownish-yellow, explosive gas, made by passing chlorine gas over warmed mercuric oxide:

$$
\begin{aligned}
& 2 \mathrm{Cl}_{2}+\mathrm{HgO} \rightarrow \mathrm{HgCl}_{2}+\mathrm{Cl}_{2} \mathrm{O} . \\
& \mathrm{Cl}_{2} \mathrm{O}+\mathrm{H}_{2} \mathrm{O} \rightarrow 2 \mathrm{HOCl} .
\end{aligned}
$$

As an acid, hypochlorous acid is very weak, being very little decomposed into its ions, $\mathrm{H}^{+}$and $(\mathrm{OCl})^{-}$.

It is unstable, exposure to sunlight being sufficient to cause it to give up oxygen, which rises in bubbles through the solution (Fig. 64):

$$
2 \mathrm{HOCl} \rightarrow 2 \mathrm{HCl}+\mathrm{O}_{2} \uparrow .
$$

Heat is given out in the action, and the stable hydrochloric acid remains.

It is a most active oxidizing agent, because of this tendency to give up oxygen with liberation of energy. Thus, its solution oxidizes organic colored substances, producing colorless or less strongly colored ones:

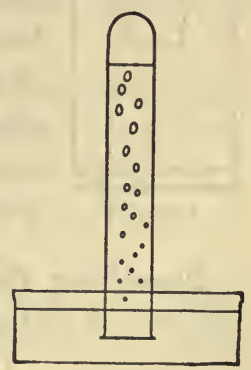

Fig. 64

$$
\underset{\text { indigo }}{\mathrm{C}_{16} \mathrm{H}_{10} \mathrm{~N}_{2} \mathrm{O}_{2}}+2 \mathrm{HOCl} \rightarrow \underset{\text { isatin }}{2 \mathrm{C}_{8} \mathrm{H}_{5} \mathrm{NO}_{2}}+2 \mathrm{HCl}
$$


Used as a disinfectant (see p. 226), it oxidizes and destroys bacteria. Hypochlorous acid is more energetic as an oxidizing agent than is ozone or hydrogen peroxide, and is used extensively in bleaching.

Chlorine-Water. - It will be recalled that chlorine acts chemically upon water (p. 145):

$$
\mathrm{Cl}_{2}+\mathrm{H}_{2} \mathrm{O} \rightleftarrows \mathrm{HCl}+\mathrm{HOCl}
$$

giving hydrochloric acid and hypochlorous acid. The action is reversible (read the equation backwards), and in half-saturated chlorine solution about one-third only of the chlorine has undergone the change shown in the equation. But, if a substance which can be oxidized, such as a dye (attached, perhaps, to cloth), is introduced into the solution, the $\mathrm{HOCl}$ which is present transfers its oxygen to the dye-stuff. This leaves $\mathrm{HCl}$ alone in the solution, and stops the backward reaction. Hence more of the chlorine acts upon the water, and more hypochlorous acid is formed. This,

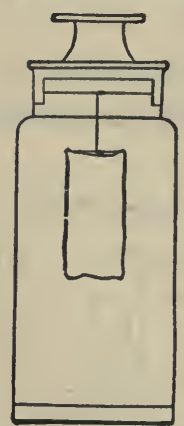

FIg. 65 in turn, is used up. Thus, in a few moments, all the free chlorine is gone, only dilute hydrochloric acid remains, and a colorless organic compound is left on the cloth or in the solution.

Chlorine itself is often, erroneously, spoken of as the bleaching agent. If a $d r y$, colored cloth be hung for a week in chlorine, dried by having sulphuric acid in the bottle (Fig. 65), little or no change in color will occur. But a wet rag is bleached as soon as the chlorine has time to dissolve in the water, and give the necessary hypochlorous acid.

Bleaching Powder.- $\mathrm{CaCl}(\mathrm{OCl})$ is made by the action of chlorine on slaked lime:

$$
\mathrm{Ca}(\mathrm{OH})_{2}+\mathrm{Cl}_{2} \rightarrow \mathrm{CaCl}(\mathrm{OCl})+\mathrm{H}_{2} \mathrm{O} \text {. }
$$

The action is not complete in practice, and the resultant product. iq always a basic salt. In solution it gives the ions $\mathrm{Ca}^{++}, \mathrm{Cl}^{-}$, and 
$(\mathrm{OCl})^{-}$. In other words, a solution of bleaching powder in water acts like mixed solutions of calcium chloride and calcium hypochlorite. Only the calcium hypochlorite is concerned in the bleaching process. When the solution is exposed to the air, it gradually absorbs carbon dioxide (see p. 336). This dissolves in the solution to form carbonic acid $\mathrm{H}_{2} \mathrm{CO}_{3}$, which by double decomposition liberates hypochlorous acid:

$$
2 \mathrm{CaCl}(\mathrm{OCl})+\mathrm{H}_{2} \mathrm{CO}_{3} \rightleftarrows \mathrm{CaCl}_{2}+\mathrm{CaCO}_{3}+2 \mathrm{HOCl} \text {. }
$$

If now materials which it is desired to bleach by oxidation are introduced into the solution, the $\mathrm{HOCl}$ is used up, stopping the backward reaction and carrying the decomposition finally to completion.

Bleaching.- - Cotton and linen, in their original states, are not pure white. Bleaching is therefore an extensive and most important industry. The yarn or cloth must first be freed from cottonwax and tannin, since the former would hinder the action of the bleaching agent, and both would also make the subsequent dyeing uneven. The material is therefore first boiled with dilute caustic soda-solution, and washed with water. The goods are then first " chemicked " in cold bleaching powder solution; next " soured" by immersion in very dilute sulphuric or hydrochloric acid; and finally washed with extreme thoroughness.

The final washing, to remove all traces of chlorine and bleaching powder, is absolutely necessary. If not removed, the hypochlorous acid acts gradually upon the cotton or linen, and "rots" it. Bleaching agents, when used in the household, carelessly, are liable to cause extensive damage from this cause. A dilute solution of sodium thiosulphate (photographers' "hypo ") is often used, as "antichlor," to interact with and remove the last traces of chlorine.

Cotton and linen $\left(\mathrm{C}_{6} \mathrm{H}_{10} \mathrm{O}_{5}\right)_{\nu}$ are rather indifferent chemical substances (p. 398), and stand brief contact with dilute chlorine- 
water without much alteration. But wool and silk contain compounds of nitrogen (proteins) and are acted upon as rapidly as are the traces of colored matter themselves. Hence sulphurous acid (see p. 259) is used for bleaching these materials.

Bleaching Powder as a Disinfectant.-A disinfectant is a substance which destroys bacteria and other minute, and often harmful organisms. Bleaching powder $\mathrm{CaCl}(\mathrm{OCl})$ has a distinct odor. This is due to the slow action of the carbon dioxide and moisture of the air upon the salt, liberating hypochlorous acid. Bleaching powder, when scattered around, will therefore disinfect the surrounding air, because the hypochlorous acid thus liberated kills all bacteria present by oxidation.

When an epidemic of typhoid fever occurs, it is usually traced to the presence of colon bacilli and typhoid organisms in the drinking water. The most effective means of destroying these bacilli is to add, at the distributing point, a small proportion of bleaching powder (about 20 pounds per million gallons of water).

Recently, chlorine-water has in many cases taken the place of bleaching powder for this purpose. Cylinders of liquid chlorine (p. 144) were used in the Great War to kill germs as well as to kill Germans, all water supplies being sterilized, whenever possible, by the addition of very minute amounts of chlorine.

Oxidations Previously Mentioned.-The simplest oxidations are reactions in which free oxygen is actually used up, for example in the union of oxygen with metals and with non-metals:

$$
\begin{aligned}
2 \mathrm{Cu}+\mathrm{O}_{2} & \rightarrow 2 \mathrm{CuO} . \\
\mathrm{S}+\mathrm{O}_{2} & \rightarrow \mathrm{SO}_{2} .
\end{aligned}
$$

The displacement of another element from a compound by oxygen is also oxidation:

$$
4 \mathrm{HCl}+\mathrm{O}_{2} \leftrightarrows 2 \mathrm{H}_{2} \mathrm{O}+2 \mathrm{Cl}_{2} .
$$


The transfer of combined oxygen from one substance to another in a reaction is again oxidation:

$$
\begin{aligned}
2 \mathrm{KMnO}_{4}+16 \mathrm{HCl} & \rightarrow 8 \mathrm{H}_{2} \mathrm{O}+2 \mathrm{KCl}+2 \mathrm{MnCl}_{2}+5 \mathrm{Cl}_{2} . \\
\mathrm{MnO}_{2} & +4 \mathrm{HCl} \rightarrow \mathrm{MnCl}_{2}+\mathrm{Cl}_{2}+2 \mathrm{H}_{2} \mathrm{O} .
\end{aligned}
$$

What is the substance oxidized in the last three reactions? It is hydrochloric acid, and the product of its oxidation is chlorine. True, we have added no oxygen to chlorine itself in any of these reactions, but we have done something which is exactly equivalent to addition of oxygen, we have taken away hydrogen, and we have not replaced that hydrogen by any other positive element.

Note that every oxidation is accompanied by reduction of the oxidizing agent. Thus in the first of the three reactions just discussed, the free oxygen is reduced to water. In the other reactions, $\mathrm{KMnO}_{4}$ and $\mathrm{MnO}_{2}$ are reduced to $\mathrm{MnCl}_{2}$. In all three cases $\mathrm{HCl}$ is the reducing agent.

The appearance of a product that could only be formed by reduction is sometimes the first thing that calls our attention to the fact that an oxidizing action has occurred. When concentrated sulphuric acid acts upon hydrogen iodide (p. 204), the iodine vapor given off on warming shows that there was oxidation, but the odor of the hydrogen sulphide is the first thing we notice when doing the experiment:

$$
\mathrm{H}_{2} \mathrm{SO}_{4}+8 \mathrm{HI} \rightarrow \mathrm{H}_{2} \mathrm{~S}+4 \mathrm{H}_{2} \mathrm{O}+4 \mathrm{I}_{2} \text {. }
$$

Removal of the elements of water from a compound is neither oxidation nor reduction, for hydrogen and oxygen are both removed:

$$
\begin{aligned}
\mathrm{H}_{2} \mathrm{CO}_{3} & \rightarrow \mathrm{CO}_{2}+\mathrm{H}_{2} \mathrm{O}: \\
\mathrm{NH}_{4} \mathrm{OH} & \rightarrow \mathrm{NH}_{3}+\mathrm{H}_{2} \mathrm{O} .
\end{aligned}
$$

We can now see that oxidation, in the above cases, consists always in adding oxygen or removing hydrogen.

Other Cases of Oxidation.-But oxygen is only one of a class of elements which we call non-metallic or negative elements, so that we do not restrict the term "oxidation" to actions involving 
oxygen. Thus, forming a sulphide, by heating a metal with sulphur, is oxidation also:

$$
\mathrm{Fe}+\mathrm{S} \rightarrow \mathrm{FeS} \text {. }
$$

Similarly, changing ferrous chloride $\mathrm{FeCl}_{2}$ to ferric chloride $\mathrm{FeCl}_{3}$ is oxidation:

$$
2 \mathrm{FeCl}_{2}+\mathrm{Cl}_{2} \rightarrow 2 \mathrm{FeCl}_{3} \text {. }
$$

In every compound one of the elements is relatively positive and the other relatively negative. Iron is positive, sulphur and chlorine are negative.

2 Oxidation, then, is introducing, or increasing the proportion of the negative element, or removing, or reducing the proportion of the positive element. Reduction is the converse.

Oxidation and Valence.-Combining a negative element with a metal raises the active valence of the latter from zero to some finite value. Metallic copper has no valence in use. In $\mathrm{CuCl}_{2}$ the copper is employing the valence II. The copper has been oxidized. Similarly, changing $\mathrm{FeCl}_{2}$ to $\mathrm{FeCl}_{3}$ increases the active valence of the iron from II to III. Conversely, changing $\mathrm{HCl}$ to $\mathrm{Cl}_{2}$ alters the active valence of the chlorine from I to zero. Hence, oxidation may be defined as increasing the active valence 3 of a positive element or decreasing that of a negative element. Reduction is the converse.

Oxidation and Electrons.-Finally, since increasing the valence of a negative atom means adding one or more electrons to that atom, and increasing the positive valence of an atom means removing one or more electrons, we reach the briefest definition by 4 saying: Oxidation is removing electrons and reduction is adding electrons.

Exercises.- 1. Why does air containing ozone lose the latter (by change into oxygen) quicker when warm than when cold?

2. Mark the valences of the radicals in barium peroxide and hypochlorous acid. 
3. What volume (at $0^{\circ}$ and $760 \mathrm{~mm}$.) of oxygen would be obtained by the decomposition of the hydrogen peroxide in 1 kilogram of the 3 per cent solution?

4. Why does a given weight of chlorine in the form of hypochlorous acid have twice as great a bleaching (or oxidizing) capacity as has the same weight of chlorine in chlorine water?

5. Write down all the oxidizing actions mentioned in chapter XVII, noting in each case the oxidizing agent and the reducing agent. 


\section{CHAPTER XX \\ CHEMICAL EQUILIBRIUM}

IN spite of its formidable title, this chapter will introduce nothing novel. Its purpose is to collect together and organize more definitely a number of scattered facts and ideas which have already come up in various connections. On this account, however, it will be all the more necessary for the reader to refresh his remembrance of these facts and ideas by re-reading all pages to which reference is made.

Reversible Actions. - In discussing Deacon's process (p. 140), it was stated that the action $4 \mathrm{HCl}+\mathrm{O}_{2} \rightarrow 2 \mathrm{H}_{2} \mathrm{O}+2 \mathrm{Cl}_{2}$ comes to rest although a large amount of both of the interacting substances (20 per cent at $345^{\circ}$ ) still remains available. Now the materials thus left unused are presumably no less capable of interacting than were the parts which have already reacted. The solution of this mystery lies in the fact that the products themselves interact to reproduce the initial substances (read the equation backwards). Thus two changes, one of which undoes the work of the other, are going on simultaneously. In consequence of this, neither action can reach completion. As we should expect, experiment shows that it makes no difference whether we start with pure chlorine and steam, or with hydrogen chloride and oxygen; the proportions of the four substances fornd in the tube, after it has been kept at $345^{\circ}$ for a sufficient time, are in both cases the same. A general statement may be founded on facts like this, to the effect that a chemical action must remain more or less incomplete when the reverse action also takes place under the same conditions. Two arrows pointing in opposite directions are used in equations representing reversible changes. 
The foregoing example of a reversible action, and the following examples which very closely resemble it, should now be looked up and studied attentively. The discussion in this and the following sections, for which they furnish the basis, cannot otherwise be understood: (1) the behavior of water vapor at the boiling-point (pp. 63-4) and at $2000^{\circ}$ (p. 67); (2) the depression of the vapor pressure of a liquid by a non-volatile solute (p. 117); (3) the action of dilute sulphuric acid on common salt (pp. 127-8); (4) the interaction of chlorine and water (pp. 145, 224); (5) the ionization of electrolytes (p. 182).

Explanation in Terms of Molecules.- Restating these reactions in terms of the molecules will enable us to reason more clearly about this variety of chemical change. Suppose we start with the materials represented on one side only of such an equation, say the hydrogen chloride and oxygen in that on p. 230. The molecules of these materials will encounter one another frequently in the course of their movements. In a certain proportion of these collisions the chemical change will take place. In the earliest stages there will be few of the new kind of molecules (say, of chlorine and steam), but, as the action goes on, these will increase in number. There will be two consequences of this. In the first place, the parent materials (in this case, hydrogen chloride and oxygen) will diminish in amount, the collisions between their molecules will become fewer, and the speed of the forward action will therefore become less and less. In the second place, the increase in the number of molecules of the products will result in more frequent collisions between them, in more frequent occurrence of the chemical change which they can undergo, and thus in an increase in the speed of the reverse acticn. The forward action begins at its maximum and decreases in speed progressively; the reverse action begins at zero and increases in speed. Finally the two speeds must become equal, and at that point perceptible change in the condition of the whole must cease. 
The most immediate inference from this mode of viewing the matter is, that the apparent halt in the progress of the action does not indicate any cessation of either chemical change. Both changes must go on, in consequence of the continued encounters of the proper molecules. But since the two changes proceed with equal speeds they produce no alteration in the mass as a whole. In fact, the final state is one of equilibrium, and not of rest, one of balanced activity and not of repose. Hence, chemical changes which are reversible lead to that condition of seemingly suspended action which we speak of as chemical equilibrium.

Chemical Equilibrium and its Characteristics. - The detailed discussion of the relations of liquid and vapor (pp. 62-64), and of saturated solution and undissolved solid (pp. 120-121), has already familiarized us with the term equilibrium and its significance. We can, in fact, apply to the discussion of any kind of reversible phenomena, the sets of ideas in regard to exchanges of molecules there elaborated.

In particular, the reader will note that the three characteristics of a state of equilibrium, developed and illustrated in the case of the physical equilibrium between a liquid and its vapor (p. 63), apply also to a typical case of chemical equilibrium, such as that in Deacon's process now before us. Thus:

1. There are the two opposing tendencies, which ultimately balance one another. Here they are the tendency of the steam and chlorine to produce hydrogen chloride and oxygen, and the tendency of the hydrogen chloride and oxygen to reproduce steam and chlorine by this interaction.

2. At equilibrium the two opposing tendencies or activities are still in full operation, although their effects then neutralize one another.

3. (and this is the chief mark of chemical, as it is of physical equilibrium). The system is in a sensitive state, so that a change 
in the conditions (temperature and pressure or concentration), even if slight, produces a corresponding change in the state of the system, and does this by favoring or disfavoring one of the two opposing tendencies or activities. Such a change is called a displacement of the equilibrium, for the system settles down in a new state of equilibrium with new proportions of the two sets of substances, corresponding to the changed conditions. Thus, in the present instance, a change from $345^{\circ}$, where there is 80 per cent of the material in the form of steam and chlorine, to $384^{\circ}$ results in the diminution of this proportion to 75 per cent. The equilibrium is affected by changes in concentration also, as we shall presently see.

Now, the foregoing facts show that the key to understanding chemical activities, their magnitudes, their changes, and especially their practical results, must lie in knowing how changes in the conditions affect them. Hence, to the chemist, familiarity with the influence of conditions on chemical phenomena must be of the greatest practical importance.

The " conditions " to be considered are familiar, - temperature, and concentration or, in the case of a gas, partial pressure. The "activity" of an action is accurately measured by the speed with which the action proceeds. Thus, if the foregoing section be re-examined, it will be seen that we spoke throughout of the speed, rather than of the tendency or activity.

Finally, temperature and other conditions influence also the activities in, and therefore the speeds of, those actions which proceed to completion, and are not reversible. Hence, unless our statements are expressly restricted to reversible actions and to states of equilibrium, they apply to all chemical changes.

The Influence of Concentration. - In the first place, let us assume that the temperature is constant, and let us confine our attention for the present to the influence of concentration upon a chemical reaction. We have seen (p. 231) that the speed of a 
chemical change is determined by the frequency with which the molecules of the interacting substances encounter one another. The frequency of the encounters amongst a given set of molecules, resulting in a definite chemical change, will in turn evidently depend entirely upon the degree to which the molecules are concentrated in each other's neighborhood. Larger amounts of one of the materials, for example, will not result in more rapid chemical action, if the larger amount of material is also scattered through a larger space. Chemical changes, therefore, are not accelerated by increasing the mere quantity of any ingredient, but only by increasing the concentration of its molecules. Thus, a large amount of a 0.1 normal solution of hydrochloric acid with a piece of zinc will generate hydrogen no faster than a smaller amount. But substitution of a normal solution of hydrochloric acid, which contains a higher concentration of hydrogen ions, will instantly increase the speed of the action. In the second case, the number of hydrogen ions reaching the zinc per second is greater, and the displacement reaction $\mathrm{Zn}+2 \mathrm{H}^{+} \rightarrow \mathrm{Zn}^{++}+\mathrm{H}_{2} \uparrow$ proceeds more rapidly. So also, iron burns faster in oxygen (100 per cent) than in air (20 per cent oxygen).

With a reversible action the effect on the speed is the same, excepting that the continued activity of the reverse action prevents the direct one from reaching completion.

Thus, if, in the action of hydrogen chloride upon oxygen, we introduce into the same space an extra amount of oxygen, this facilitates the formation of steam and chlorine by increasing the possibilities of encounter between molecules of hydrogen chloride and oxygen. At the same time it does not affect ( $c f$. p. 230) the number of encounters in a given time of steam and chlorine molecules with one another which result in the reverse transformation. The proportion of chlorine (and steam) formed, therefore, from a given amount of hydrogen chloride will be greater, although the total possible (by complete consumption of the materials) has not been altered, since the quantity of one ingredient only has been 
increased. The introduction of an excess of hydrogen chloride would have had precisely the same effect.

An Experimental Illustration.- A reaction in which the effects of different concentrations were carefully studied by Gladstone (1855) affords a good illustration. If ferric chloride and ammonium thiocyanate are mixed in aqueous solution, a liquid containing the soluble, blood-red ferric thiocyanate is produced. The compound radicals are $\left(\mathrm{NH}_{4}\right)$ and (CNS), and the action is a simple double decomposition:

\section{$\mathrm{FeCl}_{3}+3 \mathrm{NH}_{4} \mathrm{CNS} \rightleftarrows \mathrm{Fe}(\mathrm{CNS})_{3}+3 \mathrm{NH}_{4} \mathrm{Cl}$.}

The action is a reversible one, and the mixture is homogeneous, i.e., there is no precipitation. Now, if the two just-named salts are mixed in very dilute solution in the proportions required by the equation, say by adding 20 c.c. of a decinormal solution of each salt to several liters of water, a pale-reddish solution is obtained. When this is divided into four parts, and one is kept for reference, the addition of a little of a concentrated solution of ferric chloride to one jar, and of ammonium thiocyanate to another, will be found to deepen the color by producing more of the ferric thiocyanate. On the other hand, mixing a few drops of concentrated ammonium chloride solution with the fourth portion will be found to remove the color almost entirely, on account of its influence in favoring the backward change.

The Law of Molecular Concentration.- The general principle discussed and illustrated in this section may be called the law of molecular concentration, and may be stated as follows: In every chemical change the activity, and therefore the speed of the action, is proportional to the molecular concentration of each interacting substance. This holds whether the action is reversible or not.

The molecular concentration is expressed, numerically, for each substance, in terms of the number of moles (gram-molecular 
weights, p. 122) of the substance contained in a liter of the whole mixture. There is the same number of molecules in a grammolecular weight of any substance (see p. 84). Hence the number of moles per liter defines the concentration of each substance in terms of this number of molecules in a liter as the unit of concentration.

As an example, the dissociation of phosphorus pentachloride vapor into phosphorus trichloride and chlorine (p. 145):

$$
\mathrm{PCl}_{5} \rightleftharpoons \mathrm{PCl}_{3}+\mathrm{Cl}_{2}
$$

may be considered.

The law states that, in any mixture of the three substances, the speed of the forward action, or decomposition, is proportional to the concentration of $\mathrm{PCl}_{5}$ molecules. It is therefore equal to the concentration of $\mathrm{PCl}_{5}$ molecules, which we may write $\left[\mathrm{PCl}_{5}\right]$, multiplied by a constant, which we shall write $\mathrm{K}_{1}$. Mathematically expressed, if $S_{1}$ is the speed of decomposition:

$$
\mathrm{S}_{1}=\mathrm{K}_{1}\left[\mathrm{PCl}_{5}\right]
$$

In the same way the law states that the speed of the reverse action, or combination, is proportional to the concentration of $\mathrm{PCl}_{3}$ molecules and also proportional to the concentration of $\mathrm{Cl}_{2}$ molecules. It is therefore equal to the concentration of $\mathrm{PCl}_{3}$ molecules $\left[\mathrm{PCl}_{3}\right]$, multiplied by the concentration of $\mathrm{Cl}_{2}$ molecules $\left[\mathrm{Cl}_{2}\right]$, multiplied by another constant, which we shall write $K_{2}$. If $S_{2}$ is the speed of combination, we have now:

$$
\mathrm{S}_{2}=\mathrm{K}_{2}\left[\mathrm{PCl}_{3}\right] \cdot\left[\mathrm{Cl}_{2}\right]
$$

Each reaction, it will be noted, must slow up as the concentration of the reacting substances diminishes. This is true of all reactions. The further they proceed, the less rapid their speed. The burning of a candle in a confined space becomes less brilliant, as the available oxygen is used up. The evolution of hydrogen. in the action of an acid on a metal becomes very slow when the acid is nearly exhausted, owing to the diminution in the concentration of hydrogen ion. 
The Condition for Chemical Equilibrium.-As we have seen (p. 231), the characteristic of a system in chemical equilibrium is that the speeds of the forward and reverse reactions have become equal. Applying this to the case of the dissociation of phosphorus pentachloride discussed above, we see that, when we have an equilibrium mixture of the three substances, where $\mathrm{S}_{1}=$ $\mathrm{S}_{2}$, then we also have the relationship:

$$
\mathrm{K}_{1}\left[\mathrm{PCl}_{5}\right]=\mathrm{K}_{2}\left[\mathrm{PCl}_{3}\right] \cdot\left[\mathrm{Cl}_{2}\right]
$$

This may be written in the form:

$$
\frac{\mathrm{K}_{1}}{\mathrm{~K}_{2}}=\frac{\left[\mathrm{PCl}_{3}\right] \cdot\left[\mathrm{Cl}_{2}\right]}{\left[\mathrm{PCl}_{5}\right]}
$$

The ratio $K_{1} / K_{2}$ is, of course, a constant, since $K_{1}$ and $K_{2}$ are both constants. This ratio, which we may write $K$, is called the equilibrium constant of the reversible reaction.

The equilibrium constant is a very important quantity. Once we have determined it, by investigating one equilibrium mixture, we can calculate exactly what will happen to any mixture of the three substances concerned at the same temperature. However much we may vary the molecular concentrations of the three substances, whether by changing the pressure or by adding an excess amount of one of them, such as chlorine, the composition of the mixture will adjust itself until, when equilibrium is attained, the ratio $\left[\mathrm{PCl}_{3}\right] \cdot\left[\mathrm{Cl}_{2}\right] /\left[\mathrm{PCl}_{5}\right]$ has again reached the value $\mathrm{K}$. In large-scale industrial processes, therefore, a knowledge of the equilibrium constant of the reaction involved is often of inestimable value.

In many important reversible reactions, the concentrations of the reacting substances on one side of the equation are, under equilibrium conditions, very much greater than the concentrations of those on the other side. Although the reaction is reversible, it proceeds much farther towards completion in one direction than the other. This fact may often be indicated, very con- 
veniently, by modifying the thickness of the arrows used to express reversibility. Thus:

$$
\mathrm{HCl} \rightleftharpoons \mathrm{H}^{+}+\mathrm{Cl}^{-} \quad \mathrm{H}_{2} \mathrm{O} \rightleftharpoons \mathrm{H}^{+}+\mathrm{OH}^{-}
$$

In the case of phosphorus pentachloride vapor, 80 per cent of the whole weight of material in the equilibrium mixture at $250^{\circ}$ and $760 \mathrm{~mm}$. pressure is dissociated into $\mathrm{PCl}_{3}$ and $\mathrm{Cl}_{2}$.

Homogeneous and Inhomogeneous Systems.-While there are all degrees of speed in chemical actions, yet in practice we quickly distinguish two different classes. There is a class of actions of which most examples are almost instantaneously accomplished, and a class in which, frequently, the operation takes minutes or even hours. The classes overlap, but, in a general way, the following distinction may be made.

To the former, speedy class belong the explosion of hydrogen and oxygen or other gaseous mixtures, and the interactions when solutions are mixed, as in precipitations. In view of the foregoing explanations, we perceive that the rapid accomplishment of such actions is due, not so much to any especially great intrinsic affinity, as to the homogeneous state of mixture of the interacting materials. This, of course, is a purely physical, and not a chemical motive for speedy interaction. In intimate mixtures, every molecule has an equal opportunity freely to encounter every other molecule and there is therefore no mechanicai impediment to the operation of the affinities of the substances. Hence the apparent activity is great.

To the second class, comprising the slower actions, belong cases like the interaction of a piece of zine with hydrochloric acid, or of manganese dioxide (p. 142) with the same acid, whereby hydrogen and chlorine, respectively, are slowly evolved, and the solid is gradually consumed. Here the hindrance is evidently the fact that the interacting substances are not intimately mixed. In the slow actions, the system is inhomogeneous. Pulverizing the solid before use will increase the speed, indeed, by providing more 
surface and better mutual contact, but will not transfer the action to the rapid class. It is chiefly the dissolved part of the substance which interacts, for chemical action takes place between molecules, and only the dissolved part is disintegrated in such a way that the molecules are readily accessible. Thus, the action is held back by continual waiting for the slow replenishment, from the "insoluble" solid, of the supply of dissolved molecules. In the cases cited, the restraining influence of the dissolving process, which is part of the whole phenomenon, may be formulated thus:

$\mathrm{Zn}$ (solid) $\leftrightarrows \mathrm{Zn}$ (dslvd.) $+2 \mathrm{HCl} \rightarrow \mathrm{ZnCl}_{2}+\mathrm{H}_{2}$.

$\mathrm{MnO}_{2}$ (solid) $\leftrightarrows \mathrm{MnO}_{2}$ (dslvd.) $+4 \mathrm{HCl} \rightarrow \mathrm{MnCl}_{2}+2 \mathrm{H}_{2} \mathrm{O}+\mathrm{Cl}_{2}$.

Here, again, the mechanical details, depending on physical properties, have more to do with the progress of the action than has the chemical affinity. In terms of the law of concentration, the action is slow, and the apparent activity small, because the concentration of the acting molecules of one of the substances is very small, and cannot be increased because of low solubility.

Displacement of Equilibria.-We have seen (pp. 234-5) that one way in which a reversible action may be forced nearer to completion, in one direction or the other, is the introduction of an excess of one of the ingredients contributing to the forward action. This method of displacing the equilibrium point, however, cannot be very effective, unless it is possible to introduce an exceedingly large excess of the selected ingredient in a high degree of molecular concentration, since this operation does not in any way affect or, in particular, restrain the reverse action which is continually undoing the work of the forward one. A much more effective means of furthering the desired direction of such actions is found, therefore, in the restraint or practical annulment of the reverse action. A good way of accomplishing this is to allow the products of the direct action to separate into an inhomogeneous mixture. Any agency which could remove the water vapor as fast as it was formed by the interaction of hydrogen chloride and oxygen, for 
example, would entirely stop the reproduction of these substances, and so would enable the forward action $\left(4 \mathrm{HCl}+\mathrm{O}_{2} \rightarrow 2 \mathrm{H}_{2} \mathrm{O}\right.$ $+2 \mathrm{Cl}_{2}$ ) to run to completion.

This might be realized by causing one end of a sealed tube charged with the substances, after the contents had settled down to a condition of equilibrium, to project from the bath in which the whole had been kept at $345^{\circ}$ (Fig. 66, which is simply diagram-

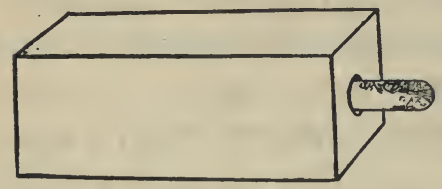

Fig. 66 matic). By cooling this end, a large part of the steam would quickly be condensed in it to the liquid form, while the other substances would remain gaseous. In other words, the concentration of the water vapor would be greatly reduced. In fact, only the trace of vapor which cold water gives would then be available to interact with the chlorine, and reproduce hydrogen chloride. Meanwhile the decomposition of the latter would go on, and thus, eventually, almost all the water would be found in one end of the tube, and the chlorine, all free, would occupy the rest. By this purely mechanical adjustment the chemical change would therefore be carried from 80 per cent completion to almost absolute completion:

$$
4 \mathrm{HCl}+\mathrm{O}_{2} \rightleftarrows 2 \mathrm{Cl}_{2}+2 \mathrm{H}_{2} \mathrm{O} \text { (vapor) } \rightleftarrows 2 \mathrm{H}_{2} \mathrm{O} \text { (liq.) }
$$

If, on the other hand, arrangements were made to have powdered marble, in a sealed bulb of thin glass, enclosed in the tube, we might imagine the very opposite of the above effect to be produced. The breaking of the bulb of marble, when equilibrium had been reached, would provide means for the removal of all the hydrogen chloride, ${ }^{*}$ while the other three substances would still be

* The hydrogen chloride would be destroyed by interaction with the marble:

$$
2 \mathrm{HCl}+\mathrm{CaCO}_{3} \rightarrow \mathrm{CaCl}_{2}+\mathrm{CO}_{2}+\mathrm{H}_{2} \mathrm{O} .
$$

The calcium chloride is a solid. The gas, carbon dioxide, does not interact with the other substances, and would not, therefore, interfere with the formation of fresh hydrogen chloride. 
gaseous. Thus, the compound $(\mathrm{HCl})$ having been reduced in concentration to the point of being removed entirely, there would be no direct action to undo the work of the reverse action. The whole chlorine would, therefore, soon have passed through the form $\mathrm{HCl}$. Hence, by another mechanical arrangement, an action which ordinarily could progress to only 20 per cent would be turned into a complete one:

$$
2 \mathrm{Cl}_{2}+2 \mathrm{H}_{2} \mathrm{O} \rightleftarrows \mathrm{O}_{2}+4 \mathrm{HCl}\left(+\mathrm{CaCO}_{3} \rightarrow \mathrm{CaCl}_{2}+\mathrm{H}_{2} \mathrm{O}+\mathrm{CO}_{2}\right) \text {. }
$$
lutenp.

Reversibility Usually Avoided.-In every-day chemical work, since our object is usually to prepare some one substance, chemists either avoid chemical changes which are notably reversible, or adjust the conditions, as is done in the foregoing illustrations, so that the reverse of the action which they desire is prevented. In consequence of this, when carrying out the directions for making familiar preparations, the fact that such actions are reversible at all very readily escapes our notice. Arranging the conditions so that the separation of a solid body by precipitation, or the liberation of a gas, takes place, are the two commonest ways of rendering a reversible action complete. Excellent examples of both of these are furnished by the chemical change used in producing hydrogen chloride by the interaction of salt and sulphuric acid, the full discussion of which (p. 127) should now be studied attentively in the light of these explanations.

Double decompositions between electrolytes in solution may also be carried to completion by arranging that one of the products of the reaction shall be a non-ionized, or practically non-ionized, substance. Neutralization of an acid by a base is a case in point (see p. 193).

The Influence of Temperature on the Speed of any Reaction.-The activity of chemical change, and therefore the speed of all chemical changes, is increased by raising the temperature and diminished by lowering it (cf. p. 27). Different actions 
are affected in different degrees, and no simple rule accurately defining the effect can be given. Roughly speaking, however, a rise of $10^{\circ}$ doubles the speed of every action. A rise of $100^{\circ}$ will therefore make the speed roughly 1024 times greater. Hence, when the chemist finds that two substances show no evidence of interaction he infers that there must be either slow action or none, and he seeks to settle the question quickly by heating the mixture.

\section{The Influence of Temperature on a System in Equilib-} rium. - In a reversible change the two opposing reactions are different actions and their speeds are therefore affected in different degrees by the same alteration in temperature. Hence, when the temperature is changed, the relative amount of the two sets of material present is altered and the equilibrium is displaced. Thus, in Deacon's process, a rise of $40^{\circ}$ in the temperature displaces the equilibrium backwards (p. 233), and diminishes the yield of chlorine by 5 per cent. In the vapor of phosphorus pentachloride (p. 236), the displacement is in the opposite direction. At $250^{\circ}$, and $760 \mathrm{~mm}$. pressure, 20 per cent of the material is present as pentachloride and 80 per cent as trichloride and chlorine. At $300^{\circ}$ only 3 per cent of the pentachloride remains while at $200^{\circ}$ only a little more than 50 per cent is dissociated. Evidently, here, raising the temperature favors the decomposition of the pentachloride, and therefore increases the speed of its dissociation more than it does the speed of the reunion of the trichloride and chlorine.

Van't Hoff's Law. - Now the facts mentioned above are connected by a law which will answer many practical questions in chemistry.

When phosphorus trichloride and chlorine combine (to form $\mathrm{PCl}_{5}$ ), heat is given out. Conversely, when phosphorus pentachloride dissociates, heat is absorbed:

$$
\mathrm{PCl}_{5}+30,000 \text { cal. } \rightleftarrows \mathrm{PCl}_{3}+\mathrm{Cl}_{2} \text {. }
$$


Now, when the temperature is raised, the action proceeds in the direction of decomposing more of the pentachloride. That is, the equilibrium is displaced in the direction which absorbs heat.

In Deacon's process, we find that the interaction of hydrogen chloride and oxygen liberates heat:

$$
4 \mathrm{HCl}+\mathrm{O}_{2} \rightleftarrows 2 \mathrm{H}_{2} \mathrm{O}+2 \mathrm{Cl}_{2}+28,000 \text { cal. }
$$

and in this action raising the temperature drives the equilibrium backwards, and a lowering in the temperature is required to increase the yield of chlorine.

The rule is obvious, and applies to all reversible reactions: When the temperature of a system in equilibrium is raised, the equilibrium point is displaced in the direction which absorbs heat. In other words, a rise in temperature favors the interaction of that one of the two sets of materials to which the heat is added ( + sign) in the equation. If the equation happens to be written with a negative heat of reaction (e.g., p. 162), the heat can, of course, be transferred to the other side with its sign changed. This law is known as Van't Hoff's law of mobile equilibrium.

This law is of practical value. More than once, in chemical factories, much time and money have been spent on trying to arrange machinery to give a better yield of some substance at a high temperature, when a reference to this law would have shown that the chief change necessary was to use a lower temperature. We shall frequently have occasion to refer to this law.

Application to Physical Equilibria.-Van't Hoff's law applies also to physical equilibria. Thus, the vaporization of a liquid absorbs heat, and so an increase in temperature will increase the pressure, and therefore the concentration of its vapor. The special case of a saturated solution may be somewhat more fully considered.

When we heat a saturated solution, with excess of solid present, more solid will dissolve as the temperature is raised, if solution is attended with absorption of heat. This is the usual case, as is 
shown by the way in which most solubilities increase with rising temperature. The solution of a solid in a liquid, indeed, may be regarded in normal cases as equivalent to the process of fusion, which, like vaporization, always absorbs heat. The precipitation of crystals from a supersaturated solution, similarly, may be considered as analogous to the solidification of a pure substance, the freezing-point being depressed below the normal va'ue, however, by the presence of the solvent (see p. 119). Freezing-point lowering and solubility phenomena are therefore identical. When we determine the depression of the freezing-point of water on addition. of sugar by noting at what temperature ice begins to separate out, we measure, simultaneously, the solubility of ice in the solution at that temperature. In the same way, when we determine the solubility of sugar in water at $25^{\circ}$, we establish also how much water must be added to a given quantity of sugar to depress the freezing-point of sugar to $25^{\circ}$. It is therefore just as correct to say that sugar melts in tea as it is to say that ice melts in iced tea.

Often, however, extensive chemical reactions occur in the process of solution (see p. 123), and the heat effect of these reactions may be considerable. Thus when water is added to concentrated sulphuric acid, so much heat is evolved that the water boils. In the case of a few salts, also, we find that solution in water takes place with evolution of heat, and we draw the conclusion that the heat of the chemical reactions involved more than counterbalances the heat absorbed in the process of fusion.

When we heat a saturated solution, with excess of solid present, crystals will separate out as the temperature is raised, if precipitation is attended by absorption of heat. This is the case with anhydrous sodium sulphate $\mathrm{Na}_{2} \mathrm{SO}_{4}$ and some calcium salts in water (see p. 113).

Le Chatelier's Law.- The above-mentioned law is really a particular case of a more general one, the law of Le Chatelier. 
If some stress (e.g., by change of temperature, pressure, or concentration) is brought to bear on a system in equilibrium, a reaction occurs, displacing the equilibrium in the direction which tends to undo the effect of the stress. Thus, raising the temperature furthers the change which absorbs heat - and therefore would tend to lower the temperature. Increasing the concentration of the molecules pushes the action in the direction which uses up these very molecules (p. 234). Again pressure causes ice to melt, because the water which is formed occupies a smaller volume, and this change tends to relieve the pressure. But pressure will not cause most substances to melt, because usually the liquid form occupies a greater volume and its production would tend to increase pressure.

The student is cautioned against applying these laws to systems not in equilibrium, for example, to unsaturated or supersaturated solutions. To such cases they do not, necessarily, apply. Thus the addition of a small quantity of cupric chloride to water is attended by evolution of heat. It would be quite wrong to reason from this, however, that the solubility must fall off as the temperature is raised. The salt is extremely soluble in water, and if we keep on adding it to the solution until the latter is saturated we find that the last portions dissolve with absorption of heat. Now it is only the saturated solution that is in equilibrium with the solid, hence it is only with respect to this solution that we can apply Van't Hoff's or Le Chatelier's law. In accordance with the behavior of this solution, we find that the solubility increases with rising temperature.

The characteristics of systems in equilibrium (pp. 63, 232) should therefore be kept in mind carefully, in order to avoid mistakes.

Summary. - In this chapter we have answered three questions: 
1. Why do some chemical actions cease, while still incomplete? Answer: They are reversible.

2. What explains the position of the equilibrium point? Answers: (a) Equal effects of opposed molecular actions; (b) Equality in speed of opposed reactions.

3. What will displace the equilibrium point? Answer: (a) Change in concentration of one (or more) of the substances; $(b)$ Change in the temperature.

Exercises.- 1. Explain the completeness of the action by which hydrogen chloride and water, respectively, are formed by direct union of the elements.

2. Explain the completeness of the action by which silver chloride (p. 131) is formed.

3. Explain why the decomposition of potassium chlorate is complete.

4. In view of the statement on p. 16, explain why mercuric oxide is completely decomposed by heating.

5. Why can magnetic oxide of iron be reduced completely by a stream of hydrogen (p. 57), and iron oxidized completely by a current of steam (p. 51)?

6. With the phosphorus pentachloride system, say at $250^{\circ}$, what effect would suddenly enlarging the space containing a given amount of the vapor produce? What would be the effect of diminishing the space? What would be the effect of introducing additional chlorine into the same space (p. 234)?

7. Are the following systems in equilibrium or not: (a) a captive balloon pulling at its rope, $(b)$ a balloon floating freely in cooling air, (c) a man wearing a heavy suit in winter only, (d) a woman changing to a velvet hat in August and to a straw hat in February? What is the stress in each case? In which systems does a reaction take place which opposes the effect of the stress? 
8. What inference should you draw from the fact that: (a) the solubilities of potassium nitrate and of Glauber's salt increase with rise in temperature; (b) those of calcium hydroxide (p. 113) and triethylamine decrease with rise in temperature? 


\section{CHAPTER XXI}

\section{SULPHUR AND HYDROGEN SULPHIDE}

SUlPHUR, the compounds of which have been so often mentioned, provides us, in sulphuric acid, with a substance which has more extensive and more important applications in commerce than any other chemical. The element sulphur, itself, enters, with potassium nitrate and charcoal, into gunpowder. Vulcanite is a compound of caoutchouc (rubber) and sulphur. Sulphur is employed to destroy fungi on grape-plants, and furnishes sulphur dioxide for bleaching and disinfecting.

Sources. - The greater part of the sulphur of commerce comes from Sicily, Louisiana and Texas. In Sicily, free sulphur is mixed with pumice and other rocks. When the lumps of rock, obtained by mining or quarrying, are heated by setting fire to the sulphur. (there is no coal in Italy), the sulphur melts and runs to the bottom of the kiln. This product is far from pure, and is distilled from iron retorts. The vapor is condensed in chambers of brick, and the liquid is run into moulds, giving roll sulphur. The first vapor condensed, while the chambers are cold, yields flowers of sulphur.

In Louisiana the sulphur occurs in a deposit over half a mile in diameter, below 900 feet of clay, quicksand, and rock. It is obtained by means of borings, which permit four pipes, one within the other, to reach the deposit. Water, previously heated under pressure to a temperature of $170^{\circ}$, is pumped down the two outside pipes ( 6 and 8 inches in diameter). After time has been allowed for the melting of a quantity of the sulphur (it melts at $114.5^{\circ}$ ), compressed air is pumped down through an inner, one-inch pipe. The melted sulphur, alone, has twice the specific gravity of the 
water in the outer pipes. But the air breaks up into small bubbles, forming with the liquid sulphur an emulsion which has a lower specific gravity, and this flows freely up a three-inch pipe which surrounds the air pipe. The sulphur runs into wooden enclosures, measuring 150 by 250 feet, in which it quickly solidifies. The product is so pure that, for most purposes, no other treatment is required. The output of Louisiana and Texas -500 tons a day from each well and, in all, over 1,000,000 tons annually - supplies the whole demand of the United States, and could easily be increased.

A number of sulphates, such as gypsum $\left(\mathrm{CaSO}_{4}, 2 \mathrm{H}_{2} \mathrm{O}\right)$ and barite $\left(\mathrm{BaSO}_{4}\right)$, and several sulphides, such as galena $(\mathrm{PbS})$, zinc blende $(\mathrm{ZnS})$, and pyrite $\left(\mathrm{FeS}_{2}\right)$, are found in large quantities as minerals. The last two sulphides are used in the manufacture of sulphuric acid.

Allotropic Forms of Sulphur.-Sulphur appears in two different liquid forms, and in two familiar and perfectly distinct solid varieties. The two latter are called, from their crystalline forms, rhombic and monoclinic sulphur.

Physical Properties of Rhombic Sulphur.-This form is yellow, with specific gravity 2.06. Natural sulphur, roll sulphur, and practically all of most specimens of flowers of sulphur are of this variety, and are identical in all physical properties. Specimens of natural sulphur often show the rhombic crystalline form very clearly. All the forms of sulphur are insoluble in water, and all the crystalline forms are soluble in carbon disulphide. Good rhombic crystals are obtained from the solution (Fig. 10, p. 14).

The rhombic form is stable when not heated above $96^{\circ}$. If kept above this temperature, it slowly changes into monoclinic sulphur.

Monoclinic Sulphur.-This form is obtained most quickly by first melting some sulphur (m.-p. $114.5^{\circ}$ ), and then allowing it 
slowly to cool. As the temperature is now above $96^{\circ}$, the crystals which grow in the liquid are of the monoclinic variety. They are long, transparent, pale-yellow needles (Fig. 67), almost rectangular in section, and bevelled at the points. The specific gravity is 1.96 . This form can be kept indefinitely above $96^{\circ}$, but, when allowed to cool below that temperature, it slowly becomes opaque, changing into particles of rhombic sulphur.

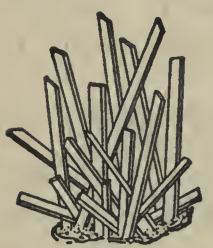

Fig. 67

The temperature at which a substance changes its crystalline form is called a transition point. It is analogous to the fusion point in the case of a solid and a liquid; only at this one point can both forms exist together in equilibrium.

\section{The Two Liquid Forms: Amorphous Sul-} phur. - When sulphur is melted, and the liquid is heated, two fluid, mutually soluble forms of sulphur are produced. These are known as $S_{\lambda}$ and $S_{\mu}$ or amorphous sulphur. As the temperature rises, the second variety increases in quantity at the expense of the first variety. When the temperature is lowered, the reverse change occurs:

$$
\mathrm{S}_{\lambda} \rightleftarrows \mathrm{S}_{\mu} \text { (amorphous). }
$$

If the temperature is lowered gradually, therefore, only monoclinic sulphur (by crystallization of the $\mathrm{S}_{\lambda}$ ) is obtained, the reaction proceeding to completion in the reverse direction owing to the removal of $S_{\lambda}$ (compare p. 239). But the change from $S_{\mu}$ to $S_{\lambda}$ takes place only very slowly, except at temperatures near the boiling-point. Consequently, if the liquid is quickly chilled, by pouring into a cold vessel or into cold water, the $S_{\mu}$ is found as a non-crystalline substance mixed with the crystalline form. The crystalline form can be dissolved out with carbon disulphide, leaving the amorphous sulphur which is not soluble. The proportion of $\mathrm{S}_{\mu}$ varies from 3.6 per cent at $120^{\circ}$, to 11 per cent at $160^{\circ}$ and about 34 per cent at $445^{\circ}$ (the boiling-point of sulphur). $\mathrm{S}_{\mu}$ 
is very viscous, so that, as its quantity increases, the whole liquid becomes thick. At $120^{\circ}$ molten sulphur is a limpid fluid, at $260^{\circ}$ a vessel containing it can be inverted without loss of material. Amorphous sulphur is a super-cooled liquid, and not a true solid, for true solids are all crystalline (see p. 94). At room temperature it changes into rhombic sulphur, but so slowly that the transformation even of a small part of it can be detected (by treating with carbon disulphide) only after the lapse of many months. At $100^{\circ}$ the change is complete in less than an hour (compare p. 242).

Elastic sulphur.-When melted sulphur is chilled, the amorphous sulphur does not at once become hard. Sulphur which has been heated to a high temperature, therefore, and then -suddenly cooled, consists at first of a sticky, transparent, elastic material, called elastic or plastic sulphur. In the course of forty eight hours, however, this becomes opaque and hard, because of the separation of the crystalline and the hardening of the amorphous varieties.

Melting and Freezing-Points.-Amorphous sulphur, like glass and other amorphous substances, softens when heated, but has no sharp melting temperature. The two crystalline forms have different melting-points, rhombic melting to form $S_{\lambda}$ at $112.8^{\circ}$, and monoclinic at $119.25^{\circ}$. But these are difficult to observe, as the rhombic begins to turn into monoclinic above $96.5^{\circ}$, and gradual transformation of $\mathrm{S}_{\lambda}$ to $\mathrm{S}_{\mu}$, to produce an equilibrium mixture of the two, occurs in both cases in the liquid state. Hence, the only temperature which is easy to observe is that at which both the solid forms melt when heated very slowly, and that at which the liquid freezes if cooled very slowly, namely $114.5^{\circ}$. This is the so-called natural freezing-point of sulphur.

Chemical Properties. - The vapor density of sulphur indicates that the vapor is a mixture of the molecules $S_{8}, S_{6}$ and $S_{2}$, 
the former diminishing and the latter increasing in number as the temperature is raised.

All the metals, excepting gold and platinum, cembine with sulphur to form sulphides, and in most cases much heat is given out during the union. Sulphur unites with chlorine to give sulphur monochloride $\mathrm{S}_{2} \mathrm{Cl}_{2}$, used in vulcanizing rubber, and burns in oxygen to give sulphur dioxide:*

$$
\mathrm{S}+\mathrm{O}_{2} \rightarrow \mathrm{SO}_{2} \text {. }
$$

In these compounds the valence of an atomic weight of sulphur appears to be one (in $\mathrm{S}_{2} \mathrm{Cl}_{2}$ ) or four (in $\mathrm{SO}_{2}$ ). These are exceptional values, however, the common valences being two (in $\mathrm{H}_{2} \mathrm{~S}$, $\mathrm{ZnS}$, etc.) and six (in $\mathrm{SO}_{3}, \mathrm{SO}_{2} \mathrm{Cl}_{2}$, etc.).

Moist sulphur is slowly oxidized at ordinary temperatures to sulphuric acid:

Skeleton:

$$
\mathrm{S}+\mathrm{H}_{2} \mathrm{O}+\mathrm{O}_{2} \rightarrow \mathrm{H}_{2} \mathrm{SO}_{4} \text {. }
$$

Balanced:

$$
2 \mathrm{~S}+2 \mathrm{H}_{2} \mathrm{O}+3 \mathrm{O}_{2} \rightarrow 2 \mathrm{H}_{2} \mathrm{SO}_{4} \text {. }
$$

In the equations, the simple formula $\mathrm{S}$ is used in place of a molecular formula. The latter is needed only when questions about the volume of the vapor are asked, and sulphur is almost always used only in solid or melted form. Then, too, the vapor contains several kinds of molecules, and using $\mathrm{S}_{8}$ or $\mathrm{S}_{6}$ would introduce large and inconvenient coefficients.

\section{HYdROGEN SUlphide $\mathrm{H}_{2} \mathrm{~S}$}

Occurrence.-Sulphur is a constituent of albumen, of which, for example, the white of an egg is composed. When decay takes place within the shell, so that air is excluded and the oxidation which accompanies ordinary decay is prevented, the sulphur gives hydrogen sulphide. The latter can be recognized by its odor. Some mineral waters contain a small amount in solution.

* Traces of sulphur trioxide are found at the same time. They give minute drops of sulphuric acid, which cause a haziness in the gas when it is formed by this action. 
Preparation.- Hydrogen and sulphur combine so slowly that at $310^{\circ}$ the completion of the union requires seven days. A trace may be obtained in a few minutes by leading hydrogen over sulphur, melted in a bulb (Fig. 68). A strip of paper, dipped in lead acetate solution and placed in the wide part of the tube, is darkened by the formation of insoluble lead sulphide PbS (black), while acetic acid is also formed:

\section{$\mathrm{Pb}\left(\mathrm{CO}_{2} \mathrm{CH}_{3}\right)_{2}+\mathrm{H}_{2} \mathrm{~S} \rightarrow \mathrm{PbS} \downarrow+2 \mathrm{HCO}_{2} \mathrm{CH}_{3}$.}

Laboratory Method. - The gas is commonly

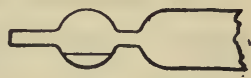

Fig. 68 made by double decomposition, using a sulphide to get the $\mathrm{S}$

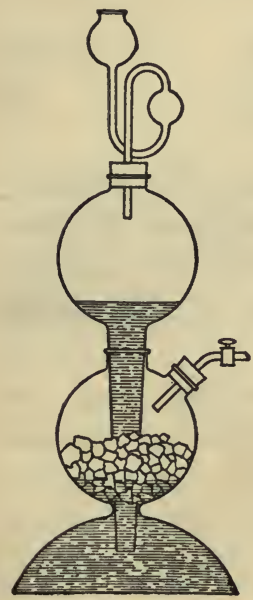

FIG. 69 radical, and an acid for the $\mathrm{H}$ radical. Eerrous sulphide, made by heating iron filings and sulphur, is the cheapest sulphide, and it interacts easily with hydrochloric acid or sulphuric acid:

$$
\mathrm{FeS}+2 \mathrm{HCl} \rightleftarrows \mathrm{FeCl}_{2}+\mathrm{H}_{2} \mathrm{~S} \uparrow .
$$

The action, like all double decompositions, is reversible. But use of an excess of hydrochloric acid forces it forward, and the escape of the gaseous hydrogen sulphide reduces the backward action almost to zero. The gas can be made in a flask fitted like that in Fig. 25 (p. 52), or in a Kipp's automatic generator (Fig. 69). It can be collected by upward displacement.

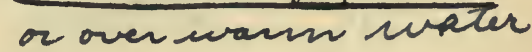

Physical Properties.- Hydrogen sulphide is a colorless gas with an odor recalling rotten eggs. It is rather easily liquefied, and the liquid boils at about $-60^{\circ}$ and freezes at $-83^{\circ}$. The density, implied in the formula $\mathrm{H}_{2} \mathrm{~S}$, shows that 22.4 liters weigh $32+2$ or $34 \mathrm{~g}$, so that the density is only one-sixth greater than that of air (of which 22.4 l. weigh $28.95 \mathrm{~g}$.). The gas is moder- 
ately saluble in water (290 vols. in 100 vols. water at $20^{\circ}$ ), a property which enables us to carry out many reactions of the gas upon substances in solution.

Physiological Properties.-Care must be taken to allow as little of the gas as possible to escape into the air, and all work with it should be done in a well-ventilated hood. The proportion must reach 1 part in 200 of air, however, before fatal results follow breathing the mixture. The best antidote is very dilute chlorine.

Chemical Properties.- 1. The gas burns in the air, giving water and sulphur dioxide:

Skeleton:

$$
\mathrm{H}_{2} \mathrm{~S}+\mathrm{O}_{2} \rightarrow \mathrm{H}_{2} \mathrm{O}+\mathrm{SO}_{2} \text {. }
$$

Balanced:

$$
2 \mathrm{H}_{2} \mathrm{~S}+3 \mathrm{O}_{2} \rightarrow 2 \mathrm{H}_{2} \mathrm{O}+2 \mathrm{SO}_{2} \text {. }
$$

2. The compound is not very stable. When heated, for example, in the interior of its own flame, it is partially decomposed into free sulphur and hydrogen. A cold porcelain dish (Fig. 70) placed in

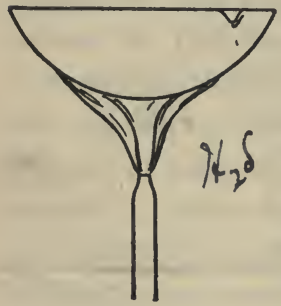

Fig. 70 the flame will condense some of the sulphur on its surface.

3. On account of its instability, and the ease with which it gives up hydrogen, the gas is a reducing agent. Thus, when jars of hydrogen suiphide and sulphur dioxide are placed mouth to mouth, a deposit of sulphur gradually appears:

$$
\mathrm{SO}_{2 .}+2 \mathrm{H}_{2} \mathrm{~S} \rightarrow 2 \mathrm{H}_{2} \mathrm{O}+3 \mathrm{~S} \text {. }
$$

Part of the free sulphur found in nature seems to be liberated by the action of these gases, both of which are found in volcanic regions. The gases must be moist, for, without water vapor as a contact agent, no interaction occurs.

In this action the sulphur dioxide loses its oxygen. We say that the $\mathrm{H}_{2} \mathrm{~S}$ was oxidized by the $\mathrm{SO}_{2}$, or that the $\mathrm{SO}_{2}$ was reduced by 
the $\mathrm{H}_{2} \mathrm{~S}$. As we have already noted, every reduction involves also an oxidation.

4. The metals, down to and including silver in the activity series, quickly receive a coating of sulphide when exposed to the gas:

$$
2 \mathrm{Ag}+\mathrm{H}_{2} \mathrm{~S} \rightarrow \mathrm{Ag}_{2} \mathrm{~S}+\mathrm{H}_{2} \uparrow .
$$

The tarnishing of silver in the household is due to the presence of a trace of hydrogen sulphide in the illuminating gas which escapes from slight leaks in the pipes.

Chemical Properties-An Acid.-The aqueous solution is an acid, and hence the compound is frequently called hydrosulphuric acid. It turns faintly tinted litmus paper distinctly pink. The poor conductivity of the solution shows the substance to be little ionized, and therefore a weak acid.

Like all acids, it enters into double decomposition with bases and salts. A number of these actions are used in analytical chemistry. Thus, with cupric sulphate solution, we get cupric sulphide (black), and with antimony trichloride antimony trisulphide (orange), both as precipitates:

$$
\begin{aligned}
& \mathrm{CuSO}_{4}+\mathrm{H}_{2} \mathrm{~S} \rightleftarrows \mathrm{CuS} \downarrow+\mathrm{H}_{2} \mathrm{SO}_{4} . \\
& 2 \mathrm{SbCl}_{3}+3 \mathrm{H}_{2} \mathrm{~S} \rightleftarrows \mathrm{Sb}_{2} \mathrm{~S}_{3} \downarrow+6 \mathrm{HCl} .
\end{aligned}
$$

Sulphides.-Many sulphides of metals are found as minerals. Most sulphides are insoluble, and can therefore be made by double decomposition. They may also be prepared by reduction of sulphates. Thus, when sodium sulphate is heated on a piece of charcoal (such as a half-burnt match) the sulphide is formed:

$$
\mathrm{Na}_{2} \mathrm{SO}_{4}+4 \mathrm{C} \rightarrow \mathrm{Na}_{2} \mathrm{~S}+4 \mathrm{CO} .
$$

It will be observed that in sulphides, $\mathrm{H}_{2} \mathrm{~S}, \mathrm{Na}_{2} \mathrm{~S}, \mathrm{ZnS}$, CuS, and so forth, sulphur is invariably bivalent.

Carbon Disulphide $\mathrm{CS}_{2}$. - This compound is an important solvent for sulphur, caoutchouc (rubber), and other substances 
which do not dissolve in water. It is manufactured by heating coke and sulphur together to a very high temperature. The sulphur (vapor) mixed with the coke combines with the latter, and carbon disulphide passes off as vapor and is condensed. The liquid boils at $46^{\circ}$, and is highly inflammable:

$$
\mathrm{CS}_{2}+3 \mathrm{O}_{2} \rightarrow 2 \mathrm{SO}_{2}+\mathrm{CO}_{2} \text {. }
$$

Large quantities are employed in the destruction of prairie dogs and for freeing grain elevators of rats and mice.

Exercises.-1. Write equations for the union of aluminium and of zinc with sulphur.

2. What experiments should you use to recognize a piece of sulphur?

3. In what proportions by volume do, (a) sulphur dioxide and hydrogen sulphide, $(b)$ oxygen and hydrogen sulphide interact?

4. Write full ionic equations for the precipitation of antimony trisulphide, and for the other double decompositions given in this chapter.

5. Is heat evolved, or absorbed, when monoclinic sulphur changes over to rhombic sulphur? Is heat evolved, or absorbed, when $S_{\lambda}$ changes over to $S_{\mu}$ ? Apply van't Hoff's law (p. 242).

6. Would equal weights of rhombic and monoclinic sulphur give out equal or different amounts of heat on burning? If different, which would give the most and which the least?

7. What would be the effect of passing hydrogen sulphide through a red-hot tube?

8. Why is chlorine an antidote for hydrogen sulphide poisoning (see p. 209)? 


\section{Anhydride Ch oxide which with wal forms an acid.}

\section{CHAPTER XXII \\ OXIDES AND OXYGEN ACIDS OF SULPHUR}

THere are two familiar oxides, namely sulphur dioxide or sulphurous anhydride $\mathrm{SO}_{2}$, and sulphur trioxide or sulphuric anhydride $\mathrm{SO}_{3}$. Each of these dissolves in water and combines with it to form an acid. The former gives sulphurous acid $\mathrm{H}_{2} \mathrm{O}, \mathrm{SO}_{2}$ or $\mathrm{H}_{2} \mathrm{SO}_{3}$, and the latter sulphuric acid $\mathrm{H}_{2} \mathrm{O}, \mathrm{SO}_{3}$ or $\mathrm{H}_{2} \mathrm{SO}_{4}$.

Acidic and Basic Oxides. - An oxide, like carbon dioxide $\mathrm{CO}_{2}$ (p. 336) or sulphur dioxide $\mathrm{SO}_{2}$, which combines with water to form an acid, is said to be the anhydride of the acid. The oxides of the non-metallic elements, when they combine with water, in so doing invariably form acids. In the next chapters we-shall meet with other examples (e.g., $\mathrm{N}_{2} \mathrm{O}_{5}, \mathrm{P}_{2} \mathrm{O}_{5}$, $\mathrm{SiO}_{2}$, etc.). On the other hand, the oxides of metallic elements, when they are able to combine with water, generally give bases (e.g. $\mathrm{Ca}(\mathrm{OH})_{2}$ from $\left.\mathrm{CaO}\right)$. For convenience, therefore, we shall often speak of an oxide as an acidic oxide or a basic oxide, as the case may be.

Nomenclature. - The acids and salts within one group are distinguished by the terminations of, and prefixes to, their names. Thus we have:

Hydrochloric acid $\mathrm{HCl}$

Hypochlorous acid $\mathrm{HOCl}$

Chlorous acid

Chloric acid

Perchloric acid
$\mathrm{HClO}_{2}$

$\mathrm{HClO}_{3}$

$\mathrm{HClO}_{4}$
Sodium chloride Sodium hypochlorite $\mathrm{NaOCl}$ Sodium chlorite

Sodium chlorate

Sodium perchlorate
$\mathrm{NaCl}$

$\mathrm{NaClO}_{2}$ $\mathrm{NaClO}_{3}$ $\mathrm{NaClO}_{4}$

The proportion of oxygen to the other elements is at the basis of the system. The terminations ous and ite indicate less oxygen than ic and ate. The prefix hypo (Greek, below) implies still less 
oxygen, the prefix hydro implies none at all. The per-acid contains the most oxygen.

The names of compounds containing only two elements (the binary compounds) end in ide: Zinc sulphide $\mathrm{ZnS}$, magnesium nitride $\mathrm{Mg}_{3} \mathrm{~N}_{2}$, calcium carbide $\mathrm{CaC}_{2}$, sodium chloride $\mathrm{NaCl}$, and the oxides $\mathrm{CaO}$, etc.

\section{Sulphur Dioxide and Sulphurous Acid}

Preparation of Sulphur Dioxide $\mathrm{SO}_{2}$. - In commercial practice sulphur dioxide is obtained in three ways:

1. By burning sulphur.

2. By burning natural sulphides, such as pyrite:

Skeleton:

$\mathrm{FeS}_{2}+\mathrm{O}_{2} \rightarrow \mathrm{Fe}_{2} \mathrm{O}_{3}+\mathrm{SO}_{2}$.

Balanced:

$$
\underline{4 \mathrm{FeS}_{2}}+11 \mathrm{O}_{2} \rightarrow 2 \mathrm{Fe}_{2} \mathrm{O}_{3}+8 \mathrm{SO}_{2} \text {. }
$$

With fairly pure pyrite the combustion has only to be started, the heat evolved in the reaction being sufficient to offset loss of heat by radiation and to keep it going of its own accord. But with some sulphides, like zinc blende $\mathrm{ZnS}$, which is used as a source of sulphur dioxide as well as of zinc, the air must be strongly heated throughout to maintain the combustion:

$$
2 \mathrm{ZnS}+3 \mathrm{O}_{2} \rightarrow 2 \mathrm{ZnO}+2 \mathrm{SO}_{2} .
$$

Forced combustion of an ore, like this, is called roasting, or calcining, and is the first stage towards obtaining the metal. The oxide is subsequently reduced by heating with coke.

3. By dropping concentrated sulphuric acid into red-hot iron retorts:

$$
2 \mathrm{H}_{2} \mathrm{SO}_{4} \rightarrow 2 \mathrm{H}_{2} \mathrm{O}+2 \mathrm{SO}_{2}+\mathrm{O}_{2} \text {. }
$$

4. In the laboratory a steady stream of the gas is easily obtained by dropping hydrochloric acid upon crystals of sodium-hydrogen sulphite (Fig. 25, p. 52):

$$
\begin{gathered}
\mathrm{NaHSO}_{3}+\mathrm{HCl} \rightleftharpoons \mathrm{NaCl}+\mathrm{H}_{2} \mathrm{SO}_{3}, \\
\mathrm{H}_{2} \mathrm{SO}_{3} \rightleftarrows \mathrm{H}_{2} \mathrm{O}+\mathrm{SO}_{2} \uparrow .
\end{gathered}
$$


This method takes advantage of the facts that sulphurous acid is only slightly ionized in solution, which renders reaction 1 practically complete (see p. 241), and that this acid is unstable and decomposes (equation 2) when there is not a large excess of water present.

Physical Properties. - The usual six physical properties may be noted: The gas is colorless, but has a characteristic taste and odor. It has a density considerably greater than that of air $\left(\mathrm{SO}_{2}\right.$ $=64$ against 28.95). It can be liquefied below $156^{\circ}$ (the crit. temp.) and the liquid boils at $-8^{\circ}$. As the pressure required at $20^{\circ}$ is only $3 \frac{1}{4}$ atmospheres, the liquid can be kept in bottles like syphons, or in sealed tin cans. It is extremely soluble in water (about 40 vols. in 1 vol. water at $15^{\circ}$ ). The solution is sulphurous acid. $2-y 3$.

Chemical Properties and Uses.-Sulphur dioxide is very stable. It combines with water giving a solution of sulphurous acid. The gas is used in bleaching straw, silk, and wool (compare p. 225). The bleaching action seems largely to consist in combination with the coloring matter, to give a colorless compound. Hence straw hats recover the yellow color of straw by exposure to light, which slowly reverses the reaction and liberates the sulphur dioxide.

To prevent the growth of fungi or other organisms, wine casks are fumigated with sulphur dioxide before being filled. Dried peaches and apples are prepared by exposing slices of the fruit on trays to sulphur dioxide. The sulphurous acid produced bleaches the fruit, keeps insects away, and prevents the formation of dark-colored substances during the subsequent drying.

Enormous quantities of sulphur dioxide are employed in the manufacture of sulphuric acid and of sulphites.

Properties of Sulphurous Acid $\mathrm{H}_{2} \mathrm{SO}_{3}$.- Sulphurous acid, in aqueous solution, shows all the properties of a transition acid. 
As already noted, concentrated solutions are very unstable. A solution of sulphurous acid therefore smells strongly of sulphur dioxide.

Being rather easily convertible into sulphuric acid $\mathrm{H}_{2} \mathrm{SO}_{4}$, sulphurous acid is a reducing agent. Thus oxygen from the air acts slowly upon the solution:

$$
2 \mathrm{H}_{2} \mathrm{SO}_{3}+\mathrm{O}_{2} \rightarrow 2 \mathrm{H}_{2} \mathrm{SO}_{4}
$$

and iodine is turned into hydrogen iodide:

$$
\mathrm{H}_{2} \mathrm{SO}_{3}+\mathrm{H}_{2} \mathrm{O}+\mathrm{I}_{2} \rightarrow \mathrm{H}_{2} \mathrm{SO}_{4}+2 \mathrm{HI} \text {. }
$$

Sulphites and Bisulphites.-Sulphites are formed by neutralization of sulphurous acid with a base:

$$
2 \mathrm{NaOH}+\mathrm{H}_{2} \mathrm{SO}_{3} \rightarrow \mathrm{Na}_{2} \mathrm{SO}_{3}+2 \mathrm{H}_{2} \mathrm{O} \text {. }
$$

With excess of sulphur dioxide passed into the solutions of the bases, the acid sulphites are formed:

$\mathrm{NaOH}+\mathrm{H}_{2} \mathrm{SO}_{3} \rightarrow \mathrm{NaHSO}_{3}+\mathrm{H}_{2} \mathrm{O}$.

$\mathrm{Ca}(\mathrm{OH})_{2}+2 \mathrm{H}_{2} \mathrm{SO}_{3} \rightarrow \mathrm{Ca}\left(\mathrm{HSO}_{3}\right)_{2}+2 \mathrm{H}_{2} \mathrm{O}$.

Such acid salts are known in commerce as bisulphites, because, the proportion of the metal being half that in a sulphite, the proportion of the sulphite radical is, relatively, twice as great. They are used extensively on paper manufacture (see p. 398).

Dibasic Acids. - Acids containing two atoms of hydrogen in each molecule are called dibasic acids. $\mathrm{H}_{2} \mathrm{CO}_{3}$ (p. 336), $\mathrm{H}_{2} \mathrm{~S}$, $\mathrm{H}_{2} \mathrm{SO}_{3}$ and $\mathrm{H}_{2} \mathrm{SO}_{4}$ are such acids. Each molecule is able to react with two molecules of a base like sodium hydroxide, as may be seen in the first of the equations in the preceding section. When half the quantity of the base is used, an acid salt (p. 192) is produced, as the two other equations show. Phosphoric acid $\mathrm{H}_{3} \mathrm{PO}_{4}$ is a tribasic acid, and forms two series of acid salts, for example $\mathrm{NaH}_{2} \mathrm{PO}_{4}$ and $\mathrm{Na}_{2} \mathrm{HPO}_{4}$. Hydrochloric and nitric acids $\mathrm{HNO}_{3}$ are monobasic. 


\section{Sulphur Trioxide and Sulphuric Acid}

Sulphur Trioxide.-Sulphur dioxide and oxygen, when heated together to $400^{\circ}$, unite very slowly with evolution of heat to give sulphur trioxide:

$$
2 \mathrm{SO}_{2}+\mathrm{O}_{2} \rightarrow 2 \mathrm{SO}_{3}+45,200 \text { calories. }
$$

This reaction, however, cannot be utilized for the manufacture of sulphur trioxide except under special conditions, for at $400^{\circ}$ the union takes place far too slowly for use in industrial work, while at higher temperatures the reverse action becomes appreciable and poor yields are obtained. If we apply van't Hoff's law to the reversible reaction: $2 \mathrm{SO}_{2}+\mathrm{O}_{2} \rightleftharpoons 2 \mathrm{SO}_{3}$ we see that, since the forward change is exothermic, raising the temperature will favor the backward change. In actual practice it is found that at $400^{\circ}, 98-99$ per cent of the materials unite; at $700^{\circ}$, only 60 per cent; at $900^{\circ}$, practically none.

Sulphur trioxide is a white solid which exists in two allotropic crystalline forms. One melts at $15^{\circ}$, and is therefore fluid at ordinary temperatures. The other vaporizes without melting at $50^{\circ}$. Both forms react vigorously with water, causing a hissing noise due to the steam produced by the heat of the union:

$$
\mathrm{SO}_{3}+\mathrm{H}_{2} \mathrm{O} \rightarrow \mathrm{H}_{2} \mathrm{SO}_{4} \text {. }
$$

Sulphur trioxide combines also with sulphuric acid to give oleum, or fuming sulphuric acid, $\mathrm{H}_{2} \mathrm{~S}_{2} \mathrm{O}_{7}$ :

$$
\mathrm{SO}_{3}+\mathrm{H}_{2} \mathrm{SO}_{4} \rightarrow \mathrm{H}_{2} \mathrm{~S}_{2} \mathrm{O}_{7} \text {. }
$$

The Contact Process for Sulphuric Acide- The interaction of sulphur dioxide and oxygen is hastened by many substances, such as glass, porcelain, ferric oxide and, more especially, finelydivided platinum, which remain themselves unchanged and simply act as contact or catalytic agents. The contact process, as this is called, is now very extensively used in the manufacture of sulphuric acid. 
The efficiency of the contact agent depends on the amount of surface it presents to the gases. The action may be illustrated by dipping asbestos in a solution of chloroplatinic acid and then heating the mineral in the Bunsen flame:

$$
\mathrm{H}_{2} \mathrm{PtCl}_{6} \rightarrow \mathrm{Pt}+2 \mathrm{HCl} \uparrow+2 \mathrm{Cl}_{2} \uparrow \text {. }
$$

The platinum is thus spread in a fine grey powder on the fibers of the asbestos. The latter is placed in a tube (Fig. 48, p. 140), where a mixture of oxygen (or air) and sulphur dioxide may be passed over the heated material. The sulphur trioxide issues as vapor at the other end of the tube, where its presence is recognized by the dense fumes (droplets of sulphuric acid), produced when it meets the moisture in the air. The vapor can be condensed to liquid form in a cooled flask.

In practice the contact agent employed is platinum, dispersed in a very finely-divided condition throughout a suitable carrying material, or base. The Grillo process uses as a base magnesium sulphate. This gives a catalytic mass just as active as platinized asbestos, and requires only one-hundreth part the amount of platinum. With silica gel (p. 360) as a base, the platinum content of the contact mass can be still further reduced. This is a very important point in the economics of the process.

It is absolutely necessary, in employing the contact process, to remove from the sulphur dioxide all traces of substances such as arsenious oxide and similar impurities derived from the calcining of pyrite or some other mineral sulphide. The most minute quantities of these substances act as poisons on the catalytic agent, and soon render it quite inoperative. The sulphur dioxide is therefore very carefully purified before reaching the contact chamber. Excess oxygen is used in the reacting mixture, in order to drive the reaction more readily towards complete formation of $\mathrm{SO}_{3}$ (compare p. 234). The temperature in the contact chamber is kept between $380^{\circ}$ and $450^{\circ}$. The system has a tendency to get hotter during the reaction, owing to the heat 
evolved. This tendency, if unchecked, would lead to a decreased yield of sulphur trioxide; the cold entering gases are therefore first led over the outside of the pipes which contain the catalyst, in order to keep the temperature constant inside.

The issuing gases, consisting mainly of sulphur trioxide vapor mixed with excess oxygen, are condensed by being led into 97-99 per cent sulphuric acid, the concentration of the liquid being maintained at this point by a regulated influx of water. If oleum, or fuming sulphuric acid, is required, the addition of water is omitted.

It would seem to be simpler to dissolve the gaseous sulphur trioxide in water, to give sulphuric acid $\mathrm{H}_{2} \mathrm{O}+\mathrm{SO}_{3} \rightarrow \mathrm{H}_{2} \mathrm{SO}_{4}$, rather than in 98 per cent sulphuric acid, but this cannot be done. The mixture $\mathrm{O}_{2}+2 \mathrm{SO}_{3}$ is very incompletely absorbed by water. When a bubble of this mixture enters water, the latter evaporates into the bubble in the attempt to saturate the space occupied by the bubble with water vapor (p. 62). The water which so evapYorates, however, combines immediately with the sulphur trioxide to form a fog, consisting of droplets of liquid sulphuric acid, and so more and more water evaporates into the bubbles. Now the molecules of $\mathrm{SO}_{3}$, so long as they remain gaseous, move with great velocity, namely 292 meters per second at room temperature, and still faster in this hot gaseous mixture (see p. 90). Hence, all the molecules that escape combination with the water vapor, strike the wall of the bubble, and combine with the water in a few seconds. The droplets of sulphuric acid, forming the fog, however, are not molecules but large aggregates of molecules. They do not therefore move like the molecules of a gas, but are relatively stationary. The chance of their striking the wall of the bubble is therefore reduced enormously. Hence, after the sulphur trioxide that escapes combination has dissolved, the droplets of fog, carried by the excess of oxygen, can be bubbled through a whole series of vessels of water in succession without any appreciable number of the droplets being dissolved. The same fog can be shaken in

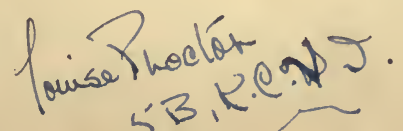




\section{Concentrated here means pailyptrongecid}

a flask with water, violently and continuously, without any appreciable solution. When the water is thrown, by the shaking, through the oxygen, the oxygen is split up by the water, and driven about, but the fog particles move with the oxygen, so that the water never reaches them. On the other hand, when the mixture of gases bubbles through $97-99$ per cent sulphuric acid, as is done in practice, there is practically no water available for evaporation, the sulphur trioxide remains gaseous, and its rapidly moving molecules in a few seconds have all plunged into the sulphuric acid and combined with it, either uniting with the 1 to 3 per cent of water present, or, when oleum is made, uniting with the sulphuric acid to form $\mathrm{H}_{2} \mathrm{~S}_{2} \mathrm{O}_{7}$.

This case affords an admirable illustration of the importance of physics in practical chemistry (p. 22). The chemical reaction occurs with water, but the physical condition of the fog of sulphuric acid prevents its dissolving and, if water were used in a factory, a large proportion of the sulphuric acid would pass with the excess of oxygen into the air and be lost. In fact, it would kill vegetation, and make life unbearable in the neighborhood.

Chamber Process for Sulphuric Acid.-Although salts of sulphuric acid, such as calcium sulphate $\mathrm{CaSO}_{4}$, are exceedingly plentiful in nature, the preparation of the acid by chemical action upon the salts is not practicable. The sulphates, indeed, interact with all acids, but the actions are reversible. The completion of the action by the plan used in making hydrogen chloride (p. 126), involving the removal of the sulphuric acid by distillation, would be difficult on account of the involatility of this acid. It boils at $330^{\circ}$; and suitable acids, less volatile still, which might be used to liberate it, do not exist. We are therefore compelled to build up sulphuric acid from its elements.

The gases, the interactions of which result in the formation of sulphuric acid, are: water vapor, sulphur dioxide, nitrous anhy- 
dride $\mathrm{N}_{2} \mathrm{O}_{3}{ }^{*}$ (see p. 314 ), and oxygen. These are obtained, the first by injection of steam, the second usually by the burning of pyrite, the third from nitric acid $\mathrm{HNO}_{3}$, and the fourth by the introduction of air. The gases are thoroughly mixed in large leaden chambers, and the sulphuric acid forms droplets which fall to the floors. In spite of elaborate investigations, instigated by the extensive scale upon which the manufacture is carried on and the immense financial interests involved, some uncertainty still exists in regard to the precise nature of the chemical changes which take place. According to Lunge, supporting the view first suggested by Berzelius, the greater part of the product is formed by two successive actions, the first of which yields a complex compound that is decomposed by excess of water in the second:

$$
\mathrm{H}_{2} \mathrm{O}+2 \mathrm{SO}_{2}+\mathrm{N}_{2} \mathrm{O}_{3}+\mathrm{O}_{2} \rightarrow 2 \mathrm{SO}_{2} \backslash \begin{aligned}
& \mathrm{O}-\mathrm{H} \\
& \mathrm{O}-\mathrm{NO}
\end{aligned}
$$

The group - NO, nitrosyl, is found in many compounds. Here, if it were displaced by hydrogen, sulphuric acid would result. Hence this compound is called nitrosylsulphuric acid:

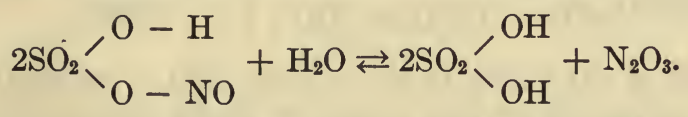

The equations (1) and (2) are not partial equations for one interaction, but represent distinct actions which can be carried out separately. In a properly operating plant, indeed, the nitrosylsulphuric acid is not observed. But when the supply of water is deficient, white " chamber crystals," consisting of this substance, collect on the walls.

The explanation of the success of this seemingly roundabout method of getting sulphuric acid is as follows: The direct union of

* This gas is unstable, breaking up in part into nitric oxide NO and nitrogen tetroxide $\mathrm{NO}_{2}: \mathrm{N}_{2} \mathrm{O}_{3} \rightleftarrows \mathrm{NO}+\mathrm{NO}_{2}$. In this process, however, the mixture behaves as if it were all $\mathrm{N}_{2} \mathrm{O}_{3}$, and so only nitrous ankydride is named in this connection. 
sulphur dioxide and water to form sulphurous acid is rapid, but the action of free oxygen upon the latter, $2 \mathrm{H}_{2} \mathrm{SO}_{3}+\mathrm{O}_{2} \rightarrow 2 \mathrm{H}_{2} \mathrm{SO}_{4}$, is exceedingly slow. Reaching sulphuric acid by the use of these two changes, although they constitute a direct route to the result, is not feasible in practice. On the other hand, both of the above actions, (1) and (2), happen to be much more speedy, and so, by their use, more rapid production of the desired substance is secured at the expense of a slight comp'exity.

The progress of the first action is marked by the disappearance of the brown nitrous anhydride and, on the introduction of water, the completion of the second stage results in the reproduction of the same substance. The nitrous anhydride takes part a large number of times in these changes, and so facilitates the conversion of a great amount of sulphur dioxide, oxygen, and water into sulphuric acid, without much diminution of its quantity. Some is unavoidably lost, however.

The loss of nitrous anhydride is made good by the introduction of nitric acid vapor into the chamber. This acid is made from concentrated sulphuric acid and commercial sodium nitrate $\mathrm{NaNO}_{3}$ :

$$
\mathrm{NaNO}_{3}+\mathrm{H}_{2} \mathrm{SO}_{4} \rightleftarrows \mathrm{HNO}_{3} \uparrow+\mathrm{NaHSO}_{4} \text {. }
$$

On account of the volatility of the nitric acid, a moderate heat is sufficient to remove it from admixture with the other substances, and its vapor is swept along with the other gases into the apparatus. The first reaction which this vapor undergoes may be written:

$$
\mathrm{H}_{2} \mathrm{O}+2 \mathrm{SO}_{2}+2 \mathrm{HNO}_{3} \rightarrow 2 \mathrm{H}_{2} \mathrm{SO}_{4}+\mathrm{N}_{2} \mathrm{O}_{3} \text {. }
$$

Details of the Chamber Process. - The sulphur dioxide is produced in a row of furnaces $A$ (Fig. 71). The gases from the various furnaces pass into one long dust-flue, in which they are mingled with the proper proportion of air, and deposit oxides of iron and of arsenic, and other materials which they transport mechanically. From this flue they enter the Glover tower $G$, in which they acquire the oxides of nitrogen. Having secured 
all the necessary constituents, excepting water, the gases next enter the first of the lead chambers, large structures lined completely with sheet lead. These measure as much as $100 \times 40 \times$ 40 feet, and have a total capacity of 150,000 to 200,000 cubic feet. As the gases drift through these chambers they are thor-

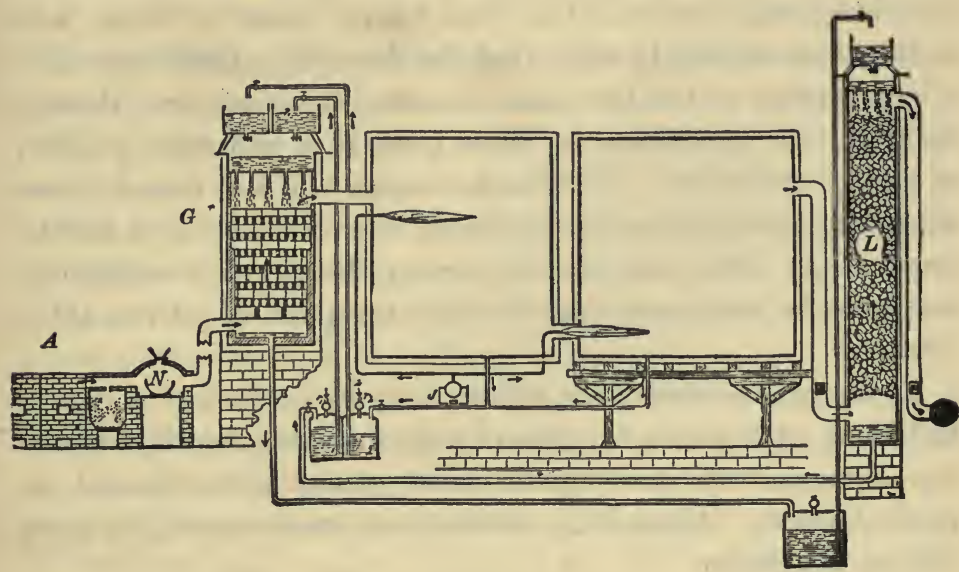

Frg. 71

oughly mixed, and an amount of water considerably in excess of that actually required is injected in the form of steam at various points. The acid, along with the excess of water, condenses and collects upon the floor of the chamber, while the unused gases, chiefly nitrous anhydride and nitrogen, the latter derived from the air originally admitted, find an exit into the Gay-Lussac tower $L$.

This is a tower about fifty feet in height, filled with tiles, over which concentrated sulphuric acid continually trickles. The object of this tower, to catch the nitrous anhydride and enable it to be reëmployed in the process, is accomplished by a reversal of action (2) above. The acid which accumulates in the vessel at the bottom of this tower contains, therefore, nitrosylsulphuric 
acid, and by means of compressed air this acid is forced through a pipe up to a vessel at the top of the Glover tower $G$. A neighboring vessel at the top of this tower is filled with dilute sulphuric acid, and when the contents of both vessels are mixed by allowing their contents to trickle down through the tower, nitrous anhydride is once more set free by the interaction of the water in the dilute acid (action (2)). The Glover tower is filled with broken flint or tiles, in order that the descending liquid may offer a large surface to the hot gases ascending the tower, and thereby facilitate the acquisition by these gases of a sufficient quantity of nitrous anhydride. Their high temperature also causes a considerable concentration of the diluted sulphuric acid as it trickles downward. This acid, after traversing this tower, is sufficiently strong to be used once more for the absorption of nitrous anhydride.

To replace the part of the nitrous anhydride which is inevitably lost, fresh nitric acid is furnished by small open vessels $N$, containing sodium nitrate and sulphuric acid, placed in the flues of the pyrite-burners. About $4 \mathrm{~kg}$. of the nitrate are consumed for every $100 \mathrm{~kg}$. of sulphur.

The acid which accumulates upon the floors contains but 60 to 70 per cent of sulphuric acid, and has a specific gravity of 1.51.62.

This crude sulphuric acid is applicable directly in some chemical manufactures, such as the preparation of superphosphates (p.412). For many purposes, however, a more concentrated acid is required. Concentration is effected by evaporating off water from the chamber acid in pans lined with lead, which are frequently placed over the pyrite-burners in order to economize fuel. The evaporation in lead is carried on until a specific gravity 1.7, corresponding to 77 per cent concentration, is reached. Up to this point the sulphate of lead formed by the action of the sulphuric acid produces a crust which protects the metal from further action. When a still more concentrated acid is 
wanted other methods of driving off the water, such as the cascade system or the Gaillard tower, must be employed.

The cascade system consists of a series of small silica or siliconiron basins set over an inclined flue, and so placed that each basin delivers by a spout into the one below. The flue is heated by gas or coke firing at the lower end, and dilute acid is fed continuously into the basin at the top end. As the acid passes from basin to basin, it meets hotter and hotter conditions and becomes more and more concentrated.

The Gaillard plant consists essentially of a large hollow tower built of acid-resisting stone, and filled with small fragments of similar material. Dilute acid is sprayed in at the top, and meets hot furnace gases injected in at the bottom. Most of the water contained in the acid is carried off by these gases, and concentrated acid collects at the bottom of the tower.

A more convenient method of obtaining very concentrated acid, which avoids the difficulties of evaporation entirely, is to add to the chamber acid the requisite quantity of oleum, prepared by the contact process already described. Commercial sulphuric acid, oil of vitriol, has a specific gravity 1.83-1.84, and contains about 93.5 per cent $\mathrm{H}_{2} \mathrm{SO}_{4}$.

Physical Properties of Sulphuric Acid.- The pure acid is a colorless, oily liquid of sp. gr. 1.84, which freezes to a solid at $10^{\circ}$. It mixes with water in all proportions, and much heat (heat of solution, see p. 244) is given out when it dissolves. It boils at $330^{\circ}$, but the vapor is largely decomposed into free water and sulphur trioxide, which recombine when it cools.

Chemical Properties. - 1. The acid is more stable than sulphurous acid, but has a slight tendency to lose $\mathrm{SO}_{3}$ even at ordinary temperatures and as already noted, decomposes largely at the boiling-point.

2. In aqueous solution, sulphuric acid is much more active Indectivil cues depend an proppectici. 
as an acid than is sulphurous acid, but is somewhat inferior in this respect to hydrochloric acid $\mathrm{HCl}$ and nitric acid $\mathrm{HNO}_{3}$. Like other active, soluble acids, its solution turns litmus red, gives hydrogen upon addition of active metals, and enters into double decomposition with bases and salts. Thus, insoluble barium sulphate is obtained as a white precipitate by the action of dilute sulphuric acid on any soluble barium salt:

$$
\mathrm{BaCl}_{2}+\mathrm{H}_{2} \mathrm{SO}_{4} \rightarrow \mathrm{BaSO}_{4} \downarrow+2 \mathrm{HCl} \text {. }
$$

Any soluble sulphate will, of course, give the same precipitate with barium chloride, and the action is used as a test for this ion. Some other salts of barium are also insoluble in water, but the sulphate is recognized by the fact that it is too insoluble to be acted upon by dilute pure hydrochloric acid or nitric acid. The other insoluble salts of barium interact with these acids and dissolve. The addition of one of these acids is therefore part of the test for $\mathrm{SO}_{4}=$ ion.

On account of its high boiling-point, the double decompositions of the concentrated acid can be used for preparing more volatile acids:

$\mathrm{NaCl}+\mathrm{H}_{2} \mathrm{SO}_{4} \rightarrow \mathrm{NaHSO}_{4}+\mathrm{HCl} \uparrow$ (gas at room temp.). $\mathrm{NaNO}_{3}+\mathrm{H}_{2} \mathrm{SO}_{4} \rightarrow \mathrm{NaHSO}_{4}+\mathrm{HNO}_{3} \uparrow$ (volatile at $86^{\circ}$ ).

3. Concentrated sulphuric acid combines with water to form a stable hydrate $\mathrm{H}_{2} \mathrm{SO}_{4}, \mathrm{H}_{2} \mathrm{O}$. Hence it removes the elements of water from many substances containing hydrogen and oxygen, and is called a dehydrating agent. Thus, paper (cellulose), moistened with the acid and warmed, turns black from the liberation of carbon. Sugar $\left(\mathrm{C}_{12} \mathrm{H}_{22} \mathrm{O}_{11}\right)$ is decomposed even more easily:

$$
\mathrm{C}_{12} \mathrm{H}_{22} \mathrm{O}_{11} \rightarrow 12 \mathrm{C}+11 \mathrm{H}_{2} \mathrm{O} \text {. }
$$

4. Finally, concentrated sulphuric acid acts as an oxidizing agent. Sulphur and carbon, boiled in it, are oxidized:

$$
\begin{aligned}
& 2 \mathrm{H}_{2} \mathrm{SO}_{4}+\mathrm{S} \rightarrow 3 \mathrm{SO}_{2}+2 \mathrm{H}_{2} \mathrm{O} . \\
& 2 \mathrm{H}_{2} \mathrm{SO}_{4}+\mathrm{C} \rightarrow 2 \mathrm{SO}_{2}+2 \mathrm{H}_{2} \mathrm{O}+\mathrm{CO}_{2} .
\end{aligned}
$$


The reducing action of $\mathrm{HBr}$ and $\mathrm{HI}$ on sulphuric acid has already been noted (pp. 202, 204). The more active metals, like zinc, reduce it to hydrogen sulphide, the less active, like copper, give sulphur dioxide. Hydrogen is not liberated, because practically no hydrogen-ion is present in concentrated sulphuric acid. Gold and platinum alone are not attacked.

$$
\begin{aligned}
4 \mathrm{Zn}+5 \mathrm{H}_{2} \mathrm{SO}_{4} & \rightarrow 4 \mathrm{ZnSO}_{4}+4 \mathrm{H}_{2} \mathrm{O}+\mathrm{H}_{2} \mathrm{~S} . \\
\mathrm{Cu}+2 \mathrm{H}_{2} \mathrm{SO}_{4} & \rightarrow \mathrm{CuSO}_{4}+2 \mathrm{H}_{2} \mathrm{O}+\mathrm{SO}_{2} .
\end{aligned}
$$

Uses of Sulphuric Acid.- The acid has innumerable applications, some of which will be taken up in detail in later chapters.

It is employed in almost every chemical industry, something like 6,000,000 tons being produced yearly in the United States alone. It is used in the manufacture of sulphates, hydrochloric acid, nitric acid, sodium carbonate, etc., in making fertilizers and dyes, in bleaching, electroplating and so on. Its dehydrating power is especially valuable in making explosives (pp. 481-2).

Other Oxygen Acids of Sulphur.-Many other oxygen acids of sulphur exist, such as hyposulphurous acid $\mathrm{H}_{2} \mathrm{~S}_{2} \mathrm{O}_{4}$ and persulphuric acid $\mathrm{H}_{2} \mathrm{~S}_{2} \mathrm{O}_{8}$. The acids themselves are very unstable, and cannot be isolated in the pure state. Some of the salts, however, are in common use, and will be dealt with later under their several positive radicals.

When acid sulphates, such as $\mathrm{NaHSO}_{4}$, are heated, water is given off and a pyrosulphate (Greek prefix, fire) remains.

$$
2 \mathrm{NaHSO}_{4} \rightarrow \underline{\mathrm{Na}_{2} \mathrm{~S}_{2} \mathrm{O}_{7}}+\mathrm{H}_{2} \mathrm{O} \text {. }
$$

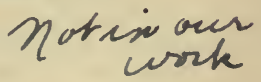

The pyrosulphates are salts of oleum, or fuming sulphuric acid, which has already been mentioned. Oleum possess all of the dehydrating and oxidizing powers of sulphuric acid in an accentuated form, and is widely used in the industries on account of these properties. 
Exercises.-1. Which contains more oxygen: (a) a phosphate or a phosphite; (b) a nitrite or a nitrate; (c) a borate or a perborate? Name the acids corresponding to these six salts.

2. Make equations for: (a) the roasting of stannic sulphide $\left(\mathrm{SnS}_{2}\right)$ giving $\mathrm{SnO}_{2}$; (b) the action of concentrated sulphuric acid on silver giving silver sulphate and $\mathrm{SO}_{2}$; (c) the dissociation of sulphuric acid vapor.

3. What are the formulæ of magnesium sulphite and bisulphite, respectively?

4. Give two reasons why boiling sulphuric acid, when spilt upon the flesh, causes most serious burns.

5. By what facts or tests could you recognize concentrated sulphuric acid?

6. Why is the wooden laboratory shelf commonly "burned" where the sulphuric acid bottle stands?

7. What would be the reaction between sodium sulphite and sulphuric acid?

8. How could you distinguish between, and recognize, the sulphide, sulphite, and sulphate of potassium?

9. Why is it not desirable to make chamber sulphuric acid of a concentration higher than $60-70$ per cent?

10. Justify the nomenclature in the case of hyposulphurous and persulphuric acids. 


\section{CHAPTER XXIII}

\section{THE PERIODIC SYSTEM}

IN an earlier chapter (p. 208) we saw that the elements fluorine, chlorine, bromine and iodine exhibited striking similarities in their chemical properties, and we grouped these four elements together under the name of the halogen family. Now there are two rather rare clements, selenium and tellurium, which resemble sulphur very markedly in their chemical properties. Both give compounds with hydrogen, hydrogen selenide $\mathrm{H}_{2} \mathrm{Se}$ and hydrogen telluride $\mathrm{H}_{2} \mathrm{Te}$, corresponding with hydrogen sulphide $\mathrm{H}_{2} \mathrm{~S}$, but less stable. Both give compounds with oxygen, selenium dioxide $\mathrm{SeO}_{2}$ and tellurium dioxide $\mathrm{TeO}_{2}$, corresponding with sulphur dioxide $\mathrm{SO}_{2}$. These dioxides dissolve in water to form weak acids similar to sulphurous acid. These acids, again, can be oxidized to yield selenic acid $\mathrm{H}_{2} \mathrm{SeO}_{4}$ and telluric acid $\mathrm{H}_{2} \mathrm{TeO}_{4}$, analogous to sulphuric acid $\mathrm{H}_{2} \mathrm{SO}_{4}$. All these compounds show gradations in properties, as we go upwards in atomic weights from $\mathrm{S}$ to $\mathrm{Te}$, which are very reminiscent of the gradations encountered in a series of halogen compounds, such as $\mathrm{HCl}, \mathrm{HBr}, \mathrm{HI}$.

Furthermore, just as we have in the halogen family a first member with rather irregular habits, fuorine, so we note in the sulphur family a corresponding light element showing decided peculiarities, oxygen. Oxygen forms a compound with hydrogen $\mathrm{H}_{2} \mathrm{O}$, which is akin to $\mathrm{H}_{2} \mathrm{~S}$ in being a weak acid and, as might be predicted, is much more stable. The metallic oxides are very similar in their properties to the metallic sulphides. Ozone may be regarded as oxygen dioxide $\mathrm{OO}_{2}$, analogous to sulphur dioxide $\mathrm{SO}_{2}$. The family resemblance in other compounds, however, is more diffcult to trace. 
The question naturally arises: can we group all of the elements into families like the halogen family and the sulphur family? If so, then we shall lighten considerably the burden of chemical facts that we need to remember, for the behavior of one element in a family will suggest to us immediately how the other members of the same group will act under similar conditions. Classification of this kind is part of the method of science, and furnishes a very useful guide in investigation.

Metallic and Non-Metallic Elements.- Thus far we have found the division into metallic and non-metallic elements very serviceable for classification in terms of chemical relations. The metallic or positive elements (p. 54), form positive radicals and ions containing no other element ( $c f$. p. 171). Thus the metals give sulphates, nitrates, carbonates, and other salts, which furnish a metallic ion, such as $\mathrm{Na}^{+}$or $\mathrm{K}^{+}$, together with the ions $\mathrm{SO}_{4}{ }^{=}, \mathrm{NO}_{3}{ }^{-}$, and $\mathrm{CO}_{3}{ }^{=}$. Their hydroxides, $\mathrm{KOH}, \mathrm{Ca}-$ $\left(\mathrm{OH}_{2}\right)$, etc., give the same metallic ion, and the rest of the molecule forms hydroxide-ion. That is to say, their hydroxides are bases and their oxides are basic. The metallic elements often enter, but only with other elements, into the composition of a negative ion, as is the case with manganese in $\mathrm{K} . \mathrm{MnO}_{4}$, with chromium in $\mathrm{K}_{2} \cdot \mathrm{Cr}_{2} \mathrm{O}_{7}$, and with silver in $\mathrm{K} \cdot \mathrm{Ag}(\mathrm{CN})_{2}$.

The non-metallic or negative elements are found chiefly in negative radicals and ions. They form no nitrates, sulphates, carbonates, etc., for they could not do so without themselves alone constituting the positive ion. We have no such salts of sulphur, carbon, or phosphorus, for example. Their hydroxides, although their formulæ may be written $\mathrm{ClO}_{2} \mathrm{OH}, \mathrm{P}(\mathrm{OH})_{3}, \mathrm{SO}_{2}(\mathrm{OH})_{2}$, furnish no hydroxyl ions, as this would involve the same consequence. These hydroxides are divided by dissociation, in fact, so that the non-metal forms part of a compound negative radical, and the other ion is hydrogen-ion, $\mathrm{ClO}_{3} \cdot \mathrm{H}, \mathrm{PO}_{3} \mathrm{H} \cdot \mathrm{H}_{2}, \mathrm{SO}_{4} \cdot \mathrm{H}_{2}$. Their oxides are acidic. Their halogen compounds, like $\mathrm{PBr}_{3}$ (p. 201) 
and $\mathrm{S}_{2} \mathrm{Cl}_{2}$ (p. 252), are completely decomposed by water, and the actions are not, in general, reversible. The halides of the typical metals are not extensively hydrolyzed (see p. 369), and with those that are not typical, the action is reversible.

The distinction is not perfectly sharp, however. Thus, zinc gives both salts like the sulphate, $\mathrm{Zn} . \mathrm{SO}_{4}$, and chloride, $\mathrm{Zn}$. $\mathrm{Cl}_{2}$, and compounds like sodium zincate $\mathrm{Na} \cdot \mathrm{HZnO}_{2}$.

Classification by Atomic Weights. - Newlands (1863-4) discovered a surprising regularity that became apparent when the elements then known were placed in the order of ascending atomic weight. Omitting hydrogen (at. wt. 1) the first seven were: lithium (7), glucinum (9), boron (11), carbon (12), nitrogen (14), oxygen (16), fluorine (19). These are all of totally different classes, and include first a metal forming a stongly basic hydroxide, then a metallic element of the less active sort, then five non-metals of increasingly negative character, the last being the most active non-metal known. The next element after fluorine (19) was sodium (23), which brings us back sharply to the elements that form strongly basic hydroxides. Omitting none, the next seven elements were sodium, (23), magnesium (24.4), aluminium (27), silicon (28.4), phosphorus (31), sulphur (32), chlorine (35.5). In this series there are three metals of diminishing positive character, followed by four non-metals of increasing negative activity, the last being a halogen very like fluorine. On account of the fact that each element resembles most closely the eighth element beyond or before it $n$ the list, the relation was called the law of octaves. After chlorine the octaves become less easy to trace. That this periodicity in chemical nature is more than a coincidence is shown by the fact that the valence and even many physical properties, such as the specific gravity, show a similar fluctuation in each series. In the first two series the compounds with other elements are of the types: 


\section{$\begin{array}{lllllllll}\mathrm{LiCl} & \mathrm{GlCl}_{2} & \mathrm{BCl}_{3} & \mathrm{CCl}_{4} ; & \mathrm{CH}_{4} & \mathrm{NH}_{3} & \mathrm{OH}_{2} & \mathrm{FH}\end{array}$ \\ $\begin{array}{llllll}\mathrm{Li}_{2} \mathrm{O} & \mathrm{GlO} & \mathrm{B}_{2} \mathrm{O}_{3} & \mathrm{CO}_{2} & \mathrm{~N}_{2} \mathrm{O}_{5} & -\end{array}$

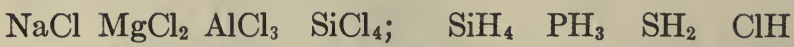

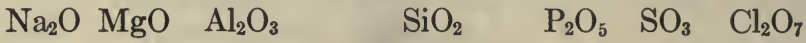

Thus the valence towards chlorine and hydrogen ascends to four and then reverts to one in each octave. The highest valence, shown in oxygen compounds, ascends from lithium to nitrogen with values one to five, and then fails because compounds are lacking. In the second octave, however, it goes up continuously from one to seven.

Again, the specific gravities of the elements in the second series, using the data for red phosphorus and liquid chlorine, are:

$\mathrm{Na}$ 0.97, Mg 1.75, Al 2.67, Si 2.49, P 2.14, S 2.06, Cl 1.33.

Mendelejeff's Scheme.- In 1869 Mendelejeff published an important contribution towards adjusting the difficulty which the elements following chlorine presented, and developed the whole conception so completely that the resulting system of classification has been connected with his name ever since. The table on page 278 , in which the atomic weights are expressed in round numbers, is a modification of one of Mendelejeff's, extended to include elements more recently discovered.

The chief change made by Mendelejeff from the arrangement in simple octaves is that the third series, beginning with potassium, is made to furnish material for two octaves, potassium to manganese and copper to bromine, and is called a long series. The valences fall in with this plan fairly well. Copper, while usually bivalent, forms also a series of compounds in which it is univalent. Iron, cobalt, and nickel fall between the two octaves, and cannot be accommodated in either.

Every long series contains three elements of this character, closely resembling one another. As will be seen from the table; these transition elements, as they are called, may be placed togethe? 
in an eighth group. At the time Mendelejeff made the table, three places in the third, long series had to be left blank, as a trivalent element $[\mathrm{Sc}]$ was lacking in the first octave of the series, and a trivalent [Ga] and a quadrivalent one [Ge] in the second. These places have since been filled, as we shall presently see.

The fourth series, which is a long series exactly similar to the third, contained many blanks at the time of Mendelejeff, but is now nearly complete. It begins with an active alkali metal, rubidium, and ends with iodine, a halogen. The rule of valence is strictly preserved throughout the series, and in general the elements fall below those which they most closely resemble.

The fifth series is still somewhat incomplete, but the order of the atomic weights and the valence enable us satisfactorily to place most of those elements which are known. The chemical relations to elements of the fourth series justify the position assigned to each. Cæsium, for example, is the most active of the alkali metals; barium has always been classed with strontium, and bismuth with antimony.

The sixth and last series contains only a few radioactive elements. No element with an atomic weight greater than that of uranium (238) has yet been discovered.

The most important change made in the table since the time of Mendelejeff is the addition of another group, the family of the inert gases of the atmosphere (see p. 296). These elements were unknown before 1894, but fall logically into a new group at the left hand side of the table as here given. 


\begin{tabular}{|c|c|c|c|c|c|c|c|}
\hline 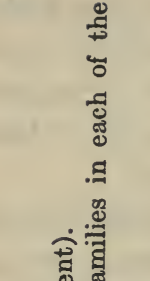 & $\sum_{0}^{\circ}$ & & & 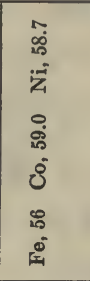 & 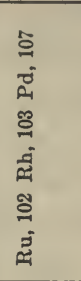 & 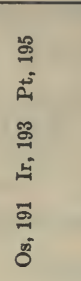 & \\
\hline 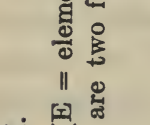 & 보롫 & $\stackrel{9}{9}$ & 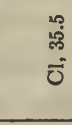 & यू के के & : & $\vdots$ & : \\
\hline 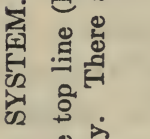 & 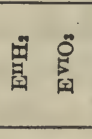 & $\frac{1}{0}$ & $\begin{array}{l}\text { लै } \\
\text { कू }\end{array}$ & . กี คี & \& & $\stackrel{D}{-~}$ & 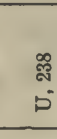 \\
\hline 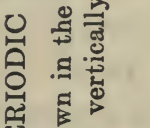 & 若 & $\begin{array}{l}\nexists \\
z\end{array}$ & के & in & 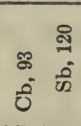 & 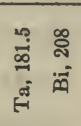 & 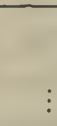 \\
\hline 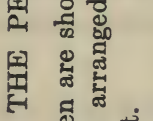 & 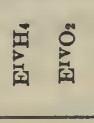 & $\underset{ن}{\tilde{0}}$ & 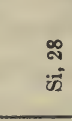 & & $\begin{array}{l}0 \\
\stackrel{8}{\Xi} \\
\text { s }\end{array}$ & 是 & $\begin{array}{l}\text { क् } \\
\text { है }\end{array}$ \\
\hline 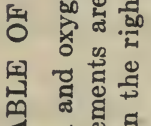 & రొ ฏ & $\begin{array}{l}\Rightarrow \\
\infty 0^{\circ}\end{array}$ & $\begin{array}{l}\text { Aิ } \\
\text { خे }\end{array}$ & 2 & $\underset{\infty}{2} \stackrel{20}{=}$ & 总 & : \\
\hline 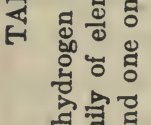 & 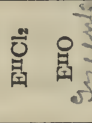 & $\ddot{v}$ & సొ & 유 & 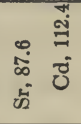 & 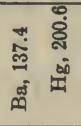 & 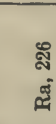 \\
\hline 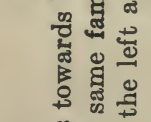 & छ & $\bar{i}$ & ¿ & 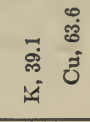 & 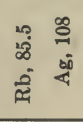 & 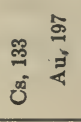 & 犃 \\
\hline 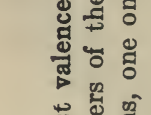 & คิ & 茴 & ฉิ & $\begin{array}{l}\stackrel{9}{0} \\
\ddot{r} \\
\dot{4}\end{array}$ & ஜ & $\stackrel{\dddot{0}}{\dddot{0}}$ & $\begin{array}{l}\text { สี } \\
\text { ż }\end{array}$ \\
\hline 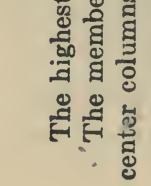 & & 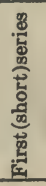 & 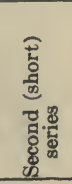 & 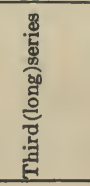 & 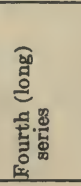 & 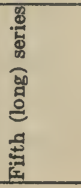 & 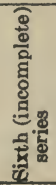 \\
\hline
\end{tabular}


General Relations in the System. - In every octave the valence towards oxygen ascends from one to seven, while that towards chlorine and hydrogen ascends to four and then reverts to one. The long series octaves therefore exhibit the same periodic changes with respect to valence as do the short series octaves already discussed (p. 276). Furthermore, the elements in the new group on the left hand side of the table fall directly into line with the rest by exhibiting zero valence. The inert gases, in other words, form no compounds with other elements. The transition elements on the right hand side of the table, similarly, justify their position by forming a few compounds in which a valence of eight is shown, for example "osmic acid" $\mathrm{OsO}_{4}$. It must be admitted, however, that lower valences are more frequently displayed by these transition elements.

The physical properties, both of the elements themselves and of corresponding compounds, fluctuate within the limits of each series in the same way. Thus the melting-points of the first eight elements in the third series are as follows:

$$
\mathrm{A}-188^{\circ}, \mathrm{K} 62^{\circ}, \mathrm{Ca} 810^{\circ}, \mathrm{Sc}-\text {, Ti } 1790^{\circ}, \mathrm{V} 1720^{\circ}, \mathrm{Cr} 1520^{\circ}, \mathrm{Mn} 1260^{\circ} \text {. }
$$

All of the elements in the same column do not show the same degree of resemblance. We find, instead, that there are two well-defined families in each of the columns forming the octaves. In each long series the element in the first octave falls into one of these families, the element in the second octave into the other. In the table on p. 278 these two families are differentiated in each column by spacing one towards the left and the other towards the right of the available space. Thus in the second column of the table we have the family of the alkali metals ( $\mathrm{Li}, \mathrm{Na}, \mathrm{K}, \mathrm{Rb}, \mathrm{Cs})$ and the copper family $(\mathrm{Cu}, \mathrm{Ag}, \mathrm{Au})$. The members of the first family, and their corresponding compounds, are all strikingly similar in physical and chemical properties. The members of the second family, on the other hand, have little in common with those of the first except in their valence, and even here abnormal values 
are shown in well-known compounds such as cupric salts, which contain the radical $\mathrm{Cu}^{\mathrm{II}}$.

The inert gases, on the left of the octaves, constitute a single family. As for the transition elements on the right, while the three members in any one series resemble one another in many respects, yet a closer relationship between elements in different series, according to the vertical arrangement shown in the table, dividing the group into three families, is also evident.

If we examine the physical properties of successive elements, or corresponding compounds of successive elements, of any one family we find a uniform gradation observable, just as in the cases of the halogens and their hydrogen compounds studied in chapter XVII. Thus the melting-points of the alkali metals are as follows:

$$
\mathrm{Li} 186^{\circ}, \mathrm{Na} 96^{\circ}, \mathrm{K} 62^{\circ}, \mathrm{Rb} 38^{\circ}, \mathrm{Cs} 26^{\circ} \text {. }
$$

As yet no exact mathematical (quantitative) relation between the values for any property and the values of the atomic weights has been discovered; only a general (qualitative) relationship can be traced. Anticipating the discovery of some more exact mode of stating the relationship in each case, and remembering that similar values of each property recur periodically, usually at intervals corresponding to the length of an octave or series, the principle which is assumed to underlie the whole, the periodic law, is stated thus: All the properties of the elements are periodic functions of their atomic weights.

That the chemical relations of the elements vary just as do the physical properties of the simple substances is easily shown. Thus, each series begins with an active metallic (positive) element, and ends with an active non-metallic (negative) element, the intervening elements showing a more or less continuous variation between these limits. Again, the elements at the top are the least metallic of their respective columns. As we descend, the members of each group are more markedly metallic (in the first columns), or, what is the same thing, less markedly non-metallic (in the later columns). 
Applications of the Periodic System.-The system has found application chiefly in four ways:

1. In the prediction of new elements. Mendelejeff (1871) drew attention to the blank then existing between calcium (40) and titanium (48). He predicted that an element to fit this place would have an atomic weight 44 and would be trivalent. From the nature of the surrounding elements, he very cleverly deduced many of the physical and chemical properties of the unknown element and of its compounds. In 1879 Nilson discovered scandium (44), and its behavior corresponded closely with that predicted. Mendelejeff described accurately two other elements, likewise unknown at the time. In 1875 Lecoque de Boisbaudran found gallium, and in 1888 Winkler discuvered germanium, and these blanks were filled.

2. By enabling us to decide on the correct values for the atomic weights of some elements, when the equivalent weights have been measured, but no volatile compound is known (cf. pp. 77 and 86). Thus, the equivalent weight of indium was 38 and, as the element was supposed to be bivalent, it received the atomic weight 76 . It was quite out of place near arsenic (75), however, being decidedly a metallic element. As a trivalent element with the atomic weight 115 , it fell between cadmium and tin. Later work fully justified the change. More recently, when radium was discovered, it was found to have the equivalent weight 113 and to resemble barium. Consequently we assume that, like barium, it is bivalent, and assign it a vacant place under this element, in the last series.

3. By suggesting problems for investigation. The periodic system has been of constant service in the course of inorganic research, and has often furnished the original stimulus to such work as well.

For example, the atomic weights of the platinum metals at first placed them in the order, Ir (197), Pt (198), Os (199), although the resemblance of osmium to iron and ruthenium would have led us to expect that this element should come first. For similar

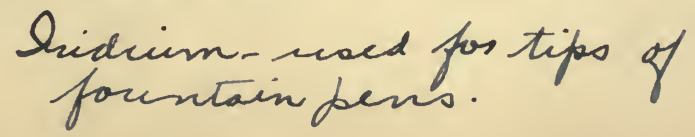


reasons platinum should have come last, under palladium. A reinvestigation of the atomic weights, suggested by these considerations, was undertaken by Seubert, and the old values were found in fact to be very inaccurate. He obtained:

$$
\text { Os }=191, \mathrm{Ir}=193, \mathrm{Pt}=195 .
$$

Originally lead, although it fell in the fourth column, possessed only one compound $\mathrm{PbO}_{2}$ in which it seemed to be undoubtedly quadrivalent. Search for salts of the same form, however, speedily yielded the tetrachloride $\mathrm{PbCl}_{4}$, tetracetate, and many others. The existence of osmic acid $\mathrm{OsO}_{4}$, and a corresponding compound of ruthenium, suggests that other compounds of the elements of the eighth group, displaying the valence eight, may be capable of preparation. The collocation of copper, silver, and gold, in the same column with the alkali metals, is not at present perfectly satisfactory, and suggests the advisability of strengthening their position, if possible, by further investigation.

In the same way, incorrect values of many physical properties have been detected, and have been rectified by more careful work.

4. By furnishing a comprehensive classification of the elements, arranging them so as to exhibit the relationships among the physical and chemical properties of the elements themselves and of their compounds. Constant use will be made of this property of the table in the succeeding chapters. Having disposed of the halogen and sulphur families, situated, respectively, in the eighth and seventh columns of the table on p. 278, we shall presently take up nitrogen and phosphorus from the right side of the sixth column. Then from the fifth column, we shall select carbon and silicon, and from the fourth boron, leaving the other, more decidedly metallic elements for later treatment.

Defects in the Periodic System.-The periodic system is often described as if it furnished a classification of the properties 
of chemical substances which was complete in its scope, and ideal in its exactness. This, however, is far from being the case.

The order of activity of the metals (p. 54) and of the non-metals (p. 209) summarizes many properties, and explains many features of the chemical behavior of the elements. This list is scattered through the periodic table (compare both), without any trace of regularity.

The periodic system concentrates attention too largely on one of the valences of each element. Thus, for manganese, it focuses attention on the septivalent form in the permanganates. But manganous salts are more like the ferrous, the cobaltous, the chromous, and other sets of salts, none of which are in the same column of the table. Similarly, the manganic salts are like the ferric salts and the salts of aluminium. Again, copper is univalent in one series of salts, but in its better known salts it is bivalent. Silver, which belongs to the same periodic family, is always univalent, while gold, also in the same family, is univalent or trivalent, and in the latter case is almost wholly a non-metallic element. If it were possible to place each element in several different columns, one for each of the valences that it shows, the table would then include far more of the properties of the elements. But this cannot be done, for, according to the order of magnitude of the atomic weights, there is but one place for each element. In other words, the periodic system largely ignores the variety of different classes of chemical relations which an element with several valences always shows.

The position of hydrogen in the system is still a matter of dispute. It is more familiar to us as a univalent positive radical resembling the alkali metals in forming compounds with negative radicals such as chlorine, but it can also function as a univalent negative radical, resembling the halogens in forming hydrides with the alkali metals which are analogous to chlorides. Most chemists shelve the difficulty by giving hydrogen a position all to itself at the top of the table. 
Between cerium (140) and tantalum (181.5) in the fifth series, there occur fourteen rare elements, called the elements of the rare earths, which have been omitted entirely from the Mendelejeff system. What is to be done with these elements is a point on which agreement has not yet been reached.

Finally, reference to the table will show that in three cases a slight displacement of the order of the elements according to atomic weights is necessary. Argon, an inert gas, is placed before potassium, an alkali metal, although its atomic weight is 0.8 higher. Cobalt is put before nickel because it resembles iron more closely. Tellurium and iodine are placed in that order to bring them into the sulphur and halogen groups, respectively. Their valence and other chemical relations both require this. These three cases constitute undoubted exceptions to the Mendelejeff system of classification. The general agreement, however, is obviously far too remarkable to be due entirely to chance.

In a later chapter it will be shown that recent work on atomic structure throws considerable light on the several abnormalities discussed above, supplying us with a more logical basis for the periodicity exhibited by the elements in respect to valence and other properties than is furnished by the use of Mendelejeff's system alone. Nevertheless the latter will be found to be of valuable service to us throughout the remainder of the book.

Exercises.- 1. There is a blank at the end of the fifth long series, where we should expect to find another halogen (see p. 278). If the element that should fill this blank were to be discovered, what would be its physical and chemical properties? What would be the properties of its compound with hydrogen?

2. How should you attempt to obtain $\mathrm{H}_{2} \mathrm{Te}$, and what physical and chemical properties should you expect it to possess?

3. Make a list of bivalent elements and criticize this method of grouping as a means of chemical classification. 
4. Write down the symbols of the elements in the fourth series (that beginning with rubidium, and ending with iodine) on $p$. 278. Record the valence of each element toward oxygen, using for reference the chapters in which the oxygen compounds are described.

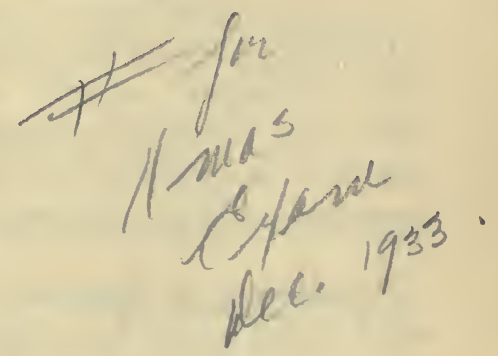




\section{CHAPTER XXIV}

\section{NITROGEN. THE ATMOSPHERE}

IT is time now to return to the atmosphere, of which the most active component, oxygen, has already been discussed. The other chief component, nitrogen, will lead us to ammonia $\mathrm{NH}_{3}$ and nitric acid $\mathrm{HNO}_{3}$, both of which are of great commercial importance, and have interesting derivatives.

Occurrence of Nitrogen.- Aside from the free nitrogen, which forms nearly four-fifths of the bulk of atmospherie air,

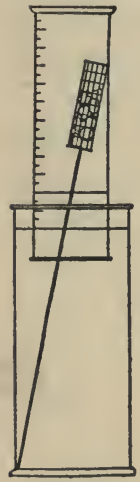

FIG. 72 much nitrogen is found in nature in combination. Potassium nitrate $\mathrm{KNO}_{2}$ is formed in the soil by the action of bacteria upon animal matter, and sodium nitrate $\mathrm{NaNO}_{3}$ is obtained from an immense deposit in Peru and Chili. Nitrogen is an essential constituent of an important class of organic substances called the proteins, which are found in plants, particularly in the fruit, and in the muscles and other tissues of the animal body.

Preparation.- Nitrogen may be obtained from the air by simply removing the oxygen. This nitrogen is not pure, however, as it retains about one per cent of other gases - the "inert gases" of the atmosphere. The oxygen can be removed by allowing pieces of moist phosphorus (Fig. 72) slowly to oxidize in an enclosed specimen of air. The phosphoric acid $\mathrm{H}_{3} \mathrm{PO}_{4}$ and other products of the oxidation of the phosphorus dissolve in the water.

Pure nitrogen can be obtained from pure compounds of nitrogen. Thus, ammonia gas may be passed over heated cupric oxide 286 
(Fig. 73), and the water removed by bubbling the gas through sulphuric acid.

Skeleton: $\quad \mathrm{NH}_{3}+\mathrm{CuO} \rightarrow \mathrm{Cu}+\mathrm{H}_{2} \mathrm{O}+\mathrm{N}_{2}$.

Balanced: $\quad 2 \mathrm{NH}_{3}+3 \mathrm{CuO} \rightarrow 3 \mathrm{Cu}+3 \mathrm{H}_{2} \mathrm{O}+\mathrm{N}_{2}$.

A steady stream of nitrogen is most easily made by heating sodium nitrite and ammonium chloride very gently along with a little water in a flask:

$$
\mathrm{NaNO}_{2}+\mathrm{NH}_{4} \mathrm{Cl} \rightleftarrows \mathrm{NaCl}+\mathrm{NH}_{4} \mathrm{NO}_{2} \rightarrow 2 \mathrm{H}_{2} \mathrm{O}+\mathrm{N}_{2} \uparrow \text {. }
$$

The double decomposition is reversible, and the first action might be expected to be only partially completed. But the ammonium nitrite $\mathrm{NH}_{4} \mathrm{NO}_{2}$ is unstable, and decomposes as fast as it is formed, so that one of

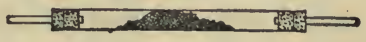

FIg. 73 the substances required to reverse the first reaction is removed, and the reversing action does not occur.

Physical Properties. - Nitrogen is a colorless, tasteless, and odorless gas. Its density is indicated in the formula $\mathrm{N}_{2}$ (mol. wt. $2 \times 14=28$ ). It is very little soluble in water. When liquefied it boils at $-194^{\circ}$.

Chemical Properties.-Nitrogen is chemically a rather indifferent gas. It unites easily with a very few elements, notably some of the most active metals, such as calcium and magnesium. When magnesium burns in the air, the white powder which is formed contains some of the nitride of magnesium $\mathrm{Mg}_{3} \mathrm{~N}_{2}$, along with much of the oxide:

$$
3 \mathrm{Mg}+\mathrm{N}_{2} \rightarrow \mathrm{Mg}_{3} \mathrm{~N}_{2} \text {. }
$$

The presence of the nitride may be shown by the odor of ammonia, given off when the ash is moistened with water:

$$
\mathrm{Mg}_{3} \mathrm{~N}_{2}+6 \mathrm{H}_{2} \mathrm{O} \rightarrow 3 \mathrm{Mg}(\mathrm{OH})_{2}+2 \mathrm{NH}_{3} \uparrow \text {. }
$$


The compounds with oxygen, such as $\mathrm{NO}$ and $\mathrm{HNO}_{3}$, and with hydrogen such as $\mathrm{NH}_{3}$, are of immense commercial value, but, not being very stable, they are formed only in traces by direct union of the elements. The processes for utilizing these tendencies to union, feeble as they are, for manufacturing purposes, will be described under the compounds themselves.

\section{The Atmosphere}

The components of the air may be conveniently divided into regular components and accidental components. The regular components, again, consist of three which are present in practically the same proportions in all samples, and three (namely water, carbon dioxide and dust) which vary markedly in quantity.

\section{Components Present in Constant Proportions. - The} components whose proportions are practically invariable are nitrogen, oxygen, and the group of inert gases. When the variable components are removed, the proportions of the constant ones are as follows:

\begin{tabular}{|c|c|c|}
\hline & By Volume & By Weight \\
\hline $\begin{array}{l}\text { Nitrogen } \ldots \ldots \ldots \ldots \ldots \ldots \ldots \ldots \\
\text { Oxygen } \ldots \ldots \ldots \ldots \ldots \ldots \ldots \ldots \\
\text { Argon } \ldots \ldots \ldots \ldots \ldots \ldots \ldots \ldots\end{array}$ & $\begin{array}{r}78.06 \\
21.00 \\
.0 .94\end{array}$ & $\begin{array}{r}75.5 \\
23.2 \\
1.3\end{array}$ \\
\hline
\end{tabular}

The inert gases, excepting argon, are present in traces only.

The Water Vapor. - The proportion of water vapor in the air is exceedingly variable. When air becomes cool, the moisture separates in cloud and fog, which are composed of minute drops of liquid water. When much moisture is condensed, the drops are larger and fall as rain. When they fall through a cold region, they freeze to hail. When condensation takes place in air already 
below $0^{\circ}$, the fog is composed of solid, and not of liquid particles. The hexagonal crystalline structures of ice which are deposited form snow.

On the other hand, when the weather becomes warm, evaporation goes on rapidly, especially in the neighborhood of seas, lakes, or moist country, and the proportion of water vapor in the air may be considerably increased.

Humidity. - The moisture is usually defined in terms of relative humidity, the standard being the quantity required to saturate the air at the existing temperature. A space filled with air can take up aqueous vapor only until the partial pressure of water vapor becomes equal to the vapor pressure of water (p. 61) at the same temperature. The humidity is then said to be 100 per cent. If the partial pressure actually reached is only half as great as the vapor pressure of water at the same temperature, the humidity is 50 per cent. The average humidity may be placed very roughly at about 66 per cent.

At $18^{\circ}\left(64.4^{\circ} \mathrm{F}\right.$.), the vapor pressure of water is $15.4 \mathrm{~mm}$. (Appendix IV). If the total pressure of the atmosphere were $760 \mathrm{~mm}$., then the air would be saturated with moisture at $18^{\circ}$, and have a humidity of 100 per cent, when $\frac{15.4}{760}$ or about 2 per cent of it by volume was water vapor. Upon cooling to $0^{\circ}$, at which temperature the vapor pressure of water is $4.6 \mathrm{~mm}$., this air would retain only $\frac{4.6}{760}$, or about 0.6 per cent of moisture. At $18^{\circ}$ there would be 16.3 grams of water in a cubic meter of air and at $0^{\circ}$ only 4.9 grams. The difference, 11.4 grams (11.4 c.c.), would be precipitated as fog or rain from each cubic meter.

Test for Moisture in Air.- The presence of moisture in air may be shown by placing any deliquescent (p. 118) salt, such as calcium chloride, in an open vessel. The quantity can be measured by driving a known volume of air slowly through a weighed 
tube containing dry calcium chloride. It may be ascertained also by noting the temperature to which the air has to be cooled before it becomes saturated and deposits fog or dew. For example, if air at $18^{\circ}$ has to be cooled to $11^{\circ}$ before it deposits dew, it contains water vapor at a partial pressure of $9.8 \mathrm{~mm}$. If saturated at $18^{\circ}$, it would have contained water vapor under a partial pressure of $15.4 \mathrm{~mm}$. Its relative humidity was therefore $9.8 / 15.4$, or 63.6 per cent.

Moisture and Comfort.-The chemical changes occurring in our bodies, and particularly the oxidation of waste and of digested food by oxygen carried by the blood, are accompanied by liberation of heat. Yet our bodies must remain at $98.4^{\circ} \mathrm{F}$. $\left(37^{\circ} \mathrm{C}\right.$.). A rise of a few tenths of a degree (F.) produces noticeable discomfort. Much of the heat is lost by radiation from the surface. The extent of this loss depends upon the surface, which is invariable, and upon the surrounding temperature, which we can not always control. Non-conducting clothes reduce the radiation, and are increased in thickness in cold weather. The real adjustment, however, is accomplished, independently of radiation, by evaporation of water at the surface of the skin. The evaporation of 1 gram of water requires about 540 calories of

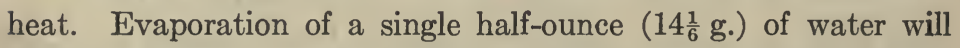
therefore lower the temperature of $76 \frac{1}{2}$ kilograms (168 pounds) of water (or of flesh, which is largely water) by one-tenth of a degree C. (nearly $0.2^{\circ} \mathrm{F}$.).

Our comfort, then, depends upon the possibility of continual, moderate evaporation from the surface of our bodies. "Much" moisture in the air means, to us, not necessarily a great absolute amount, but a near approach to the maximum possible at the existing temperature. So the ratio of the amount present, to the maximum - the humidity - is the significant fact for a practical purpose, such as feeling comfortable (or drying the wash quickly). 
Ventilation.- In winter, cold air is brought into our rooms. The amount of water vapor contained in this air, even if it is saturated with moisture, is very small (see Appendix IV). When this air has been heated, therefore, its relative humidity is too low, discomfort is felt because there is too much evaporation, and moisture has to be added artificially. Here the moisture afforded by evaporation from our bodies has little effect on the air. In summer, however, the outside air is often already nearly saturated at the temperature of the room. At such times the speed of displacement by the ventilating appliances may not be great enough to keep the relative humidity down, and discomfort will arise from the opposite cause. To relieve it, the evaporation may be promoted by electric fans. They do not remove or add any air, but they stir it, and blow away the moist, nearly saturated, layers next to the skin.

The chief purposes of ventilation are, therefore, to supply fresh air, to keep it in motion, and to maintain a humidity that is neither too low nor too high.

The Carbon Dioxide. - The breathing of animals, the combustion of coal and wood, and the decay (oxidation) of vegetable and animal matter produce carbon dioxide $\mathrm{CO}_{2}$. The same gas issues from volcanoes, and often in great quantities from the soil in regions which are no longer volcanic. The proportion in the air is therefore greatest in cities and in some volcanic regions, and least in the country and over the sea. It varies from 3.5 parts in 10,000 in the country, to 1 per cent in crowded rooms.

Its presence may be proved in any air, and very quickly in the breath itself, by bubbling the air through calcium hydroxide solution (lime-water). Calcium carbonate is precipitated (p. $336)$.

Carbon Dioxide and Respiration.- We draw about half a liter of air into our lungs at each breath, or half a cubic meter per 
hour. In the lungs some of the oxygen is removed, and some carbon dioxide is added.

\begin{tabular}{|c|c|c|}
\hline & $\begin{array}{c}\text { Fresh Air, } \\
\text { Per Cent }\end{array}$ & $\begin{array}{c}\text { Expired Air, } \\
\text { Per Cent }\end{array}$ \\
\hline 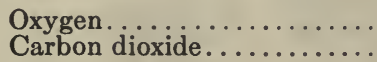 & $\begin{array}{r}21.00 \\
0.04\end{array}$ & $\begin{array}{r}15.9 \\
3.7\end{array}$ \\
\hline
\end{tabular}

A candle flame goes out when the proportion of oxygen has fallen to 16.5 per cent. But air will sustain life until the proportion has fallen to about 10 per cent.

Nearly all experts are now convinced that the unhealthiness of over-crowded, "stuffy" rooms is not due to the increase in the proportion of carbon dioxide, which is seldom great enough to do any damage. Nor is it due to "poisons" given off by the lungs or skin. In spite of many experiments the presence of such substances has never been proved - they are imaginary. The harm is caused by the stillness of the air, which, as we have seen (p. 291), prevents the removal of the water vapor near the skin, and therefore hinders evaporation.

Dust in the Air.- A beam of sunlight crossing a dark room can be seen by the light reflected from the particles of dust which all air contains. These are chiefly solid bodies, and are composed of salts, limestone, clay, and other rock materials, of soot and other particles of unburnt fuel, of bits of hay or straw, and of fragments of insects and other débris of plants and animals. They also include living particles, such as bacteria, and spores of plants such as moulds. The latter, when they settle upon food, germinate and give rise to putrefaction. Some of the bacteria also produce disease, when they enter the body at a place where the skin has been damaged by a cut or burn.

It is instructive to note that natural soil contains about 100,000 micro-organisms, and good, unfiltered river water from 6000 to 
20,000 , in each cubic centimeter. Ordinary, pure air contains only 4 to 5 micro-organisms per liter. Most of these bacteria come from the drying of soil and the dispersion of the resulting dust.

If dust were not present, we should soon notice its absence. There would be no clouds or rain. It appears that moisture will not condense to fog or rain in air which has been filtered, by being drawn through a wide tube containing a long ( 20 inches or more) plug of cotton, and has so been freed from dust. The particles act as nuclei, round which the liquid grows at the expense of the vapor. In the absence of dust, the condensation would occur directly upon the surfaces of plants, houses, and animals. Thus, in a dustless atmosphere, an open shed or shelter, or an umbrella, would afford no protection whatever against a wetting.

The formation of fog from ordinary air, and its nonformation in filtered air are easily shown in a darkened room (Fig. 74). The flask

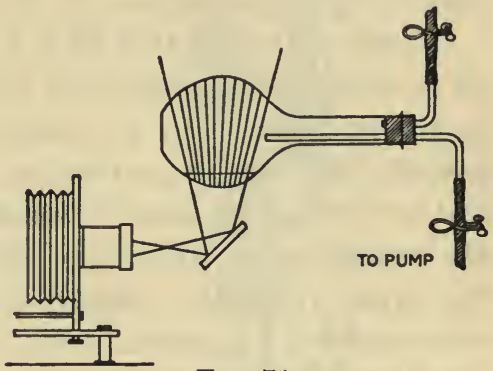

Fig. 74 contains water to saturate the air. When the tube leading to the water pump is opened for an instant, the saturated air in the flask expands and is cooled. In such circumstances, ordinary air gives a fog, brilliantly illuminated by the beam of light, while filtered air (dustless) gives none.

Air a Mixture.- The air does not contain in combined condition the various substances we have named. Each of the substances in air shows precisely the same properties which it exhibits when free, separate, and pure. This behavior is characteristic of a mixture. 
Thus, the observed density of the air is precisely that which we find by calculation from the known proportions and several densities of the components. The solubility of each gas is observed to be the same as if it were alone present.

Again, when liquefied air is allowed to evaporate in a suitable apparatus, the nitrogen, being more volatile, can be separated almost completely from the oxygen. When the oxygen is, in turn, allowed to evaporate, the carbon dioxide and water remain as solids, frozen by the low temperature.

Finally, the exact proportions can not be represented by a chemical formula. This shows that the law that, in chemical compounds, the proportions can be represented by multiples of the atomic weights by whole numbers (p. 77), does not apply to air.

In spite of the fact that air is a mixture, the composition of the air is remarkably uniform and constant. The uniformity is due to constant mixing by the winds. The steadiness of the composition from year to year is due to the fact that, although decay and combustion continually remove oxygen and add carbon dioxide, vegetation as continually consumes the latter and restores the former (p. 396). The mass of carbon dioxide in the whole atmosphere of the planet, about 2450 thousand million tons, is so great that the amounts added and removed by the agencies just mentioned are small by comparison.

\section{(Liquefaction of Gases.) The principle now used in liquefy-} ing gases depends on the fact that, although a perfect gas, when expanding into a vacuum, should suffer no fall in temperature, since it does no work, ordinary gases do become cooled very slightly. The work which they do in expanding in such circumstances is done in overcoming the cohesion between their molecules (p. 91), so that a tearing apart of the substance, which consumes heat, has to take place. Since this cohesion becomes more conspicuous the lower the temperature, the cooling effect of expansion becomes greater and greater as the temperature falls. 
The most successful apparatus for use on a small or large scale is that devised by Hampson. In this apparatus (Fig. 75), two concentric copper pipes, about 130 meters in length, are coiled closely in a cylindrical form, with non-conducting covering to prevent access of heat from the outside. Air at 130-150 atmos-

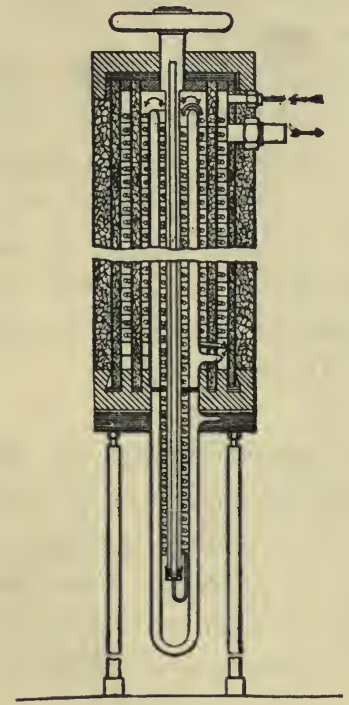

Fig. 75

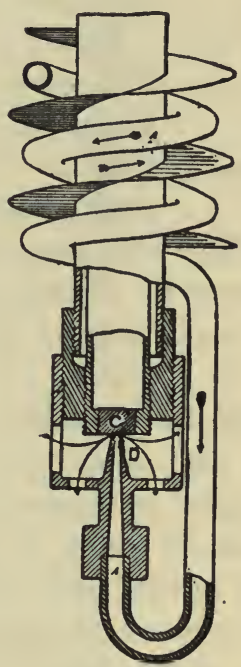

FIG. 76

pheres pressure is forced through the inner pipe (upper opening Fig. 75). When it reaches the extremity of this pipe, it suddenly escapes into a closed vessel. This expansion lowers its temperature. A spiral partition between the coils produces the outer tube of which we have spoken. The gas in the tube A (Fig. 76) is under a pressure of $130-150$ atmospheres. The distance of the nozzle $\mathrm{D}$ from the plug $\mathrm{C}$ is adjusted so that the pressure of the gas in the chamber and spiral outer tube is reduced to one atmosphere. The air can now escape only by traveling back through the outer pipe to the final, wider exit near the top. In doing so, 
it cools the highly compressed air in the inner pipe. The cooler air, on reaching the closed vessel, expands and becomes colder than ever, and in passing backwards lowers the temperature of the air in the inner pipe still further. Finally, the air in this pipe liquefies and drops of liquid air are expelled into the closed vessel.

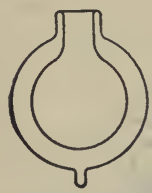

Fig. 77 This is allowed to run out through a valve, from time to time, as it accumulates.

Liquid air can be kept in Dewar flasks (Fig. 77). The space between the inner and outer flasks is evacuated, so that there is no gas to carry heat from the atmosphere in to the liquid air. The inner surface of the outer flask is often silvered, so that radiant heat, from surrounding bodies, may be reflected and not absorbed. Similar containers are in common use for keeping liquids hot or cold for a long time (Thermos flasks).

Liquid Air.- - Liquid air varies in composition, as the nitrogen (b.-p. $-194^{\circ}$ ) is less condensible than the oxygen (b.-p. $-181.4^{\circ}$ ). When liquid air evaporates, therefore, the first portions of gas that come off consist almost entirely of nitrogen. Pure nitrogen obtained in this way is used in the manufacture of ammonia by the Haber process (p. 300), and in the formation of calcium cyanamide (p. 392). By allowing evaporation to continue a liquid o sntaining 75 to 95 per cent of oxygen is obtained. This is pumped into cylinders and sold as compressed oxygen. It contains about 3 per cent of argon, and is a convenient source of this element. Cartridges made of granular charcoal and cotton waste, when saturated with oxygen-rich liquid air, are used as an explosive in mining.

\section{The Inert Gases}

Argon.- Lord Rayleigh was the first to observe that a liter of pure nitrogen weighed $1.2505 \mathrm{~g}$., while a liter of atmospheric "nitrogen" weighed $1.2572 \mathrm{~g}$. The natural inference was that 


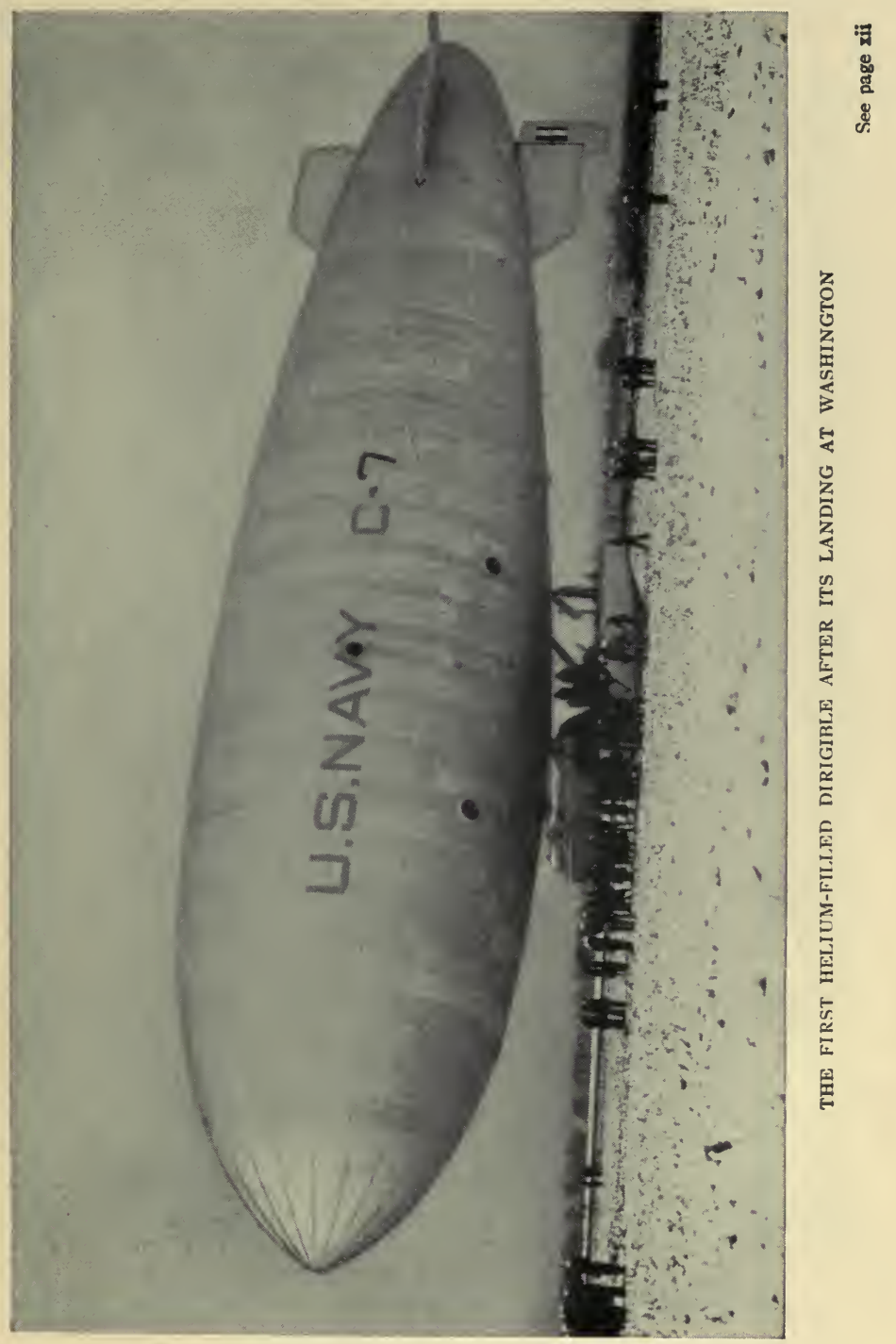




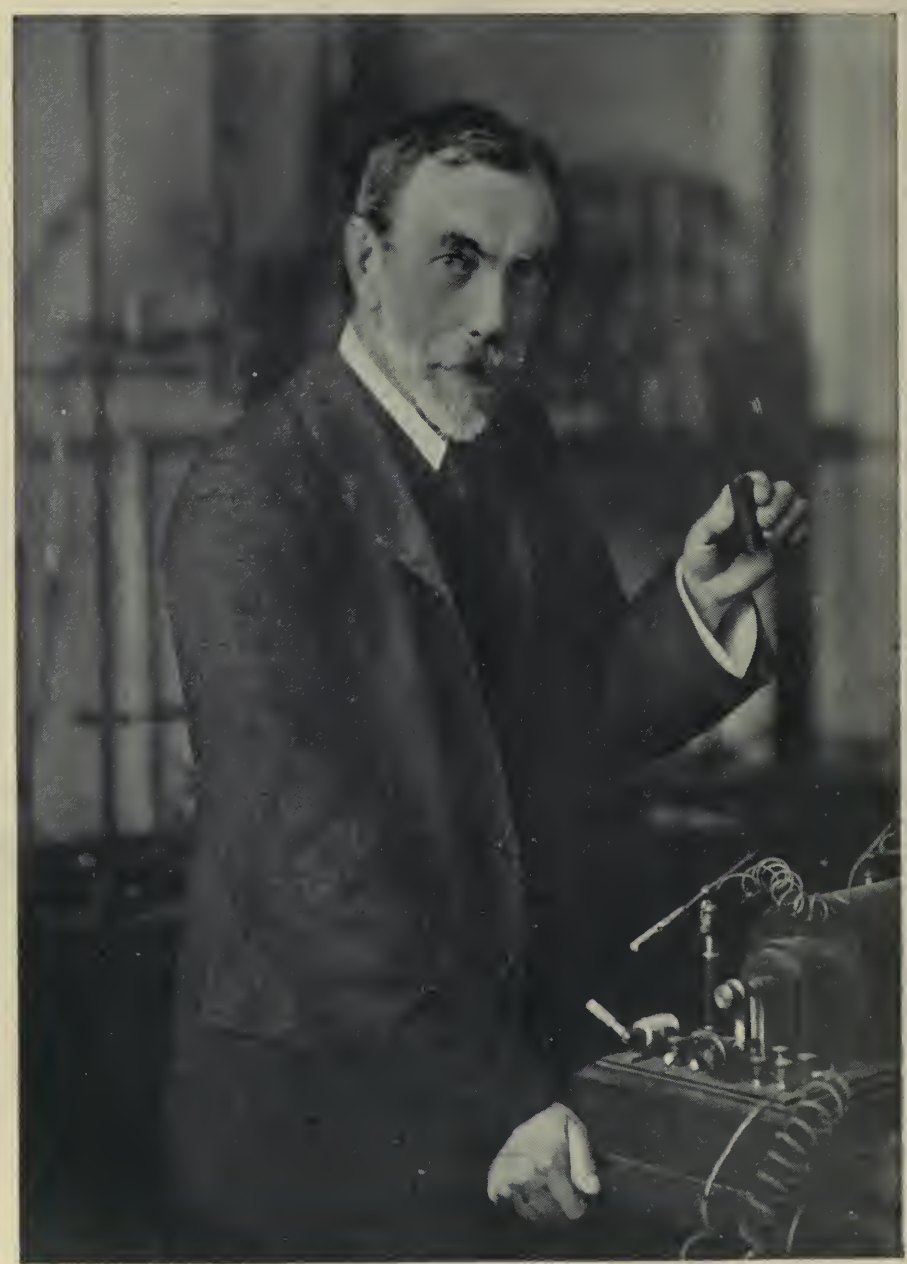

See page xii 
the latter contained a little of some heavier gas. In 1894 Ramsay, in consultation with Rayleigh, succeeded in separating this gas by passing the "nitrogen" repeatedly over heated magnesium, and so removing the real nitrogen as solid magnesium nitride $\mathrm{Mg}_{3} \mathrm{~N}_{2}$. The remaining gas, about 1 per cent of the whole, was named argon (Greek, lazy or inactive), because it would combine with no other element.

Argon has a molecular weight of 39.9 (nitrogen only 28), and when liquefied boils at $-186^{\circ}$ and freezes at $-189.5^{\circ}$. It is used in filling electric light bulbs.

Helium.-An indifferent gas, previously known to be given off when uranium ores were heated in a vacuum, was found by Ramsay (1895) to be neither nitrogen, nor yet argon. By its spectrum it was recognized to be helium (Greek, the sun), a substance shown in 1868 to be present in the sun. Its molecular weight is 4 , so that it is only twice as dense as hydrogen. It was the last gas to be liquefied (by Onnes), and the liquid boils at $-268.7^{\circ}\left(4.3^{\circ} \mathrm{Abs}.\right)$. Like argon, it does not enter into chemical combination. Helium is now being used to fill balloons, because it is not combustible.

Other Inert Gases.- When liquefied argon was allowed to evaporate, the first vapor coming off was found to contain another gas, neon (Greek, new; Mol. wt. 20), along with helium. Careful distillation of the remaining liquid gave two other gases krypton (Greek, hidden; Mol. wt. 83) and xenon (Greek, stranger; Mol. wt. 130). The total amount of these four gases, however, was only 1 part in 80 , the remaining 79 parts being pure argon. None of these gases form any compounds. They do not combine with themselves even, as do the more common gases such as $\mathbf{H}_{2}$, $\mathrm{O}_{2}, \mathrm{Cl}_{2}$. The molecule in each case is monatomic, for example $\mathrm{He}, \mathrm{A}$. The valence throughout the group is therefore zero. 
Exercises.-1. Classify (p. 132) each of the reactions represented by equations in this chapter.

2. What are the radicals of sodium nitrite, and what their valences? Justify the nomenclature.

3. At $77^{\circ} \mathrm{F}$. the air of a room contains water vapor at a partial pressure of $20 \mathrm{~mm}$. What is the percentage of humidity?

4. What weight of water is contained in a cubic meter (1000 liters) of air saturated at $10^{\circ} \mathrm{C}$. ?

5. What weight of carbon is contained in the total carbon dioxide in the earth's atmosphere?

6. Air at $18^{\circ}$ has to be cooled to $14^{\circ}$ before it deposits dew or fog. What is the percentage humidity at $18^{\circ}$ ?

7. Why is the air nearest the ground heated (by the sun) to a higher temperature than the upper air? 


\section{CHAPTER XXV}

\section{AMMONIA}

THE interest in ammonia centers largely in the use of liquefied ammonia for refrigeration, in the employment of the gas in making carbonate of soda, and in the value of its compounds as fertilizers and explosives.

Manufacture.-Ammonia is formed when nitrogenous organic matter is heated, in absence of air. It was formerly made by distilling scraps of hoofs, horns, and hides. The solution of the gas thus obtained was called "spirit of hartshorn." The pungent odor of smoldering feathers, leather, or fur is, therefore, partly due to its presence in the escaping vapors. From the proteins of the original plants, coal derives a considerable proportion of nitrogenous matter. Hence, when coal is distilled for the making of coal gas, or, on a far larger scale, for the making of coke, much ammonia can be separated, by washing with water, from the mixture of gases produced. The aqueous solution is separated from the tar, neutralized with sulphuric acid, and evaporated to give the salt, ammonium sulphate $\left(\mathrm{NH}_{4}\right)_{2} \mathrm{SO}_{4}$.

$$
\begin{aligned}
& \mathrm{NH}_{3}+\mathrm{H}_{2} \mathrm{O} \rightarrow \mathrm{NH}_{4} \mathrm{OH} \text { (ammonium hydroxide). } \\
& 2 \mathrm{NH}_{4} \mathrm{OH}+\mathrm{H}_{2} \mathrm{SO}_{4} \rightarrow\left(\mathrm{NH}_{4}\right)_{2} \mathrm{SO}_{4}+2 \mathrm{H}_{2} \mathrm{O} .
\end{aligned}
$$

The distillation of coal is the chief source of commercial ammonia. In the United States, prior to the war, nearly all the coke was made in "beehive" ovens, in which the vapors issuing from the coal are burned, uselessly, on the spot. Since the war, about 75 per cent of coke is made in "by-product" coke ovens, in which the ammonia and innumerable other by-products are collected and utilized (see p. 424). In Scotland, oil-bearing shale 
is distilled for the purpose of extracting the petroleum, and much ammonia, liberated at the same time, is collected. Formerly it was allowed to escape, but, in the absence of a protective tariff, the competition of petroleum from American and Russian wells compelled economy. Now, the profit on the sale of the ammonium sulphate pays the whole cost of mining and distilling the shale.

Synthetic Ammonia. - The latest method of manufacturing ammonia is by the direct union of hydrogen and nitrogen.

Exactly the same difficulties are encountered in the commercial operation of this reaction (Haber's process) as in the manufacture of sulphur trioxide by the contact process (p. 261), but in a greatly accentuated form. The union of the gases, which is exothermic, is exceedingly slow in the absence of a suitable catalyst:

$$
\mathrm{N}_{2}+3 \mathrm{H}_{2} \rightarrow 2 \mathrm{NH}_{3}+24,000 \text { calories. }
$$

In the presence of a contact agent - such as a specially prepared mixture of iron and molybdenum - combination is greatly hastened. Traces of other gases, however, such as carbon monoxide or hydrogen sulphide, must be very carefully eliminated from the reacting mixture, since they act as poisons on the catalyst, that is, they destroy or impair its activity.

The reaction is reversible, and much more incomplete than is the union of sulphur dioxide and oxygen under similar conditions. Since the forward action evolves heat, the reverse action is favored by raising the temperature (van't Hoff's law, p. 242), hence the yield of ammonia in the equilibrium mixture becomes less and less the higher the temperature employed. Thus, under one atmosphere pressure, the proportions of the gases that combine in a mixture of one volume nitrogen and three volumes hydrogen are as follows: at $200^{\circ}, 15.3$ per cent; at $300^{\circ}, 2.2$ per cent; at $500^{\circ}, 0.13$ per cent; at $1000^{\circ}, 0.004$ per cent.

The preponderance of this reverse reaction, or in other words the tendency of ammonia to decompose into its constituent 
elements as the temperature is raised, makes it impossible to obtain high yields of ammonia by the Haber process at high temperatures, while at lower temperatures the combination is too tardy, even in the presence of a catalyst. Fortunately we are able to make use of another of our general laws, the principle of Le Chatelier (p. 244), to improve matters. It will be noted from the equation given above that the union of hydrogen and nitrogen to form ammonia is accompanied with diminution of volume, 1 volume of nitrogen +3 volumes of hydrogen $=2$ volumes of ammonia. Consequently the forward action will be favored by increase of pressure. In fact, under 200 atmospheres pressure the yield of ammonia in the equilibrium mixture is as follows: at $200^{\circ}, 86$ per cent; at $500^{\circ}, 17.6$ per cent; at $600^{\circ}$, 8.2 per cent; at $1000^{\circ}, 0.9$ per cent.

There still remains the question of speed of combination. This decreases very rapidly as the temperature is lowered (compare p. 241), and no catalyst has yet been prepared which is sufficiently active to make the combination of nitrogen and hydrogen speedy enough to permit the process to be operated on an industrial scale much below $600^{\circ}$. A yield of about 8 per-cent ammonia, therefore, is the best that can be obtained.

Details of the Haber Process. - The hydrogen may be obtained either as a by-product in an electrolytic process (p. 166), or by the action of steam on iron (p. 51), or by careful purification of water-gas (see p. 338). The preparation of pure hydrogen, it may be noted, is the most costly feature of the whole process. The nitrogen is obtained from liquid air. After removal of all impurities injurious to the catalyst, the mixed gases are passed under high pressure into the vessel containing the catalyst. This consists of a steel "bomb" specially adapted to withstand the enormous pressure. Very serious disasters have taken place owing to the explosion of such bombs. After passing over the catalyst, the reaction mixture is cooled and its ammonia content 
( 6 to 8 per cent) removed either by refrigeration or by absorption in water. The residual nitrogen and hydrogen are returned to the plant for further treatment.

The ammonia obtained is used, in times of peace, mainly in the manufacture of fertilizers, such as ammonium sulphate. In war times, however, it is required more urgently for the production of explosives. Nitric acid $\mathrm{HNO}_{3}$, which is necessary in the preparation of most explosives, is obtained from ammonia by oxidation (see p. 313). By the neutralization of nitric acid with ammonia, ammonium nitrate $\mathrm{NH}_{4} \mathrm{NO}_{3}$ is formed. A mixture of this substance with trinitrotoluene (p. 483) was used extensively as a high explosive during the Great War, under the name of amatol. Synthetic ammonia may also be prepared by the calcium cyanamide process (see p. 392). But for these two processes, Germany would never have been able to continue fighting in the Great War beyond the first year. With all foreign supplies of nitrates (Chili saltpeter) cut off, the only other available source of ammonia was the by-product coke industry, and this was already being utilized almost to its maximum. In the allied countries, unfortunately, the Haber process during the war was not developed beyond the experimental stage.

The productive capacity of Haber process plants in 1920 was no less than one and a half million tons (calculated as ammonium sulphate).

Preparation in the Laboratory. - In the laboratory ammonia is most readily made by heating a mixture of a salt of ammonium, such as the chloride $\left(\mathrm{NH}_{4} \mathrm{Cl}\right)$ or sulphate, and slaked lime $\mathrm{Ca}(\mathrm{OH})_{2}$.

$$
\mathrm{Ca}(\mathrm{OH})_{2}+2 \mathrm{NH}_{4} \mathrm{Cl} \rightarrow \mathrm{CaCl}_{2}+2 \mathrm{NH}_{4} \mathrm{OH} \rightarrow 2 \mathrm{NH}_{3}+2 \mathrm{H}_{2} \mathrm{O} .
$$

The ammonium hydroxide, formed by the double decomposition, immediately decomposes. To free the gas from water vapor, it is passed through a tower filled with quicklime $\mathrm{CaO}$ (Fig. 78):

$$
\mathrm{CaO}+\mathrm{H}_{2} \mathrm{O} \rightarrow \mathrm{Ca}(\mathrm{OH})_{2} \text {. }
$$


Sometimes, a stream of the gas is generated by warming commercial ammonium hydroxide solution (aqua ammonia), and drying the gas as above. Liquefied ammonia is obtainable in small iron cylinders, and is a convenient source when much of the gas is required.

The liberation by hydrolysis of nitrides, already noted (p. 287), is interesting:

$\mathrm{Mg}_{3} \mathrm{~N}_{2}+6 \mathrm{H}_{2} \mathrm{O} \rightarrow 3 \mathrm{Mg}(\mathrm{OH})_{2}+2 \mathrm{NH}_{3}$.

Composition of Ammonia.That ammonia contains nitrogen may be shown by passing the gas over cupric oxide heated in a tube, and collecting the nitrogen over water:

$2 \mathrm{NH}_{3}+3 \mathrm{CuO} \rightarrow 3 \mathrm{Cu}+3 \mathrm{H}_{2} \mathrm{O}+\mathrm{N}_{2}$.

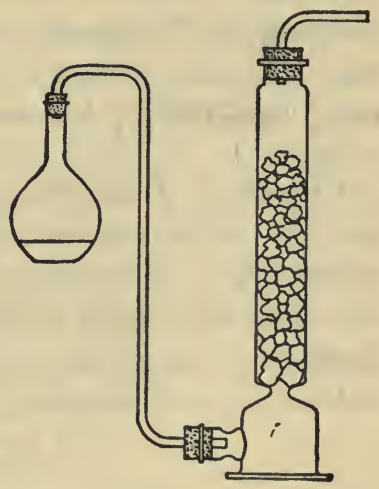

Frg. 78

The hydrogen may be liberated by drying the ammonia, if necessary, with soda-lime, and leading it through a tube containing

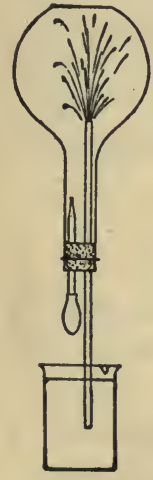

FIG. 79 heated magnesium ribbon:

$$
2 \mathrm{NH}_{3}+3 \mathrm{Mg} \rightarrow \mathrm{Mg}_{3} \mathrm{~N}_{2}+3 \mathrm{H}_{2} .
$$

Physical Properties.-Ammonia is a colorless gas. It has a soapy taste, and a very pungent odor. Its density, recorded in the formula $\mathrm{NH}_{3}$, indicates that it is only about half as heavy, bulk for bulk, as air ( $14+3=17$ against 28.95). It is easily liquefied, boiling at $-38.5^{\circ}$, and exerting a pressure of 6 atmospheres at $10^{\circ}$. The gas is exceedingly soluble in water ( 1 vol. water dissolves 1300 vol. of $\mathrm{NH}_{3}$ at $0^{\circ}$ ). A 35 per cent solution is sold as " concentrated ammonia."

The extreme solubility in water may be shown by the "fountain" experiment (Fig. 79). The flask is filled with am- 
monia by downward displacement of air. The long tube is closed by a short rubber tube and a clip at the bottom (not shown). The "dropper" contains water, and is closed at the tip with soft wax. A few drops of water, squirted into the flask by pinching the "dropper," dissolve at once so much of the gas that the water rushes in, like a fountain, through the longer tube, when the clip is opened.

Chemical Properties.-Ammonia, as we have seen, is not very stable, and decomposes rapidly and almost completely above $700^{\circ}$. A discharge of sparks from an induction coil has the same effect more gradually, and so a sample of the gas confined over mercury in a closed tube may be shown to double in volume when decomposed. Every two molecules give four:

$$
2 \mathrm{NH}_{3} \rightarrow 3 \mathrm{H}_{2}+\mathrm{N}_{2} \text {. }
$$

The most characteristic property of ammonia is that it combines directly with acids, giving ammonium salts:

$\mathrm{NH}_{3}$ (gas) $+\mathrm{HCl}$ (gas) $\rightarrow \mathrm{NH}_{4} \mathrm{Cl}$ (solid particles).

It combines also with water at low temperatures to give ammonium hydroxide $\mathrm{NH}_{4} \mathrm{OH}$ and ammonium oxide $\left(\mathrm{NH}_{4}\right)_{2} \mathrm{O}$, white solids melting around $-80^{\circ}$. These compounds are unstable at ordinary temperatures, so that a solution of the gas, in a great excess of water, is the only form of ammonium hydroxide convenient for use:

\section{$\mathrm{NH}_{3}+\mathrm{H}_{2} \mathrm{O} \rightleftharpoons \mathrm{NH}_{4} \mathrm{OH}$.}

Ammonium Hydroxide.-This substance, as indicated by the way in which we have written its formula, is a base. The ions are $(\mathrm{OH})^{-}$, given by all bases, and $\left(\mathrm{NH}_{4}\right)^{+}$, ammonium-ion, which is found also in the salts mentioned above. The latter is a compound positive radical, playing the part of a univalent metallic element, such as $\mathrm{Na}$ or $\mathrm{K}$.

As a base, ammonium hydroxide, although rather weak (little 
ionized), turns red litmus blue, possesses the characteristic soapy taste and feeling, and enters into double decomposition with acids, neutralizing them:

\section{$\mathrm{NH}_{4} \mathrm{OH}+\mathrm{HCl} \rightarrow \mathrm{H}_{2} \mathrm{O}+\mathrm{NH}_{4} \mathrm{Cl}$.}

The salts, obtained by evaporation, are, of course, identical with those formed by union of ammonia with the same acids.

Ammonium hydroxide used to be known as " volatile alkali," in reference to the fact that it decomposes into its constituents $\left(\mathrm{NH}_{3}+\mathrm{H}_{2} \mathrm{O}\right)$, both of which are volatile, while the other alkalies $(\mathrm{NaOH}$, etc.) are not volatile ("fixed"). This property was utilized in the laboratory method of making ammonia (p. 302).

The Salts of Ammonium.- The salts are ionized in aqueous solution, giving $\mathrm{NH}_{4}$ as the positive ion:

$$
\left(\mathrm{NH}_{4}\right)_{2} \mathrm{SO}_{4} \rightarrow 2 \mathrm{NH}_{4}++\mathrm{SO}_{4}=\text {. }
$$

When heated, dry, in a tube, they are decomposed. Most of them give ammonia and an acid:

$$
\mathrm{NH}_{4} \mathrm{Cl} \rightleftarrows \mathrm{NH}_{3} \uparrow+\mathrm{HCl} \uparrow \text {. }
$$

If the acid is also volatile at, or below, a red heat, like sulphuric acid $\mathrm{H}_{2} \mathrm{SO}_{4}$, the whole salt usually vaporizes. These actions are reversible (read the equation backwards). Hence the acid and the ammonia recombine, and the salt condenses again in a cold part of the tube. This behavior helps us to recognize a salt of ammonium, for the salts of mercury are the only others which behave in this way.

Uses of Ammonia.- Some of the uses have already been mentioned. The ammonia process for making carbonate of soda is described under the latter substance (p. 366).

Refrigeration by liquid ammonia depends upon the fact that liquid ammonia, like any other liquid, absorbs heat in evaporating. It absorbs 260 cal. per gram. To freeze one gram of water at $0^{\circ}, 80$ calories have to be subtracted. Thus, 1 gram of liquid ammonia in evaporating will convert about 3 grams of 
water to ice. The same principle is also largely used for cooling air (in storage rooms for meat, etc.).

The machinery is represented diagrammatically in Fig. 80. The ammonia, first admitted from a bomb of liquefied ammonia, is driven by the pump $F$ along the tube $E$ and condenses to liquid

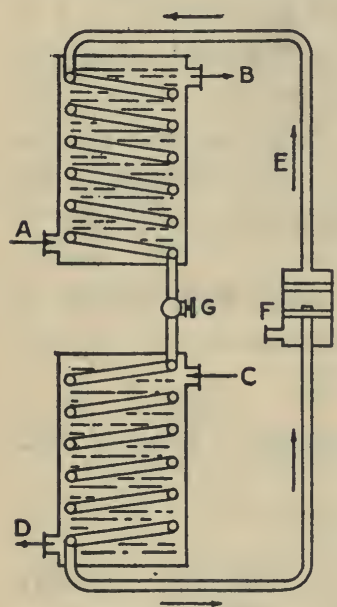

Fig. 80 form in the tube coiled in the $\operatorname{tank} A B$. Cold water circulates through $A B$, and removes the heat produced by the compression and liquefaction of the gas. The liquid ammonia is allowed to drip through the stopcock $G$ into the lower coil. This is kept exhausted by the compresser $F$, and the liquid ammonia evaporates. In doing this, it takes heat from a 30 per cent solution of calcium chloride in water, which does not freeze even at $-12^{\circ}$. This cooled brine leaves the tank at $D$, circulates through another tank (not shown) in which the water-filled ice moulds are suspended, and returns to $C$. When used for cooling refrigerating chambers, the brine passes through a system of pipes suitably placed in the cold-room. The whole machinery is made of iron, as copper and brass are corroded by the ammonia.

Ammonium hydroxide solution is sold under the name of household ammonia, and is used, in washing and cleaning, to soften the water.

Exercises.-1. Classify the reactions shown by equations in this chapter.

2. How could you recognize the nitrogen and the hydrogen obtained in the decomposition of ammonia (p. 304)?

3. Why can we not dry ammonia gas with concentrated sulphuric acid or with phosphorus pentoxide?

4. How could you separate a mixture of oxygen and ammonia? 
5. In what relative volumes do ammonia and hydrogen chloride unite?

6. Write in ionic form the equations for the interaction of ammonium hydroxide: (a) with sulphuric acid; $(b)$ with hydrochloric acid (p. 305). 


\section{CHAPTER XXVI}

\section{NITRIC ACID}

NITRIC acid $\mathrm{HNO}_{3}$ is used in large quantities for making explosives like guncotton, picric acid and TNT, and plastics like celluloid, as well as innumerable drugs and dyes. Nitrates are largely used as fertilizers (p. 410).

Manufacture.- Nitric acid is obtained in two ways, namely, by the action of sulphuric acid upon natural sodium nitrate and by oxidation of the nitrogen of the atmosphere. The processes which utilize the latter method will be referred to in a later section.

Sodium nitrate, Chile saltpeter, is found in an immense deposit ( 2 by 220 miles) on the boundary of Chile and Peru. This salt is mixed with concentrated sulphuric acid in iron retorts and gently heated to drive off the nitric acid. The sodium-hydrogen sulphate romains in the retort:

$$
\mathrm{NaNO}_{3}+\mathrm{H}_{2} \mathrm{SO}_{4} \rightleftarrows \mathrm{NaHSO}_{4}+\mathrm{HNO}_{3} \uparrow .
$$

The vapor is condensed in glass tubes (cooled with water) and the

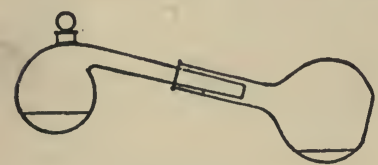

Fig. 81 acid collected in vessels of earthenware. Sulphuric acid (b.-p. $330^{\circ}$ ) is used because it is much less volatile than nitric acid, and so only the latter is vaporized. The acid boils at $86^{\circ}$ (760 mm.), but, to prevent loss by decomposition, a lower boiling-point is secured by reducing the pressure in the whole apparatus.

In the laboratory the same action is employed, without, however, the reduction in the pressure (Fig. 81).

Physical Properties.- Pure nitric acid is a colorless liquid, boiling at $36^{\circ}$. It is miscible in all proportions with water. The 
vapor, like hydrogen chloride gas, condenses moisture from the air, giving a fog of droplets of the solution. The concentrated nitric acid of commerce contains 68 per cent of the acid and boils at $120.5^{\circ}$.

\section{Chemical Properties-Decomposition of Nitric Acid.} - The acid is not very stable. It decomposes, in part, even when simply distilled $\left(86^{\circ}\right)$, giving a red gas, nitrogen tetroxide $\mathrm{NO}_{2}$, water and oxygen:

Skeleton: $\quad \mathrm{HNO}_{3} \rightarrow \mathrm{NO}_{2}+\mathrm{O}_{2}+\mathrm{H}_{2} \mathrm{O}$.

Balanced: $\quad 4 \mathrm{HNO}_{3} \rightarrow 4 \mathrm{NO}_{2}+\mathrm{O}_{2}+2 \mathrm{H}_{2} \mathrm{O}$.

When a reducing agent is present, oxides containing less oxygen than $\mathrm{NO}_{2}$ may be formed, such as nitric oxide NO.

Nitric Acid as an Acid.- An aqueous solution of nitric acid turns blue litmus red, and is a very active, highly ionized acid. With bases it gives nitrates, which can be obtained from the solution by evaporation:

$$
\begin{gathered}
\mathrm{NaOH}+\underset{\mathrm{HNO}_{3}}{\rightarrow} \rightarrow \mathrm{H}_{2} \mathrm{O}+\mathrm{NaNO}_{3} . \\
\mathrm{Ca}(\mathrm{OH})_{2}+2 \mathrm{HNO}_{3} \rightarrow 2 \mathrm{H}_{2} \mathrm{O}+\mathrm{Ca}\left(\mathrm{NO}_{3}\right)_{2} .
\end{gathered}
$$

Nitric Acid as an Oxidizing Agent.- Since nitric acid gives up oxygen with liberation of energy, it is also active as an oxidizing agent. Glowing charcoal, as a powder or in the form of a stick, will burn when pure nitric acid is

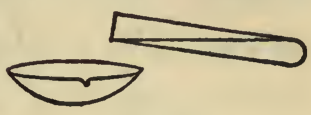

FIG. 82 poured upon it (Fig. 82). Carbon dioxide and red nitrogen tetroxide $\mathrm{NO}_{2}$ are evolved.

Skeleton: $\mathrm{HNO}_{3}+\mathrm{C} \rightarrow \mathrm{NO}_{2}+\mathrm{CO}_{2}+\mathrm{H}_{2} \mathrm{O}$.

Balanced: $4 \mathrm{HNO}_{3}+\mathrm{C} \rightarrow 4 \mathrm{NO}_{2} \uparrow+\mathrm{CO}_{2} \uparrow+2 \mathrm{H}_{2} \mathrm{O}$.

Nitric acid oxidizes indigo and other colored organic compounds, in the same way as do the three oxidizing agents described in Chapter XIX. It also oxidizes hydrochloric acid, upon which 
hydrogen peroxide does not act, so that it is a more active oxidizing agent than is that substance:

$$
\mathrm{HNO}_{3}+3 \mathrm{HCl} \rightarrow \mathrm{NOCl}+2 \mathrm{H}_{2} \mathrm{O}+\mathrm{Cl}_{2} \text {. }
$$

The mixture of concentrated hydrochloric acid, nitric acid, and water is called aqua regia, and has strong oxidizing properties, due to the presence of hypochlorous acid (from $\mathrm{Cl}_{2}+\mathrm{H}_{2} \mathrm{O}$ ) as well as nitric acid.

Action of Nitric Acid on Metals.-Magnesium, and metals above it in the activity list, will displace hydrogen freely, especially from diluted nitric acid:

$$
\mathrm{Mg}+2 \mathrm{HNO}_{3} \rightarrow \mathrm{Mg}\left(\mathrm{NO}_{3}\right)_{2}+\mathrm{H}_{2} \uparrow .
$$

But, with the less active metals, oxidation takes place, and instead of hydrogen, we get water and, of course, a reduction product of the nitric acid. The nitrate of the metal, however, is formed also. Even metals, like copper and silver, which do not displace hydrogen, are acted upon by nitric acid in the same way (compare sulphuric acid, p. 271). For example, diluted nitric acid acts vigorously upon copper, giving nitric oxide $\mathrm{NO}$ as the reduction product:

Skeleton: $\mathrm{Cu}+\mathrm{HNO}_{3} \rightarrow \mathrm{Cu}\left(\mathrm{NO}_{3}\right)_{2}+\mathrm{NO}+\mathrm{H}_{2} \mathrm{O}$.

Balanced: $3 \mathrm{Cu}+8 \mathrm{HNO}_{3} \rightarrow 3 \mathrm{Cu}\left(\mathrm{NO}_{3}\right)_{2}+4 \mathrm{H}_{2} \mathrm{O}+2 \mathrm{NO}$.

The nitric oxide is a colorless gas, but unites with oxygen in the air to give $\mathrm{NO}_{2}$.

A test for a nitrate may be founded on this action. To the nitrate sulphuric acid is added to liberate nitric acid. Then copper turnings are thrown in to give NO. A gas turning red as it meets the air shows that a nitrate was present.

Action Upon Organic Compounds. - Nitric acid stains the skin and nails yellow, by giving colored compounds with the proteins. It gives similar compounds with wool (a protein), and 
therefore produces, on clothing, yellow stains which cannot be removed.

The explosives made by the use of nitric acid are discussed in Chapter XL.

Nitric Oxide NO.- This oxide is made by the action of diluted nitric acid upon copper, by the action already discussed (p. 310).

It is a colorless gas, and almost insoluble in water.

- Vigorously burning phosphorus continues to burn in it:

$$
4 \mathrm{P}+10 \mathrm{NO} \rightarrow 2 \mathrm{P}_{2} \mathrm{O}_{5}+5 \mathrm{~N}_{2} .
$$

Its most important property is that of uniting with oxygen to give nitrogen tetroxide;

$$
2 \mathrm{NO}+\mathrm{O}_{2} \rightarrow 2 \mathrm{NO}_{2} \text {. }
$$

This reaction is reversible at high temperatures.

Nitrogen Tetroxide $\mathrm{NO}_{2}$. - This oxide is formed by the union of oxygen with nitric oxide. It is given off, also, when concentrated nitric acid acts upon metals and other reducing substances. It is further produced, along with oxygen, when nitrates, excepting those of potassium, sodium and ammonium are heated, dry:

$$
2 \mathrm{Cu}\left(\mathrm{NO}_{3}\right)_{2} \rightarrow 2 \mathrm{CuO}+4 \mathrm{NO}_{2}+\mathrm{O}_{2} \text {. }
$$

Potassium and sodium nitrates, when heated, give off only oxygen, and leave the nitrites:

$$
2 \mathrm{KNO}_{3} \rightarrow \mathrm{O}_{2}+2 \mathrm{KNO}_{2} \text {. }
$$

Nitrogen tetroxide is a red gas, with a choking odor. When heated strongly, it becomes colorless, being dissociated into NO and oxygen. When cooled, it becomes pale yellow, and its density becomes twice as great. This is due to the formation of molecules of the formula $\mathrm{N}_{2} \mathrm{O}_{4}$ :

$$
2 \mathrm{NO}_{2} \rightleftarrows \mathrm{N}_{2} \mathrm{O}_{4} \text {. }
$$


The most interesting property of nitrogen tetroxide is its action upon water, whereby nitric acid is formed, and nitric oxide escapes:

$$
3 \mathrm{NO}_{2}+\mathrm{H}_{2} \mathrm{O} \rightarrow 2 \mathrm{HNO}_{3}+\mathrm{NO} \text {. }
$$

When oxygen is present also, then the $\mathrm{NO}$ gives more $\mathrm{NO}_{2}$, and this in turn gives more nitric acid. This action plays an important part in the making of nitric acid from the nitrogen of the air (see next section).

IThe gas is sometimes used for bleaching flour, but traces of the oxide remain in the bread.)

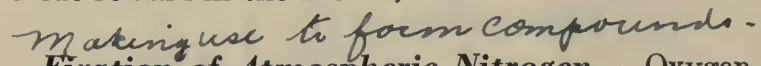

Fixation of Atmospheric Nitrogen.- Oxygen and nitrogen have no natural tendency to combine at the ordinary temperature, but rather the reverse - the compounds tend to decompose with

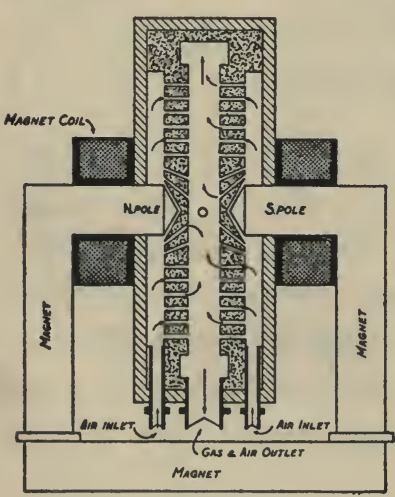

FIG. 83 evolution of heat. But a high temperature will supply the necessary energy. Even so, however, the union extends to only 1 per cent of the mixture at $2000^{\circ}$ and 5 per cent at $3000^{\circ}$ :

$$
\mathrm{N}_{2}+\mathrm{O}_{2} \rightleftarrows 2 \mathrm{NO} \text { - heat }
$$

Note that, since the formation of $\mathrm{NO}$ is an endothermic reaction, the yield of NO is increased by raising the temperature (van't Hoff's law, p. 242). In spite of the poor yield obtainable under the best conditions, the supply of natural nitrates is so limited that machinery has been devised, and is now in successful use, for carrying on the combination on a commercial scale. Three devices are in use, and all employ hydro-electric power.

In the Birkeland-Eyde process (Fig. 83), used at Notodden and elsewhere in Norway, an arc discharge between rods of carbon is spread, by the influence of powerful electromagnets, into a circular The action takes hear - mou hear more wetion yees right-endothenmie. 
brush discharge several feet in diameter. The figure shows a cross section of the space filled by the discharge. In the center is a section of one of the carbon rods. Air is blown through the flame, giving 1 per cent of $\mathrm{NO}$, and is cooled to permit of union of the nitric oxide with oxygen, to give the tetroxide, $\mathrm{NO}_{2}$. The air containing $\mathrm{NO}_{2}$ is then passed through absorbing towers down which water trickles. Here the action mentioned in the last section takes place, and an queous solution of nitric acid is produced. In peace times, the nitric acid is mixed with calcium hydroxide (slaked lime):

$$
\mathrm{Ca}(\mathrm{OH})_{2}+2 \mathrm{HNO}_{3} \rightarrow \mathrm{Ca}\left(\mathrm{NO}_{3}\right)_{2}+2 \mathrm{H}_{2} \mathrm{O}
$$

to give calcium nitrate, which, being very soluble, is sold for use as a fertilizer. In war times, the acid is concentrated for use in the manufacture of explosives.

The Schönherr process, used in the same factories in Norway, employs a discharge through a tube 22 feet long (Fig. 84). The column of air rotates as it traverses the tube and so every part is exposed to the discharge.

The Pauling process, used in Italy and Austria, uses preheated air, and a different arrangement of the discharge. The principles employed are, however, the same.

The productive capacity of plants employing these arc processes in 1920 was three

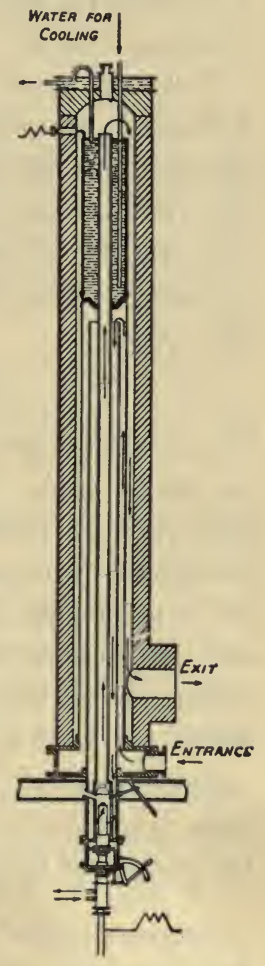

Fig. 84 hundred thousand tons (calculated as nitrate of lime).

Nitric Acid from Ammonia. - The ammonia oxidation process for the production of nitric acid was developed on a large scale during the Great War, particularly in Germany. Gaseous ammonia in the presence of air and a suitable catalyst undergoes 
oxidation, with the formation of axides of nitrogen and water vapor. The oxides of nitrogen can be recovered by absorption in water, yielding dilute nitric acid. This can be concentrated further if desired, or neutralized with a base for the production of nitrates for use as explosives (ammonium nitrate) or fertilizers (calcium nitrate).

Platinum gauze is almost universally employed as a catalyst. A mixture of ammonia and oxygen-enriched air passes through one or more layers of the gauze, which is heated electrically to $650-700^{\circ}$ to start the reaction and which is maintained at that temperature subsequently by the heat of combustion of the ammonia. The reaction that takes place may be represented by the equation:

$$
4 \mathrm{NH}_{3}+5 \mathrm{O}_{2} \rightarrow 4 \mathrm{NO}+6 \mathrm{H}_{2} \mathrm{O} \text {. }
$$

The excess oxygen present converts the $\mathrm{NO}$ into $\mathrm{NO}_{2}$ as the issuing gases cool. Unless the reaction is very nicely regulated, however, the yield of oxides of nitrogen is diminished either by incomplete combustion of ammonia, or by the dissociation of $\mathrm{NO}$ into nitrogen and oxygen (see p. 312). The time of contact with the catalyst must not be too long, or this latter effect will be appreciable. With proper precautions, a conversion efficiency of 90-95 per cent is obtained. Poisoning of the catalyst must be guarded against by careful purification of the gases, especially from non-volatile impurities such as dust particles, which choke the surface of the gauze and render it inoperative.

Nitrous Acid $\mathrm{HNO}_{2}$ and Nitrous Anhydride $\mathrm{N}_{2} \mathrm{O}_{3}$.When an acid, such as sulphuric acid, is added to a solution of a nitrite, like potassium nitrite (p. 311), nitrous acid $\mathrm{HNO}_{2}$ is formed:

$$
2 \mathrm{KNO}_{2}+\mathrm{H}_{2} \mathrm{SO}_{4} \rightarrow \mathrm{K}_{2} \mathrm{SO}_{4}+2 \mathrm{HNO}_{2} .
$$

Nitrous acid, however, like sulphurous acid, is unstable and nitrous anhydride $\mathrm{N}_{2} \mathrm{O}_{3}$ is at once liberated and escapes as a gas:

$$
2 \mathrm{HNO}_{2} \rightarrow \mathrm{H}_{2} \mathrm{O}+\mathrm{N}_{2} \mathrm{O}_{3} \uparrow \text {. }
$$


This gas is used as a catalytic agent in the chamber process ( $p$. 264) for making sulphuric acid. The acid and its anhydride are employed in the manufacture of many dyes.

Nitrous Oxide $\mathrm{N}_{2} \mathrm{O}$ - When ammonium nitrate, a white salt, is heated, it decomposes into steam and nitrous oxide:

$$
\mathrm{NH}_{4} \mathrm{NO}_{3} \rightarrow 2 \mathrm{H}_{2} \mathrm{O} \uparrow+\mathrm{N}_{2} \mathrm{O} \uparrow \text {. }
$$

Nitrous oxide is somewhat more easily liquefied (b.-p. $-90^{\circ}$ ) than is carbon dioxide. At $12^{\circ}$ its vapor pressure is 41 atmospheres. It is sold, as a liquid, in steel cylinders, and used as an anæsthetic for minor operations, chiefly in dentistry. The hysterical symptoms which accompany its use caused it to be named "laughing gas."

Like oxygen, it relights a glowing splinter of wood, and supports combustion brilliantly. It does not interact with nitric oxide ( $p$. 311 ), as does oxygen, however, to form nitrogen tetroxide:

The Writing of Equations. - The reader will have discovered that some of the equations in the present chapter are rather harder than usual to balance correctly, even when the products of the reaction are all known. The following sections should be read through carefully, in order to obtain a thorough understanding of the points under discussion. The hints given will be found of general assistance in writing difficult equations.

Points About Oxygen Acids. - In dealing with an acid that contains oxygen, like nitric acid, there are some things which we must acquire the habit of keeping in mind.

Thus, an oxygen acid, as we have seen (p. 257), can be deprived of water, leaving the anhydride. The chemist always thinks of the one as soon as the other is mentioned. If the acid is named, he instantly subtracts water from its formula to get the formula of the anhydride:

$$
\mathrm{H}_{2} \mathrm{CO}_{3} \rightarrow \mathrm{H}_{2} \mathrm{O}+\mathrm{CO}_{2} \text {. }
$$

Skeleton:

$$
\mathrm{HOCl} \rightarrow \mathrm{H}_{2} \mathrm{O}+\text { ? }
$$


To balance the second equation, hydrogen atoms must be supplied in pairs, so the $\mathrm{HOCl}$ must be multiplied by two:

Balanced:

$$
2 \mathrm{HOCl} \rightarrow \mathrm{H}_{2} \mathrm{O}+\mathrm{Cl}_{2} \mathrm{O} .
$$

With nitric acid the operation is similar:

Skeleton: $\quad \mathrm{HNO}_{3} \rightarrow \mathrm{H}_{2} \mathrm{O}+$ ?

Balanced: $\quad 2 \mathrm{HNO}_{3} \rightarrow \mathrm{H}_{2} \mathrm{O}+\mathrm{N}_{2} \mathrm{O}_{5}$.

This operation should be practiced:

Skeleton: $\quad \mathrm{HClO}_{4} \rightarrow \mathrm{H}_{2} \mathrm{O}+? \quad \mathrm{H}_{3} \mathrm{PO}_{4} \rightarrow \mathrm{H}_{2} \mathrm{O}+$ ?

Balanced: $\quad 2 \mathrm{HClO}_{4} \rightarrow \mathrm{H}_{2} \mathrm{O}+$ ? $\quad 2 \mathrm{H}_{3} \mathrm{PO}_{4} \rightarrow 3 \mathrm{H}_{2} \mathrm{O}+$ ?

The valence of the characteristic non-metal is ascertained by this process. What is the valence of $\mathrm{Cl}$ in $\mathrm{HOCl}$ ? The anhydride is $\mathrm{Cl}_{2} \mathrm{O}$. The valence is $\mathrm{Cl}^{\mathrm{r}}$. What is the valence of $\mathrm{S}$ in $\mathrm{H}_{2} \mathrm{SO}_{4}$ ? The anhydride is $\mathrm{SO}_{3}$. The valence is $\mathrm{S}^{\mathrm{vr}}$. What is the valence of $\mathrm{N}$ in $\mathrm{HNO}_{3}$ ? The anhydride $\mathrm{N}_{2} \mathrm{O}_{5}$ shows the valence to be $\mathrm{N}^{\mathrm{v}}$. What are the valences of $\mathrm{Cl}$ in $\mathrm{HClO}_{4}$ and of $\mathrm{P}$ in $\mathrm{H}_{3} \mathrm{PO}_{4}$ and in $\mathrm{HPO}_{3}$ ?

When we get $\mathrm{SO}_{2}$ from sulphuric acid, by a chemical action, how do we know that the acid has been reduced (and something else oxidized)? Because in sulphuric acid we have $\mathrm{S}^{\mathrm{vr}} \mathrm{O}_{3} \mathrm{II}$, and in the product $\mathrm{S}^{\mathrm{rv}} \mathrm{O}_{2}{ }^{\mathrm{II}}$. The valence of $\mathrm{S}$ has been lowered from VI to IV. When from nitric acid we get $\mathrm{N}_{2} \mathrm{O}_{5}$, has there been reduction? No, because $\mathrm{N}_{2} \mathrm{O}_{5}+\mathrm{H}_{2} \mathrm{O}=2 \mathrm{HNO}_{3}$ (nitric acid). If we get $\mathrm{NO}_{2}$ or NO, has there been reduction of the acid? Yes, because the valence has been reduced: $\mathrm{N}^{\mathrm{IV}} \mathrm{O}_{2}, \mathrm{~N}^{\mathrm{II}} \mathrm{O}$. We then proceed to pick out the other substance that has been oxidized.

Analyzing the formula of the acid, to get that of the anhydride, also aids us to balance equations.

Balancing Equations. - When dilute nitric acid acts upon copper, the following skeleton equation represents the products of the reaction.

Skeleton: $\quad \mathrm{Cu}+\mathrm{HNO}_{3} \rightarrow \mathrm{Cu}\left(\mathrm{NO}_{3}\right)_{2}+\mathrm{NO}+\mathrm{H}_{2} \mathrm{O}$. 
The valence of $\mathrm{N}$ in $\mathrm{HNO}_{3}$, from its anhydride $\mathrm{N}_{2} \mathrm{O}_{5}$, is $\mathrm{N}^{\mathrm{v}}$. In $\mathrm{Cu}\left(\mathrm{NO}_{3}\right)_{2}$ the valence of $\mathrm{N}$ is unchanged; all that has happened here is the displacement of $2 \mathrm{H}^{\mathrm{I}}$ by $\mathrm{Cu}^{\mathrm{II}}$. The valence of $\mathrm{N}$ in $\mathrm{NO}$, however, has been reduced to $\mathrm{NII}$.

Let us see how we can utilize these facts to balance our equation. The formation of one molecule of $\mathrm{Cu}\left(\mathrm{NO}_{3}\right)_{2}$ involves the displacement of 2 atoms of hydrogen. The reduction of one molecule of $\mathrm{N}_{2} \mathrm{O}_{5}$ to $2 \mathrm{NO}$ involves the liberation of 3 atoms of oxygen. Both reactions are occuring simultaneously, so that neither hydrogen nor oxygen is actually obtained in the free state, they will of course unite to form water. But 3 atoms of oxygen are equivalent to 6 atoms of hydrogen, therefore 3 molecules of $\mathrm{Cu}\left(\mathrm{NO}_{3}\right)_{2}$ must be formed for every molecule of $\mathrm{N}_{2} \mathrm{O}_{5}$ that is reduced. To obtain $3 \mathrm{Cu}\left(\mathrm{NO}_{3}\right)_{2}$, we must use $3 \mathrm{Cu}$ and $6 \mathrm{HNO}_{3}$. To reduce $1 \mathrm{~N}_{2} \mathrm{O}_{5}$, we must use 2 more $\mathrm{HNO}_{3}$, that is, $8 \mathrm{HNO}_{3}$ in all. The balanced equation therefore becomes:

Balanced: $\quad 3 \mathrm{Cu}+8 \mathrm{HNO}_{3} \rightarrow 3 \mathrm{Cu}\left(\mathrm{NO}_{3}\right)_{2}+2 \mathrm{NO}+4 \mathrm{H}_{2} \mathrm{O}$.

These operations should be practiced on the following skeleton equations:

$$
\begin{aligned}
& \mathrm{HNO}_{3}+\mathrm{C} \rightarrow \mathrm{NO}_{2}+\mathrm{CO}_{2}+\mathrm{H}_{2} \mathrm{O} \text { (p. 309). } \\
& \mathrm{H}_{2} \mathrm{SO}_{4}+\mathrm{HI} \rightarrow \mathrm{H}_{2} \mathrm{~S}+\mathrm{H}_{2} \mathrm{O}+\mathrm{I}_{2} \text { (p. 204). } \\
& \mathrm{Zn}+\mathrm{H}_{2} \mathrm{SO}_{4} \rightarrow \mathrm{ZnSO}_{4}+\mathrm{H}_{2} \mathrm{O}+\mathrm{H}_{2} \mathrm{~S} \text { (p. 271). }
\end{aligned}
$$

These are typical examples of rections which it is very difficult to write correctly without some such guide as is given above.

Exercises. - 1. What is the valence of carbon in carbonic acid $\mathrm{H}_{2} \mathrm{CO}_{3}$ ?

2. What is the anhydride of nitrous acid $\mathrm{HNO}_{2}$, and what the valence of nitrogen in this compound?

3. When nitric acid acts upon copper, which substance is oxidized and which reduced?

4. How could you show experimentally that both nitrogen tetroxide and oxygen are formed when cupric nitrate is heated? 
5. How could you distinguish nitric oxide, (a) from hydrogen, (b) from oxygen?

6. How could you distinguish nitrous oxide from oxygen?

7. Make a list of the names and formulæ of the oxides of nitrogen, arranging them in the order of increasing proportions of oxygen.

8. Write full ionic equations for the preparation of ammonium nitrate (p. 309)? 


\section{CHAPTER XXVII}

\section{PHOSPHORUS, ARSENIC, ANTIMONY, BISMUTH}

Inese betong ts group of-docuble valency

We now take up the elements which, with nitrogen, form the nitrogen family. These elements all have two regular valences, being trivalent and quinquivalent. Nitrogen, phosphorus and arsenic are non-metallic elements, that is, they do not form positive radicals of salts. Antimony is non-metallic, although in its trivalent condition it acts also as a metallic element. Bismuth is metallic.
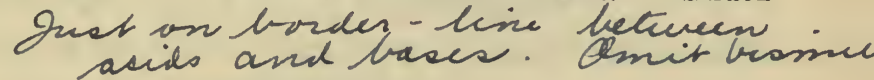

Phosphorus $\mathrm{P}$

Occurrence.-Calcium phosphate $\mathrm{Ca}_{3}\left(\mathrm{PO}_{4}\right)_{2}$ forms about 25 to 27 per cent of the material of the bones and teeth of animals. The same salt occurs in deposits, as a mineral, and is found scattered through all fertile soils. Complex organic compounds of phosphorus, such as lecithin, are essential constituents of the muscles, nerves and brains of animals and are found also in plants. The average man's skeleton contains $1400 \mathrm{~g}$. of phosphorus, his muscles $130 \mathrm{~g}$., and his nerves and brain $12 \mathrm{~g}$. Amongst foods, egg-yolks and beans contain an unusually large proportion, nuts, peas, and wheat (entire grain) coming next.

Phosphorus was discovered by Brand in 1669, and by Kunkel in 1670 , by distilling at a white heat the solid residue from evaporated animal matter. They were both searching for the philosophers' stone. Scheele in Sweden prepared it from bones in 1771. The element is used chiefly in the manufacture of matches and, to a small extent, in roach paste and rat poison.

Manufacture of Phosphorus.-Phosphorus is now manufactured by mixing natural calcium phosphate with sand $\left(\mathrm{SiO}_{2}\right)$ 
and coke, and heating the mixture in an electric furnace (Fig. 85). The mixture is admitted by moving the traps below the hopper, and is carried into the furnace by the worm conveyor. The resistance of the mass between the electrodes causes great development of heat. The actions may be shown by partial equations, which, when added together, give the complete equation:

$$
\begin{aligned}
& \mathrm{Ca}_{3}\left(\mathrm{PO}_{4}\right)_{2} \rightarrow 3 \mathrm{CaO}+\mathrm{P}_{2} \mathrm{O}_{5} \\
& 3 \mathrm{CaO}+\underline{3 \mathrm{SiO}_{2}} \rightarrow 3 \mathrm{CaSiO} \text { (calcium silicate) } \\
& \mathrm{P}_{2} \mathrm{O}_{5}+5 \mathrm{C} \rightarrow 2 \mathrm{P}+5 \mathrm{CO} \\
& \frac{\mathrm{Ca}_{3}\left(\mathrm{PO}_{4}\right)_{2}+3 \mathrm{SiO}_{2}+5 \mathrm{C}}{\rightarrow} \rightarrow 3 \mathrm{CaSiO}_{3} \downarrow+2 \mathrm{P} \uparrow+5 \mathrm{CO} \uparrow
\end{aligned}
$$

The calcium silicate is melted and runs out below as a slag. The phosphorus (vapor) and carbon monoxide

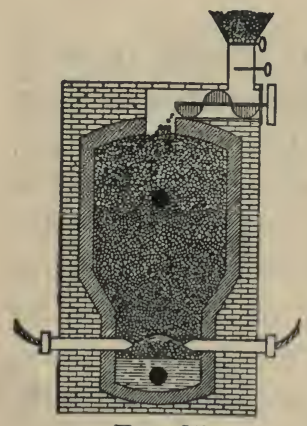

FIG. 85 (gas) pass off through the opening near the top. The phosphorus vapor is condensed under cold water.

White Phosphorus. - The product, after purification, is a colorless, transparent waxy solid (sp. gr. 1.83), which melts at $44^{\circ}$ and boils at $287^{\circ}$. It is insoluble in water, but dissolves in carbon disulphide. It has a strong odor, resembling ozone.

White phosphorus oxidizes in the air, giving, when moist, phosphorous acid and phosphoric acid, and emitting a faint light from which the element derives its name (Greek, light bearer). It catches fire at a low temperature (about $35^{\circ}$ ), and in burning forms a cloud of the solid phosphorus pentoxide $\mathrm{P}_{2} \mathrm{O}_{5}$. It combines readily, even when cold, with the halogens, and when heated it unites with sulphur and the more active metals.

White phosphorus is a very active poison (fatal dose, $0.15 \mathrm{~g}$.). When traces of the vapor are breathed day after day, a disease, 
frequently shown by workers in match-factories and consisting in ulceration of the bones of the jaw, makes its appearance. The use of white phosphorus is forbidden by law in Sweden, France, Great Britain, and Switzerland and is penalized by a special tax in the United States.

Red Phosphorus. - When white phosphorus is heated at 230 to $300^{\circ}$ in a tightly closed vessel (air excluded) it changes into red phosphorus. This material is composed of small crystals, of dull red color, and variable specific gravity 2.19 to 2.34. It is insoluble in carbon disulphide, has no odor, and is not poisonous. On distillation the vapor condenses to white phosphorus.

This allotropic form of phosphorus is formed from the white variety with liberation of much heat. It thus contains less energy, and is much less active. It burns to form the pentoxide, but has to be heated to about $240^{\circ}$ before it will catch fire in the air. It combines also with elements other than oxygen much less readily than does white-phosphorus.

Manufacture of Matches. - These are of two kinds, ordinary matches, which strike on any rough surface, and "safety" matches. Ordinary matches are still made in some countries by dipping the splints of wood in melted paraffin, and then in a paste made of 4 to 7 per cent of white phosphorus, lead dioxide about 50 per cent, water, and dextrin (paste). The head, when dry, is dipped in varnish to exclude air and moisture. Where the use of white phosphorus is very properly forbidden, a sulphide $\mathrm{P}_{4} \mathrm{~S}_{3}$ is substituted. When the match is struck, the friction explodes the mixture of phosphorus trisulphide (combustible) and lead dioxide (or other oxidizing agent), and the resulting heat sets fire to the paraffin and this, in turn, to the wood.

Safety matches carry no phosphorus, but only a mixture of substances containing oxygen, such as potassium chlorate or potassium chromate, with a combustible, like antimony trisul- 
phide, some dextrin and a filling (e.g., chalk). The box is coated with a mixture of red phosphorus, antimony trisulphide, dextrin and filling. The friction converts a trace of the red phosphorus into the white variety, and the latter sets fire to the head.

Phosphorus Pentoxide $\mathrm{P}_{2} \mathrm{O}_{5}$.-When phosphorus is burned in $d r y$ air or oxygen, under a bell jar, the cloud of pentoxide slowly settles as a white powder. If the pentoxide is thrown into cold water, chemical union takes place. The heat developed produces a hissing sound, caused by the formation and condensation of minute bubbles of steam. The solution, when concentrated at a low temperature, gives crystals of orthophosphoric acid $\mathrm{H}_{3} \mathrm{PO}_{4}$ (m.-p. $42^{\circ}$ ).

$$
\mathrm{P}_{2} \mathrm{O}_{5}+3 \mathrm{H}_{2} \mathrm{O} \rightarrow 2 \mathrm{H}_{3} \mathrm{PO}_{4} \text {. Phorphovic }
$$

On account of its tendency to unite with water, the pentoxide is used for drying gases.

When phosphorus is burnt in moist air, the cloud of pentoxide forms tiny droplets, consisting of a concentrated solution of phos phoric acid, which remain suspended in the atmosphere as a fog (compare sulphur trioxide, p. 263). Burning phosphorus was therefore used in the war for screening the movement of vessels. In land warfare, shells containing white phosphorus were also employed for incendiary purposes. Such shells produced terrible, and usually fatal, burns on any enemy within the radius of their explosion.

As a tribasic acid, phosphoric acid $\mathrm{H}_{3} \mathrm{PO}_{4}$ gives three series of salts by interaction with bases. In the ortho or normal phosphates, such as $\mathrm{Na}_{3} \mathrm{PO}_{4}$, all of the hydrogen is replaced by a metallic radical. Two series of acid salts are also known (see p. 277). The phosphates of calcium are of particular value as fertilizers (see p. 411).

Test for a Phosphate.- Most phosphates (and phosphoric acid), when mixed intimately with dry sodium carbonate, char- 
salt. They are green, insoluble compounds used, as insecticides, for spraying plants. On account of their poisonous character, they are no longer employed as ingredients in paints.

Arsenic as a Member of the Nitrogen Family. - While free arsenic is as different physically from phosphorus as the latter is from nitrogen, the compounds have much in common. Arsenic forms arsine $\mathrm{AsH}_{3}$ (corresponding to $\mathrm{NH}_{3}$ and $\mathrm{PH}_{3}$ ), arsenious oxide $\mathrm{As}_{2} \mathrm{O}_{3}$, arsenic oxide $\mathrm{As}_{2} \mathrm{O}_{5}$, and the acids $\mathrm{H}_{3} \mathrm{AsO}_{3}$ and $\mathrm{H}_{3} \mathrm{AsO}_{4}$. In these compounds it is non-metallic, and shows the valences three and five.

\section{Antimony $\mathrm{Sb}$}

Preparation and Properties.-Antimony is found free in nature. The sulphide, stibnite $\mathrm{Sb}_{2} \mathrm{~S}_{3}$, is also a well-known mineral. When the latter is melted with iron, ferrous sulphide and free antimony are formed:

$$
3 \mathrm{Fe}+\mathrm{Sb}_{2} \mathrm{~S}_{3} \rightarrow 2 \mathrm{Sb}+3 \mathrm{FeS} .
$$

The molten ferrous sulphide (sp. gr. 4.8) floats upon the molten antimony (sp. gr. 6.5), and the products, being mutually insoluble, are easily separated.

The metal is brilliantly silvery and non-tarnishing. It is brittle and the black powder obtained by pulverizing it, "antimony black," is rubbed on plaster casts to give them a dull, metallic appearance. When heated, antimony volatilizes and burns in the air with a brilliant white light to form the white trioxide $\mathrm{Sb}_{2} \mathrm{O}_{3}$.

The trisulphide $\mathrm{Sb}_{2} \mathrm{~S}_{3}$ (orange-colored when precipitated) is used in making matches and fireworks.

Alloys Containing Antimony. - The metal is chiefly used as an ingredient in alloys. Lead, when solidifying, shrinks and antimony counteracts this tendency. Hence type metal, which, when cold, must fill the mould completely, is made by melting together 15 to 25 per cent of antimony, 10 to 20 per cent tin, and

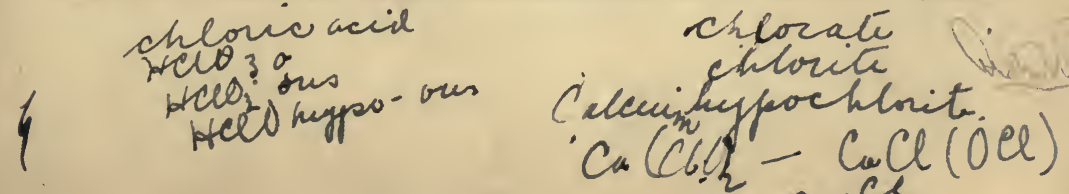


the rest lead. The alloy is also harder than lead, and is less quickly deformed by handling and by use in the printing press.

Babbitt's metal ( $\mathrm{Sb} \mathrm{3,} \mathrm{Zn} \mathrm{69,} \mathrm{As} \mathrm{4,} \mathrm{Pb} \mathrm{5,} \mathrm{Sn} \mathrm{19),} \mathrm{and} \mathrm{other}$ anti-friction alloys, used in lining bearings, contain antimony along with zinc, copper, and other metals. Molten mixtures of metals (alloys), when solidifying, do not always form a homogeneous, solid mass. In an anti-friction alloy, what is wanted is a mass, in general soft, but containing hard particles. The latter bear most of the pressure, yet, as the alloy wears, they are pressed into the softer matrix so that a smooth surface is always presented. An alloy which has the opposite composition, that is, which gives a hard mass containing softer particles, develops heat by friction much more rapidly.

\section{Bismuth $\mathrm{Bi}$}

As the atomic weight increases, the members of this family become more like metallic elements in their chemical properties. Thus bismuth is a true metallic element. Its oxides are basic, and its compounds give positive ions $\mathrm{Bi}^{+++}$and include salts like the carbonate, sulphate, chloride, and phosphate.

Metallic Bismuth. - The metal occurs free in nature. It is a brittle metal, with a pink metallic luster. It melts at $270^{\circ}$ and vaporizes at a high temperature. It does not tarnish. It is used in preparing alloys with very low melting-points. Thus Wood's metal contains bismuth (m.-p. 270 4 parts, lead (m.-p. $\left.326^{\circ}\right) 2$ parts, tin (m.-p. $233^{\circ}$ ) 1 part, and cadmium (m.-p. $320^{\circ}$ ) 1 part. As is the case with other solutions, the melting-point is lower than that of any of the components, namely $60^{\circ}$. Alloys of this class are used as plugs in sprinkler systems and stops to hold steel fire-doors open. When, in consequence of a fire, the temperature rises, the alloy melts, the water exits are opened and the fire-doors swing shut. Safety piugs in steam boilers, made of a similar, but less fusible alloy, melt when, as the result of failure 
of the safety valve, the steam pressure, and therefore the temperature, exceeds a predetermined value. They behave in the same way when the water is dangerously low and the metal above the water becomes too hot.

Compounds of $\boldsymbol{B}$ smuth. - When strongly heated, the metal burns to form a brown trioxide $\mathrm{Bi}_{2} \mathrm{O}_{3}$. This oxide gives salts with acids. Thus, with nitric acid it dissolves to form a solution of bismuth nitrate:

$$
\mathrm{Bi}_{2} \mathrm{O}_{3}+6 \mathrm{HNO}_{3} \rightarrow 2 \mathrm{Bi}\left(\mathrm{NO}_{3}\right)_{3}+3 \mathrm{H}_{2} \mathrm{O} .
$$

When the solution is evaporated, the nitrate appears in colorless crystals. If the crystals are placed in water, a white, insoluble basic nitrate is formed:

$$
\mathrm{Bi}\left(\mathrm{NO}_{3}\right)_{3}+\mathrm{H}_{2} \mathrm{O} \rightleftarrows \mathrm{BiONO}_{3} \downarrow+2 \mathrm{HNO}_{3} \text {. }
$$

This is used in medicine under the name of bismuth subnitrate, to cure stomach troubles.

Exercises.-1. By what chemical experiments could you recognize red phosphorus?

2. Explain the fact that solid Wood's metal floats upon melted Wood's metal.

3. Why is white phosphorus always kept under water? 


\section{CHAPTER XXVIII}

\section{CARBON AND THE OXIDES OF CARBON}

THE majority of the substances composing, or produced by, living organisms, such as starch, fat, and sugar, are compounds of carbon. Hence the chemistry of these compounds is known as organic chemistry. It was at first supposed that the artificial production of such compounds, e.g., without the intervention of life, was impossible. But many natural organic products have now been made from simpler ones or from the elements, and the preparation of the others is delayed only in consequence of difficulties caused by their instability and complexity. On the other hand, hundreds of compounds unknown to animal or vegetable life, including many valuable drugs and dyes, have now been added to the catalogue of chemical compounds. Hundreds of thousands of different compounds containing carbon are known, and thousands more are added every year.

The elements entering into carbon compounds are chiefly hydrogen and oxygen. After these, nitrogen, phosphorus, the halogens and sulphur may be named.

\section{Carbon C}

Occurrence.-Large quantities of carbon are found in the free condition in nature. The diamond is the purest natural carbon. Graphite, or plumbago, which is the next purest, is found in limited amounts, and is a valuable mineral. Coal occurs in numerous forms containing greatly varying proportions of free carbon. Small quantities of the free element have been found in meteorites.

In combination, carbon is found in marsh-gas, or methane $\mathrm{CH}_{4}$, which is the chief component of natural gas. The numerous 
compounds found in plants and animals have already been mentioned. The mineral oils consist almost entirely of mixtures of various compounds of carbon and hydrogen (hydrocarbons). Whole geological formations are composed of carbonates of common metats, particularly calcium carbonate or limestone.

Diamond.- This allotropic modification of carbon is distinguished by its natural crystalline form, which often resembles the octahedron (Fig. 39, p. 94). The ultimate structure of the diamond crystal is represented in Fig. 45 (p. 96). Its specific gravity is 3.5. For ornamental purposes the diamond is " cut" by grinding new faces so as to give artificial forms called "brilliants" (Fig. 86) and "rosettes." It is the hardest of familiar substances, and can be scratched or polished only by rubbing with diamond powder. The colorless stones and those with special tints are valuable.

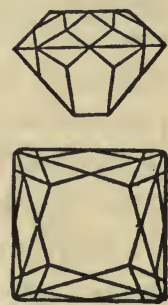

FIG. 86 The black ("carbonado") and badly colored specimens are less valuable and are used for grinding, for glass-cutting, and on the points of drills.

Diamonds are found chiefly in South Africa and Brazil. They are separated from the rock by weathering and washing. They are sold by the carat ( 1 international carat $=200 \mathrm{mg}$.) and the value increases with the size. The largest known specimen, the Cullinan, weighed 3032 carats before being cut.

Small synthetic diamonds are obtained when molten iron containing dissolved carbon is suddenly chilled. If the fused mass is allowed to cool gradually, however, the carbon separates out in the form of graphite.

Graphite is found in nature in Siberia, Cumberland, Brazil, Ceylon and elsewhere. It forms dark grey or black hexagonal tablets, and, when pulverized, it gives slippery scales of microscopic size. Unlike the diamond, it is quite soft, has a specific 
gravity of 2.3, and conducts electricity. Natural graphite is usually mixed with foreign matter, and even the purest specimen leaves, when burned, from 2 to 5 per cent of ash. It is called also plumbage, or black lead.

Graphite (Greek, I write), moulded into blocks, is sawn into rods for the cores of "lead" pencils (first used in the 16th century). Clay is added in varying proportions to give different degrees of hardness. Because of its infusibility, it is used to make crucibles. Smeared on a plaster cast (non-conductor), it gives a conducting surface on which metals (copper or silver) can be deposited by electrolysis. A thin layer, used as stove-polish, protects the iron from rusting. In electro-chemical industries it is used for electrodes at which chlorine is to be liberated; all other conductors interact chemically with this element and are destroyed. It is employed also as a lubricant, when wooden beams slide upon one another.

Large amounts of pure graphite are now manufactured by heating coke with some pitch and a little sand or ferric oxide (Acheson's process). The mixture (3 to $3 \frac{1}{2}$ tons) is piled (Fig. 87, p. 333 ) between the electrodes connected with a dynamo, and, on account of its high resistance, becomes strongly heated. The operation is complete in from 24 to 30 hours.

Other Forms of Carbon.-The apparently amorphous varieties of carbon are numerous. They include wood-charcoal, lampblack, animal charcoal, coal (e.g., bituminous coal and anthracite) and coke. All of these substances will come up for discussion in later chapters. None of them, it may be noted here, is composed of pure carbon, other elements being present, mostly. in combination with carbon, in very variable amounts.

Examination of "amorphous" charcoal by X-ray methods indicates that it possesses a crystalline structure identical with that of graphite. Charcoal is not to be regarded, therefore, as a supercooled liquid (see p. 94) like glass. It consists of tiny frag- 
ments of graphite. The amorphous appearance is due to the extreme minuteness of the crystals, which are interspersed, with attendant impurities, through a highly porous mass.

Chemical Properties of Carbon.- 1. Carbon unites vigorously with oxygen. With an excess of oxygen it forms carbon dioxide, a gas:

$$
\mathrm{C}+\mathrm{O}_{2} \rightarrow \mathrm{CO}_{2} \text {. }
$$

With a limited supply of oxygen, it forms carbon monoxide, also a gas:

$$
2 \mathrm{C}+\mathrm{O}_{2} \rightarrow 2 \mathrm{CO} \text {. }
$$

2. In consequence of this tendency to unite with oxygen, carbon is much used as a reducing agent. Thus, when oxide of copper is heated with pulverized charcoal, carbon dioxide is formed, and the metal is liberated:

$$
2 \mathrm{CuO}+\mathrm{C} \rightarrow 2 \mathrm{Cu}+\mathrm{CO}_{2} \text {. }
$$

In the same way the oxides of tin, of lead, and of many other metals may be reduced. Copper, tin, and lead are manufactured from the ores in this way.

3. Carbon unites directly with some elements, particularly with sulphur to form carbon disulphide $\mathrm{CS}_{2}$ (p. 255) and with certain of the metals. Thus, when dissolved in molten iron, it forms iron carbide $\mathrm{Fe}_{3} \mathrm{C}$.

The union with hydrogen is ordinarily too slow to be observed. But when the carbon is mixed with pulverized nickel (contact agent) and hydrogen is passed over the mixture at $250^{\circ}$, methane $\mathrm{CH}_{4}$ is formed (99 per cent). The action is reversible and exothermal, and is therefore, at higher temperatures, less complete (compare p. 242), at $850^{\circ}$ reaching only 1.5 per cent. On the other hand, an electric arc, between carbon poles in an atmosphere of hydrogen, gives traces of acetylene $\mathrm{C}_{2} \mathrm{H}_{2}$, this action being endothermal. The other compounds of carbon and hydrogen are all obtained by indirect reactions. 
The valence of carbon is almost always four. This is clearly seen in $\mathrm{C}^{\mathrm{IV}} \mathrm{O}_{2}{ }^{\mathrm{II}}$ and $\mathrm{C}^{\mathrm{rvH}} \mathrm{H}_{4}^{\mathrm{I}}$. In a few compounds, of which $\mathrm{CO}$ is the commonest, carbon is bivalent.

Carbon Tetrachloride $\mathrm{CCl}_{4}$. - This compound is manufactured by leading dry chlorine into carbon disulphide, in which a little iodine (contact agent) is dissolved:

$$
\mathrm{CS}_{2}+3 \mathrm{Cl}_{2} \rightarrow \mathrm{CCl}_{4}+\mathrm{S}_{2} \mathrm{Cl}_{2} \text {. }
$$

On distilling the resulting mixture, the carbon tetrachloride $\mathrm{CCl}_{4}$ (b.-p. $77^{\circ}$ ) passes off and is condensed, while the sulphur monochloride $\mathrm{S}_{2} \mathrm{Cl}_{2}$ (b.-p. $136^{\circ}$ ) remains.

Carbon tetrachloride is a colorless liquid. It dissolves fats and tars and other organic compounds, and has the advantage over benzine and gasoline of being non-inflammable. It is therefore used in taking the grease out of wool, linen cloth, oil-bearing seeds, and bones. Many liquids sold for dry cleaning and removing stains from clothing, gloves, etc., consist of carbon tetrachloride diluted with a cheaper material, such as benzine. Although the proportion of such material originally added may be insufficient to render the mixture inflammable or explosive, the risk exists that evaporation of the more volatile carbon tetrachloride may eventually bring the composition of the residue into the dangerous region. Products containing no benzine are therefore to be preferred. Many fire extinguishers also enntain a liquid which is mainly carbon tetrachloride.

Carbides and the Electric Furnace.-Chemical actions which proceed only at very high temperatures are most economically carried out by using electricity as the source of heat. In such cases the electricity has no electrolytic or other chemical action. There are two types of electric furnaces. In the making of graphite (p. 330) and of carbon disulphide (p. 255), which illustrates one of them, the resistance of the carbon furnishes the occasion for the rise in temperature.

Of the same type is the furnace used for making carborundum 
(SiC, silicon carbide), manufactured in large quantities at Niagara Falls (Acheson's process). The coke and sand (silicon dioxide $\mathrm{SiO}_{2}$ ) are piled between the terminals, and the resistance of the former causes the production of the heat (Fig. 87):

$3 \mathrm{C}+\mathrm{SiO}_{2} \rightarrow \mathrm{SiC}+2 \mathrm{CO} \uparrow$.

Here the carbon reduces the oxide, and combines with the element ( $\mathrm{Si}$ ) as well. The

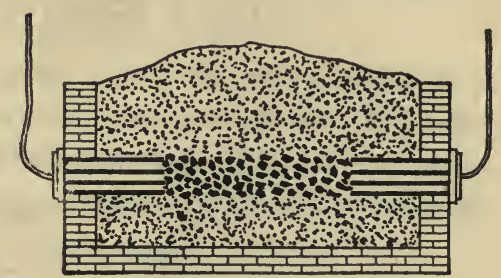

FIG. 87 product ( $\mathrm{SiC}$ ) is exceedingly hard, and, after pulverization and mixing with other materials, is moulded into grinding wheels.

In the other type of furnace the air between the terminals furnishes the resistance, and the arc (a discharge carried by the badly conducting air and carbon vapor) furnishes the heat.

The arc is used in making calcium carbide $\left(\mathrm{CaC}_{2}\right)$, by heating a mixture of lime $(\mathrm{CaO})$ and coke:

$$
\mathrm{CaO}+3 \mathrm{C} \rightarrow \mathrm{CO}+\mathrm{CaC}_{2} \text {. }
$$

Cold water acts vigorously with calcium carbide, giving acetylene gas $\mathrm{C}_{2} \mathrm{H}_{2}$ (see p. 351) and calcium hydroxide (slaked lime):

$$
\text { . } \mathrm{CaC}_{2}+2 \mathrm{H}_{2} \mathrm{O} \rightarrow \mathrm{Ca}(\mathrm{OH})_{2}+\mathrm{C}_{2} \mathrm{H}_{2} \uparrow \text {. }
$$

\section{Carbon Dioxide}

Occurrence.- Carbon dioxide is found in nature issuing from the ground, especially in volcanic neighborhoods, and dissolved in effervescing natural waters, such as Saratoga and Vichy. It is found in the air (3.5 liters in every 10,000 liters of air) and in the breath (37 liters per 1000 liters).

Preparation.- 1. Carbon dioxide is most easily prepared in the laboratory by the action of an acid such as hydrochloric acid upon a natural carbonate like calcium carbonate (marble or limestone). The action occurs in two stages. The first is a double 
decomposition, such as all acids and salts exhibit when brought in contact with one another:

$$
\mathrm{CaCO}_{3}+2 \mathrm{HCl} \rightleftarrows \mathrm{CaCl}_{2}+\mathrm{H}_{2} \mathrm{CO}_{3} \text {. }
$$

The calcium chloride $\mathrm{CaCl}_{2}$ remains dissolved in the water contained in the hydrochloric acid. The carbonic acid $\mathrm{H}_{2} \mathrm{CO}_{3}$ is unstable, however, and immediately dissociates into water, which remains, and carbon dioxide gas, which escapes:

$$
\mathrm{H}_{2} \mathrm{CO}_{3} \rightleftarrows \mathrm{H}_{2} \mathrm{O}+\mathrm{CO}_{2} \uparrow \text {. }
$$

The apparatus used is similar to that employed in making chlorine (Fig. 49, p. 141).

2. For commercial purposes the carbon dioxide is either used as it is produced, or else it is compressed into wrought-iron cylinders and shipped in the form of a liquid. Three sources of such commercial carbon dioxide are in use:

When carbon, for example, in the form of coke, is burned with a plentiful supply of air, all the carbon is converted into carbon dioxide:

$$
\mathrm{C}+\mathrm{O}_{2} \rightarrow \mathrm{CO}_{2} \text {. }
$$

Since, however, there are four volumes of nitrogen to one of oxygen in the air, this carbon dioxide is diluted with nitrogen in the same proportion. The gases must therefore be separated by leading them through potassium carbonate solution, which absorbs the carbon dioxide:

$$
\mathrm{CO}_{2}+\mathrm{H}_{2} \mathrm{O} \rightleftarrows \mathrm{H}_{2} \mathrm{CO}_{3}+\mathrm{K}_{2} \mathrm{CO}_{3} \rightleftarrows 2 \mathrm{KHCO}_{3} .
$$

The bicarbonate of potassium $\mathrm{KHCO}_{3}$ thus produced is subsequently decomposed by heating.

3. Calcium carbonate (limestone) $\mathrm{CaCO}_{3}$, or, more easily, magnesium carbonate (magnesite) $\mathrm{MgCO}_{3}$, may be decomposed by heating in a kiln:

$$
\begin{aligned}
\mathrm{CaCO}_{3} & \rightarrow \mathrm{CaO}+\mathrm{CO}_{2} \uparrow . \\
\mathrm{MgCO}_{3} & \rightarrow \mathrm{MgO}+\mathrm{CO}_{2} \uparrow .
\end{aligned}
$$


In the former case the quicklime $(\mathrm{CaO})$, which is formed at the same time, is a valuable product also.

4. Carbon dioxide is formed in fermentation, and so is collected from the vats in which beer is brewed (see p. 417).

Physical Properties. - Carbon dioxide is a colorless, odorless, almost tasteless gas. As the molecular weight $\left(\mathrm{CO}_{2}=44\right)$ shows, it is one-half heavier than air. Its greater specific gravity may easily be shown by pouring it from one jar into another, or into a beaker placed on one pan of a balance with an equipoise of shot on the other pan. It is much more soluble in water than is air. One volume of water at $15^{\circ}$ dissolves an equal volume of the gas. At two atmospheres pressure, two volumes are dissolved, at three atmospheres, three volumes. Pure water, charged at 3 or 4 atmospheres pressure is known as soda water. Effervescent waters, such as Selters and Vichy, contain dissolved salts in addition.

The gas can be liquefied at any temperature below $31.35^{\circ}$ (see $p$. 91). At $20^{\circ}$ the pressure required is 60 atmospheres and this is therefore the pressure in a cylinder of liquid carbon dioxide at that temperature. To withstand the pressure very massive cylinders are required, and they weigh, when empty, about twice as much as does the liquid they will hold when full.

When liquid carbon dioxide is allowed to run from a cylinder nto a cloth bag (non-conductor of heat), the rapid evaporation of a part of the liquid consumes so much heat that the rest of the liquid freezes to a snow-like mass of solid carbon dioxide. In the laboratory this solid is used as a cooling agent, being mixed with ether or alcohol to secure closer contact with the object to be cooled.

Chemical Properties.-Stability. - 1. Carbon dioxide is very stable (only 7.5 per cent dissociated at $2000^{\circ}$ ), and so, although it contains much oxygen, it will not support combustion. Mag- 
nesium or aluminium powder, however, will burn when placed on a cake of solid carbon dioxide and set on fire with burning magnesium ribbon:

$$
2 \mathrm{Mg}+\mathrm{CO}_{2} \rightarrow 2 \mathrm{MgO}+\mathrm{C} .
$$

The gas extinguishes burning wood, oil, or candles, and 15 to 16 per cent of it in air is sufficient to extinguish ordinary combustibles. For this reason some fire extinguishers contain a dilute solution of bicarbonate of sodium $\left(\mathrm{NaHCO}_{3}\right.$, p. 366) and sulphuric acid:

$$
2 \mathrm{NaHCO}_{3}+\mathrm{H}_{2} \mathrm{SO}_{4} \rightleftarrows \mathrm{Na}_{2} \mathrm{SO}_{4}+2 \mathrm{H}_{2} \mathrm{CO}_{3} \rightarrow 2 \mathrm{H}_{2} \mathrm{O}+2 \mathrm{CO}_{2} \uparrow \text {. }
$$

When the instrument is inverted, these materials are mixed, and water and carbon dioxide are forced out by the pressure of the gas.

Chemical Properties - Carbonic Acid.-2. Carbon dioxide, when dissolved in water, combines in part to form carbonic acid:

$$
\mathrm{H}_{2} \mathrm{O}+\mathrm{CO}_{2} \rightleftarrows \mathrm{H}_{2} \mathrm{CO}_{3} \text {. }
$$

The gas is therefore often called the anhydride (Greek, without water) of carbonic acid. The solution has all the properties of an acid, although, as the acid is very little ionized (p. 189), it exhibits them rather feebly. It tastes slightly sour, turns blue litmus faintly red, and neutralizes bases. The last action is easily shown by shaking the gas with limewater (solution of calcium hydroxide, a base):

$$
\mathrm{Ca}(\mathrm{OH})_{2}+\mathrm{H}_{2} \mathrm{CO}_{3} \rightarrow \mathrm{CaCO}_{3} \downarrow+2 \mathrm{H}_{2} \mathrm{O} .
$$

The carbonate of calcium is precipitated and the liquid becomes milky in appearance. This action is used by sugar refiners for removing the lime employed in purifying the sugar. Manufacturers of white lead (carbonate of lead) also employ carbon dioxide, because of its entering into double decomposition to give carbonates. The same property is utilized in making bicarbonate of sodium and washing soda (carbonate of sodium, p. 367). 
Since the molecule of carbonic acid $\left(\mathrm{H}_{2} \mathrm{CO}_{3}\right)$ contains two atoms of hydrogen, either one or both of these atoms may be replaced by a metal - the acid is dibasic. Thus we may have sodium carbonate $\mathrm{Na}_{2} \mathrm{CO}_{3}$, or its hydrate, washing soda $\mathrm{Na}_{2} \mathrm{CO}_{3}, 10 \mathrm{H}_{2} \mathrm{O}$, and also sodium bicarbonate (baking soda, sodium-hydrogen carbonate) $\mathrm{NaHCO}_{3}$ (see pp. 366-7).

3. The most marvellous chemical action into which carbon dioxide enters is the action by which plants use the gas as food. This important action is discussed in detail in a later chapter.

\section{Carbon Monoxide}

Preparation.- Carbon monoxide CO is most easily prepared in the laboratory by heating formic acid (or sodium formate, a white crystalline solid) with concentrated sulphuric acid. The latter combines with the water, but is not otherwise changed:

$$
\mathrm{HCO}_{2} \mathrm{H} \rightarrow \mathrm{CO}+\mathrm{H}_{2} \mathrm{O} \text {. }
$$

When coke, or any form of carbon burns with a limited supply of air, or oxygen, the same gas is produced:

$$
2 \mathrm{C}+\mathrm{O}_{2} \rightarrow 2 \mathrm{CO} \text {. }
$$

The gas therefore rises from the surface of a coal fire, sometimes escaping unburned, but often burning with a blue flame above the coal.

Producer Gas and Water Gas.-When air is, led through burning coke, the mixture of carbon monoxide (39 per cent) with nitrogen (60 per cent) obtained is called producer gas. It is combustible, and is used in industrial establishments for heating and to drive gas engines for power.

Commercially, large amounts of carbon monoxide mixed with hydrogen (water gas), are manufactured by blowing steam over white hot coke or anthracite:

$$
\mathrm{C}+\mathrm{H}_{2} \mathrm{O} \rightarrow \mathrm{CO}+\mathrm{H}_{2}-29,100 \text { calories. }
$$


The coke is first set on fire in a brick-lined cylindrical structure and brought to vigorous combustion by blowing in air for ten minutes. Then steam is substituted for the air.

The interaction, as the equation shows, takes place with absorption of heat. Hence, at the end of a few minutes, the coke becomes too cool. It is then necessary to turn the steam off and to turn the air on again, and so on alternately. The mixture of carbon monoxide (40 to 50 per cent) and hydrogen ( 45 to 50 per cent), containing also some carbon dioxide ( 4 to 7 per cent), nitrogen ( 4 to 5 per cent), and oxygen ( 1 per cent), is known as water gas. It is almost wholly combustible, burning with a blue flame, and is used as a source of heat and, by driving internal combustion engines, to furnish power. It is used also in manufacturing illuminating gas (see p. 356).

If both air and steam are driven together over the burning coke, the air enables the coke to burn continuously, and a fuel gas which is a cross between producer gas and water gas is obtained.

Fuel gases are employed on a large scale in steel works, and other industrial plants. They give a uniform and eacily regulated heat, they leave no ash, and their use involves no labor for stoking.

Industrial Hydrogen from Water Gas.-Hydrogen is required in large quantities in chemical industry for the manufacture of ammonia (p. 300) and for hydrogenating oils (p. 433). It is essential that this hydrogen should be carefully purified from traces of other gases, such as carbon monoxide and sulphuretted hydrogen, which act as poisons on the cata-ysts employed in the above processes. The cheapest source of industrial hydrogen is water-gas, and much work has been done to devise a method for eliminating the undesirable carbon monoxide from this.

When a mixture of water-gas (substantially $\mathrm{H}_{2}+\mathrm{CO}$ ) and superheated steam is passed over a suitable catalyst, such as iron oxide, a reaction occurs as follows.

$$
\mathrm{CO}+\mathrm{H}_{2} \mathrm{O} \rightleftharpoons \mathrm{CO}_{2}+\mathrm{H}_{2}+9,800 \text { calories. }
$$


The reaction being reversible, and exothermic in the forward direction, its equilibrium point is displaced towards the left, favoring the backward reaction, as the temperature is raised. It is therefore desirable to work the process at as low a temperature as possible. With iron oxide alone as a catalyst, however, the interaction between the gases becomes too slow to be effective at temperatures much below $600^{\circ}$. The addition of small quantities of other oxides, such as nickel oxide and chromiun 1 oxide, has been found to increase the activity of the catalyst very considerably. Substances which act in this manner (catalyzing a catalyst, so to speak) are termed promoters.

In actual practice the reaction, carried out at $450-500^{\circ}$, gives a mixture of gases containing only about 2 per cent residual CO. Excess of steam is employed to drive the equilibrium as far as possible towards the right, but the excess of hydrogen present in the original water-gas favors, of course, the opposite reaction (compare p. 234). It should be noted that nearly twice as much hydrogen as was contained in this water-gas is obtained by the process, the second half being derived from the decomposition of the steam.

The bulk of the carbon dioxide present in the final mixture (approximately 30 per cent by volume) is removed by washing the gas with water under pressure. The last traces of carbon dioxide are absorbed by means of lime or alkalies. The removal of the 2 per cent residual CO presents difficulties. Absorption of $\mathrm{CO}$ by hot caustic soda solutions and by ammoniacal solutions of cuprous salts has been employed. The most efficient method, however, consists of preferential combustion of $\mathrm{CO}$ to $\mathrm{CO}_{2}$ with the requisite quantity of air or oxygen in the presence of a second oxide catalyst. If due precautions are taken, $\mathrm{CO}$ burns almost quantitatively to $\mathrm{CO}_{2}$ without any $\mathrm{H}_{2}$ present burning to $\mathrm{H}_{2} \mathrm{O}$. The small amount of $\mathrm{CO}_{2}$ formed is then removed as already described. 
Physical Properties of Carbon Monoxide.- Carbon monoxide is a colorless, odorless, and tasteless gas. It is a little lighter than air (mol. wt. 28), and is very slightly soluble in water. It is difficult to liquefy. Its boiling-point, when liquid, is $-190^{\circ}$, close to that of liquid air.

Chemical Properties. - When set on fire, the gas burns in air or oxygen with a blue flame. Carbon dioxide is formed, and the presence of the latter may be shown with lime-water (p. 336):

$$
2 \mathrm{CO}+\mathrm{O}_{2} \rightarrow 2 \mathrm{CO}_{2} \text {. }
$$

On account of this property, carbon monoxide reduces the oxides of the less active metals, such as those of iron and of the metals below iron in the order of activity. Commercially, the ores of iron are reduced by this gas (essentially producer gas) in the blast furnace. The oxides of the metals above iron are not reduced.

Physiological Properties. - The gas is an active poison, and 1 volume in 100,000 volumes of air produces symptoms of poison. ing, while one volume in 750 to 800 volumes produces death in about thirty minutes. The gas combines with the hæmoglobin of the blood corpuscles, forming a stable compound, and thus preventing the absorption of oxygen by the blood (p. 34). This gas is the chief poisonous substance in illuminating gas. The poisonous effect of tobacco smoke, particularly when inhaled, is due mainly to the carbon monoxide produced by the necessarily incomplete combustion.

Combustions or explosions in confined spaces (such as in a mineshaft, or in the interior of a warship during an engagement) may cause many deaths through $\mathrm{CO}$ poisoning. Gas masks for use in rescue work in such cases are fitted with canisters containing a mixture of metallic oxides, as $\mathrm{MnO}_{2}, \mathrm{CuO}, \mathrm{Co}_{2} \mathrm{O}_{3}$ and $\mathrm{Ag}_{2} \mathrm{O}$ (hopcalite). A mixture of this kind acts catalytically, any carbon monoxide passing into the canister being oxidized to carbon dioxide by the oxygen of the air. 
Exercises. - 1. (a) What physical property of graphite enables it to cover the surface of a stove so effectively? (b) How does "polishing" with a brush contribute to the result? (c) Why not use paint on a stove? (d) Explain why graphite can be used as a lubricant.

2. If a metal formed the positive electrode (anode) in electrolyzing sodium chloride solution, what chemical change might it undergo (p. 54), and which metals would be least rapidly attacked? What objection is there to using the latter metals in practice?

3. When one cubic meter of oxygen acts upon carbon, what volumes (at the same temperature and pressure): (a) of carbon dioxide; (b) of carbon monoxide can be obtained?

4. Make the equation: (a) for the formation of methane by union of carbon and hydrogen; (b) for the reduction of stannic oxide $\left(\mathrm{SnO}_{2}\right)$ by carbon.

5. Make equations for: (a) the action of sulphuric acid upon calcium carbonate; (b) carbon dioxide on sodium hydroxide solution (p. 336); (c) the burning of aluminium in carbon dioxide.

6. From the fact that the molecular weight of carbon dioxide is 44, how do we infer that it is one-half heavier than air?

7. Why does soda water remain quiescent in the closed bottle, and why does it effervesce when the bottle is opened?

8. Rewrite the equations on p. 336 in full ionic form.

9. Name the variety of chemical change (p. 132) to which belongs the reaction shown in each equation in this chapter.

10. Assuming that air contains oxygen and nitrogen in the proportion of $1: 4$ by volume, what are the theoretical proportions of carbon monoxide and nitrogen in producer gas?

11. (a) What volume of water gas is produced from each liter of steam, and (b) what is the proportion of the component gases in the product? (c) What impurities should you expect to find in water gas? (d) How should you attempt to separate the components of water gas? 
12. Why is water gas an especially valuable source of heat when high temperatures are required?

13. Make a list of metals the oxides of which would be reduced by carbon monoxide. 


\section{CHAPTER XXIX}

\section{THE HYDROCARBONS AND THEIR DERIVATIVES. FLAME}

THE compounds of carbon and hydrogen are called the hydrocarbons. Hundreds of different hydrocarbons, containing different proportions of the two elements, are known. The natural oil petroleum is a mixture of many substances of this class.

The hydrocarbons fall into several distinct series, the chief one of which contains methane $\mathrm{CH}_{4}$ as its simplest member. On account of the fact that certain members of this set are found in paraffin, it is commonly known as the paraffin series. For the reason that in this series the carbon has all its four valences employed, the members are also called the saturated hydrocarbons.

Paraffin or Saturated Series of Hydrocarbons.-The following is a list of the names, formulæ, and boiling-points of seven of the simplest hydrocarbons of this series, and of two of the higher members of the series:

$\begin{array}{lcllc}\text { Methane } \mathrm{CH}_{4} & \text { b.-p. } & -164^{\circ} & \text { Hexane } \mathrm{C}_{6} \mathrm{H}_{14} & \text { b.-p. } 71^{\circ} \\ \text { Ethane } \mathrm{C}_{2} \mathrm{H}_{6} & -89.5^{\circ} & \text { Heptane } \mathrm{C}_{7} \mathrm{H}_{16} & 99^{\circ} \\ \text { Propane } \mathrm{C}_{3} \mathrm{H}_{8} & -37^{\circ} & \text { Hexadecane } \mathrm{C}_{16} \mathrm{H}_{84} & 287.5^{\circ} \\ \text { Butane } \mathrm{C}_{4} \mathrm{H}_{10} & +1^{\circ} & & \text { m.-p. } 18^{\circ} \\ \text { Pentane } \mathrm{C}_{5} \mathrm{H}_{12} & 35^{\circ} & \text { Pentatriacontane } \mathrm{C}_{36} \mathrm{H}_{72} & \text { m.-p. } 74.7^{\circ}\end{array}$

After the first four, the names are based on the Greek numerals corresponding to the number of carbon atoms in the molecule. On comparing the formulæ, we observe that in each, the number of units of hydrogen is equal to twice the number of carbon units plus two. The general formula is therefore $\mathrm{C}_{n} \mathrm{H}_{2 n+2}$. The series affords a striking illustration of the law of multiple proportions. We note, further, that the first four are gases at the ordinary tem- 
perature. The members of the series from pentane to pentadecane $\left(\mathrm{C}_{15} \mathrm{H}_{32}\right)$ are liquid under ordinary conditions. From hexadecane onwards they are solids, with higher and higher melting-points.

In these compounds the carbon is quadrivalent, and each substance is related to the preceding one by containing the additional units $\mathrm{CH}_{2}$. The formulæ of the first three members may be written graphically to illustrate these two facts:

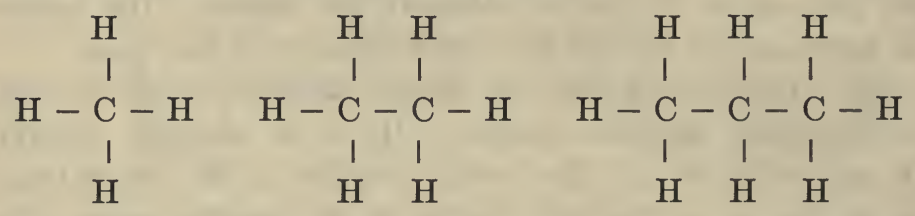

Petroleum.- Petroleum is a thick, greenish-brown oil. When borings are made into the oil-bearing strata, the oil either gushes up, or is pumped to the surface. In the United States many thousands of miles of pipe-lines are used to transport the oil, with the aid of force pumps, to the refineries, and in 1920 nearly 450 million barrels (42 gal. each) were produced. The world's production in 1920 was 700 million barrels.

After the United States, Mexico and Russia are the chief producers of petroleum.

Oil Refining.-The natural oil is a complex mixture, and is partially separated by distillation (p. 67) into products which are still mixtures, but are suited to special purposes. The components of lower boiling-point come off first and the temperature rises steadily as these components are eliminated and those of higher and higher boiling-point enter the vapor. As certain temperatures are reached (or as the sp. gr. of the distillate attains certain values) the condensed liquid is diverted into different vessels, so as to collect together the "fractions" of the same kind. This is called fractional distillation. 
At some suitable stage, the residual oil is chilled, and a quantity of the solid members of the series $\left(\mathrm{C}_{22} \mathrm{H}_{46}\right.$ to $\left.\mathrm{C}_{28} \mathrm{H}_{58}\right)$ crystallizes in flakes (solid paraffin) and is separated by filtration in presses. The final residue is used for lubricants and for fuel. The fractions are still mixtures, but contain mainly compounds lying close together in the series. Some of the products are as follows:

\begin{tabular}{|c|c|c|c|}
\hline Name & Main Components & B.-P. & Uses \\
\hline Petroleum ether & Pentane, hexane. & $40^{\circ}-70^{\circ}$ & Solvent, gas-making \\
\hline Gasoline... & Hexane, heptane. & $70^{\circ}-90^{\circ}$ & Solvent, fuel \\
\hline Naphtha. & Heptane, octane. & $80^{\circ}-120^{\circ}$ & Solvent, fuel \\
\hline Benzine. & Octane, nonane... & $120^{\circ}-150^{\circ}$ & Solvent \\
\hline Kerosene & Decane-hexadecane.. & $150^{\circ}-300^{\circ}$ & Illuminating oil \\
\hline
\end{tabular}

Vaseline, $\mathrm{C}_{22} \mathrm{H}_{46}$ to $\mathrm{C}_{23} \mathrm{H}_{48}$, is separated in some refineries. Solid paraffin is employed for waterproofing paper, as an ingredient in candles, and in making chewing gum.

Asphalt, a natural mixture of the solid hydrocarbons, found particularly in Trinidad, is used in road-making.

Oil Shale.- In Scotland, petroleum is also obtained by heating shale. The shale is a clay deposit, which contains no oil as such, but which when heated gives off fuel gas, ammonia (see p. 299), petroleum oils, and many valuable hydrocarbon derivatives. The richer shales yield from 30 to 40 gallons of oil per ton. The oil usually contains a much larger percentage of unsaturated hydrocarbons than well petroleum. The yield can be somewhat increased, and the proportion of undesirable unsaturated hydrocarbons diminished, by blowing superheated steam into the retorts during the distillation.

Natural Gas: Methane $\mathrm{CH}_{4}$. - Natural gas is obtained from wells, tapping strata close to those which contain petroleum, and in the same localities. It often issues under very high pressure. 
It owes its combustibility to its chief component (over 90 per cent), methane $\mathrm{CH}_{4}$. It is largely used as a fuel in the regions in which it is found and, in the United States, the annual value of the gas so consumed is nearly $\$ 160,000,000(1919)$. The same gas issues from many coal seams ("fire-damp"), and forms explosive mixtures with the air of mines. It rises to the surface when stagnant pools containing decomposing vegetable matter are stirred ("marsh-gas").

The formation of methane by direct union of carbon and hydrogen has already been discussed (p. 331).

Chemical Properties of the Hydrocarbons. - The hydrocarbons, whether pure or in solution, show no conductivity for electricity. They have none of the chemical properties of acids, bases, or salts, and therefore do not enter into double decompositions with substances of these classes. The saturated hydrocarbons are in fact quite indifferent to the presence of most chemical reagents.

All the hydrocarbons burn with oxygen or air to form carbon dioxide and water:

$$
\begin{aligned}
\mathrm{CH}_{4}+2 \mathrm{O}_{2} & \rightarrow \mathrm{CO}_{2}+2 \mathrm{H}_{2} \mathrm{O} . \\
\mathrm{C}_{7} \mathrm{H}_{16}+11 \mathrm{O}_{2} & \rightarrow 7 \mathrm{CO}_{2}+8 \mathrm{H}_{2} \mathrm{O} .
\end{aligned}
$$

The water can be shown by its condensation on a cold vessel held over the flame. The carbon dioxide gives a precipitate of calcium carbonate (p. 336) when the gases rising from the flame are drawn through lime-water.

All the hydrocarbons, when heated strongly (air excluded), decompose or crack. They usually lose a part of their hydrogen and become unsaturated. These of high molecular weight break up to give a mixture of hydrocarbons of low molecular weight. Ethylene $\mathrm{C}_{2} \mathrm{H}_{4}$, for example, is produced in large amounts by heating the higher members of the series to a red heat. On the other hand, the lower members of the series, when heated, often 
give compounds of higher molecular weight. Thus, methane gives ethylene and acetylene, along with hydrogen:

$$
\begin{aligned}
& 2 \mathrm{CH}_{4} \rightarrow \mathrm{C}_{2} \mathrm{H}_{4}+2 \mathrm{H}_{2} . \\
& 2 \mathrm{CH}_{4} \rightarrow \mathrm{C}_{2} \mathrm{H}_{2}+3 \mathrm{H}_{2} .
\end{aligned}
$$

At a white heat all the hydrocarbons decompose into hydrogen and free carbon.

The latter is deposited in a dense form called gas-carbon, which is used in making carbon rods for arc lights and electric furnaces, and carbon plates for batteries, and for the electrodes employed in electrolysis. The carbon is ground up, moistened with petroleum residues, subjected to hydraulic pressure and finally heated strongly to expel volatile matter.

Derivatives of the Hydrocarbons.-Although the hydrocarbons are themselves almost inert chemically, yet many important classes of organic substances may be regarded as their derivatives, one or more atoms of hydrogen in the graphic formula (p. 344) being replaced by other elements. The following tabulation should be carefully studied, and the graphic formula of each compound mentioned should be written down by the student.

1. Halogen Derivatives. When a mixture of methane and chlorine is exposed to sunlight several successive changes occur:

$$
\begin{aligned}
& \mathrm{CH}_{4}+\mathrm{Cl}_{2} \rightarrow \mathrm{HCl}+\mathrm{CH}_{3} \mathrm{Cl} \text { (methyl chloride) } \\
& \mathrm{CH}_{3} \mathrm{Cl}+\mathrm{Cl}_{2} \rightarrow \mathrm{HCl}+\mathrm{CH}_{2} \mathrm{Cl}_{2} \text { (methylene chloride) } \\
& \mathrm{CH}_{2} \mathrm{Cl}_{2}+\mathrm{Cl}_{2} \rightarrow \mathrm{HCl}+\mathrm{CHCl}_{3} \text { (chloroform) } \\
& \mathrm{CHCl}_{3}+\mathrm{Cl}_{2} \rightarrow \mathrm{HCl}+\mathrm{CCl}_{4} \text { (carbon tetrachloride). }
\end{aligned}
$$

Chloroform $\mathrm{CHCl}_{3}$, used as an anæsthetic, and carbon tetrachloride $\mathrm{CCl}_{4}$ (p. 332) are familiar substances. Iodoform $\mathrm{CHI}_{3}$ is employed in surgical dressing. These substances are not salts, and are not ionized in solution. 
2. Hydroxyl Derivatives (Alcohols). Water acts slowly upon methyl chloride, according to the equation:

$\mathrm{CH}_{3} \mathrm{Cl}+\mathrm{H}_{2} \mathrm{O} \rightleftharpoons \mathrm{HCl}+\mathrm{CH}_{3} \mathrm{OH}$ (methyl alcohol).

The reaction is reversible and incomplete, but can be accelerated and carried to completion by addition of a base, which removes the $\mathrm{HCl}$ as fast as it is formed. Ethyl chloride $\mathrm{C}_{2} \mathrm{H}_{5} \mathrm{Cl}$ gives ethyl alcohol $\mathrm{C}_{2} \mathrm{H}_{5} \mathrm{OH}$.

Although containing the radical $\mathrm{OH}$, the alcohols are not ionized in solution, and are therefore not bases. They are extensively used as solvents for other organic substances (see also p. 418).

3. Oxygen Derivatives (Ethers). When ethyl alcohol and eoncentrated sulphuric acid are heated to $140^{\circ}$, water and ether $\left(\mathrm{C}_{2} \mathrm{H}_{5}\right)_{2} \mathrm{O}$ distil off. The action occurs in two stages:
(1) $\mathrm{C}_{2} \mathrm{H}_{5} \mathrm{OH}+\mathrm{H}_{2} \mathrm{SO}_{4} \rightarrow \mathrm{H}_{2} \mathrm{O}+\mathrm{C}_{2} \mathrm{H}_{5} \cdot \mathrm{HSO}_{4}$
(2) $\mathrm{C}_{2} \mathrm{H}_{5} \cdot \mathrm{HSO}_{4}+\mathrm{C}_{2} \mathrm{H}_{5} \mathrm{OH} \rightarrow \mathrm{H}_{2} \mathrm{SO}_{4}+\left(\mathrm{C}_{2} \mathrm{H}_{5}\right)_{2} \mathrm{O}$.

Ether $\left(\mathrm{C}_{2} \mathrm{H}_{5}\right)_{2} \mathrm{O}$ is a very volatile and inflammable liquid, used as an anæsthetic and as a solvent for resins, fats and oils.

4. Aldehydes. By fractional combustion of methyl alcohol (passage of a heated mixture of alcohol vapor and air over a metal catalyst), formaldehyde $\mathrm{H} . \mathrm{CHO}$ is obtained:

$$
2 \mathrm{CH}_{3} . \mathrm{OH}+\mathrm{O}_{2} \rightarrow 2 \mathrm{CH}_{2} \mathrm{O}+2 \mathrm{H}_{2} \mathrm{O} \text {. }
$$

Formaldehyde is a gas. Its solution in water (formalin) is employed as an antiseptic and disinfectant. Its property of hardening gelatins makes it valuable in the leather industry and in the manufacture of artificial silk. It is also used in making dyes and in the production of bakelite (p. 481). The corresponding derivative of ethyl alcohol is acetaldehyde $\mathrm{CH}_{3} . \mathrm{CHO}$. The group .CHO is characteristic of aldehydes.

5. Acids. By further partial oxidation, alcohols or aldehydes give members of the fatty acid series. Thus ethyl alcohol can 
THE HYDROCARBONS AND THEIR DERIVATIVES. FLAME 349

be oxidized directly to acetic acid $\mathrm{CH}_{3} \mathrm{COOH}$ by passing a mixture of the vapor, with air, over specially prepared platinum as a catalyst:

$$
\mathrm{C}_{2} \mathrm{H}_{5} \cdot \mathrm{OH}+\mathrm{O}_{2} \rightarrow \mathrm{CH}_{3} \cdot \mathrm{COOH}+\mathrm{H}_{2} \mathrm{O} \text {. }
$$

The first acid of this series is formic acid H.COOH, a corrosive liquid secreted by red ants and present in stinging nettles. Acetic acid (see p. 419) has many industrial uses.

The lower members of the fatty acid series are perfectly miscible with water, and are slightly ionized in aqueous solution. By neutralization with bases we obtain salts, such as the formates and the acetates. Only the hydrogen of the characteristic . $\mathrm{COOH}$ group, it must be noted, is replaceable by metals.

6. Ketones. When calcium acetate $\mathrm{Ca}\left(\mathrm{CH}_{3} \cdot \mathrm{COO}\right)_{2}$ is heated, acetone $\left(\mathrm{CH}_{3}\right)_{2}$. $\mathrm{CO}$ distils off :

$$
\mathrm{Ca}\left(\mathrm{CH}_{3} \cdot \mathrm{COO}\right)_{2} \rightarrow \mathrm{CaCO}_{3}+\left(\mathrm{CH}_{3}\right)_{2} \cdot \mathrm{CO}
$$

The ketones resemble the aldehydes in many respects, but their characteristic group : $\mathrm{CO}$ is not directly combined with hydrogen. Acetone is a liquid boiling at $56^{\circ}$, used in large quantities in the industries as a solvent.

7. Esters. Alcohols and acids interact slowly and incompletely to form esters. Thus when ethyl alcohol and acetic acid are used, we obtain ethyl acetate $\mathrm{C}_{2} \mathrm{H}_{5} \cdot \mathrm{COO} \cdot \mathrm{CH}_{3}$ :

$$
\mathrm{C}_{2} \mathrm{H}_{5} \cdot \mathrm{OH}+\mathrm{CH}_{3} \cdot \mathrm{COOH} \leftrightarrows \mathrm{H}_{2} \mathrm{O}+\mathrm{C}_{2} \mathrm{H}_{5} \cdot \mathrm{COO} \cdot \mathrm{CH}_{3}
$$

The action may be catalyzed by the addition of a little sulphuric acid.

The equation, as given above, bears certain resemblances to a neutralization. It differs sharply, however, from a true neutralization in several respects. An alcohol is not a base, neither is an ester a salt. Both classes of substances are non-ionized in solution. True neutralization takes place instantaneously, while the foregoing action, and all like it, proceed very slowly. 
The esters form the sweet-smelling constituents of plants. Many are now produced synthetically as substitutes for natural and fruit essences.

A few additional classes of hydrocarbon derivatives will be given later (p. 353).

Unsaturated Hydrocarbons. - In addition to the saturated series of hydrocarbons, several other series are known in which smaller proportions of hydrogen are present. Thus, ethylene $\mathrm{C}_{2} \mathrm{H}_{4}$, to which illuminating gas largely owes the luminosity of its flame, belongs to a series $\mathrm{C}_{n} \mathrm{H}_{2 n}$, all the members of which contain two atoms of hydrogen less than the corresponding compounds of the first series. Again, acetylene $\mathrm{C}_{2} \mathrm{H}_{2}$ is the first member of a series $\mathrm{C}_{n} \mathrm{H}_{2 n-2}$, and benzene $\mathrm{C}_{6} \mathrm{H}_{6}$ begins a series $\mathrm{C}_{n} \mathrm{H}_{2 n-6}$. These are all unsaturated because the full valence of the carbon is not in use, and these compounds, therefore, unite more or less readily with hydrogen, chlorine, bromine, and concentrated sulphuric acid. The hydrocarbons of all the series are mutually soluble, but none of them dissolve in water.

Members of the ethylene and acetylene series are found in petroleum, and are formed also to some extent by decomposition during the distillation. As oil containing them acquires darkcolored products by chemical change, the oils are always refined before being sold. They are agitated with concentrated sulphuric acid, which unites with the unsaturated substances and, being insoluble in the oil, collects in a layer below it. The oil is finally washed free from the acid with dilute alkali and with water.

Ethylene $\mathrm{C}_{2} \mathrm{H}_{4}$ - The formation of one molecule of ethylene from two molecules of methane with elimination of two molecules of hydrogen suggests its graphic formula:

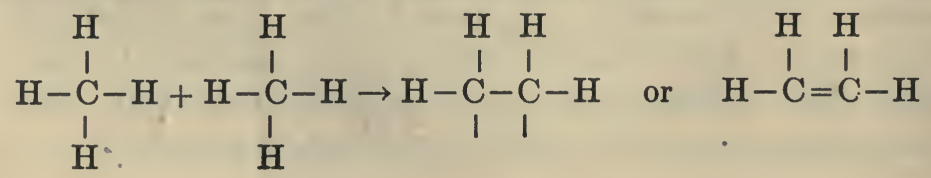


THE HYDROCARBONS AND THEIR DERIVATIVES. FLAME 351

Ethylene is a gas which burns in the air with a highly luminous flame (see p. 356). It combines directly with bromine to form ethylene bromide $\mathrm{C}_{2} \mathrm{H}_{4} \mathrm{Br}_{2}$.

The hydrocarbons of the ethylene series are known as olefines. They are of value as illuminants. Their derivatives are similar in character to those of the paraffin hydrocarbons, but are more active chemically in view of their unsaturation. When heated with hydrogen in the presence of a catalyst, such as finely-divided nickel, they give the corresponding saturated derivatives (compare pp. 433-4).

Acetylene $\mathrm{C}_{2} \mathrm{H}_{2}$. - A mixture, containing acetylene, is formed when any hydrocarbon is heated strongly (p. 346), air being excluded. As in the case of ethylene, the formation from methane by loss of hydrogen (p. 347) suggests the graphic formula:

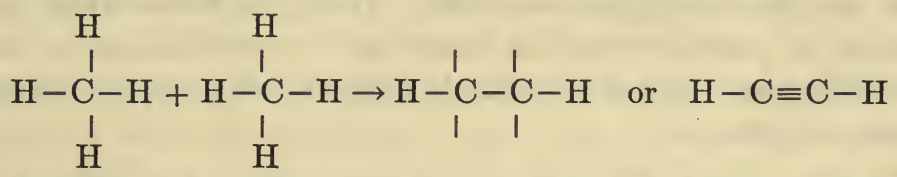

Pure acetylene is prepared by the action of water on calcium carbide (p. 333):

$\mathrm{CaC}_{2}+2 \mathrm{H}_{2} \mathrm{O} \rightarrow \mathrm{Ca}(\mathrm{OH})_{2}+\mathrm{C}_{2} \mathrm{H}_{2} \uparrow$.

Calcium hydroxide (slaked lime) remains. The gas burns with a flame even more luminous than that of ethylene. It is therefore made in generators by the foregoing action for use on automobiles and for lighting buildings remote from a public supply of illuminating gas. Acetylene tanks, which are also in use, contain acetylene dissolved, under high pressure, in acetone.

The Acetylene Blowpipe or Torch.-Acetylene decomposes, when heated, with liberation of heat:

$$
\mathrm{C}_{2} \mathrm{H}_{2} \rightarrow 2 \mathrm{C}+\mathrm{H}_{2}+58,100 \text { cal. }
$$

When acetylene burns with oxygen, therefore,

$$
2 \mathrm{C}_{2} \mathrm{H}_{2}+5 \mathrm{O}_{2} \rightarrow 4 \mathrm{CO}_{2}+2 \mathrm{H}_{2} \mathrm{O} \text {, } 619680
$$


we obtain not only the heat due to the combustion (p. 162) of the carbon to carbon dioxide $(4 \times 96,900 \mathrm{cal}$.) and of the hydrogen to water $(116,200$ cal.) but also the heat due to the decomposition of the gas $(2 \times 58,100$ cal.). The temperature of the flame is, therefore, the highest that can be reached by the combustion of any easily obtainable gaseous mixture. The oxy-acetylene flame, produced by means of a suitable burner (Fig. 29, p. 56), the gases being furnished from small, portable tanks, is now used for cutting metals. Such a flame will melt its way through a 6-inch shaft of steel, or a heavy steel plate several feet wide, in less than one minute, cutting the object in two. Steel buildings have been taken apart rapidly by this device.

Blau gas and oil gas, mixtures of hydrocarbons made by "cracking" (see p. 346) heavy oils, 'are now largely displacing acetylene for uses like those just mentioned. They give flames which are almost as effective, and are more easily controlled. Even the oxy-hydrogen torch is remarkably efficient, when applied to the same purposes.

Benzene $\mathrm{C}_{6} \mathrm{H}_{6}$. - This is the first member of the aromatic hydrocarbon series. It may by synthesized by heating acetylene in a closed vessel at a moderately low temperature:

$$
3 \mathrm{C}_{2} \mathrm{H}_{2} \rightarrow \mathrm{C}_{6} \mathrm{H}_{6} \text {. }
$$

In practice it is obtained, with many of its valuable derivatives, as a by-product in the production of coke (p. 424).

The graphic formula of benzene is represented as a closed ring structure:

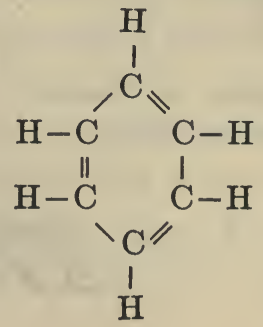


The hydrocarbons of the benzene series exhibit, in fact, many of the properties of olefines, combining directly with hydrogen and with the halogens to form saturated compounds such as hexahydrobenzene $\mathrm{C}_{6} \mathrm{H}_{12}$ and benzene hexabromide $\mathrm{C}_{6} \mathrm{H}_{6} \mathrm{Br}_{6}$.

The second member of the aromatic series is toluene $\mathrm{C}_{6} \mathrm{H}_{5} . \mathrm{CH}_{3}$. The third is xylene $\mathrm{C}_{6} \mathrm{H}_{4} \cdot\left(\mathrm{CH}_{3}\right)_{2}$. Naphthalene $\mathrm{C}_{10} \mathrm{H}_{8}$ and anthracene $\mathrm{C}_{14} \mathrm{H}_{10}$ are members of more complex series, containing more than one ring.

Of all hydrocarbons, those of the aromatic series, with their derivatives, are the most important. They are of particular significance in the dye-stuff and explosive industries, and in the manufacture of synthetic perfumes and drugs. They give aldehydes, acids, esters, etc., similar to those listed under the paraffin hydrocarbons (pp. 347-50). In addition to these, the following extremely valuable classes of derivatives should be noted:

(1). Phenols. The substitution of hydroxyl for a hydrogen atom in benzeriè gives phenol $\mathrm{C}_{6} \mathrm{H}_{5} \mathrm{OH}$, a substance quite different in many of its properties from an alcohol, although it resembles the alcohols in forming esters with acids (see p. 349). Phenol, when pure, is a colorless solid melting around $40^{\circ}$, with a characteristic odor. It is strongly antiseptic, corrosive and poisonous. In solution it is a weak acid.

(2). Nitro-compounds. Nitrobenzene $\mathrm{C}_{6} \mathrm{H}_{5} \mathrm{NO}_{2}$ is obtained by the action of a mixture of concentrated nitric and sulphuric acids upon benzene in the cold:

$$
\mathrm{C}_{6} \mathrm{H}_{6}+\mathrm{HNO}_{3} \rightarrow \mathrm{C}_{6} \mathrm{H}_{5} \cdot \mathrm{NO}_{2}+\mathrm{H}_{2} \mathrm{O} \text {. }
$$

It is a pale yellow liquid, with a smell resembling bitter almonds, and is used in scenting cheap soap.

(3). Amino-compounds. Reduction of nitrobenzene by a metal in acid solution gives aniline $\mathrm{C}_{6} \mathrm{H}_{5} \cdot \mathrm{NH}_{2}$. The aminocompounds are derivatives of ammonia, and their solutions accordingly are weakly basic. Aniline, when pure, is a colorless, oily liquid, boiling at $185^{\circ}$. It is the parent substance of the countless aniline dyes. 
In subsequent chapters we shall return to the different classes of organic substances, tabulated here and on pp. 347-50, and discuss their properties and industrial uses in greater detail.

\section{Flame}

We have encountered a variety of flames, from the simple one of hydrogen burning in air to the more complicated case of the luminous flame of ethylene or acetylene. The subject will now repay a somewhat closer study.

The Simple Flame.- The flame of hydrogen (giving water), or of carbon monoxide (forming carbon dioxide) is very simple in structure (Fig. 88). We find that there is a tapering column of unburnt gas in the interior, surrounded by a layer of hot

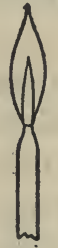
gas - the flame itself. The flame is therefore a hollow cone. That the flame is hollow is easily shown by holding a wooden match across it. The match is charred at the two points at which it crosses the flame, and remains unheated in the middle. These flames are simple, because only one chemical change occurs in them. The FIG. 88

flames are rather large, because sufficient oxygen to burn all the gas does not reach the latter at once, and the gas travels upwards and diffuses outwards a certain distance before being all consumed.

If oxygen is substituted for air, by lowering the jet into a jar of that gas, the flame becomes much smaller. In the absence of atmospheric nitrogen, there is now five times as much oxygen within a given range of the center of the jet as before. This chemical union, like any other, proceeds more rapidly with an increase in the concentration of the interacting substances (p. 233). It is therefore completed before the gas has time to diffuse very far from the opening of the jet.

The Candle Flame.-A candle is made of a mixture of paraffin and stearic acid (one of the higher members of the fatty acid 
series (p. 348), with the formula $\mathrm{C}_{17} \mathrm{H}_{35} . \mathrm{COOH}$, made from fat). When it burns, the whole phenomenon is vastly more complicated than the burning of hydrogen. The following are some of the stages in the process, which is operated by the flame's own heat. To start with, the wax is melted and ascends the wick by capillary action. This is merely a physical phenomenon. Then the chemical changes begin. (1) The melted compounds of carbon are decomposed by the heat (cracked, p. 346), being turned into more volatile compounds and gases which occupy the central hollow of the flame. (2) The compounds forming the gases and vapors are further decomposed at a white heat, giving free carbon and hydrogen (p. 347). (3) All the materials finally reach a sufficient supply of oxygen and are burned to water and carbon dioxide. There are thus three chemical changes, each of which takes place in a definite region that can be observed by the eye (Fig. 89). The formation of the gases from the melted wax (without gas, there would be no flame) takes place in the dark central region where there is no oxygen. The carbon is set free and glows brilliantly in the luminous cone that surrounds the gas and extends far above it. The final combustion occurs in a fainter cone of flame covering the whole exterior.

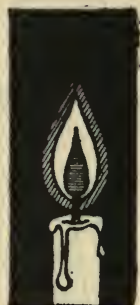

Fig. 89

That there is unburnt gas (produced by decomposition of the wax) in the center is easily shown by inserting a narrow tube, through which some of the gas will ascend. The free carbon in the luminous zone will show its presence by blackening a cold dish placed across the flame.

Lampblack. - When an iron vessel, cooled by a stream of water circulating through it, is suspended in the luminous flame of natural gas or burning petroleum, the carbon (soot) is deposited on the vessel. By rotating the latter, the soot can be continuously scraped off by a stationary piece of metal. The product, 
lampblack, mainly very finely divided carbon, is used in making printers' ink, India ink, and black varnish.

Carburetted Water Gas. - To fit water gas, essentially $\mathrm{H}_{2}+\mathrm{CO}$ (p. 337), which burns with a pale blue flame, for public service as an illuminating gas, unsaturated hydrocarbons, and particularly ethylene $\mathrm{C}_{2} \mathrm{H}_{4}$, which burn with a highly luminous flame, must be added. The water gas is passed through a tower, filled with strongly heated brick on which oil is continually sprayed. Mixed with the vapor of the oil, the gas goes into the "superheater" where, at a higher temperature, the decomposition into unsaturated hydrocarbons (cracking) takes place. The gas is then cooled and washed to remove the condensible hydrocarbons, which would otherwise collect in the service pipes with resulting waste of combustible material as well as obstruction in the delivery of the gas. A typical carburetted water gas has the composition: Illuminants (largely ethylene) 16.6 per cent; heating gases - methane 19.8 per cent, hydrogen 32.1

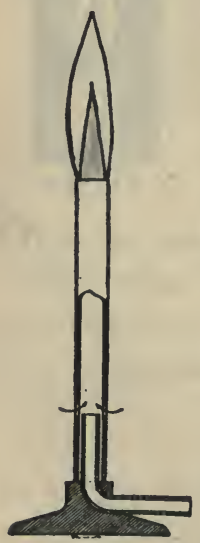

FIG. 90 per cent, carbon monoxide 26.1 per cent; impurities (nitrogen and carbon dioxide) 5.4 per cent.

Carburetted water gas has now largely supplanted coal gas (p. 423) for lighting and heating purposes.

Non-luminous Gas Flames.-When gas is to be used for heating, the complete combustion of the gas, without any intermediate liberation of free carbon, is desirable. This is achieved in the Bunsen burner (Fig. 90) by admitting air at the bottom of the burner, in such a way that the air mixes with the gas before the latter reaches the flame. The air cools the middle zone of the flame, so that at this point the temperature required for dissociating the ethy- 
THE HYDROCARBONS AND THEIR DERIVATIVES. FLAME 357

lene, and liberating carbon, is not reached. The oxygen in the air plays no part - mixing carbon dioxide or pure nitrogen with the gas has exactly the same effect. A flame of this kind is non-luminous.

Although the middle zone of the non-luminous flame is cooler than that of the luminous flame, the average temperature of the flame as a whole is higher. This is the case because the same total amount of heat is liberated in both cases, but the nonluminous flame as a whole is smaller in size.

The Bunsen type of burner, placed in a horizontal position (Fig. 91) is used in the ordinary gas cooking range. As with the Bunsen burner, some care is required to get good results. The holes which admit the air to the mixer must be kept clear of obstructions, as otherwise luminous flames are produced, smoke and soot are formed, and less heat is generated. The size of the openings must also be adjusted so_that the admission of too much air will not cause the flame to flash down the burner, and set fire to the gas within the mixer.

\section{Flames with Incandescent Mantles.} - When gas is burned in a Bunsen burner, a bright light may still be obtained from the flame. This is managed by suspending in the flame a structure ("mantle") made of the oxides of thorium (99 per cent) and of cerium (1 per cent). These oxides act as a contact agent, hastening the combustion and liberation of heat close to their surface, which thereby becomes incandescent.

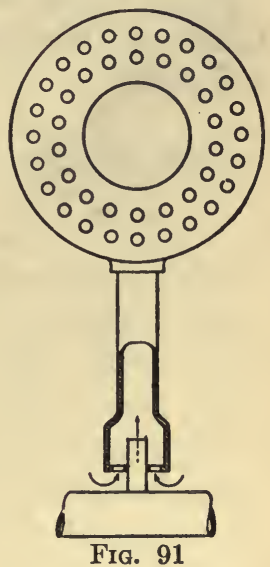
The light has about ten times the illuminating power of a flat flame burner using the same amount of gas.

Exercises.-1. When vegetable matter decays in the air the carbon it contains is finally all turned into carbon dioxide. 
When the same matter decays under water, it gives methane (p. 346). Explain the difference in the result.

2. What is the density (air =1) of (a) methane, (b) ethylene?

3. Write the graphic formulæ for propane, propyl chloride, propyl alcohol, dipropyl ether, propionaldehyde, propionic acid, dipropyl ketone, propyl propionate.

4. Write the graphic formulæ for toluene, phenol, nitrobenzene, aniline.

5. (a) Given a flame of hydrogen burning in a jar of air, what would be the effect on the flame of lowering the pressure of the air by means of an air pump? (b) What would be the effect on the average temperature of the flame? (c) How about the heat produced by burning $1 \mathrm{~g}$. of hydrogen in each case? (d) What differences would be observed in using an alcohol lamp at the bottom and on the top of a high mountain?

6. (a) In the candle or gas flame, what is the source of the light? (b) Why does such a flame become smoky when placed in a draft? 


\section{CHAPTER XXX}

\section{SILICON; BORON}

Silicon belongs to the carbon family, being, like carbon, quadrivalent and non-metallic.

Although silicon does not occur free in nature, yet its compounds are so plentiful that about 26 per cent of the terrestrial globe is silicon. Instead of naming all the rocks which contain it, such as sandstone, basalt, granite, and so forth, it is easier to say that limestone is the only common rock which is not siliceous.

Silicon Si.-The element is now manufactured at Niagara Falls and elsewhere, by heating sand $\left(\mathrm{SiO}_{2}\right)$ with coke in an electric furnace. The process closely resembles that for making carborundum (p. 333), except that less coke is used:

$$
2 \mathrm{C}+\mathrm{SiO}_{2} \rightarrow 2 \mathrm{CO} \uparrow+\mathrm{Si} \text {. }
$$

The element, as prepared in this way, is a grey, crystalline material.

\section{Silicon Dioxide $\mathrm{SiO}_{2}$ (Silica), Physical Properties.- Color-} less rock-crystal, often showing large hexagonal crystals, is pure silicon dioxide, deposited from natural solutions. When impurities enter into it, smoky quartz, rose quartz (pink), and amethyst (violet) are formed. Often the impurity changes during the growth of the deposit, and beautifully variegated specimens, like jasper, catseye, and agate are produced. Chalcedony, opal, and flint contain a small amount of water in combination. The nodules (rounded masses) of flint break in splinters, when struck, and our prehistoric ancestors dexterously fashioned their implements and weapons from this material. The solid structure of sponges and diatoms is also hydrated silicon dioxide. 
Diatomaceous earth (Tripoli powder) is used in making polishing powders and for removing coloring matters from oils.

Chemical Properties.- Silicon dioxide (sand) is not acted upon by acids, with the exception of hydrofluoric acid, which gives silicon tetrafluoride $\mathrm{SiF}_{4}$ and water:

$$
\mathrm{SiO}_{2}+4 \mathrm{HF} \rightarrow \mathrm{SiF}_{4} \uparrow+2 \mathrm{H}_{2} \mathrm{O} .
$$

When silicon dioxide is fused with sodium carbonate, carbon dioxide gas is liberated, and sodium silicate $\mathrm{Na}_{2} \mathrm{SiO}_{3}$ is formed:

$$
\mathrm{SiO}_{2}+\mathrm{Na}_{2} \mathrm{CO}_{3} \rightarrow \mathrm{CO}_{2} \uparrow+\mathrm{Na}_{2} \mathrm{SiO}_{3} \text {. }
$$

The resulting salt is very soluble and a concentrated aqueous solution is sold under the name of water-glass or soluble glass. This material is used as a filler in cheap soaps, as an ingredient in artificial stone, a coating to render wood or cloth fireproof, and a cement for uniting glass or porcelain. Eggs are preserved by being submerged in a solution of this salt.

When an acid is added to sodium silicate solution, silicic acid $\mathrm{H}_{4} \mathrm{SiO}_{4}$ or $\mathrm{H}_{2} \mathrm{SiO}_{3}$ (gelatinous, the degree of hydration varies with the conditions) is precipitated. If this acid is heated strongly, silica $\mathrm{SiO}_{2}$ remains as a powder.

Incompletely dehydrated silicic acid, containing 5-7 per cent of water (silica gel), is employed as an adsorbent material for recovering valuable vapors (such as sulphur dioxide, oxides of nitrogen, and volatile organic solvents) from the issuing gases in many large-scale industrial processes. The adsorbed vapors are given up on heating, and the gel is ready for renewed use. Silica gel is also of service in deodorizing petroleum oils, and as a catalyst (see p. 262).

Silicon Tetrachloride $\mathrm{SiCl}_{4}$. - This compound is made by direct union of the free elements. It is more conveniently prepared by passing chlorine over a strongly heated mixture of silicon 


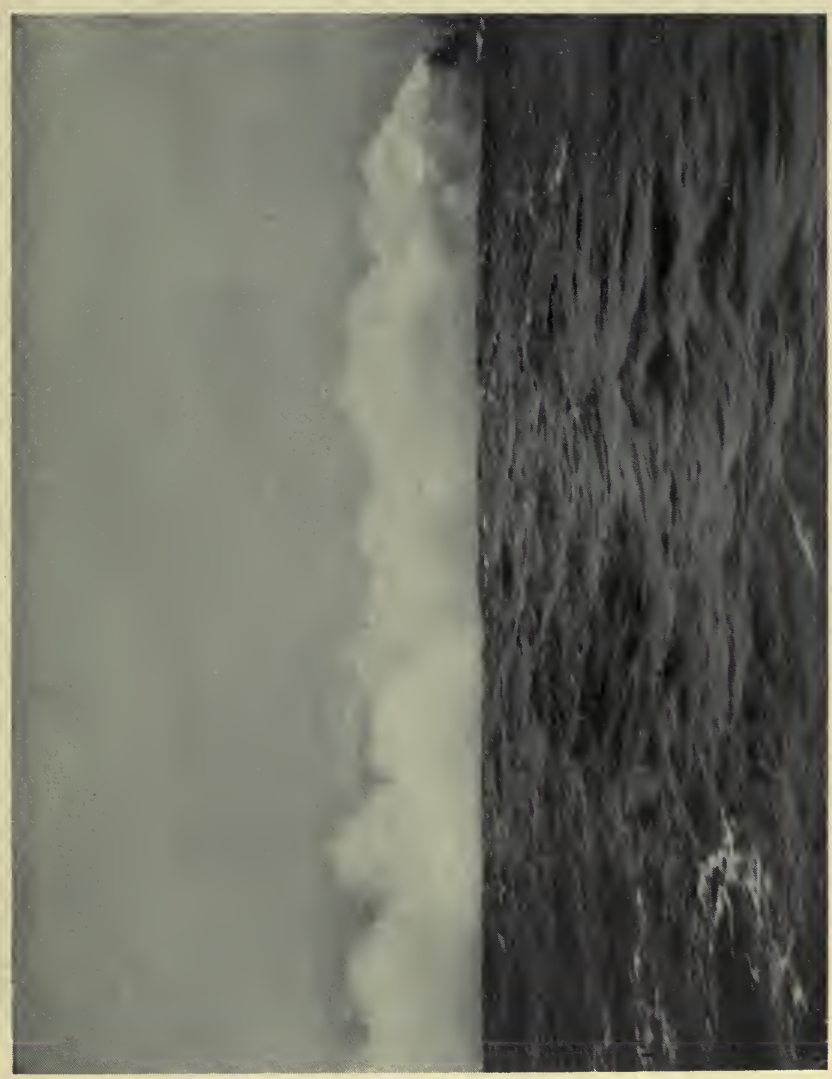

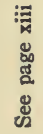

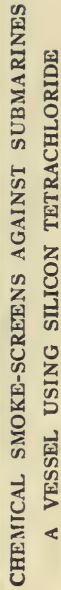




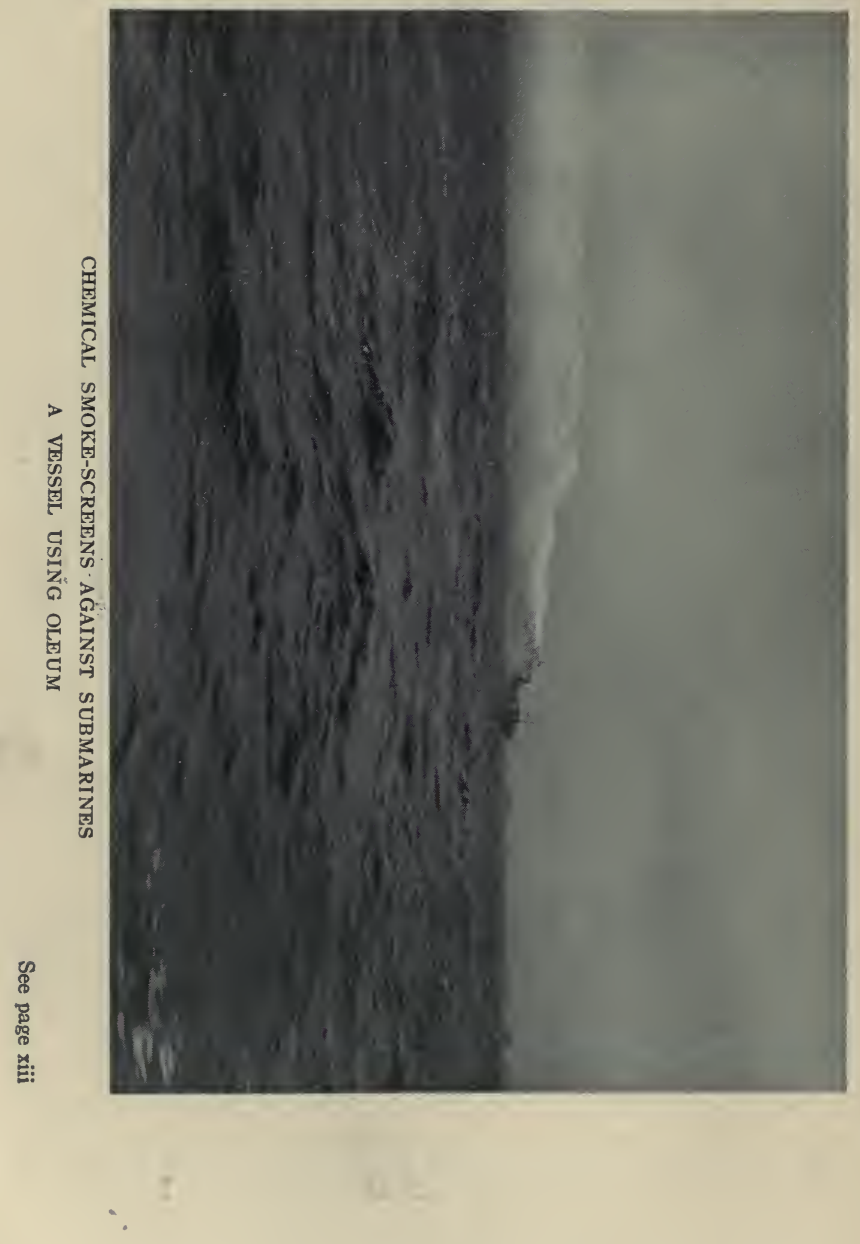


dioxide and carbon. The gaseous products enter a condenser in which the tetrachloride assumes the liquid form:

$$
2 \mathrm{Cl}_{2}+\mathrm{SiO}_{2}+2 \mathrm{C} \rightarrow \mathrm{SiCl}_{4}+2 \mathrm{CO} \text {. }
$$

Silicon tetrachloride is a colorless liquid (b.-p. $59^{\circ}$ ) which fumes strongly in moist air, giving silicic acid and $\mathrm{HCl}$. Mixed vapors of $\mathrm{SiCl}_{4}, \mathrm{NH}_{3}$ and $\mathrm{H}_{2} \mathrm{O}$ produce a very dense white smoke, consisting of minute particles of $\mathrm{NH}_{4} \mathrm{Cl}$ and silicic acid. This smoke was utilized during the war for screening vessels from submarines.

Glass.-Calcium carbonate (limestone) interacts at a high temperature with sand in the same way as does sodium carbonate:

$$
\mathrm{SiO}_{2}+\mathrm{CaCO}_{3} \rightarrow \mathrm{CO}_{2} \uparrow+\mathrm{CaSiO}_{3}
$$

giving calcium silicate. Now sodium silicate, when alone, is soluble in water. Calcium silicate is insoluble, but forms a brittle, crystalline mass. By using both sodium and calcium carbonates, and employing a larger proportion of sand than that shown in the equation, a material is obtained which has the qualities required in glass. When cooled, the molten mass becomes viscous and finally, for all practical purposes, solid. Yet it does not crystallize - it is amorphous. It is also practically insoluble in water.

By pouring the viscous material into moulds, or stamping it with dies, articles of pressed glass are obtained. Bottles are blown, by taking up a sufficient mass of the hot, thick liquid on the end of an iron tube, inserting it in a mould, and blowing until the outline of the mould is filled. Window glass is made by blowing an immense, elongated bubble ( 6 by $1 \frac{1}{2} \mathrm{ft}$.), ripping it while still hot and soft, and flattening it out. Plate glass for windows and mirrors is manufactured by pouring out the material upon a cast-iron table, with a raised rim, and passing a large, heated iron roller over it. The plate is subsequently ground flat on both sides and polished with rouge $\left(\mathrm{Fe}_{2} \mathrm{O}_{3}\right)$. 
Soda-calcium glass is called soft glass, because it is easily softened by heating. When potassium carbonate is substituted for sodium carbonate, a less fusible substance, used in making some chemical apparatus, and called hard glass, is obtained. When lead oxide is employed in place of the limestone, a potassium-lead silicate $\mathrm{K}_{2} \mathrm{SiO}_{3}, \mathrm{PbSiO}_{3}, x \mathrm{SiO}_{2}$ is formed which, on being cooled, gives flint glass. This glass has a higher density and greater brilliancy than soft glass, and is used in making vessels of cut glass and lamp chimneys. The cutting is done with a revolving grinding wheel.

When glass is allowed to cool quickly, the product is very brittle and apt to crumble to pieces on receiving a shock or scratch. Glassware is therefore all annealed, by being passed on a slowly moving frame through a long furnace, which is very hot at the entrance and much cooler at the exit.

Colored glass is made by adding oxides of metals which, with the silica, give colored silicates. Oxide of chromium gives green silicates, oxides of copper and of cobalt blue silicates, and oxide of manganese violet. Gold oxide is reduced to the metal, which goes into colloidal solution and gives ruby glass. Milky glass is made by adding calcium fluoride, or stannic oxide. The green color of bottle glass is due to iron (ferrous silicate) derived from impure sand or limestone.

The rough surface of ground glass is produced with a sand blast. For engraved glass, the surface is covered by a stencil to protect it from the sand blast, and only the pattern is left exposed.

In granite iron ware the surface is covered with a thin layer of easily fusible glass (enamel, see borax).

Pure quartz can be melted in the oxy-hydrogen blowpipe, and recently chemical apparatus (silica ware) has been made out of it. It has the advantage of being less soluble than glass, and of not breaking even when it is heated white hot and quenched in cold water. Glass breaks when chilled, because the parts first cooled shrink considerably and a great strain is produced. Quartz 
suffers very little change in volume with change in temperature, and so unequal cooling causes almost no strain.

Pyrex glass, a borosilicate, has also come into extensive use lately for laboratory ware and for cooking vessels. Its low coefficient of expansion renders it much less liable to crack under sudden temperature changes. Like pure silica, it is very resistant to chemicals. It possesses the further advantage of withstanding much greater mechanical shocks.

\section{Boron B}

The element boron resembles silicon and graphite in appearance. It has no applications.

Boric Acid $\mathrm{H}_{3} \mathrm{BO}_{3}$. - This acid is contained in the steam which issues from the ground in certain parts of Tuscany. It is caught in water, placed in basins built over the " soffioni," and separated by evaporation. Much of it is also made from borax.

Boric acid crystallizes in white, slippery scales. It dissolves somewhat in water $\left(4: 100\right.$ at $\left.18^{\circ}\right)$, and the saturated solution, mixed with an equal volume of water, is used as an eye-wash. Boric acid is an exceedingly weak acid; its solution scarcely affects litmus. It is a mild antiseptic, and preserves foods by preventing the development of moulds and bacteria. It is often added to talcum powder to prevent infection of irritated skin.

When heated, it loses water and gives tetraboric acid:

Skeleton: $\quad \mathrm{H}_{3} \mathrm{BO}_{3} \rightarrow \mathrm{H}_{2} \mathrm{~B}_{4} \mathrm{O}_{7}+\mathrm{H}_{2} \mathrm{O}$

Balanced: $\quad 4 \mathrm{H}_{3} \mathrm{BO}_{3} \rightarrow \mathrm{H}_{2} \mathrm{~B}_{4} \mathrm{O}_{7}+5 \mathrm{H}_{2} \mathrm{O}$

and eventually boric anhydride $\mathrm{B}_{2} \mathrm{O}_{3}$.

Borax.-This salt is the decahydrate of sodium tetraborate $\mathrm{Na}_{2} \mathrm{~B}_{4} \mathrm{O}_{7}, 10 \mathrm{H}_{2} \mathrm{O}$.

It is made by adding calcium borate, found in California, to sodium carbonate solution. The precipitate of calcium car- 
bonate is separated by filtration, and the solution is concentrated until crystals appear upon cooling.

It is a white crystalline salt. It is added to the glass, used for enamelling and glazing, to make it more fusible and easier to spread in a thin layer. It is a preservative. Since it contains but a small proportion of the metallic oxide $\left(\mathrm{Na}_{2} \mathrm{O}, 2 \mathrm{~B}_{2} \mathrm{O}_{3}\right)$, it combines with other metallic oxides when fused with them. For this reason the powdered salt is sometimes sprinkled on tarnished metallic surfaces which are to be soldered or brazed. The heat of the bolt or blowpipe melts the borax, and the latter removes the oxides and permits perfect running of the solder over the surface. The borates thus formed are often colored, and the colors afford a means of recognizing the metallic compound which produced them. In chemical analyses a bead of borax, produced by fusion on a platinum wire, is heated with a particle of the unknown compound and its color then examined. The colors are similar to those already described under colored glass (p. 362).

When hydrochloric or nitric acid is added to a hot, concentrated solution of borax, boric acid crystallizes out:

$$
\mathrm{Na}_{2} \mathrm{~B}_{4} \mathrm{O}_{7}+2 \mathrm{HCl}+5 \mathrm{H}_{2} \mathrm{O} \rightarrow 2 \mathrm{NaCl}+4 \mathrm{H}_{3} \mathrm{BO}_{3} \downarrow \text {. }
$$

Boric acid may be recognized by the green tint which it confers on the Bunsen or alcohol flame.

Exercises.- 1. Make an equation for the preparation of, (a) lead silicate $\mathrm{PbSiO}_{3}$ by fusion of litharge $\mathrm{PbO}$ and sand, (b) potassium silicate $\mathrm{K}_{2} \mathrm{SiO}_{3}$.

2. The inside surface of the bottle of sodium hydroxide solution becomes etched and dull. To what is this due?

3 . What is the valence of boron?

4. Why is not all of the boric acid deposited from a hot solution containing it?

5. Write an equation for the effect of heat upon borax.

6. Why does the addition of borax render a glass more easily fusible? 


\section{CHAPTER XXXI \\ COMPOUNDS OF SODIUM AND POTASSIUM}

$\mathrm{WE}_{\mathrm{E}}$ have already considered sodium and sodium hydroxide (Chap. XIV). In this chapter we take up the other important compounds of sodium and their uses, and we devote some space also to potassium and its more useful compounds.

In general, we shall find that these metals and their corresponding compounds are very much alike in properties. The chief differences are that the sodium compounds are usually cheaper, and that, on account of the difference in the atomic weights of the two elements (sodium 23, potassium 39), smaller weights of the sodium compounds suffice for a given use involving chemical interaction. For these reasons the sodium compounds find, in most cases, more applications.

Sodium and potassium are univalent, and both are very active as metallic elements. Their hydroxides being strongly alkaline, the elements are often called the metals of the alkalies.

\section{Sodium Na}

Sodium derives its symbol Na from its German name, natrium. All compounds of sodium, when heated with a Bunsen burner, confer a strong yellow tint upon the flame.

Sodium Chloride $\mathrm{NaCl}$. - Sea water contains about 2.5 per cent of sodium chloride $\mathrm{NaCl}$. The same compound is found in extensive deposits at Stassfurt in Germany, in Cheshire (England), at Syracuse (New York), at Salina (Kansas), in Utah, California and many other parts of the United States.

The pure salt is obtained from these deposits by re-crystal365 
lization from water. A certain amount is made, by evaporation, from sea water.

The salt crystallizes in white cubes. Its chief application is in the manufacture of other compounds of sodium.

Sodium Bicarbonate $\mathrm{NaHCO}_{3}$, Manufacture.-This salt is manufactured by the interaction of sodium chloride and ammonium-hydrogen carbonate in the Solvay or ammonia-soda process. Very concentrated solutions are used, and from them a great part of the sodium-hydrogen carbonate, which is a much less soluble salt, is precipitated:

$$
\mathrm{NaCl}+\mathrm{NH}_{4} \mathrm{HCO}_{3} \rightleftarrows \mathrm{NaHCO}_{3} \downarrow+\mathrm{NH}_{4} \mathrm{Cl} \text {. }
$$

In practice salt is dissolved in water and the solution is saturated with ammonia gas. The mixture is placed in an iron tower filled with perforated shelves. Carbon dioxide, made by heating limestone in special kilns, is forced in at the bottom. The perforations split up the gas into small bubbles, and facilitate its solution to form carbonic acid $\mathrm{H}_{2} \mathrm{CO}_{3}$. With the ammonium hydroxide $\mathrm{NH}_{4} \mathrm{OH}$ in the liquid it gives ammonium-hydrogen carbonate:

$$
\mathrm{NH}_{4} \mathrm{OH}+\mathrm{H}_{2} \mathrm{CO}_{3} \rightarrow \mathrm{NH}_{4} \mathrm{HCO}_{3}+\mathrm{H}_{2} \mathrm{O} \text {. }
$$

This product interacts as in equation (1). The sodium bicarbonate is precipitated and is freed from the liquor in filter-presses. The ammonia is recovered for use by treating the residual liquor, containing ammonium chloride, with the quicklime $\mathrm{CaO}$ from the kilns. The quicklime, with the water, gives slaked lime $\mathrm{Ca}(\mathrm{OH})_{2}$ and the latter liberates the ammonia (p. 302):

$2 \mathrm{NH}_{4} \mathrm{Cl}+\mathrm{Ca}(\mathrm{OH})_{2} \rightarrow \mathrm{CaCl}_{2}+2 \mathrm{NH}_{4} \mathrm{OH} \rightarrow 2 \mathrm{NH}_{3} \uparrow+2 \mathrm{H}_{2} \mathrm{O}$.

Properties of Sodium Bicarbonate $\mathrm{NaHCO}_{3}$.- This salt is a fine, white, not obviously crystalline powder, which is only slightly soluble in water. It is commonly known as baking soda. It decomposes slowly in an open vessel, even when cold. When 
heated, it rapidly gives off carbon dioxide and water, and leaves sodium carbonate:

Skeleton: $\quad \mathrm{NaHCO}_{3} \rightleftarrows \mathrm{Na}_{2} \mathrm{CO}_{3}+\mathrm{H}_{2} \mathrm{CO}_{3} \rightleftarrows \mathrm{H}_{2} \mathrm{O}+\mathrm{CO}_{2}$.

Balanced: $\quad 2 \mathrm{NaHCO}_{3} \rightleftarrows \mathrm{Na}_{2} \mathrm{CO}_{3}+\mathrm{H}_{2} \mathrm{O}+\mathrm{CO}_{2}$.

Like all carbonates, when treated with an acid, it gives carbonic acid and this, in turn, gives water and carbon dioxide.

$$
\mathrm{NaHCO}_{3}+\mathrm{HCl} \rightleftarrows \mathrm{NaCl}+\mathrm{H}_{2} \mathrm{CO}_{3} \rightleftarrows \mathrm{H}_{2} \mathrm{O}+\mathrm{CO}_{2} \uparrow \text {. }
$$

This property leads to its use in fire extinguishers.

Sodium Carbonate $\mathrm{Na}_{2} \mathrm{CO}_{3}$. - This salt is manufactured by heating sodium bicarbonate. It is made also by the Le Blanc process. Sodium chloride is treated at a red heat with an equivalent amount of sulphuric acid, giving sodium sulphate $\mathrm{Na}_{2} \mathrm{SO}_{4}$. The latter is roasted with powdered coal and limestone. The coal reduces the sulphate to sodium sulphide $\mathrm{Na}_{2} \mathrm{~S}$, and the latter interacts with the limestone giving sodium carbonate and calcium sulphide CaS. The carbonate is separated by solution in water. The solution, when concentrated, gives crystals of the decahydrate, washing soda $\mathrm{Na}_{2} \mathrm{CO}_{3}, 10 \mathrm{H}_{2} \mathrm{O}$. The latter, when heated, leaves "soda-ash" or " calcined soda" $\mathrm{Na}_{2} \mathrm{CO}_{3}$.

$$
\begin{aligned}
\mathrm{NaCl}+\mathrm{H}_{2} \mathrm{SO}_{4} & \rightarrow \mathrm{Na}_{2} \mathrm{SO}_{4}+2 \mathrm{HCl} \uparrow \\
\mathrm{Na}_{2} \mathrm{SO}_{4}+2 \mathrm{C} & \rightarrow \mathrm{Na}_{2} \mathrm{~S}+2 \mathrm{CO}_{2} \uparrow \\
\mathrm{Na}_{2} \mathrm{~S}+\mathrm{CaCO}_{3} & \rightarrow \mathrm{Na}_{2} \mathrm{CO}_{3}+\mathrm{CaS} .
\end{aligned}
$$

Sodium carbonate $\mathrm{Na}_{2} \mathrm{CO}_{3}$ is used in making sodium hydroxide (p. 166) and glass (p. 361), and to soften water (see p. 389). As about two-thirds of washing soda is water, it is cheaper to ship the anhydrous form, except when the hydrate is wanted, as for washing.

Properties of Sodium Carbonate Solution.- The solution is not neutral to litmus, as we might expect, but distinctly alkaline. The explanation by the ionization theory is of general interest. 
Water, it will be remembered, is ionized to a very small extent. When any salt is dissolved in water, therefore, there exists the possibility of a double decomposition taking place. Thus with sodium chloride:

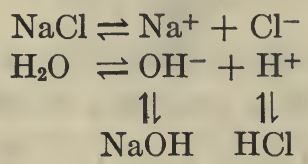

In this case, however, the extent of formation of $\mathrm{NaOH}$ and $\mathrm{HCl}$ is altogether negligible. $\mathrm{NaOH}$ and $\mathrm{HCl}$ are both very highly ionized in aqueous solution, their existence in appreciable amount would involve the presence of both $\mathrm{OH}^{-}$and $\mathrm{H}^{+}$in quantity, and these ions would immediately combine to form water.

When a salt like sodium carbonate is dissolved in water, the state of affairs is different, as may be seen by studying the arrows in the ionic equations:

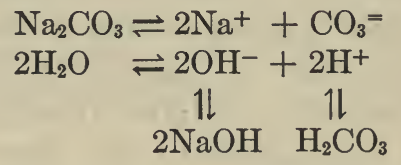

Double decomposition with the ions of water here involves the formation of some $\mathrm{NaOH}$ and some $\mathrm{H}_{2} \mathrm{CO}_{3}$. Now the latter substance is an exceedingly weak acid. While, therefore, any $\mathrm{NaOH}$ formed is almost entirely ionized, furnishing $\mathrm{OH}^{-}$to the solution; any $\mathrm{H}_{2} \mathrm{CO}_{3}$ formed remains, on the contrary, almost entirelyin the non-ionized state. Now we have learnt (p. 241) that one way of driving a reversible reaction to completion is to remove one product as a non-ionized substance. This reaction cannot be driven to completion in this way, since we have the formation of another practically non-ionized substance, water, tending to drive the reverse reaction to completion. In such circumstances, obviously, a balance must be struck between the two conflicting tendencies, and we will be left with a solution in which partial 
double decomposition has occurred. But such a solution will contain a much higher concentration of $\mathrm{OH}^{-}$from the highly ionized $\mathrm{NaOH}$ than of $\mathrm{H}^{+}$from the practically non-ionized $\mathrm{H}_{2} \mathrm{CO}_{3}$. It will, therefore, react like an alkali.

(1). Hydrolysis of Salts. - This interaction of a salt with water is called hydrolysis (Greek, decomposition by water). All salts are hydrolyzed, at least to a slight extent. The action is the reverse of neutralization (p. 192), water and a salt giving, by double decomposition, an acid and a base. The effect is noticeable, however, only when the acid and base are of very unequal activity. A salt which, by hydrolysis, gives an active base and a weak acid, furnishes a solution the reaction of which is basic.

Conversely, if the salt gives, by hydrolysis, a weak base and an active acid, then the solution is acid in reaction. Thus, the solution of cupric sulphate is acidic because cupric hydroxide is a feeble base.

The extent of hydrolysis, even in cases where it is distinctly observable, such as sodium carbonate and cupric sulphate mentioned above, is in general only small. Borax, a salt of an extremely weak acid (p. 363), reacts distinctly alkaline in water, but the degree of hydrolysis in a $0.1 \mathrm{~N}$ solution is only one-half of one per cent. The reason, of course, lies in the fact that water is very much less ionized even than exceedingly weak acids like boric acid or exceedingly weak bases like cupric hydroxide. The tendency towards the completion of neutralization, therefore, preponderates considerably over the tendency towards the completion of hydrolysis.

Sodium Nitrate $\mathrm{NaNO}_{3}$. - This salt is prepared by recrystallizing Chile saltpeter (p. 308). When heated, it gives off oxygen, leaving sodium nitrite $\mathrm{NaNO}_{2}$, a compound much used as a source of nitrous acid in the manufacture of organic dyestuffs.

Sodium nitrate is at present the chief source of nitric acid ( $p$. 
308). On account of its solubility and its nitrogen content, it is valuable as a fertilizer (p. 410). It is used also in the manufacture of cheap grades of gunpowder. Much of it is converted into potassium nitrate, as this salt is less deliquescent, and the gunpowder made from it keeps better.

Other Salts of Sodium. - Several of them, such as the peroxide (p. 221), the silicate (p. 360), and the tetraborate (p. 363 ), have been described already. Sodium sulphate $\mathrm{Na}_{2} \mathrm{SO}_{4}$ is used as a substitute for sodium carbonate in making cheap glass. Sodium sulphite $\mathrm{Na}_{2} \mathrm{SO}_{3}$ (p. 260), made by the action of sulphur dioxide on an aqueous solution of sodium hydroxide, is a convenient source of sulphur dioxide and is also used as a preservative. Sodium thiosulphate $\mathrm{Na}_{2} \mathrm{~S}_{2} \mathrm{O}_{3}$ (" hypo ") "can be obtained readily by boiling sodium sulphite solution with sulphur:

$$
\mathrm{Na}_{2} \mathrm{SO}_{3}+\mathrm{S} \rightarrow \mathrm{Na}_{2} \mathrm{~S}_{2} \mathrm{O}_{3}
$$

and is used in fixing photographs. Sodium cyanide NaNC (preparation, see p. 393) is used in extracting gold from its ores.

\section{Potassium $\mathrm{K}$}

Potassium receives its symbol from the initial of the German word for it, kalium (related to the word alkali). All compounds of potassium confer a violet color upon the Bunsen flame.

The metal itself may be prepared from its hydroxide, as in the case of sodium (p. 164), and exhibits similar properties. It has no uses. Certain of its salts, however, are of the greatest value, chiefly as fertilizers (p. 412) and explosives.

Occurrence.- Silicates containing potassium, such as felspar and mica, are common constituents of volcanic rocks. These minerals have not yet been brought into commercial use as sources of potassium compounds. Many salt deposits (p. 412) contain potassium chloride, alone (sylvite) and in combination with other salts, and most of the compounds of potassium are manu- 
factured from this material. Potassium sulphate occurs also in the salt layers, and is used directly as a fertilizer.

\section{Potassium Carbonate $\mathrm{K}_{2} \mathrm{CO}_{3}$, Preparation.-The water} used in scouring wool leaves, when evaporated, the potassium salts of organic acids. When the residue is roasted, potassium carbonate remains. Wood ashes contain considerable amounts of potassium carbonate, and were indeed originally the only source of this compound. The sugar beet takes up exceptional quantities of potassium and, after all the sugar has been removed from the extract, potassium carbonate is obtained by evaporating the liquid and calcining the residue. Much of the salt is also manufactured direct from potassium chloride.

Potassium carbonate is used in making soft soap and difficultly fusible glass (p. 362).

Potassium Bicarbonate $\mathrm{KHCO}_{3}$. - When carbon dioxide is led into potassium carbonate solution potassium bicarbonate $\mathrm{KHCO}_{3}$ is formed:

$$
\mathrm{CO}_{2}+\mathrm{H}_{2} \mathrm{O} \rightleftarrows \mathrm{H}_{2} \mathrm{CO}_{3}+\mathrm{K}_{2} \mathrm{CO}_{3} \rightleftarrows 2 \mathrm{KHCO}_{3} \text {. }
$$

The bicarbonate decomposés easily, especially when warmed, reversing the above action (read the equation backwards). This occurs even in the solution. Hence carbon dioxide can be forced by pressure in large amounts into a warm solution of potassium carbonate, and liberated again by pumping so as to create a vacuum. This plan is used as a means of purifying carbon dioxide (p. 334). The same quantity of potassium carbonate can be used over and over again.

Potassium Hydroxide $\mathrm{KOH}$.- This alkali is made like sodium hydroxide. Either potassium carbonate is treated with slaked lime, or potassium chloride solution is electrolyzed in a Nelson cell (see p. 166). The solution is evaporated, and the substance cast in slender sticks.

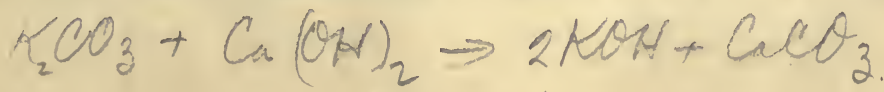


Its properties are much like those of sodium hydroxide. It is used in making soft soap and other compounds of potassium.

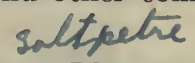

Potassium Nitrate $\mathrm{KNO}_{3}$. - The supply of the natural nitrate being insufficient, the salt is made by double decomposition from the Chile saltpeter $\mathrm{NaNO}_{3}$ :

$$
\mathrm{NaNO}_{3}+\mathrm{KCl} \rightleftarrows \mathrm{KNO}_{3}+\mathrm{NaCl} \downarrow \text {. }
$$

Sodium chloride is not much more soluble in hot water than in cold. The three other salts, however, become very soluble as the temperature rises. Hence, when sodium nitrate and potassium chloride are heated with very little water, they dissolve, sodium chloride is precipitated, and potassium nitrate remains in solution. The mass is filtered quickly through canvas to separate the precipitate, and potassium nitrate crystallizes from the filtrate as it cools.

The salt is used in making gunpowder and fireworks. It is employed also in preserving ham and corned beef.

Gunpowder.- Gunpowder is composed of potassium nitrate (75 per cent), charcoal (15 per cent) and sulphur (10 per cent). The ingredients are moistened with water, and intimately mixed by grinding under the heavy rollers of a mill. The "mill cake" is then broken up and granulated to the required size.

The explosion results largely from the union of the charcoal with the oxygen from the nitrate and of the sulphur with the potassium. One gram of powder yields 264 c.c. of gas $\left(\mathrm{CO}_{2}, \mathrm{CO}\right.$, and $\left.\mathrm{N}_{2}\right)$ measured at $0^{\circ}$ and $760 \mathrm{~mm}$., and a much larger volume at the temperature of the explosion. One gram produces about 660 calories of heat. The explosion is due to the suddenness with which the gases are generated and the heat is developed. The smoke is composed of particles of solid compounds of potassium and is therefore very slow in dissipating itself. Smokeless powder (p. 483) produces no solids when it explodes. 
Gunpowder is still used in mining, and for detonating charges of smokeless powder.

Other Compounds of Potassium.- Potassium bromide $\mathrm{KBr}$ (p. 202), used in medicine to relieve nervousness and to produce sleep, potassium iodide KI (p. 206), used also in medicine, and potassium chlorate $\mathrm{KClO}_{3}$, employed in making matches and fireworks (p. 321), have already been mentioned. Potassium sul- of phate $\mathrm{K}_{2} \mathrm{SO}_{4}$, made by the action of sulphuric acid upon potassium chloride at a red heat, is employed in making alum (p. 469).

Exercises.-1. In the Solvay process why is the ammonia dissolved in the salt solution, and not separately in water? Make the equation for the action of heat on limestone.

2. What is the exact percentage of water in washing soda?

3. What will be the reactions to litmus of aqueous solutions of: (a) sodium phosphate, (b) sodium bromide, (c) sodium silicate, (d) sodium peroxide, (e) sodium nitrate?

4. Write full ionic equations to show why cupric sulphate solution reacts acid to litmus.

5. How will a solution of ammonium acetate react towards litmus?

6. Write full ionic equations for the Solvay process (p. 366).

7. Why does a piece of glass, when strongly heated, confer a yellow color on the Bunsen flame? 


\section{CHAPTER XXXII}

THE RECOGNITION OF SUBSTANCES, I. - A REVIEW OF THE NON-METALLIC ELEMENTS

IN the chapters preceding we have classified our substances under onc of the chemical elements they contained. Thus, chloroform, alcohol, and ether were put under carbon. Hydrogen sulphide and sulphuric acid were considered as compounds of sulphur. Now this classification is of a theoretical nature. That chloroform, alcohol, and ether all contain carbon can not be told by mere inspection. We have to make experiments, and to reason about the results, before we reach this inference. Thus we put our inference as the title of the chapter, and distributed the observations and data through it. There is, however, another way of classifying the facts, which is just the opposite of this one. It is the practical classification. When we obtain a specimen, or when a substance appears in the course of an experiment, we must be able to tell what particular substance it is. If it is a white powder, it may contain almost any of the whole list of elements. It may be any one of several thousands of substances. We can recognize it only by its physical properties (p. 6) and by the physical properties of other substances that we can get from it by interaction with known chemical compounds. We need, therefore, a plan of operation, and this plan must be based upon a classification by physical properties, not by constituents.

One benefit of the discussion of such a plan is that it will afford us a review of some of the facts already mentioned, by presenting the same facts from a different view-point, and by showing the uses to which they may be put. To avoid unnecessary repeti- 
tion, we shall refer frequently to the previous statements of the facts. To understand what is here said, therefore, the reader should look up every reference and reread the statement.

Is the Material a Mixture? - The first question that occurs to us is, whether the material is a single substance or a mixture. When it is a mixture, we can often, though not always, very quickly find this out.

If it is a solid mass or powder, we examine it with the naked eye and with the help of a lens. If we see two or more kinds of particles, as in granite (p. 4), in a mixture of sand and sugar, or in a piece of rusty iron, the kinds differing in form or color or both, then it probably is a mixture.

Whether it appears to the eye to be a mixture, or not, we can next try a solvent, such as water, ether, or carbon tetrachloride. If a part of a small sample refuses to dissolve (e.g. sand), while the rest dissolves (e.g. sugar), we have shown that there are two different sets of physical properties, and therefore two different substances (p. 13) present. As there may be very little of the soluble substance in the mixture, we may not perceive at once that anything has dissolved. So we allow a drop of the liquid to evaporate on a clean watch crystal, and observe whether any residue remains.

If the material is a liquid, we depend largely on differences in the volatility of different substances to find out whether it is a mixture. If a drop evaporates on a watch crystal, leaving a residue (solid or liquid) which does not evaporate, then it is a mixture. If this test fails, because all, or none, evaporates, then we must distil the liquid, with the bulb and most of the stem of a thermometer in the vapor (Fig. 35, p. 67), and note whether the whole comes over at one temperature (single substance, in most cases) or whether the temperature changes as the distillation proceeds (mixture, such as petroleum, p. 344).

These are simply a few examples intended to show how, when 
a. practical problem is before us for solution, we use physical properties as the basis of our reasoning and classification.

The Recognition of a Single Substance.-The majority of the substances we have met with are aoids, bases, or salts. In identifying a substance of one of these classes, it is convenient to attempt the recognition of the positive and of the negative radicals (or ions) as two almost separate problems. In other words, we investigate one radical at a time. On the other hand, substances which do not belong to any of these classes, such as simple substances (sulphur, carbon, chlorine, etc.), oxides (sulphur dioxide, carbon monoxide, etc.), and many organic substances (e.g. carbon disulphide, ethyl alcohol), are investigated as a single problem.

Scope of this Chapter.-In discussing the recognition of a single substance we shall, for the present, limit ourselves to the non-metallic elements. We shall consider the elementary substances themselves (sulphur, oxygen, etc.), the oxides of such elements, the few organic compounds described, the non-metallic negative ions, and ammonium-ion. We shall leave out of consideration until later (Chap. XLVI) the metallic elements (including $\mathrm{As}, \mathrm{Sb}, \mathrm{Bi}, \mathrm{Na}$ and $\mathrm{K}$ ). We shall also ignore the metallic positive radicals, although one of these (or hydrogen-ion) must inevitably be combined with the negative radical under consideration.

External Examination (Solids). - The specimen may be a solid, a liquid, or a gas. We should note in which of these states it exists under room conditions. What follows applies only to solids - the liquids and gases will be taken up later (pp. 380, $381)$.

Without training in crystallography we can tell little about the crystalline form (p. 94) of the specimen. But anything con- 
spicuous, such as needle-shape or cubical formation of the particles, must be noted.

The color, if any, is significant. If yellow, the specimen may be sulphur (p. 249), if black, carbon (p. 330), if black and crystalline, with violet vapor, iodine (p. 203). Most substances are colorless.

The odor, if any, must be noted. Many salts of ammonium (carbonate, sulphide, etc.) smell of ammonia (p. 303). Some sulphides (of sodium, potassium, ammonium, etc.) smell of hydrogen sulphide (p. 253). Some acetates smell of acetic acid and hypochlorites of hypochlorous acid (p. 223).* Some chlorides (e.g. $\mathrm{PCl}_{5}$ and $\mathrm{AlCl}_{3}$ ), in moist air, give hydrogen chloride, with the odor and fumes characteristic of that substance.

Effect of Heating (Solids).- A great deal may be learned by heating as much of the specimen as will fill the rounded bottom of a small, dry test-tube (Fig. 92).

The substance may fuse. Many substances, such as some chlorides and most hydrates, do so. Continue heating.

A sublimate (solid deposit on the cold part of the tube) may appear. Black crystals (from violet vapor) are iodine: white crystals, with the limitations we have set, show the substance to be a salt of ammonium (p. 305).

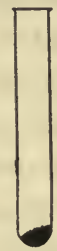
Confirm by smelling, and by adding sodium hydroxide to the original substance (p. 302). (Salts of mercury sublime also.)

A reddish-brown liquid condenses, becoming, when cold, a yellow solid. The substance was sulphur from a sulphide (such as $\mathrm{FeS}_{2}$, p. 486).

Water condenses. Hydrates, acid-salts, and some organic compounds. Test the water with litmus paper. An acid reaction in-

* Many experimental details, essential for the successful performance by a beginner of the tests described in this chapter are here omitted. They will be found in the Authors' Intermediate Outline of Elementary Chemistry. 
dicates an acid-salt (p. 192), an easily hydrolyzed salt (e.g. $\mathrm{FeCl}_{3}$, $6 \mathrm{H}_{2} \mathrm{O}$ ) or organic acid. Continue heating, inclining the tube mouth downwards, removing condensed water with filter paper until no more comes off, and heat the residue.

A gas is given off. The gas may be violet (some iodides) or brown (some nitrates, p. 311, and some bromides). If brown, lower a glass rod dipped in silver nitrate solution into the gas in the tube. Bromine will give a white precipitate $(\mathrm{AgBr}, \mathrm{p}$. 202). In case of a negative result try the test for nitrates.

The gas may be colorless. If it has an odor, it may be ammonia from a salt of ammonium, sulphur dioxide from a bisulphite (p. 258) or from oxidation of a sulphide (p. 258). A stifling odor with fumes may be sulphur trioxide from some sulphates or decomposition products from some organic matters.

The gas is colorless and odorless. It may be oxygen (test with long, glowing splinter of wood) from a peroxide, chlorate, or nitrate (of $\mathrm{K}$ or $\mathrm{Na}$ ). To learn which of these it is, dissolve or suspend a little of the substance in water, add dilute sulphuric acid, and test for hydrogen peroxide (p. 223). In case of a negative result examine the residue (as in p. 379).

A colorless, odorless gas may be carbon dioxide, coming from a bicarbonate or a carbonate (except of $\mathrm{K}$ or $\mathrm{Na}$ ). Lower a glass rod dipped in lime-water into the gas in the test-tube (white precipitate, $\mathrm{CaCO}_{3} \downarrow$ ).

The substance carbonizes or chars and gives an odor of smoldering wood or burning flesh. The compound is organic. Identify, by properties (pp. 346-50, 353).

Heating may produce no effect. On the other hand, more than one of these effects (e.g. both water and sulphur dioxide from a bisulphite) may be given by the same specimens.

If heating produces any effect, continue heating until all change ceases, and preserve the residue for use in p. 379.

In most cases other distinctive properties and tests will be found on the pages referred to. 
Effect of Sulphuric Acid on Solids. - Fill the rounded bottom (only) of a test-tube with the substance, add just enough concentrated sulphuric acid to moisten the sample, and warm slightly.

A gas (effervescence) which fumes in the breath may be given off. If the gas is brown or yellow, it may be bromine (bleaches litmus paper) mixed with hydrogen bromide from a bromide (p. 202). It may be nitrogen tetroxide (odor) from a nitrate (p. 311). If violet, with brown deposit, accompanied by an odor of hydrogen sulphide, it is iodine from an iodide (p. 204).

If the gas fumes, but is colorless, it may be hydrogen chloride . from a chloride (add manganese dioxide to get chlorine, p. 142). It may be hydrogen fluoride from a fluoride (a moistened glass rod acquires white precipitate of silicic acid produced by decomposition of silicon fluoride, p. 207).

The gas does not fume. If yellow it may be chlorine from bleaching powder (p. 224) or nitrous anhydride from a nitrite (p. 314). A chlorate also gives a yellow gas (chlorine dioxide $\mathrm{ClO}_{2}$ ) when heated with concentrated sulphuric acid. In this case oxygen will have obtained in the test on page 378. Heat very carefully, since chlorine dioxide is explosive (stop heating when material in tube begins to crackle!).

The gas does not fume and is colorless. An odor of sulphur dioxide indicates a sulphite (p. 258). An odor of hydrogen sulphide indicates a sulphide. If the gas is odorless, it may be carbon monoxide (burns, leaving carbon dioxide) from a formate (p. 337), or oxygen from some oxides or a peroxide, or nitrous oxide from ammonium nitrate (p. 315), or carbon dioxide from a carbonate.

No gas evolved indicates a silicate (p. 360), sulphate (p. 270), phosphate (p. 322), or a basic oxide.

Sulphuric Acid on the Residue from p. 378.- If the substance gave off oxygen when heated alone (p. 378), add a drop 
or two of concentrated sulphuric acid to the residue. If the specimen now gives a yellow gas (nitrous anhydride), the original substance was a nitrate (of $\mathrm{K}$ or $\mathrm{Na}$ ), from which the nitrite was formed by heating (p. 311). If it gives a colorless, fuming gas $(\mathrm{HCl})$, the original substance was a chlorate (p. 30).

Examination of a Liquid.- Test the specimen with litmus paper. A marked acid reaction may be due to an acid, such as concentrated or dilute sulphuric acid (p. 270), concentrated or dilute nitric acid (p. 309), concentrated or dilute hydrochloric acid (p. 131), hydrobromic acid (p. 202), hydriodic acid (p. 205), phosphoric acid (p. 322), sulphurous acid (p. 259), or an organic acid (p. 348); also an acid-salt (p. 192), or a hydrolyzed salt (p. $369)$.

If it bleaches litmus paper, it may be chlorine-water or brominewater (odor).

If it is markedly alkaline in reaction, it may be a solution of a base $\left(\mathrm{NaOH}, \mathrm{KOH}, \mathrm{NH}_{4} \mathrm{OH}\right.$, etc.) or a hydrolyzed salt (p. $355)$.

Note the odor. Ammonium hydroxide, hydrogen sulphide solution, sulphurous acid, concentrated nitric acid and concentrated halide acids all have odors. Alcohol, acetic acid, carbon disulphide, carbon tetrachloride, and hydrocarbons (e.g. gasoline) have odors easily distinguished from those of the foregoing.

Evaporate a few drops to dryness on a watch crystal. A solid residue shows that the original substance was a solution in water (or possibly alcohol or some other solvent, if the vapor has an odor indicating this). If there is a solid residue, a quantity of it may be obtained by evaporating a larger amount of the liquid, and may then be treated as a solid (pp. 377-9).

If the specimen leaves no residue, and is not acid or alkaline but has an odor, it may be one of the volatile organic compounds named above. If it is odorless, it may be a solution of hydrogen peroxide (p. 222) or simply pure water. 
Examination of a Gas.- The gas has a color. A brown gas may be bromine or nitrogen tetroxide. The former liberates iodine from potassium iodide solution (p. 205), but not bromine from a bromide (insert rods moistened with solution of an iodide and a bromide). The latter becomes deeper brown on warming (p. 311). A greenish-yellow gas is chlorine. It bleaches, and displaces bromine from a solution of a bromide (p. 202).

The gas may become colored (yellow or brown) on admitting air. It is nitric oxide (p. 311).

The gas may have a distinctive odor. Sulphur dioxide, hydrogen sulphide, nitrogen tetroxide, and ammonia are of this kind.

The gas may fume in the breath. The chloride, bromide, and iodide of hydrogen do so. Distinguish by dissolving in little water and adding manganese dioxide.

The gas may be combustible. Burning with a blue flame indicates hydrogen (vessel bedewed with moisture), or carbon monoxide (leaving carbon dioxide, test, p. 336). Burning with a slightly luminous flame indicates methane (p. 345) and a very luminous flame (often depositing carbon) indicates ethylene (p. $350)$ or acetylene (p. 351).

The gas may relight a glowing splinter of wood. This is oxygen, or nitrous oxide (p. 315). The former, with nitric oxide, gives a brown gas (p. 311), the latter does not.

The gas may give a white precipitate $\left(\mathrm{CaCO}_{3}\right)$ with limewater. This is carbon dioxide.

The gas having none of these properties is nitrogen (p. 287).

In most cases other distinctive properties will be found on the pages referred to.

Exercises.- 1. Look up the references, and give the properties (physical as well as chemical), other than those mentioned in p. 377, by which you should recognize: (a) sulphur, (b) carbon, and (c) iodine. 
2. Same question in regard to:

(a) ammonia, (b) hydrogen sulphide, and (c) hypochlorous acid.

3. Same question in regard to: (a) an acid salt, (b) a hydrate, (c) nitrogen tetroxide, (d) bromine, (e) sulphur trioxide, (f) hydrogen peroxide, (g) ammonium nitrate, (h) carbon dioxide. 4. Same question in regard to (p. 379): (a) a bromide, (b) an iodide, (c) a nitrate, (d) bleaching powder, (e) sulphur dioxide, (f) carbon monoxide, (g) a silicate, (h) a sulphate, and (i) a phosphate.

5. Same question in regard to (p. 380): (a) conc. and (b) dil. sulphuric acid, (c) conc. and (d) dil. nitric acid, (e) conc. and (f) dil. hydrochloric acid, (g) hydrobromic acid, (h) hydriodic acid, (i) phosphoric acid, (j) sulphurous acid.

6. Same question in regard to: (a) chlorine-water, (b) alcohol, (c) acetic acid, (d) carbon disulphide, (e) carbon tetrachloride, (f) a hydrocarbon.

7. Same question in regard to: (a) nitric oxide (b) hydrogen, (c) methane, (d) ethylene, (e) acetylene, (f) nitrous oxide, (g) ozone, (h) nitrogen.

8. Why does sodium sulphide smell of $\mathrm{H}_{2} \mathrm{~S}$ (see pp. 118, 369)?

9. When ammonium nitrate is heated in a test-tube, the gases evolved do not relight a glowing splinter. How can this be reconciled with p. 315 ? 


\section{CHAPTER XXXIII}

\section{CALCIUM AND ITS COMPOUNDS}

CaLCIUM belongs to a family of metallic elements which includes also strontium, barium, and radium. This family resembles that to which sodium and potassium belong in so far that the metals are second in activity only to the two last named and that the hydroxides are active bases. The chief differences are that the metals of the present group are bivalent and that all the carbonates and many other single compounds are insoluble.

Compounds of calcium confer a brick-red color upon the Bunsen flame.

Calcium. - The metal is made by electrolyzing melted-oalcium chloride in a graphite crucible, which forms the anode. The cathode is a rod of iron, one end of which dips into the fused salt. The calcium, liberated at this point, adheres to the rod. The latter is slowly raised, in such a way that the calcium always remains in contact with the liquid. In this fashion a long " cabbage-stalk" of calcium is finally produced.

The metal is slightly harder than lead and has a silver-white luster. It decomposes cold water, liberating hydrogen (p. 50).

Calcium Carbonate $\mathrm{CaCO}_{3}$.- The carbonate is the commonest compound of calcium. White marble is a pure variety, composed of crystals compactly wedged together. Limestone does not show much crystalline structure and usually contains clay and other impurities. Chalk is made up of shells of minute marine organisms. Shells, coral, and pearls are likewise mainly calcium carbonate. Well-formed crystals (calcite, or Iceland spar - Fig. 93 - and aragonite - Fig. 94) are common. 
Limestone is used for building and in road-making. Much of it is employed in making quicklime, cement (p. 471), and glass (p. 361), and as a flux in metallurgical operations (see p. 486). Marble, often variegated by the presence of impurities, is used in building and sculpture.

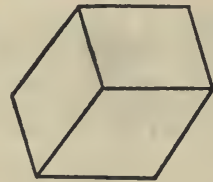

FIG. 93

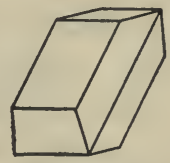

FIG. 94

As we have seen (p. 333), calcium carbonate reacts with acids to give carbonic acid:

$$
\mathrm{CaCO}_{3}+2 \mathrm{HCl} \rightarrow \mathrm{CaCl}_{2}+\mathrm{H}_{2} \mathrm{CO}_{3} \rightarrow \mathrm{H}_{2} \mathrm{O}+\mathrm{CO}_{2} \uparrow \text {. }
$$

When heated, all forms of calcium carbonate give off carbon dioxide, and leave calcium oxide:

$$
\mathrm{CaCO}_{3} \rightleftarrows \mathrm{CaO}+\mathrm{CO}_{2} \text {. }
$$

Calcium Oxide $\mathrm{CaO}$, Manufacture. - The manufacture of

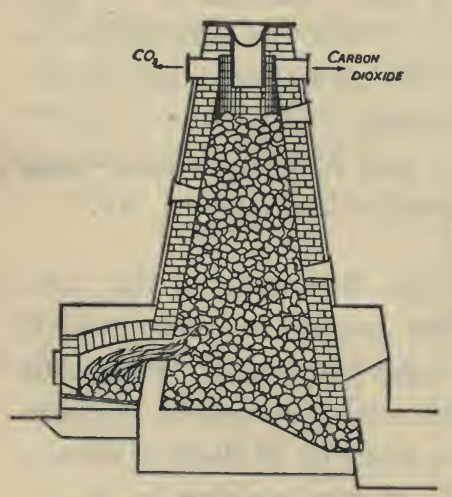

FIG. 95 calcium oxide or quicklime (i.e. live lime) is one of the most ancient chemical industries. The limestone is thrown into a kiln lined with brickwork (Fig. 95). The flames and heated gases from the fire pass through the limestone and the carbon dioxide is liberated and carried off by the draft. When this gas is to be used, as in the Solvay process or in the refining of sugar, coke (smokeless) is chosen as the fuel. When no use is to be made of the escaping gas, coal may be employed.

The use of as low a temperature as possible is important. A 
high temperature causes the impurities in the limestone (the clay, etc.) to interact with the quicklime and form fusible silicates, which fill the pores and retard the subsequent action of water in slaking the lime. Calcium carbonate gives a pressure of only $25 \mathrm{~mm}$. of carbon dioxide at $700^{\circ}$ and one atmosphere at about $900^{\circ}$. The action is reversible (see equation), and if the gas accumulates in the kiln, the carbon dioxide recombines with the quicklime as fast as it is liberated - unless a temperature above $900^{\circ}$ is used. When, however, the gas is continually removed, the backward action is prevented, and a lower temperature suffices to complete the dissociation of the compound. Hence a low temperature and a good draft of air through the kiln are essential.

Properties and Uses of Quicklime.-Calcium oxide is a white, amorphous material. It melts only in the electric arc. When heated strongly, it glows with an unusually brilliant and white light. The Drummond, or oxy-hydrogen light, more commonly called the lime light or calcium light, is produced by allowing a flame of burning oxygen and hydrogen or illuminating gas to play upon a cylinder of calcium oxide (Fig. 96). The gases are contained in iron cylinders, under pressure, and so the apparatus can be used for illumination or projection where neither electricity nor a local supply of gas is available.

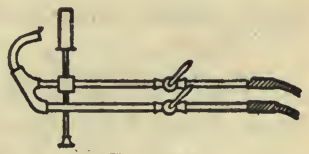

FIG. 96

When water is poured upon quicklime, it is at first absorbed, and then enters into combination to form calcium hydroxide (slaked lime):

$$
\mathrm{CaO}+\mathrm{H}_{2} \mathrm{O} \rightarrow \mathrm{Ca}(\mathrm{OH})_{2} .
$$

So much heat is given out that the excess of water is converted into steam. The quicklime swells and falls to powder.

Quicklime is used most largely in making slaked lime for mor- 
tar, and also in the manufacture of bleaching powder. Other uses are mentioned under the hydroxide.

Quicklime deteriorates when exposed to the air. It combines both with the moisture and carbon dioxide in the atmosphere and becomes air-slaked.

Calcium Hydroxide $\mathrm{Ca}(\mathrm{OH})_{2}$. - The hydroxide is a white, amorphous powder. It is slightly soluble in water (about $1: 600$ at $18^{\circ}$ ), giving lime-water. The solution has a strong alkaline reaction, however, showing that so much as is dissolved is very largely ionized. Milk of lime, a saturated solution with a large excess of calcium hydroxide suspended in it, is employed in many operations (see e.g. pp. 166, 371). As the dissolved part undergoes chemical change, more goes into solution. Being cheap, it is used whenever an alkali is needed, provided a dilute alkali will serve the purpose. It interacts with acids giving salts of calcium, and shows the other properties of a base (pp. 167, 192).

Slaked lime is used in making mortar (see below) and alkalies (p. 166) and in purifying sugar (p. 402). It is employed to remove the hair from hides, before tanning, an action which recalls the solubility of wool (sheep's hair) in an alkali (p. 1). It finds application, also, in softening water (p. 389) and as whitewash.

Mortar. - Mortar is made by mixing slaked lime with three or four times its bulk of sand, and making the whole into a paste with water. When the water evaporates, a porous, rather crumbly material remains. This, however, at once begins to harden, owing to the action of the carbon dioxide in the air upon the lime:

$$
\mathrm{Ca}(\mathrm{OH})_{2}+\mathrm{CO}_{2} \rightarrow \mathrm{CaCO}_{3}+\mathrm{H}_{2} \mathrm{O} \uparrow \text {. }
$$

The crystalline calcite $\left(\mathrm{CaCO}_{3}\right)$ adheres to, and is interlaced with the sand, and gives a rigid, though porous, structure attached firmly to the brick or stone. The pores facilitate the penetration of the air into the deeper parts and thus provide, both for the fresh supplies of carbon dioxide required for the continuance of 
the action shown in the equation, and for a considerable amount of useful ventilation through walls of the building.

Calcium Sulphate $\mathrm{CaSO}_{4}$, Various Forms.-Calcium sulphate is a very common mineral. It occurs, as anhydrite $\mathrm{CaSO}_{4}$, in salt deposits. Gypsum $\mathrm{CaSO}_{4}, 2 \mathrm{H}_{2} \mathrm{O}$ is found in masses, and also in single crystals (selenite, Fig. 42, p. 94). Alabaster is highly crystalline gypsum, tinted by impurities.

Gypsum $\mathrm{CaSO}_{4}, 2 \mathrm{H}_{2} \mathrm{O}$ is the commonest form, and is the one produced when calcium sulphate is precipitated. It is white and much softer than calcite. It is only slightly soluble in water $\left(1: 500\right.$ at $\left.18^{\circ}\right)$. It is used as a fertilizer and in making plaster of Paris and is the chief component of blackboard crayon or " chalk."

When gypsum is heated, the vapor pressure of the water it gives off soon exceeds that of the moisture in the atmosphere, and the compound begins to decompose:

$$
2\left[\mathrm{CaSO}_{4}, 2 \mathrm{H}_{2} \mathrm{O}\right] \rightleftarrows\left(\mathrm{CaSO}_{4}\right)_{2}, \mathrm{H}_{2} \mathrm{O}+3 \mathrm{H}_{2} \mathrm{O} \uparrow \text {. }
$$

The hemi-hydrate which remains (plaster of Paris) gives a much lower pressure of water vapor and is more stable. Plaster of Paris is manufactured in large quantities by heating gypsum in kilns. When moistened with water, it sets in about half an hour to a solid mass of gypsum. The temperature used in making it must not exceed $125^{\circ}$, otherwise the hemi-hydrate is itself decomposed, the plaster is "dead burnt," and it no longer sets readily. The setting involves, simply, the reversal of the equation given above.

Plaster of Paris swells somewhat, in setting, and so fills out completely every detail of a mould and applies itself closely to the outline of an object on which it is spread. It is used in making casts, and in surgical bandages where movable parts are to be held rigidly in place. SStucco is made with sizing or glue instead of pure water.) 
Casts are made smooth and non-porous ("ivory" surface) by a coating of paraffin which fills the pores. Excellent imitations of bronze or other castings are produced by rubbing with pulverized metals.

What Makes Water Hard.- All natural waters except rain water, which is "soft," contain salts of calcium and magnesium in solution and are more or less " hard." These salts are dissolved by the water in its passage over and through the soil.

Although limestone is very insoluble in pure water $(0.013 \mathrm{~g}$. per liter), yet it interacts with the carbonic acid contained in all natural waters, giving calcium bicarbonate which is about thirty times more soluble under atmospheric conditions:

$$
\mathrm{CO}_{2}+\mathrm{H}_{2} \mathrm{O} \rightleftarrows \mathrm{H}_{2} \mathrm{CO}_{3}+\mathrm{CaCO}_{3} \rightleftarrows \mathrm{Ca}\left(\mathrm{HCO}_{3}\right)_{2} \text {. }
$$

When the water is boiled, these actions are all reversed. The carbon dioxide is driven out of solution, the carbonic acid is decomposed, and the calcium bicarbonate gives calcium carbonate, most of which is at once precipitated. Iron carbonate is also held in solution as bicarbonate $\mathrm{Fe}\left(\mathrm{HCO}_{3}\right)_{2}$ and is precipitated as $\mathrm{FeCO}_{3}$ by boiling. These two bicarbonates constitute temporary hardness. Their decomposition causes the "fur" in a kettle.

The sulphates of calcium (solubility $2 \mathrm{~g}$. per liter) and of magnesium (very soluble) are also commonly found in natural waters. These salts are not altered by boiling and, along with magnesium carbonate (sol'ty $1 \mathrm{~g}$. per l.) and calcium carbonate (sol'ty $0.013 \mathrm{~g}$. per 1.), give permanent hardness to the water.

Consequences of Hardness in Water.- When hard water is used in a steam boiler, the salts, of course, are not carried off with the steam, but accumulate amazingly as fresh water is injected and steam alone is drawn off. In time, heavy deposits of boiler crust settle on the tubes of the boiler, and interfere with . the transfcrence of heat from the metal to the water. One-fourth 
of an inch oi crust will increase the bill for fuel by 50 per cent. In addition to this the iron is heated to a higher temperature and may even become red hot. In consequence, it combines more rapidly with oxygen on the outside and displaces hydrogen from the water (p. 51) on the inside, giving in both cases $\mathrm{Fe}_{3} \mathrm{O}_{4}$. Thus the life of the boiler is shortened. If the formation of the crust is not prevented, or if the crust is not removed, the boiler may explode and great damage may be done.

When hard water is used for washing, in the household or laund$r y$, much soap has to be dissolved before the necessary lather can be secured. Soap, which consists of a mixture of the sodium salts of several organic acids, such as palmitic acid $\mathrm{H} \cdot \mathrm{CO}_{2} \mathrm{C}_{15} \mathrm{H}_{31}$ (see p. 438), interacts by double decomposition with the salts of calcium and magnesium giving palmitates etc. of these metals. These salts are insoluble and form a "curd." With sodium palmitate $\mathrm{Na}\left(\mathrm{CO}_{2} \mathrm{C}_{15} \mathrm{H}_{31}\right)$, for example, the action is

$$
\mathrm{CaSO}_{4}+2 \mathrm{Na}\left(\mathrm{CO}_{2} \mathrm{C}_{15} \mathrm{H}_{31}\right) \rightarrow \mathrm{Ca}\left(\mathrm{CO}_{2} \mathrm{C}_{15} \mathrm{H}_{31}\right)_{2} \downarrow+\mathrm{Na}_{2} \mathrm{SO}_{4} \text {. }
$$

Not until all the salts causing the hardness have been decomposed, does the permanent solution of soap which is required for washing begin to be formed. The waste thus involved is often very great and expensive.

Treatment of Hard Water.-Temporary hardness is commonly removed, on a large scale, by adding slaked lime (made into milk of lime) in exactly the quantity shown by an analysis of the water to be required, and stirring for a considerable time:

$$
\mathrm{Ca}\left(\mathrm{HCO}_{3}\right)_{2}+\mathrm{Ca}(\mathrm{OH})_{2} \rightarrow 2 \mathrm{CaCO}_{3} \downarrow+2 \mathrm{H}_{2} \mathrm{O} .
$$

The bicarbonate is neutralized and all the lime precipitated. The latter is removed by filtration.

Permanent hardness is not affected by slaked lime, but is removed by adding sodium carbonate in the necessary proportion:

$$
\mathrm{CaSO}_{4}+\mathrm{Na}_{2} \mathrm{CO}_{3} \rightarrow \mathrm{CaCO}_{3} \downarrow+\mathrm{Na}_{2} \mathrm{SO}_{4} .
$$


When both kinds of hardness are present, crude caustic soda (sodium hydroxide) may be employed. It neutralizes the bicarbonate, precipitating $\mathrm{CaCO}_{3}$ :

$$
\mathrm{Ca}\left(\mathrm{HCO}_{3}\right)_{2}+2 \mathrm{NaOH} \rightarrow \mathrm{CaCO}_{3} \downarrow+\mathrm{Na}_{2} \mathrm{CO}_{3}+2 \mathrm{H}_{2} \mathrm{O}
$$

and giving sodium carbonate. The latter then acts as in equation (2).

Instead of this, the treatments indicated in equations (1) and (2) may be applied in combination (Porter-Clark process).

In the new permutite process the water is simply filtered through an artificial sodium silico-aluminate (permutite) which is supplied in the form of a coarse sand. The calcium, etc., in the water is exchanged for sodium, which does no harm. If we use for permutite the abbreviated formula $\mathrm{Na} \overline{\mathrm{P}}$, we may write the reaction thus:

$$
\mathrm{Ca}\left(\mathrm{HCO}_{3}\right)_{2}+2 \mathrm{Na} \overline{\mathrm{P}} \rightarrow 2 \mathrm{NaHCO}_{3}+\mathrm{Ca} \overline{\mathrm{P}}_{2} .
$$

After twelve hours' use, the permutite is covered with 10 per cent salt solution and allowed to remain for the other twelve hours of the day, when it is ready for employment once more:

$$
2 \mathrm{NaCl}+\mathrm{Ca} \overline{\mathrm{P}}_{2} \rightarrow \mathrm{CaCl}_{2}+2 \mathrm{Na} \overline{\mathrm{P}} .
$$

Only salt, which is inexpensive, is consumed, and calcium chloride solution is thrown away. Permutite removes magnesium, iron, manganese, and other elements in the same way. The life of a charge is said to be over twenty years.

Hard Water in the Laundry. - As we have seen (p. 389), soap will soften water, but the calcium and magnesium salts of the soap acids, which are precipitated, are sticky, and soil the goods being washed. Other substances that soften water not only give non-adhesive precipitates, but are also much cheaper, and an attempt is generally made to utilize them. The use of slaked lime is impracticable on a small scale. 
Washing soda $\mathrm{Na}_{2} \mathrm{CO}_{3}, 10 \mathrm{H}_{2} \mathrm{O}$ is added to precipitate both kinds of hardness:

$$
\begin{gathered}
\mathrm{Ca}\left(\mathrm{HCO}_{3}\right)_{2}+\mathrm{Na}_{2} \mathrm{CO}_{3} \rightarrow \mathrm{CaCO}_{3} \downarrow+2 \mathrm{NaHCO}_{3} \\
\mathrm{CaSO}_{4}+\mathrm{Na}_{2} \mathrm{CO}_{3} \rightarrow \mathrm{CaCO}_{3} \downarrow+\mathrm{Na}_{2} \mathrm{SO}_{4} .
\end{gathered}
$$

The small amounts of salts of sodium which remain in the water have no action on soap.

Household Ammonia $\mathrm{NH}_{4} \mathrm{OH}$ acts like sodium hydroxide (p. 390):

$$
\begin{gathered}
\mathrm{Ca}\left(\mathrm{HCO}_{3}\right)_{2}+2 \mathrm{NH}_{4} \mathrm{OH} \rightarrow \mathrm{CaCO}_{3} \downarrow+\left(\mathrm{NH}_{4}\right)_{2} \mathrm{CO}_{3}+2 \mathrm{H}_{2} \mathrm{O} \\
\mathrm{CaSO}_{4}+\left(\mathrm{NH}_{4}\right)_{2} \mathrm{CO}_{3} \rightarrow \mathrm{CaCO}_{3} \downarrow+\left(\mathrm{NH}_{4}\right)_{2} \mathrm{SO}_{4}
\end{gathered}
$$

except that it will not precipitate magnesium-ion (see p. 539).

When borax $\mathrm{Na}_{2} \mathrm{~B}_{4} \mathrm{O}_{7}, 10 \mathrm{H}_{2} \mathrm{O}$ (p. 363) is added, it is hydrolyzed and the sodium hydroxide contained in its solution acts as already described.

The supposed bleaching or whitening action of borax or soda is a myth; these salts prevent staining by the iron in the water. They simply precipitate the iron (present as $\left.\mathrm{Fe}\left(\mathrm{HCO}_{3}\right)_{2}\right)$, which almost all waters contain, as $\mathrm{FeCO}_{3}$ before the goods are put in. This precipitate is easily washed out in rinsing. The palmitate, etc., of iron, however, which the soap itself would throw down, is sticky and adheres to the cloth. The air subsequently oxidizes it and gives hydrated ferric oxide (rust), which is brownish-red.

It is evident that, properly to achieve their purpose, the soda and borax must be added, must be completely dissolved, and must be allowed to produce the precipitation of $\mathrm{FeCO}_{3}, \mathrm{CaCO}_{3}$, etc., all before the soap, or the goods, is introduced. If the soap is dissolved before or with the soda, it will take part in the precipitation, and give sticky particles containing the iron and calcium salts of the soap acids.

Washing powders are, or ought to be, mainly sodium carbonate, mixed with more or less pulverized soap. 
Calcium Chloride, $\mathrm{CaCl}_{2}$. - Chloride of calcium is obtained as a by-product in the Solvay process (p. 366) and in other industries. It crystallizes from water as the white hexahydrate, $\mathrm{CaCl}_{2}, 6 \mathrm{H}_{2} \mathrm{O}$, and is very soluble. The porous, granular variety, used for drying gases, is made by driving most of the wator out of the hexahydrate by heat. The granular form is used in large amounts for sprinkling on dusty roads. The salt, being deliquescent (p. 118), attracts water from the air and moistens the dust with calcium chloride solution. The saturated solution does not freeze until $-48^{\circ}$ is reached, so that chilled calcium chloride brine is used in refrigerating appliances (p. 306).

Calcium Cyanamide $\mathrm{CaCN}_{2}$. - Calcium carbide, when strongly heated, absorbs nitrogen, giving a mixture of calcium cyanamide and carbon (nitro-lime):

$$
\mathrm{CaC}_{2}+\mathrm{N}_{2} \rightarrow \mathrm{CaCN}_{2}+\mathrm{C} .
$$

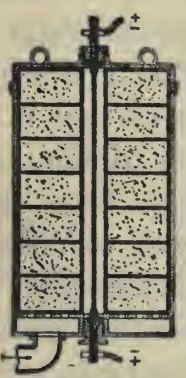

FIg. 97

The carbide is pulverized and placed in a cylindrical furnace (Fig. 97), holding 300 to $450 \mathrm{~kg}$. The heat $\left(800\right.$ to $\left.1000^{\circ}\right)$ is furnished by the passage of a current of electricity through a thin carbon rod, which passes through the axis. The tube surrounding the rod and the other partitions are of cardboard, which burns up and leaves openings for the circulation of the nitrogen. The latter is made by the fractionation of liquid air and is introduced under pressure. In thirty-five hours nitrogen ceases to be absorbed, and the product is pulverized when cold.

Calcium cyanamide is now manufactured in large quantities at Niagara Falls (Ontario) and Odda (Norway) for use as a fertilizer. It is also a valuable source of ammonia, and was utilized very extensively as such for the manufacture of explosives during 
the Great War. When treated with hot water, calcium cyanamide is decomposed as follows:

$$
\mathrm{CaCN}_{2}+3 \mathrm{H}_{2} \mathrm{O} \rightarrow \mathrm{CaCO}_{3}+2 \mathrm{NH}_{3} \text {. }
$$

In practice, pulverized nitro-lime is fed into an autoclave charged with water. Impurities such as free calcium carbide and calcium phosphide are immediately decomposed, and the gases evolved allowed to escape. Small amounts of alkali are then added to facilitate the evolution of ammonia and to prevent the formation of complex nitrogen compounds. The autoclave is closed and steam admitted until the pressure rises to 3-4 atmospheres, the heat evolved by the decomposition of the cyanamide being sufficient to carry the reaction to rapid completion. The ammonia given off is absorbed in water, or converted directly to ammonium sulphate. When the gas ceases to be given off, steam is blown through the liquor in the autoclave until all residual dissolved ammonia is expelled.

The productive capacity of cyanamide plants in 1920 exceeded $1,750,000$ tons, calculated as nitro-lime.

Nitro-lime, when fused with sodium carbonate, gives sodium cyanide $\mathrm{NaNC}$, used in the extraction of gold:

$$
\mathrm{CaCN}_{2}+\mathrm{C}+\mathrm{Na}_{2} \mathrm{CO}_{3} \rightarrow \mathrm{CaCO}_{3}+2 \mathrm{NaNC} .
$$

Cther Compounds of Calcium.-Calcium fluoride $\mathrm{CaF}_{2}$ occurs as a mineral (fluorite). It is our source of hydrofluoric acid (p. 207), and is used in metallurgy to lower the melting-point of slags. The phosphates (p. 411) and bisulphite (p. 398) of calcium, and bleaching powder $\mathrm{CaCl}(\mathrm{OCl})$ (p. 224) are elsewhere discussed.

Strontium Sr and Barium Ba.- The compounds of these elements closely resemble those of calcium in physical properties and chemical behavior. Strontium salts confer a carmine-red color to the Bunsen flame, barium salts a green color. Both are 
used as ingredients in fireworks and signal lights. Barium sulphate is used in making white paint ("permanent white").

Exercises.-1. Why can not lime-water be kept in an open bottle?

2. Why does whitewash become so firmly attached to the wall?

3. What is the percentage of nitrogen in nitro-lime, assuming 100 per cent efficiency of conversion?

4. Make equations for the action of sodium palmitate: (a) upon calcium bicarbonate; (b) upon magnesium sulphate.

5. In softening water: (a) what would be the objection to using an excess of milk of lime; (b) why is prolonged stirring required (p. 389); (c) why must the precipitate be removed by filtration?

6. Explain why wood ashes are sometimes used to soften water, and how they act.

7. Make equations for the action of chlorine upon quicklime-

8. Why does fluorite lower the melting-point of a slag? 


\section{CHAPTER XXXIV}

\section{PLANT LIFE. CELLULOSE, STARCH AND SUGAR}

Plants and animals are similar in composition. They contain much the same elements, and these are present in the form of similar compounds. They differ sharply, however, in the foods they use in constructing these compounds. Plants use simple, inorganic materials; animals absolutely require complex, organic substances as food. The main chemical processes, therefore, are very different in the two groups.

How the Plant Feeds. - The walls of the cells which form the frame-work of a plant are made of cellulose $\left(\mathrm{C}_{6} \mathrm{H}_{10} \mathrm{O}_{5}\right)_{y}$. In the cells, especially in certain parts of the plant, granules of starch $\left(\mathrm{C}_{6} \mathrm{H}_{10} \mathrm{O}_{5}\right)_{x}$ are found. These complex substances differ in properties, although they have the same composition. The plant juice (sap) contains sugars, such as cane-sugar or sucrose, $\mathrm{C}_{12} \mathrm{H}_{22} \mathrm{O}_{11}$, in variable amounts, and also esters (vegetable oils, p. 432) and alkaloids (vegetable bases, p. 479) in much smaller quantities. The plant cells also contain still more complex substances, known as proteins. Gluten, the sticky portion of wheat flour (p. 5), is a typical protein. These proteins are the chief components of the protoplasm, a semi-liquid substance lining each active plantcell, and the real seat of life of the plant. Now all these substances contain carbon, hydrogen, and oxygen, and plant food must furnish these elements, which constitute over 95 per cent, on the average, of all plants. Hence, in addition to large quantities of water ascending from the soil through the roots and stem, and sufficient amounts of compounds of nitrogen, potassium, phosphorus, and other elements, all plants require an abundant supply of carbon in absorbable form. This carbon is practically all 
taken up by plants in the form of atmospheric carbon dioxide. It is admitted through minute openings (stomata), situated mainly in the surface of the leaves.

The Reaction Involved.-Comparison of the formulæ of carbon dioxide $\mathrm{CO}_{2}$ and of any plant substance, like starch $\left(\mathrm{C}_{6} \mathrm{H}_{10} \mathrm{O}_{5}\right)_{x}$, shows at once that the latter contains a far smaller proportion of oxygen, relatively to the amount of carbon, than

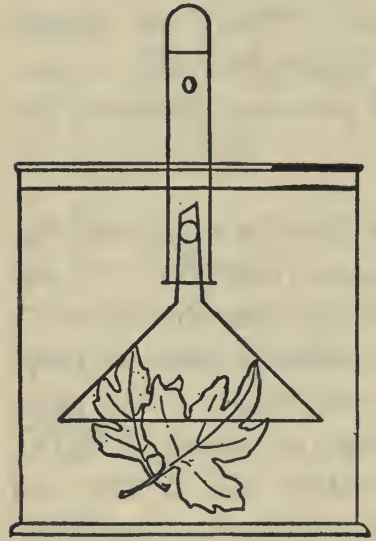

FIG. 98 does the former. Hence, during the digestion or assimilation of the carbon dioxide by the plant, this compound must be reduced. In point of fact, the chlorophyll (green coloring matter) and protoplasm in the leaves act upon the carbon dioxide, causing oxygen to be liberated:

$$
6 \mathrm{CO}_{2}+5 \mathrm{H}_{2} \mathrm{O} \rightarrow \mathrm{C}_{6} \mathrm{H}_{10} \mathrm{O}_{5}+6 \mathrm{O}_{2} \uparrow \text {. }
$$

This action goes on only in the sunlight. The steps by which sugar, starch, and cellulose are manufactured by the plant out of water and carbon dioxide, are not yet perfectly understood. But the liberation of the oxygen is easily shown by placing a green plant under water in a jar, and setting the jar in the sunlight (Fig. 98). Bubbles of gas appear on the leaves, grow larger, and then detach themselves and rise to the top. The gas relights a glowing splinter of wood, and is pure oxygen.

The results of recent investigations suggest the following stages of the reaction:

(1) Carbonic acid, formed by the union of water and carbon dioxide, is reduced to formaldehyde ( $p .348$ ), oxygen being liberated:

$$
\mathrm{H}_{2} \mathrm{CO}_{3} \rightarrow \mathrm{H} . \mathrm{CHO}+\mathrm{O}_{2} \text {. }
$$


PLANT LIFE. CELlULOSE, STARCH AND SUGAR

(2) Formaldehyde molecules quickly combine together, or polymerize, to give simple sugars with the formula $\mathrm{C}_{6} \mathrm{H}_{12} \mathrm{O}_{6}$ :

$$
6 \mathrm{H} . \mathrm{CHO} \rightarrow \mathrm{C}_{6} \mathrm{H}_{12} \mathrm{O}_{6} \text {. }
$$

(3) These sugars lose a molecule of water, and polymerize further to form starch and cellulose:

$$
n \mathrm{C}_{6} \mathrm{H}_{12} \mathrm{O}_{6} \rightarrow n \mathrm{H}_{2} \mathrm{O}+\left(\mathrm{C}_{6} \mathrm{H}_{10} \mathrm{O}_{5}\right)_{n} \text {. }
$$

Reactions (1) and (2) have been carried out in the laboratory with ultra-violet light as a catalyst. Chlorophyll thus appears to act in the rôle of a promoter (compare p. 339), in the presence of which the reactions are able to proceed in visible light.

Reverse reactions also take place in the plant. Thus starch, which first accumulates in the leaves, is later turned back into a sugar soluble in the sap, and is thus able to pass to parts of the plant requiring new material. Some carbon dioxide is also liberated from plant surfaces by oxidation of sugars.

\section{The Thermochemistry of the Reaction.- In the combina-} tion of carbon and oxygen, during combustion of wood or coal, much heat is liberated. Hence, when oxygen is taken out of carbon dioxide again, heat or energy in some form must be supplied. When this takes place in a plant, the energy is evidently furnished by the sunlight, for the action proceeds more slowly in the shade, and ceases in the dark.

The energy required can be measured, and may be expressed in calories. The energy required to produce one simple formulaweight of cellulose $\left(\mathrm{C}_{6} \mathrm{H}_{10} \mathrm{O}_{5}=6 \times 12+10 \times 1+5 \times 16=162\right.$ g.) is 671,000 calories. The whole may be represented in a rough equation, in which the unknown intermediate steps are left out, and only the starting substances and the final products are shown:

$$
6 \mathrm{CO}_{2}+5 \mathrm{H}_{2} \mathrm{O}+671,000 \text { cal. } \rightarrow \mathrm{C}_{6} \mathrm{H}_{10} \mathrm{O}_{5}+6 \mathrm{O}_{2} \text {. }
$$

Cellulose $\left(\mathrm{C}_{6} \mathrm{H}_{10} \mathrm{O}_{5}\right)_{y}$. - This substance, named cellulose because it forms the walls of the cells, composes much of the frame- 
work and intricate structure of plants. We are familiar with pure cellulose in the forms of filter paper and cotton. The latter consists of fine, hollow tubes of cellulose (see Fig. 2, p. 2), large tufts of which surround the seed of the cotton plant. Linen is almost pure cellulose, wood is largely cellulose, and paper pulp is practically all cellulose.

Cellulose interacts with very few chemical substances. It is because it thus remains unchanged, by most substances that come in contact with it, that it can be used as a filter paper. When it does undergo chemical change, it acts as if it contained hydroxyl $(\mathrm{OH})$ groups, and behaves therefore chemically like an alcohol (see p. 480) forming valuable derivatives (explosives and plastics) which will be discussed later (Chap. XL).

Paper Manufacture.-Paper is composed of cellulose $\left(\mathrm{C}_{6} \mathrm{H}_{10} \mathrm{O}_{5}\right)_{y}$ and is made from a mixture of cotton or linen pulp and wood pulp - the cheapest varieties from the latter alone. The wood is cut into chips and heated (" cooked") with a solution of calcium bisulphite $\mathrm{Ca}\left(\mathrm{HSO}_{3}\right)_{2}$. This dissolves out the lignin, which, together with cellulose, makes up the solid part of its structure. The pulpy material is then washed, beaten with water to reduce it to minute shreds, and bleached with very dilute chlorine-water. The pure cellulose, now paper pulp, suspended in water, is spread on screens, drained, pressed, and dried. During the process other substances are usually added. Thus size (glue or gelatine) prevents the ink from running; pulverized calcium sulphate (gypsum), and other white solids ("loading ") give body to the paper and make possible the subsequent production of a smooth surface by rolling ("calendering "). Ultramarine (blue) and other colored powders are added to the pulp when special tints are required.

Other Uses of Cellulose.-Cellulose dissolves in hot, concentrated zinc chloride solution. When the liquid is pressed through 
a small orifice into alcohol, the cellulose is reprecipitated in the form of a thread. By carbonizing, this is made into filaments for incandescent electric lamps.

Cellulose is solubla also in a solution of cupric hydroxide in excess of ammonium hydroxide, and is reprecipitated by dilute sulphuric acid. Paper or cotton goods can be passed through first one and then the other of these liquids, and so receive a tough, waterproof surface. Artificial silk is made by pressing the solution through dies into the precipitant. It can be dyed to any desired tint, and is at least as brilliant in appearance as the natural article. Its strength, however, does not equal that of the natural silk fibre, especially when wet.

Cotton, when dipped in concentrated sodium hydroxide solution, and then stretched while drying to prevent the shrinkage which otherwise occurs, acquires a brilliant luster and is used in enormous quantities under the name of mercerized cotton.

Finally, mercerized cotton, or wood pulp treated with caustic soda, combines with carbon disulphide to give viscose. Viscose dissolves in water, and decomposes in solution giving a plastic form of cellulose. This can be rolled into transparent sheets, made into caps for bottles, moulded into any form, or pressed through dies into solutions of salts of ammonium to give another form of artificial silk.

Starch $\left(\mathrm{C}_{6} \mathrm{H}_{10} \mathrm{O}_{5}\right)_{x}$. - Starch is found in plants in little colorless granules of various rounded shapes (Fig. 99) which may readily be seen under the microscope. These granules are massed in large numbers in the ears of wheat and oats, in the tubers of potatoes, in the grains of maize (corn) and in peas and beans. Even in the leaves they can be seen, immediately after the plant has been ex-
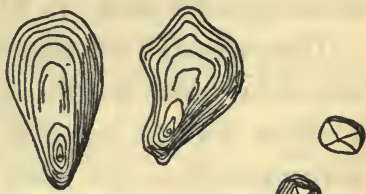
posed to sunlight. They gradually disappear from the leaves 
in the dark. They can be recognized, not only by their appearance, but, without a microscope, by the iodine test. When a drop of a potassium iodide solution, rendered brown by the addition of a little free iodine, is placed on the leaf or other part of the plant, the granules of starch become blue while the other parts are not affected.

Preparation of Starch. - If flour, which is made by grinding wheat, and is three-fourths starch, is placed in a muslin bag and kneaded under water, the granules of starch are washed out and render the water milky (p. 5). After a time the granules settle and the water can be poured off. Starch is manufactured by washing disintegrated potatoes (in Europe) or maize (in America) on sieves, and collecting and drying the white powder deposited in the water used for the washing.

Starch is not soluble in water. If it be boiled with water, however, the granules swell and break, and the starch becomes finely diffused through the water, forming a clear liquid. With little water, a sort of transparent jelly is produced. When the liquid is poured through a filter, a large part of the starch goes through the paper as if it were truly dissolved. Such a liquid is called a colloidal suspension (p. 109). Imitation solutions like this are constantly met with in using complex organic compounds such as enter into jellies, glues, soaps, and the juices of the bodies of animals. Even inorganic substances, of the insoluble class, give such suspensions. A description of their peculiarities must be noticed under soap (p. 440).

The colloidal suspension of starch is used in the laundry, for stiffening white goods. Glucose is manufactured from it.

Since neither cellulose nor starch can be vaporized without decomposition, and since they do not form true solutions in any of the common solvents, we have no means of determining their molecular weights, and are therefore forced to write their formulæ in the indeterminate forms $\left(\mathrm{C}_{6} \mathrm{H}_{10} \mathrm{O}_{5}\right)_{\nu}$ and $\left(\mathrm{C}_{6} \mathrm{H}_{10} \mathrm{O}_{5}\right)_{x}$ respectively. 
The molecule in each case is, in all probability, exceedingly complex.

muade by hydrolisis of

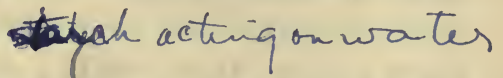

Glucose $\mathrm{C}_{6} \mathrm{H}_{12} \mathrm{O}_{6}$ from Starch. - When starch is boiled with water, to which a few drops of an acid (catalyst) such as hydrochloric acid have been added, the liquid, after neutralization of the acid, is found to be sweet in taste.) A kind of sugar, glucose $\mathrm{C}_{6} \mathrm{H}_{12} \mathrm{O}_{6}$, can be obtained in crystals by evaporation. In commerce the evaporation is stopped before crystallization begins, and the syrup ("corn-syrup," if maize is the source of the starch) is sold for making candy and for preserving fruits.

$$
\left(\mathrm{C}_{6} \mathrm{H}_{10} \mathrm{O}_{5}\right)_{x}+x \mathrm{H}_{2} \mathrm{O} \rightarrow x \mathrm{C}_{6} \mathrm{H}_{12} \mathrm{O}_{6} \text {. }
$$

Glucose is known also as dextrose, and as grape sugar. Brownish crystalline granules found in dried grapes (raisins) are mainly composed of it. When pure, it is almost colorless. It reduces cupric hydroxide, in Fehling's solution (p. 513), to cuprous oxide.

The Sugars. - The common sugars are divided into two classes. There are several sugars, having the same formula, $\mathrm{C}_{6} \mathrm{H}_{12} \mathrm{O}_{6}$, but different properties, which are called monosaccharides. Other sugars, having twice as many carbon units in the formula $\mathrm{C}_{12} \mathrm{H}_{22} \mathrm{O}_{11}$, are called disaccharides. The sugars we have occasion to mention here are the following:

Monosaccharides: Glucose (dextrose or grape sugar) $\mathrm{C}_{6} \mathrm{H}_{12} \mathrm{O}_{6}$. Fructose (fruit sugar) $\mathrm{C}_{6} \mathrm{H}_{12} \mathrm{O}_{6}$.

Disaccharides: Sucrose (cane-sugar, beet-sugar, saccharose) $\mathrm{C}_{12} \mathrm{H}_{22} \mathrm{O}_{11}$.

Maltose (formed by action of malt on starch) $\mathrm{C}_{12} \mathrm{H}_{22} \mathrm{O}_{11}$.

Lactose (milk-sugar, found only in animals) $\mathrm{C}_{12} \mathrm{H}_{22} \mathrm{O}_{11}$.

Carbohydrates. - Since cellulose, starch and the sugars are freely changed, one into another, they are grouped together in one 
class, the carbohydrates. The word refers to the fact that they contain hydrogen and oxygen in the proportions required to form water, and are, therefore, in a sense, hydrates of carbon. When dehydrating agents like concentrated sulphuric acid (p. 270) act on the carbohydrates, a black mass of carbon is left.

Sucrose or Cane-Sugar $\mathrm{C}_{12} \mathrm{H}_{22} \mathrm{O}_{11}$. - The sugar-cane and the beet produce exceptionally large amounts of this sugar, which is the one commonly used as table sugar. Maple sugar, obtained by evaporating the sap of the tree, is composed mainly of the same substance.

The sugar-cane forms stalks from ten to twelve feet high. The juices are extracted by crushing the plants between rollers. The liquid is evaporated in losed pans. A vacuum maintained in the pans permits the boiling of the solution at a low temperature (about 65 degrees) and prevents the decomposition of a part of the sugar which would otherwise occur. When the syrup cools, the sugar crystallizes and the crystals are freed from the liquid in centrifugal machines. The crystals are brown in color. At the sugar refinery they are dissolved, and the solution is passed through a column of bone charcoal. This adsorbs the coloring matter, and the filtrate is once more evaporated and allowed to crystallize. Refined cane-sugar has a faint yellow tint, and a small amount of ultramarine is added to cover up this tint, and give the white appearance which is popularly connected with purity in sugar.

The sugar beets, which contain 16 per cent or more of canesugar, are sliced and steeped in water to extract the sugar. The liquid contains gummy material in colloidal suspension. This is coagulated and precipitated by adding "milk of lime" (calcium hydroxide $\mathrm{Ca}(\mathrm{OH})_{2}$ suspended in water) and boiling. Carbon dioxide is then passed through the solution to precipitate the excess of lime:

$$
\mathrm{Ca}(\mathrm{OH})_{2}+\mathrm{CO}_{2} \rightarrow \mathrm{CaCO}_{3} \downarrow+\mathrm{H}_{2} \mathrm{O} .
$$


PLANT LIFE. CELLULOSE, STARCH AND SUGAR 403

The solution is decolorized with charcoal and evaporated to crystallization in the same way as is the extract from the sugarcane.

Properties of Sucrose.-Sucrose crystallizes in four-sided prisms, the form of which is seen in "rock-candy." It melts at $160^{\circ}$. It does not reduce Fehling's solution (p. 401). When heated to 200 to $210^{\circ}$ it begins to decompose, slowly losing water and leaving a brown, soluble mass called caramel, used in coloring whiskey and soups.

When boiled with water, to which a trace of an acid catalyst has been added, it is hydrolyzed, giving a mixture of the two monosaccharides, glucose and fructose:

$$
\mathrm{C}_{12} \mathrm{H}_{22} \mathrm{O}_{11}+\mathrm{H}_{2} \mathrm{O} \rightarrow \mathrm{C}_{6} \mathrm{H}_{12} \mathrm{O}_{6}+\mathrm{C}_{6} \mathrm{H}_{12} \mathrm{O}_{6} \text {. }
$$

This mixture of glucose and fructose is called invert sugar and is found in many sweet fruits and in honey. Each sugar interferes with the crystallization of the other, by lowering the freezing-point (p. 119), and so invert sugar is added in making "fondant" candy and candy that is to be "pulled," both of which are intended to remain soft for some time. With the same object in view, vinegar, lemon juice, or cream of tartar is added to a syrup made from cane-sugar, in order that the acid contained in them may produce some invert sugar and so give a less crystallizable mixture (icing for cakes). Prolonged heating has the same effect.

Exercises.-1. What inference do you draw as to the composition of tapioca, sago, and rice from the facts that they are plant products and when boiled with water and cooled give a jelly-like mass? How should you confirm your inference?

2. (a) Why does a concentrated solution of sugar boil at a temperature far above that of boiling water? (b) In evaporation why is the boiling-point lower in a vacuum than in air? 
3. In what relative volumes are carbon dioxide used and oxygen produced by a plant?

4. What products must be formed when paper is burned? Make the equation. 
How the Plant Feeds. - We have mentioned (p. 395) that the plant juice or sap contains soluble sugars, which travel to those parts of the plant which require new material for their growth. The moist walls of the root-hairs of the plant are freely permeable to water, the entrance of which into the plant from the soil is necessary to offset evaporation from the leaves and stems. Soluble salts present in the soil water are also able to pass, although less freely, through these walls, and are incorporated in the sap. If they are of nutritive value to the plant, they react, as they circulate through the plant from cell to cell, with the organic constituents there present, forming more complex compounds which are unable to permeate the boundary walls of the cells, and thus become permanently fixed in the growing parts. If they are not of nutritive value, they complete the circuit unchanged.

Now the passage of salts from the soil water into the plant continues only until the sap inside contains the same concentration of each salt as the solution outside. Hence while nutritive constituents, which are removed from the sap during its circulation through the plant, can continuously enter to keep up the supply necessary for growth, non-nutritive constituents soon reach their equilibrium concentration and are thereafter rejected.

Osmosis. - The membranes lining the cell walls and the roothairs of plants exercise, as we saw above, a selective action with regard to the passage of different substances through them. Water is able to permeate the membranes quite freely, dissolved salts pass through less readily, while the movement of complex organic substances, such as proteins, is completely blocked. Upon this selective flow of materials through the plant membranes, it must be noted, the life of a plant is absolutely dependent. If the membranes were freely permeable to the organic materials contained in the plant sap, the plant would soon lose these materials to the outside soil and die of exhaustion. 
The selective flow of certain components of a solution through a membrane is known as osmosis. Osmosis and its consequence, osmotic pressure, are phenomena which may be very clearly illustrated in the laboratory by means of a solution of cane sugar and an artificial membrane of precipitated cupric ferrocyanide $\mathrm{Cu}_{2} \cdot \mathrm{Fe}(\mathrm{CN})_{6}$.

Osmotic Pressure.-A suitable semi-permeable membrane may be obtained by soaking a clean porous pot in a solution of

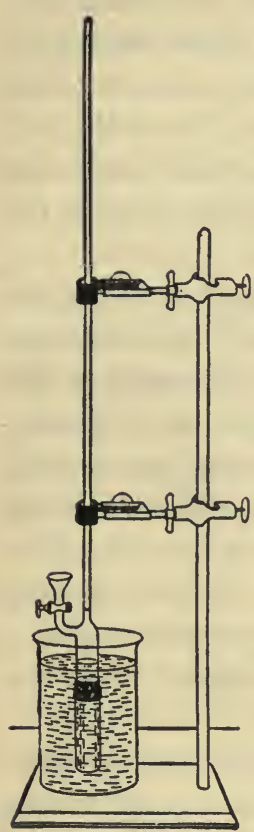

FIG. 100 potassium ferrocyanide $\mathrm{K}_{4} \cdot \mathrm{Fe}(\mathrm{CN})_{6}$ (p. 497), rinsing in water, and then allowing to stand in a solution of cupric sulphate. The diffusion of the latter substance into the cell produces, by double decomposition, a film of insoluble copper ferrocyanide within its walls. This film is freely permeable to water molecules, but not to molecules of sugar dissolved in the water.

A simpler method is to use a diffusion shell of specially treated parchment, of test-tube form. This, however, is not entirely impermeable to sugar molecules.

The porous pot or diffusion shell, filled with sugar solution, is securely attached to a long glass tube, and suspended in pure water (Fig. 100). It is found that the level of the liquid in the tube gradually rises until, if the membrane remains intact and is truly impermeable to sugar, a definite hydrostatic pressure is established, the magnitude of this osmotic pressure depending only upon the temperature and upon the fraction of sugar molecules in the solution within the cell. 
Explanation of Osmotic Pressure.-For a complete discussion of osmotic pressure, the reader is referred to a modern text-book of physical chemistry. A brief explanation by means of the molecular hypothesis, however, may be given here.

The molecules of water in the pure water outside the cell, and the molecules of water and of sugar in the solution inside the cell, are all in rapid motion (p. 94). When, in consequence of this motion, they strike the membrane, water molecules have a chance of passing through, but sugar molecules are all turned back. Now the concentration of water molecules in the pure water outside, striking the membrane and attempting to enter the cell, is greater than the concentration of water molecules in the solution inside, striking the membrane and attempting to leave the cell (compare vapor pressures, p. 117). Hence more water molecules will be entering than leaving, and the level of the liquid inside the tube must rise in consequence.

Why does the level of the liquid stop rising when a definite hydrostatic head has been established? Because now, although there is still a greater concentration of water molecules in the pure water outside than in the solution inside, water molecules attempting to enter the cell through the membrane are opposed by the hydrostatic pressure, while water molecules attempting to leave are assisted in their passage. We have on one side of the membrane more water molecules with a smaller chance of getting through, on the other side fewer water molecules with a greater chance of getting through. Equilibrium is reached, evidently, when these two factors counterbalance.

The student should note very carefully the fact that the sugar molecules are not directly concerned in the phenomenon of osmotic pressure. Their function is merely to reduce the concentration of water molecules in the solution inside the cell. Any solute, with respect to which the membrane is similarly impermeable, will give the same effect, the osmotic pressure at any given temperature being dependent only upon the fraction of solute molecules in 
the solution, that is, upon the extent to which the concentration of water molecules has been reduced (compare, again, vapor pressure depression of solutions, p. 117).

A solution containing $1 \mathrm{~g}$. molecular weight of an inert solute dissolved in $1000 \mathrm{~g}$. water should give at $20^{\circ}$, with a perfectly semi-permeable membrane, an osmotic pressure of 23.6 atmospheres.

Osmotic Pressure in Plant Life-- Osmotic pressure was first studied by Pfeffer, a botanist, in 1877. De Vries (1878) used plant cells for the same purpose. The cell content included a liquid containing various salts in solution, and a protoplasmic layer lining, but not firmly attached to, the cell wall. This protoplasmic layer behaved like an imperfect semi-permeable membrane. When such cells were immersed in a concentrated solution of any substance, the water passed from the interior of the cell to the solution, and by means of a microscope a shrinkage of the protoplasmic layer away from the cell wall could be observed. Conversely, when such cells were placed in pure water, or a solution of a very dilute nature, water passed from the outside into the interior, and the protoplasmic layer was distended so as to cause the cell to become turgid.

Osmotic pressure is, therefore, a subject of great interest in connection with the physiology of plants. It aids in explaining why a withered flower, containing a solution in its cells, revives when placed in pure water. The latter enters through the walls of the cells, and the pressure thus produced distends the structure and stiffens it. Similarly, the wilting of plants, when too high a concentration of salts as fertilizers is added to the soil, is explained. In the animal body also, osmosis plays a large part.

Fertilizers.-Many soils are either naturally deficient in one or more of the necessary plant foods, or the supply may have been exhausted by repeated cropping. Every crop removes 
permanently a certain part of the supply. Thus, in the case of nitrogen, an average crop of maize or corn ( 45 bushels) removes 63 pounds per acre, a crop of cabbage ( 15 tons) removes 100 pounds per acre, clover hay ( 2 tons) 82 pounds, and wheat (15 bushels) 31 pounds. When the supply becomes reduced, the crops become poor. Moreover, the necessary elements must be present in soluble form, or they cannot enter into the plant system.

Felspar $\mathrm{KAlSi}_{3} \mathrm{O}_{8}$ is a common constituent of many rocks, such as granite (p. 4). When such rock material, contained in the soil, is decomposed by weathering, through the action of carbonic acid from the atmosphere, the felspar gives clay $\mathrm{HAlSiO}_{4}$ and soluble compounds of potassium. There are immense quantities of felspar available, but the process of weathering is very slow, and in many agricultural regions the soil is therefore deficient in soluble salts of potassium.

It is just as necessary to feed crops as to feed cattle, and equally foolish to starve either of them. Fertilizers are used to make good the original, or acquired deficiences of the soil in the most important elements, nitrogen, phosphorus, potassium and calcium. It is absolutely necessary, in addition, to keep up a sufficient supply of fresh organic material in the soil, or the use of fertiliz-, ers may result in more harm than benefit.

The value of the systematic use of fertilizers is indicated by comparison of the average crop of wheat per acre in different countries. The average of ten successive years is: Denmark 40 bushels, Great Britain 33, Germany 29, United States 14.

\section{2nl.}

Nitrogen.- The nitrogen is supplied as sodium nitrate or guano (p. 369), calcium nitrate (p. 313), ammonium sulphate (p. 299), calcium cyanamide (p. 392), manure or the offal (" tankage ") and ground bones from slaughter houses.

Over every acre of the earth's surface there are 34,000 tons of free atmospheric nitrogen. Plants in general are incapable of drawing upon this immense store for the nitrogen necessary for 
their growth, but peas, beans, clover, alfalfa and other leguminous plants bear round their roots colonies of a special kind of bacteria which has the power to bring free nitrogen into combination. In these root nodules (Fig. 101) the bacteria first produce proteins, which later decompose, and ultimately, by bacterial action, yield nitric acid. In this way a crop of clover will fertilize the soil, not only for itself, but also for the following crop. The advantage of rotation of crops is therefore explained.

The beneficial action of bacteria upon plant growth is not limited to this special case. The soil is crowded with bacteria which assist in the decomposition or rotting of vegetable and animal matter. The product, the black, gummy stuff of a fertile soil, is called humus. Certain varieties of bacteria convert insoluble

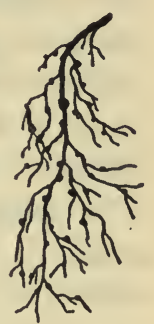

FIG. 101 carbohydrates such as cellulose into simpler soluble materials, immediately available for plant use. Other species liberate the nitrogen from proteins in the form of ammonia and amino-compounds (p. 353). Others, again, oxidize these compounds to nitrous acid and nitrites. Still others, finally, oxidize these products to nitric acid and nitrates.

The actual assimilation of nitrogen by plants, in whatever form that element is originally present in or added to the soil, takes place almost exclusively through soluble nitrates. The co-ordinated team-work of all of the above classes of bacteria is therefore an essential point in the efficient utilization of other nitrogen-containing fertilizers.

Phosphorus. Natural calcium phosphate $\mathrm{Ca}_{3}\left(\mathrm{PO}_{4}\right)_{2}$ is the orthophosphate of calcium. It is found in considerable deposits in S. Carolina, Florida, Tennessee, and several western states, and in Algeria and Tunis. This insoluble salt, however, affords only an exceedingly dilute phosphorus diet for plants. Hence, a more soluble compound is to be preferred. This is found in 
calcium acid-phosphate ("superphosphate") $\mathrm{CaH}_{4}\left(\mathrm{PO}_{4}\right)_{2}$, which is made by heating pulverized natural calcium phosphate with sulphuric acid, containing the requisite proportion of water:

$$
\mathrm{Ca}_{3}\left(\mathrm{PO}_{4}\right)_{2}+2 \mathrm{H}_{2} \mathrm{SO}_{4}+4 \mathrm{H}_{2} \mathrm{O} \rightarrow \mathrm{CaH}_{4}\left(\mathrm{PO}_{4}\right)_{2}+2 \mathrm{CaSO}_{4}, 2 \mathrm{H}_{2} \mathrm{O} \text {. }
$$

The whole turns into a dry mixture, consisting of the superphosphate and gypsum (hydrated calcium sulphate). The latter does not interfere with the fertilizing power of the soluble superphosphate, so the mixture is placed directly in sacks and sold as " superphosphate of lime."

In slaughter houses the bones, after being deprived of fat and gelatin, give a residue containing much calcium phosphate. This residue is treated with sulphuric acid and made into fertilizer.

Potassium. $\rightarrow$ Wood ashes contain much potassium carbonate, and are used as fertilizers for this reason. The giant sea-weeds (Kelp) of the Pacific coast have also been found to contain an unusually large proportion of salts of potassium. By far the most important source of this element, however, is potassium chloride, obtained from natural salt deposits.

The average production of the German deposits at Stassfurt in the ten years preceding the war exceeded 1,000,000 tons (calculated as $\mathrm{K}_{2} \mathrm{O}$ ). These deposits constituted practically a world monopoly, and the shortage of potassium salts for fertilizer purposes during the war was consequently extreme. The most strenuous efforts to develop the potassium resources of the United States (natural brines, Kelp, recoverable by-products from the cement and molasses industries, etc.) culminated in a production of only 50,000 tons $\mathrm{K}_{2} \mathrm{O}$ in 1918. Fortunately, the cession of Alsace to France has destroyed the German monopoly, since very extensive deposits exist near Mulhouse. The working of these deposits has been greatly hampered by the damage done to shafts and machinery by the Germans before their evacuation, but production in 1920 already exceeded 200,000 tons $\mathrm{K}_{2} \mathrm{O}$. 
Potassium chloride occurs in the Stassfurt deposits as sylvine $\mathrm{KCl}$, but is chiefly associated with magnesium chloride as carnallite $\mathrm{KCl}, \mathrm{MgCl}_{2}, 6 \mathrm{H}_{2} \mathrm{O}$. When water is added to carnallite, a large part of the potassium chloride, which is much less soluble, separates out. Complete extraction and purification of the salt involves a series of recrystallizations. The Alsace deposits contain very little magnesium salts, and the separation of the potassium chloride from the sodium chloride with which it is mixed is comparatively simple.

Potassium sulphate is also obtained from salt deposits, and is substituted for potassium chloride as a fertilizer for certain crops, such as tobacco. Chlorides, in general, melt at lower temperatures than sulphates, and the presence of a chloride in tobacco results in an ash that fuses on burning. This is, obviously, an undesirable property, especially for cigars.

Calcium.-Calcium is naturally present in many soils as calcium carbonate $\mathrm{CaCO}_{3}$. In contact with water containing carbonic acid (see p. 400), this gives a solution of the more soluble bicarbonate $\mathrm{Ca}\left(\mathrm{HCO}_{3}\right)_{2}$. Other compounds of calcium which are used as fertilizers include lime $\mathrm{CaO}$ or $\mathrm{Ca}(\mathrm{OH})_{2}$, calcium phosphate and acid phosphate, calcium sulphate or gypsum (p. 387), and calcium cyanamide.

Manure.- One ton of farm manure contains about 10 pounds of nitrogen (chiefly as urea $\mathrm{CO}\left(\mathrm{NH}_{2}\right)_{2}$ and proteins), 5 pounds of phosphoric acid and 10 pounds of potash. The manure is mixed with the soil just before planting seed, or is used as top dressing.

The bacteria in the air and in the soil assist materially in the changes in the manure. Thus urea is hydrolyzed to ammonium carbonate $\left(\mathrm{NH}_{4}\right)_{2} \mathrm{CO}_{3}$ :

$\mathrm{CO}\left(\mathrm{NH}_{2}\right)_{2}+2 \mathrm{H}_{2} \mathrm{O} \rightarrow\left(\mathrm{NH}_{4}\right)_{2} \mathrm{CO}_{3}$. 
The proteins are changed by air bacteria into ammonia. The formation of nitrates seldom happens in manure piles, but scattered manure, with the help of soil bacteria, develops nitrates.

The potassium compounds form potassium hydrogen carbonate $\mathrm{KHCO}_{3}$. The phosphorus and sulphur compounds become soluble phosphates and sulphates.

Indirect Fertilizers. - Not all substances which are added to the soil are employed with the direct object of their assimilation for plant growth. Often indirect effects induced by their presence are of greater importance. A few cases where calcium salts are of service as indirect fertilizers may be briefly presented as illustrations of this point.

(1) Gypsum $\mathrm{CaSO}_{4}, 2 \mathrm{H}_{2} \mathrm{O}$ is added to manure at the rate of 100 pounds per ton. This slightly soluble salt reacts in the soil solution with the ammonium carbonate produced by the hy. drolysis of urea, precipitating the much less soluble calcium carbonate and leaving ammonium sulphate in solution:

$$
\left(\mathrm{NH}_{4}\right)_{2} \mathrm{CO}_{3}+\mathrm{CaSO}_{4} \rightarrow\left(\mathrm{NH}_{4}\right)_{2} \mathrm{SO}_{4}+\mathrm{CaCO}_{3} \downarrow \text {. }
$$

Now ammonium sulphate, being a salt of a strong acid with a weak base, is only very slightly hydrolyzed in solution (see p. 369). Ammonium carbonate, however, is a salt of a weak acid and a weak base, and is extensively hydrolyzed. This hydrolysis, unless a large excess of water is present, would lead to rapid loss of ammonia from the manure. The smell of free ammonia, indeed, is often very noticeable in manure piles. The addition of gypsum fixes this valuable constituent in the fertilizer for plant use. Lime, on the other hand, would assist in the liberation of ammonia.

(2) Gypsum is often added, also, to clay soils with the object of converting insoluble compounds of potassium into more soluble compounds. Lime and calcium carbonate are employed to 
change insoluble phosphates of iron and aluminium into more soluble calcium phosphates.

(3) Some soils are either naturally acidic or sour, or have become so by excessive use of sulphate fertilizers, and are hence unfavorable to plant growth. This acidity is corrected by addition of lime or calcium carbonate, but not gypsum.

(4) Salts which are injurious to plants, such as soluble magnesium compounds or "black alkali" (sodium "carbonate), are converted into less soluble compounds, such as magnesium hydroxide or carbonate, by addition of lime or calcium carbonate, or into non-poisonous compounds, such as sodium sulphate, by gypsum.

Adsorption in Soils.-As we have seen, fertilizers must contain the elements necessary for plant growth in soluble form. It is undesirable, however, to use salts which are exceedingly soluble in water as fertilizers, since they will obviously be rapidly washed away from the surface soil. A large proportion of all fertilizers is unavoidably wasted in this manner. The minute particles of the soil, however, possess the power of conserving dissolved substances in the soil solution by concentrating and holding them upon their moist surfaces. This phenomenon, adsorption, is of considerable interest and importance in other connections, and will be taken up in detail in a later chapter (p. 421).

Exercises.-1. Given an osmotic pressure cell and an unknown substance, soluble in water, to which the membrane is perfectly impermeable, how could you determine the molecular weight of the substance?

2. What would be the effect of putting fresh flowers in a vase containing a concentrated salt solution?

3. At $20^{\circ} 100$ c.c. of water, shaken up with excess $\mathrm{NaCl}$ and $\mathrm{KCl}$, dissolve $30 \mathrm{~g}$. $\mathrm{NaCl}$ and $15 \mathrm{~g}$. $\mathrm{KCl}$. At $100^{\circ}$ the same 
amount of water, in equilibrium with the same two solids, contains $20 \mathrm{~g}$. $\mathrm{NaCl}$ and $40 \mathrm{~g}$. $\mathrm{KCl}$. On the basis of these figures, devise a method for separating $\mathrm{KCl}$ from $\mathrm{NaCl}$ in the Alsace deposits.

4. The use of ammonium sulphate as a fertilizer is apt to result in an acid soil. Explain why.

5. What are the three elements most needed in fertilizers? Of which does every country have a free and unlimited supply? How can it be made available?

6. What valuable soil ingredients are lost to a locality when a carload of wheat is shipped away? A carload of pure sugar? Df cotton, (a) ginned or (b) unginned? Of peanut oil? 


\section{CHAPTER XXXVI}

\section{PLANT PRODUCTS. FERMENTATION AND FUELS}

Having described the chemistry of plant life, we may now proceed to the chemistry of substances resulting from plant life and growth. Foods will be taken up separately in the following chapter. In the present chapter we shall restrict ourselves to two other main branches, fermentation products and fuels.

Enzymes. - All fermentations are brought about either directly or indirectly by the activities of animal or vegetable organisms. The most familiar ferment, of course, is yeast.

Yeast belongs to a low order of plants and consists of minute cells. Its value lies in the fact that, while growing and multiplying, it secretes within each cell small amounts of two very active chemical substances which are dissolved in the cell contents. These substances are known as zymase and invertase (or sucrase), and belong to the class of organic materials called enzymes. Enzymes produce remarkable chemical changes in organic materials by their mere presence (contact actions). These changes are specific, each enzyme acting only on certain carbohydrates, for example, and being quite inert towards others.

Fermentation of Sugars.- When a cake of yeast is broken into an aqueous solution of glucose or grape-sugar (p. 401), the small amount of zymase present causes the gradual decomposition of the sugar. The most favorable temperature is about $30^{\circ}$. Bubbles of carbon dioxide soon begin to rise to the surface, and the gas can be led off (Fig. 102) to exhibit its characteristic action (p. 336) on limewater. At the same time alcohol $\mathrm{C}_{2} \mathrm{H}_{5} \mathrm{OH}$ accumulates in the liquid as the sugar disappears:

$$
\mathrm{C}_{6} \mathrm{H}_{12} \mathrm{O}_{6} \rightarrow 2 \mathrm{CO}_{2} \uparrow+2 \mathrm{C}_{2} \mathrm{H}_{5} \mathrm{OH} \text {. }
$$


The liquid extracted from the yeast cells works as well as does the plant itself.

Yeast will ferment fructose (fruit sugar, p. 401), with the same

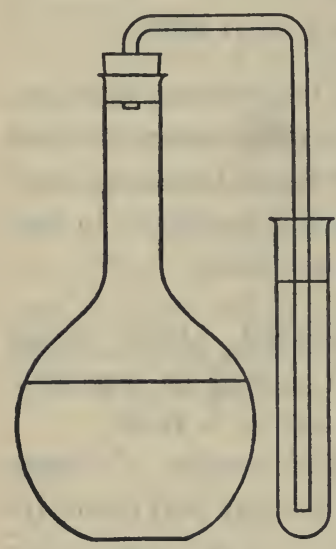

Frg. 102 result, but more slowly.

Zymase does not act upon canesugar (sucrose $\mathrm{C}_{12} \mathrm{H}_{22} \mathrm{O}_{11}$ ). But the invertase (sucrase), which is also contained in the yeast, hydrolyzes the sucrose in the same way as does a dilute acid, giving invert sugar (p. 403). The latter is then decomposed by the zymase. Hence cane-sugar in solution is decomposed by yeast into alcohol and carbon dioxide, just as is grape-sugar, only more slowly.

In the manufacture of wines the glucose contained in the grape juice is fermented by a species of yeast always found on the skins.

Fermentation of Starch. Barley, which has been allowed to sprout, and is then dried, is called malt. This contains an enzyme, diastase (or amylase), which is able to hydrolyze starch into maltose $\mathrm{C}_{12} \mathrm{H}_{22} \mathrm{O}_{11}$ (p. 401). Maltose is further hydrolyzed by another enzyme, maltase, to form glucose, and the latter is then decomposed by zymase into alcohol and carbon dioxide.

Whisky is made by treating the starch of rye, maize, or barley in the above way, with subsequent distillation. Beer is made similarly from various kinds of grain, particularly barley, except that the fermented liquid is not distilled.

Industrial Alcohol.- Alcohol has very extensive uses, apart from its historic value as a beverage. It is employed as a solvents in making varnishes for wood and lacquers for metal, as well as for plastics like celluloid, collodion and artificial silk (p. 399). 
It is of service in the purification of many natural organic products, such as turpentine, and in the preparation of many synthetic organic products, such as dyes. It is also rapidly coming into use as a fuel, its smokeless flame and efficiency of combustion making it of special importance for aeroplane and motor engines. $\Gamma$ Solidified alcohol, obtained by the addition of cellulose esters (p. 480), is now largely employed for cooking purgoses. Another new development is the catalytic production of ethylene for cutting and welding purposes (p. 352).

Alcohol, as used in the industries, is denatured, or rendered unsuitable for drinking purposes, by addition of small quantities of beazine (p. 345), pyridine bases, or other disagreeable and non-removable organic liquids. The exact formula of denatured alcohol depends upon the use for which it is intended.

The cheap production of industrial alcohol is rendered possible by the utilization of certain waste materials rich in carbohydrates. When the price of food is high, grains are employed in the manufacture of alcohol only when a crop has been damaged in some manner so that it cannot be sold as a food material. When saw-dust or wood refuse is heated with dilute sulphuric acid under pressure, thellulose is converted into fermentable sugars by hydrolysis (compare p. 401). At the present time, however, the most important source of industrial alcohol in the United States is molasses. Only a few years ago the disposal of molasses furnished a very troublesome problem to the sugar mills, but in 1918 nearly 120,000,000 gallons of industrial alcohol were obtained from this "waste product" in the United States alone.

(Acetic Acid $\mathrm{CH}_{3} \mathrm{COOH}$ - This acid is formed by the partial oxidation of alcohol (p. 348). Vinegar (crude acetic acid) is manufactured by oxidizing alcohol with atmospheric oxygen, using a bacterium (B. Aceti, "mother of vinegar"), or more probably an enzyme which it secretes, as a contact agent. The 
dilute alcohol, in the form, for example, of "hard" cider (fermented apple juice), is allowed to trickle over shavings in a barrel. The shavings are inoculated with the $B$. aceti by preliminary wetting with vinegar. Holes in the sides admit a plentiful supply of air, to the action of the oxygen of which the liquid is exposed by being spread over the surface of the shavings:

$$
\mathrm{C}_{2} \mathrm{H}_{5} \mathrm{OH}+\mathrm{O}_{2} \rightarrow \mathrm{CH}_{3} \cdot \mathrm{COOH}+\mathrm{H}_{2} \mathrm{O} \text {. }
$$

The liquid (vinegar), which issues at the bottom, contains from 5 to 15 per cent of acetic acid, besides coloring and flavoring matters derived from the fruit juices.

Pure acetic acid may be prepared by distilling the vinegar repeatedly. It is derived more cheaply, however, from the liquid distillate obtained by heating wood in the manufacture of charcoal. Large quantities are used in the manufacture of various synthetic organic products (see, for example, p. 480).

\section{FUELS}

Pestructive Distillation of Wood. 7 When dry wood is heated in iron retorts in absence of air. the compounds which

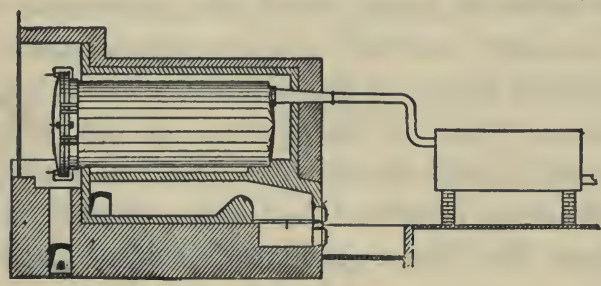

FIg. 103. it contains are decomposed. Much of the carbon remains in the form of charcoal. The vapors which pass off (through the pipe on the right, Fig. 103) deposit, when cooled, much liquid material. The uncondensed gases are combustible and are used for heating the retorts or other similar purposes. Hard wood furnishes, approximately, 25 per cent of its weight of charcoal, 25 per cent of gases, and 50 per cent of liquids. The liquid contains acetic acid (10 per cent), methyl alcohol $\mathrm{CH}_{3} \mathrm{OH}$ or wood spirit (3 per cent), a complex, tarry mix- 
ture, used in road-making (10 per cent), water (77 per cent), and a little acetone (p. 349). The distillate from resinous wood also contains valuable quantities of turpentine, $\mathrm{C}_{10} \mathrm{H}_{16}$, an unsaturated hydrocarbon used extensively as a solvent. The gases evolved contain a large part of the nitrogen of the original proteins in the form of ammonia, which is dissolved out with water.

When charcoal only is desired, the wood is stacked, covered with turf (Fig. 104), and set on fire. A part is burned, the rest is converted into charcoal, and all the valuable volatile products are lost.

\section{Properties of Wood Charcoal.} Adsorption. - The charcoal retains the structure - a complex network of

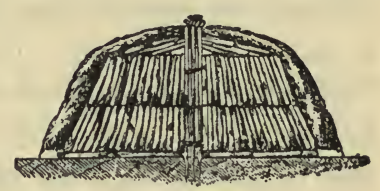

Frg. 104 minute cells - of the original wood, and therefore has a surface which is vast in proportion to the amount of material it contains. Upon this surface it is capable of taking up or of adsorbing many times its own volume of gases, especially of the more condensible ones. Thus, boxwood charcoal takes up ammonia (90 volumes), hydrogen sulphide ( 55 volumes), and oxygen (9 volumes).

The adsorption is extremely rapid and, in the case of a condensible gas contained in small quantity in air, practically complete. For this reason, charcoal and other substances with very finely divided surfaces are used as adsorbent materials for industrial gases and vapors (compare silica gel, p. 360).

The toxic gases employed in the Great War are also readily adsorbed by charcoal. Hence the canisters of gas masks contain layers of porous charcoal, together with granulated sodalime and potassium permanganate, which react chemically with certain of the noxious gases liable to be present. During the war vast strides were made in increasing the adsorptive power of various kinds of charcoal by modifications in methods of carbonization, the most efficient of all charcoals being dense varieties derived from cocoanut shells and fruit pits. Canisters packé
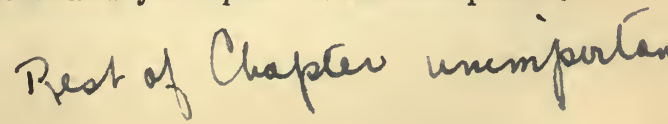
with such charcoal reduced the concentration of all toxic gases employed in the war (see Chapter XL) below the danger limit. Toxic smokes, however, were not satisfactorily adsorbed. The explanation is similar to that advanced in a preceding chapter (p. 263) for the persistence of the fog obtained when a mixture of sulphur trioxide and oxygen is bubbled through water. The molecules of a gas are in such rapid motion that they are practically certain to strike the surface of the charcoal while passing through the canister, and to be adsorbed on this surface if the gas is easily condensible. The dimensions of the solid smoke particles, however, are much larger than molecular, and the particles are relatively stationary. Most of them, in consequence, are able to pass through the air channels between the charcoal granules without touching.

Pulverized charcoal, when shaken with a liquid, is also able to extract from it any dissolved substances, and to concentrate them upon its surface. Other finely-divided materials, such as soil particles (p. 415), possess the same power of adsorbing substances from solution. Salts are, in general, only partially taken up; organic solutes are removed more completely. This property of charcoal is made use of in water purification and in sugar refining (p. 402). Charcoal is used, also, in making gunpowder, in reducing ores, and as a fuel (smokeless).

Coal.-When wood burns with a plentiful supply of oxygen, it gives nothing but carbon dioxide, water, free nitrogen, and a certain amount of ash (oxides and carbonates of the metals). What happens when it is heated in absence of oxygen, we have just seen. In nature, however, the intermediate case of slow decomposition of vegetable matter, without much heating and without access of oxygen, takes place on a large scale. Clay and sand, or even simply water, cover the vegetation and exclude the air, and the products are anthracite coal, bituminous coal, or peat. Little is known of the actual compounds contained in coal. We 
are concerned mainly with the products obtained by heating it in the absence of air, and with its use as a fuel.

Bituminous coals give much, and widely varying amounts of volatile matter; anthracite coals give very little. The ash is the mineral matter of the original plants, with additional rock materials in some specimens. The coal is selected according to the purpose for which it is to be used. For coal gas, and even for coke, a variety high in volatile matter is chosen. For water gas (p. 203) anthracite or coke itself is employed.

Coal Gas. - The gas plant (Fig. 105) includes (1) the fire-brick retorts in which the coal is heated (externally) to $1300^{\circ},(2)$ the hydraulic main (a wide iron pipe) immediately above them in

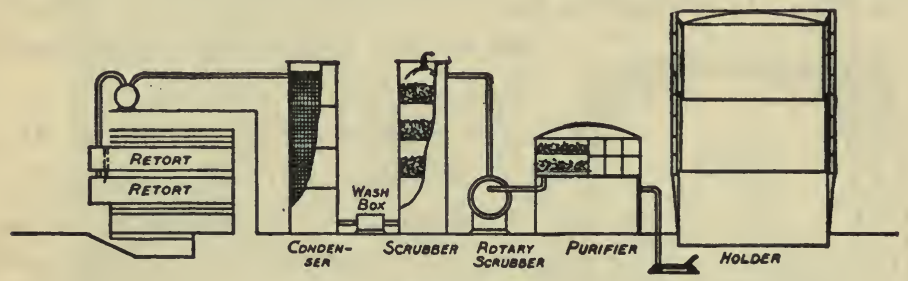

Fig. 105

which most of the tar collects, (3) the condenser and wash box for cooling, condensing, and removing oils, (4) the scrubbers (vertical and rotary) where the ammonia is taken out by water dripping over strips of wood and by stirring the gas with water, (5) the purifier where hydrogen sulphide is taken up by hydrated ferric oxide, and (6) the holder in which the gas collects.

The yield of gas varies considerably with the type of coal used. One ton of good bituminous coal should produce approximately 10,000 cubic feet of coal gas, 1300 pounds of coke, 5 pounds of ammonia and 12 gallons of tar. The average composition of coal gas is: Illuminants 4 per cent, carbon monoxide 8 per cent, hydrogen 50 per cent, methane 29 per cent, ethane 3 per cent, carbon dioxide 2 per cent, oxygen and nitrogen 4 per cent. 
The ammonia is made into ammonium sulphate. The tar may be used for road-making, as a waterproof material in building, and wherever pitch is applicable. More frequently it is separated by distillation, and other forms of treatment, and yields benzene $\mathrm{C}_{6} \mathrm{H}_{6}$, naphthalene $\mathrm{C}_{10} \mathrm{H}_{8}$, anthracene $\mathrm{C}_{14} \mathrm{H}_{10}$, phenol or carbolic acid $\mathrm{C}_{6} \mathrm{H}_{5} \mathrm{OH}$ and innumerable other valuable substances.

Coke Ovens. - The by-product coke oven is very much like the plant used for making coal gas. The difference is thai the heating is arranged so as to decompose the volatile matter and cause it to leave as much as possible of its carbon behind. The resulting gas is consequently poor in illuminants, but excellent as a fuel. The ammonia and tar are also diminished in amount,

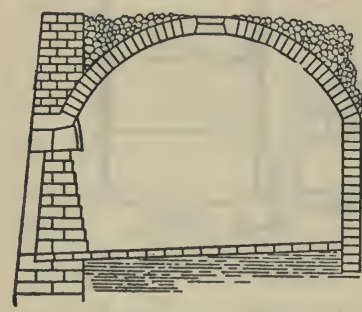

Fig. 106 but are still produced in paying quantities.

The beehive coke oven (Fig. 106), now largely discarded, is a primitive device of fire-brick, shaped like a beehive. It is simply filled with coal, part of which is allowed to burn with a limited supply of air. It yields 66 per cent coke, against 73 per cent from the by-product oven. All the volatile matter, with its gas, ammonia, and tar, escapes through an opening at the top, where it burns in a large flame and is wasted.

Properties and Uses of Coke.-Coke is a grey-black, hard material of spongy texture. It burns without flame, and gives a higher temperature than does coal, because no heat is used in vaporizing moisture and volatile matter. On account of these and other properties, it is used in immense quantities in reducing ores of iron and other metals, and in smaller amounts in electric furnace work and in making electric light carbons. 


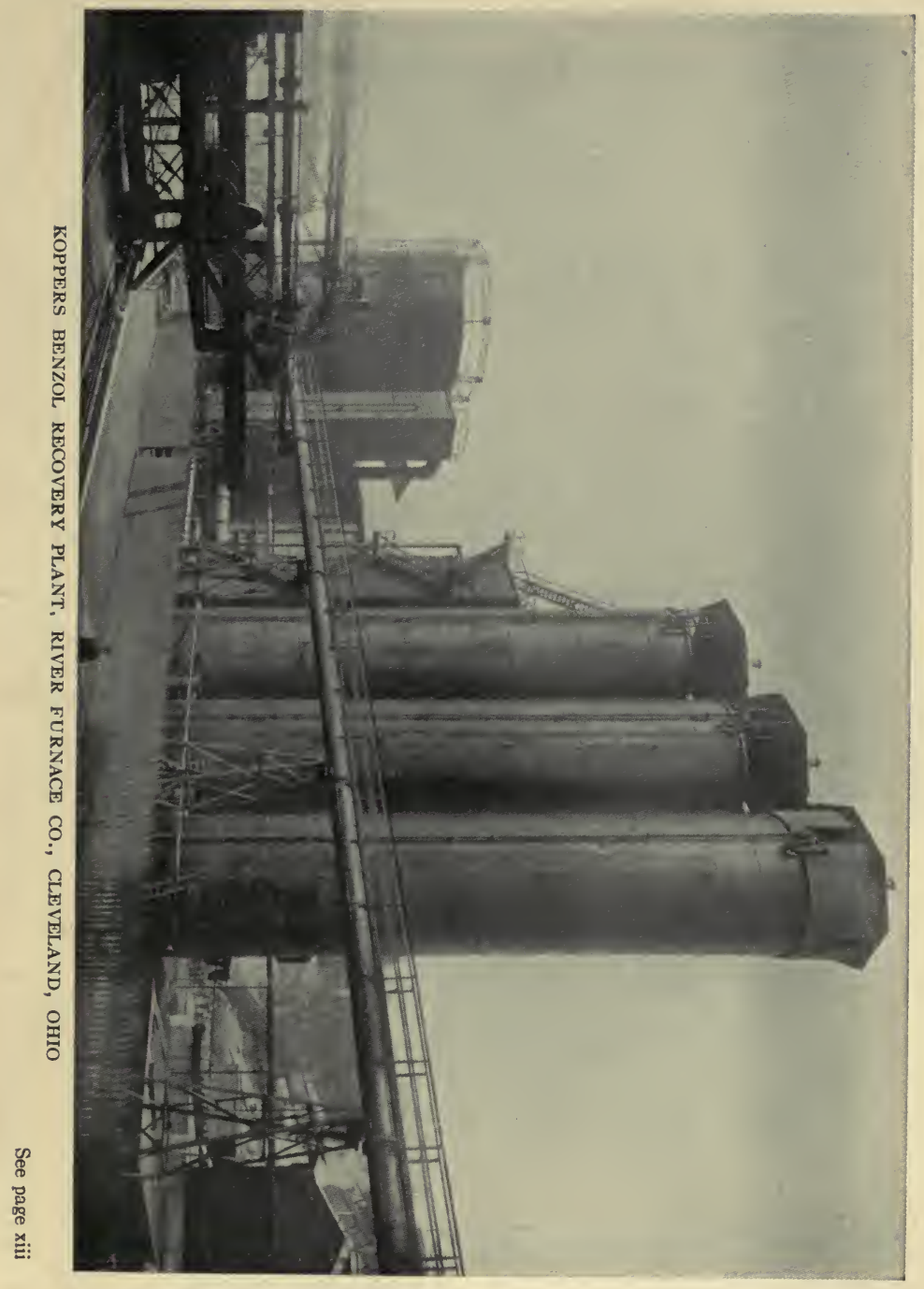




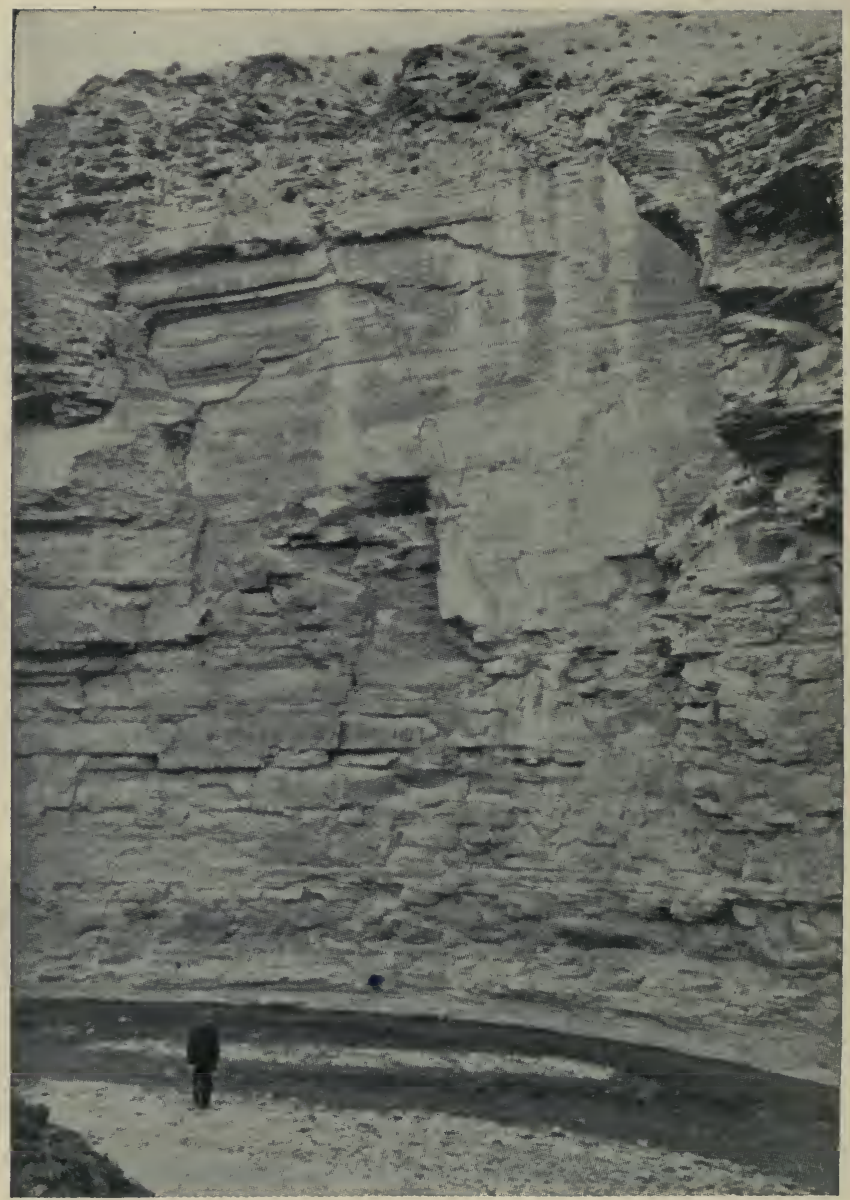

OIL SHALE CLIFF, UTAH

See page xiii 
Coal as Fuel.- The quality of a fuel coal, and whether it is worth its price, is learned by measuring its calorific (heating) power. A sample (about $1 \mathrm{~g}$.) is burned in a bomb calorimeter. This is a closed, metal vessel, filled with oxygen and submerged in a known weight of water. The coal is set on fire by a wire heated electrically and, after it has burned, the increase in temperature of the water is read off. Hence the heat in calories (p. 162) evolved by the burning of $1 \mathrm{~g}$. of coal is obtained. In engineering practice they use the number of British Thermal Units ( 1 B.T.U. $=$ heat required to raise 1 pound of water $1^{\circ} \mathrm{F}$.) developed by 1 pound of coal, and call the result the calorific power.

\begin{tabular}{|c|c|c|c|c|c|}
\hline 1 & $\begin{array}{l}\text { Calories } \\
\text { per. } 1 \mathrm{~g} .\end{array}$ & $\begin{array}{l}\text { B.T.U. } \\
\text { per } 1 \mathrm{lb} \text {. }\end{array}$ & I & $\begin{array}{l}\text { Calories } \\
\text { per } 1 \mathrm{~g} .\end{array}$ & $\begin{array}{l}\text { B.T.U. } \\
\text { per } 1 \mathrm{lb} \text {. }\end{array}$ \\
\hline $\begin{array}{l}\text { Hydrogen ...... } \\
\text { Charcoal (to } \mathrm{CO}_{2} \text { ) } \\
\text { Wood (seasoned) }\end{array}$ & $\begin{array}{r}28,800 \\
8,080 \\
4,750\end{array}$ & $\begin{array}{r}51,840 \\
14,544 \\
8,550\end{array}$ & $\begin{array}{l}\text { Bituminous coal } \\
\text { Anthracite....... } \\
\text { Petroleum...... }\end{array}$ & $\begin{array}{r}7,800 \\
8,000 \\
11,000\end{array}$ & $\begin{array}{l}14,040 \\
14,400 \\
19,800\end{array}$ \\
\hline
\end{tabular}

Knowing that 100 cal. will raise $1 \mathrm{~g}$. of water from $0^{\circ} \mathrm{C}$. to $100^{\circ} \mathrm{C}$., and $539 \mathrm{cal}$. more will convert it into steam, it is possible to calculate how much steam should be furnished by 100 kilog. of coal of known heat of combustion. If the quantity falls short, then the furnace, draft, or method of firing may be defective. Too much draft, for example, merely introduces additional, useless air to be heated. Thus, if the flue gas, upon analysis, is found to contain, not 12 per cent carbon dioxide (normal), but only 3 per cent, then for every ton of coal burned, 52 tons of unnecessary air have been raised to the temperature of the furnace. By chemical tests, made in ways like this, the efficiency of every device in the modern factory is (or ought to be) controlled. If the coal is bought without heed to its calorific value, and used without experimental checks, the boiler house alone may easily waste the whole profit earned by the rest of the plant. 
Source of the World's Energy. - The energy that does the world's work comes mainly from two sources, namely, water power and the combustion of wood, or of coal (which is fossil wood). The water comes from vapor, generated by the sun's heat, condensed as rain, and collected in lakes or reservoirs. The source of the energy of coal or wood is a little less obvious. When wood (which is largely cellulose) burns, it gives carbon dioxide, water, and heat. In fact, its combustion is represented by the equation given on p. 397 , when the equation is read backwards. Thus the sunlight, working through the machinery of the plant, takes the carbon dioxide and water, furnishes the energy (as light), and gives us wood and oxygen. And the wood and oxygen, when burned, give us back the original substances, and the equivalent of the original energy in the form of heat. Hence, our other main source of energy turns out to be the same as the first - the sun's rays - although the route by which the energy comes to us is a little less direct.

If, instead of burning the starch of the plant, we consume it as food, it goes through a series of changes instead of only one. But the end products are the same, namely, carbon dioxide and moisture issuing from our lungs, and heat and other forms of energy such as are developed in living organisms. Thus, whether we use our muscles, a steam engine, or a waterwheel to do work, sunlight is in each case the ultimate source of energy employed.

Exercises.-1. In fermentation, why does not carbon dioxide appear in bubbles at once?

2. How do we ascertain that acetic acid in aqueous solution is only slightly ionized? Give as many methods as possible.

3. (a) Why are charcoal and coke smokeless fuels? (b) Explain why bituminous coal burns with flames while anthracite does not.

4. Point out the analogies between the processes used in making coke and charcoal, and between their properties and uses. 
5. A gas of sp. gr. 0.43 (air =1) gives, on burning, 610 B.T.U. per cu. ft. How many B.T.U. is this per pound?

6. How many kilog. of steam, from water at $20^{\circ}$, can be made by burning 100 kilog. of coal, the heat of combustion of $1 \mathrm{~g}$. of which is 8500 cal.?

7. At 5 atmos. pressure and $152^{\circ} \mathrm{C}$, how many cubic meters will 190 kilog. of steam occupy?

8. What is " conservation?" What four industries or operations (or ways of performing operations) that are wasteful have been mentioned in this chapter (compare p. 424)? 


\section{CHAPTER XXXVII}

\section{ANIMAL LIFE AND ANIMAL PRODUCTS. FOODS}

ONLY a few of the more important points in the chemistry of animal life and growth can be touched upon here. In the same way, the chemistry of foods is presented merely in outline. Some food products, derived from plants, have been described in earlier chapters. The single animal product dealt with in any detail in the present chapter is soap. In connection with soap, the subject of colloids is also briefly discussed.

Composition of the Human Body. - The following gives, roughly, the percentage of each element in the human body.

\begin{tabular}{|c|c|c|c|c|}
\hline $\begin{array}{l}\mathrm{O} \ldots \ldots \ldots 65 \\
\mathrm{C} \ldots \ldots \ldots .18 \\
\mathrm{H} \ldots \ldots \ldots .10\end{array}$ & $\begin{array}{l}\mathrm{N} . . \\
\mathrm{Ca} \\
\mathrm{P} . .\end{array}$ & $\begin{array}{l}\mathrm{K} \ldots \ldots .0 .35 \\
\mathrm{~S} \ldots \ldots \ldots 0.25 \\
\mathrm{Na} \ldots \ldots .0 .15\end{array}$ & $\begin{array}{l}\mathrm{Cl} \ldots \ldots 0.15 \\
\mathrm{Mg} \ldots \ldots .0000 \\
\mathrm{Fe} \ldots \ldots .0 .004\end{array}$ & $\begin{array}{l}\text { trace } \\
\text { trace } \\
\text { trace }\end{array}$ \\
\hline
\end{tabular}

We have already learned that the calcium and phosphorus are chiefly in the bones (p. 412). The nitrogen, sulphur, and iron are in the proteins. The sodium is largely present as salts, in the fluids of the body. The potassium is in the soft tissues and in special secretions like milk. As in the plant, the carbon, hydrogen, and oxygen are in the form of carbohydrates, proteins, and fats, and there is also much water.

Certain amounts of all these elements leave the system daily. Water evaporates from the lungs and skin. The carbon leaves in large amounts, chiefly from the lungs as carbon dioxide, and also as excreted fats, proteins and carbohydrates. Much of the nitrogen is eliminated, chiefly as urea $\mathrm{CO}\left(\mathrm{NH}_{2}\right)_{2}$. The salts are removed in the same way. 
Animal Nutrition.- Since there is continual loss, there must be continual replacement. The animal resembles the plant, in the fact that it can take up into its system only dissolved material. It differs from the plant, however, in the fact that it is provided with a wonderful laboratory in which insoluble substances are changed into soluble ones. This is the digestive tract, consisting of the mouth, stomach, and intestine. The production of soluble substances of suitable composition is called digestion.

The processes are too complex for detailed treatment here. Only a few typical illustrations can be given. The principles concerned have all been used and illustrated already, and many of the facts are contained in previous chapters.

Foods. First, let us examine the table showing the percentage composition of the edible portion of several articles of food:

\begin{tabular}{|c|c|c|c|c|c|}
\hline Food material & Water & Protein & Fat & $\begin{array}{l}\text { Carbo- } \\
\text { hydrate }\end{array}$ & As \\
\hline Beef (lean). & 73.8 & 22.1 & 2.9 & & 1.2 \\
\hline Cod.. & 82.6 & 15.8 & 0.4 & & 1.2 \\
\hline Eggs. . & 73.7 & 14.8 & 10.5 & & 1.0 \\
\hline Milk*. & 87.0 & 3.3 & 4.0 & 5.0 & 0.7 \\
\hline Butter. . & 11.0 & 1.0 & 85.0 & & 3.0 \\
\hline Cheese (cheddar). & 27.4 & 27.7 & 36.8 & 4.1 & 4.0 \\
\hline Oatmeal......... & 7.3 & 16.1 & 7.2 & 67.5 & 1.9 \\
\hline flour & 11.9 & 13.3 & 1.5 & 72.7 & 0.6 \\
\hline (dried). & 12.6 & 22.5 & 1.8 & $59: 6$ & 3.5 \\
\hline ads. . . . . & 4.8 & 421.0 & 54.9 & 17.3 & 2.0 \\
\hline Maize (green corn). & 75.4 & 3.1 & 1.1 & 19.7 & 0.7 \\
\hline Potatoes.......... & 78.3 & 2.2 & 0.1 & 18.4 & 1.0 \\
\hline 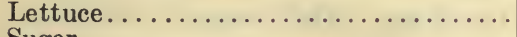 & 94.7 & 1.2 & 0.3 & 2.9 & 0.9 \\
\hline Sugar ........ & - & $\cdots$ & $\cdots$ & 100.0 & $\cdots$ \\
\hline
\end{tabular}

* The emulsified fat separates slowly as the cream; the protein (casein, colloidally suspended in the skim milk) is coagulated by rennet and constitutes cheese; the carbohydrates (lactose, a sugar) are then left in the water, along with inorganic salts.

We note, at once, that there is little more water in milk than in cod; that the animal foods, except milk (carrying lactose, p. 401), contain no carbohydrates; that potatoes and corn when dried 
are nearly all carbohydrate (starch); that lean beef when dry is nearly all protein; that some seeds (wheat and beans) contain almost no fat, some (oats) much more, and some (almonds and nuts) a very large amount; and that lettuce and other leaves are mainly water, with dissolved inorganic salts (valuable), contained in a light framework of cellulose (non-digestible).

Digestion of Starch. - The carbohydrates, in most foods which contain a large proportion of them, are mainly in the form of starch. The exceptions are milk, sweet fruits, and sugar itself. Starch is insoluble in water, and can not be directly absorbed. But we have seen (p. 401) that, when boiled with a dilute acid, it is hydrolyzed, giving glucose. When bread and potatoes are masticated, an enzyme (p. 417), named ptyalin, contained in the saliva (alkaline) turns a part of it, by hydrolysis, into a soluble sugar, maltose. Later, in the small intestine, amylopsin completes this process. Here also another enzyme, maltase, splits the maltose into glucose. The glucose then passes through the intestinal wall and so goes into the circulation, where most of it is oxidized.

The cooking of starch (baked bread, boiled potatoes, etc.) breaks up the grains and makes the mixing with the enzyme more perfect and the digestion more rapid and complete.

Baking Powders.- The purpose of the powder is to generate carbon dioxide in the dough. The bubbles of the gas are retained by the sticky gluten of the flour. They expand when the dough is baked, and give to it the open texture which, when the bread is eaten, facilitates access of the saliva to every particle.

Baking soda $\mathrm{NaHCO}_{3}$, if used alone, will give off, when heated, half the carbon dioxide it contains (p. 367). The sodium carbonate which remains in the bread, however, has an acrid taste. By its action on the gluten in the flour, it gives also a yellow color and an unpleasant odor. Finally, the carbonate of soda tends to 
neutralize the gastric juice (acid) of the stomach and so to interfere with digestion.

To obviate these difficulties sour milk (containing lactic acid) is sometimes used in making the dough. Occasionally vinegar (p. 420) is added. Most frequently a baking powder, containing an acid substance along with the soda, is employed. The acid substances contained in baking powders are alum (p. 469), acidphosphate of calcium or sodium, or potassium-hydrogen tartrate. The last, known commonly as cream of tartar $\mathrm{HKC}_{4} \mathrm{H}_{4} \mathrm{O}_{6}$, acts as follows:

$\mathrm{HKC}_{4} \mathrm{H}_{4} \mathrm{O}_{6}+\mathrm{NaHCO}_{3} \rightarrow \mathrm{NaKC}_{4} \mathrm{H}_{4} \mathrm{O}_{6}+\mathrm{H}_{2} \mathrm{CO}_{3} \rightarrow \mathrm{H}_{2} \mathrm{O}+\mathrm{CO}_{2}$.

The sodium-potassium tartrate which remains is better known under the name of Rochelle Salt.

It is important that the soda and the acid substance should be used in the correct proportions, which can be calculated from the equation. In commercial baking powders a little corn-flour is added, to keep the particles of the other compounds apart and prevent that gradual interaction which otherwise would be bound to occur. The acid substance should also be somewhat insoluble, so that, even when wet, it will not act upon the soda until ample time has been allowed for complete mixing with the dough.

Bakers' Bread.- The "raising" of bakers' bread is effected by adding yeast. The batch is " set" in a warm place for some hours to permit the yeast to propagate and to act upon the sugar in the flour. In this action, as we have seen (p. 417), carbon dioxide and alcohol are produced. A little sugar, molasses, or malt extract is added to the dough, to afford a larger supply of the sugar required for the production of the carbon dioxide.

The whites of eggs " raise" cake, without the presence of any soda, because of the expansion under heat of the bubbles of air entangled with the albumen when the eggs are "whipped." 
Fats and Oils. - The fats and oils found in the bodies of animals, or pressed from the seeds of plants, are composed mainly of various esters (p.349). As such, they are formed by the interaction of a tribasic alcohol, glycerine $\mathrm{C}_{3} \mathrm{H}_{5}(\mathrm{OH})_{3}$, with higher acids of the paraffin and olefine series of hydrocarbons, such as the saturated acids $\mathrm{C}_{15} \mathrm{H}_{31}$. $\mathrm{COOH}$ (palmitic acid) and $\mathrm{C}_{17} \mathrm{H}_{35} . \mathrm{COOH}$ (stearic acid), and the unsaturated acid $\mathrm{C}_{17} \mathrm{H}_{33}$. $\mathrm{COOH}$ (oleic acid). For example:

$$
\underbrace{\mathrm{C}_{3} \mathrm{H}_{5}(\mathrm{OH})_{3}}_{\text {glycerine }}+\underset{\text { palmitic acid }}{3 \mathrm{H}}\left(\underset{\left.\mathrm{COO} . \mathrm{C}_{15} \mathrm{H}_{31}\right)}{\mathrm{C}_{3} \mathrm{H}_{5}\left(\mathrm{COO}_{\text {glyceryl palmitate }} \mathrm{C}_{15} \mathrm{H}_{31}\right)_{3}}+3 \mathrm{H}_{2} \mathrm{O}\right.
$$

The glycerine esters of the saturated acids are solid at ordinary temperatures (fats), while those of the unsaturated acids are liquid (oils). Beef suet is a mixture of about three-fourths glyceryl palmitate (palmitin) $\mathrm{C}_{3} \mathrm{H}_{5}\left(\mathrm{CO}_{2} \mathrm{C}_{15} \mathrm{H}_{31}\right)_{3}$ and glyceryl stearate (stearin) $\mathrm{C}_{3} \mathrm{H}_{5}\left(\mathrm{CO}_{2} \mathrm{C}_{17} \mathrm{H}_{35}\right)_{3}$, and one-fourth glyceryl oleate (olein) $\mathrm{C}_{3} \mathrm{H}_{5}\left(\mathrm{CO}_{2} \mathrm{C}_{17} \mathrm{H}_{33}\right)_{3}$. Hog lard contains about 40 per cent of the former and 60 per cent of the latter, and is therefore softer. Butter includes the same esters, with about 14 per cent of water. When butter is dried, the remaining fat contains about 8 per cent of glyceryl butyrate (butyrin) $\mathrm{C}_{3} \mathrm{H}_{5}\left(\mathrm{CO}_{2} \mathrm{C}_{3} \mathrm{H}_{7}\right)_{3}$. Olive oil contains 75 per cent of olein. Cotton seed oil is similar in nature.

All these fats and oils contain, also, a small amount of the free acids. They must not be confused with mineral oils like petroleum, which are mixtures of hydrocarbons.

Oleomargarine is an artificial butter. It is made by straining melted beef fat and allowing it to stand at $24^{\circ}$. Much of the stearin crystallizes out and the remaining liquid (the "oleomargarine ") is pressed out and allowed to solidify. The solid is finally mixed with a little of some oil, to render it softer, and is churned with milk to impart the proper flavor. Although it lacks the butyrin, the product is similar in chemical nature to butter, and just as nutritious and wholesome. 
Hydrolysis of Fats and Oils. - The chief chemical property of the fats and oils, and in fact of all esters, is that each can be decomposed, or hydrolyzed, to give back the alcohol and acid from which it is derived. Thus, when ethyl acetate is boiled with water, it is slowly decomposed into ethyl alcohol and acetic acid:

$$
\mathrm{C}_{2} \mathrm{H}_{5}\left(\mathrm{CO}_{2} \mathrm{CH}_{3}\right)+\mathrm{H}_{2} \mathrm{O} \rightarrow \mathrm{C}_{2} \mathrm{H}_{5} \mathrm{OH}+\mathrm{HCO}_{2} \mathrm{CH}_{3} \text {. }
$$

In the case of the fats and oils, if water alone is used, they must be heated in a closed vessel, or autoclave, under pressure so as to secure a high temperature (about $200^{\circ}$ ):

$$
\underset{\text { palmitin }}{\mathrm{C}_{3} \mathrm{H}_{5}\left(\mathrm{CO}_{2} \mathrm{C}_{15} \mathrm{H}_{31}\right)_{3}}+\underset{\text { glycerine }}{3 \mathrm{H}_{2} \mathrm{O}} \rightarrow \underset{\text { palmitic acid }}{\mathrm{C}_{3} \mathrm{H}_{5}(\mathrm{OH})_{3}}+\underset{\mathrm{CO}_{2} \mathrm{C}_{15} \mathrm{H}_{31}}{3 \mathrm{HC}_{\text {g }}}
$$

When the mixture has cooled, the acid, which is insoluble in water, forms a solid cake, while the glycerine is dissolved in the water.

With water alone as a hydrolyzing agent, however, the reaction is slow and incomplete and, at the high temperatures which it is necessary to employ, some destruction of fatty matter is apt to occur. In the presence of dilute sulphuric acid as a catalyst, the hydrolysis can be carried out much more satisfactorily and rapidly, even at $100^{\circ}$. Sulpho-derivatives of the fatty acids are still more effective as catalysts, since they are freely soluble both in fats and oils and in water, and hence promote the miscibility of the two layers (Twitchell process).

When tallow (beef fat) is treated in this way, the solid is a mixture of palmitic, stearic, and oleic acids. The latter, being liquid, is pressed out, and the solid material is used with paraffin in making candles. The glycerine is separated from the water and used in making nitroglycerine (p. 481) and in medicine.

Hydrogenation of Oils. - The market value of solid fats is much higher than that of liquid oils. Many natural oils, indeed, possess disagreeable characteristics (taste, odor, etc.) which render them totally unsuitable for edible purposes. They may be converted into more appetizing edible fats; however, by 
hydrogenation. At $200^{\circ}$, in the presence of finely divided nickel as a catalyst, these unsaturated compounds take up hydrogen and become saturated, the oil hardening to a fat in the process.

Many substances which were formerly waste products, such as cotton-seed oil, are now treated in enormous quantites in this way. The hydrogenation of low grade oils (for example, fish oil) is also of great industrial importance in the manufacture of soap (see p. 438) and candles.

Digestion of Fats. - At body temperature, the fats and oils present in foods are all insoluble in water, and therefore cannot be directly absorbed into the system. But fats, if already emulsified (p. 110), as in milk, are hydrolyzed by a lipase (enzyme for fat) in the gastric juice of the stomach, and are decomposed into the acid and glycerine (p. 433). Fat in larger masses is hydrolyzed by lipases in the bile and here the acid (insoluble in water) is dissolved. The acid and the glycerine then diffuse through the intestinal wall and finally recombine to form fat in the blood. Some of this fat is deposited in the tissues and some is oxidized (giving muscular energy and heat).

Cooking (application of heat) does not affect the digestibility of fat. However, when fat is heated too strongly, the beginning of destructive distillation produces unsaturated compounds. These are intensely irritating to the digestive organs - as the way their vapors bring tears to the eyes would lead us to expect.

Digestion of Proteins. - The proteins contained in foods, of which the white of an egg (albumen) is a typical example, are not affected by saliva, but, when mixed with the gastric juice of the stomach, they are changed by the free hydrochloric acid it contains into syntonin. This in turn is hydrolyzed by the pepsin (enzyme), also contained in the gastric juice, into peptones which are soluble in water. These changes, only partly carried 
out in the stomach, are completed in the small intestine by the trypsin of the pancreatic juice, and the peptones (or amino-acids into which they are split) pass through the intestinal wall into the circulation. The casein of milk, being in colloidal suspension, is completely hydrolyzed to peptones in the stomach.

When heated, as in cooking, the proteins do not behave alike. Some, like albumen (white of egg) become coagulated, though probably not less digestible. The same is true of the blood proteins (hæmoglobin, etc.) of beef. On the other hand, the connective tissue of meat (chiefly collagen) is insoluble in cold water, but in hot water goes into colloidal suspension as gelatine. It is therefore softened by judicious roasting (under-done meat), provided the operation is not carried so far (over-done meat) that the water in the meat is largely evaporated.

Fuel Value. - While food is needed primarily to replace the material which is continually eliminated from the system, the organism also requires energy to maintain the routine motions of the heart, intestines, lungs, and other organs, and the normal muscular tension, as well as the movements of the muscles in walking and working. If the heat derived from routine changes is not sufficient to maintain the temperature $\left(37^{\circ} \mathrm{C}\right.$.) of the body, then additional food material is oxidized by the system for this specific purpose (compare p. 426). The values of foods are therefore conveniently estimated in terms of the heat they produce when burned - their fuel values.

The average fuel values, as measured in the calorimeter, with certain necessary corrections, and expressed, as is usual in this work, in large calories* per gram, are: Carbohydrates 4 Cal., fats 9 Cal., proteins 4 Cal. The fuel values per pound ( $=453.6$ g.) are 453.6 times greater: Carbohydrates $1800 \mathrm{Cal}$., fats $4080 \mathrm{Cal}$., proteins 1800 Cal.

* One large calorie (1 Cal.) is equal to one thousand small calories (1000 cal.), as hitherto defined (p. 162) and used. 
Normal Diet. - There is much uncertainty, as yet, in regard to the best choice of foods, in respect to the exact distribution in kind and quantity. We know, however, that life cannot be maintained on one kind (say, sugar or gelatine) alone. A mixed diet is necessary. In general, it appears that $100 \mathrm{~g}$. of proteins (giving $4 \times 100 \mathrm{Cal}$.) per day, and a sufficient amount of other foods to bring the total fuel value up to 2200 Cal. per day, is sufficient for a person leading a strictly sedentary life. For work involving physical exercise, larger values, up to about $3800 \mathrm{Cal}$., are required.

From the data given in the table (p. 429) the fuel value of 100 g. of each kind of food can easily be calculated.

Fuel Values and Prices of Foods. - If the current prices are considered, one can also readily calculate the fuel value obtainable for a given sum of money invested in each kind of food. Thus: lean beefsteak contains 22.1 per cent of protein, or 0.221 pounds per pound of meat. The fuel value of this protein is $0.221 \times 1800$, or 398 Cal. per pound.

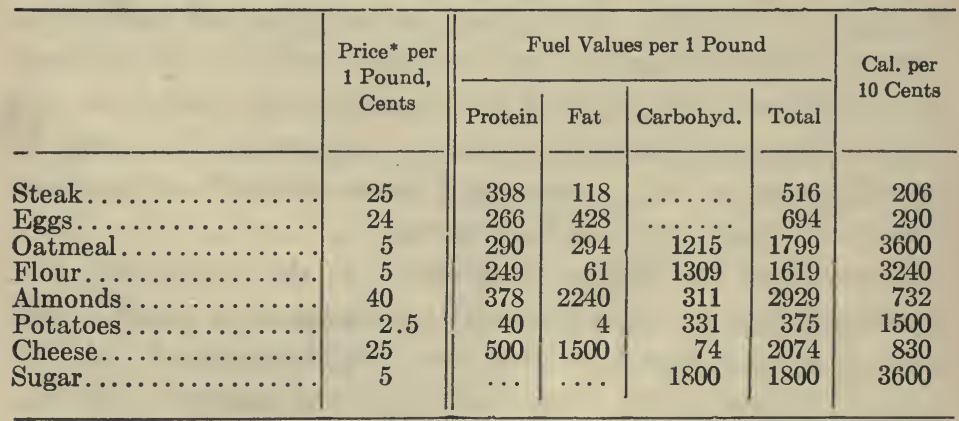

* The prices vary gr atly with the quality, the season of the year, the demand, the supply, etc.

Vitamins. - A diet may be carefully balanced with respect to carbohydrates, fats and proteins, and yet lead to malnutrition 
through deficiency in vitamins. These are substances present in minute quantities in most fresh or unsterilized foods, particularly in milk and green vegetables, without the aid of which certain parts of the animal mechanism either cease to develop or fail to perform their functions entirely. The three known types are:

(1) Fat-soluble vitamin A, plentiful in milk, butter, the yolks of eggs, cod-liver oil and the leaves of green plants, but not found in grains, sugars, or refined vegetable fats and oils. This vitamin is needed to promote the growth of children; its absence leads to rickets and a disease of the eye called xerophthalmia.

(2) Water-soluble vitamin B, plentiful in the outer hull of grains, beans, green leaves and fruit and yeast, but not in the kernels of grains, such as polished or milled rice. Deficiency of this vitamin in the diet leads to boils and skin eruptions, and in extreme instances to beriberi.

(3) Water-soluble vitamin C, plentiful in citrous fruits, tomatoes, cabbage, lettuce and other fresh fruits and vegetables. The value of this vitamin lies in the prevention and cure of scurvy. It is easily destroyed, except in the case of acid foods, by heating, drying or ageing. Since milk is neutral, infants fed entirely on pasteurized milk are almost sure to develop a mild case of scurvy unless the diet also includes a little orange juice or some other source of water-soluble vitamin C. Owing to the acid nature of tomatoes, even the canned product is rich in this vitamin. A knowledge of this fact was put to good use by the authorities of the British armies in Mesopotamia and Palestine during the war, when fresh fruits and vegetables were unobtainable.

Animal Products. - Many valuable products, apart from foods, are derived from animal life. The use of animal products in fertilizers (calcium phosphate from bones, nitrogen compounds from manure, etc.) has already been discussed (Chapter XXXV). 
When bones or dried blood are subjected to destructive distillation (compare p. 420), the residue consists of animal charcoal. The charcoal from bones (bone black) contains 90 per cent of mineral matter, largely calcium phosphate, and only 10 per cent of carbon. Animal charcoal, being a very active adsorbent (p. 421), is used in sugar refining.

The basis of wool, and of hair fibers in general, is a protein called keratin. The silk fiber is also of animal origin, but differs very widely from wool in its structure and properties. As spun by the silkworm in the preparation of its cocoon, it consists of two filaments composed of a protein called fibroin surrounded and cemented together by a gluey substance known as sericin or silkgum.

Glue, an impure form of gelatine (p. 435), is obtained from the skin and bones of animals by extraction with water under pressure. Leather is prepared from the hides by tanning (see p. 533).

Casein, a protein contained in milk and precipitated therefrom by dilute acids, has recently found many interesting applications. In the modified form of cheese, of course, it has long been of value as a food. Mixed with various alkalies it gives glues, cements and putties. It is used as an ingredient in paints, and for sizing or enameling paper. Paper bottles are made waterproof by impregnating them with casein and then exposing them to the vapor of formaldehyde (p. 348). Galalith (milk-stone) is a plastic, harder than celluloid (p. 480) and non-inflammable, made by the same hardening action of formaldehyde on casein.

Soap. - When fats are hydrolyzed by heating with a solution of caustic soda $\mathrm{NaOH}$, instead of water, the sodium salts of the acids are obtained. These sodium salts are known as soaps and the operation is called saponification:

$$
\underset{\text { palmitin }}{\mathrm{C}_{3} \mathrm{H}_{5}\left(\mathrm{CO}_{2} \mathrm{C}_{15} \mathrm{H}_{31}\right)_{3}}+\underset{\text { glycerine }}{3 \mathrm{NaOH}} \rightarrow \underset{\text { sodium palmitate }}{\mathrm{C}_{3} \mathrm{H}_{5}(\mathrm{OH})_{3}}+\underset{\text { (a soap) }}{3 \mathrm{Na}}\left(\mathrm{CO}_{2} \mathrm{C}_{15} \mathrm{H}_{31}\right)
$$


The sodium palmitate or other soap is soluble in the water. When common salt is added, however, the soap coagulates and separates into a floating layer which solidifies on cooling.

Soft soap is made with potassium hydroxide, and is composed of the potassium salts of the organic acids.

Manufacture of Soap.- Soap is made in large iron caldrons. These contain closed steam coils for heating, and pipes delivering live steam, when needed, for stirring the mass. The fat is mixed with caustic soda solution containing, at first, only about onefourth of the total amount of the base that the above equation requires. When, after heating and stirring, a uniform mixture has been produced, the rest of the alkali is added gradually and the heating is continued until the reaction appears to be complete. Salt is now dissolved in the mixture and the soap separates as a curd. The curd floats, leaving the "spent lye," containing the salt solution and much of the glycerine, in the lower layer. This process is called salting out. )

When the process stops at this point, the upper layer is known as curd soap, and may be dipped out and allowed to cool and solidify. Most "Marseilles" soaps are curd soaps made in this way. A large part of the imported "Castile" soaps are of the same kind.

Curd soaps contain salt, glycerine, adhering lye, and other impurities. To prepare a purer soap, the spent lye is run off, dilute brine is added to the soap, and the curd and brine are stirred up. When separation has again occurred, the brine is run off and the process repeated. Finally, some water is added, and steam is run in until the curd mixes completely with the water. When the solution stands, it " settles," that is, resolves itself once more into two distinct layers. The upper layer is called settled soap. The washing with brine and temporary dissolving in water remove the impurities, and hence settled soap is the purest variety. The greater part of the soap made in the United States 
and in Great Britain, and much of that made in other countries, is of this kind.

The qualities of soaps are varied by adding "fillers," such as sodium carbonate, borax, or sodium silicate. Soap powders are often made of ground soap mixed with sodium carbonate. Dyes and perfumes are sometimes added to soaps. Air bubbles are mixed with the soap, by beating, to give the floating varieties. Soap for scouring contains fine sand. Transparent soaps are made by dissolving the soap in alcohol, or by the addition of glycerine or sugar.

Chemical Properties of Soaps.- The soaps, being sodium salts, dissolve in water and have the usual properties of salts. Thus, when an acid is added, double decomposition takes place:

$$
\begin{aligned}
& \mathrm{Na}\left(\mathrm{CO}_{2} \mathrm{C}_{15} \mathrm{H}_{31}\right)+\mathrm{HCl} \rightarrow \mathrm{NaCl}+\mathrm{H}\left(\mathrm{CO}_{2} \mathrm{C}_{15} \mathrm{H}_{31}\right) \downarrow \text {. } \\
& \text { sodium palmitate palmitic acid }
\end{aligned}
$$

The acids, being insoluble, are thrown down as white precipitates.

When other salts are added, similar actions occur. Thus with calcium chloride solution, calcium palmitate is formed, and being insoluble, is precipitated:

$$
2 \mathrm{Na}\left(\mathrm{CO}_{2} \mathrm{C}_{15} \mathrm{H}_{31}\right)+\mathrm{CaCl}_{2} \rightarrow 2 \mathrm{NaCl}+\mathrm{Ca}\left(\mathrm{CO}_{2} \mathrm{C}_{15} \mathrm{H}_{31}\right)_{2} \downarrow \text {. }
$$

This action is important in connection with " hardness " in water (p. 389).

Colloidal Suspension.-We have seen (pp. 109, 400) that starch can be suspended in water in such a fine state of division that the liquid is transparent, and runs through a filter without leaving any solid on the paper. Yet this "suspension" lacks some of the characteristic properties of a solution.

The simplest proof, that this is a case of imitation solution, and not of true solution (molecular dispersion, p. 108), is obtained by examining the liquid with the ultra-microscope. A converg- 
ing beam of strong light is sent through the liquid horizontally (Fig. 107), and the illuminated place is viewed from above through a microscope. When the room is dark, and the only light comes from the horizontal beam, a colloidal solution shows minute points of light. A true solution - such as one of common salt or of alcohol - remains perfectly dark. The points of light are produced by myriads of suspended

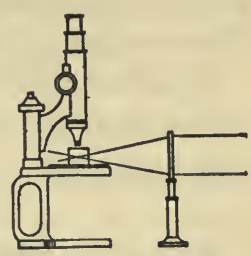

FIG. 107 particles, which although extremely minute, are of far larger than molecular dimensions. Solutions of soap, gelatine, and many dyes, blood serum, and innumerable other liquids contain such suspended particles.

The particles of a colloid (like starch), when viewed in this way, show a continual unordered, zig-zag movement (Brownian movement), which is more rapid the smaller the particles, and is due to the impacts of the molecules of the solvent.

Other Properties of Colloidal Suspensions.- The freezingpoint, boiling-point and vapor pressure of a liquid containing a colloid in suspension are all practically identical with those of the pure solvent. This is quite different from what we have seen to be the case with true solutions (p. 117), and indicates that the fraction of the "solute" particles present is substantially zero.

The unit particles of suspended material, indeed, are complex aggregates so much larger than the ultimate molecules into which true solutes are broken up that the number of them present, compared with the number of solvent molecules, is entirely negligible.

When a "solution" of a colloid is placed in a "diffusionshell" (test-tube shaped) of parchment, surrounded by pure water, none of the colloid escapes through the minute pores of the shell. Ordinary, non-colloidal solutes do escape, more or less rapidly (salt rapidly, sugar slowly, see p. 407). In this way a mixture of colloid and non-colloid (say, starch and salt) can be 
separated, if the water surrounding the shell is replaced by pure water at intervals until all the non-colloid has been removed. This method of separation is called dialysis.

Finally, matter in colloidal suspension may be coagulated (or flocculated) by addition of electrolytes or other colloids, and is then precipitated.

\section{Colloidal Matter in Soap Solutions. Explanation of} Salting Out.-Soaps, being salts of weak acids, are somewhat hydrolyzed in solution. Letting $\mathrm{R}$ stand for the hydrocarbon part of the acid radical:

$$
\mathrm{Na}\left(\mathrm{CO}_{2} \mathrm{R}\right)+\mathrm{H}_{2} \mathrm{O} \rightleftarrows \mathrm{H}\left(\mathrm{CO}_{2} \mathrm{R}\right)+\mathrm{NaOH} .
$$

The free acid $\mathrm{HCO}_{2} \mathrm{R}$ thus produced combines with the original salt $\mathrm{NaCO}_{2} \mathrm{R}$ to form an acid salt $\mathrm{NaH}\left(\mathrm{CO}_{2} \mathrm{R}\right)_{2}$. This acid salt is a colloidal substance, and exists in colloidal suspension in the soap solution, in equilibrium with the ions and molecules of the original salt and the $\mathrm{NaOH}$.

The capacity for being coagulated and precipitated, which is characteristic of colloidal matter, is shown very clearly by soap solutions. Most sodium salts will coagulate a soap solution and precipitate the soap as a curd. The acid salt $\mathrm{NaH}\left(\mathrm{CO}_{2} \mathrm{R}\right)_{2}$ seems to collect (adsorb) and carry down with it the most of the sodium hydroxide. As both of the substances on the right of the equation (above) are thus precipitated, the equilibrium is displaced to the right, and the precipitation becomes complete. This explains the process of "salting out" (p. 439) which plays so large a part in the manufacture of soap.

Causes of the Cleansing Action of Soap.- The chief use of soap solution is in removing grease and dirt from yarn, cloth, or clothing, and from woodwork and kitchen utensils. Soap solution has two more or less distinct properties, one of which enables it to remove oil or grease (viscous, insoluble liquids), and the 
other of which enables it to remove dust and dirt (largely minute, solid particles of carbon - soot). The former is its emulsifying power, the latter is probably connected with its nature as a colloidal suspension.

\section{The Emulsifying Action of Soap. How Soap Removes} Grease.-When an insoluble oil, such as kerosene or lubricating oil, is shaken with water it is divided into minute droplets separated by water from one another. When the shaking ceases, however, the droplets begin to run together and soon the oil and water have separated once more into two layers of transparent liquid. When very dilute soap solution and oil are shaken together, however, the droplets do not run together, but remain permanently suspended. The mixture is opaque and more or less viscous. Such a permanent mixture of two insoluble liquids is called an emulsion (compare p. 110).

Soap solution, when rubbed on oily or greasy goods, emulsifies the grease, converts it into droplets, surrounded by soap solution and separated from the cloth, and so permits it to be washed off.

In mayonnaise dressing, which is a thick, almost solid, permanent emulsion, the olive oil is emulsified by the colloidal matter of the yolks of the eggs which have been dissolved in the vinegar.

How Soap Removes Dirt.- When a solution containing colloidal substances, such as many dyestuffs and organic coloring matters, is shaken with finely pulverized charcoal, the colloidal substance adheres to the surface of the powder (is adsorbed) and the liquid is consequently decolorized (see p. 422).

When dilute soap solution is shaken with infusorial earth and the mixture is filtered, the clear liquid is found to have been deprived of the soap. The soap is evidently precipitated (adsorbed) on the surface of the particles of the solid.

Clearly there is a tendency to cohesion between the colloid in a soap solution and the particles of a fine powder. When there is 
much of the powder, and little of the soap in solution, the powder takes the soap out of the solution. When, however, there is much of the colloid in the form of soap solution, and little of the solid, and that very finely divided, the same tendency to adsorption exists, only, in this case, the colloidal particles carry off the powder. In short, the dirt is removed by adsorption into the solution.

Possible Objections to the Foregoing Explanation.- Formerly soap solution was supposed to remove grease (and soot?) because of its slight alkaline reaction, due to hydrolysis. This explanation must be given up because: (1) an alkali so dilute that it exists in equilibrium with the free fatty acid, can not possibly saponify the ester contained in a grease spot. (2) Pure alkali of the same concentration (or stronger) has no more emulsifying power than water. Such an alkaline solution will indeed emulsify an animal or vegetable oil (cod-liver oil, cotton oil, castor oil), but it does so by interacting with the free fatty acid always present in such oil (p. 432) and forming therefrom a soap. Such an alkaline solution does not emulsify kerosene, although soap solution does. The emulsifying agency is always a soap. (3) Very dilute alkali has no more effect upon soot than has water - but soap solution takes clean (greaseless) soot instantly into permanent suspension.

The power of forming an emulsion depends, theoretically, upon the abnormally low surface tension of dilute soap solution. Very dilute alkali has the same high surface tension as has pure water.

Exercises. - 1. Make equations for the formation: (a) of maltose from starch; (b) of glucose from maltose.

2. Make a connected statement showing the stages in the digestion of milk.

3. Why does fat appropriately form a larger proportion of the diet in the Arctic regions than elsewhere?

4. Give the weights of carbohydrate, protein, and fat which 
would supply a menu, such that the total food value was 3000 Cal., and that $100 \mathrm{~g}$. of protein was included, and the remaining fuel value was divided equally between carbohydrates and fats.

5. Calculate the calorific value of 1 kilog. of: (a) wheat flour, (b) oatmeal.

6. (a) In what proportions by weight should baking soda and cream of tartar be used in raising bread? (b) What is the objection to using too large a proportion of baking soda? (c) Why must baking powder be kept in a dry, cool place?

7. Why does vinegar liberate carbon dioxide from baking soda? Make the equation for the action.

8. Make equations for the formation of: (a) ethyl formate; (b) glyceryl formate; (c) ethyl stearate.

9. Make equations for the action of superheated water on: (a) stearin, and (b) olein; and for the action of caustic soda on: (c) stearin, and (d) ethyl acetate.

10. Summarize the facts which show soaps to be salts.

11. (a) Write the full equation for the hydrolysis of sodium palmitate. (b) What reaction (acid or alkaline) should soap solution possess, and why (p. 369)?

12. Why does "French dressing" (vinegar, salt, and olive oil) give an emulsion, which is much less durable than mayonnaise dressing? 


\section{CHAPTER XXXVIII}

\section{MAGNESIUM AND ZINC. IONIC EQUILIBRIA}

We shall now return to a consideration of the metallic elements. We can most easily remember magnesium and zinc by the facts that they are silver-white metals with a markedly crystalline structure, and that they displace hydrogen from dilute acids. In these respects they resemble aluminium, but the latter is trivalent in all its compounds, while the present two elements are bivalent exclusively (see Periodic System).

\section{Magnesium $\mathrm{Mg}$}

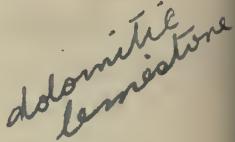

Occurrence.-Magnesium carbonate is found in dolomite $\mathrm{CaCO}_{3}, \mathrm{MgCO}_{3}$, a common rock, and in small amounts as magnesite $\mathrm{MgCO}_{3}$. The sulphate and chloride are found at Stassfurt. Several natural silicates of magnesium, such as meerschaum, asbestos, talc or soapstone, and olivine, are familiar minerals.

Asbestos, a fibrous material, is used in making fireproof cloth and cardboard. Soapstone is made into sinks and table tops for use, for example, in laboratories.

The Metal Magnesium. - The metal is made by electrolyzing a molten mixture of magnesium, potassium, and sodium chlorides. A carbon rod forms the anode, and the iron crucible the cathode, on which the metal collects in globules. The mass, when cold, is broken up and the metal is recast in bars. The metal can be drawn, through a die, into ribbon or wire.

Magnesium rusts in the air, gradually crumbling to a white powder of a basic magnesium carbonate. It burns in air, with a brilliant white light, producing a mixture of the oxide $\mathrm{MgO}$ and nitride $\mathrm{Mg}_{3} \mathrm{~N}_{2}$ (see argon, p. 297). Magnesium filings, mixed 
with potassium chlorate, give flash-light powder. Signal lights are made of shellac, barium nitrate and magnesium powder.

Oxide $\mathrm{MgO}$ and Hydroxide $\mathrm{Mg}(\mathrm{OH})_{2}$. - The oxide is made by heating magnesium carbonate, and is therefore called calcined magnesia. Being very infusible ("refractory") it is used in lining electric furnaces. On account of its poor heat conductance, it is also employed very extensively for insulating pipes and boilers, and so reducing heat losses ( $85 \%$ Magnesia). It combines slowly with water, giving the hydroxide $\mathrm{Mg}(\mathrm{OH})_{2}$, which with water gives a mortar that hardens under the action of the carbon dioxide of the air (see mortar, p. 386). The oxide is basic, and with acids gives salts by double decomposition.

Magnesium hydroxide $\mathrm{Mg}(\mathrm{OH})_{2}$, being insoluble, is easily precipitated by adding sodium hydroxide to a solution of a salt of magnesium. When moistened and mixed with a little magnesium chloride, it sets to a hard basic chloride of variable composition. The mixture, to which sawdust is sometimes added, is used as a plaster in house decoration.

Salts of Magnesium. - Magnesium carbonate $\mathrm{MgCO}_{8}$ is found in nature. That made by precipitation is a basic carbonate $3 \mathrm{MgCO}_{3}, \mathrm{Mg}(\mathrm{OH})_{2}$, magnesia alba, which is used in tooth powder and for polishing silver.

Magnesium sulphate $\mathrm{MgSO}_{4}$ is commonly sold as the heptahydrate, $\mathrm{MgSO}_{4}, 7 \mathrm{H}_{2} \mathrm{O}$, Epsom salt. It is found in the salt deposits and in many aperient mineral waters. Thus Hunyadi water contains little beside $47 \mathrm{~g}$. Epsom salt and $52 \mathrm{~g}$. sodium sulphate $\left(\mathrm{Na}_{2} \mathrm{SO}_{4}, 10 \mathrm{H}_{2} \mathrm{O}\right)$ and $1 \mathrm{~g}$. sodium bicarbonate per liter. The salt is used for loading cotton goods and as a purgative.

Magnesium chloride $\mathrm{MgCl}_{2}$ is found in sea water and in some natural waters. It is very deliquescent (p. 118) and, being present in impure table salt, causes the latter to cake or even become moist in damp weather. Addition of a very little sodium bicarbonate 
to the salt remedies this difficulty. Magnesium chloride is a very objectionable form of hardness in water, because hot water partially hydrolyzes the salt and liberates hydrochloric acid, which attacks and corrodes the iron of the boiler and tubes. Hence sea water can not be used in marine boilers.

\section{Zinc Zn}

Occurrence and Manufacture.-Zine is found as zinc blende $\mathrm{ZnS}$ (in large amounts in Missouri) and smithsonite $\mathrm{ZnCO}_{3}$ (Spain and U. S.).

In the case of the carbonate ore, the oxide $\mathrm{ZnO}$ is first obtained by heating. When zinc blende $\mathrm{ZnS}$ is the ore, it is crushed and pulverized, and then roasted (p. 258) to remove the sulphur and leave the oxide:

$$
2 \mathrm{ZnS}+3 \mathrm{O}_{2} \rightarrow 2 \mathrm{ZnO}+2 \mathrm{SO}_{2} .
$$

The ore is fed in at the top of a huge, box-like furnace (Fig. 108, diagrammatic) through which rush the flames and heated gases

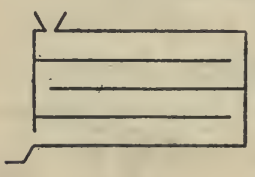

FIg. 108 from fuel gas burning with an excess of air. The ore is turned over and gradually displaced forward by moving rakes until, at the end, it drops to the next level. Here it is raked in the opposite direction, until it falls to the third level. The ore collects at the bottom fully oxidized, while the sulphur dioxide in the gases is made into sulphuric acid. The oxide from either ore is then reduced by heating with powdered coal:

$$
\mathrm{ZnO}+\mathrm{C} \rightarrow \mathrm{Zn}+\mathrm{CO} .
$$

This treatment of zinc ores should be carefully considered. Since ores of most metals consist of the carbonate, sulphide, or oxide of the metal, these steps are common to most metallurgical processes. In the case of other metals, only the forms of the furnaces and other details vary. 
In the case of zinc, because it is a volatile metal, the heating of the mixture of oxide and coal is conducted in retorts, from which the metal issues as vapor. The mixture is placed in fire-clay cylinders (4 to $5 \mathrm{ft}$. long), which are arranged in several tiers in an oblong, gas-heated furnace (Fig. 109). A fire-clay receiver is

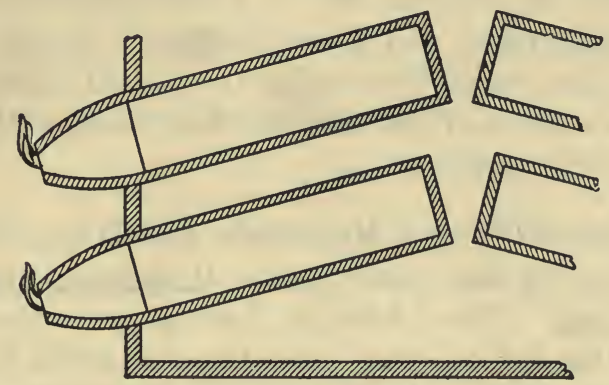

FIG. 109

luted on with clay. The carbon monoxide burns with a blue flame at the nozzle of each receiver, while the zinc condenses to liquid within it. From time to time the liquid metal is raked out into a traveling iron pot, from which it is poured into moulds.

Properties and Uses. - The metal is silvery and crystalline. At 120 to $150^{\circ}$ it can be rolled into sheets between hot rollers, at 200 to $300^{\circ}$ it becomes brittle, at $419^{\circ}$ it melts, and at $950^{\circ}$ it boils. The density of the vapor shows it to be monatomic. Zinc vapor burns with a bluish flame, giving $\mathrm{ZnO}$. In air the metal does not rust, being protected by a non-porous coating of a basic carbonate which adheres closely to the surface.

Sheet zinc is used for gutters and cornices. Iron is coated (galvanized) with zinc by thorough cleaning with dilute sulphuric acrd or the sand blast, and dipping in melted zinc. Iron netting, corrugated iron for sheds and roofing, and iron gutters, tanks, and pipes are coated with zinc, either in this way, or by electroplating (see p. 515). Sherardized iron is made by covering the article

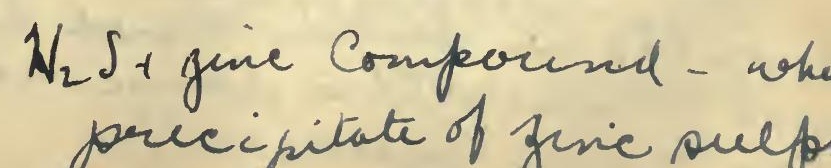


with zinc dust and baking it. The zinc protects the iron, primarily because it excludes the air from the surface, and secondarily because, even when the coating is broken, the zinc, being the more active metal of the two (p. 54), is rusted first. Zinc is used also in extracting silver from crude lead (p. 519), as the active metal (anode) in batteries, and in several alloys (e.g. Babbitt's metal, p. 326, brass, and German silver). In the laboratory granulated zinc, made by pouring the melted metal in a thin stream into water, and zinc dust (impure, contains $\mathrm{ZnO}$ ), are the forms commonly employed.

Zinc Oxide $\mathrm{ZnO}$ and Hydroxide $\mathrm{Zn}(\mathrm{OH})_{2}$. - The oxide is made by burning zinc vapor in air. It is yellow while hot, and white when cold. Mixed with oil, it is used as a paint (Chinese white). It has less covering power than has white lead paint (4 coats of the former equal 3 of the latter), but it does not darken from exposure to hydrogen sulphide in the air ( $\mathrm{ZnS}$ is white, $\mathrm{PbS}$

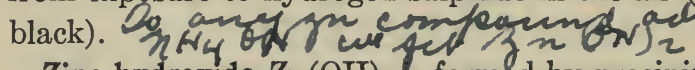

Zinc hydroxide $\mathrm{Zn}(\mathrm{OH})_{2}$ Is formed by precipitation. Both the oxide and hydroxide are. weakly basic, and give salts with acids. But with respect to strong bases they are weakly qcidic, dissolving for example in excess 'of sodium hydroxide, giving sodium zincate $\mathrm{Na} \cdot \mathrm{HZnO}_{2}$.

\section{Ineatain of wood - verevents decay.}

Zinc Chloride $\mathrm{ZnCl}_{2}$. - The chloride is formed in the action of zinc or zinc oxide on hydrochloric acid. It is a white, deliquescent solid. Its aqueous solution gelatinizes cellulose and dissolves it (p. 398), and thus is used in parchmentizing paper and in impregnating wood to prevent decay. The aqueous solution is acid (hydrolysis) and is used for cleaning the surface of metals before soldering. Solder "runs" on a hot brass, copper, or lead surface, provided the latter is clean, and adheres perfectly when cold. But it does not dissolve oxides, or melt them, and therefore cannot even reach the surface of the metal, much less adhere to it, if the slightest tarnish is present. 
Other Compounds. - Zinc sulphate, $\mathrm{ZnSO}_{4}, 7 \mathrm{H}_{2} \mathrm{O}$, is made by the action of sulphuric acid on zinc or zinc oxide. It is used in preserving hides and as a mordant in cotton printing (see dyeing, p. 475). Zinc sulphide $\mathrm{ZnS}$ (white) is precipitated practically completely when a solution of ammonium sulphide $\left(\mathrm{NH}_{4}\right)_{2} \mathrm{~S}$ is added to a salt of zinc:

$$
\mathrm{ZnSO}_{4}+\left(\mathrm{NH}_{4}\right)_{2} \mathrm{~S} \rightarrow \mathrm{ZnS} \downarrow+\left(\mathrm{NH}_{4}\right)_{2} \mathrm{SO}_{4} .
$$

When hydrogen sulphide is employed instead

$$
\mathrm{ZnSO}_{4}+\mathrm{H}_{2} \mathrm{~S} \rightleftarrows \mathrm{ZnS}+\mathrm{H}_{2} \mathrm{SO}_{4}
$$

only a small part of the zinc is precipitated. The cause of this difference will come up for discussion in a subsequent section ( $p$. 459).

A mixture of zinc sulphide and barium sulphate $\mathrm{BaSO}_{4}$, prepared in a special way, is called lithopone. Used as a white pigment, it has greater covering power than has white lead and is, besides, non-poisonous.

Cadmium.- Aside from the rare mineral greenockite CdS, this element is found only in small amounts (about 0.5 per cent), as carbonate and sulphide, in the corresponding ores of zinc. During the reduction, being more volatile than zinc, it distils over first (b.-p. $778^{\circ}$ ). The metal is white, and is much more malleable than is zinc. It melts at $320^{\circ}$.

It displaces hydrogen from dilute acids, but is itself displaced from solutions of its compounds by zinc, since it is less electropositive. It is used in making fusible alloys.

\section{IONIC EQUILIBRIA}

In view of the importance of ionic actions in the chemistry of the metals, we must now consider more closely the subject of ionic equilibria. The whole basis for this exact consideration has already been supplied, and only more specific application of the

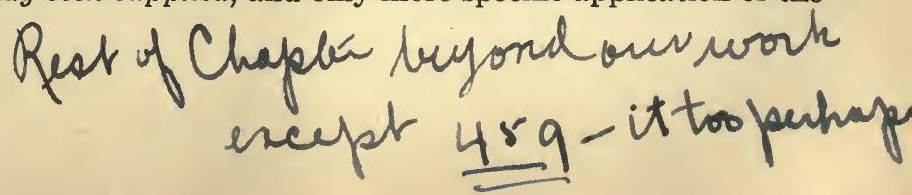


principles is demanded. The basis referred to, which should now be re-read as a preliminary to what follows, is contained in the discussion of chemical equilibrium in general (pp. 230-241). One important point may be briefly recapitulated here, as an aid to the reader.

Equilibrium in Reversible Reactions.-We saw (p. 237) that the point of equilibrium in the dissociation of phosphorus pentachloride:

$$
\mathrm{PCl}_{5} \rightleftharpoons \mathrm{PCl}_{3}+\mathrm{Cl}_{2}
$$

is dependent upon the molecular concentrations of the reacting substances, and may be represented by the equation:

$$
\frac{\left[\mathrm{PCl}_{3}\right] \cdot\left[\mathrm{Cl}_{2}\right]}{\left[\mathrm{PCl}_{5}\right]}=\mathrm{K} \text {. }
$$

In this equation the quantities within the brackets represent the equilibrium concentrations of the respective substances, and $\mathrm{K}$ is the equilibrium constant of the reversible reaction. However much we may vary the molecular concentration of any one substance at any fixed temperature (say, by adding excess of $\mathrm{Cl}_{2}$ to the gaseous mixture), the only effect will be a re-adjustment of the equilibrium point such that the final ratio $\left[\mathrm{PCl}_{3}\right] \cdot\left[\mathrm{Cl}_{2}\right] /$ $\left[\mathrm{PCl}_{5}\right]$ is unchanged. In other words, the equilibrium constant $\mathrm{K}$ is a fixed quantity.

Application to Ionization.- Exactly the same principles can be applied to the ionic dissociation of an electrolyte. The behavior of acetic acid, a weak, slightly ionized acid, will serve as an illustration.

In normal solution (60 g. in 11.) acetic acid is only 0.004 ionized (p. 190), so that, in the equation for the equilibrium,

$$
\text { (0.996) } \mathrm{HC}_{2} \mathrm{H}_{3} \mathrm{O}_{2} \leftrightarrows \mathrm{H}^{+}(0.004)+\mathrm{C}_{2} \mathrm{H}_{3} \mathrm{O}_{2}^{-}(0.004),
$$

the relative proportions are as shown by the numbers in parentheses. If the whole of the acid (60 g.) were ionized, there would 
be $1 \mathrm{~g}$. of hydrogen-ion per liter. Yet, even in the much smaller concentration actually present $(0.004 \mathrm{~g}$. per liter), the acid taste of the $\mathrm{H}^{+}$and its effect upon indicators can be distinctly recognized. If, now, solid sodium acetate is dissolved in the solution, the liquid no longer gives an acid reaction with one of the less delicate indicators, like methyl orange (an organic dye which turns pink in the presence of a fair amount of hydrogen-ion). The explanation is simple. Sodium acetate is highly ionized. It gives, therefore, a large concentration of acetate-ion to a liquid formerly containing very little. This causes a greatly increased union of the $\mathrm{H}^{+}$ ions and $\mathrm{C}_{2} \mathrm{H}_{3} \mathrm{O}_{2}{ }^{-}$ions to occur, and the former, being already very few in number, disappear almost entirely. Hence the solution becomes, to all intents and purposes, neutral. There is no less acetic acid present than before, but the concentration of hydrogen-ion is very much smaller.

Formulation and Quantitative Treatment of the Case of Excess of One Ion.- If $\left[\mathrm{H}^{+}\right]$and $\left[\mathrm{C}_{2} \mathrm{H}_{3} \mathrm{O}_{2}^{-}\right]$represent the molecular concentrations of hydrogen-ion and acetate-ion, respectively, and $\left[\mathrm{HC}_{2} \mathrm{H}_{3} \mathrm{O}_{2}\right]$ that of the acetic acid molecules at equilibrium, then:

$$
\frac{\left[\mathrm{H}^{+}\right] \times\left[\mathrm{C}_{2} \mathrm{H}_{3} \mathrm{O}_{2}^{-}\right]}{\left[\mathrm{HC}_{2} \mathrm{H}_{3} \mathrm{O}_{2}\right]}=\mathrm{K}
$$

The value of the ionization constant $\mathrm{K}$ is unchanged, whether the concentration of the solution of acetic acid is great or small, and even when another substance with a common ion is present. In the latter case, $\left[\mathrm{C}_{2} \mathrm{H}_{3} \mathrm{O}_{2}^{-}\right]$and $\left[\mathrm{H}^{+}\right]$stand for the whole concentrations of each of these ionic substances from both souroes.

Now, in normal acetic acid $\left[\mathrm{H}^{+}\right] \doteq 0.004,\left[\mathrm{C}_{2} \mathrm{H}_{3} \mathrm{O}_{2}{ }^{-}\right]=0.004$ (for the number of each kind of ions is the same), and $\left[\mathrm{HC}_{2} \mathrm{H}_{3} \mathrm{O}_{2}\right]=$ 0.996 , practically 1 . Substituting in the formula:

$$
\frac{0.004 \times 0.004}{1}=K\left(=0.0_{4} 16\right) \text {. }
$$


When, however, sodium acetate is dissolved in the liquid until the solution is normal in respect to this substance also, which is 0.528 ionized in normal solution, the following additional equilibrium has to be considered:

$$
\text { (0.472) } \mathrm{NaC}_{2} \mathrm{H}_{3} \mathrm{O}_{2} \rightleftarrows \mathrm{Na}^{+}(0.528)+\mathrm{C}_{2} \mathrm{H}_{3} \mathrm{O}_{2}^{-} \text {(0.528). }
$$

The concentration of acetate-ion from this source is 0.528 , so that, in the mixture of acid and salt, the concentration of acetate-ion $\left[\mathrm{C}_{2} \mathrm{H}_{3} \mathrm{O}_{2}^{-}\right]$will be $0.528+0.004=0.532$, or 133 times larger than in the acid alone. Hence, in order that the ionization constant $\mathrm{K}$ may recover, as it must, its original value, $\left[\mathrm{H}^{+}\right]$must be diminished to something like $\frac{1}{13}$ of its former magnitude. That is, $\left[\mathrm{H}^{+}\right]$will become equal to about 0.00003 ,

$$
\frac{0.00003 \times 0.532}{1}=\mathrm{K}\left(=0.0_{4} 16\right)
$$

the rest of the hydrogen-ion uniting with a corresponding amount of the acetate-ion to form molecular acetic acid. The concentration of this increases only from 0.996 to 0.99997 ; in other words, so much is already present that its concentration is practically unchanged. The chief effect of adding this amount of sodium acetate therefore is, as we have seen, to reduce the concentration of the hydrogen-ion below the amount which can be detected by use of an indicator like methyl-orange.

This effect is of course reciprocal, and the ionization of the sodium acetate will be reduced also. But the acetate-ion furnished by the acetic acid is relatively so small in amount that the effect it produces on the ionization of the salt is imperceptible.

Although, therefore, acetate-ion and hydrogen-ion must disappear in equivalent quantities, for they unite, there is, however, so much of the former that the loss it sustains goes unremarked, while there is so little of the latter that almost none of it remains. When substances of more nearly equal degrees of ionization are 
used, both effects are relatively inconspicuous. It is altogether impossible, for example, to reduce the concentration of the hydrogen-ion given by an active acid like hydrochloric acid, by addition of a salt containing a common ion, like sodium chloride, below the limit at which indicators are affected, for there is no way of introducing the enormous concentration of the other ion which the theory demands.

With more crude means of observation than indicators afford, effects like this last, however, may sometimes be rendered visible. This is the case with cupric bromide solution, to which potassium bromide is added. The blue of the cupric-ion disappears from view, while much cupric-ion is still present, because the brown color of the molecular cupric bromide covers it up completely.

The color changes discussed on p. 235 in connection with the reaction $\mathrm{FeCl}_{3}+3 \mathrm{NH}_{4} \cdot \mathrm{CHS} \rightleftharpoons \mathrm{Fe}(\mathrm{CNS})_{3}+3 \mathrm{NH}_{4} \mathrm{Cl}$ also illustrate this point. The red color is due entirely to undissociated ferric thiocyanate. The reader should re-examine this equilibrium carefully, writing the equation in full ionic form. He will then be in a position to understand why the addition of any ferric salt or of any thiocyanate to a given mixture will deepen the red color, while the addition of any chloride (except ferrio chloride) or any ammonium salt (except ammonium thiocyanate) will tend to lighten it.

Special Case of Saturated Solutions. - The commonest as well as the most interesting application of the conceptions developed above is met with in connection with saturated solutions, especially those of relatively insoluble substances.

The situation in a system consisting of the saturated solution and excess of the solute has been discussed already (read p. 120). In the case of potassium chlorate, for example, we have the following scheme of equilibria:

$\mathrm{KClO}_{3}$ (solid) $\rightleftarrows \mathrm{KClO}_{3}$ (dslvd.) $\rightleftarrows \mathrm{K}^{+}+\mathrm{ClO}_{3}^{-}$. 
If we apply to the two reversible reactions represented in this scheme the principles which have been developed above, we obtain the two equations:

$$
\begin{gathered}
\frac{\left[\mathrm{KClO}_{3} \text { (dissolved) }\right]}{\left[\mathrm{KClO}_{3}(\text { solid })\right]}=\mathrm{K}_{1} \\
\frac{\left[\mathrm{K}^{+}\right] \cdot[\mathrm{Cl}-]}{\left[\mathrm{KClO}_{3} \text { (dissolved) }\right]}=\mathrm{K}_{2}
\end{gathered}
$$

where $K_{1}$ and $K_{2}$ are the equilibrium constants of the two reactions. By multiplying equation (1) by equation (2), we obtain a third relationship:

$$
\frac{\left[\mathrm{K}^{+}\right]\left[\mathrm{ClO}_{3}^{-}\right]}{\left[\mathrm{KClO}_{3} \text { (solid) }\right]}=\mathrm{K}_{1} \cdot \mathrm{K}_{2}
$$

Now $\left[\mathrm{KClO}_{3}\right.$ (solid)], the concentration of $\mathrm{KClO}_{3}$ in the solid state, is invariable at any fixed temperature. Equation (3) consequently reduces to the form:

$$
\left[\mathrm{K}^{+}\right] \cdot\left[\mathrm{ClO}_{3}^{-}\right]=\mathrm{K}_{1} \mathrm{~K}_{2} \cdot\left[\mathrm{KClO}_{3} \text { (solid) }\right]=\text { a constant quantity. }
$$

In other words, in a saturated solution, the product of the molar concentrations of the ions is a constant. This product is called the solubility product of the substance. The law of the constancy of the ion-product in a saturated solution is one of the most useful of the principles of chemistry. It enables us to explain all the varied phenomena of precipitation and of the solution of precipitates in a consistent manner, as will be seen below. Upon the accuracy of the law of the constancy of the solubility product our whole system of quantitative analysis is based. It is of importance therefore to mention the fact that, although experimental test has shown that both equation (1) and equation (2), by means of which the law was derived, are grossly inaccurate for all strong electrolytes (neither $K_{1}$ nor $K_{2}$ remaining by any means constant as conditions are varied), yet equation (3), the law itself, always approximates very closely to the truth. 


\section{Illustration of the Principle of Solubility-Product Con-} stancy. - When, to a saturated solution of one of the less soluble salts, a concentrated solution of a very soluble salt having one ion in common with the first salt is added, partial precipitation of the first salt is found to take place. This happens, for example, with a saturated solution of potassium chlorate, which is not very soluble (molar solubility 0.52 , see Table). The concentrations $\left[\mathrm{K}^{+}\right]$and $\left[\mathrm{ClO}_{3}^{-}\right]$being small, one may easily increase the value for one of the ions, say $\left[\mathrm{ClO}_{3}^{-}\right]$, fivefold, by adding a chlorate which is sufficiently soluble. To preserve the value of the product $\left[\mathrm{K}^{+}\right] \times\left[\mathrm{ClO}_{3}{ }^{-}\right]$, the value of $\left[\mathrm{K}^{+}\right]$will then have to be diminished at once to one-fifth of its former value. This can occur only by union of the ionic material it represents with an equivalent amount of that for which $\left[\mathrm{ClO}_{3}^{-}\right]$stands. The molecular material so produced will thus tend at first to swell the value of $\left[\mathrm{KClO}_{3}\right]$. But the value of $\left[\mathrm{KClO}_{3}\right]$ cannot be increased, for the solution is already saturated with molecules, so that the new supply of molecules, or others in equal numbers, will be precipitated. Hence the ionic part of the dissolved substance may be diminished, the equilibria (p. 455) may be partially reversed, and we may actually precipitate a part of the dissolved material without introducing any substance, which, in the ordinary sense, can interact with it.

In point of fact, when, to a saturated solution of potassium chlorate there is added a saturated solution of potassium chloride $\mathrm{KCl}$ (molar solubility, 3.9) or of sodium chlorate $\mathrm{NaClO}_{3}$ (molar solubility, 6.4), a precipitate of potassium chlorate is thrown down.

The precipitation of sodium chloride from a saturated solution, by the introduction of gaseous hydrogen chloride (p. 127), is to be explained in the same manner. The equilibria:

$$
\mathrm{NaCl} \text { (solid) } \rightleftarrows \mathrm{NaCl} \text { (dslvd) } \rightleftarrows \mathrm{Na}^{+}+\mathrm{Cl}^{-}
$$

are reversed by the introduction of additional $\mathrm{Cl}^{-}$from the very soluble, and highly ionized $\mathrm{HCl}$. 
Precipitation by Double Decomposition.- The mechanism of this type of reaction has already been discussed in some detail in an earlier chapter (p. 185), to which reference should here be made by the reader. The solubility-product law, however, throws much additional light on the subject, and enables us to forecast the completeness of any given precipitation under given conditions.

The first thing to be remembered is that the precipitate which we observe, however insoluble its material may be, does not include all of the substance, but only the excess beyond what is required to saturate the water. The liquid surrounding the precipitate is always a saturated solution of the substance precipitated. If it were not so, some of the precipitate would dissolve until the liquid became saturated. Thus, for example, when we add ammonium sulphide solution to zinc chloride solution (p. 451):

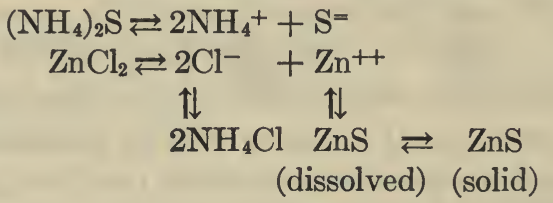

the liquid is a saturated solution of zinc sulphide, with the excess of this salt suspended in it as a precipitate.

Looking at the matter from this viewpoint, we perceive the application of the rule of solubility-product constancy. In this saturated solution the product of the ion-concentrations, $\left[\mathrm{Zn}^{++}\right] \times$ $[S=$, is constant. If the original solutions had been so very dilute that, when they were mixed, the product of the concentrations of these two ions had not reached the value of this constant, no precipitation would have occurred. As a matter of fact, the original salts are so extensively ionized in solution, and the solubility-product of zinc sulphide is so small, that in all ordinary mixtures the product $\left[\mathrm{Zn}^{++}\right] \times\left[\mathrm{S}^{=}\right]$considerably exceeds the requisite value, and hence the salt is thrown down until the balance remaining gives the value in question. The rule for precipitation, then, is as follows: Whenever the product of the con- 
centrations of any two ions in a mixture exceeds the value of the ion-product in a saturated solution of the compound formed by their union, this compound will be precipitated. Naturally the substances with small solubilities, and therefore small solubilityproduct constants, are the ones most frequently formed as precipitates.

Completeness of Precipitation.- In the above case, we precipitate zinc sulphide practically completely from solution by adding excess of ammonium sulphide. This substance, like all salts, is highly ionized in solution, and therefore a solution which contains it in excess contains a high concentration of sulphide ion $\mathrm{S}^{=}$. The amount of zinc ion $\mathrm{Zn}^{++}$that can exist simultaneously in such a solution is negligible, since otherwise the solubility-product of zinc sulphide would be exceeded. Zinc is therefore precipitated quantitatively as zinc sulphide by addition of excess of ammonium sulphide to a solution of any zinc salt. This is a fact which is turned to practical use in quantitative analysis.

If we attempt to carry_out the precipitation with hydrogen sulphide instead of ammonium sulphide, however, we find that only partial precipitation of the zinc as sulphide occurs (p. 451). However great an excess of $\mathrm{H}_{2} \mathrm{~S}$ we add, precipitation is incomplete. The reason for this is immediately apparent from a study of the full ionic equations:

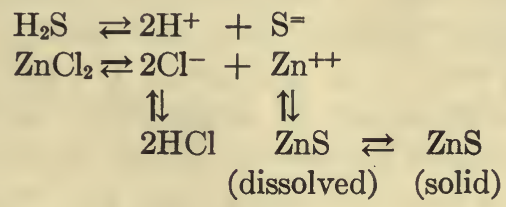

Hydrogen sulphide is so slightly ionized in solution that the concentration of sulphide ion $S=$ that it supplies is extremely small. A large amount of zinc ion $\mathrm{Zn}^{++}$can therefore remain in solution without the solubility-product of zinc sulphide being exceeded. 
Rule for Solution of Substances. - The rule for solution of any electrolyte follows at once from the foregoing considerations, and may be formulated by changing a few of the words in the rule just given: Whenever the product of the concentrations of any two ions in a mixture is less than the value of the ion-product in a saturated solution of the compound formed by their union, this compound, if present in the solid form, will be dissolved. When applied to the simplest case, this rule means that a substance will dissolve in a liquid not yet saturated with it, but will not dissolve in a liquid already saturated with the same material. The main value of the rule lies, however, in its application to the less simple, but equally common cases, such as when an insoluble body is dissolved by interaction with another electrolyte.

\section{Applications of the Rule for Solution to the Solution of} Insoluble Substances.- So long as a substance remains in pure water its solubility is fixed. Thus, with calcium hydroxide, the system comes to equilibrium at $18^{\circ}$ when 0.17 g. per 100 c.c. of water ( 0.02 moles per liter) have gone into solution:

$$
\mathrm{Ca}(\mathrm{OH})_{2} \text { (solid) } \leftrightarrows \mathrm{Ca}(\mathrm{OH})_{2} \text { (dslvd.) } \leftrightarrows \mathrm{Ca}^{++}+2 \mathrm{OH}^{-} \text {. }
$$

But if an additional reagent which can combine with either one of the ions is added, the concentration of this ion at once becomes less, the actual numerical value of the ion-product therefore begins to diminish, and further solution must take place to restore its value. Thus, if a little of an acid (giving $\mathrm{H}^{+}$) be added to the solution of calcium hydroxide, the union of $\mathrm{OH}^{-}$and $\mathrm{H}^{+}$to form water removes almost all the $\mathrm{OH}^{-}$(see p. 368), and solution of the hydroxide proceeds until the acid is used up. There are now more $\mathrm{Ca}^{++}$than $\mathrm{OH}^{-}$ions present, but the ion-product reaches the same value as before, and then the change ceases. If a further supply of acid is added, the removal of $\mathrm{OH}^{-}$to form $\mathrm{H}_{2} \mathrm{O}$ begins again. Hence, with excess of acid, the calcium hydroxide finally all dissolves. 
This particular action is a neutralization of an insoluble base. But the other kinds of actions by which insoluble electrolytes pass into solution all resemble it closely, and differ only in details. The general outlines of the explanation are the same in every case. We proceed now to apply it to the common phenomenon of the solution of an insoluble salt by an acid.

\section{Interaction of Insoluble Salts with Acids, Resulting in} Solution of the Salt.-Zine sulphide passes into solution when in contact with acids, especially active acids. Thus, with hydrochloric acid, it gives zinc chloride and hydrosulphuric acid, both of which are soluble:

$$
\mathrm{ZnS} \uparrow+2 \mathrm{HCl} \leftrightarrows \mathrm{ZnCl}_{2}+\mathrm{H}_{2} \mathrm{~S} .
$$

The action of acids upon insoluble salts is so frequently mentioned in chemistry and is so important a factor in analytical operations that it demands separate discussion. This example is a typical one and may be used as an illustration.

According to the rules already explained (p. 457), zinc sulphide (or any other salt) is precipitated when the numerical value of the product of the concentrations of the two requisite ions $\left[\mathrm{Zn}^{++}\right] \times\left[\mathrm{S}^{=}\right]$exceeds the value of the ion-product for a saturated solution of zinc sulphide in pure water. When, on the contrary, the product of the concentrations of the two ions falls below the limiting value, a condition which may arise from the removal in some way either of the $\mathrm{Zn}^{++}$or of the $\mathrm{S}^{=}$ions, the solid will dissolve to replace them until the ionic concentrations necessary for equilibrium with molecules have been restored or until the whole of the solid present is consumed. Here the sulphide-ion from the zinc sulphide combines with the hydrogen-ion of the acid (usually an active one) which has been added, and forms molecular $\mathrm{H}_{2} \mathrm{~S}$ :

$$
\mathrm{S}^{=}+2 \mathrm{H}^{+} \leftrightarrows \mathrm{H}_{2} \mathrm{~S}
$$


It will be seen that the removal of the ions in this fashion can result in considerable solution of the salt only when the acid produced is a feebly ionized one. Here, to be specific, the concentration of the $S^{=}$in the hydrosulphuric acid equilibrium (2) above must be less than that of the same ion in a saturated zinc sulphide solution. Now hydrosulphuric acid belongs to the least active class of acids, and its pure solution contains only a very small concentration of $S=$ (p. 189). There is, however, another factor in the situation which we have not yet taken into account. The hydro chloric acid which we used for dissolving the precipitate is a very highly ionized acid and gives an enormously greater concentration of hydrogen-ion than does hydrosulphuric acid. Hence the hydrogen-ion is in large excess in equation (2), and the condition for equilibrium, $\frac{\left[\mathrm{H}^{+}\right]^{2} \times\left[\mathrm{S}^{=}\right]}{\left[\mathrm{H}_{2} \mathrm{~S}\right]}=\mathrm{K}$, will be satisfied by a correspondingly much smaller concentration of $S^{=}$. In this particular case, therefore, the $\left[\mathrm{S}^{=}\right]$of the hydrosulphuric acid is far less than that given by the zinc sulphide. The whole change, therefore, depends for its accomplishment, not only on the mere presence of hydrogen-ion, but on the repression of the ionization of the hydrosulphuric acid by the great excess of hydrogen-ion furnished by the active acid that has been used. The whole scheme of the equilibria is as follows:

$$
\begin{aligned}
& \mathrm{ZnS} \text { (solid) } \leftrightarrows \mathrm{ZnS} \text { (dissolved) } \leftrightarrows \mathrm{Zn}^{++}+\mathrm{S}^{=} \\
& 2 \mathrm{HCl} \\
& \leftrightarrows 2 \mathrm{Cl}^{-}+2 \mathrm{H}^{+}
\end{aligned}
$$

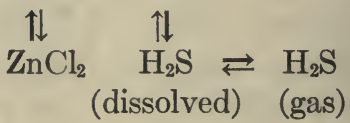

A generalization may be based on these considerations: an insoluble salt of a given acid will in general interact and dissolve when treated with a solution containing another acid, provided that the latter acid is a much more highly ionized (more active) one than the former. 
Precipitates Insoluble in Acids.-But even active acids frequently fail to bring salts of weak acids into solution. Here the cause lies in the fact that such salts are even less soluble than those of the zinc sulphide type. Thus, even hydrochloric acid (normal) will not appreciably dissolve cupric sulphide. The solubility product $\left[\mathrm{Cu}^{++}\right] \times\left[\mathrm{S}^{=}\right]$for this salt is so small that, after an infinitesimal amount has gone into solution, the sulphide-ion concentration is sufficient, in spite of the repressive action of the large hydrogen-ion concentration furnished by the $\mathrm{HCl}$, to bring the product $\left[\mathrm{Cu}^{++}\right] \times\left[\mathrm{S}^{=}\right]$up to its maximum possible value. In this case the first link in the chain of equilibria:

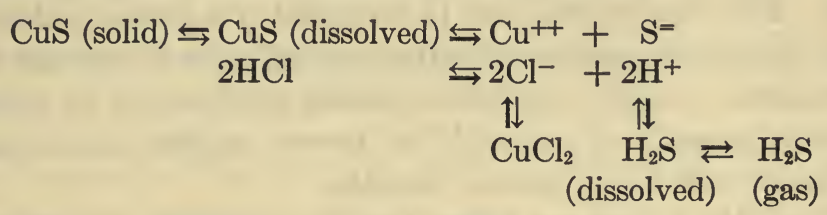

tends so decidedly backward that only the use of very concentrated acid will increase the concentration of the $\mathrm{H}^{+}$to an extent sufficient to secure even a slight advance of the whole action. We must add, therefore, to the above rule: provided also that the salt is not one of extreme insolubility.

Illustrations of the application of these generalizations are countless. Carbonic acid is made from marble (p. 333), hydrogen sulphide from ferrous sulphide (p. 253), hydrogen peroxide from sodium peroxide (p. 221), and phosphoric acid from calcium phosphate (p.412). In each case the acid employed to decompose the salt is more active than the acid to be liberated. On the other hand, calcium phosphate is insoluble in acetic acid because this acid is weaker than is phosphoric acid. We have thus only to examine the list of acids showing their degrees of ionization (p. 189) in order to be able to tell which salts, if insoluble in water, will be dissolved by acids and, in general, what acids will be sufficiently active in each case for the purpose. In chemical 
analysis we discriminate between salts soluble in water, those soluble in acetic acid (the insoluble carbonates and some sulphides, $\mathrm{FeS}$ and $\mathrm{MnS}$, for example), those requiring active mineral acids for their solution (calcium phosphate, zinc sulphide and the more insoluble sulphides, for example), and those insoluble in all acids (barium sulphate and other insoluble salts of active acids).

\section{Precipitation of Insoluble Salts in Presence of Acids.-} The converse of solution, namely, precipitation, depends upon the same conditions: an insoluble salt which is dissolved by a given acid cannot be formed by precipitation in the presence of this acid. Thus zinc sulphide can be precipitated in presence of acetic acid, but not in presence of active mineral acids in ordinary concentrations. Cupric sulphide or barium sulphate can be precipitated in presence of any acid, but ferrous sulphide and calcium carbonate only in the absence of acids.

From this it does not follow that zinc sulphide, for example, cannot be precipitated if once an active acid has been added to the mixture. To secure precipitation, all that is necessary is to remove the excess of hydrogen-ion which is repressing the ionization of the hydrosulphuric acid. This can be done by adding a base, or by adding ammonium sulphide.

Exercises.-1. (a) How do the electrolytic methods of making calcium and magnesium differ? (b) Why not electrolyze an aqueous solution of magnesium chloride in making magnesium? (c) Why use both potassium and sodium chlorides in making magnesium? (d) Why is magnesium, but not potassium or sodium, liberated?

2. Why are magnesium and zinc not found free in nature?

3. Why does magnesium rust completely (in time), while zinc does not?

4. Make equations for: (a) the action of magnesium on hydrochloric acid; (b) the burning of magnesium in air; (c) the heating 
of magnesium carbonate; (d) the precipitation of magnesium hydroxide from the sulphate; (e) the hydrolysis of magnesium chloride.

5. Why is salt containing magnesium chloride, after mixing with sodium bicarbonate, no longer deliquescent?

6. What is the density of zinc vapor (air $=1$ )?

7. Make equations for: (a) the action of hydrogen sulphide on zinc oxide; (b) the precipitation of zinc hydroxide; (c) the action of sodium hydroxide on zinc hydroxide.

8. What will be the effect of adding a concentrated solution of silver nitrate to a saturated solution of silver sulphate (see table of solubilities)?

9. Write full ionic equations for the reactions mentioned on $\mathrm{p}$. 463. 
From time to time more of the oxide is added and the melted aluminium (m. p. 650 to $700^{\circ}$ ) is tapped off. The oxide must be made from carefully purified bauxite, as the metal itself can not be purified commercially. In 1866 it cost $\$ 250$ to $\$ 750$ per kilogram but now sells at about 50 cents.

Properties.-The metal has a lower specific gravity (2.6) than any other metal that could be put to the same uses (sp. gr. iron 7.8, copper 8.8). It has malleability and the foil is taking the place of tinfoil to some extent for wrapping foods. It has considerable tensile strength, and is a good conductor of electricity. When heated, the metal burns brilliantly.

In the air it acquires only a slight film of closely adhering oxide. This film prevents it from actıng upon water (hot, or cold). When the surface is cleaned and amalgamated with mercury, by dipping in mercuric chloride solution, however, this metal acts as a contact agent, and hydrogen is rapidly displaced:

$$
2 \mathrm{Al}+6 \mathrm{H}_{2} \mathrm{O} \rightarrow 3 \mathrm{H}_{2} \uparrow+2 \mathrm{Al}(\mathrm{OH})_{3} \downarrow .
$$

Uses.-The largest quantity of aluminium is consumed by steel-makers. When added in small amount (less than $1: 1000$ ) to molten steel, it combines with the gases, and gives sound ingots free from blow holes. Next to this comes its use for long distance transmission of electricity. A cable of the requisite capacity is larger than one of copper for the same current, but is lighter and puts less strain on the supports. Cooking vessels of aluminium are not corroded and are largely used. Cameras and opera glasses are made of it. On account of its lightness, it is used extensively in the metal parts of dirigibles and aeroplanes. Pulverized aluminium, mixed with oil, gives a paint which protects iron admirably.

Aluminium bronze (copper, with 5 to 12 per cent aluminium) has a brilliant golden yellow color and is stable in air and easily worked. Magnalium (containing 1 to 2 per cent of magnesium) 
can be filed, turned, or polished like a mirror, and is better for many purposes than the pure metal.

On account of its great chemical activity ( $p .54$ ), aluminium displaces many other metals (e.g. iron, manganese, and chromium) from combination. Thus powdered aluminium and oxide of iron when mixed "(thermite) in a crucible, and started by a burning magnesium ribbon, interact with great violence:

$$
2 \mathrm{Al}+\mathrm{Fe}_{2} \mathrm{O}_{3} \rightarrow \mathrm{Al}_{2} \mathrm{O}_{3}+2 \mathrm{Fe} .
$$

A temperature of 3000 to $3500^{\circ}$ is reached, the molten iron (m.-p. $1500^{\circ}$ ) collects at the bottom, and the molten aluminium oxide (m.-p. $2050^{\circ}$ ) floats to the top. Steel rails are welded together, and large objects of steel like broken propeller shafts are mended, by enclosing a mass of thermite round the joint and firing it.

Aluminium Hydroxide $\mathrm{Al}(\mathrm{OH})_{3}$. - The hydroxide is precipitated when ammonium hydroxide, or other alkaline hydroxide, is added to a solution of a salt of aluminium:

$\mathrm{Al}_{2}\left(\mathrm{SO}_{4}\right)_{3}+6 \mathrm{NH}_{4} \mathrm{OH} \rightarrow 2 \mathrm{Al}(\mathrm{OH})_{3} \downarrow+3\left(\mathrm{NH}_{4}\right)_{2} \mathrm{SO}_{4}$ (dslvd.).

It tends to remain in colloidal suspension (p. 441), and forms a white gelatinous precipitate. It is both weakly basic and feebly acidic in chemical properties. In acids it dissolves forming salts of aluminium, such as the chloride $\mathrm{AlCl}_{3}$ or sulphate $\mathrm{Al}_{2}\left(\mathrm{SO}_{4}\right)_{3}$. Solutions of these salts in water give an acid reaction, owing to hydrolysis (p. 369).

Aluminium hydroxide dissolves also in sodium hydroxide solution, to form sodium aluminate $\mathrm{Na}_{3} \mathrm{AlO}_{3}$ :

$$
3 \mathrm{NaOH}+\mathrm{H}_{3} \mathrm{AlO}_{3} \rightarrow \mathrm{Na}_{3} \mathrm{AlO}_{3}+3 \mathrm{H}_{2} \mathrm{O} \text {. }
$$

The aluminates are hydrolyzed by water, and their solutions have an alkaline reaction.

Aluminium hydroxide, precipitated from the sulphate, is used in sizing paper (to fill the capillaries and pores), in purifying water (see p. 470), and as a mordant (see p. 475) in dyeing. Deli- 
cate fabrics (cloth) are rendered waterproof by saturating them with aluminium acetate solution and boiling to promote the hydrolysis. The aluminium hydroxide is precipitated in the capillaries of the cotton or linen, rendering them non-absorbent.

Aluminium Oxide (Alumina) $\mathrm{Al}_{2} \mathrm{O}_{3} .-$ Corundum, and the impure variety emery, are next to the diamond in the scale of hardness, and are used as abrasives. Ruby and sapphire are also crystallized aluminium oxide, containing traces of impurities (iron and titanium in the one case and chromium in the other) to which they owe their colors. By ingenious methods of fusing the oxide, "synthetic" sapphires to the extent of six million carats and rubies to the extent of ten million carats are now made annually. The artificial gems are chemically identical with the natural ones, and can be distinguished only by the fact that they are free from microscopic bubbles and other defects. Alundum, an artificial abrasive, and refractory material for crucibles and muffles, is made by barely melting the oxide in the electric furnace.

Aluminium Sulphate $\mathrm{Al}_{2}\left(\mathrm{SO}_{4}\right)_{3}, 18 \mathrm{H}_{2} \mathrm{O}$. - The sulphate is manufactured by the action of sulphuric acid on bauxite. It crystallizes in leaflets, which usually have a faint yellow tinge due to the presence of iron $\left(\mathrm{Fe}(\mathrm{OH})_{3}\right)$ derived from the mineral. The salt is used in fireproofing cloth, since, when heated, it melts in its water of hydration. It is used as a source for precipitated aluminium hydroxide in paper-making, water purification, and dyeing.

Alums.-When aluminium sulphate and potassium sulphate are dissolved together in molecular proportions, the solution deposits transparent octahedral (Fig. 39, p. 94) crystals of potash-alum $\mathrm{K}_{2} \mathrm{SO}_{4}, \mathrm{Al}_{2}\left(\mathrm{SO}_{4}\right)_{3}, 24 \mathrm{H}_{2} \mathrm{O}$. This salt is more easily freed from impurities (e.g. compounds of iron) by recrystallization than is aluminium sulphate, and is therefore used instead of the 
latter in medicine, in dyeing (delicate shades), and to replace the cream of tartar ( $\mathrm{p} .431$ ) in making baking powder.

Sodium - alum, $\mathrm{Na}_{2} \mathrm{SO}_{4}, \mathrm{Al}_{2}\left(\mathrm{SO}_{4}\right)_{3}, 24 \mathrm{H}_{2} \mathrm{O}$, ammonium - alum $\left(\mathrm{NH}_{4}\right)_{2} \mathrm{SO}_{4}, \mathrm{Al}_{2}\left(\mathrm{SO}_{4}\right)_{3}, 24 \mathrm{H}_{2} \mathrm{O}$ and chrome-alum $\mathrm{K}_{2} \mathrm{SO}_{4}, \mathrm{Cr}_{2}\left(\mathrm{SO}_{4}\right)_{3}$, $24 \mathrm{H}_{2} \mathrm{O}$ are made in the same way, and crystallize in the same form. The first two are used as sources of aluminium hydroxide, and the last in the " fixing bath" to harden the gelatine on photographic films and plates.

- Water Purification-Coagulation Process.-The suspended matter in water to be used for a domestic supply can be coagulated into larger particles by introducing a small amount of the gelatinous precipitate of aluminium hydroxide. These larger particles, which adsorb also the greater part of the bacteria, settle rapidly and the process therefore permits the use of relatively small settling ponds. Aluminium sulphate, made from crude bauxite, and lime are added to the water:

$$
3 \mathrm{Ca}(\mathrm{OH})_{2}+\mathrm{Al}_{2}\left(\mathrm{SO}_{4}\right)_{3} \rightarrow 3 \mathrm{CaSO}_{4}+2 \mathrm{Al}(\mathrm{OH})_{3} \text {. }
$$

If the water has much temporary hardness, lime is not required:

$$
3 \mathrm{Ca}\left(\mathrm{HCO}_{3}\right)_{2}+\mathrm{Al}_{2}\left(\mathrm{SO}_{4}\right)_{3} \rightarrow 3 \mathrm{CaSO}_{4}+2 \mathrm{Al}\left(\mathrm{HCO}_{3}\right)_{3} \text {. }
$$

The carbonate of aluminium, being a salt of both a very weak acid and a very weak base, if formed, would be instantly hydrolyzed:

$$
\mathrm{Al}\left(\mathrm{HCO}_{3}\right)_{3}+3 \mathrm{H}_{2} \mathrm{O} \rightarrow \mathrm{Al}(\mathrm{OH})_{3} \downarrow+3 \mathrm{H}_{2} \mathrm{CO}_{3}
$$

so that aluminium hydroxide is precipitated.

The few remaining bacteria are destroyed by the addition of bleaching powder (p. 224).

Crude ferrous sulphate $\mathrm{FeSO}_{4}$ (copperas), being in many places cheaper than aluminium sulphate, is often used instead of the latter. The lime precipitates ferrous hydroxide $\mathrm{Fe}(\mathrm{OH})_{2}$. This is quickly oxidized to the red ferric hydroxide $\mathrm{Fe}(\mathrm{OH})_{3}$, which coagulates the suspended matter. 
Clay and Pottery - Pure clay (kaolin) is white. It is hydrogen-aluminum silicate $\mathrm{HAISiO}_{4}$, derived from the weathering of felspar (p. 410). Common clay contains impurities such as sand (silica), limestone, and compounds of iron. Both kinds are plastic when wet and can be moulded. When heated strongly the material shrinks (so that the products are porous) and becomes hard. Bricks, and tiling for roofs and drains, are made of common clay and, when red, owe their color to oxide of iron $\left(\mathrm{Fe}_{2} \mathrm{O}_{3}\right)$. The firing is done with fuel gas in ovens or kilns of brickwork. To glaze drain pipes and some bricks, salt is thrown into the kiln. The vapor of the salt produces a more fusible sodium-aluminium silicate, which fills the surface pores. Clay for fire-brick (infusible) must contain free silica, but no lime.

China and porcelain are white, translucent and non-porous. They are made of pure clay to which a little of the more fusible felspar is added. After firing, the articles are dipped in water, in which the materals for the "glaze," namely finely ground felspar and silica, are suspended. Having thus acquired a thin coating of these substances, they are fired again at a higher temperature and for a longer time. Colored decoration is done with materials which melt (third firing) to colored enamels (p. 362).

Ultramarine.-This material is made by heating together kaolin, sodium carbonate, sulphur, and charcoal, pulverizing the green mass, and heating it again with more sulphur. The product is used as laundry blueing, and in making blue-tinted paper. It is added also to correct the yellow shade of linen, starch, sugar (p. 402), and paper stock.

Cement.-Portland cement is made by heating a pulverized mixture of a material rich in lime, such as limestone $\mathrm{CaCO}_{3}$, with one in which silica, iron oxide and alumina are the main constituents, such as common clay. Some natural rocks contain all of the necessary elements in suitable proportions. The finely 
powdered material is first burnt or raised to a temperature of $1400-1600^{\circ}$, at which temperature it fuses partially and forms lumps or clinkers. When these have cooled, they are mixed with 2-3 per cent of gypsum and pulverized again. The resulting product is Portland cement, the manufacture of which in the United States alone now exceeds $90,000,000$ barrels (of 380 pounds) yearly.

Portland cement is essentially a mixture of calcium silicate and calcium aluminate, with excess lime. The calcium silicate is simply a filler. The calcium aluminate is hydrolyzed on addition of water, according to the equation:

$$
\mathrm{Ca}_{3}\left(\mathrm{AlO}_{3}\right)_{2}+6 \mathrm{H}_{2} \mathrm{O} \rightarrow 3 \mathrm{Ca}(\mathrm{OH})_{2}+2 \mathrm{H}_{3} \mathrm{AlO}_{3} \text {. }
$$

The calcium hydroxide thus formed slowly crystallizes, connecting the particles of the calcium silicate. The aluminium hydroxide fills the interstices and renders the whole compact and impervious.

The small amount of gypsum added regulates the setting time of the cement. The iron oxide is necessary to assist in the burning and to lower the temperature at which the mixture begins to fuse. If too small a quantity of excess lime is present, the cement will be unsound and crack on drying. Too little excess lime gives a cement which sets too quickly and is lacking in strength.

Concrete is a mixture of cement with sand and crushed stone or gravel, all made into a paste with water. It sets to a solid mass, suitable for walks, and for the foundations, walls, and floors of buildings. Since no carbon dioxide from the air is required in the hardening process (contrast with p. 386), it sets equally well under water (hence hydraulic cement), and is employed in constructing dams, levees, and the foundations of bridges. Reinforced concrete contains twisted rods of iron, embedded in the mass, and is much used in building construction.

Blast-furnace slag, when pulverized and heated with limestone, has been found to yield an excellent quality of cement, and a valuable use has thus been found for what was formerly an annoying encumbrance. 
Exercises. - 1. Make equations for the following actions: (a) aluminium on hydrochloric acid; (b) aluminium on mercuric chloride $\mathrm{HgCl}_{2}$; (c) displacement of manganese from manganese dioxide by aluminium.

2. How must aluminium hydroxide be ionized so as to behave both as an acid and a base?

3. Make the equation for heating calcium carbonate with: (a) aluminium hydroxide; (b) aluminium oxide.

4. Explain why the reaction of solutions of aluminates is alkaline (p. 369) and that of alums is acid.

5. Make the equation: (a) for the action of sulphuric acid on bauxite, assuming the formula of the latter to be $\mathrm{Al}_{2} \mathrm{O}(\mathrm{OH})_{4}$; (b) for the formation of potash-alum.

6. Why is the tarnish on aluminium the oxide, and not the carbonate (as on $\mathrm{Zn}$ and $\mathrm{Mg}$ )? What qualities in a tarnish enable it to protect the metal from further oxidation (p. 467)?

7. Make equations for the action of bicarbonate of soda and aluminium sulphate (alum baking powder) when heated. Explain what raises the bread. 


\section{CHAPTER XL}

\section{SYNTHETIC ORGANIC PRODUCTS}

Mention was made several times in the preceding chapter of the use of salts of aluminium in dyeing. A brief summary of the chemistry of dyeing and dyestuffs is all that can be presented here. A still more restricted résumé of some other important synthetic organic products is also given. The products included are, on the constructive side, perfumes, drugs and plastics (including rubber); on the destructive side, explosives and toxic gases.

Some dyes, perfumes and drugs are still, of course, obtained from natural sources, and synthetic rubber continues to show but little promise of superseding plantation rubber in the near future. Nevertheless, the interest of the chemist in the fields here under discussion is almost entirely synthetic, seeking to duplicate natural products by laboratory methods and to discover new products of even greater use to humanity.

Dyeing. - The problem of the dyer is to confer the desired color upon a fabric made, usually, of cotton, linen, wool, or silk, and to do this in such a way that the dye is fast to (i.e., is not removed or destroyed by) rubbing and light, and often, also, to washing with soap. To understand the means by which this is achieved, it must be noted that cotton and linen consist of smooth hollow fibers (Fig. 2, p. 2) of cellulose. Wool is made of hollow fibers with a scaley surface, and silk of solid filaments, but these are composed of proteins (p. 438). Now, the proteins are much more active chemically than is cellulose, and also, as colloidal materials, seem to have a much greater tendency to adsorb other substances (see pp. 422, 443) than has cellulose. Hence, accidental stains on wool or silk are much less often remov- 
able than are those on cotton, and when samples of the three materials are dipped in a solution of a dye, the first two are permanently dyed, while from the last most dyes can be completely washed out with water.

Three modes of dyeing may be mentioned:

1. Insoluble Dyes. If the colored body can be produced by precipitation, after the solution has filled the capillary and wall of every fiber of the goods, then, if the dye is sufficiently insoluble, it is mechanically imprisoned in every fiber and cannot be washed out. This plan may be applied to any kind of goods. For example, if cotton, silk, or wool is first boiled in a solution of lead acetate, and is then soaked in a boiling solution of potassium chromate $\mathrm{K}_{2} \mathrm{CrO}_{4}$, it is dyed a brilliant, permanent yellow. Lead chromate is the colored body:

$$
\mathrm{Pb}\left(\mathrm{CO}_{2} \mathrm{CH}_{3}\right)_{2}+\mathrm{K}_{2} \mathrm{CrO}_{4} \leftrightarrows 2 \mathrm{~K}\left(\mathrm{CO}_{2} \mathrm{CH}_{3}\right)+\mathrm{PbCrO}_{4} \downarrow \text {. }
$$

The part precipitated on the outside of the goods can be, and is, at once washed off by rubbing in water, but the particles inside the fibers can come out only by being dissolved, and they are insoluble in water. Indigo $\mathrm{C}_{16} \mathrm{H}_{10} \mathrm{~N}_{2} \mathrm{O}_{2}$, which is used in larger amounts than any other dye, belongs to this class. The cloth is saturated with an alkaline solution of indigo white $\mathrm{C}_{16} \mathrm{H}_{12} \mathrm{~N}_{2} \mathrm{O}_{2}$, a soluble, slightly acid substance, and the oxygen of the air subsequently oxidizes this and deposits the insoluble indigo blue within the fibers:

$$
2 \mathrm{C}_{16} \mathrm{H}_{12} \mathrm{~N}_{2} \mathrm{O}_{2}+\mathrm{O}_{2} \rightarrow 2 \mathrm{C}_{16} \mathrm{H}_{10} \mathrm{~N}_{2} \mathrm{O}_{2} \downarrow+2 \mathrm{H}_{2} \mathrm{O} \text {. }
$$

2. Mordant or Adjective Dyes. Since cotton is inactive chemically and has but a slight tendency to adsorb dyes, it is usually necessary first to introduce into the fibers of cotton some colloidal substance with greater adsorptive powers. Substances of this kind are tannic acid (p. 499) for basic dyes, and gelatinous colloidal hydroxides, such as those of aluminium, tin, iron and chromium, for non-basic (including acid) dyes. They are called mordants (Lat. mordere, to bite). Thus, if in three jars we place 
very dilute solutions of aluminium sulphate, ferric chloride $\mathrm{FeCl}_{3}$ and chromous acetate $\mathrm{Cr}\left(\mathrm{CO}_{2} \mathrm{CH}_{3}\right)_{2}$, then add a few drops of a solution of a dye to each, and finally introduce a little of a base (like sodium hydroxide) to precipitate the hydroxide of the metal, this hydroxide vill adsorb the dye and carry it into the precipitate. Such a precipitate of mordant and dye is called a lake. With the same dye, the three lakes have different colors. Thus, in the above-mentioned experiment, if alizarin (madder) is used as the dye, the colors are red (Turkey red), violet, and maroon, respectively. This is probably due to the different degrees of dispersion in the three colloidal materials. If aluminium hydroxide is to be used, by first saturating the cloth with hot aluminium acetate solution (p. 468), or by using first aluminium sulphate and then ammonium hydroxide, the aluminium hydroxide is precipitated within the fibers of the goods. When the material is then dyed, the coloring matter is adsorbed by the mordant, with which it forms an insoluble lake, within the fibers. Basic dyes, like Malachite green and Methylene blue, behave similarly with tannic acid, or an insoluble salt of tannic acid, as mordant. It will be seen that, so far as the fabric is concerned, this process, like the first, is a mechanical one, and is independent of the chemical nature of the goods.

3. Direct or Substantive Dyes. Most organic dyes are direct dyes on silk or wool, and require no mordant with these materials. The actions seem to be sometimes chemical, but more often cases of adsorption by the silk or wool (both colloids) themselves. A few dyes are also fast on cotton. Congo-red is fast both on cotton and wool, but is no longer much used. Chrysophenin is now one of the commonest dyes of this class. These dyes, which are sodium salts of complex organic acids, are colloids like soap ( $p$. 442 ), and are salted out within the fibers of the goods by adding sodium sulphate to coagulate them and assist the adsorption by the cotton. Once adsorbed in this way, unlike soap, they cannot be washed out. 
Dye-stuffs. - Natural dye-stuffs have now been almost entirely superseded by manufactured products, which can be prepared more cheaply and are of superior quality. Logwood, still used as a black mordant dye for silk, is the only important exception. The total value of natural dyes imported into the United States in 1919 was only $\$ 1,250,000$, while the artificial dyes made in the country in the same year were worth over $\$ 70$,000,000 .

The vast bulk of synthetic dyes are built up from ring hydrocarbons (p. 352) and their derivatives, extracted from coal tar (p. 424). By substituting suitable groups into the molecule of the simpler colorless products, more complex derivatives with great brilliancy of color are obtained. Thus indigo, formerly the most extensively used of all natural dyes, is now manufactured most conveniently with aniline (p. 353) as a starting-point. Alizarin (Turkey red), once extracted from madder root, is obtained from anthracene.

By varying the position of the substituted groups in the molecule, the most delicate variations in color can be effected. The most precious of all dyes in ancient times was Tyrian purple, obtained from certain species of sea snails (Murex). The secret of preparing this substance was lost for centuries, but in 1909 Friedlaender gathered 12,000 of these mollusks and succeeded in isolating 1.5 grammes of the coloring material for analysis. $\mathrm{He}$ showed it to be a derivative of indigo, containing two bromine atoms in place of two of the hydrogens. This identical substance had been prepared synthetically five years earlier, but found to be inferior to another dye containing the bromine atoms in different positions in the molecule!

Preparation of Typical Dyes. - The student is recommended to attempt the following preparations, which are at the same time easy and instructive.

(a) Phenolphthalein. Take 0.1 gram of phthalic anhydride (the 
anhydride of phthalic acid, $\left.\mathrm{C}_{6} \mathrm{H}_{4} \cdot(\mathrm{COOH})_{2}\right)$ and 0.1 gram of phenol $\mathrm{C}_{6} \mathrm{H}_{5} . \mathrm{OH}$ in a test-tube, add 2 drops of concentrated sulphuric acid, and heat carefully for a minute over a small flame. The mixture, which should be well shaken during the heating, will turn dark-red in color. Allow to cool, add a few c.c. of water, and then add drop by drop a dilute solution of sodium hydroxide until a pink color persists on shaking.

Take a portion of this solution, and test the action of phenolphthalein as an indicator by adding first dilute hydrochloric acid, then dilute sodium hydroxide (see p. 168).

(b) Fluorescein. To 0.1 gram of phthalic anhydride and 0.1 gram of resorcinol $\mathrm{C}_{6} \mathrm{H}_{4}(\mathrm{OH})_{2}$ add 3 drops of concentrated sulphuric acid, and heat carefully for a minute. Allow to cool, add a few c.c. of water, then add sodium hydroxide until alkaline (use litmus paper as a test).

Shake up a few drops of this solution with a test-tube of water. The dye imparts to the solution a brilliant green fluorescence, hence its name.

Perfumes.- Many natural perfumes and fruit flavors can also be produced synthetically. The basis of most of these consists of esters (p. 349). The fragrance of ripe apples is due to minute amounts of the amyl esters of formic, acetic and caproic acids. In bananas the characteristic ester is amyl acetate; in grapes it is methyl anthranilate. Almond flavor is due to benzaldehyde, $\mathrm{C}_{6} \mathrm{H}_{5}$. $\mathrm{CHO}$; the smell of geraniums to diphenyl ether, $\left(\mathrm{C}_{6} \mathrm{H}_{5}\right)_{2} \mathrm{O}$. The chief ingredient of otto of roses is geraniol; of the vanilla bean, vanillin; of the perfume sold as " new mown hay," coumarin; all complex hydrocarbon derivatives which are now commercial products. Some of the synthetic flavors and perfumes on the market are identical with the substances that give odor to fruits and flowers; others are merely more or less adequate imitations. Thus, natural oil of wintergreen is essentially the same as synthetic methyl salicylate. On the other 
hand amyl valerate, which is sold as apple essence, is not contained in the fruit, though it smells like it. Most perfumery nowadays, including the most expensive, consists of mixtures of natural and synthetic products.

Preparation of Typical Perfumes.-Take 0.1 gram of beta-naphthol $\mathrm{C}_{10} \mathrm{H}_{7} . \mathrm{OH}$ and 10 drops of methyl alcohol in a test-tube, add 2 drops of concentrated sulphuric acid, and warm gently for a few minutes. The methyl ether of beta-naphthol, which is formed, has a most powerful odor, reminiscent of acacia blossoms.

Repeat the experiment with ethyl alcohol instead of methyl alcohol. The ethyl ether of beta-naphthol is produced; its odor recalls the perfume of orange flowers.

Drugs. - In the same way, many drugs formerly obtained from natural sources are now built up in the laboratory, and many new compounds have been made which possess as valuable medicinal properties as any found in nature. The first stage in this work consisted in determining the constitution of the active ingredients of plant products. Quinine was isolated from cinchona bark, morphine from the seed capsules of the opium poppy, strychnine from the seeds of nux vomica. These and other substances of similar character are now classed together as alkaloids, complex nitrogeneous substances possessing basic properties. The structure of many of the alkaloids is now completely worked out. It is not necessary, however, to construct the entire complicated molecule if the same results can be secured with simpler substances, and this has been demonstrated already in many instances.

Aspirin is an ester of salicylic acid $\mathrm{C}_{6} \mathrm{H}_{4} \cdot \mathrm{OH} . \mathrm{COOH}$; acetanilide is a derivative of aniline $\mathrm{C}_{6} \mathrm{H}_{5} \cdot \mathrm{NH}_{2}$. Acetophenone (hypnone), $\mathrm{C}_{6} \mathrm{H}_{5} \cdot \mathrm{CO} . \mathrm{CH}_{3}$, is used as a hypnotic. Novocaine, prepared from diethylaniline, has practically replaced the natural alkaloid cocaine in dentistry. Saccharin is obtained from toluene; 
its solution in water has an intensely sweet taste, hence it is used as a substitute for sugar in war times and in cases of diabetes. It has no food value, however, and hence cannot replace sugar in nutrition.

Plastics. - These are substances, like celluloid, which can be moulded or shaped into any desired form. Among natural plastics may be mentioned resins, gums, and rubber. Synthetic rubber equal to the natural product in durability and cheapness has still to be prepared (see p. 165), but many other plastics are now of considerable industrial importance.

Certain cellulose plastics have already been described (p. 399). Cellulose behaves chemically like an alcohol, and as such forms esters with acids (see p. 349). When cotton is treated with acetic acid (in the form of acetic anhydride $\left(\mathrm{CH}_{3} \cdot \mathrm{CO}\right)_{2} \mathrm{O}$, see p. 315 ) it gives cellulose acetate. The viscous liquid dries to a tenacious film. On account of its waterproof character, non-inflammability and non-conductance of electricity, it is now used for coating the wings of aeroplanes, for making moving picture films, and for insulating electric wires. Artificial horsehair (e.g. for making women's hats) and bristles for hair brushes are made of it.

By the action of nitric acid upon cellulose, various cellulose nitrates may be formed, according to the number of $\mathrm{OH}$ groups replaced by $\mathrm{NO}_{3}$ (see guncotton, below). An incompletely nitrated ester, when worked between rollers with camphor* and a little alcohol, forms a viscous solution. When the alcohol evaporates, a transparent colorless solid, celluloid, remains. Photographic films are made by rolling the dough into sheets. Fillers and dyes can be added to the dough and the latter can be moulded to any form. In this way ivory-like or black combs and brush handles, opaque white knife handles, articles of " artificial amber" and so forth can be made.

*A white solid with the formula $\mathrm{C}_{10} \mathrm{H}_{10} \mathrm{O}$, obtained commercially by distilling with steam the wood of the camphor tree, but recently also prepared synthetically. 
The same sort of guncotton dissolves in a mixture of alcohol and ether, giving a solution called collodion, used in photography and in medicine.

When collodion is forced under great pressure through minute holes in a steel die, the threads dry as they issue from the openings and can be wound on spools. The product is treated with an alkali, which decomposes the ester, leaving a material of the composition of the original cotton. The product is another form of artificial silk (p. 399).

Another plastic, not chemically related to the preceding, is bakelite, prepared from formaldehyde $\mathrm{CH}_{2} \mathrm{O}$ and phenol $\mathrm{C}_{6} \mathrm{H}_{5} \mathrm{OH}$ (carbolic acid). Under suitable heat treatment the mixture gradually sets to a solid, hard, infusible, resinous mass, which is insoluble in all common solvents. Before it sets, it can be dyed or " filled," and it can be applied as lacquer, or moulded to any form. Switchboards, dolls, ornamental buttons, artificial jewels, phonograph records, billiard balls, and stereotyping matrices are amongst the objects into which it is now fashioned.

Explosives. Nitroglycerine.-Some inorganic explosives, gunpowder (p. 372) and ammonium nitrate (p. 302), have been discussed in earlier chapters. The main organic explosives are also compounds of nitrogen.

As already mentioned, the alcohols interact with inorganic acids, as well as with organic ones, to produce esters. A familiar illustration is met with in the manufacture of nitroglycerine (glyceryl trinitrate) by the action of glycerine and nitric acid:

$$
\mathrm{C}_{3} \mathrm{H}_{5}(\mathrm{OH})_{3}+3 \mathrm{HNO}_{3} \rightarrow \mathrm{C}_{3} \mathrm{H}_{5}\left(\mathrm{NO}_{3}\right)_{3}+3 \mathrm{H}_{2} \mathrm{O} \text {. }
$$

To assist in the liberation of the water, the nitric acid is mixed with a dehydrating agent. The glycerine then is added slowly to the cooled reagents. The nitroglycerine is an almost colorless oil which floats to the surface of the acid mixture. It is shaken repeatedly with water, in which it is insoluble, and then 
with sodium carbonate solution, in order to free it from all traces of the acids.

Nitroglycerine explodes violently, often from the slightest shock. It owes this power to the fact that its carbon and hydrogen can combine with the oxygen it contains to form carbon dioxide and water:

$$
2 \mathrm{C}_{3} \mathrm{H}_{5}\left(\mathrm{NO}_{3}\right)_{3} \rightarrow 6 \mathrm{CO}_{2}+5 \mathrm{H}_{2} \mathrm{O}+3 \mathrm{~N}_{2}(+\mathrm{O}) .
$$

The latter are very stable substances and much heat is liberated in forming them. They are both produced as gases and, at the high temperature of the action, they and the nitrogen tend to occupy a great volume - or to exert an enormous pressure in the effort to do so.

The explosion is also so sudden, compared with that of gunpowder, that nitroglycerine would shatter the breech of a rifle before the bullet had time to move. It also pulverizes rock, instead of breaking it into fragments of usable size. For these reasons, as well as on account of the danger in handling, and impossibility of safely transporting the substance, it is made into blasting gelatine (see below). The old form of dynamite was made by soaking a porous earth (infusorial earth, kieselguhr) with nitroglycerine.

Guncotton.-When cotton is steeped for half an hour in a cooled mixture of nitric and sulphuric acids, it is converted into cellulose trinitrate or guncotton:

$$
\mathrm{C}_{6} \mathrm{H}_{7} \mathrm{O}_{2}(\mathrm{OH})_{3}+3 \mathrm{HNO}_{3} \rightarrow \mathrm{C}_{6} \mathrm{H}_{7} \mathrm{O}_{2}\left(\mathrm{NO}_{3}\right)_{3}+3 \mathrm{H}_{2} \mathrm{O} \text {. }
$$

The equation, as written above, shows that three hydroxyl groups $\mathrm{OH}$ in the empirical cellulose formula $\mathrm{C}_{6} \mathrm{H}_{10} \mathrm{O}_{5}$ are replaced by three nitrate groups $\mathrm{NO}_{3}$, with simultaneous formation of three molecules of water. The sulphuric acid hastens the reaction and carries it to completion by acting as a dehydrating agent and removing this water (see p. 270). The fibers have the same appear- 
ance as before, but are crisper to the touch. The guncotton is washed thoroughly with water to remove the acids, which would cause slow decomposition and perhaps accidental explosion.

Dried guncotton burns briskly (deflagrates) when set on fire. While wet, it can be moulded and cut without danger. It explodes only when "set off" by a small amount of another explosive. Fulminate of mercury $\mathrm{Hg}(\mathrm{ONC})_{2}$, used in percussion caps, is commonly employed. By such means the explosion is brought about in wet guncotton as easily as in dry.

In pure form guncotton is used only in torpedoes and submarine mines. It explodes too rapidly to be used in fire-arms or for blasting.

Smokeless Powder and Dynamite.-The violence of guncotton is reduced by compressing it, and still more by dissolving it and allowing the solvent to evaporate. Thus, cordite is made by dissolving guncotton ( 65 parts), nitroglycerinc (30 parts) and vaseline (5 parts) in acetone. The resulting paste is rolled and cut into pieces of different dimensions, according to the rate of explosion desired. When the acetone evaporates, the horny cordite remains. These explosives are smokeless because they differ from gunpowder (p. 372) in yielding no solids when they decompose.

Blasting gelatine, giant powder, and other forms of dynamite are made by dissolving guncotton in nitroglycerine. Substances like nitrate of sodium or of ammonium and sawdust or flour are added to adjust the rate of explosion so that, for example, coal may be split up, but not shattered.

High Explosives. - These are substances which develop their explosive affect at an extremely rapid rate, and are used therefore when a shattering effect of great violence is required, for example in bursting shells or in anti-submarine mines. Trinitro-toluene (TNT) is made by nitrating toluene (p. 353), picric acid (trinitro- 
phenol) by nitrating phenol. Picric acid* is a strong acid and forms salts which, with the exception of ammonium picrate, are more sensitive to shock and friction than the acid itself. The action of this explosive on the metals with which it comes in contact must therefore be guarded against. TNT, however, is inert towards metals, and is insensitive towards ordinary shocks. It melts at a low temperature $\left(80^{\circ}\right)$, and so can be readily liquefied and poured into shells. Mixed with ammonium nitrate, it gives amatol (p. 302).

Toxic Gases.- The poisonous substances employed in the Great War were mainly synthetic organic products. Most of these, it is true, were not gases, but liquids or solids of low volatility. Their vaporization was, however, favored by the explosion of the shell in which they were contained, part of the material being converted to gas by the heat of the explosion and the remainder being scattered around in a finely-divided condition. Some of the substances used were so powerful in their action upon the human system that a concentration of 1 part in 10,000,000 in the air was sufficient to incapacitate anyone unprotected with a gas-mask.

The first method employed in gas warfare was to release a highly-volatile poisonous substance (chlorine, or a mixture of chlorine and phosgene $\mathrm{COCl}_{2}$ ) from cylinders under pressure. A cloud of gas was thus evolved, which under favorable wind conditions was carried over the enemy's lines. This cloud method was soon abandoned in favor of the shell method described above. Among the substances thus employed may be mentioned chlorpicrin $\mathrm{CCl}_{3} \cdot \mathrm{NO}_{2}$ (made by the action of bleaching powder and lime upon picric acid) and mustard gas $\left(\mathrm{CH}_{2} \mathrm{Cl} \cdot \mathrm{CH}_{2}\right)_{2} \mathrm{~S}$. The

* Picric acid is an interesting example of the close interrelation of synthetic organic compounds. It is used in dyeing to give a yellow color, in warfare as an explosive and in medicine as an antiseptic. It may happen that picric acid is used in a base hospital to cure the wound that picric acid caused. 
latter substance, the chemical name of which is dichlordiethyl sulphide, may be regarded as diethylether (p. 348) in which oxygen is replaced by sulphur and two hydrogen atoms are replaced by chlorine. It was produced by the action of ethylene $\mathrm{C}_{2} \mathrm{H}_{4}$ upon sulphur monochloride $\mathrm{S}_{2} \mathrm{Cl}_{2}$.

Both of the above substances are actually toxic, that is, a sufficient concentration will induce death. Many other substances were used, however, which merely put the victim temporarily out of action. Among these were lachrymatories (tearproducing substances) such as benzyl bromide $\mathrm{C}_{6} \mathrm{H}_{5} . \mathrm{CH}_{2} \mathrm{Br}$, and sternutatories (sneeze-producing substances) such as diphenylchlorarsine $\left(\mathrm{C}_{6} \mathrm{H}_{5}\right)_{2} \mathrm{AsCl}$. The latter substance is a derivative of arsine $\mathrm{AsH}_{3}$, the three hydrogen atoms being replaced by two phenyl groups $\mathrm{C}_{6} \mathrm{H}_{5}$ and one chlorine. Mustard gas in very low concentration also acted as a skin irritant.

The methods employed for obtaining protection against toxic gases have already been discussed (p. 421).

Exercises.-1. Write graphic formulæ (see Chapter XXIX) for the following synthetic essences: ethyl formate, ethyl butyrate, benzaldehyde, diphenyl ether.

2. What chemical change would occur after mixing nitroglycerine with sodium hydroxide solution? Name the kind of reaction and give the equation.

3. When nitroglycerine explodes, in what relative volumes are steam, carbon dioxide, and nitrogen produced? What principle is used in answering this question?

4. Make an equation for the decomposition of guncotton, similar to that given for nitroglycerine (p. 482).

5. Make an equation for the denitration of guncotton by an alkali.

6. Write the graphic formulæ for the following substances: acetic anhydride, trinitrotoluene, picric acid, phosgene, chlorpicrin, mustard gas, benzyl bromide, diphenylchlorarsine. 


\section{CHAPTER XLI \\ IRON, NICKEL, COBALT \\ IroN $\mathrm{Fe}$}

Occurrence. - Iron as a free metal is rarer than gold (see p. 54). Masses of iron are found in meteorites, lately arrived on earth from airless space. Minute particles of the metal may be detected in igneous rocks, freshly broken open. Compounds of iron are very widely distributed. Pyrite $\mathrm{FeS}_{2}$ (fools' gold) is used mainly as a source of sulphur for sulphuric acid. The ores which yield iron itself are:

$\mathrm{Fe}_{2} \mathrm{O}_{3}$ (ferric oxide), red hæmatite. Red when pulverized.

$2 \mathrm{Fe}_{2} \mathrm{O}_{3}, 3 \mathrm{H}_{2} \mathrm{O}$ (hydrated ferric oxide), brown hæmatite.

$\mathrm{Fe}_{3} \mathrm{O}_{4}$ (magnetic oxide of iron), magnetite. Black when pulverized.

$\mathrm{FeCO}_{3}$ (ferrous carbonate), spathic iron ore.

The carbonate, mixed with clay (clay iron-stone), furnishes most - of the iron in Great Britain, but less than one per cent of it in the United States. The ore is first calcined to produce the oxide.

The Blast Furnace.- Coke is used to reduce the oxides and as fuel. The carbon monoxide $\mathrm{CO}$, produced by the burning of the coke and air, is the actual reducing agent:

$$
\begin{aligned}
& \mathrm{Fe}_{3} \mathrm{O}_{4}+\mathrm{CO} \rightleftarrows 3 \mathrm{FeO}+\mathrm{CO}_{2} . \\
& \mathrm{FeO}+\mathrm{CO} \rightleftarrows \mathrm{Fe}+\mathrm{CO}_{2} .
\end{aligned}
$$

Since the ores contain rocky material (gangue), such as silica $\mathrm{SiO}_{2}$ and silicates of aluminium, limestone is added in the proportion required to give a fusible slag. 
The blast furnace (Fig. 111) is an iron structure 40 to 100 feet high, lined with fire-brick. A circular pipe delivers a blast of pre-heated compressed air to several nozzles (tuyères) near the foot. The ore, coke and limestone are admitted at the top. Reduction of the ore occurs continuously as the solid materials, passing downwards, become hotter and hotter through combustion of the coke. The melted iron and slag (immiscible) finally collect in two layers in the hearth or crucible at the bottom. From time to time the slag is allowed to flow from an opening near the top of the crucible, and the iron from a similar opening at the bottom. Plugs of wet clay close the openings and are instantly baked hard. The iron is taken in ladles to other parts of the plant, or is cast into "pigs" in steel moulds and chilled in water.

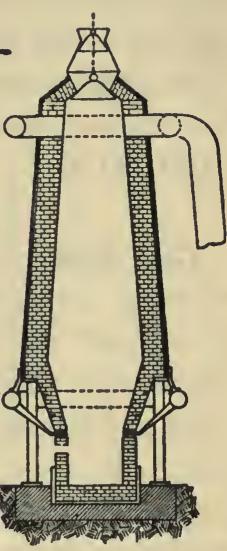

FIG. 111

Reactions in the Blast Furnace.-The actions (see equations, above) are both reversible and the carbon dioxide formed tends to react to reproduce the original materials. At any particular temperature it is necessary therefore to keep the proportion of $\mathrm{CO}_{2}$ to $\mathrm{CO}$ in the furnace gases below a certain value in order to prevent the reversal of the reactions. This proportion of $\mathrm{CO}_{2}$ to $\mathrm{CO}$ is regulated, however, by a third reversible reaction:

$$
\mathrm{C}+\mathrm{CO}_{2} \rightleftharpoons 2 \mathrm{CO} \text {. }
$$

At high temperatures (above $1000^{\circ}$ ) this reaction is almost complete in the forward direction. As the temperature falls, the proportion of $\mathrm{CO}_{2}$ in the equilibrium mixture increases rapidly.

If all three reactions had time to attain equilibrium conditions in the blast furnace, reduction of $\mathrm{Fe}_{3} \mathrm{O}_{4}$ to $\mathrm{FeO}$, and of $\mathrm{FeO}$ to $\mathrm{Fe}$, would occur at about $645^{\circ}$ and $700^{\circ}$ respectively, and the residual gases would contain very little $\mathrm{CO}$. In practice, however, the 
slowness of the reactions necessitates the use of much higher temperatures and a large excess of $\mathrm{CO}$. The gases that escape are therefore combustible, and are led off through openings near the top of the furnace and used for pre-heating the air-blast.

In 1920, nearly $40,000,000$ tons of pig iron were produced in the United States. The production in Great Britain exceeded $8,000,000$ tons.

Cast Iron.- Pig iron contains 4 to 5 per cent of carbon and varying amounts of silicon (as silicide of iron), phosphorus (as phosphide) and sulphur (as sulphide). These impurities lower the melting-point from $1510^{\circ}$ to about $1100^{\circ}$. The material is hard and brittle. Most of it is made into wrought iron or steel, but some is used in making objects of cast iron, such as ranges, stoves, pipes, and radiators, which are not to be subjected to shocks or strains. Cast iron expands on solidifying and forces itself into the details of the mould.

By adding pyrolusite $\mathrm{MnO}_{2}$ in the blast furnace, cast iron containing from 20 per cent of manganese (spiegeleisen) up to 80 per. cent (ferromanganese), and carbon up to 6 per cent, is made for use in steel manufacture.

Wrought Iron- Wrought iron is commercially pure iron. The broken pigs are placed in a reverberatory furnace (Fig. 112), the hearth of which is covered with a bed of hæmatite ore $\mathrm{Fe}_{2} \mathrm{O}_{3}$ and silicates. The flames and heated gases, deflected by the low roof, play upon the iron and melt it. The oxygen in the hæmatite combines with the carbon, phosphorus, sulphur, and silicon, giving the oxides. The mass is worked vigorously with iron rods upon the bed of hæmatite (puddled), carbon monoxide escapes, and the iron becomes more viscous as its melting-point rises on account of the removal of the impurities. Finally, it is collected in balls (blooms) on the iron rods. The treatment occupies an hour and a half. To press out the slag, the blooms are first passed 
through a squeezer and then put through the rolls. The resulting bars are repeatedly cut, "piled" in a bundle, reheated, and rolled. These treatments, and the presence of a little slag distributed through the mass, give wrought iron the valuable properties which distinguish it from all other iron products, namely

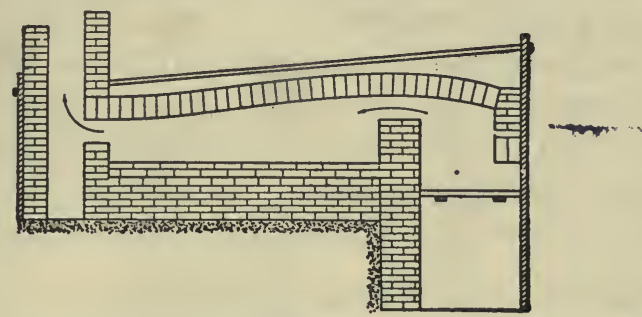

FIG. 112

its fibrous structure and its extreme toughness. On account of these properties it is used for anchors, chains, and bolts. It is drawn into wire, and, when heated, can be cut, shaped, and welded under the hammer. The impurities having been greatly reduced (to 0.1 or 0.2 per cent), this iron is much less fusible than cast iron, and is used for fire bars.

Crucible Steel.- Steel contains 0.75 to 1.5 per cent of carbon, and is freed as far as possible from other impurities. Small lots, for special purposes, are made in clay (or graphite and clay) crucibles in melts of 60 to 100 pounds. The charge in Sheffield consists of blister steel, i.e., carburized Swedish wrought iron of varying carbon content. The modern method is to melt "melting bar," a very pure open hearth steel with charcoal, or even pure pig iron. Crucible steel is used in making razors (1.5 per cent $\mathrm{C}$ ), tools ( 1 per cent $\mathrm{C})$, dies ( 0.75 per cent $\mathrm{C})$, pens, needles, and cutlery.

Electric heating (e.g. in the Héroult furnace), recently introduced, permits the steel maker, first to wash the molten iron with 
basic slags of high oxidizing power until perfectly pure, and then by suitable additions to give it any required final composition.

Properties of Steel.- Cast iron can be melted and cast, is hard when chilled, but can not be forged or rolled. Wrought iron is slag-bearing and malleable, and is not hardened by quenching from a high temperature. It is never cast. Steel is free from slag, being cast from an originally liquid condition. If its carbon content is high enough, it can be hardened by quenching. Steel has also greater tensile strength* than wrought iron, and it can be permanently magnetized. In addition, high carbon steel can also be tempered to the required degree of hardness.

Tempering. - To understand the last fact, it must be noted that carbon dissolves readily in molten iron, and is partly converted to a carbide of iron ( $\mathrm{Fe}_{3} \mathrm{C}, 6.6$ per cent $\mathrm{C}$ by weight) in solution. As the temperature falls, the solubility of carbon in iron diminishes. When white hot steel (up to 2 per cent C) is suddenly chilled, there is no time for any changes to occur during the cooling, and a supersaturated solid solution is obtained which is very hard and brittle. When, however, the cooling is slow, some of the carbon separates in minute crystals of cementite, carbide of iron $\mathrm{Fe}_{3} \mathrm{C}$, until at about $700^{\circ}$ there remains only 0.9 per cent of carbon in solution. At this temperature the solid solution breaks down into a mechanical mixture of pure iron which is soft, and carbide of iron which is hard. Steel is thus a mixture, and not homogeneous, when slowly cooled. When therefore hard, chilled steel is heated once more for the purpose of tempering, the extent to which the softer material is formed depends on the temperature reached, and on the rate and duration of the cooling permitted. By varying these the degree of hardness allowed to remain can be adjusted.

* Tensile strength or tenacity is measured by the weight (in kilograms) required to break a wire of the metal $1 \mathrm{sq}$. mm. in section. Lead 2.6, copper 51, iron 71, steel 91 . 


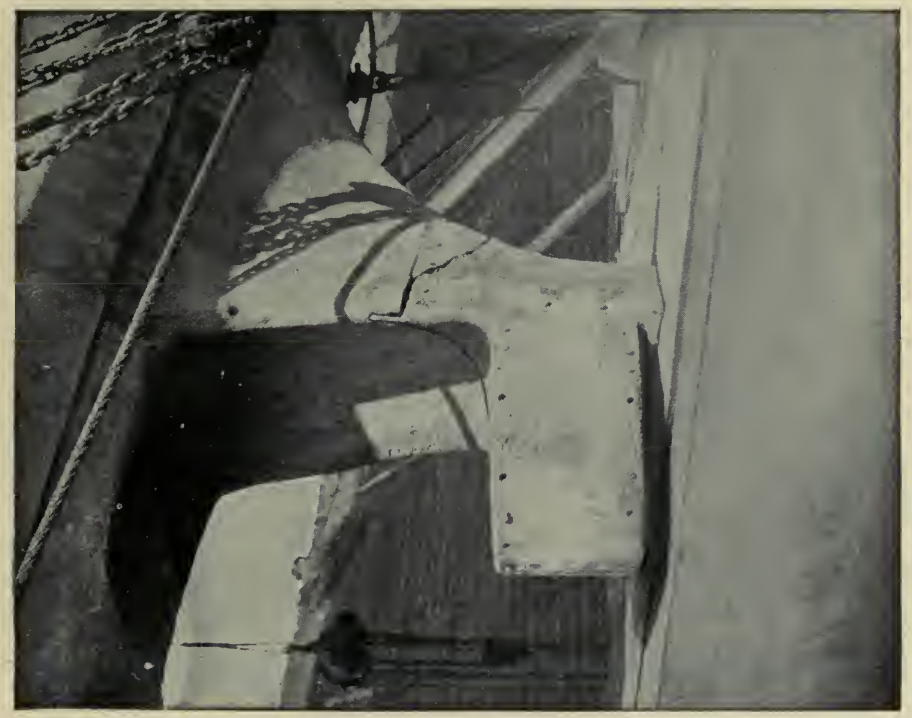

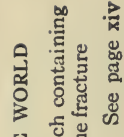

国

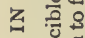

갠

茯

3 芩

雚

就药

ज्ञ

원

일

国焉

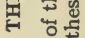

บ ณั

氜 ह

这

乙 ⿹ㅛㅇ

密 豆

先 हू

乙

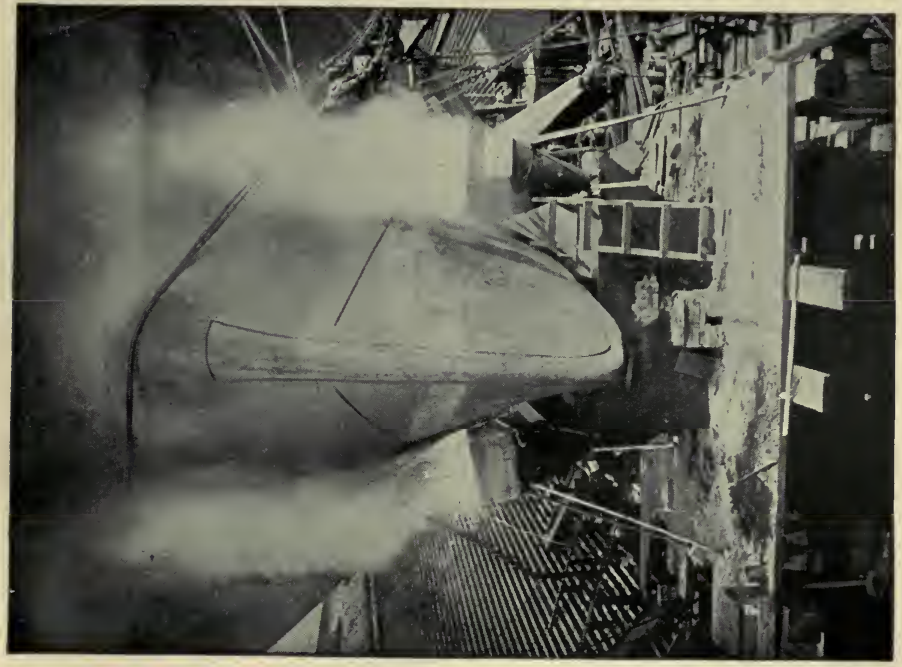

乙. छี

⿰讠)

ن స

D

- 1 奥

ह5:

z

¿

융 응

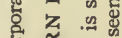

ठํํㅇ

感告

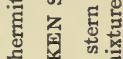

है ठ है

매

สี

党

205

‡

을

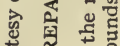

究

วี

동 

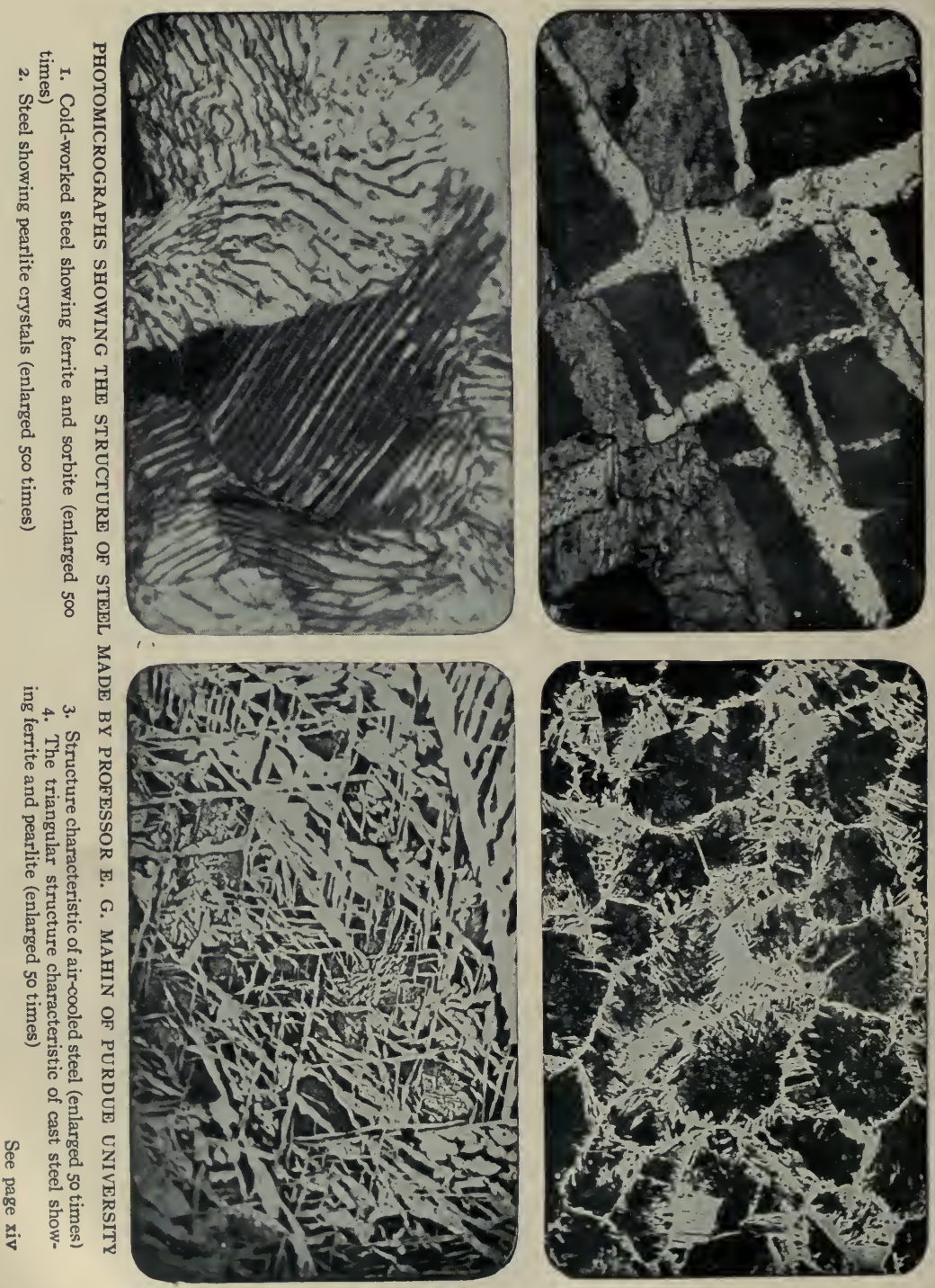
Phosphide of iron makes steel brittle when cold (" cold short "). Sulphide of iron makes it brittle when hot ("red short"), and unsuitable for forging. Hence phosphorus and sulphur are reduced to the lowest possible amounts.

Bessemer Process.- Pig iron is melted and run into the converter (Fig. 113), which is lined with fire-brick, measures about 15 by 8 feet, and holds 15 tons. An air-blast, entering through one axle, blows through the metal from tuyères at the bottom. The oxidation of the carbon and silicon, which raises the temperature above the melting-point of pure iron, is over in 20 minutes. Spiegeleisen is then added to give the desired
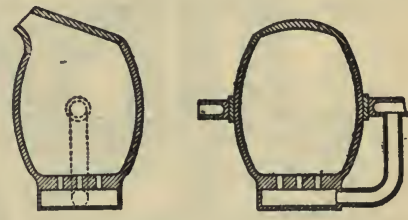

Fig. 113 percentage of carbon and manganese, and the liquids, first the slag and then the iron, are poured into ladles, and the metal is cast.

Sulphur and phosphorus are not removed by the air. If present in too great amounts, they are removed by lining the converter with basic material such as magnesium and calcium carbonates (Thomas-Gilchrist process). The slag then contains phosphates, and is valuable as a fertilizer.

Bessemer steel is used for heavy and light machinery castings, and is rolled into bridge and structural iron. It contains from 0.1 per cent (soft) to 1 per cent (hard) of carbon.

\section{0}

Open-hearth (Siemens-Martin) Process.-In this process pig iron and scrap iron (up to 75 tons) are melted on a hearth lined with fire-brick and sand (Fig. 114). At a later stage hæmatite is added to furnish oxygen (as in puddling). To secure economically the temperature necessary to keep the pure product (iron) fused, Siemens contributed the idea of preheating the fuel gas and air by a regenerative device. The spent air and gas pass out through a
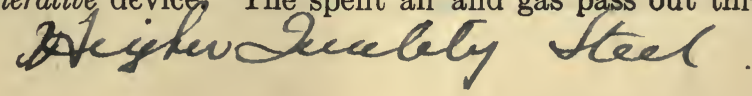
checkerwork of brick. When this has become hot, the valves are reversed, the gas and air now enter through the hot brickwork and pass out through the checkerwork on the opposite side, raising its temperature. The direction of the gases is changed every twenty minutes or so, and the whole operation lasts 8 to 12 hours.

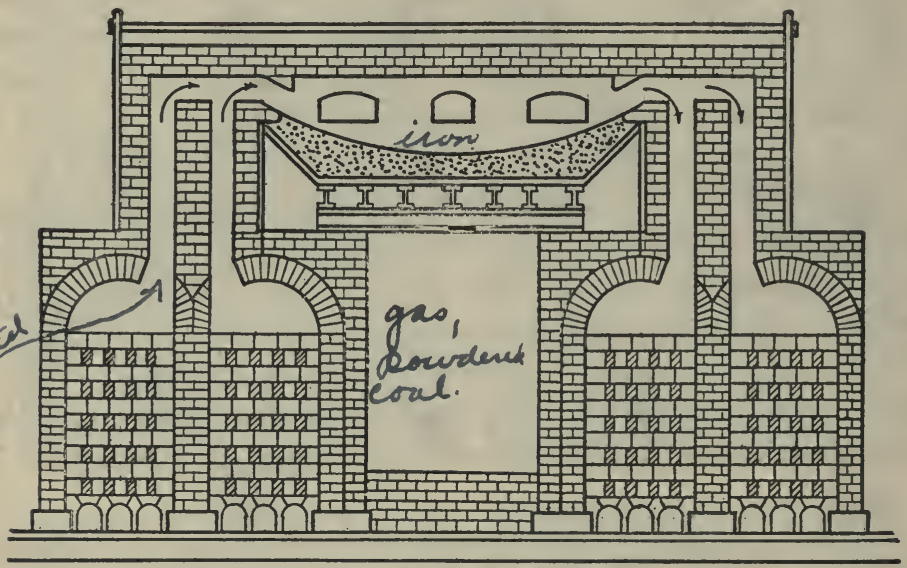

FIG. 114

Towards the end some aluminium is added to combine with oxygen (present as $\mathrm{CO}$ ), and give sounder ingots. Recently, iron fontaining 10 to 15 per cent of titanium has been added instead. Whe titanium combines with both nitrogen and oxygen, and passes into the slag. Rails made of steel purified with this element are less liable to breakage and are 40 per cent more durable than ordinary open-hearth rails.

The advantage of this process over that of Bessemer is that it is not hurried and is therefore under better control. The material can be tested by sample at intervals, and defects corrected. The product is of better and more uniform quality.

As in the Bessemer process, phosphorus and sulphur are removed by using a basic lining. 
Open-hearth steel is used for the better class of rails, for railway bridges, for shafts, armor-plate, and heavy guns, and wherever the steel is subject to much vibration.

Steel Alloys. - We must distinguish between manganese, aluminium, silicon, or titanium added in small amounts ("medicine") to purify the iron, and passing (in combination) into the slag, as described in preceding sections, and the present subject, which concerns metals added so as to produce regular alloys.

Manganese steel ( 7 to 20 per cent $\mathrm{Mn}$ ) is exceedingly hard even when cooled slowly. It therefore does not lose its temper readily when heated by friction. It is used for the jaws of rock-crushing machinery and for burglar-proof safes.

Chromium-vanadium steel ( 1 per cent $\mathrm{Cr}, 0.15$ per cent Va) has great tensile strength, can be bent double while cold, and offers great resistance to changes of stress, and to torsion. It is used for frames and axles of automobiles, and for connecting rods.

Tungsten steel (tungsten 8 to 20 per cent, and chromium 3 to 5 per cent) is used for tools in high-speed metal turning, as it can become red hot (from friction) without loss of temper.

Nickel steel (containing 2 to 4 per cent of nickel) resists corrosion, and has a very high limit of elasticity and great hardness. It is used for armor-plate, wire cables, and propeller shafts. The alloy with 36 per cent nickel, called invar, is practically nonexpansive when heated and is valuable for meter-scales and pendulum rods.

Duriron or tantiron (15 per cent $\mathrm{Si}$ ) is rustproof and is not attacked by sulphuric, nitric or acetic acid, hot or cold, dilute or concentrated. Vessels made of this alloy are therefore used industrially in acid concentrations.

Properties of Pure Iron.- Pure iron may be made by electrolysis, or by reduction of a pure salt by hydrogen. It has 
a white luster, is very tough, and melts at about $1510^{\circ}$. The purest iron does not rust in cold water.

Ordinary iron rusts in moist air or under water, forming a hydrated ferric oxide $3 \mathrm{Fe}_{2} \mathrm{O}_{3}, \mathrm{H}_{2} \mathrm{O}$. The impurities act as contact agents. The rust is a brittle, porous, non-adherent coating, which does not protect the metal below. Oil protects iron from rusting because, although oxygen penetrates the oil, being soluble in it, moisture does not. Iron displaces hydrogen from hydrochloric and sulphuric acids, giving ferrous salts:

$$
\mathrm{Fe}+2 \mathrm{H}^{+} \rightarrow \mathrm{Fe}^{++}+\mathrm{H}_{2} \uparrow \text {. }
$$

The impurities - carbide, sulphide, and phosphide - produce hydrocarbons, hydrogen sulphide, and phosphine $\mathrm{PH}_{3}$, and the last two confer an odor on the escaping gas.

Iron burns in oxygen, and acts when heated upon steam, in both cases producing magnetic oxide of iron $\mathrm{Fe}_{3} \mathrm{O}_{4}$ (p. 51). A thin film of this oxide is adherent, and protects the iron ("Russia" iron). The articles to be treated are put into a closed retort and exposed first to a current of superheated steam and then to a current of producer gas (p. 337) to reduce any higher oxides that may have been formed. Watch hands, buckles and the like may be given a protective coating by dipping them in an oxidizing bath such as melted saltpeter. Another method of rust proofing is to immerse iron articles in a hot solution of ferrous phosphate. This salt is appreciably hydrolyzed in solution, and the free acid acting upon the iron converts its surfaces into an adherent film of basic phosphate.

Iron has Two Valences.-One atomic weight of iron may hold two or three atomic weights of a univalent element in combination. Thus, we have ferrous chloride $\mathrm{FeCl}_{2}$ and ferric chloride $\mathrm{FeCl}_{3}$, the bromides $\mathrm{FeBr}_{2}$ and $\mathrm{FeBr}_{3}$, the oxides $\mathrm{FeO}$ and $\mathrm{Fe}_{2} \mathrm{O}_{3}$, ferrous sulphate $\mathrm{FeSO}_{4}$ and ferric sulphate $\mathrm{Fe}_{2}\left(\mathrm{SO}_{4}\right)_{3}$, and so forth. It may therefore be bivalent or trivalent, according to the chemical conditions. 
The ferrous salts are pale green and give colorless solutions, containing ferrous-ion $\mathrm{Fe}^{++}$. The ferric salts, containing the ion $\mathrm{Fe}^{++}$, are usually yellow in solution, on account of ferric hydroxide produced by hydrolysis.

Ni/. Ferrous Sulphate $\mathrm{FeSO}_{4}$ - When the bath of dilute sulphuric acid, used in cleaning iron for making tin-plate (p. 508), and galvanized iron (p. 449), is becoming exhausted, scrap iron is thrown in to use up the rest of the acid. The solution gives, on evaporation, pale green crystals of ferrous sulphate, $\mathrm{FeSO}_{4}, 7 \mathrm{H}_{2} \mathrm{O}$ (copperas or green vitriol). The salt is used in making ink (see p. 499) and rouge (see p. 496), and in purifying water (p. 470).

N. 5 . Chlorides of Iron.- Ferrous chloride $\mathrm{FeCl}_{2}$ is obtained in solution when iron displaces hydrogen from hydrochloric acid ( $p$. 52 ), and is isolated by evaporation. The hydrate $\mathrm{FeCl}_{2}, 4 \mathrm{H}_{2} \mathrm{O}$ is pale green, the anhydrous salt colorless. When chlorine is dissolved in the solution, or when the latter, acidified with hydrochloric acid, is exposed to the air, ferric chloride $\mathrm{FeCl}_{3}$ is produced:

$$
\begin{gathered}
2 \mathrm{FeCl}_{2}+\mathrm{Cl}_{2} \rightarrow 2 \mathrm{FeCl}_{3} \text { or } 2 \mathrm{Fe}^{++}+\mathrm{Cl}_{2} \rightarrow 2 \mathrm{Fe}^{+1+}+2 \mathrm{Cl}^{-} \\
4 \mathrm{FeCl}_{2}+\mathrm{O}_{2}+4 \mathrm{HCl} \rightarrow 4 \mathrm{FeCl}_{3}+2 \mathrm{H}_{2} \mathrm{O}
\end{gathered}
$$

and is familiar in the form of a yellow hydrate $\mathrm{FeCl}_{3}, 6 \mathrm{H}_{2} \mathrm{O}$ obtained by evaporation. Other oxidizing agents, such as nitric acid, produce the same change.

Ferric chloride, in solution, has an acid reaction, due to hydrolysis. It is reduced to ferrous chloride by shaking the solution, or more quickly by boiling it, with iron filings:

$$
2 \mathrm{FeCl}_{3}+\mathrm{Fe} \rightarrow 3 \mathrm{FeCl}_{2} \text { or } 2 \mathrm{Fe}^{+++}+\mathrm{Fe} \rightarrow 3 \mathrm{Fe}^{++} \text {. }
$$

Hydroxides of Iron.-Ferric hydroxide $\mathrm{Fe}(\mathrm{OH})_{3}$ appears as a brown precipitate when an equivalent amount of sodium hydroxide is added to a solution of a ferric salt:

$\mathrm{FeCl}_{3}+3 \mathrm{NaOH} \rightarrow \mathrm{Fe}(\mathrm{OH})_{3} \downarrow+3 \mathrm{NaCl}$. 
When only a little sodium hydroxide is added, the brown procipitate, formed locally, redissolves to give a deep reddish solution. This contains ferric hydroxide in colloidal suspension. The sodium chloride and unused ferric chloride can be separated by dialysis (p. 442), and a pure colloidal suspension of the hydroxide obtained.

Ferrous hydroxide $\mathrm{Fe}(\mathrm{OH})_{2}$ produced by precipitation, is white when pure, but becomes quickly green and then brown by oxidation.

Oxides of Iron.- When ferric hydroxide is heated, ferric oxide $\mathrm{Fe}_{2} \mathrm{O}_{3}$ remains as a red mass:

$$
2 \mathrm{Fe}(\mathrm{OH})_{3} \rightarrow \mathrm{Fe}_{2} \mathrm{O}_{3}+3 \mathrm{H}_{2} \mathrm{O} \uparrow \text {. }
$$

It is made by calcining (roasting) ferrous carbonate, ferrous sulphide, or ferrous sulphate:

$$
\begin{aligned}
4 \mathrm{FeSO}_{4}+\mathrm{O}_{2} & \rightarrow 2 \mathrm{Fe}_{2} \mathrm{O}_{3}+4 \mathrm{SO}_{3} . \\
4 \mathrm{FeS}+7 \mathrm{O}_{2} & \rightarrow 2 \mathrm{Fe}_{2} \mathrm{O}_{3}+4 \mathrm{SO}_{2} .
\end{aligned}
$$

The oxide, when pulverized in a ball mill, gives a powder of more or less brilliant red color commonly used in paints (Venetian red and Indian red). That from ferrous sulphate is rouge, used in polishing plate glass and lenses, and as a pigment. Yellow ochre is a natural hydrated ferric oxide $2 \mathrm{Fe}_{2} \mathrm{O}_{3}, 3 \mathrm{H}_{2} \mathrm{O}$, which acquires various depths of color during calcination, and constitutes the sienna and umber used for paints.

Magnetic Oxide of Iron $\mathrm{Fe}_{3} \mathrm{O}_{4}$ is regarded as a compound of ferrous and ferric oxides $\mathrm{FeO}, \mathrm{Fe}_{2} \mathrm{O}_{3}$. It is formed by strongly heating ferric oxide:

$$
6 \mathrm{Fe}_{2} \mathrm{O}_{3} \rightarrow 4 \mathrm{Fe}_{3} \mathrm{O}_{4}+\mathrm{O}_{2}
$$

and is formed when iron is oxidized at a high temperature by oxygen, air (blacksmith's scale), or steam. It can be magnetized, and natural specimens are often strongly magnetic (lodestone). 
Ferrous oxide $\mathrm{FeO}$ is a black substance made by cautious reduction of ferric oxide by a stream of hydrogen.

Ferrous Carbonate $\mathrm{FeCO}_{3}$. - The carbonate occurs in nature as an impurity in clay, in clay iron stone, and pure as siderite. Water containing carbonic acid dissolves it, giving the bicarbonate:

$$
\mathrm{FeCO}_{3}+\mathrm{H}_{2} \mathrm{CO}_{3} \rightarrow \mathrm{Fe}\left(\mathrm{HCO}_{3}\right)_{2} \text {. }
$$

Thus well and river waters all contain at least traces of ferrous bicarbonate as a part of their hardness. Exposure to the air causes oxidation and, as ferric carbonate is not stable, rust (ferric hydroxide or a hydrated ferric oxide) is deposited:

$$
4 \mathrm{Fe}\left(\mathrm{HCO}_{3}\right)_{2}+\mathrm{O}_{2}+10 \mathrm{H}_{2} \mathrm{O} \rightarrow 4 \mathrm{Fe}(\mathrm{OH})_{3}+8 \mathrm{H}_{2} \mathrm{CO}_{3} \text {. }
$$

This red deposit is seen in white vessels in which such water drips or stands. It also "yellows" goods washed in such water, if the carbonate of iron is not previously precipitated by soda or some other softening agent (see p. 391).

Ferro- and Ferri-cyanides.- Potassium ferrocyanide $\mathrm{K}_{4} \mathrm{Fe}$ $(\mathrm{CN})_{6}$, or yellow prussiate of potash, is a pale yellow, soluble salt. The iron is contained in the negative radical and ion $\left[\mathrm{Fe}(\mathrm{CN})_{6}\right]^{==}$, and the solution therefore gives the reactions of this ion, and not of ferrous- or ferric-ion. One of the double decompositions of this salt - namely, that with ferric salts - is important because it gives a gelatinous precipitate of Prussian blue (ferric ferrocyanide):

$$
4 \mathrm{FeCl}_{3}+3 \mathrm{~K}_{4} \mathrm{Fe}(\mathrm{CN})_{6} \rightarrow \mathrm{Fe}_{4}{ }^{\mathrm{III}}\left(\mathrm{Fe}(\mathrm{CN})_{6}\right)_{3^{\mathrm{VV}}} \downarrow+12 \mathrm{KCl} \text {. }
$$

Prussian blue is employed in making paints, and is the usual pigment in laundry blueing. Although insoluble, it is such a fine powder that it appears to dissolve in the water. It is used in the laundry to correct the yellowish tint derived from the ferrous bicarbonate in the water (p. 391). If the goods are not freed by rinsing from soap and soda, however, the alkali liberated by 
hydrolysis of the latter enters into double decomposition with the Prussian blue and produces more rust:

$$
\mathrm{Fe}_{4}\left(\mathrm{Fe}(\mathrm{CN})_{6}\right)_{3}+12 \mathrm{NaOH} \rightarrow 4 \mathrm{Fe}(\mathrm{OH})_{3} \downarrow+3 \mathrm{Na}_{4} \mathrm{Fe}(\mathrm{CN})_{6} .
$$

Potassium Ferricyanide $\left.\mathrm{K}_{3} \mathrm{I}\left[\mathrm{Fe}(\mathrm{CN})_{6}\right]\right]^{\mathrm{III}}$. - The difference between this and the preceding salt can be seen by writing the formulæ thus: $4 \mathrm{KCN}, \mathrm{Fe}(\mathrm{CN})_{2}$ and $3 \mathrm{KCN}, \mathrm{Fe}(\mathrm{CN})_{3}$. In the ferricyanide the iron is trivalent and the negative ion $\mathrm{Fe}(\mathrm{CN})_{6} \equiv$ is also trivalent as a whole. It is a soluble salt, of red color, made by oxidizing the ferrocyanide. With ferric salts it gives only a brown solution, but with ferrous salts it gives a deep blue precipitate of ferrous ferricyanide - Turnbull's blue:

$$
3 \mathrm{FeCl}_{2}+2 \mathrm{~K}_{3} \mathrm{Fe}(\mathrm{CN})_{6} \rightarrow \mathrm{Fe}_{3}\left(\mathrm{Fe}(\mathrm{CN})_{6}\right)_{2} \downarrow+6 \mathrm{KCl} \text {. }
$$

We can distinguish ferrous-ion $\mathrm{Fe}^{++}$from ferric-ion $\mathrm{Fe}^{+++}$by this reaction. An equally sharp distinction is obtained by adding potassium thiocyanate, for, although the ferrous and ferric thiocyanates are both soluble, the latter is blood red in color (see p. 235).

j Blue-Prints. - Some ferric salts are reduced to ferrous salts when exposed to light. Thus ferric oxalate $\mathrm{Fe}_{2}\left(\mathrm{C}_{2} \mathrm{O}_{4}\right)_{3}$ will keep in the dark, but in light gives ferrous oxalate $\mathrm{FeC}_{2} \mathrm{O}_{4}$ :

$$
\mathrm{Fe}_{2}\left(\mathrm{C}_{2} \mathrm{O}_{4}\right)_{3} \rightarrow 2 \mathrm{FeC}_{2} \mathrm{O}_{4}+2 \mathrm{CO}_{2} \uparrow \text {. }
$$

When paper is dipped in ferric oxalate solution and dried, and a fern (or ink drawing on transparent paper) is placed over the prepared sheet, sunlight will reduce the iron to the ferrous condition excepting where the fern or ink lines protect it from the light. When the sheet is now dipped in potassium ferricyanide solution (developer), the ferric oxalate gives only the brown substance which can be washed out. But the parts exposed to the light turn deep blue from the precipitation of ferrous ferricyanide in the paper. The pattern is white on a blue ground. In regular blueprint paper ammonium-ferric citrate takes the place of the oxa-

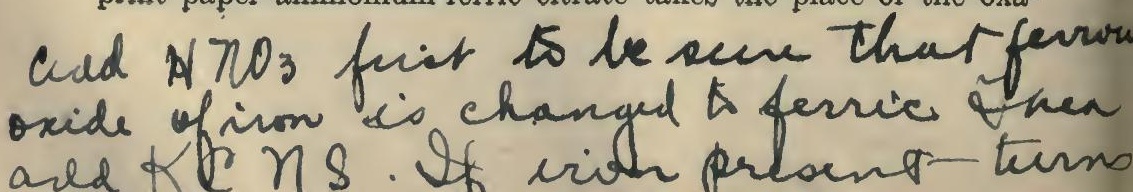


late, and the ferricyanide has already been applied to the paper, so that only exposure and washing remain to be done.

The student may make blue-prints for his own use as follows. Dissolve $10 \mathrm{~g}$. of potassium ferricyanide in 100 c.c. of water, and $13 \mathrm{~g}$. of ammonium-ferric citrate in a second 100 c.c. Mix equal volumes of the two solutions and filter if there is any precipitate. Paint evenly over the paper with a clean camel's-hair brush, dry, and keep in a dark place until required.

Ink.-Writing ink is commonly made by adding ferrous sulphate to an extract of nut-galls. The active constituent of this extract is tannic acid, useful also in dyeing (p. 475) and tanning leather (p.533). Tannic acid is not, strictly speaking, a single substance, but a mixture of complex phenolic acids (see p. 353). Eerrous tannate is soluble and almost colorless, but is slowly oxidized, when exposed to air, to the insoluble, black ferric tannate. To make the writing visible from the first, a blue or black dye is added to the ink.

Stains of fresh writing ink, being soluble, can usually be washed out with water, if the latter is used at once. After the oxidation has occurred, the ferric tannate must be reduced again, by soaking the part for 12 hours or longer in fmmonium oxalate solution, and the ink can then be washed out. Rust stains are often rendered soluble by ammonium oxalate also.

Q cuit

Cobalt Co and Nickel Ni

These metals, like iron, are attracted by a magnet. Cobalt, To like iron, has two series of compounds, in which it is bivalent and trivalent, respectively. In its salts, nickel is bivalent only, but the oxide and hydroxide $\mathrm{Ni}_{2} \mathrm{O}_{3}$ and $\mathrm{Ni}(\mathrm{OH})_{3}$ are also known.

In olden times ores containing cobalt and nickel were frequently mistaken for iron and copper ores, and were treated accordingly. Failure to isolate the expected metals was regarded as due to supernatural influences, hence the word cobalt is derived from the 
German Kobald, an evil spirit (akin to the English "goblin "), while the connection between nickel and the chief of evil spirits is obvious. Cobalt continued to justify its name until very recently; only within the last few years have any applications of the metal been discovered. Nickel, on the other hand, has long ago found many uses.

Cobalt. - The metal has a silvery luster, tinged faintly with pink, and does not tarnish. It displaces hydrogen very slowly from dilute acids, but is acted upon rapidly by nitric acid.

An alloy of cobalt, chromium and tungsten (stellite) is used for high-speed tools. When heated, it keeps its temper better than the steel alloys (p. 493).

The oxide is used as a pigment in the glass and china industries (p. 362).

Cobaltous chloride $\mathrm{CoCl}_{2}, 6 \mathrm{H}_{2} \mathrm{O}$ is red in color, and when partially or wholly dehydrated becomes deep blue. Writing made with a dilute solution of this salt leaves pink traces so faint as to be invisible. But, when the paper is warmed, the hexahydrate is decomposed, and the writing appears blue. When the breath is now blown on the writing, it disappears once more (sympathetic ink).

Metallurgy of Nickel.- Nickel occurs in all iron meteorites. The chief source of nickel is pentlandite, a mixture of nickel, copper and iron sulphides, from Sudbury, Ontario. The ore is roasted and smelted and finally bessemerized (p. 491). The result is an alloy of nickel and copper which is much used for sheet metal work under the name of monel metal. Pure nickel is separated from the copper by an electrolytic process (see copper, p. 515), or by the Mond process (see below). In 1918, more than $1,500,000$ tons of nickel ore (approximating 3 per cent $\mathrm{Ni}$ ) were smelted.

Properties and Uses of the Metal. - Nickel is a white, hard, malleable metal which takes, and keeps, a high polish even in 
moist air. Nickel-plating, deposited electrolytically on iron, has the same qualities. The metal is used also in alloys such as monel metal (copper, nickel, approximately $1: 1$ ), German silver (copper, zinc, nickel, $2: 1: 1$ ), and nickel coinage (copper, nickel, $3: 1$ ). Nickel steel is used for armor-plate.

Compounds of Nickel.-These salts are green, and give green solutions $\left(\mathrm{Ni}^{++}\right)$. The sulphate $\mathrm{NiSO}_{4}, 7 \mathrm{H}_{2} \mathrm{O}$ is familiar, as is also the double salt, ammonium-nickel sulphate $\left(\mathrm{NH}_{4}\right)_{2} \mathrm{SO}_{4}$, $\mathrm{NiSO}_{4}, 6 \mathrm{H}_{2} \mathrm{O}$, used in nickel-plating. Nickel carbonyl $\mathrm{Ni}(\mathrm{CO})_{4}$ is a volatile, colorless liquid (b.-p. $43^{\circ}$ ). It is formed by passing carbon monoxide over warm, finely divided nickel, and is decomposed again, yielding nickel 99.6 per cent pure, by heating at $180^{\circ}$. These actions are the basis of the Mond process for separating nickel.

Exercises. - 1. Why does paint protect iron from rusting?

2. Why does iron, in time, turn completely into rust, while zinc and aluminium receive only a slight film of tarnish?

3. How could it be ascertained that ferric hydroxide is in colloidal suspension, and not dissolved?

4. Make equations for: (a) the interaction of potassium ferrocyanide and cupric sulphate $\mathrm{CuSO}_{4}$, giving a brown precipitate of cupric ferrocyanide; (b) the action of hydrochloric acid on ferric hydroxide (or rust). 


\section{CHAPTER XLII}

\section{LEAD AND TIN}

LEAD and tin are the best-known metallic members of the family to which the non-metals silicon and carbon also belong. In their compounds they are bivalent or quadrivalent.

\section{LEAD $\mathrm{Pb}$}

Metallurgy of Lead.-The chief ore of lead is galenite PbS. The ore, if rich, is roasted in a reverberatory furnace (Fig. 112, p. 489) until a part has been converted into the oxide $\mathrm{PbO}$ and sulphate $\mathrm{PbSO}_{4}$. The air is then shut off, and the temperature raised, so that these products may oxidize the remaining galenite:

$$
\begin{aligned}
& \mathrm{PbS}+2 \mathrm{PbO} \rightarrow 3 \mathrm{~Pb}+\mathrm{SO}_{2} \uparrow \\
& \mathrm{PbS}+\mathrm{PbSO}_{4} \rightarrow 2 \mathrm{~Pb}+2 \mathrm{SO}_{2} \uparrow .
\end{aligned}
$$

The melted lead flows out.

Ores poorer in lead are sometimes reduced by heating with scrap iron, or with a mixture of iron ore and coke.

The lead usually contains silver and some gold, which are recovered by Parke's process (see silver, p. 519).

Properties of Lead.- Lead is a soft malleable metal, with a bluish-white or grey luster when freshly cut. It is quickly, but only superficially, oxidized by the air. It acts very slowly upon hydrochloric acid, but not upon sulphuric acid (see p. 268). With nitric acid it gives lead nitrate $\mathrm{Pb}\left(\mathrm{NO}_{3}\right)_{2}$ and oxides of nitrogen. Soft water, in presence of air, dissolves it in appreciable amounts as hydroxide $\mathrm{Pb}(\mathrm{OH})_{2}$, and carbonic acid assists the process. Hard water, however, produces a skin of carbonate and sulphate (insoluble) which protects the surface. Hence, lead pipes may 502 
be used for hard water, but not for rain water. In presence of air, acids (even feeble vegetable acids) interact with the metal, which is therefore unsuitable for kitchen utensils.

Uses of Lead. - The metal is rolled into sheets, which are used for lining tanks. Lead pipes are made by pressing the metal, while hot, through dies. Their pliability, and the ease with which they can be cut and soldered, fits them for use in plumbing and for covering electric cables.

Type metal, containing 20 per cent of antimony, is harder than lead, and expands on solidifying. Small shot (p. 340) contain 0.5 per cent of arsenic. Solder (lead, tin $1: 1$ ) remains melted at a lower temperature than pure lead (m.-p. $326^{\circ}$ ) and so can be applied to a lead joint without danger of melting the lead itself.

Oxides.-Lead monoxide $\mathrm{PbO}$ is made by oxidizing melted lead in a current of air. At low temperatures a buff colored powder, massicot, is obtained. When the oxide is allowed to melt, it solidifies to a reddish-yellow, scaley mass - litharge. The oxide is predominantly basic, absorbing carbon dioxide from the air and, with acids, giving salts. It is used in making glass and enamels. Stone and glass can be cemented with a mixture of massicot and glycerine.

Minium or red lead $\mathrm{Pb}_{3} \mathrm{O}_{4}$ is a bright-red powder formed by oxidizing the monoxide in air at 470 to $480^{\circ}$ :

$$
6 \mathrm{PbO}+\mathrm{O}_{2} \rightleftarrows 2 \mathrm{~Pb}_{3} \mathrm{O}_{4} \text {. }
$$

Bed lead is used in making flint glass and in paints.

Lead dioxide $\mathrm{PbO}_{2}$ is a brown powder, made by treating red lead with diluted nitric acid.

Paints.-A paint usually contains three ingredients: 1 . The oil, which hardens ("dries") to a tough resin, being oxidized by the air, and adheres firmly to the surface being painted. 
2. The body, a fine powder which makes the paint opaque. Since the powder does not shrink, it also "fills" the paint and prevents the formation of minute pores which otherwise would appear in the oil after drying. White lead (see below) is the most common material for the body ${ }_{2}$ but zinc oxide and other substances are also used.

3. Except in the case of white paint, a pigment is added. Various oxides, such as minium, colored salts, and lakes (p. 476) are used as coloring matters.

The oil does not "dry" by evaporation but gives a resin by oxidation (see p. 41). Linseed oil and hemp oil are commonly used. They contain glyceryl esters (p. 432) of unsaturated acids, such as that of linoleic acid, $\left(\mathrm{C}_{3} \mathrm{H}_{5}\left(\mathrm{CO}_{2} \mathrm{C}_{17} \mathrm{H}_{31}\right)_{3}\right)$. The unsaturated part of the molecule takes up the oxygen. By previously boiling the oil with manganese dioxide and other oxides, it is rendered more active, and "dries" more quickly.

Plumbers use a cement made of minium and linseed oil, in which the former oxidizes the latter, without access of air being necessary, to make joints tight.

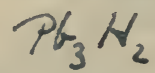

White Lead. - White lead is a basic carbonate $2 \mathrm{PbCO}_{3}, \mathrm{~Pb}-$ $(\mathrm{OH})_{2}$. It is a heavy, white, insoluble, amorphous substance. Mixed with linseed oil, it forms a white paint valued for its " body" or covering power (opacity). Its disadvantage is the darkening, due to formation of the black lead sulphide PbS, which is produced by the hydrogen sulphide in the air (see p. 223). Its poisonous character is also objectionable.

The old Dutch process for making white lead is still used extensively. Gratings (" buckles ") of lead are placed above a little vinegar in small pots. The pots are covered with boards, on which manure or spent tan bark is spread. Other tiers of pots, boards and bark are placed on the first, until the shed is full. Carbon dioxide, warmth and moisture are furnished by the decaying bark. The gratings, by the end of three months, are converted 
into cakes of white lead. The vapor of acetic acid arising from the vinegar may be regarded as a catalytic agent.

In Mild's process melted lead is atomized by a jet of steam, and the lead dust is beaten with vinegar, air, and carbon dioxide in a vat for about seven days. In the French process white lead is precipitated by a stream of carbon dioxide from a solution of the basic acetate.

\section{3}

Other Compounds of Lead.-Lead chromate $\mathrm{PbCrO}_{4}$ is precipitated by adding potassium chromate solution to a solution of a salt of lead. It is used as a pigment (chrome-yellow). Lead chloride $\mathrm{PbCl}_{2}$ (white) is very little soluble in cold water and the iodide $\mathrm{PbI}_{2}$ (yellow) is insoluble. Both are formed by precipitation. Lead sulphide PbS (black) is precipitated by hydrogen sulphide, even from acid solutions (see p. 253). The sulphate $\mathrm{PbSO}_{4}$ is a very insoluble salt. On this account, the workmen in white lead works add a little sulphuric acid to the water they drink.

Zinc, or any of the metals more active than lead, when placed in a solution of a soluble salt of lead, will displace the metal, and deposit it in a mossy form ("lead-tree "):

$$
\mathrm{Zn}+\mathrm{Pb}^{++} \rightarrow \mathrm{Zn}^{++}+\mathrm{Pb} \downarrow \text {. }
$$

The Storage Battery. - In the ordinary lead accumulator the plates consist of leaden gratings. The openings in these gratings are filled with finely divided lead in one plate and with lead dioxide in the other. These, and the dilute sulphuric acid in the cell, are the active substances when the cell is charged. When the battery is used, the $\mathrm{SO}_{4}=$ ions migrate towards the plate filled with the lead (Fig. 115), and convert this lead into a mass of the insoluble lead sulphate: $\mathrm{SO}_{4}=+\mathrm{Pb} \rightarrow \mathrm{PbSO}_{4}+2 \Theta$. This plate therefore becomes negatively charged. Simultaneously, the $\mathrm{H}^{+}$ions move towards the other plate and there reduce to monoxide the lead dioxide with which it is filled.

$$
\mathrm{PbO}_{2}+2 \mathrm{H}^{+} \rightarrow \mathrm{H}_{2} \mathrm{O}+\mathrm{PbO}+2 \oplus \text {. }
$$


This plate consequently becomes positively charged and, by interaction of the lead monoxide with the sulphuric acid, becomes filled, like the negative plate, with lead sulphate. During the discharge, much sulphuric acid is thus removed from the cell fluid, and the approaching exhaustion of the cells can therefore be ascertained

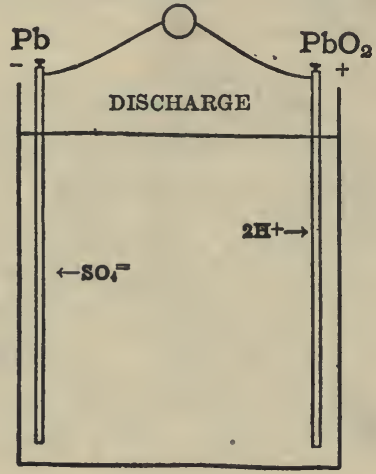

Frg. 115

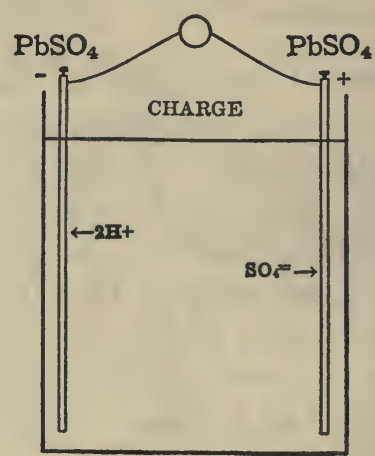

FIG. 116

by measuring the specific gravity of the fluid. The E.M.F. of the current is a little over 2 volts.

The cell may be recharged by passing a high-voltage current through the cell, in the opposite direction (Fig. 116). The $\mathrm{H}^{+}$ ions are attracted to the negative plate and an equivalent number of $\mathrm{SO}_{4}=$ ions are formed, so that only lead remains:

$$
\mathrm{PbSO}_{4}+2 \mathrm{H}^{+}+2 \Theta \rightarrow \mathrm{Pb}+2 \mathrm{H}^{+}+\mathrm{SO}_{4}=\text {. }
$$

Simultaneously, the $\mathrm{SO}_{4}=$ is attracted by the positive plate and, with the lead sulphate there present, forms lead disulphate: $\mathrm{SO}_{4}=+\mathrm{PbSO}_{4}+2 \oplus \rightarrow \mathrm{Pb}\left(\mathrm{SO}_{4}\right)_{2}$. The disulphate is at once hydrolyzed and the filling of this plate is thus changed into lead dioxide: $\mathrm{Pb}\left(\mathrm{SO}_{4}\right)_{2}+2 \mathrm{H}_{2} \mathrm{O} \rightarrow \mathrm{PbO}_{2}+2 \mathrm{H}_{2} \mathrm{SO}_{4}$. Both plates are thus brought back to the condition in which they were before the discharge. 
The last set of charges consumes energy, while the first set liberates energy. Both may be stated in a single equation:

$$
\begin{aligned}
& 2 \mathrm{PbSO}_{4}+2 \mathrm{H}_{2} \mathrm{O} \underset{ }{\rightleftarrows} \mathrm{Pb}+2 \mathrm{H}_{2} \mathrm{SO}_{4}+\mathrm{PbO}_{2} . \\
& \leftarrow \text { discharge }
\end{aligned}
$$

In the Edison cell, when charged, one plate is of iron and the other contains nickelic oxide $\mathrm{Ni}_{2} \mathrm{O}_{3}$. The cell liquid is a solution of potassium hydroxide. When the cell operates, the nickelic oxide is reduced to $\mathrm{Ni}(\mathrm{OH})_{2}$ and the iron is oxidized to $\mathrm{Fe}(\mathrm{OH})_{2}$, an action which delivers energy:

$$
\mathrm{Fe}+3 \mathrm{H}_{3} \mathrm{O}+\mathrm{Ni}_{2} \mathrm{O}_{3} \rightleftarrows \mathrm{Fe}(\mathrm{OH})_{2}+2 \mathrm{Ni}(\mathrm{OH})_{2} .
$$

When the cell is recharged, the nickel is reoxidized and the iron reduced.

\section{Tin Sn}

Metallurgy. - Tin is obtained from cassiterite or tin-stone $\mathrm{SnO}_{2}$ (stannic oxide). The world's production averages 120,000 tons annually. Formerly the mines in Cornwall (England) constituted the chief source of the metal, but now the largest supply comes from the East Indies, the next largest from Bolivia. The ore is roasted to expel sulphur and arsenic, and reduced with coal in the reverberatory furnace. The melted metal is cast in blocks (" block tin "). The metal was well known to the ancients (found in Egyptian tombs).

Properties. - The metal is white, and markedly crystalline. It is soft and malleable (tinfoil), and melts at $232^{\circ}$.

Tin does not tarnish in the air. With concentrated acids it acts rapidly. Hydrochloric acid gives stannous chloride $\mathrm{SnCl}_{2}$ and hydrogen. Concentrated sulphuric acid gives stannous sulphate $\mathrm{SnSO}_{4}$, sulphur dioxide (p. 271) and water:

$$
\mathrm{Sn}+2 \mathrm{H}_{2} \mathrm{SO}_{4} \rightarrow \mathrm{SnSO}_{4}+\mathrm{SO}_{2} \uparrow+2 \mathrm{H}_{2} \mathrm{O} .
$$


Nitric acid gives the insoluble, white meta-stannic acid $\mathrm{H}_{2} \mathrm{SnO}_{3}$ :

$$
4 \mathrm{HNO}_{3}+\mathrm{Sn} \rightarrow \mathrm{H}_{2} \mathrm{SnO}_{3} \downarrow+4 \mathrm{NO}_{2}+\mathrm{H}_{2} \mathrm{O} .
$$

Uses.-Tin plate, used in making "tin" cans, is produced by dipping cleaned sheets of mild steel in melted tin. So long as the layer of tin remains intact, the iron is protected from rusting. But, if the tin layer is damaged, the iron rusts. The iron being the more active metal of the two, the tin acts as a contact agent and actually hastens the rusting.

Tin is sufficiently valuable to render the detinning of scrap tin plate from can factories, bearing 3 to 5 per cent of tin, a paying process. In the Goldschmidt process the scrap is cleaned, dried, and exposed to dry chlorine, which converts the tin into the liquid stannic chloride $\mathrm{SnCl}_{4}$, but leaves the iron unaffected. The chloride is used in mordanting.

Copper vessels for cooking and brass pins are also coated with tin, to preserve them from the action of air and moisture. Tin pipes are used where lead would be unsafe, as, for example, for beverages.

Tin enters into many useful alloys, such as bronze (with copper) and solder.

Compounds of Tin.- Stannous chloride $\mathrm{SnCl}_{2}, 2 \mathrm{H}_{2} \mathrm{O}$, "tin crystals," and a hydrate of stannic chloride $\mathrm{SnCl}_{4}, 5 \mathrm{H}_{2} \mathrm{O}$ are used in mordanting (p. 475). With soda the former gives stannous hydroxide $\mathrm{Sn}(\mathrm{OH})_{2}$ and the latter stannic acid $\mathrm{Sn}(\mathrm{OH})_{4}$, both gelatinous substances which are precipitated in the goods to be dyed. Stannic acid is also sometimes precipitated in flannelette (a material made of cotton) to render it non-inflammable, and always in silk to "weight" it (the increase may be from 25 per cent to 300 per cent or more).

Stannous sulphide $\mathrm{SnS}$ (brown) and stannic sulphide $\mathrm{SnS}_{2}$ (yellow) are precipitated by hydrogen sulphide, even from acid solutions of stannous and stannic salts, respectively. 
Exercises.-1. Make equations for: (a) the action of air and water on lead to form the hydroxide; (b) the precipitation of lead chromate by adding $\mathrm{K}_{2} \mathrm{CrO}_{4}$ to lead nitrate solution; (c) the action of hydrogen sulphide on white lead; (d) the precipitation of $\mathrm{PbCl}_{2}$ and of $\mathrm{PbI}_{2}$.

2. Tin melts at $232^{\circ}$ and lead at $326^{\circ}$. Solder $(1: 1)$ melts (and solidifies) at $210^{\circ}$. Why is this? What is the advantage of using solder?

3. Make equations for: (a) the action of soda on solutions of stannous and stannic chlorides, respectively: (b) the action or hydrogen sulphide on the same salts. 


\section{CHAPTER XLIII \\ COPPER AND MERCURY}

IN this chapter we encounter the first metals (if we except As and $\mathrm{Sb}$ ) which are below hydrogen in the activity list, and do not displace that element from dilute acids. Copper and mercury both have two valences, so that we have cupric-ion $\mathrm{Cu}^{++}$and cuprous-ion $\mathrm{Cu}^{+}$and mercuric-ion $\mathrm{Hg}^{++}$and mercurous-ion $\mathrm{Hg}^{+}$. All the soluble compounds of both are poisonous.

\section{Copper $\mathrm{Cu}$}

Occurrence.-Copper occurs free in considerable amounts, particularly on the Michigan shore of Lake Superior. Cuprous oxide $\mathrm{Cu}_{2} \mathrm{O}$ and basic carbonates, like malachite $\mathrm{CuCO}_{3}, \mathrm{Cu}(\mathrm{OH})_{2}$, are less common. The latter is often used as an ornamental stone. A large proportion of commercial copper is obtained from chalcopyrite $\mathrm{Cu}_{2} \mathrm{~S}_{3} \mathrm{Fe}_{2} \mathrm{~S}_{3}$.

Metallurgy. - The free copper, after being concentrated (freed from gangue) by washing, is smelted with a flux. The carbonate is roasted, leaving the oxide. The oxides are reduced with coal.

The sulphide ores are more difficult to reduce, and the presence of so much iron complicates the process. They are first roasted. This removes much of the sulphur as sulphur dioxide, leaving $\mathrm{Cu}_{2} \mathrm{O}$ and $\mathrm{Fe}_{2} \mathrm{O}_{3}$. Next the roasted material is treated in a blast furnace, along with "green" (unroasted) ore, sand (if silica is not present in the ore), and coke. Some of the iron is oxidized and removed in the slag (as silicate). The product, known as copper " matte," is a mixture of cuprous sulphide $\mathrm{Cu}_{2} \mathrm{~S}$ with ferrous sulphide FeS. The third stage is to bessemerize the melted matte 510

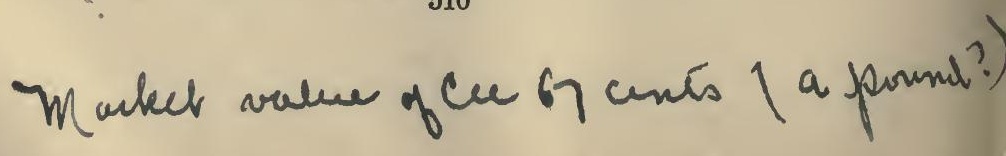


with sand in a converter. Here the rest of the iron is oxidized and eiiminated as silicate in the slag, and the sulphur escapes as $\mathrm{SO}_{2}$. The slag and metallic copper are poured separately. The latter gives off some dissolved sulphur dioxide in bubbles as it solidifies and, from its appearance, is named blister copper. Finally, since the copper now contains dissolved cuprous oxide $\mathrm{Cu}_{2} \mathrm{O}$, the blister copper is melted and " poled," by stirring with green wood. The gases (hydrocarbons, etc., p. 435) given off by the heated wood reduce the oxide to copper. If the copper is to be refined electrolytically (p. 485), it is then cast in plates 3 feet square and $\frac{3}{4}$ inch thick.

The old methods of concentrating copper sulphide ores by simple washing left large amounts of copper in the rejected gangue. Recent flotation processes prevent this loss. The ore is finely ground and beaten up with water containing a small quantity of oil. The particles of copper sulphide become wetted by the oil, the particles of gangue are preferentially wetted by the water. When air is forced through the pulp, the copper sulphide particles float with the froth to the top and are scraped off, while the gangue sinks to the bottom. Sixty million tons of ore per year are now treated by this method.

Properties.- Copper has a characteristic bright yellow-pink luster, quickly darkened by oxidation. It is second only to silver in electrical conductivity and to iron in tenacity. It is third in order of malleability. It melts at $1057^{\circ}$.

In moist air copper acquires a green coating of basic carbonate, which protects the metal. It is not affected by dilute hydrochloric or sulphuric acids, when air is excluded. Hot concentrated sulphuric acid gives cupric sulphate and sulphur dioxide (p. 271), and nitric acid gives cupric nitrate and oxides of nitrogen (p. 310).

Uses.- Pure copper is used for electric wires and cables. Traces of other metals greatly reduce the conductivity. Kettles,

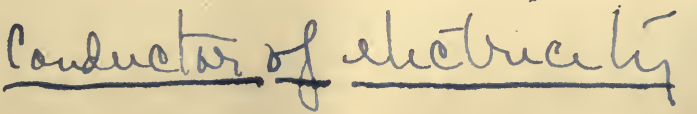


stills, and evaporating pans are made of copper. It is used for sheathing ships and for bolts, because it resists corrosion by sea water.

It enters into important alloys, such as brass (18 to 40 per cent of zinc), and bronze for coins (4 per cent tin and 1 per cent zinc), for gun-metal (10 per cent tin), and for bell-metal (20 to 24 per cent tin). Aluminium bronze ( 5 to 10 per cent aluminium) is used for the hulls of yachts. All of these are composed in part of compounds, such as $\mathrm{Cu}_{3} \mathrm{Sn}$ and $\mathrm{Cu}_{2} \mathrm{Zn}_{3}$.

Cupric Sulphate $\mathrm{CuSO}_{4}, 5 \mathrm{H}_{2} \mathrm{O}$. - The hydrated sulphate, bluestone or blue vitriol, is made by continuously agitating granulated copper with air and warm dilute sulphuric acid:

$$
2 \mathrm{Cu}+\mathrm{O}_{2}+2 \mathrm{H}_{2} \mathrm{SO}_{4} \rightarrow 2 \mathrm{CuSO}_{4}+2 \mathrm{H}_{2} \mathrm{O} \text {. }
$$

The blue crystals form on strips of lead suspended in the warm, saturated solution.

Because of slight hydrolysis, giving an active acid and a weak base $\mathrm{Cu}(\mathrm{OH})_{2}$, the aqueous solution is acid in reaction.

Cupric sulphate is used in battery solutions. The salt is employed in minute proportions to destroy algæ, which otherwise confer a disagreeable taste and odor on water that has been standing in reservoirs. Seed for cereals is moistened with a dilute solution to prevent "smuts."

Cupric Hydroxide $\mathrm{Cu}(\mathrm{OH})_{2}$. - The hydroxide is a blue, gelatinous precipitate, formed when an alkali is added to cupric sulphate solution. It is used as a mordant. A mixture of cupric sulphate solution and milk of lime $\left(\mathrm{Ca}(\mathrm{OH})_{2}\right)$, - Bordeaux mixture - containing this precipitate, is used extensively as a spray on grape vines and other plants, to prevent the growth of fungi.

Cupric hydroxide dissolves in ammonium hydroxide solution, giving a liquid of deep blue color. The explanation of this, according to the ionic hypothesis, is as follows. The solubility product 
of copper hydroxide in water is very small; in other words, we cannot have a high concentration of $\mathrm{Cu}^{++}$and $\mathrm{OH}^{-}$simultaneously in solution. But when ammonia is added to a solution containing the very small amount of $\mathrm{Cu}^{++}$and $\mathrm{OH}^{-}$requisite to be in equilibrium with precipitated $\mathrm{Cu}(\mathrm{OH})_{2}$, it combines with the $\mathrm{Cu}^{++}$to form complex ions of the formula $\left[\mathrm{Cu}\left(\mathrm{NH}_{3}\right)_{4}\right]^{++}$. To replace $\mathrm{Cu}^{++}$, more $\mathrm{Cu}(\mathrm{OH})_{2}$ goes into solution, and the process continues, if enough ammonia is added, until all $\mathrm{Cu}(\mathrm{OH})_{2}$ has been dissolved. We shall meet with other cases of a similar character later; in fact the principle involved - the formation of a complex ion - is used very extensively in analysis (see p. 538) and in industrial processes (see p. 523).

An ammoniacal solution of cupric hydroxide is employed as a solvent for cellulose. It dissolves also in a solution of sodiumpotassium tartrate $\mathrm{NaKC}_{4} \mathrm{H}_{4} \mathrm{O}_{6}$ (p. 354), giving Fehling's solution, a reagent used in testing for glucose and similar reducing agents. When this reagent is added to a liquid containing glucose (p. 401), red cuprous oxide $\mathrm{Cu}_{2} \mathrm{O}$ is precipitated.

Cuprous Oxide $\mathrm{Cu}_{2} \mathrm{O}$.- This oxide, mixed with $\mathrm{CuO}$, is formed by gentle heating of copper in air, and is best prepared by use of Fehling's solution. It is employed in making ruby glass and in coloring porcelain.

Cupric Oxide CuO. - When the liquid containing the blue precipitate of cupric hydroxide is boiled, the blue color changes to black and cupric oxide is thrown down:

$$
\mathrm{Cu}(\mathrm{OH})_{2} \rightarrow \mathrm{CuO}+\mathrm{H}_{2} \mathrm{O} \text {. }
$$

This oxide is used in the laboratory to ascertain the compositions (and formulæ) of organic compounds (determination of carbon and hydrogen). A weighed amount of the organic compound is placed in a horizontal tube, between heated masses of the oxide. A stream of oxygen or air carries the vapor of the organic com-

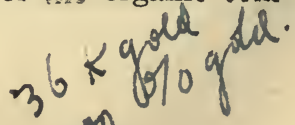


pound over the cupric oxide, which oxidizes it to water and carbon dioxide. The first is absorbed in a weighed U-tube filled with calcium chloride, and the second is caught in a weighed vessel containing potassium hydroxide. From the increase in weight in each case, the corresponding weights of hydrogen and carbon (derived from the weighed portion of the organic compound) are calculated.

Tests for Copper. - The blue color of cupric salts in dilute solution is distinctive (p. 175). Hydrogen sulphide precipitates cupric sulphide CuS (brownish-black) even from acid solutions of cupric salts (see p. 463).

More active metals, such as zinc or iron, displace copper from solutions of its salts, so that a blade of a knife, for example, receives instantly a red coating of copper when immersed in such a solution:

$$
\mathrm{Fe}+\mathrm{Cu}^{++} \rightarrow \mathrm{Fe}^{++}+\mathrm{Cu} \downarrow
$$

Copper Plating. - When platinum or carbon plates, connected with a battery, are immersed in a solution of cupric sulphate, copper is deposited on the negative plate (cathode). The $\mathrm{SO}_{4}=$ migrates towards the positive wire (anode) and there produces oxygen and sulphuric acid:

$$
2 \mathrm{SO}_{4}+2 \mathrm{H}_{2} \mathrm{O} \rightarrow 2 \mathrm{H}_{2} \mathrm{SO}_{4}+\mathrm{O}_{2} \uparrow \text {. }
$$

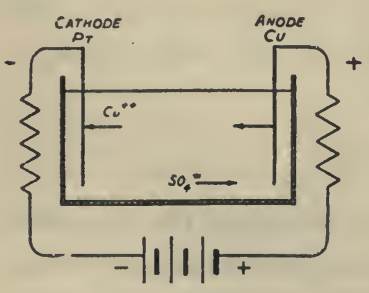

Frg. 117

If the anode is made of copper itself, however, the $\mathrm{SO}_{4}=$ migrates, but is not discharged. Instead, copper goes into solution (Fig. 117) as $\mathrm{Cu}^{++}$, in amount equal to that deposited on the other plate. Thus the quantity of cupric sulphate in solution remains unchanged, and the effect is, virtually, to transfer copper from the copper anode to the cathode. 
Electrotypes.-A copper electrotype of an object like a medal is made by first preparing a cast of the medal in plaster of Paris or wax. The surface of this is rubbed with graphite, to render it a conductor, and the cast is used as the cathode in a cell like that just described. The deposit of copper, when stripped off, is found to show an exact reproduction of the engraving, etc., on the object.

Book plates are made by taking a cast of each page of type, preparing the copper electrotype, and then strengthening and thickening it by filling the back with melted lead. The printing is then done from the electrotype. For newspapers this process is too slow, and the plate is made from the cast by means of melted stereotype-metal (lead, antimony, tin; $82: 15: 3$ ).

Copper Refining. - The copper, as obtained from the ores by the treatment already described (p. 510), contains a certain amount of silver, gold, and baser metals. The former pay for the cost of refining, and the simultaneous removal of the latter gives pure copper suitable for electrical purposes.

The principle is the same as that used in electroplating. The heavy plates of poled copper (p. 511) are hung at intervals in large, lead-lined vats of copper sulphate solution and form the anodes (Fig. 118, diagrammatic, view from above). The metal is deposited on thin sheets of copper, which are coated with graphite to permit the deposit to be easily stripped off. These sheets hang in the vat between the anodes,

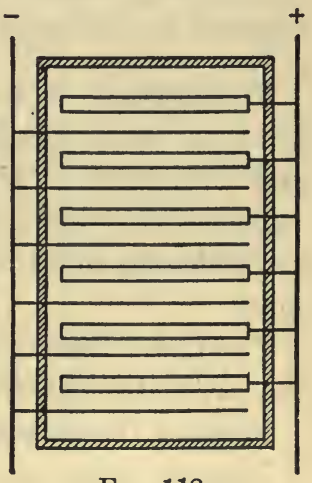

FrG. 118 and are connected with the negative wire. The copper, along with such traces of more active metals, like zinc, as are present, is ionized and goes into solution, until the anode is reduced to a skeleton and is exchanged for a fresh one. The less active metals, 
such as silver and gold, as well as traces of sulphides, are not ionized. They fall to the bottom of the vat, as a sort of heavy mud. At the cathode copper ions alone are discharged, and deposited, because copper is the least active of the metals present in ionic form. In this way copper, 99.8 per cent pure, can be obtained, and gold and silver are recovered from the mud.

Nickel Plating.- Here the bath contains an ammoniacal solution of ammonium-nickel sulphate (p. 501), and a plate of nickel forms the anode. The article to be plated is carefully cleaned, to secure a uniform deposit, and is suspended as cathode in the vat. The surface of the deposit is afterwards burnished.

\section{Mercury $\mathrm{Hg}$}

Metallurgy.- Mercury occurs both free and as mercuric sulphide HgS, cinnabar. Most of the ore comes from California and Spain.

The ore is roasted, sulphur dioxide escapes, and the vapor of mercury is condensed in long, tortuous flues.

Properties.-Mercury is a liquid at ordinary temperatures, hence its name, quicksilver (i.e. live silver). It freezes at $-40^{\circ}$, and boils, giving an invisible, non-conducting vapor, at $357^{\circ}$. The vapor density shows the molecules to be monotomic, as indeed are the molecules of all metals of which the densities have been measured.

The metal has a silvery metallic luster, which is not affected by the air, and a high specific gravity.

Mercury dissolves other metals, forming alloys or amalgams.

Mercury, when moderately heated, combines with oxygen, forming mercuric oxide (red), but the action is reversible, and the oxide is decomposed by stronger heating ( $p$. 15). It combines readily with sulphur and the halogens. Dilute hydrochloric and sulphuric acids are not attacked by mercury. Concentrated nitric acid attacks and dissolves it readily. 
Uses.-Mercury is used in filling thermometers and barometers. Sodium amalgam is used in the laboratory and the zinc plates of batteries are amalgamated superficially to protect them. when the battery is not in use. Dentists fill teeth with mixtures of mercury with silver, copper, cadmium and other metals, which quickly set to a solid amalgam. The pulverized ores of gold and silver, mixed with water, are allowed to trickle over thin layers of mercury. The latter dissolve the particles of the precious metals, while the sand passes on.

Compounds of Mercury.- As in the case of copper, there are two sets of compounds - mercurous $\mathrm{Hg}^{\mathrm{I}}$ and mercuric $\mathrm{Hg}^{\mathrm{II}}$. All the common salts are completely volatile, with or without decomposition, when strongly heated (vapor poisonous!).

Mercuric chloride or corrosive sublimate $\mathrm{HgCl}_{2}$ is made by subliming a mixture of mercuric sulphate and sodium chloride. It is a white substance, soluble in water. Hydrogen sulphide precipitates mercuric sulphide HgS (black) from the solution. Mercuric chloride is a violent poison. Albumen (white of eggs) forms insoluble compounds with it, and is used as an antidote.

Mercurous chloride or calomel $\mathrm{HgCl}$ is precipitated as an amorphous white powder when a chloride is added to a solution of a mercurous salt. It is used in medicine to stimulate all organs producing secretions. Mercuric fulminate $\mathrm{Hg}(\mathrm{ONC})_{2}$ is used in percussion caps (p. 483).

Baser metals precipitate mercury from solutions of its salts. The grey deposit is best seen on a clean strip of copper foil:

$$
\mathrm{Cu}+\mathrm{Hg}^{++} \rightarrow \mathrm{Cu}^{++}+\mathrm{Hg} \downarrow \text {. }
$$

Exercises.-1. Make an equation: (a) for the oxidation of ethyl alcohol by heated cupric oxide; (b) for the precipitation of cupric sulphide from cupric sulphate solution.

2. When we electrolyzed sodium chloride solution (p. 139), hydrogen was liberated at the cathode. What principle, used in 
the electrolytic refining of copper, does this phenomenon illustrate?

3. How could you recognize cupric sulphate solution by showing that it contained, (a) cupric-ion, (b) sulphate-ion (p. 270)?

4. Make an equrition for the liberation of mercury from cinnabar.

5. What gases should you collect over mercury?

6. What other salts, beside those of mercury, are volatile?

7. $\mathrm{H}_{\mathrm{O}} \mathrm{I}$ should you recognize a salt of mercury in solution? 


\section{CHAPTER XLIV \\ SILVER, GOLD, PLATINUM}

DESCENDING the activity list, we now reach the "noble" metals, which are the least active. They do not displace hydrogen from dilute acids. They do not combine with oxygen, even when heated.

\section{Silver Ag}

Occurrence.- Native silver is found in many localities, usually in small amounts. The chief supply of the metal is obtained from the ores of lead and copper, which contain silver sulphide $\mathrm{Ag}_{2} \mathrm{~S}$. The Rocky Mountains, Ontario and Mexico are the main sources.

Metallurgy.-After the lead (bearing silver) has been extracted from the ore (p. 502), it is melted in large caldrons, a small proportion of zinc is added, and the whole is vigorously stirred (Parke's process). Zinc is only slightly soluble in lead, but it combines with silver in several proportions. The zincsilver alloy rises to the surface, solidifies (while the lead is still molten), partly as alloy and partly as a compound (usually $\mathrm{Ag}_{2} \mathrm{Zn}_{5}$ ), and is skimmed off. The most of the adhering lead is pressed out, and the compound (or mixture) is placed in graphite retorts, in which the zinc is removed by distillation. The silver and lead which remain are heated in a blast of air (cupelled) to oxidize the lead. The melted litharge flows off and the silver is then cast.

The gold, which accompanies the silver through this treatment, is separated electrolytically (see copper, p. 515). The silver-gold alloy forms the anode and silver nitrate the vat-liquid. The silver, being the more active metal, is ionized and deposited 519 
on the cathode, while the gold collects as a powder in a bag which surrounds the cathode.

An older method, still used, is to heat the silver with concentrated sulphuric or nitric acid, either of which will dissolve the silver and leave the gold. From the solution the silver is displaced by the action of plates of copper:

$$
\mathrm{Cu}+2 \mathrm{Ag}^{+} \rightarrow \mathrm{Cu}^{++}+2 \mathrm{Ag} \downarrow \text {. }
$$

The world's production of silver in 1920 was $170,000,000$ ounces (troy), of which the mines of Mexico were responsible for 65,000,000, and those of the United States for $55,000,000$.

Properties. - Silver is fairly hard, considering its great ductility and malleability. It is the best conductor of electricity.

When an electric discharge passes between the ends of two silver wires, held under water, silver is dispersed at the points and forms a colloidal solution. The color of the solution varies from brownish to pink, according to the conditions. Colloidal solutions of gold and platinum can be made in the same way.

The metal is oxidized by ozone (p. 221), although not by oxygen. Sulphur compounds in the air tarnish the surface $\left(\mathrm{Ag}_{2} \mathrm{~S}\right.$, see p. 255), as do also eggs, secretions from the skin and rubber which contains sulphur.

Cold nitric acid and hot concentrated sulphuric acid are attacked by it, giving the nitrate and oxides of nitrogen, and the sulphate and sulphur dioxide, respectively:

$$
3 \mathrm{Ag}+4 \mathrm{HNO}_{3} \text { (dil.) } \rightarrow 3 \mathrm{AgNO}_{3}+2 \mathrm{H}_{2} \mathrm{O}+\mathrm{NO} \uparrow \text {. }
$$

Uses.-For silver ware and coins the metal is alloyed with copper. American coins contain 90 per cent of silver (" 900 fine"). British coins formerly contained 92.5 per cent, which is the proportion in "sterling silver," but the rise in price of the metal has recently necessitated a reduction to 50 per cent. Articles of baser metal are plated with silver. The bath contains potassium argenticyanide $\mathrm{KAg}(\mathrm{CN})_{2}$, made by adding potassium cyanide 
in excess to silver nitrate solution. This solution gives a compact deposit on electrolysis. The anode is of silver, so that the silver in the solution is replenished as quickly as it is deposited.

Mirrors are silvered by cleaning the surface and pouring over it a solution containing silver nitrate, ammonium hydroxide, and a reducing agent like formaldehyde $\mathrm{CH}_{2} \mathrm{O}$, or grape sugar:

$$
4 \mathrm{AgOH}+\mathrm{CH}_{2} \mathrm{O} \rightarrow 3 \mathrm{H}_{2} \mathrm{O}+4 \mathrm{Ag} \downarrow+\mathrm{CO}_{2} \text {. }
$$

The film of silver adheres to the glass and is washed, dried, and varnished.

Silver Nitrate $\mathrm{AgNO}_{3}$. - This exceedingly soluble salt is deposited from hot solution (p. 520) in colorless crystals. Its solution is neutral in reaction, which shows that silver hydroxide is not a weak base. It melts easily and is cast in sticks (lunar caustic), which are used in cauterizing sores. It is the chief source of the other compounds of silver. It is used in some hair dyes, and in indelible ink. In the latter case the organic matter in the goods reduces it, with the help of light, to metallic silver.

Silver Halides. - Silver chloride $\mathrm{AgCl}$ is precipitated (white) when a soluble chloride is added to a solution of a salt of silver:

$$
\mathrm{AgNO}_{3}+\mathrm{KCl} \rightarrow \mathrm{AgCl} \downarrow+\mathrm{KNO}_{3} \text {. }
$$

Silver bromide $\mathrm{AgBr}$ is precipitated with bromide-ion and silver iodide AgI with iodide-ion. These compounds have a yellowish tinge. The chloride and bromide are easily dissolved by ammonium hydroxide solution, giving the complex ion $\mathrm{Ag}\left(\mathrm{NH}_{3}\right)_{2}{ }^{+}$ (see p. 513), and also by sodium thiosulphate solution $\mathrm{Na}_{2} \mathrm{~S}_{2} \mathrm{O}_{3}$ (" hypo ").

All the halides of silver are decomposed by light, which liberates the halogen, and finally leaves metallic silver.

Photography. - The taking of a photograph involves four processes - preparation of the plate, exposure, development, and fixing. 
In preparing the plate, silver bromide is first precipitated in water containing gelatine. The mixture is kept warm, to permit the precipitate to become more sensitive to light by acquiring a coarser grain (" ripen "). The "emulsion " is applied to plates of glass or strips of transparent celluloid (films).

The brief exposure of the plate to the image of the object, well-focussed in the camera, produces no visible effect. But the bromide is thereafter more easily reduced to metallic silver, in proportion to the intensity of the light that fell upon each part.

Development consists in applying a reducing agent, of such slight activity that its effect during the process on non-illuminated parts of the bromide is practically zero. Ferrous oxalate, or an alkaline solution of pyrogallol $\mathrm{C}_{6} \mathrm{H}_{3}(\mathrm{OH})_{3}$ or of quinol $\mathrm{C}_{6} \mathrm{H}_{4}(\mathrm{OH})_{2}$ (two substances belonging to the class of phenols, see p. 353) may be used. The reduction goes fastest and deposits most silver where the illumination was most intense. Thus, the plate becomes most opaque where the object was brightest, and vice versa. On account of this reversal, the plate is called a negative. With the potassium salt of quinol, quinone $\mathrm{C}_{6} \mathrm{H}_{4} \mathrm{O}_{2}$ is formed:

$$
2 \mathrm{AgBr}+\mathrm{C}_{6} \mathrm{H}_{4}(\mathrm{OK})_{2} \rightarrow 2 \mathrm{Ag}+2 \mathrm{KBr}+\mathrm{C}_{6} \mathrm{H}_{4} \mathrm{O}_{2} .
$$

The foregoing processes are all carried out in a faint red light, which is almost without action on silver bromide. To prevent the gradual reduction of the remaining, unchanged bromide to silver by daylight, it is dissolved out by soaking the plate in sodium thiosulphate (fixing). The plate is now clear where no silver was deposited. The negative is finally washed thoroughly to remove all except the gelatine and the silver image, and is then dried.

In printing, the prepared paper is illuminated through the negative, and light and dark are again reversed. The denser parts of the negative protect the paper below them, and leave these parts white. On printing paper, silver chloride suspended in egg albumen is the sensitive substance, and the silver is liberated in a 
reddish, colloidal condition. The color is improved by toning with a solution containing gold chloride, as part of the silver goes into solution and gold (purplish) is deposited in its place. The print is fixed with hypo, washed, and dried. Papers like velox (invented by Baekeland) are essentially like plates (silver bromide in gelatine), and are exposed, developed, and fixed in the same way.

\section{GoLd $\mathrm{Au}$}

Occurrence and Extraction.- Gold is found in the free condition in veins of quartz and in alluvial deposits, resulting from the breaking up of such rock. It is found also in combination with tellurium (p. 273).

tecunie

In vein mining (e.g. in the Transvaal) the rock is pulverized with iron stamps working in an iron trough. The powder is washed in the form of mud over plates of copper amalgamated with mercury, in which 55 per cent of the gold dissolves. The amalgam is afterwards scraped off, the mercury removed by distillation, and the gold residue refined. The tailings still contain 45 per cent of the gold, adhering to the particles of rock. They are covered with sodium cyanide (p. 393) solution, and exposed to the air, until the gold has been dissolved as sodium aurocyanide $\mathrm{NaAu}(\mathrm{CN})_{2}$. From this solution the gold is deposited by electrolysis, or displaced by zinc.

The alluvial deposits are washed, on a small scale, in " cradles" (shallow pans) and, on a large scale, by being carried by water down a long trough (Placer Mining). The gold, having a much higher specific gravity than the rock, sinks to the bottom, while the rock particles are carried away. In the trough the gold settles between cleets nailed across the bottom. In hydraulic mining, a modification of placer mining, very heavy streams of water are thrown against the deposit.

In 1920 , the gold production of the world exceeded $\$ 330,000,000$ in value. $\$ 165,000,000$ of this came from the Transvaal, and 
$\$ 50,000,000$ from the United States. In 1912 the world yield was $\$ 475,000,000$, but the increase in the cost of working has caused many mines to be shut down.

Properties.- Gold is yellow in color. It is the most malleable and ductile of metals. It melts at $1075^{\circ}$. To enable it to resist wear, it is alloyed with copper. Pure gold is " 24 karat" fine. British gold coins are 22 karat, and American coins 21.6 karat (90 per cent gold).

Gold is not affected by oxygen or by hydrogen sulphide. It does not interact with any single acid. It combines directly, however, with chlorine and bromine. It dissolves in aqua regia (hydrochloric and nitric acids, mixed). This happens, not because aqua regia is more active as an oxidizing agent than the substances it contains, but because it oxidizes, and also furnishes the chloride-ion $\mathrm{Cl}^{-}$required to produce the exceedingly stable negative ion of chlorauric acid $\mathrm{HAuCl}_{4}$, namely $\mathrm{AuCl}_{4}-$.

Uses.- Most of the metal is used in coins and bars as a medium of exchange. It is beaten into gold leaf. It is employed in making potassium chloraurate $\mathrm{KAuCl}_{4}$ for toning photographs. Gold plating on silver and other metals is carried out by using a gold anode and a bath of sodium aurocyanide $\mathrm{NaAu}(\mathrm{CN})_{2}$ solution.

\section{Platinum Pt}

Occurrence and Extraction.- Platinum is found in the free condition in alluvial sand, chiefly in the streams of the Ural Mountains and the Caucasus and in Colombia. The separation from osmium, iridium, and other metals, which accompany it, is a complex operation.

Properties.-Platinum is a malleable and ductile metal with a greyish-white luster. It melts in the oxy-hydrogen flame (m.-p. about $1780^{\circ}$ ), but not in the Bunsen flame. 
The metal is not affected by air, water, or acids, excepting aqua regia. In the latter instance, chloroplatinic acid $\mathrm{H}_{2} \mathrm{PtCl}_{6}$ is formed.

Platinum adsorbs hydrogen and oxygen. When finely divided, so as to present a large surface, it catalyzes powerfully many chemical actions (pp. 262,314). The forms used are platinum black, a powder precipitated from chloroplatinic acid by an active metal (e.g. zinc); platinum sponge, a porous mass made by decomposing ammonium chloroplatinate by heat; and platinized asbestos, made by dipping the asbestos in chloroplatinic acid solution and heating.

It unites, slowly, with chlorine and bromine. It combines also with carbon, phosphorus, and silicon, and alloys itself with many metals, so that reactions which liberate any of these elements can not be carried out in vessels of platinum. It also acts upon fused caustic alkalies giving platinates.

Uses.- Since the metal does not melt in the Bunsen flame, and is not affected by many substances, it is employed in laboratory operations in the forms of wire, foil, and crucibles. Much of the metal is used by dentists, and in photography. Having the same expansibility as glass, it is fused through the bottoms of incandescent lamps, to connect the filament with the exterior. Since the metal is not oxidized by the air, even when heated, it is used for electrical contacts. The metal is employed most extensively for jewelry.

On account of the increasing demand, and the failure of the Russian supply, platinum is now (1922) worth $\$ 93.00$ per ounce troy, or more than four times the value of gold (nominally $\$ 20.67$ per ounce).

Other Metals of the Platinum Group.-Associated in small quantities with platinum in nature are five other metals - ruthenium, rhodium, palladium, osmium and iridium. These 
metals all fall in the last group of the periodic system (see pp. $278,282)$. Iridium is harder than platinum, and is alloyed with it for special purposes (pen-points and vessels to resist fluorine). A platinum-iridium alloy is used for the international standards of length and mass, kept in Paris. A palladium-gold alloy (palau) has recently been devised as a substitute for platinum in laboratory crucibles.

Exercises.-1. Make an equation for : (a) the action of concentrated sulphuric acid on silver; (b) the decomposition of silver chloride by light.

2. Make an equation for: (a) the displacement of gold from potassium chloraurate by silver; (b) the decomposition of chloroplatinic acid, and of (c) ammonium chloroplatinate by heat; (d) the action of zinc on chloroplatinic acid.

3. What would be the advantages and disadvantages of using gold instead of platinum for crucibles? 


\section{CHAPTER XLV \\ MANGANESE AND CHROMIUM}

The first metallic elements we considered form simple positive ions (e.g. $\left.\mathrm{Na}^{+}, \mathrm{Ca}^{++}\right)$only. The last two, on the other hand, appear almost exclusively in complex, negative ions, just as do the non-metallic elements $\left(\mathrm{AuCl}_{4}^{-}, \mathrm{Au}(\mathrm{CN})_{2}{ }^{-}, \mathrm{PtCl}_{6}=\right)$. Some of the intermediate metals can give negative ions (e.g. $\left.\mathrm{ZnO}_{2}=, \mathrm{AlO}_{3} \equiv\right)$, although in most of their compounds they are positive $\left(\mathrm{Zn}^{++}\right.$, $\left.\mathrm{Al}^{++}\right)$. The two elements taken up in the present chapter are equally familiar in both rôles.

\section{Manganese Mn}

Ore and Preparation.- The commonest ore of manganese is pyrolusite $\mathrm{MnO}_{2}$, a soft, black mineral. The metal is obtained in pure form by mixing the pulverized dioxide with aluminium (Goldschmidt's process, $p, 468$ ) in a clay crucible and starting the reaction with magnesium:

$$
3 \mathrm{MnO}_{2}+4 \mathrm{Al} \rightarrow 3 \mathrm{Mn}+2 \mathrm{Al}_{2} \mathrm{O}_{3} .
$$

At the high temperature (over $3000^{\circ}$ ) the molten manganese sinks to the bottom, and the alumina floats above it.

Properties and Uses.-Manganese is hard and crystalline, with a greyish-white luster. It is tarnished superficially by moist air. In fine powder it slowly displaces hydrogen from boiling water. It acts vigorously on dilute hydrochloric and sulphuric acids, giving manganous salts:

$$
\mathrm{Mn}+2 \mathrm{H}^{+} \rightarrow \mathrm{Mn}^{++}+\mathrm{H}_{2} \uparrow .
$$

The preparation and uses of spiegeleisen and ferro-manganese (p. 488), and of manganese steel (p. 493) have already been de- 
scribed. Traces of manganese seem to be essential to the growth of plants.

The Chemical Relations of the Element.-Manganese stands, at present, alone on the left side of the eighth column of the periodic table. The right side is occupied by the halogens. It is never univalent, as are the halogens, but its heptoxide $\mathrm{Mn}_{2} \mathrm{O}_{7}$ and the corresponding acid, permanganic acid $\mathrm{HMnO}_{4}$, are in many ways closely related to the heptoxide of chlorine and perchloric acid $\mathrm{HClO}_{4}$. Of the lower oxides of manganese, $\mathrm{MnO}$ is basic, and $\mathrm{Mn}_{2} \mathrm{O}_{3}$ feebly basic. $\mathrm{MnO}_{2}$ is feebly acidic, $\mathrm{MnO}_{3}$ more strongly so, and permanganic acid (from $\mathrm{Mn}_{2} \mathrm{O}_{7}$ ) is a very active acid. Contrary to the habit of feebly acidic and feebly basic oxides, such as those of zinc, aluminium, and tin, the basic oxides of manganese are not at all acidic, and the acidic oxides, with the exception of $\mathrm{MnO}_{2}$, are not also basic. There are thus the five following, rather well-defined sets of compounds, showing five different valences of the element. Of these the first, fourth, and fifth are the most stable and the most important.

1. Manganous compounds, $\mathrm{MnO}, \mathrm{Mn}(\mathrm{OH})_{2}, \mathrm{MnSO}_{4}$ etc. These compounds resemble those of the magnesium family (and those of $\mathrm{Fe}^{++}$). The salts of weak acids, such as the carbonate and sulphide, are easily made, and there is little hydrolysis of the halides. The salts are pale-pink in color.

2. Manganic compounds, $\mathrm{Mn}_{2} \mathrm{O}_{3}, \mathrm{Mn}(\mathrm{OH})_{3}, \mathrm{Mn}_{2}\left(\mathrm{SO}_{4}\right)_{3},\left[\mathrm{MnCl}_{3}\right]$. The salts resemble aluminium salts in behavior, but are very unstable (see p. 142) and are completely hydrolyzed by water. The salts are violet in color.

3. Manganites, $\mathrm{MnO}_{2}, \mathrm{H}_{2} \mathrm{MnO}_{3}, \mathrm{CaMnO}_{3}$. The alkali manganites are strongly hydrolyzed, like the silicates.

4. Manganates, $\mathrm{MnO}_{3}, \mathrm{H}_{2} \mathrm{MnO}_{4}, \mathrm{~K}_{2} \mathrm{MnO}_{4}$. The salts resemble the sulphates and chromates, but are much more easily hydrolyzed. They are green in color. 
5. Permanganates, $\mathrm{Mn}_{2} \mathrm{O}_{7}, \mathrm{HMnO}_{4}$ (hydrated), $\mathrm{KMnO}_{4}$. The salts resemble the perchlorates, and are not hydrolyzed by water. They are reddish-purple in color.

It will be seen that the element manganese changes its character totally with change in valence, and in each form of combination resembles some set of elements of valence identical with that which it has itself assumed. Since the valence represents the number of electrons gained or lost by each atom (p. 217), it is thus evident that the chemical properties of an element depend more upon the electrical constitution of its atom than upon the atomic weight (see p. 553).

Compounds of Manganese.-Manganese dioxide $\mathrm{MnO}_{2}$ is an oxidizing agent, and is mixed with black paints to make them "dry" rapidly. For the same reason, and because it is a conductor, it is used as a depolarizer in dry batteries and in the Leclanché cell. It is added to molten glass to remove the green color due to compounds of iron (p. 362).

Potassium permanganate $\mathrm{KMnO}_{4}$ crystallizes in needles which have a deep purple color with a greenish luster by reflected light. The purple color of the solution is the color of the ion $\mathrm{MnO}_{4}^{-}$.

The solution of potassium permanganate, especially when, by addition of an acid, permanganic acid $\mathrm{HMnO}_{4}$ has been formed, is an active oxidizing agent. We employed it in oxidizing hydrochloric acid to make chlorine (p. 141). For the same reason a dilute solution of potassium prrmanganate is used as an antiseptic and disinfectant.

\section{Chromium $\mathrm{Cr}$}

Ore and Preparation.-The chief ore of chromium is chromite $\mathrm{FeO}, \mathrm{Cr}_{2} \mathrm{O}_{3}$, which is mined chiefly in California, Rhodesia and New Caledonia. The U. S. production in 1918, stimulated by war requirements, exceeded 80,000 tons. In 1921 it sank to less than 300 tons. 
The metal is easily obtained, by reducing chromic oxide $\mathrm{Cr}_{2} \mathrm{O}_{3}$ with aluminium (Goldschmidt process, p. 468).

Properties.-Chromium is a lustrous crystalline metal. It does not tarnish in air. It displaces hydrogen, however, from dilute acids, giving chromous salts $\mathrm{CrCl}_{2}, \mathrm{CrSO}_{4}$, etc. Chromium ( 3 per cent) gives a hard steel, and with nickel is used in armorpiercing shells and in armor plate.

Alloys which, although composed entirely of active metals, are hardly affected even by boiling acids (including nitric acid), usually contain chromium (e.g. 60 per cent $\mathrm{Cr}, 36$ per cent $\mathrm{Fe}$, 4 per cent Mo, no C). "Stainless" cutlery contains 12 to 14 per cent of chromium.

\section{The Chemical Relations of the Element.-Chromium} gives four classes of compounds, and most of them are colored substances (Greek, chroma, color). The chromates are derived from chromic acid $\mathrm{H}_{2} \mathrm{CrO}_{4}$, which is analogous to sulphuric acid $\mathrm{H}_{2} \mathrm{SO}_{4}$. The free acid, however, is itself unstable, and leaves the anhydride $\mathrm{CrO}_{3}$ when its solution is evaporated. The oxide and hydroxide in which the element is trivalent, namely $\mathrm{Cr}_{2} \mathrm{O}_{3}$ and $\mathrm{Cr}(\mathrm{OH})_{3}$, are weakly basic and still more weakly acidic. Hence we have chromic salts such as $\mathrm{CrCl}_{3}$ and $\mathrm{Cr}_{2}\left(\mathrm{SO}_{4}\right)_{3}$ which are somewhat hydrolyzed, but no carbonate, and no sulphide which is stable in water. The compounds in which the same hydroxide acts as an acid are the chromites, and are derived from the less completely hydrated form of the oxide $\mathrm{CrO}(\mathrm{OH})$. Potassium chromite $\mathrm{K} \cdot \mathrm{CrO}_{2}$ is more easily hydrolyzed, however, than is potassium zincate or potassium aluminate. Finally, the chromous salts, such as $\mathrm{CrCl}_{2}$ and $\mathrm{CrSO}_{4}$, correspond to chromous hydroxide $\operatorname{Cr}(\mathrm{OH})_{2}$, in which the element is bivalent. This hydroxide is more distinctly basic than is chromic hydroxide, and forms a carbonate and sulphide which can be precipitated in aqueous solution. The chromous salts resemble the ferrous salts in being easily oxidized by the air. 
Potassium Chromate $\mathrm{K}_{2} \mathrm{CrO}_{4} .-$ Powdered chromite is roasted with potash and lime:

$$
4 \mathrm{FeO}, \mathrm{Cr}_{2} \mathrm{O}_{3}+8 \mathrm{~K}_{2} \mathrm{CO}_{3}+7 \mathrm{O}_{2} \rightarrow 2 \mathrm{Fe}_{2} \mathrm{O}_{3}+8 \mathrm{~K}_{2} \mathrm{CrO}_{4}+8 \mathrm{CO}_{2} \uparrow
$$

and the potassium chromate dissolved out of the residue. It is a yellow, soluble salt (the ion $\mathrm{CrO}_{4}=$ is yellow), with which insoluble chromates of other metals, such as lead chromate $\mathrm{PbCrO}_{4}$, can be precipitated.

The Dichromates. - When a solution of potassium sulphate is mixed with an equivalent amount of sulphuric acid, potassium bisulphate is obtainable by evaporation: $\mathrm{K}_{2} \mathrm{SO}_{4}+\mathrm{H}_{2} \mathrm{SO}_{4} \rightarrow$ $2 \mathrm{KHSO}_{4}$. The dry acid salt, when heated, loses water (p. 271), giving the pyrosulphate (or disulphate): $2 \mathrm{KHSO}_{4} \rightleftarrows \mathrm{K}_{2} \mathrm{~S}_{2} \mathrm{O}_{7}+\mathrm{H}_{2} \mathrm{O}$, but the latter, when redissolved, returns to the condition of acid sulphate. The second action is instantly reversed in presence of water. Now, when an acid is added to a chromate we should expect the chromic acid $\mathrm{H}_{2} \mathrm{CrO}_{4}$, thus liberated, to interact, giving an acid chromate (say, $\mathrm{KHCrO}_{4}$ ). No acid chromates are known, however, and instead of them, pyrochromates or dichromates are produced, with elimination of water. In other words, the second of the above actions is not appreciably reversible in presence of water when chromates are in question:

$$
\begin{aligned}
& \mathrm{K}_{2} \mathrm{CrO}_{4}+\mathrm{H}_{2} \mathrm{SO}_{4} \rightarrow\left(\mathrm{H}_{2} \mathrm{CrO}_{4}\right)+\mathrm{K}_{2} \mathrm{SO}_{4} . \\
& \mathrm{K}_{2} \mathrm{CrO}_{4}\left(+\mathrm{H}_{2} \mathrm{CrO}_{4}\right) \rightarrow \mathrm{K}_{2} \mathrm{Cr}_{2} \mathrm{O}_{7}+\mathrm{H}_{2} \mathrm{O} . \\
& \hline 2 \mathrm{~K}_{2} \mathrm{CrO}_{4}+\mathrm{H}_{2} \mathrm{SO}_{4} \rightarrow \mathrm{K}_{2} \mathrm{Cr}_{2} \mathrm{O}_{7}+\mathrm{H}_{2} \mathrm{O}+\mathrm{K}_{2} \mathrm{SO}_{4} .
\end{aligned}
$$

In terms of the ionic hypothesis, $\mathrm{S}_{2} \mathrm{O}_{7}=$ is unstable in water, and interacts with the $\mathrm{OH}^{-}$ion it contains, giving water and sulphateion, while $\mathrm{Cr}_{2} \mathrm{O}_{7}=$ is stable in water and is formed from the inter action of water and chromate-ion:

$$
\begin{aligned}
& \mathrm{S}_{2} \mathrm{O}_{7}=+2 \mathrm{OH}^{-} \leftrightarrows \mathrm{H}_{2} \mathrm{O}+2 \mathrm{SO}_{4}= \\
& \mathrm{Cr}_{2} \mathrm{O}_{7}=+2 \mathrm{OH}^{-} \leftrightarrows \mathrm{H}_{2} \mathrm{O}+2 \mathrm{CrO}_{4}=.
\end{aligned}
$$


The dichromates of potassium and sodium are made by adding sulphuric acid to the crude solution of the chromate obtained from chromite (p. 531). They crystallize when the liquid cools, and the mother-liquor, containing the potassium sulphate and undeposited dichromate, is used for extracting a fresh portion of cinder. As the dichromates are much less soluble than the chromates, they crystallize from less concentrated solutions, and can therefore be obtained in purer condition. For this reason the extract is always treated for dichromate.

Chemical Properties of the Dichromates.-1. When concentrated sulphuric acid is added to a dichromate, chromic anhydride $\mathrm{CrO}_{3}$ separates in red needles:

$$
\mathrm{Na}_{2} \mathrm{Cr}_{2} \mathrm{O}_{7}+\mathrm{H}_{2} \mathrm{SO}_{4} \rightarrow \mathrm{Na}_{2} \mathrm{SO}_{4}+\mathrm{H}_{2} \mathrm{O}+2 \mathrm{CrO}_{3} \downarrow \text {. }
$$

2. Although a dichromate lacks the hydrogen, it is essentially of the nature of an acid salt. Hence, when potassium hydroxide is added to a solution of potassium dichromate, potassium chromate is formed:

$$
\mathrm{K}_{2} \mathrm{Cr}_{2} \mathrm{O}_{7}+2 \mathrm{KOH} \rightarrow 2 \mathrm{~K}_{2} \mathrm{CrO}_{4}+\mathrm{H}_{2} \mathrm{O} .
$$

The solution changes from red to yellow, and the chromate is obtained by evaporation. In this way the pure alkali chromates are made.

3. With free acids the dichromates give powerful oxidizing mixtures, in consequence of their tendency to form chromic salts (see below).

Uses of Dichromates. - When paper is coated with gelatine containing a soluble chromate or dichromate and, after being dried, is exposed to light, chromic oxide is formed by reduction, and combines with the gelatine. This product will not swell up or dissolve in tepid water, as does pure gelatine. This action is used in many ways for purposes of artistic reproduction. Thus, 
if the gelatine mixture is made up with lampblack and, after the coating has dried, is covered with a negative and exposed to light, the parts which were protected from illumination may afterwards be washed away, while the carbon print remains. The gelatine layer can be transferred to wood or copper before washing. When materials of different colors are substituted for the lampblack, prints of any desired tint may be made by the same process.

Sodium dichromate is used, instead of tan-bark, in tanning kid and glove leathers. A reducing agent is employed to precipitate chromic hydroxide $\mathrm{Cr}(\mathrm{OH})_{3}$ in the leather. Its use diminishes the time required for the process from 8 or 10 months to a few hours. The hide is a mixture of colloidal materials, and the hydroxide is adsorbed.

Chromic Salts: Chrome-Alum.-The chromic salts are made by reduction from chromates or dichromates. These latter are derived from the anhydride $\mathrm{CrO}_{3}$, in which chromium is sexivalent, while the chromic salts are obtained from the oxide $\mathrm{Cr}_{2} \mathrm{O}_{3}$, in which the element is trivalent.

When concentrated $\mathrm{HCl}$ is heated with a dichromate, it is oxidized to chlorine (compare p. 142):

$$
\mathrm{K}_{2} \mathrm{Cr}_{2} \mathrm{O}_{7}+14 \mathrm{HCl} \rightarrow 2 \mathrm{KCl}+2 \mathrm{CrCl}_{3}+7 \mathrm{H}_{2} \mathrm{O}+3 \mathrm{Cl}_{2} \text {. }
$$

The most important chromic salt is chrome-alum, a double sulphate of potassium and chromium, which crystallizes from solution as beautiful purple octahedra $\mathrm{K}_{2} \mathrm{SO}_{4}, \mathrm{Cr}_{2}\left(\mathrm{SO}_{4}\right)_{3}, 24 \mathrm{H}_{2} \mathrm{O}$ (see p. 470).

Chromic Hydroxide $\mathrm{Cr}(\mathrm{OH})_{3}$ and Oxide $\mathrm{Cr}_{2} \mathrm{O}_{3}$. - When an alkali is added to chrome-alum solution, chromic hydroxide $\mathrm{Cr}(\mathrm{OH})_{3}$ (green) is precipitated:

$$
\mathrm{Cr}_{2}\left(\mathrm{SO}_{4}\right)_{3}+6 \mathrm{NaOH} \rightarrow 2 \mathrm{Cr}(\mathrm{OH})_{3} \downarrow+3 \mathrm{Na}_{2} \mathrm{SO}_{4} .
$$

Chromic hydroxide is the substance precipitated in the leather in chrome tanning. It is used also as a mordant in calico printing. 
When the hydroxide is heated, chromic oxide $\mathrm{Cr}_{2} \mathrm{O}_{3}$ remains as a green powder.

Other Elements of the Chromium Family.-Reference to the periodic system of the elements (p. 278) shows three elements - molybdenum, tungsten and uranium - which should resemble chromium in their properties and derivatives. Molybdenum and tungsten, indeed, give acid anhydrides $\mathrm{MoO}_{3}$ and $\mathrm{WO}_{3}$, and the salts derived therefrom correspond to the chromates and dichromates. The metals are used, like chromium, in making special steel alloys (p. 493). Tungsten has a higher meltingpoint $\left(3540^{\circ}\right)$ than any other metal and, on this account, and because it is less volatile than carbon, is now used for filaments in electric lamps. A carbon filament also requires 3.25 watts per candle power while a tungsten filament uses only 1.25 watts per 1 c.p. The powdered metal obtained by reduction can be pressed into wire form and then rolled while strongly heated by an electric current until a compact wire is obtained.

Uranium, besides giving uranates and diuranates, also exhibits base-forming properties. Pitchblende, which contains the oxide $\mathrm{U}_{3} \mathrm{O}_{8}$ along with smaller amounts of many other elements, is found mainly in Joachimsthal (Bohemia) and in Cornwall. Carnotite, a uranate and vanadate of potassium, $\mathrm{K}_{2} \mathrm{O}, 2 \mathrm{UO}_{3}$, $\mathrm{V}_{2} \mathrm{O}_{5}, 3 \mathrm{H}_{2} \mathrm{O}$, occurs in Colorado. Pitchblende is roasted with lime, the calcium uranate $\mathrm{CaUO}_{4}$ thus formed is decomposed with sulphuric acid, giving uranyl sulphate $\mathrm{UO}_{2} \mathrm{SO}_{4}$. When excess of sodium carbonate is added to the solution of the latter, the foreign metals are precipitated and sodium diuranate $\mathrm{Na}_{2} \mathrm{U}_{2} \mathrm{O}_{7}, 7 \mathrm{H}_{2} \mathrm{O}$, which is also thrown down, dissolves in the excess as $\mathrm{Na}_{2} \mathrm{UO}_{4}$.

After filtration, the diuranate of sodium is reprecipitated by neutralizing with sulphuric acid and boiling. This salt is used in making uranium glass, which shows a yellowish-green fluorescence.

The most striking property exhibited by uranium and its compounds, radioactivity, will be dealt with in the final chapter. 
Exercises.-1. Give a list of the classes of manganese compounds and, in each class, the formulæ of several compounds, the prevailing color, and the valence of manganese in that class.

2. Make equations for the formation of: (a) manganous sulphate; (b) manganous carbonate (insol.); (c) sodium manganate; (d) the oxidation of hydrochloric acid by potassium permanganate. 3. What action did we find to be catalyzed by manganese dioxide? 


\section{CHAPTER XLVI}

\section{THE RECOGNITION OF SUBSTANCES, II. - A REVIEW OF THE}

METALLIC ELEMENTS

As in Chapter XXXII, so here, we assume that the specimen to be identified contains a single substance. We consider first the metallic elements, and limit ourselves to those that have been described in the context. Our review will cover, mainly, the properties of each simple metallic positive radical.

Although arsenic has been stated to be a non-metallic element, and antimony to be partially non-metallic, it is more convenient in the problem of recognition to classify them with the metallic elements.

External Examination.-The color is often significant. Most of the common compounds of iron, nickel, cobalt, copper, gold, manganese and chromium are colored (see text). A metallic luster (scrape off the tarnish) usually, though not always, indicates a free metal or an alloy. The crystalline form should be noted. The odor usually gives information about non-metallic constituents ( $p .377$ ) only. As regards state, the vast majority of the metals and their compounds are solids. When a liquid presents itself, therefore, it is usually an aqueous solution of some compound. Obtain the solid by evaporation.

Solubility and Reaction of the Solution.- Ascertain whether the substance is soluble in water (p. 111). Note whether the solution is acid, alkaline, or neutral in reaction (p. 369). No substance can be identified by the preceding observations alone, but the final conclusion as to the nature of the specimen must be in harmony with them. 
A salt which gives an acid reaction must be an acid salt of a polybasic acid (p. 260), or a derivative of a strong acid and a weak base. Similarly a salt which reacts alkaline must be a basic salt, or a derivative of a weak acid and a strong base.

Recognition by React ons in Solution.- Starting with the substance in solution, its identity can be ascertained by using reactions involving mainly precipitations and oxidations or reductions, which separate the metals into five distinct groups.

The following plan, taken in conjunction with the statements in the context, shows how a single cation may be identified. What will be said applies only to the case of a solution containing salts like the chlorides, nitrates, or sulphates of one or more cations, and leaves the oxalates, phosphates, cyanides, and some other salts, out of consideration.

Before attempting to understand this plan, the student should turn to the discussion on ionic equilibria (Chapter XXXVIII) and read it carefully through. The sections on the solubility of precipitates (pp. 460-4) should be particularly studied, since upon the principles therein formulated the whole plan is directly based.*

Group I.- Add to the solution hydrochloric acid. A precipitate indicates that cations giving insoluble chlorides are present. Silver, mercurous and lead salts give the white $\mathrm{AgCl}, \mathrm{HgCl}$, and $\mathrm{PbCl}_{2}$ respectively. The last-named salt, being appreciably soluble, will be only incompletely precipitated.

These three chlorides can be distinguished from one another very easily. When excess of ammonium hydroxide is added to the precipitate, silver chloride dissolves (p. 521). Mercurous chloride turns black, owing to the formation of a finely divided mixture of free mercury and mercuric amidochloride $\mathrm{Hg}\left(\mathrm{NH}_{2}\right) \mathrm{Cl}$. Lead chloride remains apparently unchanged.

* Many experimental details, essential for the successful performance of the tests described in this chapter, are here omitted. They will be found in the Authors' Laboratory Outline of Intermediate Chemistry. 
Group II.- If no precipitate appears on addition of hydrochloric acid, hydrogen sulphide is led into the solution. The sulphides insoluble in active acids, namely, $\mathrm{HgS}$, CuS, $\mathrm{PbS}, \mathrm{Bi}_{2} \mathrm{~S}_{3}$, $\mathrm{CdS}, \mathrm{As}_{2} \mathrm{~S}_{3}, \mathrm{Sb}_{2} \mathrm{~S}_{3}, \mathrm{SnS}$, are therefore thrown down. The first four are black or dark brown, the next two are yellow, and the last two are orange and brown respectively. If too much $\mathrm{HCl}$ is present, the precipitation of several of these sulphides will be incomplete. On the other hand, if too little $\mathrm{HCl}$ is used, zinc sulphide may be partially precipitated (see pp. 461-3).

This group is readily sub-divided. The last three sulphides pass into solution when warmed with yellow ammonium sulphide, for they give soluble complex sulphides. The first five sulphides will be unaffected.

Group IIa.- HgS, CuS, $\mathrm{PbS}, \mathrm{Bi}_{2} \mathrm{~S}_{3}$, CdS. A yellow precipitate insoluble in ammonium sulphide indicates cadmium. To distinguish between the remaining four sulphides, boil with $\mathrm{HNO}_{3}$; $\mathrm{HgS}$ alone does not go into solution. Dilute the solution, and add $\mathrm{H}_{2} \mathrm{SO}_{4}$. Lead gives a white precipitate of $\mathrm{PbSO}_{4}$. If no precipitate is obtained, add $\mathrm{NH}_{4} \mathrm{OH}$ till alkaline. Bismuth gives a white precipitate of $\mathrm{Bi}(\mathrm{OH})_{3}$, copper a blue solution.

Group IIb.- $\mathrm{As}_{2} \mathrm{~S}_{3}, \mathrm{Sb}_{2} \mathrm{~S}_{3}$, SnS. The color distinction is not always certain. Reprecipitate the sulphides by adding $\mathrm{HCl}$, and boil the precipitate with concentrated $\mathrm{HCl} . \quad \mathrm{As}_{2} \mathrm{~S}_{3}$ does not dissolve. If the precipitate does dissolve, cool the solution and place in it a piece of bright tin. A black deposit forming on the surface (by displacement, see p. 54) indicates Sb.

Group IIIa. - If no precipitate is obtained with $\mathrm{H}_{2} \mathrm{~S}$, the solution is boiled, and a few drops of concentrated $\mathrm{HNO}_{3}$ added to oxidize any ferrous salt to the ferric state. Ammonium chloride is added, and then ammonium hydroxide in excess. A white, gelatinous precipitate of $\mathrm{Al}(\mathrm{OH})_{3}$ indicates aluminium, a bluish- 
green precipitate of $\mathrm{Cr}(\mathrm{OH})_{3}$ indicates chromium, a reddish-brown precipitate of $\mathrm{Fe}(\mathrm{OH})_{3}$ indicates iron. The presence of $\mathrm{NH}_{4} \mathrm{Cl}$ is necessary to reduce the concentration of $\mathrm{OH}^{-}$furnished by the $\mathrm{NH}_{4} \mathrm{OH}$ below the point at which other less insoluble hydroxides (such as $\mathrm{Mn}(\mathrm{OH})_{2}$ ) would be precipitated. To tell whether iron was originally present in the ferrous or the ferric condition, the ferricyanide test (p. 498) should be applied.

Group IIIb.- If, still, no precipitate is obtained, hydrogen sulphide is led into the alkaline solution. Sulphides which are insoluble in water, but soluble in active acids (see pp. 460-4), now appear. They are CoS and NiS (both black), MnS (fleshcolored) and $\mathrm{ZnS}$ (white). To distinguish between $\mathrm{Co}$ and $\mathrm{Ni}$, add $\mathrm{NaOH}$ to the original solution. Cobalt gives a blue precipitate of a basic salt, changing to pink $\mathrm{Co}(\mathrm{OH})_{2}$ on boiling; nickel gives a light-green precipitate of $\mathrm{Ni}(\mathrm{OH})_{2}$.

Group IV. - If negative results are still obtained, add $\left(\mathrm{NH}_{4}\right)_{2} \mathrm{CO}_{3}$. A white precipitate is given by three of the remaining metals whose carbonates are insoluble, calcium, strontium and barium. Distinction between these three may be made by the flame test. A small portion of the precipitate is taken up on a platinum wire and held in the Bunsen flame. A brick-red coloration signifies $\mathrm{Ca}$, a crimson-red $\mathrm{Sr}$, a green $\mathrm{Ba}$.

Group $\boldsymbol{V}$.- The only other common positive radicals are $\mathrm{Mg}, \mathrm{NH}_{4}, \mathrm{~K}$ and $\mathrm{Na}$. On addition of ammonium phosphate to the solution from Group IV, magnesium, if present, is precipitated in the form $\mathrm{NH}_{4} \mathrm{MgPO}_{4}$ (white). An ammonium salt may be recognized by boiling some of the original solution with $\mathrm{NaOH}$, when ammonia is evolved. Potassium salts confer a violet coloration to the Bunsen flame; sodium salts a bright yellow.

Confirmatory Tests. - From the context in earlier chapters, the student will be able to pick out for each particular metal other 
tests which may serve to confirm the conclusions arrived at in the course of the above analysis. Often the metallic radical can be reiognized by a displacement reaction (compare pp. 514, 517). Often, again, color changes which occur during the course of the scheme of operations outlined above give indications of value. Thus a yellow solution changing to green during the passage of $\mathrm{H}_{2} \mathrm{~S}$ in Group II signifies a chromate (p. 533), a purple solution becoming colorless signifies a permanganate (p. 529).

Tests for Negative Radicals.-Precipitation reactions similar in nature to those outlined above may be utilized as additional tests for the negative radical of an unknown substance (see chapter XXXII). Thus a chloride gives with silver nitrate a white precipitate, soluble in ammonia. A sulphate gives a white precipitate with barium chloride, insoluble in hydrochloric acid. From the context, the student will be able to discover for himself confirmatory tests of this kind for most of the common negative radicals discussed in this book. For a complete scheme, however, a manual of qualitative analysis should be consulted.

Insoluble Substances. - If the unknown substance is insoluble in water, try to bring it into solution by boiling successively with dilute $\mathrm{HNO}_{3}$, concentrated $\mathrm{HNO}_{3}$, and aqua regia (p. 310). In case it dissolves, evaporate off the excess of acid and proceed with the analysis as above. The only common substances which are still insoluble are: the sulphates of $\mathrm{Pb}, \mathrm{Sr}, \mathrm{Ba}$; certain mineral oxides such as $\mathrm{Al}_{2} \mathrm{O}_{3}, \mathrm{Cr}_{2} \mathrm{O}_{3}, \mathrm{Fe}_{2} \mathrm{O}_{3}, \mathrm{SnO}_{2}$; some silicates; $\mathrm{CaF}_{2}$; $\mathrm{AgCl}$ (soluble in $\mathrm{NH}_{4} \mathrm{OH}$ ). Fuse with $\mathrm{Na}_{2} \mathrm{CO}_{3}$ in a crucible, cool, extract with water, and filter.

The residue contains the positive radical as carbonate, and may be analyzed for this after dissolving in $\mathrm{HNO}_{3}$. The filtrate contains the negative radical as sodium salt, and may be examined as in Chapter XXXII. 
Exercises.-1. Name some substances that have a metallic luster, but are not metals or alloys.

2. Name the metals whose salts with active acids will give: neutral aqueous solutions; (b) acid aqueous solutions. What classes of salts will give alkaline solutions?

3. Write full ionic equations for the chloride precipitations mentioned in Group I. Why is silver chloride soluble in ammonia?

4. Write full ionic equations for the sulphide precipitations mentioned in Group II. Which is more soluble in water, mercuric sulphide or cadmium sulphide?

5. Write full ionic equations for the hydroxide precipitations mentioned in Group IIIa. Which is the more soluble in water, aluminium hydroxide or magnesium hydroxide?

6. Show, by means of ionic equations, why it is possible to prevent the precipitation of magnesium hydroxide when ammonium hydroxide is added to a soluble magnesium salt, by prior addition of ammonium chloride.

7. Why is it not possible, in the same way, to prevent the precipitation of magnesium hydroxide when sodium hydroxide is added to a soluble magnesium salt, by prior addition of sodium chloride?

8. Write full ionic equations for the sulphide precipitations mentioned in Group IIIb. Which is the more soluble $\mathrm{n}$ water, nickel sulphide or zinc sulphide?

9. Write full ionic equations for the carbonate precipitations mentioned in Group IV.

10. Write full ionic equations for the reactions mentioned in Group V.

11. Give a precipitation test for the negative radical in each of the following substances: sodium hydroxide, potassium carbonate, sodium sulphide, sodium phosphate. Write full ionic equations in each case. 


\section{CHAPTER XLVII}

\section{RADIUM, ATOMIC ENERGY, AND ATOMIC STRUCTURE}

The Discovery of Radium.- In 1896 Henri Becquerel discovered that a crystal of a salt of uranium could, in the dark,

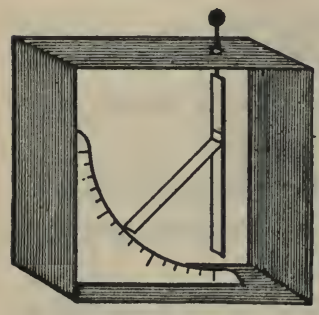

FIG. 119 reduce the silver bromide on a photographic plate, even when a sheet of black paper (impervious to light) was placed between. Evidently a radiation, different from light, was given out by the salt. Next he discovered that an electrometer (Fig. 119), in which the go.d leaves had been caused to separate by charging with electricity, lost its charge rapidly when a salt of uranium was brought near to the knob connected with the leaves. Evidently the salt rendered the air a conductor (" ionized" the air), and this permitted the escape of the electricity. These discoveries, in the hands of a multitude of observers, have led to the development of an entirely new branch of our science, namely radio-chemistry.

The radioactivity of every pure uranium compound is proportional to its uranium content. The ores are, however, relatively four times as active. This fact led M. and Mme. Curie, just after 1896, to the discovery that the pitchblende residues, from which practically all of the uranium had been extracted, were nevertheless quite active. About a ton of the very complex residues having been separated laboriously into the components, it was found that a large part of the radioactivity remained with the sulphate of barium. From this a product free from barium, and at least one million times more active than uranium, was finally secured in the form of the bromide. The nature of the spectrum and the chemical relations of the element, now named radium, 


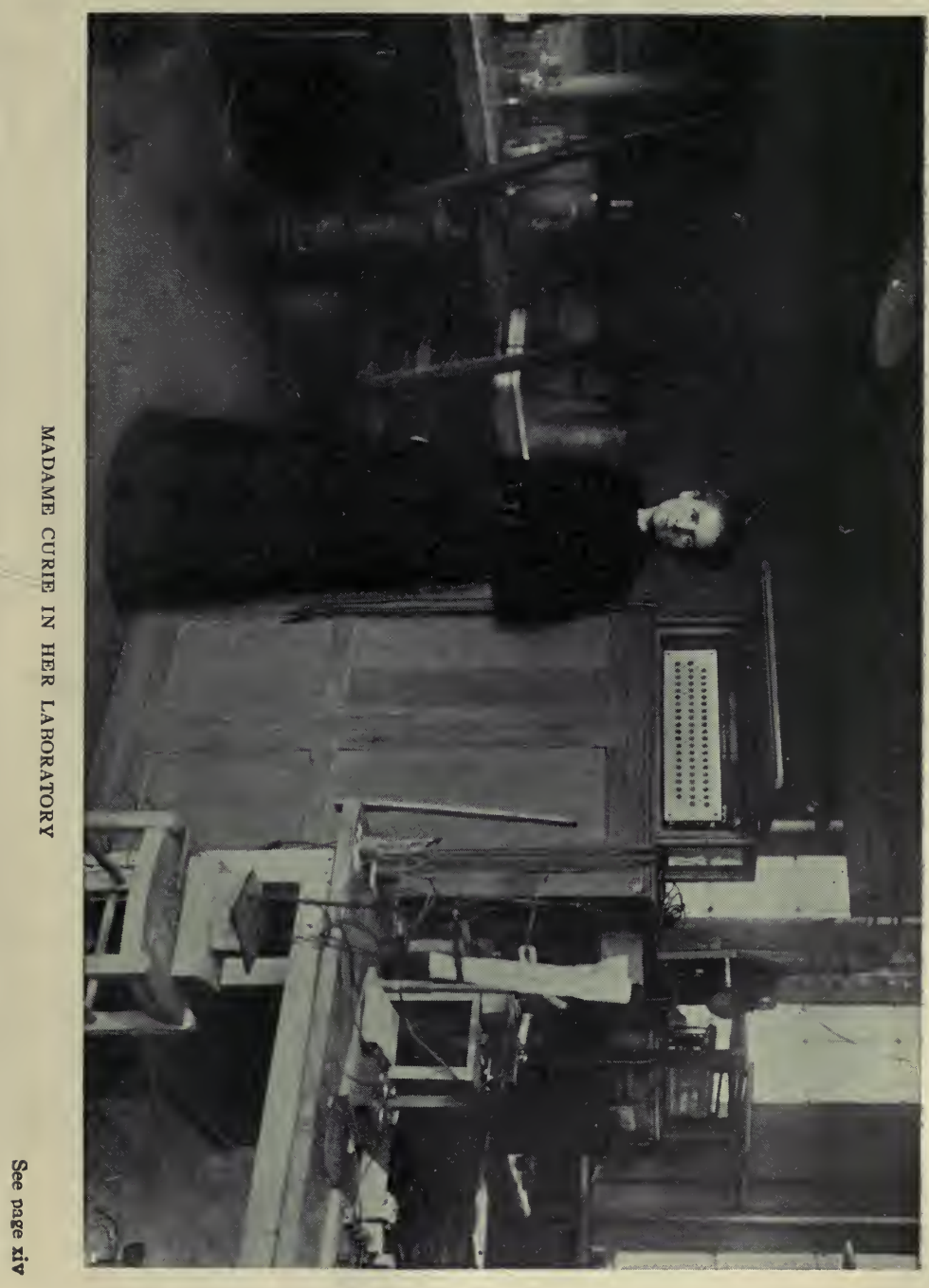



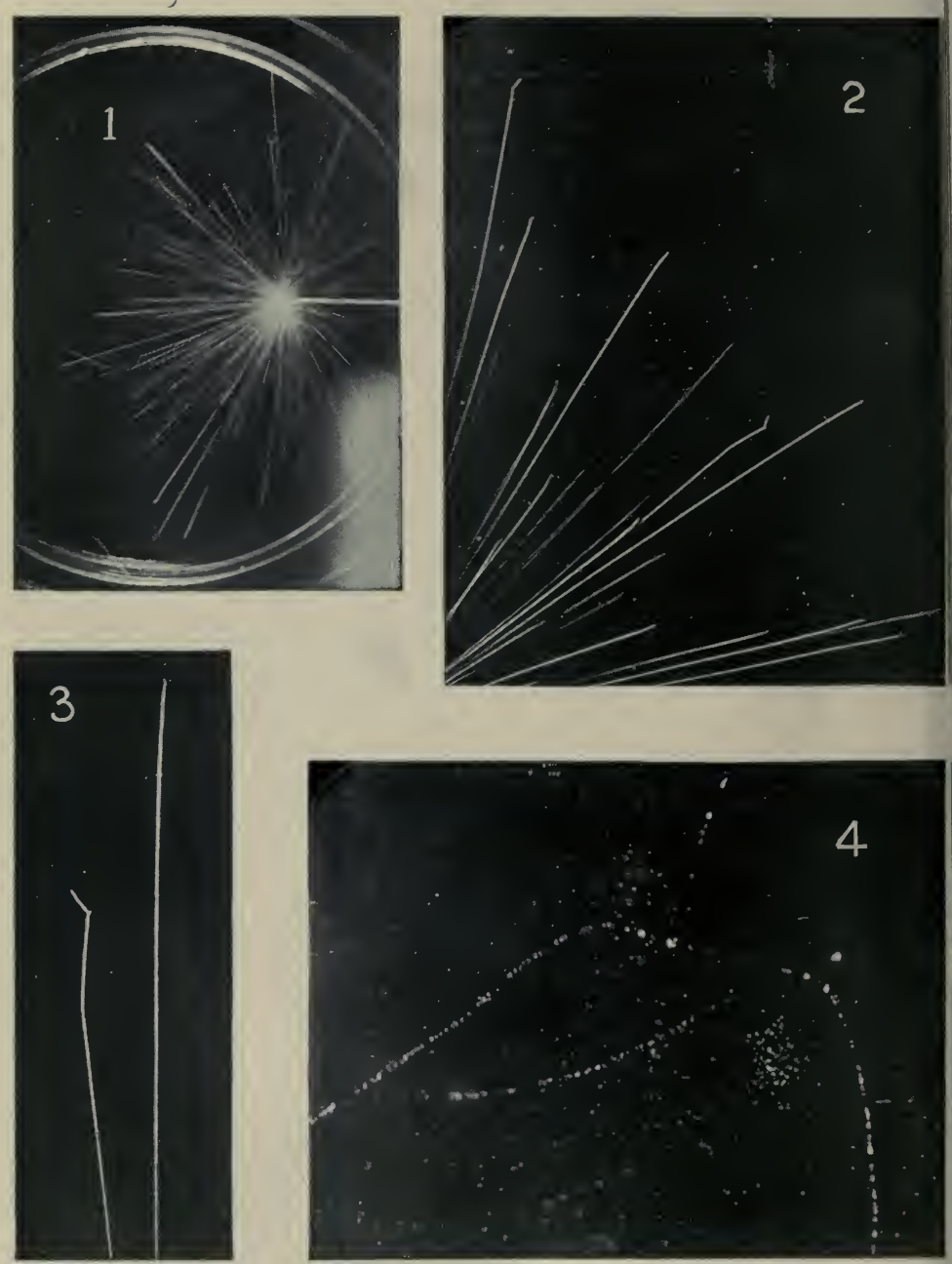

\section{FOG-TRACKS FROM RADIUM (C. T. R. WILSON)}
I, 2. Paths of helium atoms.
3. Part of 2 , enlarged.

4. Paths of electrons.

See page xiv 
placed it with the metals of the alkaline earths The ratio by weight of chlorine to radium in the chloride is $35.46: \mathrm{i} 13$, so that, on the assumption that the element is bivalent, its chloride is $\mathrm{RaCl}_{2}$ and its atomic weight is 226 . With this value it occupies a place formerly vacant in the periodic table.

In $1910 \mathrm{Mme}$. Curie obtained metallic radium by electrolyzing a solution of radium chloride, using a mercury cathode, and expelling the mercury by distillation. It was a white metal (m.-p. $700^{\circ}$ ) which, like calcium, quickly tarnished in the air and displaced hydrogen from water.

The Nature of the "Rays."- Many properties show that the "rays" emitted by compounds of uranium and of radium are of three kinds. They are most sharply distinguished from one another when allowed to pass through a powerful magnetic field. The alpha-rays are positively charged and are bent in one direction while the beta-rays are negative and are bent in the other. The gamma-rays are not affected.

The alpha-rays are atoms of helium (p. 297) thrown off in straight lines with varying initial velocities, averaging about onetenth that of light. Each such atom bears a double positive charge (the unit being the charge on a univalent positive ion), and a delicate electrometer readily indicates the impact of a single atom. These alpha-particles, being each four times as heavy as an atom of hydrogen, plough their way through tens of thousands of air-molecules and usually go about $3-8 \mathrm{~cm}$. before being stopped. The emission of atoms of helium can be detected by means of Crookes' spintharoscope (Fig. 120). The particle of radium bromide is at $\mathrm{B}$, and some of the charged helium atoms strike a surface $\mathrm{C}$. covered with zinc sulphide, producing faint flashes of light. The lens A magnifies the flashes, which can be seen in a dark room after

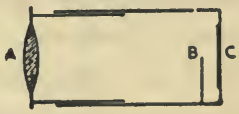

Frg. 120 the eye has become thoroughly rested (15-20 minutes). The 
helium gas given off by radium compounds was collected by Soddy working with Ramsay and identified, and its rate of production was measured. The amount was equal to 158 cubic $\mathrm{mm}$. per $1 \mathrm{~g}$. of radium per year.

The beta-particles are electrons (p. 195), or unit charges of negative electricity, and are shot out with a velocity approaching that of light (300,000 kiloms. per sec.). Their apparent mass is very small (about $\frac{1}{1830}$ that of an atom of hydrogen). Owing to collisions with the relatively ponderous air-molecules, half of them are lost after going about $4 \mathrm{~cm}$.

The gamma-rays are identical with X-rays (see p. 548), and are presumably produced like the latter by the impacts of the electrons on the surrounding matter.

The helium atoms are almost all stopped by a sheet of paper or by aluminium foil $0.1 \mathrm{~mm}$. thick. The electrons have greater penetrating power, many passing through gold-leaf, but being practically all blocked by a sheet of aluminium $1 \mathrm{~cm}$. thick. The gamma-rays (X-rays), however, are able to penetrate relatively thick layers of metals and other materials of low atomic weight.

One of the most striking facts is that the stoppage by the air of so many rapidly moving particles results in the production of much heat. One gram of radium would produce about $120 \mathrm{cal}$. per hour.

Disintegration.- The emission of atoms of helium and of electrons was first explained by Rutherford (1902-3), then of McGill University, Montreal, as being due to the spontaneous disintegration of the atoms of uranium, radium, and other radioactive elements. Thus, Rutherford was the first to show that radium compounds produced a gaseous substance called the radium emanation (niton), which was the residue left after the emission of one atom of helium from an atom of radium. This gas was itself radioactive and underwent further disintegration, 
RADIUM, ATOMIC ENERGY, AND ATOMIC STRUCTURE 545

depositing a solid radioactive residue on bodies in contact with it. Furthermore, every known uranium ore contains radium (McCoy) and radium emanation (Boltwood) in amounts proportional to the uranium content. Also, after the radium has been removed, the pure uranium compound gives off at first only $\alpha$-particles, but gradually recovers its whole radioactivity and is then found to contain radium emanation once more (Soddy). It thus appears that uranium is the starting point, and that the disintegration proceeds by steps, producing a number of different products. Each of these is formed from one such product and by disintegration furnishes another.

Unlike ordinary chemical change, the rate of disintegration is not affected by conditions. It can neither be started nor stopped at will. It is no more vigorous at $2000^{\circ}$ than at $-200^{\circ}$. Other changes occur between atoms, these within each atom.

The law, due also to Rutherford, describing the rate at which any one radioactive element disintegrates is simple. Only a certain fraction of the whole of any one specimen undergoes the change in unit time. Thus, as the total amount diminishes because of the change, the amount changing during the next unit of time, being a constant fraction of the whole, must be less. Hence an infinite time would be required for the complete disintegration of any one specimen. For convenience in expressing the rate of disintegration, however, we calculate and tabulate the average life of the element.

Radium emits helium atoms at the rate of $3.4 \times 10^{10}$ per gram per second. From this fact, we can calculate its average life to be about 2400 years. Hence, if it were not continuously being produced (from uranium), the whole supply would have been exhausted long before the earth reached a habitable condition.

The Uranium Group of Radioactive Elements.- The following shows the various elements produced from uranium by successive disintegrations. When a helium atom or an electron 
is expelled, the fact is shown by the symbols $\mathrm{He}$ and $\epsilon$, respectively. The first number below each element is the average life of that member of the series ( $y=$ year, $d=$ day, $h=$ hour, $m=$ minute, $s=$ second). The second number is the atomic weight, obtained by subtracting from the at. wt. of uranium (238) the weight (4) of each helium atom emitted.

$$
\begin{aligned}
& \mathrm{U}_{1} \rightarrow \mathrm{He}+\mathrm{U}-\mathrm{X}_{1} \rightarrow \epsilon+\mathrm{U}-\mathrm{X}_{2} \rightarrow \epsilon+\mathrm{U}_{2} \rightarrow \mathrm{He}+\text { Ionium } \\
& 8 \times 10^{9} \mathrm{y} . \quad 35.5 \mathrm{~d} . \quad 1.65 \mathrm{~m} . \quad 3 \times 10^{6} \mathrm{y} . \quad 2 \times 10^{5} \mathrm{y} \text {. } \\
& \begin{array}{lllll}
238 & 234 & 234 & 234 & 230
\end{array} \\
& \begin{array}{crrr}
\rightarrow \mathrm{He}+\mathrm{Ra} & \rightarrow \mathrm{He}+\mathrm{Niton} & \rightarrow \mathrm{He}+\mathrm{Ra}-\mathrm{A} & \rightarrow \mathrm{He}+\mathrm{Ra}-\mathrm{B} \\
2440 \mathrm{y} . & 5.55 \mathrm{~d} . & 4.3 \mathrm{~m} . & 38.5 \mathrm{~m} . \\
226 & 222 . & 218 & 214
\end{array}
\end{aligned}
$$

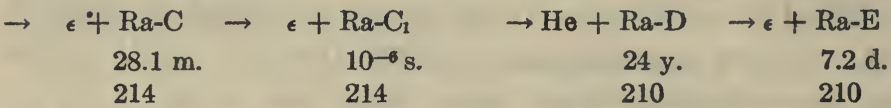

$$
\begin{aligned}
& \rightarrow \epsilon+\mathrm{Ra}-\mathrm{F} \quad \rightarrow \mathrm{He}+\mathrm{Pb} \text { (end) } \\
& 196 \mathrm{~d} \text {. } \\
& 210
\end{aligned}
$$

The radium emanation was shown by Ramsay to be one of the inert gases (p. 296), and was renamed niton. Its density was determined experimentally with a small sample, using a microbalance capable of weighing $1 / 500,000 \mathrm{mgm}$., and found to be 222.4 (density of oxygen $=32$ ).

The end-product of the disintegration is lead, and all uranium ores contain lead (see p. 550).

Thorium, found as phosphate in monazite sand, is also radioactive and furnishes a similar series of disintegration products. The final material is again lead.

Actinium and polonium are other radioactive elements, which have not yet been fully investigated.

Transmutation of Elements; Atomic Energy.-The phenomena of radioactivity establish the transmutation of elements, long regarded as a delusion of the alchemists, as an in- 
disputable fact. It is true that we have not yet discovered any simple means of disintegrating the more common elements (see, however, p. 17). We cannot even control in any way the rate of disintegration of radioactive elements (see p. 545). If, however, some method of inducing or hastening radioactive changes on a large scale is devised in the future, a wonderful new source of power will be put into our hands, namely, atomic energy.

The energy change in radioactive disintegrations is enormously greater than in ordinary chemical reactions. One gram of radium, as already mentioned, would evolve about 120 cal. per hour, and would continue to evolve this heat, at a gradually decreasing rate, for centuries. The total heat available would be over $2,000,000,000$ cals. per gram, whereas a gram of carbon burning to $\mathrm{CO}_{2}^{-}$gives only 8040 cals. The disintegration of a pound of uranium salts would furnish enough power to drive an ocean liner across the Atlantic, but $8,000,000,000$ years is entirely too long to wait for the completion of the trip. Chemists are already looking forward, however, to the possibility of using the enormous stores of energy here available so soon as a catalyst for the reaction is obtained.

Another interesting by-product of this subject is the calculation that the heat given off by the disintegration of the radium known to exist in the earth (niton is found in the soil and in well waters) is sufficient alone amply to account for the maintenance of its temperature. A globe the size of the earth, possessing originally only heat energy, and cooling from a white-hot condition to the temperature of interstellar space, would have passed through the stage of habitable temperatures in a much shorter time than that which geological deposits and fossils show to have been actually available. The discovery of the enormous, but gradually released, disintegration energy of radium, enables us now to explain the prolonged period during which life has existed in the earth.

This energy is derived from within the atom itself, by re-arrange- 
ments of the protons and electrons of which it is constructed (see p. 195). Building up a more complex atom from its disintegration products would require just as much energy as is evolved in the disintegration. This is another step which remains for the future.

We may now proceed to examine more intimately the question of atomic structure, already discussed in brief in earlier chapters (pp. 195, 217).

Atomic Numbers. - Visible light, X-rays, and wireless electric waves are all vibrations of the same nature in the ether. They differ only in wave-length, the order of the wave-lengths being $10^{-5} \mathrm{~cm}$., $10^{-8} \mathrm{~cm}$., and $10^{6} \mathrm{~cm}$. (10 kilometers), respectively. Now, just as the spectrum of visible light is obtained by using a grating, on which the rulings are separated by distances of the order of the wave-length of such light, so ordinary crystals give spectra of X-rays, because they are composed of particles arranged in rows about one thousand times closer and so form a suitable

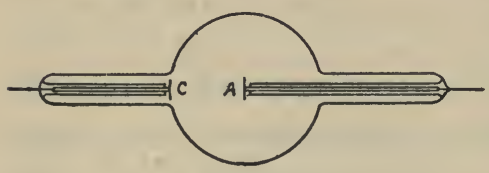

FIG. 121 grating for X-rays. This fact was first discovered by Dr. Laue of the University of Zurich (1912). The X-rays are produced in an evacuated tube by cathode rays, which are streams of electrons emanating from the cathode (C, Fig. 121), when they strike the anticathode (A).

With different elements on the anti-cathode, $\mathrm{X}$-rays of slightly different wave-lengths, and therefore giving different $\mathrm{X}$-ray spectra, are produced. The greater the number of free protons (unit positive charges) in the nucleus of the atom, the shorter should be the wave-length of the characteristic X-rays. It was shown by Moseley (a brilliant young English physicist, killed at Gallipoli) that when the elements are arranged in the order of these wave-lengths, whole numbers can be assigned to each which 
are inversely proportional to the wave-lengths of corresponding lines in their X-ray spectra. These atomic numbers have been determined for most of the elements, the atomic weights of which lic between those of aluminium and uranium. In the following table, the atomic numbers for these elements are given and, for the sake of greater completeness, numbers for the twelve elements preceding $\mathrm{Al}$ have been inserted also.

\section{ATOMIC NUMBERS (MOSELEY)}

\begin{tabular}{|c|c|c|c|c|c|c|c|c|c|c|c|c|c|c|c|c|c|}
\hline 1 & $\mathrm{He} 2$ & $\mathrm{Li}$ & 3 & Gl & & B & 5 & C & 6 & $\mathrm{~N}$ & 0 & 8 & $\mathrm{~F}$ & 9 & & & \\
\hline & $\mathrm{Ne} 10$ & $\mathrm{Na}$ & 11 & $\mathrm{Mg}$ & 12 & Al & 13 & $\mathrm{Si}$ & 14 & P & $\mathbf{S}$ & 16 & $\mathrm{Cl}$ & 17 & & & \\
\hline & A 18 & K & 19 & $\mathrm{Ca}$ & 20 & $\mathrm{Sc}$ & 21 & $\mathbf{T i}$ & 22 & V & $\mathrm{Cr}$ & 24 & Mn & 25 & $\mathrm{Fe} \quad 26$ & Co 27 & $\mathrm{Ni}$ \\
\hline & $\ldots \ldots \ldots$ & $\mathrm{Cu}$ & 29 & $\mathrm{Zn}$ & 30 & $\mathrm{Ga}$ & 31 & $\mathrm{Ge}$ & 32 & As 3 & Se & 34 & $\mathrm{Br}$ & 35 & $\ldots$ & .... & .. \\
\hline & $\mathrm{Kr} \quad 36$ & $\mathrm{Rb}$ & 37 & $\mathrm{Sr}$ & 38 & $\mathrm{Y}$ & 39 & $\mathrm{Zr}$ & 40 & $\mathrm{Mb}$ & Mo & 42 & 19 & 43 & $\mathrm{Ru} 44$ & $\mathrm{Rh} 45$ & $\mathrm{Pd}$ \\
\hline & $\ldots$ & Ag & 47 & $\mathrm{Cd}$ & 48 & In & 49 & Sn & 50 & $\mathrm{Sb}$ & Te & 52 & I & 53 & & & $\ldots$ \\
\hline & Xe 54 & $\mathrm{Cs}$ & 55 & $\mathrm{Ba}$ & 56 & La & 57 & $\mathrm{Ce}$ & 58 & Ta 73 & W & 74 & Be & 75 & Os 76 & Ir 77 & Pt 78 \\
\hline & & $\mathrm{Au}$ & 79 & $\mathrm{Hg}$ & 80 & $\mathrm{Tl}$ & 81 & $\mathrm{~Pb}$ & 82 & $\mathrm{Bi} \quad 8$ & Po & 84 & - & 85 & & . & ... \\
\hline & $\mathrm{Nt} \quad 86$ & & 87 & $\mathbf{R a}$ & 88 & Ac & 89 & Th & 90 & $\mathrm{U}-\mathrm{X}_{2} 9$ & $\mathrm{U}$ & 92 & & & & & \\
\hline
\end{tabular}

* The atomic numbers 59-72 are those of the metals of the rare earths: $\operatorname{Pr} 59, \mathrm{Nd} 60,-61$, Sa 62, Eu 63, Gd 64, Tb 65, Dy 66, Ho 67, Er 68, Tm 69, Yb 70, Lu 71, - 72.

It will be seen that there is a wholc number available for every known element, up to and including uranium, and not omitting the rare e.rths which have no satisfactory place in the periodic system. There are four blank numbers in the table, which correspond to three spaces below $\mathrm{Mn}$ in the periodic system and one under gold, and two more amongst the rare earths, indicating only six elements with atomic weights less than that of uranium yet to be discovered. The atomic numbers of argon and potassium place them in the chemically correct order, while the atomic weights do not. The same is true of cobalt and nickel and of tellurium and iodine.

The atomic numbers represent the number of free positive charges of electricity in the nucleus of the atom of each element. It must be noted that the nucleus also contains, in all cases except hydrogen, a number of bound positive charges associated with an 
equal number of electrons, indicated by the difference between the atomic weight and the atomic number.

The atomic numbers apparently determine all the properties of each element, and are more fundamental than the atomic weights. The latter are secondary properties, in most cases modified by other factors, and in a few cases actually thrown out of order by such factors.

\section{Atomic Numbers of Radioactive Elements; Isotopes.-} When an atom of a radioactive element loses an atom of helium, it also loses two free positive charges from its nucleus. Its atomic number is consequently reduced by two (for example, Radium $=88$, Niton $=86$ ). When, on the other hand, a radioactive change takes place involving the loss of an electron, a positive charge in the nucleus, previously bound, becomes free, and the atomic number is found to be increased by one.

With these facts in mind, an examination of the uranium disintegration series discloses that several elements (for example, Radium-B, Radium-D, Radium-G and Lead) must exist which possess the same atomic numbers, but different atomic weights. Such elements are known as isotopes. Isotopes are identical in all of their chemical properties, although they differ in atomic mass (see p. 20). This shows conclusively that atomic weight is not a fundamental property, but atomic number.

Ordinary lead chloride contains the elements lead and chlorine combined in the following proportions by weight:

Lead (207.2) - Chlorine (70.9) Lead chloride (278.1).

Richards, however, has found that the lead contained in uranium ores gives a chloride in which as little as 206.1 parts by weight of lead may be combined with 70.9 parts of chlorine, while Soddy has shown that the lead extracted from thorium ores gives a chloride which contains as much as 208.4 parts by weight of lead to 70.9 parts of chlorine. We have, therefore, three lead chlorides, all possessing the same specific properties, and being therefore the 
RADIUM, ATOMIC ENERGY, AND ATOMIC STRUCTURE 551

same substance, yet differing in composition. Other cases of a similar nature undoubtedly exist, although not yet encountered in actual practice.

Isotopes of Common Elements. - It has recently been shown by Aston that many common elements also are isotopic, or contain chemically identical atoms of different weight. The method employed by Aston was that of positive ray analysis (Fig. 122). The positive rays frorn a discharge tube are sorted

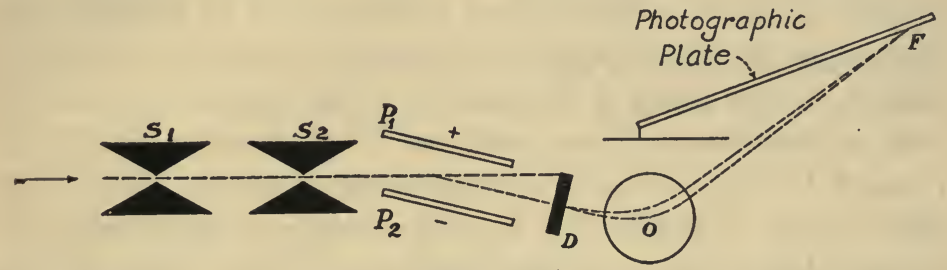

FIG. 122

out into a thin ribbon by means of the two parallel slits $S_{1}$ and $S_{2}$, and are then passed between the oppositely charged plates $\mathrm{P}_{1}$ and $P_{2}$. The rays are deflected towards the negative plate $P_{2}$, and are spread out into an electric spectrum. A portion of this spectrum deflected through a given angle is selected by the diaphragm $\mathrm{D}$ and passed between the circular poles of a powerful electromagnet $\mathrm{O}$, the field of which is such as to bend the rays back again to fall on a photographic plate placed as shown. If all the rays with a single charge have the same mass, they will converge to a focus at $\mathrm{F}$. If, however, the rays are derived from an element which consists of a mixture of isotopes, each isotope is distinguished by a separate band on the photographic plate, and from the relative position of each band the mass of the atom to which it corresponds can be obtained.

Chlorine (at. wt., 35.46), examined in this way, showed itself to be a mixture of two isotopes with atomic weights exactly 35 and exactly 37. Bromine (at. wt., 79.92) gives isotopes with 
atomic weights exactly 79 and exactly 81. Mercury (at. wt., 200.6) appears to exist in as many as six forms, with atomic weights ranging from 197 to 204 . Other elements, however, such as oxygen, nitrogen, and iodine, give no indications of isotopes.

The fundamental atomic weights obtained by Aston are with one exception whole numbers, within the limits of experimental error. The single exception is hydrogen (at. wt., 1.008).

Atomic Structure.- On the basis of the above results, general theories of atomic structure have been built up by Harkins and Rutherford, postulating hydrogen and helium atoms as the "bricks" building up the atoms of all elements. The elements with atomic weights divisible by 4 are considered as constructed entirely of charged helium nuclei, with surrounding electrons; thus $\mathrm{C}=$ $3 \mathrm{He}^{++}+6 \epsilon, \mathrm{O}=4 \mathrm{He}^{++}+8 \epsilon$, etc. Other elements must be assumed to contain hydrogen atoms also in their structure (see p. 17). The decrease in the mass of the hydrogen atom from 1.008 in hydrogen itself to exactly 1 in all other atomic types has been ascribed to a "packing effect."

Valence and Atomic Structure.- The electrons surrounding the nucleus are arranged, according to a theory recently developed by Lewis and Langmuir, in successive concentric shells. The total number of electrons in these shells must be equal, since the atom as a whole is electrically neutral, to the number of free protons in the nucleus, in other words, to the atomic number.

The case of the hydrogen atom (atomic number $=1$ ) has already been considered (p. 195). The helium atom (atomic number $=$ 2) has two electrons, which are supposed to be situated on opposite sides of the nucleus - a very stable arrangement.

No more electrons can be contained in the first shell, hence in succeeding elements the additional electrons begin to build up a second outer shell. Only the electrons in this outer shell can be added to or lost in interactions with other atoms (see p. 217), 
RADIUM, ATOMIC ENERGY, AND ATOMIC STRUCTURE 553

and it is found that, for all of the next 8 elements from lithium to neon, the tendency is either to lose electrons until none are left, or to gain electrons until a stable ring of 8 is formed. The arrangement of the electrons in this second shell has been pictured by Lewis as shown in Fig. 123. The electrons are assumed to occupy the corners of an imaginary cube, in the center of which is the atomic nucleus.

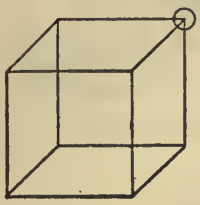

LI

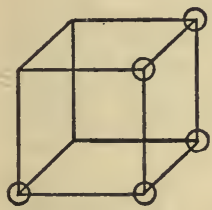

$\mathrm{N}$

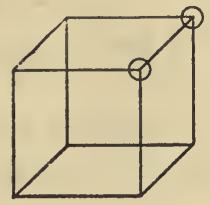

$\mathrm{Be}$

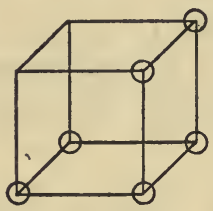

0

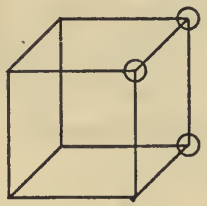

B

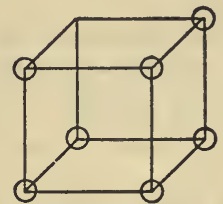

F

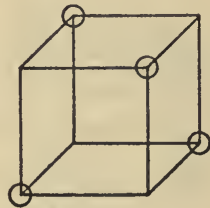

C

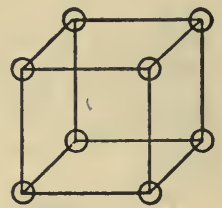

$\mathrm{Ne}$

FIG. 123

The valence of any one of these elements is therefore represented either by the number of electrons that can be lost (positive valence) or by the number required to form a stable ring of 8 (negative valence). The next eight elements (sodium to argon) exhibit the same behavior. We have not the space here to discuss in detail the electron arrangement in these and later elements. It will suffice to mention that not only are all of the relationships suggested by the Periodic System (Chapter XXIII) confirmed, but many of the points of difficulty in Mendelejeff's tabulation are satisfactorily explained.

Co-Valence.-When combination takes place between two atoms (e.g., $\mathrm{Li}$ and $\mathrm{F}$ ) by loss and gain of electrons, we are left 
with a compound, such as LiF (Fig. 124), in which the constituent atoms are apparently separate, being held together only by the attraction of their opposite charges. An atom may also complete its stable ring of eight, however, by sharing electrons with another atom, as in the case of the fluorine molecule $\mathrm{F}_{2}$ (Fig. 125).

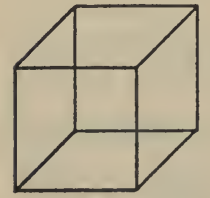

$\mathrm{Li}+$

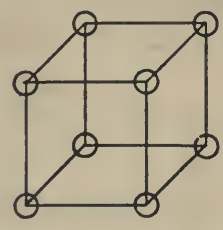

F-

FIG. 124

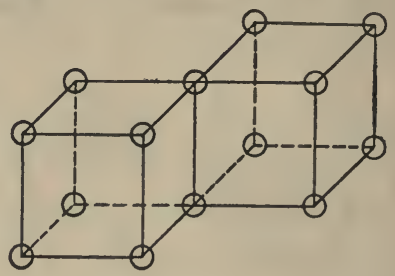

$\mathrm{F}_{2}$

Fig. 125

Similarly in carbon tetrachloride $\mathrm{CCl}_{4}$, we may assume that all five atoms have completed their "octets," each chlorine atom holding a pair of electrons in common with the central carbon atom.

The number of pairs of electrons which an atom of an element can thus share with other atoms is called its co-valence.

Polar and Non-Polar Compounds.- Compounds like LiF or $\mathrm{NaCl}$, in which an electron has already passed from one atom to another, are evidently potentially ionized, and if we can diminish the attractive forces between the two charged atoms sufficiently to enable them to break away from one another, we obtain immediately the free ions (as in aqueous solution). Such compounds are termed polar. In substances like $\mathrm{CCl}_{4}$, on the other hand, where electrons are held in common, the molecule will not tend to break up in this way. Such substances do not, consequently, ionize in solution, and are termed non-polar.

Strictly speaking, however, the distinction between polar and non-polar substances is not fundamental, but one of degree only. In no non-polar compound, probably, are pairs of elec- 
trons held in common so impartially that they will not tend, to some (albeit very small) extent, to pass over to one atom rather than to the other. In the same way, in no polar compound has the electron passed from one atom to another completely; the attractive forces between the two atoms, tending to restore it to its original position, must induce some distortion of the cubical arrangement.

Atomic Structure and Chemical Affinity. - In cases where combination between two elements produces a molecule (e.g., $\mathrm{LiF}$ ) in which the arrangement of electrons is much more stable than in the original substances, we shall clearly obtain a considerable diminution in the internal energy of the system (see p. $160)$ as the result of the interaction. Chemical activity or affinity hence appears to depend, finally, upon atomic structure. Where the electron arrangement is extremely stable, as with the inert gases, the element will be inert. Where electrons are readily gained or lost, as with the halogens or the alkali metals, the element will be active, and will form very stable compounds with such elements as assist most readily in the interchange.

Chemistry "within the atom" is still in its infancy, but it cannot be doubted that its development will lead to results of the greatest importance in the near future. The facts presented in this volume were almost all derived on a purely experimental basis, and the construction of hypotheses to correlate and explain these facts has been a long and painful process. On the basis of atomic structure, however, the next generation of chemists will be able to predict physical and chemical properties in advance. A multitude of new facts will thus be brought to light, and many new applications of chemistry to industry will become evident.

Exercises.-1. What justification has been obtained in this chapter for the use of $\mathrm{O}=16$ rather than $\mathrm{H}=1$ as a basis for atomic weights? 
2. If there are two isotopes of chlorine, and six of mercury, how many varieties of mercuric chloride $\mathrm{HgCl}_{2}$ are possible? In what respects would these varieties be different? In what respects would they be identical?

3. Draw diagrams showing the electron arrangements in the following molecules: hydrogen fluoride, water, oxygen $\left(\mathrm{O}_{2}\right)$, carbon dioxide, sodium chloride.

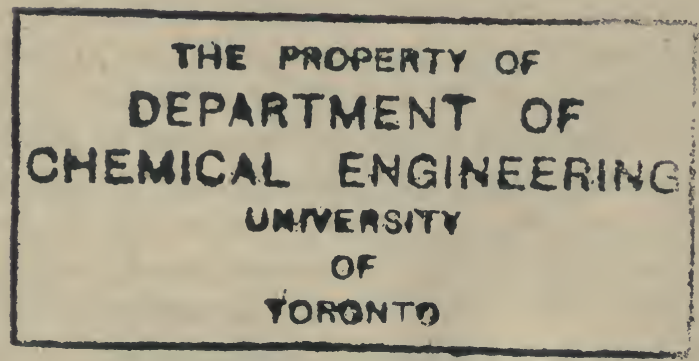




\section{APPENDIX}

\section{The Metric System}

Length. 1 meter $=10$ decimeters $=100$ centimeters $(100 \mathrm{~cm}$.) $=1000$ millimeters $(1000 \mathrm{~mm}$.).

1 decimeter $=10$ centimeters $=$ nearly 4 inches.

Volume. 1 liter $=1000$ cubic centimeters $(1000$ c.c. $)=$ a cube $10 \mathrm{~cm} . \times 10 \mathrm{~cm} . \times 10 \mathrm{~cm}$.

1 liter $=1.057$ quarts (U. S.) or 1.136 quarts (Brit.).

1 fl. ounce (U. S.) $=29.57$ c.c. 1 ounce (Brit.) $=28.4$ c.c.

Weight. 1 gram '́g.) $=$ wt. of 1 c.c. of water at $4^{\circ}$ C. 1 kilogram $=1000 \mathrm{~g}$.

1 kilogram $=2.2$ lbs. avoird. (U. S. and Brit.).

1 ounce avoird. (U. S. and Brit.) $=28.35 \mathrm{~g}$.

1 nickel (U. S.) weighs 5 g. 1 halfpenny (Brit.) weighs 5 to $5.7 \mathrm{~g}$.

\section{Scale of Hardness}

Each of the following minerals will scrateh the surface of a specimen of any one preceding jt.jn the list $t_{t}$

1. Talc

6. Felspar

2. Gypsum (or NaG)

7: Qúurtż

3. Calcite (or $\mathrm{Cu}) \cdots$ : 8 ? Topaz

4. Fluorite

$\because 9$. Corundum

5. Apatite $\because$ s $\because 6 \cdot 10$. Diamond

Glass is slightly scratched by 5 , and easily by those following. Glass will not scratch 5 distinctly, but will scratch those preceding 5 .

A good knife scratches 6 slightly, but not those following.

A file will scratch 7 , but not those following. 


\section{Temperatures Centigrade and Fahrenheit}

Upon the centigrade scale, the freezing-point of water is $0^{\circ} \mathrm{C}$. and the boiling-point $100^{\circ} \mathrm{C}$. Upon the Fahrenheit scale, the same points are $32^{\circ} \mathrm{F}$. and $212^{\circ} \mathrm{F}$., respectively. The same interval is thus $100^{\circ}$ on the one scale and $180^{\circ}$ on the other. The degree Fahrenheit is therefore $\frac{100}{180}$ or $\frac{5}{9}$ of $1^{\circ}$ Centigrade. Any temperatures can be converted by using the formulæ:

$$
\mathrm{C}^{\circ}=\frac{5}{9}\left(\mathrm{~F} .^{\circ}-32\right), \quad \mathrm{F} \bullet^{\circ}=\frac{9}{5}\left(\mathrm{C} .^{\circ}\right)+32 .
$$

The following table (IV) contains the temperatures from $0^{\circ} \mathrm{C}$. to $35^{\circ} \mathrm{C}$, with the corresponding values on the Fahrenheit scale $\left(32^{\circ} \mathrm{F}\right.$. to $95^{\circ} \mathrm{F}$.).

\section{Vapor Pressures of Water}

Both the Fahrenheit (F) or ordinary and the Centigrade (C) temperatures are given.

\begin{tabular}{|c|c|c|c|c|c|}
\hline \multicolumn{2}{|c|}{ Temperature } & \multirow{2}{*}{ Pressure, mm. } & \multicolumn{2}{|c|}{ Temperature } & \multirow{2}{*}{ Pressure, mm. } \\
\hline & C. & & F. & C. & \\
\hline $\begin{array}{l}32^{\circ} \\
41 \\
46.4 \\
48.2 \\
50.0 \\
51.8 \\
53.6 \\
55.4 \\
57.2 \\
59.0 \\
60.8 \\
62.6 \\
64.4 \\
66.2 \\
68.0 \\
69.8\end{array}$ & $\begin{array}{l}0^{\circ} \\
5 \\
8 \\
9 \\
10 \\
11 \\
12 \\
13 \\
14 \\
15 \\
16 \\
17 \\
18 \\
19 \\
20 \\
21\end{array}$ & $\begin{array}{r}4.6 \\
6.5 \\
8.0 \\
8.6 \\
9.2 \\
9.8 \\
10.5 \\
11.2 \\
11.9 \\
12.7 \\
13.5 \\
14.4 \\
15.4 \\
16.3 \\
17.4 \\
18.5\end{array}$ & $\begin{array}{r}71.6^{\circ} \\
73.4 \\
75.2 \\
77.0 \\
78.8 \\
80.6 \\
82.4 \\
84.2 \\
86.0 \\
87.8 \\
89.6 \\
91.4 \\
93.2 \\
95.0 \\
212.0\end{array}$ & $\begin{array}{l}22^{\circ} \\
23 \\
24 \\
25 \\
26 \\
27 \\
28 \\
29 \\
30 \\
31 \\
32 \\
33 \\
34 \\
35 \\
\\
100\end{array}$ & $\begin{array}{r}19.7 \\
20.9 \\
22.2 \\
23.6 \\
25.1 \\
26.5 \\
28.1 \\
29.8 \\
31.5 \\
33.4 \\
35.4 \\
37.4 \\
39.6 \\
41.8 \\
\\
760.0\end{array}$ \\
\hline
\end{tabular}




\section{INDEX}

* Acids are all listed under "acid," and salts under the positive radical.

Acetylene, 333, 351

Acid, acetic, 419

boric, 363

carbonic, 336

hydrochloric, 125-31

composition of, 146-7

hydrosulphuric, 255

hypochlorous, $223-6$

nitric, 308-11, 313-4

nitrosylsulphuric, 265

nitrous, 314

phosphoric, 322

picric, 484

silicic, 360

sulphuric, 261-71

chamber process, 264

concentration, 269

contact process, 261

fuming, 271

sulphurous, 259

Acids, properties of, 131, 170, 191 organic, 348

Activity, 27, 159-61

order of metals, 54

order of non-metals, 209

Adsorption, 421

Affinity, 159-60

Air, 26, 288-96

a mixture, 293

carbon dioxide in, 291-2

dust in, 292-3

liquid, 29, 294-6

water vapor in, 288-90

Alcohol, industrial, 418

Alcohols, 348
Aldehydes, 348

Alkalies, 168

Allotropic modifications, 220, 249

Alloys, anti-friction, 326

fusible, 326

steel, 493

Aluminates, 468

Aluminium, 466-8

salts of, $468-70$

Alums, 469

Amalgams, 516-7

Ammonia, 299-306

-oxidation process, 313

Ammonium salts, 304-5

Anhydrides, 257

Animal life and nutrition, 428-9

Anthracene, 353

Antimony, 325-6

Appendix, 557-8

Aqueous tension, correction for, 47

Argon, 296

Arsenic, 323-5 .

Atmosphere, 288-96

Atomic disintegration, 544

energy, 546-8

numbers, $548-50$

structure, 552-3

weights, $76,77,85,281$

Atoms, 85

Attributes, 7

BABBITT's metal, 326

Bakelite, 481

Baking powders, 430

soda, 366 
Barium, 393

Barometer, 44, 61

Bases, properties of, 167, 192

Battery, storage, 505-7

Benzene, 352

Bessemer process, 491

Birkeland-Eyde process, 312

Bismuth, 326-7

Blast furnace, 486-7

Bleaching, 221, 225, 259

powder, 224-6

Blue-prints, 498

Boiling-point, of liquids, 61 of solutions, 118, 177, 190

Borax, 363-4

Boron, 363

Brass, 512

Bromine, 199-201

Bronze, 512

Bunsen burner, 356

Cadmium, 451

Calcium, 383

bisulphite, 398

carbide, 333

carbonate, 383-4

chloride, 392

cyanamide, 392

fluoride, 207

hydroxide, 386

oxide, 384

phosphates, 411

salts as fertilizers, $413-5$

sulphate, 387

Calculations, 133, 148

Calorie, 61, 162

large, 435

Candle flame, 355

Cane-sugar, 401-3

Carbides, 332-3

Carbohydrates, 401, 429, 435

Carbon, 328-32

allotropic forms, $328-30$

dioxide, 333-7
Carbon dioxide, as plant food, 396 in air, 291-2

disulphide, 255

monoxide, $337-40$

tetrachloride, 332

Carborundum, 332

Casein, 438

Catalysis, 32-3

Celluloid, 480

Cellulose, 397-9, 480, 482

Cement, 471

Chamber process, 264

Charcoal, 330, 420-2, 438

Chemical change, and energy, 155 effect of temperature, 27, 241 physical accompaniments, 153 varieties of, 132

Chemical equilibrium, 230-46 properties, 33 units of weight, 74,78

Chlorine, 139-45 molecular formula of, 148 -water, 224

Chromium, 529-30

- salts, 530-4

Clay, 471

Coal, 422-5

gas, 423

Cobalt, 500

Coke ovens, 424

Collodion, 385

Colloidal suspensions, 109, 440-2

Combination, 14, 132

Combining proportions, 36 weights, 76-7

Combustion, preferential, 339 spontaneous, 41

Complex ions, 513

Components, 6

Composition, 38, 134

Compounds, 16

Conditions, 7

Conductivity, electrical, 175, 187

Constituents, 8 
Contact action, 33 process, 261

Copper, 510-12 plating, 514 refining, 515 salts, 512-4

Cotton, 1, 398

Cracking of oils, $346,352,35 €$

Crystallization, 114

Crystal structure, 94-6

Deacon's process, 140, 230

Decantation, 13

Decomposition, 16, 132

Deliquescence, 118

Density, 6, 85, 148

Dextrose, 401

Dialysis, 442

Diamond, 95, 329

Diet, normal, 436

Diffusion, 90

Digestion of foods, 430,434

Displacement, 51, 132, 172 valence by, 213

Dissociation, 104, 133

Distillation, 66 fractional, 344

Double decomposition, 132, 173, 185

Drugs, 479

Dust in air, 292-3

Dyeing, 474-6

Dyestuffs, $477-8$

Dynamite, 483

EDison cell, 507

Efflorescence, 68

Electrons, 195 and oxidation, 228 and valence, 217

Elements, 17, Inside back cover common, 19 periodic system, 273-84 transmutation of, 546

Electric furnace, 332
Electrolysis, 55, 139, 183

Electrolytes, conductivity of, 175

Electrotypes, 515

Emulsions, 110

Endothermal changes, 161

Energy, 156, 158, 426 and chemical activity, 159 atomic, 546-8 conservation of, 158 internal, 158

Enzymes, 417

Equations, 81, 135

balancing of, 101, 316-7

ionic, 182

making of, 100

thermochemical, 162

Equilibrium, 63, 69, 230-46 displacement of, 239

Equivalent weights, 53, 122

Esters, 349, 432

Ethers, 348

Ethylene, 350

Evaporation, 14

Exothermal changes, 161

Explosives, 481-4

Extraction, 112

FAts and oils, 432 hydrolysis of, 433

Fehling's solution, 401, 513

Felspar, 5, 410

Fermentation, 417

Fertilizers, 409-15

Filtration, 13, 66

Fixation of nitrogen, 300, 312

Flame, 354-7

Flotation process, 511

Flour, 5

Fluorine, 206-7 molecular structure, 554

Foods, 429

fuel value of, $435-6$

Formaldehyde, 348

Formulæ, 78 
Formulæ, in calculations, 133

making of, 99 molecular, 104-5

Freezing mixtures, 119 point of liquids, 60 of solutions, 119, 177, 190

Fructose, 401

Fuels, 435

GaIllaRd tower, 269

Gases, facts about, 93 inert, 296-7

liquefaction of, 91, 294-6

measurement of, 44

perfect, 92

properties of, 88-93

Gasoline, 213

Gay-Lussac tower, 267

Gelatine, 435

Glass, 361-3

Glover tower, 266

Glucose, 401

Gluten, 5

Gold, 523-4

Gram-molecular volume, 83

Granite, 4

Graphite, 329

Guncotton, 482

Gunpowder, 372

Gypsum, 387, 414

HABER process, 300-2

Halogen derivatives of hydrocarbons, 347

family, 199, 208-9

Hard water, 388-91

Hardness, scale of, 557

Helium, 297, 543

Homogeneous systems, 238

Humidity, 289

Hydrates, 67

Hydrocarbons, 343-53

saturated, 343-7
Hydrocarbons, unsaturated, 350

Hydrogen, 50-6

from water gas, 338

molecular formula, 105, 148

position in periodic system, 283

structure of atom, 195

Hydrogen bromide, 201-2

chloride, 129-31

fluoride, 207-8

iodide, 204-5

peroxide, 221-3

sulphide, 252-5

Hydrolyte, 56

Hypothesis, Avogadro's, 82 ionic, 181

molecular, 48, 88-93

IcE, 64

Impurities, 6

Inert gases, 296-7

Ink, 499

Iodine, 203-4

Iodoform, 205

Ion-product constancy, 456

Ionic equations, 182 equilibria, 451-64

Ionization, 181-98 and chemical activity, 189

Ions, 182

and conductivity, 187

and displacement, 184

and double decomposition, 185

and electrolysis, 183

and electrons, 195

and valence, 212

complex, 513

Iron, 486-94

Bessemer process, 491

cast, 488

galvanized, 449

open-hearth process, 492

salts, 495-8

Isotopes, 550-2 
Ketones, 349

Kindling conditions, 39

Krypton, 297

\section{LACTOSE, 401}

Lampblack, 355

Laundry, hard water in, 390-1

Law, Avogadro's, 82

Boyle's, 45, 89

Charles', 46, 90

Dalton's, 47, 112

definition of, 8,23

Dulong and Petit's, 86

Gay-Lussac's, 60, 72

Henry's, 112

Le Chatelier's, 244

Newland's, 275

of chemical change, 7 combining weights, 77

component substances, 6

definite proportions, 19,86

effect of heating, 27, 241

molecular concentration, 235

partition, 112

solubility product, 456

periodic, 280

van't Hoff's, 242

Lead, 502-3

accumulator, 505-7

salts, 503-5

white, 504

Le Blanc process, 367

Lewis-Langmuir theory, 552

Lime, 384-6

Liquids, properties of, 93

Lithopone, 451

Magnesium, 446

salts of, $447-8$

Maltose, 401,418

Manganese, 527

dioxide, $31,142,529$

salts of, 528-9

Manure, 413
Mass, conservation of, 20

Matches, 321

Matter, states of, 64 structure and behavior of, 88-97

Mendelejeff's system of elements, 276

Mercury, 516-7 salts, of, 517

Metals, order of activity, 54

Methane, 346

Metric system, 557

Mica, 4, 466

Mixtures, 6, 12

Molar solution, 122

Molecular concentration, law of, 235 formulæ, 104-6, 148

hypothesis, $48,88-93$

weights, $75,83,84$

Monel metal, 500

Mortar, 386

Mustard gas, 484

Naphthalene, 353

Natural gas, 345

Neon, 297

Neutralization, 192

Nickel, 500

plating, 516

salts of, 501

Nitrogen, 286-7

compounds as fertilizers, 410

tetroxide, 309, 311

Nitroglycerine, 481

Nitrous anhydride, 265, 314 oxide, 315

Non-metals, order of activity, 209

Normal solution, 121

Octaves, law of, 275

Oil refining, 344

shale, 345

Olefines, 351

Oleum, 261, 271

Osmosis, 406

Osmotic pressure, 407-9 
Oxidation, 40, 226-8

Oxides, acidic and basic, 257

Oxone, 31

Oxygen, 27-42 member of sulphur family, 273 molecular formula, 105

Ozone, 219-21

PaInTs, 305-4

Paper manufacture, 398

Paraffin series, 343-7

Parke's process, 519

Pasteur filter, 66

Pauling process, 313

Perfumes, 478-9

Periodic system, 273-80 applications of, 281-2 defects of, 282-4

Permutite process, 390

Petroleum, 344

Phenols, 353

Phosphine, 323

Phosphorus, 319-23 compounds as fertilizers, $4 \mathbf{1 1}$ pentachloride, 236 pentoxide, 322

Photography, 521-3

Physical properties, 33

Physics in chemistry, 22

Plant life, 395-7, 405-9

Plaster of Paris, 387

Plastics, 480

Platinum, 524-5 group of metals, 281, 525-6

Polar and non-polar compounds, 554

Portland cement, 471

Potassium, 370

bicarbonate, 371

carbonate, 371

chlorate, 30

chloride, 413

chromate, 531

dichromate, 531

ferricyanide, 498
Potassium ferrocyanide, 497

hydroxide, 371

nitrate, 30,372

permanganate, 141,529

salts as fertilizers, 412

Precipitation, 129

theory of, 458-9

Preferential combustion, 339

Pressure, atmospheric, 44 osmotic, 407-9

Producer gas, 337

Promoters, 339

Proteins, 434

Protons, 195

Pyrex, 363

Quartz, 4

Radicals, 170

positive and negative, 171

valence of, 212

Radioactivity, 542-8

Radium, 542-3

Recognition of substances, metallic, 536

non-metallic, 374

Reduction, 57, 228

Refrigeration, 306

Respiration, 291-2

Reverberatory furnace, 489

Reversible reactions, 69, 127, 230

Rouge, 496

Rusting, 9, 26, 494

Salts, 170, 192

Saponification, 438

Saturated solutions, 120, 243, 455

Saturation, 110, 120

Schönherr process, 313

Scientific method, 24

Selenium, 273

Shale, 345

Silica gel, 360

Silicon, 359 
Silicon dioxide, 359

tetrachloride, 360

tetrafluoride, 207, 360

Silk, 438

artificial, 399, 481

Silver, 519-21

salts, 521

Soap, 438-44

Sodium, 164-5

bicarbonate, 366

carbonate, 367

chloride, $95,125,365$

cyanide, 393

hydroxide, $166-8$

nitrate, 369

peroxide, 31, 221

silicate, 360

Solder, 503

Solids, properties of, 94-7

Solubilities, table of, Inside front cover

Solubility and temperature, 113, 243

of a gas, 111

-product law, 456

Solution, 108

heat of, 115

of insoluble precipitates, $460-2$

saturated, 110, 120

supersaturated, 115, 121

volume change in, 116

Solutions, boiling-points of, 119, 177

freezing-points of, 119, 177, 190

molar, 122

normal, 121

osmotic pressure of, 407-9

vapor pressure of, 117, 177, 190

Solvay process, 366

Solvents, 110

influence of solute on, 116

part in ionization, 193

Specific properties, 3,33

reed of chemical reactions, 161, 238

gct of temperature, 27,241
Spintharoscope, 543

Stability, 27

Starch, 5, 399-401

digestion of, 430

fermentation of, 418

iodine test for, 400

Steam, 61

Steel, 489-93

Stellite, 500

Strontium, 393

Sublimation, 204

Substance, 3

Substances, amorphous, 95

compound and simple, 16

Sucrose, 401-3

Sugar refining, 402

Sugars, fermentation of, 417

list of common, 401

Sulphur, 248-52

dioxide, 258-9

trioxide, 261

Supersaturation, 115, 121

Suspensions, 109

colloidal, 400, 440-2

Symbols, 78

Synthesis, 98, 300

Telluridm, 273

Temperature, absolute, 46 critical, 92

effect on solubility, 113

Temperatures, Centigrade and Fahrenheit, 558

Test, 36

Thermite, 468

Thermochemistry, 161

Tin, 507-8

salts of, 508

Toluene, 353

Toxic gases, 421, 484-5

smokes, 422

Transition point, 250

Trinitrotoluene, 483

Twitchell process, $\mathbf{4 3 3}$ 
ULTRAMARINE, 471

Ultra-microscope, 440

Uranium, 534, 545-6

VALENCE, 211-7, 228 and atomic structure, 552

Vapor pressure, of solutions, 117,177 190 of water, $47,63,558$

Vaporization, 61

Ventilation, 291

Vinegar, 419

Viscose, 399

Vitamins, 436-7

Volume, standard $73,79,83$

W ASHING soda, 367,391

Water, 58-70

as a solvent, $65-122$

association of, 122

coagulation process, 470

composition by volume, 59,105

by weight, 58
Water, hardness, 388-9 ionization of, 190 purification, 66 softening, 389-91 vapor in air, 288-90 pressure of, $47,63,558$

Water-gas, 337-8

Waters, carburetted, 356 natural, 65

Weights, atomic, 76-7, 85, 281 combining, 76-7 equivalent, 53,122 formula, 134 molecular, 75, 83-4

Wood, distillation of, 420

Wood's metal, 326

Wool, 1, 438

Xenon, 297

Xylene, 353

ZiNc, 448-5

salts of, $450-1$ 

Molar aden is a gm. molecular sol in. nom wait

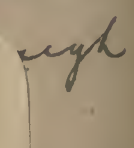

PLEASE DO NOT REMOVE CARDS OR SLIPS FROM THIS POCKET

\section{UNIVERSITY OF TORONTO LIBRARY}

S\&M

A

501 


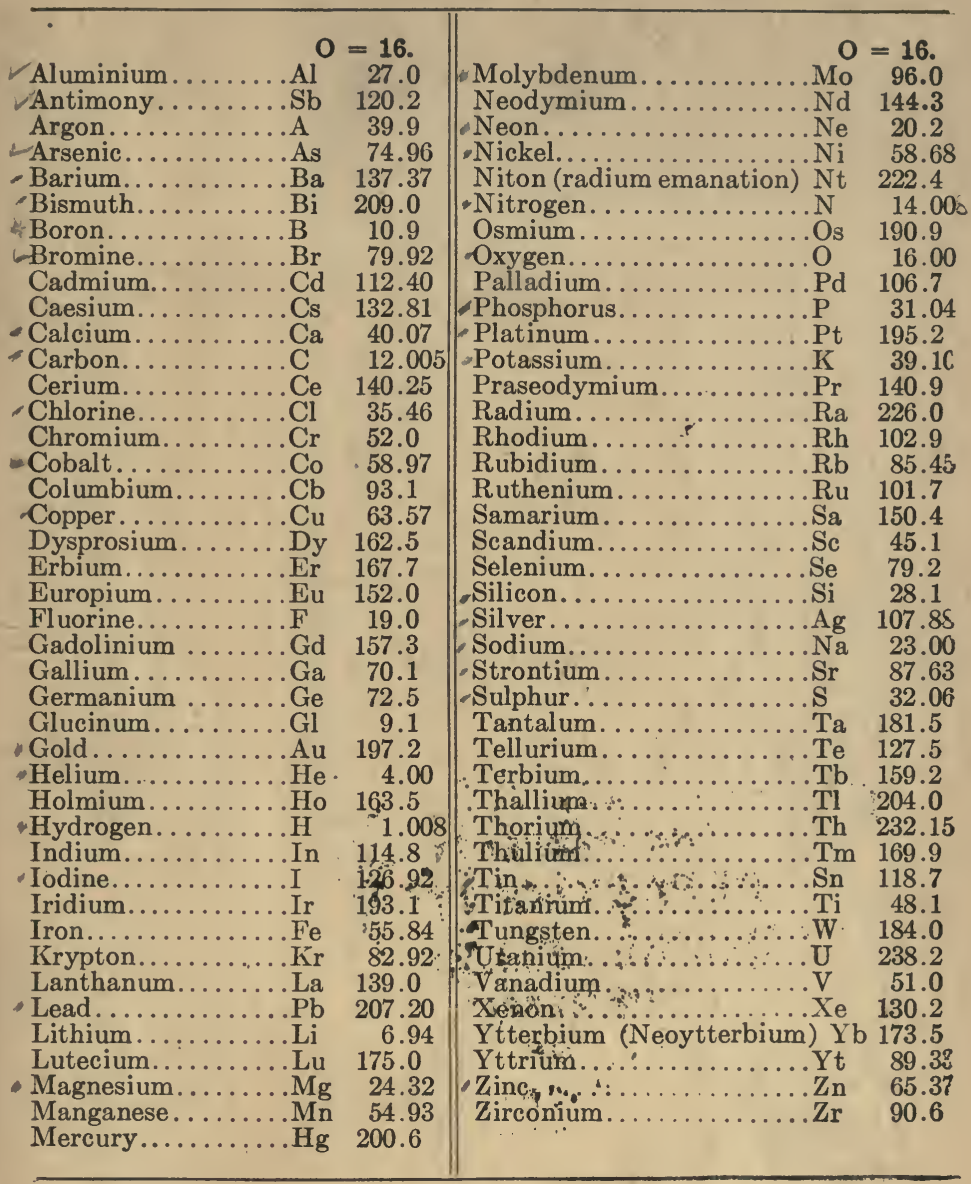

For a table of the Periodic System, see p. 278.

For a table of Atomic Numbers, see p. 549. 
